\author{
Universidade de São Paulo \\ Instituto de Astronomia, Geofísica e Ciências Atmosféricas \\ Departamento de Astronomia
}

Henrique Marques Reggiani

Differential abundances in metal-poor stars:

impact in the study of the Galaxy, stellar evolution and Big Bang nucleosynthesis

São Paulo 

Henrique Marques Reggiani

\section{Differential abundances in metal-poor stars: impact in the study of the Galaxy, stellar evolution and Big Bang nucleosynthesis}

Tese apresentada ao Departamento de Astronomia do Instituto de Astronomia, Geofísica e Ciências Atmosféricas, departamento de Astronomia, da Universidade de São Paulo, como requisito parcial para a obtenção do título de Doutor em Ciências. Versão corrigida. O original encontra-se disponível na Unidade.

Área de Concentração: Astronomia Orientador: Prof. Dr. Jorge Meléndez

São Paulo 

I dedicate this work to my wife, my mother, and my father! 



\section{Acknowledgements}

Since I finished my undergrad there is one person that stood by my side, helping both in the good and bad moments. Thank you for all the help, for reading both my dissertation and thesis, which must have been excruciatingly boring for a person that studies languages, thank you for acompaning me to other countries, both in the past and in the future. Thank you for always being there for me. Thank you Maysa!

Thanks to both my parents, without you I would never have had the oportunity, or conditions, to go to a good school and into a public university, which allowed me to get here, trying to get a $\mathrm{PhD}$ title. Thanks for the education, both formal and social, and helping me become who I am today.

Thanks to all my friends, old and new ones, for the jokes and laughs, for the beers, barbecues and parties.

Thank you Jorge, for all the advice and help during these long years. You certainly helped me become a better researcher. Hopefully I will still be able to learn much from you in our future projects together.

Thank you to everyone, not named here, that helped during my life. I could not possibly mention everyone here, but I am sure you know who you are! 

"Somewhere, something incredible is waiting to be known."

Carl Sagan

"There is a great deal of difference between an eager man who wants to read a book and the tired man who wants a book to read"

GK Chesterton 



\section{Resumo}

Nesta tese apresentamos os resultados do trabalho de doutoramento desenvolvido no Instituto de Astronomia, Geofísica e Ciências Atmosféricas, da Universidade de São Paulo, publicados em quatro artigos científicos como primeiro autor, e um artigo arbitrado em "proceedings". Todos os artigos estão anexados no Apêndice A. O interesse principal deste trabalho de doutorado é estudar a formação e evolução química da Galáxia através de análises precisas de abundâncias químicas de estrelas pobres em metais do componente interno do halo galáctico. Exploramos a viabilidade do uso da técnica de análise diferencial e mostramos que o aumento em precisão fornecido pelo uso da técnica é capaz de revelar inomogeneidades não observáveis em uma análise clássica. Também é discutido como a resolução e o sinal/ruído dos espectros, pode influenciar a precisão final das abundâncias obtidas, analisando espectros de diferentes qualidades de forma sistemática. Em seguida, é apresentado um trabalho de análise química através da técnica diferencial, onde são discutidas a formação do componente interno do halo galático e a evolução química de diversos elementos, com implicações para processos de nucleossíntese e modelos de evolução química da Galáxia. Como subproduto deste estudo apresentamos uma análise da evolução de estrelas binárias através da análise química de duas estrelas Blue Stragglers, com implicações para a nucleossíntese em estrelas AGB e para a evolução de sistemas binários. Também mostramos resultados de um estudo de um par de estrelas binárias, focado na formação do halo e no uso da técnica de "chemical tagging" para a identificação do local de nascimento de duas estrelas extra-galáticas. Uma diferença entre as abundâncias do par de estrelas é evidência de formação planetária em estrelas de baixa metalicidade. Finalizando a tese, mostramos o desenvolvimento de um modelo atômico para estudar a formação das linhas espectroscópicas de potássio, e a aplicação deste modelo no estudo da evolução química 
do elemento, mostrando que, possivelmente, estrelas massivas em alta rotação podem ser uma importante fonte nucleossintética de potássio. 


\section{Abstract}

This thesis presents the main results of the studies developed during the $\mathrm{PhD}$ at the Instituto de Astronomia, Geofísica e Ciências Atmosféricas, of the Universidade de São Paulo, that resulted in four papers as the first author and one peer-reviewed proceeding. All papers are attached in Appendix A. The main interest throughout the $\mathrm{PhD}$ is to study the Formation and early Chemical Evolution of the Galaxy via precise chemical abundances of the metal-poor component of the Galactic inner halo. We explored the viability of a chemical analysis via line-by-line differential abundance analysis in low metallicity stars, and showed that the increased precision might reveal small differences and inhomogeneities that can not be seen in a regular spectroscopic analysis. For this, we employed extremely high-resolution, and high $\mathrm{S} / \mathrm{N}$ spectra, and we also showed how the quality of our data influences the final abundance precision. The line-by-line differential abundance technique was employed in a larger sample of high-resolution, high $\mathrm{S} / \mathrm{N}$, spectra, to study the formation of the inner halo and put constraints on chemical evolution models and the nucleosynthesis processes therein. As byproduct of our analysis, we studied binary stellar evolution through Blue Straggler Stars, which were used to constrain both nucleosynthesis in AGB stars and Blue Straggler formation. We also present the results of a study focused on a pair of binary stars with implications for the formation of the inner halo and the use of chemical tagging to constrain the birth environment of the stars. As a byproduct of this analysis we found implications of possible planetary formation in metal-poor stars. At last, we show the development of an atomic model for a non-LTE analysis of potassium line formation, and its application for the study of the chemical evolution of potassium through cosmic history, with implications for the nucleosynthesis of potassium and Galactic chemical evolution models with yields from massive rotating stars. 



\section{List of Figures}

1.1 Spectra of stars with different metallicities . . . . . . . . . . 25

1.2 Geometry for the definition of radiative intensity . . . . . . . . . . 27

1.3 Geometry of a star . . . . . . . . . . . . . . . . 28

1.4 Neutral hydrogen bound-free absorption cross-sections . . . . . . . . . . . . 33

1.5 Natural atomic transition . . . . . . . . . . . . . . . . . 37

1.6 Energy of atomic levels as a function of the distance to the perturber . . . 39

1.7 The growth of the Hjerting function for different damping parameters. . . . 42

1.8 Equivalent width definition . . . . . . . . . . . . . . . . 46

1.9 Curve of Growth and line profile . . . . . . . . . . . . . . 47

1.10 Spectra reduction example for a differential analysis . . . . . . . . . . . . . 49

2.1 The stellar parameters of CD -24 17504 determined using $\mathrm{q}^{2} \ldots \ldots$. . . . 557

2.2 Error ratios in stellar parameters from a classical (absolute) and a differential

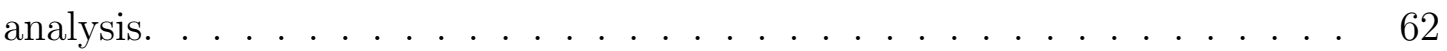

2.3 Error ratios in chemical abundances from a classical (absolute) and a differential analysis. ......................... 63

2.4 Differential $[\mathrm{X} / \mathrm{H}]$ abundances of stars G 64-37 and CD -2417504. . . . . . 65

2.5 Differential [X/Fe] abundances of stars G 64-37 and CD -24 17504. . . . . 68

2.6 Progenitor of star CD -24 17504 via abundance pattern fit. . . . . . . . . . 72

3.1 Kinematics of the dual halo. . . . . . . . . . . . . . . 77

3.2 Metallicity distribution function of the stellar halo. . . . . . . . . . . . 78

3.3 Chemical abundances of $\alpha$-elements in the solar neighborhood. . . . . . . . 80

3.4 Toomre diagram for the high- $\alpha$ and low- $\alpha$ stars. . . . . . . . . . . 81 
3.5 Chemical abundances of $\mathrm{Na}, \mathrm{Mg}, \mathrm{Si}, \mathrm{Ca}, \mathrm{Ti}, \mathrm{Cr}$, and $\mathrm{Ni}$ as a function of $[\mathrm{Fe} / \mathrm{H}] \ldots \ldots \ldots \ldots \ldots \ldots$

3.6 Metallicity distribution function of the Solar neighborhood. . . . . . . . 86

3.7 Supernovae yields as a function of initial stellar masses. . . . . . . . . . 88

3.8 Nickel abundances as a function of metallicity. . . . . . . . . . . . . . . . 90

3.9 Abundances as a function of metallicity. . . . . . . . . . . . . . 95

3.10 Lithium abundances as a function of $[\mathrm{Fe} / \mathrm{H}] \ldots \ldots \ldots$

3.11 Lithium abundances as a function of $T_{\text {eff }} \ldots \ldots \ldots$. . . . . . . . . . .

3.12 HR diagram of the globular cluster M80. . . . . . . . . . . . . . . . 100

3.13 Lithium line in our BSS and in "normal" turn-off stars. . . . . . . . . . . 102

4.1 Differential equilibrium plots for star HD 134440. . . . . . . . . . . . . 109

4.2 Chemical abundances of stars HD 134439/134440 and samples of comparison.113

4.3 Chemical abundances as a function of condensation temperature. . . . . . 114

4.4 Chemical abundances as a function of condensation temperature and atomic number, in a differential analysis of the binary pair 16 Cyg. . . . . . . . 115

4.5 Chemical abundances as a function of condensation temperature of stars HD 134439/134440, relative to the solar abundance, from Chen and Zhao

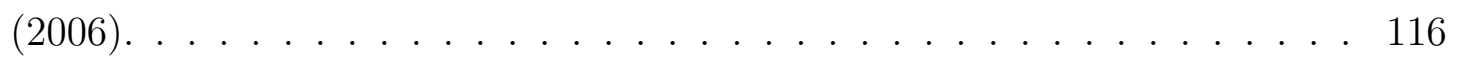

4.6 Comparison between the chemical abundances of HD 134439 and HD 134440, as a function of condensation temperature. . . . . . . . . . . . 118

5.1 Potassium nucleosynthesis in Supernovae explosions. . . . . . . . . . . . 120

5.2 Potassium abundances and GCE prediction. . . . . . . . . . . . 121

5.3 Potassium GCE models. . . . . . . . . . . . . . . . . . . 122

5.4 The Potassium $7698 \AA$ resonance line in the Sun, HD 192263, and Procyon. 123

5.5 Chemical evolution of the elements as a function of stellar age, using solar

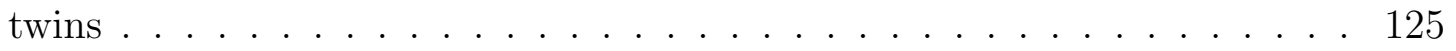

5.6 Differential abundances between the binary stars 16 Cyg $\mathrm{A}$ and B, as a function of condensation temperature. . . . . . . . . . . 126

5.7 Synthetic $12522 \stackrel{\circ}{A}$ potassium line modeled with different numbers of frequency points. . . . . . . . . . . . . . . . . 131

5.8 Grotian diagram of our potassium atom. . . . . . . . . . . . . 132 
5.9 Comparison of photoionization cross-sections. . . . . . . . . . . 137

5.10 Potassium departure coefficients. . . . . . . . . . . . . . . 139

5.11 Line source functions versus wavelength for different departure coefficients. 141

5.12 Departure coefficients for different configurations of the model atom. . . . . 143

5.13 Radiative brackets as a function of wavelength. . . . . . . . . . . 144

5.14 Synthetic non-LTE lines under different atomic models. . . . . . . . . . . 147

5.15 Synthetic LTE and non-LTE lines, and observed lines in the Sun. . . . . . 148

5.16 Synthetic LTE and non-LTE lines, and observed line in Procyon. . . . . . . 149

5.17 3D non-LTE analysis of the $7698 \AA$ line. . . . . . . . . . . . . . . 150

5.18 Abundance corrections for the $7698 \AA$ line as a function of EW and metallicity. 152

5.19 Abundance corrections for the $7664 \AA$ line as a function of EW and metallicity. 153

5.20 LTE and non-LTE potassium as a function of metallicity. Models and Observations .............................. 155

5.21 LTE and non-LTE potassium abundances of solar twins. . . . . . . . 156 



\section{List of Tables}

1.1 Nomenclature of stars based on their metallicities (Beers and Christlieb, 2005) 25

2.1 Stellar parameters and errors of stars G 64-12, G 64-37, and CD -24 17504 . 58

2.2 Differential abundances of stars G 64-37 and CD -2417504. . . . . . . . . 66

2.3 Nomenclature of stars based on their carbon enhancement (Beers and Ch-

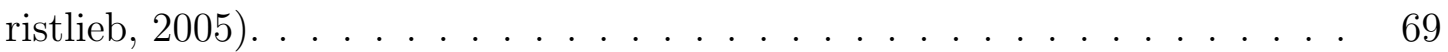

4.1 Stellar parameters and errors of stars HD 103095, HD 134439, HD 134440

and HD $163810 \ldots \ldots \ldots \ldots$. . . . . . . . . . . . . . . . . . . . . . . . . . 



\section{Contents}

1. Introduction . . . . . . . . . . . . . . . . . 23

1.1 Metal-poor Stars . . . . . . . . . . . . . . . . 23

1.1.1 Definition and classes of metal-poor stars . . . . . . . . . 24

1.2 Spectral formation and abundance determinations . . . . . . . . . . . . 25

1.2.1 Spectral formation .................. 26

1.2.2 Continuous Absorption . . . . . . . . . . . . . . . 31

1.2.3 The line absorption coefficient . . . . . . . . . . . 34

1.2.3.1 Atomic natural absorption . . . . . . . . . . 35

1.2.3.2 Pressure Broadening . . . . . . . . . . . . . 38

1.2.3.3 Doppler Broadening . . . . . . . . . . . . . 39

1.2.3.4 Microturbulence Broadening . . . . . . . . . . . . 40

1.2.4 Mass Absorption Coefficient . . . . . . . . . . . . 40

1.2.5 The LTE assumption . . . . . . . . . . . . . . . . 41

1.2.6 Abundance determination . . . . . . . . . . . . 44

1.2.7 The differential analysis . . . . . . . . . . . . . . 48

1.3 Model atmospheres and radiative transfer code . . . . . . . . . . . . . 51

2. Differential analysis of metal-poor stars . . . . . . . . . . . . . 53

2.1 The first differential analysis in extremely metal-poor stars . . . . . . . . 53

2.2 Data and stellar parameters . . . . . . . . . . . . 54

2.3 Errors in a differential analysis . . . . . . . . . . . . . . . . . 58

2.3.1 Discussion of the error ratios . . . . . . . . . . . . 61

2.4 Abundance analysis . . . . . . . . . . . . . . . . . . . 64 
3. Chemical evolution of the galaxy . . . . . . . . . . . . . . 75

3.1 Formation of the Galactic halo . . . . . . . . . . . . . 75

3.1 .1 Observational data . . . . . . . . . . . . . 81

3.1.2 Very metal-poor stars in the solar neighborhood halo . . . . . . 82

3.2 The Galactic Chemical Evolution model . . . . . . . . . . . . 85

3.2.1 Model vs Observations . . . . . . . . . . . . . . . 89

3.3 Constraining GCE with line-by-line differential abundances . . . . . . . . 90

3.4 Lithium and the Spite plateau . . . . . . . . . . . . . . 94

3.5 Blue Straggler Stars . . . . . . . . . . . . . . . . . . 99

4. Chemical tagging and Planet signature . . . . . . . . . . . . 105

4.1 Chemical Tagging . . . . . . . . . . . . . . . 105

4.2 Data, stellar parameters and chemical abundances. . . . . . . . . . 107

4.2 .1 Additional data . . . . . . . . . . . . . . . . . . 108

4.3 The birth environment of HD 134439 and HD 134440 . . . . . . . . . 110

4.4 The Planet/Star connection . . . . . . . . . . . . . . . 112

5. Non-LTE analysis of Potassium . . . . . . . . . . . . . . . . . . . . 119

5.1 Galactic Chemical Evolution of Potassium . . . . . . . . . . . . . 119

5.1.1 Theoretical chemical evolution of potassium . . . . . . . . . . 119

5.1 .2 The observational data . . . . . . . . . . . . . . 122

5.2 The non-LTE formulation . . . . . . . . . . . . . . . . 127

5.2 .1 Non-LTE radiative transfer . . . . . . . . . . . . . 128

5.2 .2 Atomic levels . . . . . . . . . . . . . . . . 129

5.2 .3 Bound-bound transitions . . . . . . . . . . . . . . . 130

5.2.3.1 Bound-Bound radiative transitions . . . . . . . . 131

5.2 .3 .2 Collisional excitation . . . . . . . . . . . . 132

$5.2 .3 .3 \mathrm{e}+\mathrm{K}$ rates . . . . . . . . . . . . . . . . 133

5.2.3.4 $\mathrm{H}+\mathrm{K}$ rates . . . . . . . . . . . . . . . . 134

5.2 .4 Bound-Free transitions . . . . . . . . . . . . . . . 135

5.2.4.1 Photoionization ................ 136 
5.2.4.2 Collisional ionization . . . . . . . . . . . . . 136

5.2 .5 Departure Coefficients . . . . . . . . . . . . . 138

5.3 Discussion . . . . . . . . . . . . . . . . . . . . . . . . . 141

$5.3 .1 \quad$ Non-LTE effects . . . . . . . . . . . . . . . . . . . . . . . 141

5.3.1.1 Non-LTE synthetic lines under different cross-sections assumptions . . . . . . . . . . . . . 144

5.3.1.2 Non-LTE synthetic lines in benchmark stars and 3D effects 146

5.3.1.3 Grid of corrections . . . . . . . . . . . . . . 150

5.3.1.4 Analysis of GCE models and data under the non-LTE assumption .................. 151

6. Conclusions . . . . . . . . . . . . . . . . . 157

Bibliography . . . . . . . . . . . . . . . . . . 161

$\begin{array}{ll}\text { Appendix } & 189\end{array}$

A. Apendix A . . . . . . . . . . . . . . . . . . . . . . . . 191 

Chapter 1

\section{Introduction}

\subsection{Metal-poor Stars}

The origin of all chemical elements began at the Big Bang. This huge event that originated our Universe produced three chemical elements: hydrogen $(\sim 75 \%)$, helium $(\sim 25 \%)$ and lithium $\left(\approx 2 \times 10^{-7 \%}\right)$ (Cyburt et al., 2016). About 50 to 100 million years after that, the first stars began to take shape (Yoshida, 2019). Formed solely from these three elements, the primordial gas did not cool efficiently, which implied large Jeans masses and therefore only massive stars formed. These stars are known as Population III (Pop III) stars and, as they were likely massive, they had a short life. So far, hydrodinamical simulations of Pop III stars formation support this picture and find characteristic masses of $\mathrm{M} \geq 100 \mathrm{M}_{\odot}$ (e.g., Silk, 1983; Tegmark et al., 1997; Bromm et al., 1999, 2002; Bromm and Larson, 2004; Abel et al., 2000, 2002; Yoshida et al., 2006, O'Shea and Norman, 2007; Christlieb et al., 2008). These simulations also indicate that Pop III stars were born as single isolated stars. Although many researchers have been extensively searching for Pop III stars (e.g, Bond, 1970; Beers et al., 1985, 1992; Cayrel et al., 2004; Beers and Christlieb, 2005; Frebel et al., 2005; Cohen et al., 2008; Norris et al., 2013; Placco et al., 2015; Starkenburg et al., 2017; Aguado et al., 2018), they are yet to be found. However, if Pop III stars were exclusively massive stars we would not be able to observe them due to their short lifetimes.

After their short life, these massive Pop III stars exploded as Supernovae, and the interstellar medium (ISM) was enriched with the first chemical elements heavier than lithium. From this newly enriched ISM came the second generation of stars. Known as Pop II stars, these stars were born in an ISM richer with heavy elements, and thus able to 
cool much more efficiently. More efficient cooling mechanisms means that the stars formed under these new conditions can have much lower masses $\left(\leq 0.8 \mathrm{M}_{\odot}\right)$, and the less massive a star is the longer they live $\left(\mathrm{a} 0.8 \mathrm{M}_{\odot}\right.$ star has a lifetime longer than the estimated age of the Universe; 13.8 Gyrs), thus Pop II stars are still observable today (Frebel and Norris, 2013). These are the stars known as metal-poor stars (their metal content being smaller than the metal content, or metallicity, estimated for our Sun). Just like the dinosaur fossils, that are used by scientists to uncover details of a long past era in our planet, astronomers can study these stars as fossils from another era of the Universe. They can help us understand details of stellar evolution, chemical evolution and formation of the Milky Way, details of the initial mass function (IMF), and the nature of the first stars themselves, as their chemical composition points directly to the nucleosynthetic products of the Pop III stars. Quoting from Frebel (2008): "metal-poor stars are the local equivalent of the high-redshift Universe".

\subsubsection{Definition and classes of metal-poor stars}

Astronomers commonly talk about the abundance ratio of a chemical element in comparison to a second element, and also compared to this same ratio in the Sun (e.g., $[\mathrm{Fe} / \mathrm{H}]$, which is commonly called "metallicity"). These are reflections of the photospheric abundances of elements in the stars, and are given by: $[A / B]=\log _{10}\left(N_{A} / N_{B}\right)-\log _{10}\left(N_{A} / N_{B}\right) \odot$, where $\mathrm{A}$ and $\mathrm{B}$ are the respective elements and $N_{A}$ and $N_{B}$ the number of atoms in the stellar photosphere of each element.

This definition is important as metal-poor stars are traditionally defined by the ratio of iron to hydrogen in the stellar photosphere, compared to this same ratio in the Sun $([\mathrm{Fe} / \mathrm{H}]-$ metallicity). Iron is historically used as the trace element of metallicity because in solar-type stars the number of absorption lines in the optical wavelength is the richest of all elements. Thus, it is the easier chemical element to find in an optical spectrum and it is (almost) always possible to find a Fe absorption line in a stellar spectrum.

Beers and Christlieb (2005) proposed a nomenclature to classify stars by their metallicity, and their classification is shown in Table 1.1. As the metallicity decreases it becomes increasingly difficult to find and to measure absorption lines of any chemical element, including iron. An example of spectra of stars with different metallicities can be seen in Figure 1.1. Throughout the thesis we will use the definitions in Table 1.1 when discussing 
metal-poor stars.

Table 1.1 - Nomenclature of stars based on their metallicities (Beers and Christlieb, 2005)

\begin{tabular}{lrc}
\hline \hline$[\mathrm{Fe} / \mathrm{H}]$ & Nomenclature & Identification \\
\hline$>+0.5$ & Super metal-rich & SMR \\
\hline$\approx 0$ & Solar & - \\
\hline$<-1.0$ & Metal-poor & MP \\
\hline$<-2.0$ & Very metal-poor & VMP \\
\hline$<-3.0$ & Extremely metal-poor & EMP \\
\hline$<-4.0$ & Ultra metal-poor & UMP \\
\hline$<-5.0$ & Hyper metal-poor & HMP \\
\hline$<-6.0$ & Mega metal-poor & MMP \\
\hline
\end{tabular}

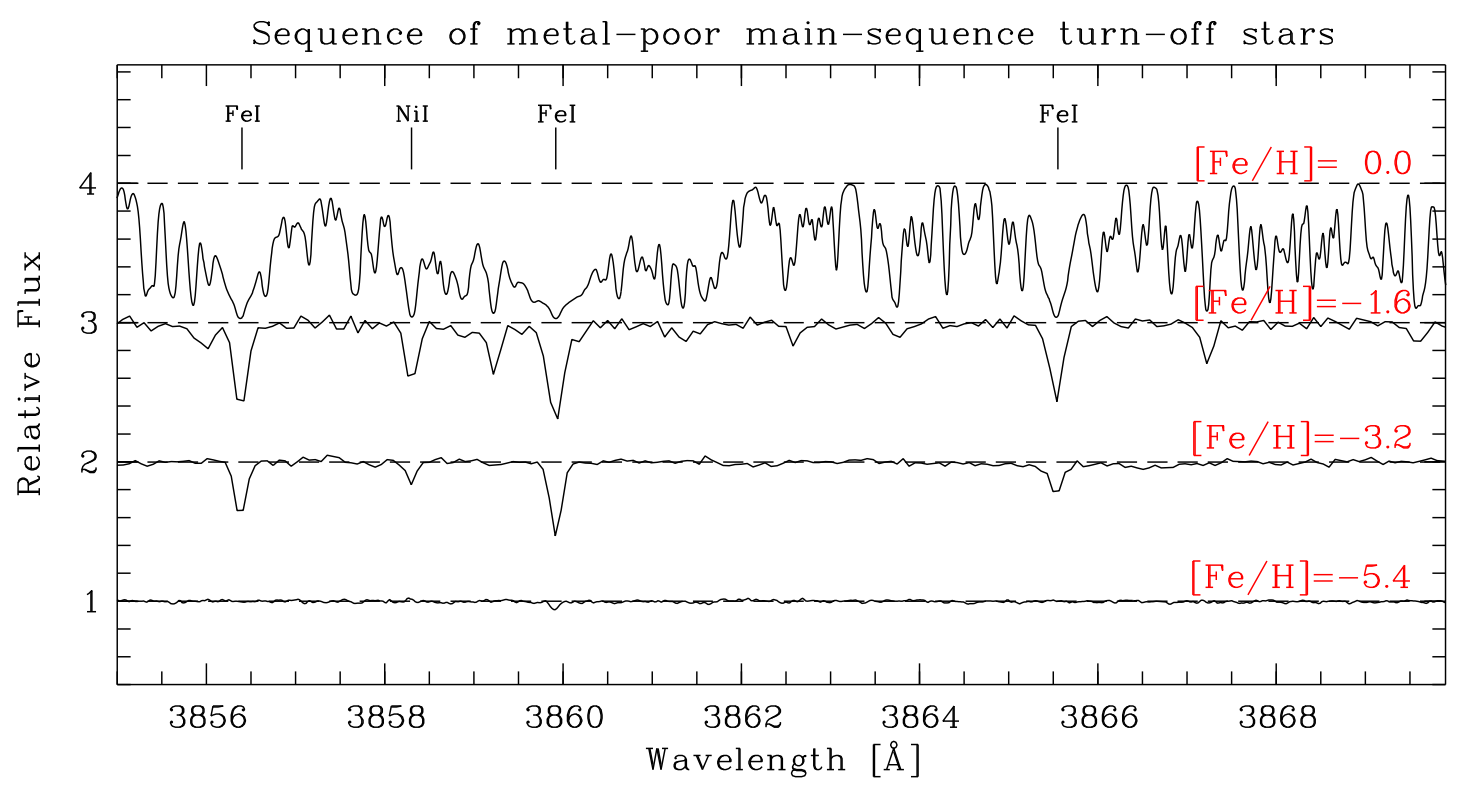

Figure 1.1: Spectra of stars with different metallicities. The difference in line depth reflects the different metallicities. Figure extracted from Frebel (2010).

\subsection{Spectral formation and abundance determinations}

Almost every bit of information that humanity has gathered about our Universe came from light (electromagnetic waves), except for neutrinos from the Sun and a few from Supernova 1987A, cosmic rays, samples collected from bodies in the Solar System (e.g., Moon), and since 2017 gravitational waves. It is impressive how much one can learn using light. We can use the light of a star to find out how it rotates, its mass, radius, or even the 
number of atoms of a certain element in the photosphere of a star. When the light comes from a star we can break it into pieces, we can break it in frequencies (wavelengths), and we can analyze each small piece of stellar spectra to figure out all sorts of information. In this work, we will use this "broken" light, the stellar spectra, and thus it is important to see how the spectrum of a star is formed. The following discussion is mainly based on chapters 7-13 of Gray (2005), chapter 2 of de Boer and Seggewiss (2008), and chapter 3 of Rutten (2003), where mathematical developments not fully shown here can be found. The readers already familiarized with the basic concepts of stellar atmosphere, can jump to Section 1.2.6.

\subsubsection{Spectral formation}

What we observe as the spectra of stars, is formed due to radiative processes in the stellar atmosphere. We see just a small portion of the photons generated within the star, as most of them are trapped in the stellar regions where the optical depth is too high to allow almost any photons to escape. Those that escape are transported, through radiative processes, until they leave the star. Our telescopes and instruments convert the photons into an observed flux, a measure of the net energy flow across an area, over a period of time, in a frequency (or wavelength) range. As will be shown, the flux is directly related to the intensity of the radiation that is observed, and first one must define the upcoming radiative intensity.

The radiative intensity $I_{\nu}$ is defined as the energy $d E_{\nu}$ within a frequency interval, or wavelength interval, passing per unit of time through a surface $d \sigma \cos \theta$ (perpendicular to the radiation) and being directed into a solid angle $d \omega$ :

$$
I(\theta, \varphi)=\frac{d E_{\nu}}{\cos \theta d t d \nu d \omega d \sigma}
$$

in which $\varphi$ and $\theta$ are the angles of orientation of $d \omega$, as shown in Figure 1.2 .

The intensity change (in a given frequency interval) over an increment of path length, $d s$, is the sum of the photons that were lost that can be expressed as an absorption coefficient $\left(\kappa_{\nu}\right)$ multiplied by the density $(\rho)$, and photons gained that are expressed as an emission coefficient $j_{\nu}$.

$$
d I_{\nu}=-\kappa_{\nu} \rho I_{\nu} d s+j_{\nu} \rho d s
$$




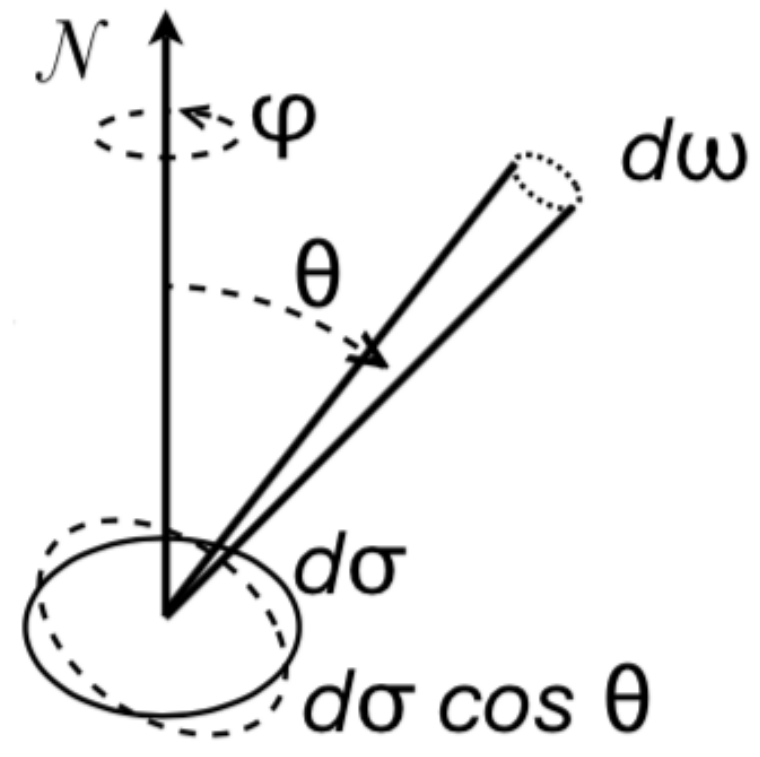

Figure 1.2: Geometry for the definition of radiative intensity in equation 1.1 Figure extracted from de Boer and Seggewiss (2008)

If we were to analyze a region without any emission, we could remove the second term of equation 1.2. In this case, the only dependence is with the absorption coefficient, which is a mass absorption coefficient. This absorption, photon losses, is due to two physical processes: true absorption, when the photon is destroyed and its energy thermalized, and scattering, where the photon is deviated in direction and removed from the solid angle under consideration.

As the output intensity does not differentiate between the two processes, we define the optical depth as the integral of both absorption processes in a certain length (or depth) within the stellar atmosphere:

$$
\tau_{\nu}=\int_{0}^{L} \kappa_{\nu} \rho d s
$$

There are also two processes that emit photons in a stellar atmosphere: The first is real emission, when a photon is created, and the second is the scattering of photons into the direction being considered.

The ratio between emission and absorption has the same unit as $I_{\nu}$ and can be interpreted as a specific intensity in the stellar atmosphere (or any other physical environment where there is emission and absorption of photons). This ratio is called "the source function". 


$$
S_{\nu}=\frac{j_{\nu}}{\kappa_{\nu}}
$$

Now the radiative transport (or transfer) equation, equation 1.2 , can be rewritten as

$$
\frac{d I_{\nu}}{d \tau_{\nu}}=-I_{\nu}+S_{\nu}
$$

To solve equation 1.5, it is necessary to consider the geometry of the problem. As the interest here is to solve it to obtain the intensity of a spectrum (more specifically the continuum intensity at any given frequency), it is necessary to consider the spherical geometry of a star, portrayed in Figure 1.3 .

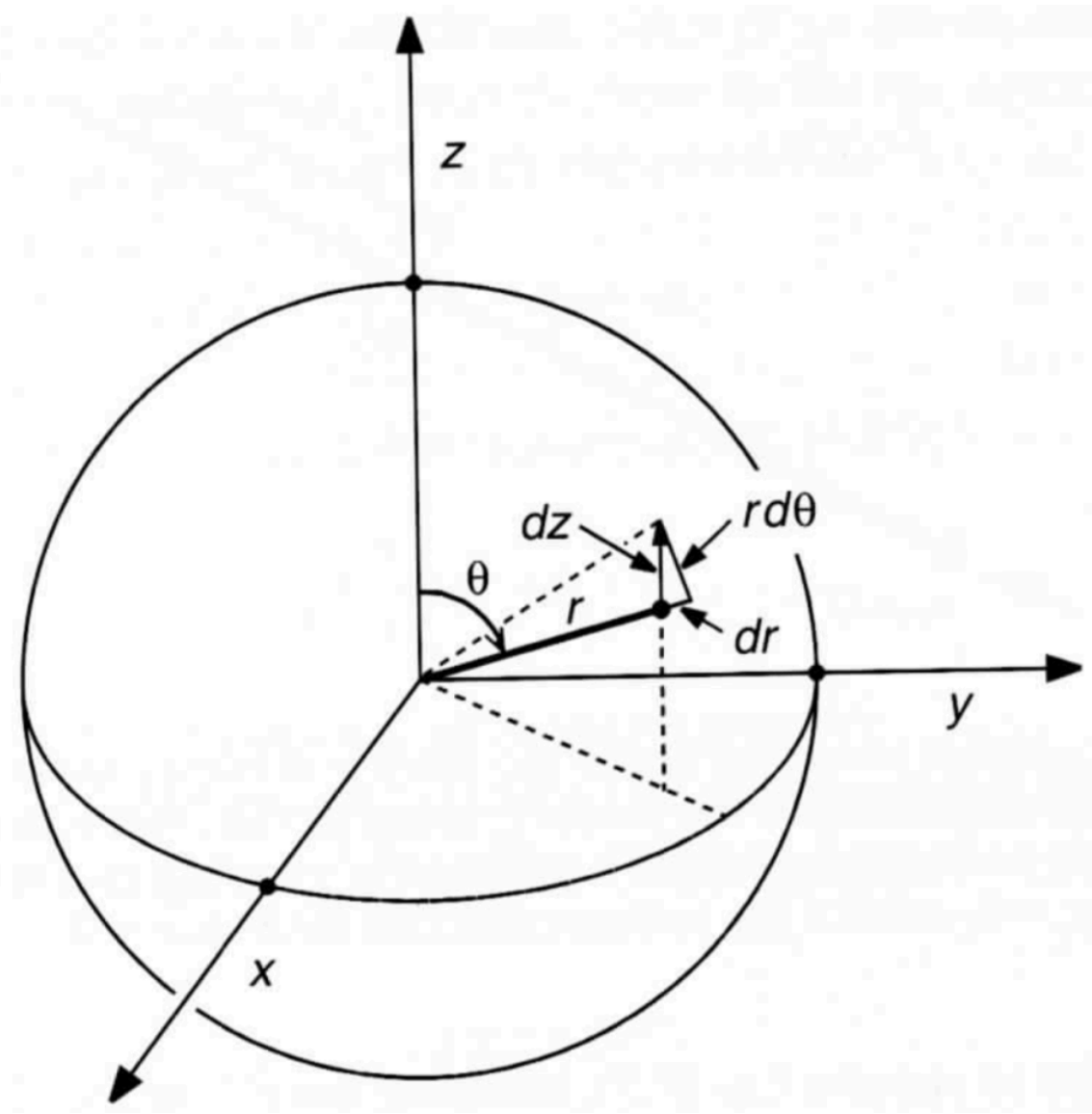

Figure 1.3: Spherical geometry (portraying a star) to solve the radiative transport equation. Figure extracted from Gray (2005)

We will consider the $Z$ axis as the line of sight to the observer. Transforming into spherical coordinates:

$$
\begin{aligned}
& d r=\cos \theta d z \\
& r d \theta=-\sin \theta d z
\end{aligned}
$$


and substituting it in equation 1.5 .

$$
\frac{\partial I_{\nu}}{\partial r} \frac{\cos \theta}{\kappa_{\nu} \rho}-\frac{\partial I_{\nu}}{\partial \theta} \frac{\sin \theta}{\kappa_{\nu} \rho r}=-I_{\nu}+S_{\nu}
$$

Now it is necessary to make the first important approximation to solve the transfer equation: As the thickness of the stellar photosphere, the region of the star that is actually observed through the spectrum, is usually much smaller than the stellar radius $(\sim 0.1 \%)$, we approximate the stellar atmosphere as a plane parallel geometry, where $\theta$ does not depend upon $z$, so the second term on the left side of the equation 1.7 can be disregarded. From this point forward this approximation will be used not only to demonstrate the production of a spectrum but also in all the atmospheric models used throughout this work.

We will also adopt a geometrical depth $d x=-d r$, and the convention of writing $d \tau_{\nu}=\kappa_{\nu} \rho d x$. The radiative transfer equation is now

$$
\cos \theta \frac{I_{\nu}}{d \tau_{\nu}}=I_{\nu}-S_{\nu}
$$

Solving equation 1.8 we obtain the intensity at a single frequency

$$
\begin{aligned}
& I_{\nu}\left(\tau_{\nu}\right)=I_{\nu}^{\text {out }}\left(\tau_{\nu}\right)+I_{\nu}^{\text {in }}\left(\tau_{\nu}\right) \\
& =\int_{\tau_{\nu}}^{\infty} S_{\nu} e^{-\left(t_{\nu}-\tau \nu\right) \sec \theta} \sec \theta d t_{\nu} \\
& -\int_{0}^{\tau_{\nu}} S_{\nu} e^{-\left(t_{\nu}-\tau \nu\right) \sec \theta} \sec \theta d t_{\nu} .
\end{aligned}
$$

The second important approximation is that at the stellar surface $\tau_{\nu}=0$. In this condition, any radiation from other sources is negligible compared to the star's own radiation. In this case, $I_{\nu}^{\text {in }}(0)=0$ and the intensity observed is

$$
I_{\nu}(0)=I_{\nu}^{\text {out }}(0)=\int_{0}^{\infty} S_{\nu} e^{-t_{\nu} \sec \theta} \sec \theta d t_{\nu} .
$$

Now we have an equation of the intensity of light in any given frequency being emitted from the stellar photosphere and coming to the observer. It must be converted into the observed flux, which is the actual observed quantity at any given frequency.

Again, the flux is a measure of the net energy flow across an area, over a period of time, in a certain frequency:

$$
F_{\nu}=\lim \frac{\Sigma \Delta E_{\nu}}{\Delta A \Delta t \Delta \nu}=\frac{\oint d E_{\nu}}{d A d t d \nu} .
$$


We can relate the flux to the radiative intensity using equation 1.1 and the flux becomes:

$$
F_{\nu}=\oint I_{\nu} \cos \theta d \omega
$$

We expand this definition in spherical coordinates and assume no azimuthal dependence in $I_{\nu}$ :

$$
F_{\nu}=2 \pi \int_{0}^{\pi} I_{\nu} \cos \theta \sin \theta d \theta=2 \pi\left(\int_{0}^{\frac{\pi}{2}} I_{\nu}^{\mathrm{out}} \cos \theta \sin \theta d \theta+\int_{\frac{\pi}{2}}^{\pi} I_{\nu}^{\mathrm{in}} \cos \theta \sin \theta d \theta\right) .
$$

To solve it one must use the $I_{\nu}^{\text {out }}$ and $I_{\nu}^{\text {in }}$ definitions, and assume that the source function $\left(S_{\nu}\right)$ is isotropic.

$$
F_{\nu}=2 \pi\left(\int_{\tau_{\nu}}^{\infty} S_{\nu} \int_{0}^{\frac{\pi}{2}} e^{-\left(t_{\nu}-\tau_{\nu}\right) \sec \theta} \sin \theta d t_{\nu} d \theta-\int_{0}^{\tau_{\nu}} S_{\nu} \int_{\frac{\pi}{2}}^{\pi} e^{-\left(t_{\nu}-\tau_{\nu}\right) \sec \theta} \sin \theta d t_{\nu} d \theta\right)
$$

To solve the inner integral we adopt $\sec \theta=w$, and $t_{\nu}-\tau_{\nu}=x$

$$
\int_{0}^{\frac{\pi}{2}} e^{-\left(t_{\nu}-\tau_{\nu}\right) \sec \theta} \sin \theta d \theta=\int_{1}^{\infty} \frac{e^{-x w}}{w^{2}} d w=E_{2}(x)
$$

Finaly, the observed flux of a given frequency is

$$
F_{\nu}\left(\tau_{\nu}\right)=2 \pi\left(\int_{\tau_{\nu}}^{\infty} S_{\nu} E_{2}\left(t_{\nu}-\tau_{\nu}\right) d t_{\nu}-\int_{0}^{\tau_{\nu}} S_{\nu} E_{2}\left(\tau_{\nu}-t_{\nu}\right) d t_{\nu}\right)
$$

Now a theoretical stellar spectrum can be calculated, as it is the observed flux from the surface of the $\operatorname{star}\left(\tau_{\nu}=0\right)$ :

$$
F_{\nu}(0)=2 \pi \int_{0}^{\infty} S_{\nu}\left(t_{\nu}\right) E_{2}\left(t_{\nu}\right) d t_{\nu}
$$

That is the flux per unit area, and the total radiation is this flux multiplied by $4 \pi R^{2}$, $R$ being the radius of the star.

This equation shows that the surface flux is the sum of the source function at each depth multiplied by an extinction factor, appropriate to that depth below the surface, and the sum is taken over all depths contributing a significant amount of radiation at the surface. 


\subsubsection{Continuous Absorption}

Equation 1.17 gives us the means to calculate the flux at any given frequency and, by integrating over all frequencies, obtain a theoretical spectrum of a star. To solve that equation one must know the form of the source function. The first step to calculate the source function is estimating the continuous absorption coefficient. As the name states, it functions as a decreasing factor of the flux at the continuous level of the spectra; the more absorption there is, the smaller the quantity of photons arriving at our telescopes and eyes.

If we wish to match the theoretical spectra with observed ones, we need to understand how the continuum behaves and, more than that, the strength of the spectral lines. In that regard, the continuum absorption shapes the line because the more absorption at the continuum means that there are fewer atoms to make the lines themselves.

The continuous absorption can be fully described by two different processes: a) A bound-free transition; and b) A free-free transition. There is also the possibility of boundbound transitions being included in the continuous absorption. This is true when many lines, usually blended, act like a continuous absorption rather than a line in the spectra (Coelho, 2014). This particular effect can be more easily observed in the ultraviolet region of the spectra, a region so crowded with overlapping lines that, at times, it is quite difficult to visualize any continuum.

Nevertheless, most of the continuum opacity comes from bound-free and free-free transitions involving hydrogen. Hydrogen is the main source of the continuum opacity because stars are mostly composed of this element, and the overwhelming number of $\mathrm{H}$ atoms in the star makes up for most of the interactions taking place at the radiative zone of virtually all stars.

The wavelength of the bound-bound transitions in neutral hydrogen atoms can be found by

$$
\frac{1}{\lambda}=R\left(\frac{1}{n^{2}}-\frac{1}{m^{2}}\right)
$$

where $\mathrm{n}$ and $\mathrm{m}$ are the lower and upper levels of the transition, and $R$ is the Rydberg constant. If $m=\infty$ the electron is lifted beyond the last possible orbit and instead of a bound-bound transition we have a bound-free transition. From that, we can calculate the energy necessary to ionize the atom as $h c / \lambda=h c R / n^{2}$, where $h$ is the Planck constant, 
and $c$ the speed of light. Any photon with energy higher than that threshold can ionize the atom. From that, we define the excitation potential $\chi$ from any level as

$$
\chi=h R c-h R c / n^{2}=h R c\left(1-1 / n^{2}\right)=13.598\left(1-\frac{1}{n^{2}}\right) .
$$

Back to the transitions, we have to define how probable they are at a given wavelength. For simple atoms the bound-free absorption coefficients (or absorption cross-sections) were described by Kramers (1923) and latter updated by Gaunt and Fowler (1930), and we show the updated absorption coefficient:

$$
\alpha_{\mathrm{bf}}(\mathrm{H})=\alpha_{0} \mathrm{~g}_{\mathrm{bf}} \frac{\lambda^{3}}{\mathrm{n}^{5}}
$$

where $\alpha_{\mathrm{bf}}$ is in $\mathrm{cm}^{2}$ per neutral hydrogen atom, $\alpha_{0}=1.0449 \times 10^{-26}$ for $\lambda$ in $\AA$ and $g_{\mathrm{bf}}$ is the gaunt factor for bound-free transitions. Below we show the gaunt factors for both bound-free and free-free transitions:

$$
\begin{aligned}
& g_{\mathrm{bf}}=1-\frac{0.3456}{(\lambda R)^{\frac{1}{3}}}\left(\frac{\lambda R}{n^{2}}-\frac{1}{2}\right) \\
& g_{\mathrm{ff}}=1+\frac{0.3456}{(\lambda R)^{\frac{1}{3}}}\left(\frac{\lambda k T}{h c}+\frac{1}{2}\right) .
\end{aligned}
$$

In the above equations $k$ is the Boltzmann constant, $\mathrm{T}$ the temperature and $c$ the speed of light. In Figure 1.4 we show the behavior of $\alpha_{\mathrm{bf}}$.

To estimate the total absorption coefficients it is necessary to know the number of atoms excited to level $n$ per neutral hydrogen atom:

$$
\frac{N_{n}}{N}=\frac{g_{n}}{u_{0}(T)} e^{-\frac{\chi}{k T}}
$$

where $g_{n}=2 n^{2}$ is the statistical weight, $u_{0}(T)$ is the partition function, and $\chi$ the excitation potential. The absorption coefficient, in $\mathrm{cm}^{2}$ per neutral hydrogen atom, for all continua starting at $n_{0}$ is

$$
\kappa\left(H_{\mathrm{bf}}\right)=\sum_{n_{0}}^{\infty} \frac{\alpha_{\mathrm{bf}}(H) N_{n}}{N}=\alpha_{0} \sum_{n_{0}}^{\infty} \frac{\lambda^{3}}{n^{3}} g_{\mathrm{bf}} 10^{\frac{\chi}{k T}} .
$$

The continuous opacity is also affected by free-free collisions, and the hydrogen absorption coefficient associated with this physical process is considerably smaller. When a free electron collides with a proton, its orbit is altered. A photon might be absorbed in such a collision and the orbital energy of the electron increased in the process. In this case, an 


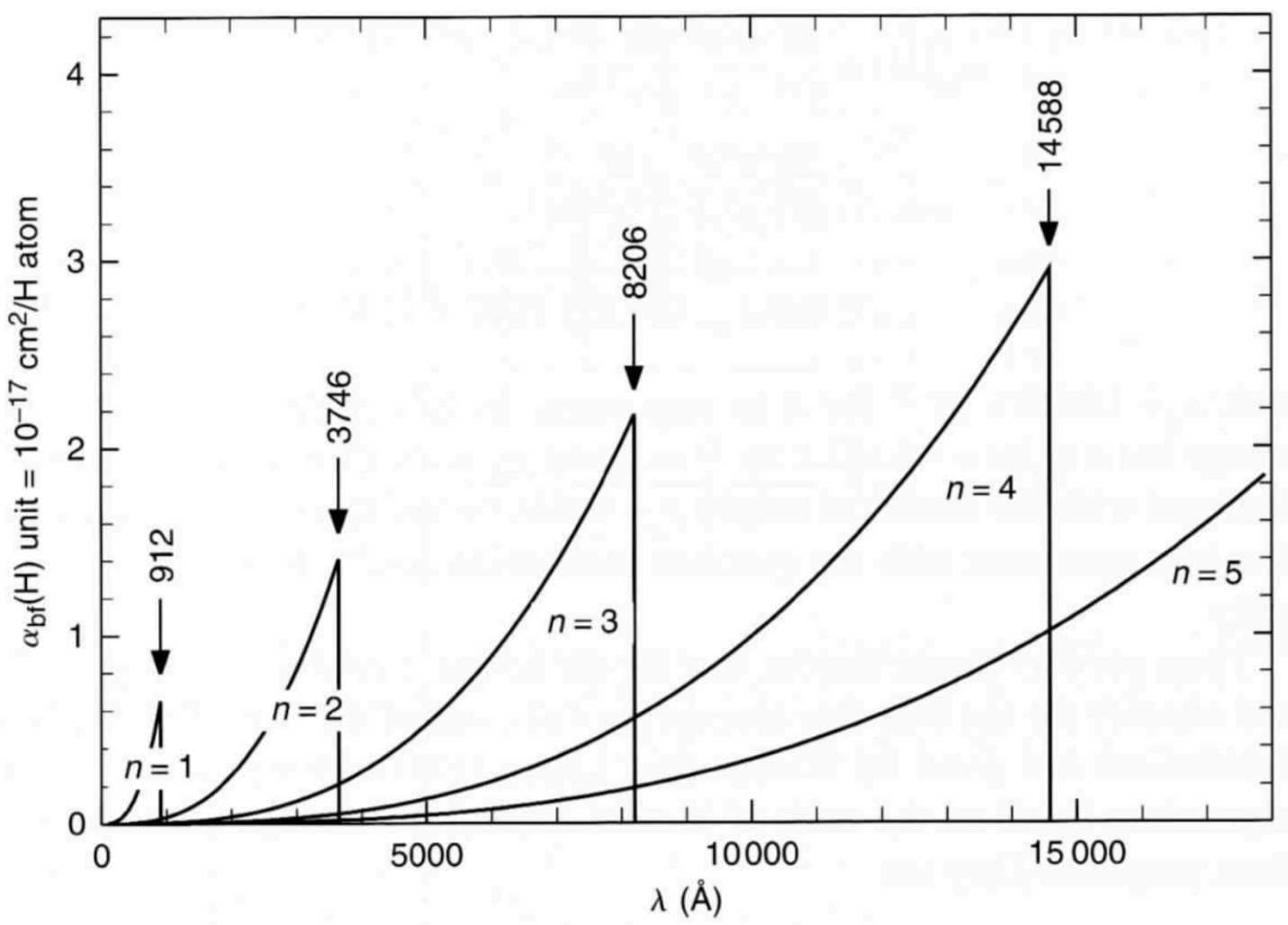

Figure 1.4: Neutral hydrogen bound-free absorption cross-sections. Figure extracted from Gray 2005.

important variable is the speed of the electron. The faster the electron, the more likely it will be for the collision to take place; in slower encounters it is more likely that the photon will pass by without interacting. According to Kramers (1923), the atomic absorption coefficient of such an encounter is

$$
d \alpha_{\mathrm{ff}}(H)=\frac{2}{3^{\frac{3}{2}}} \frac{h^{2} e^{2} R}{\pi m_{e}^{3}} \frac{1}{\nu^{3} v} d v
$$

that is the collisional cross-section in $\mathrm{cm}^{2}$ per atom of $\mathrm{H}$ in the velocity interval $v+d v$, and where $m_{e}$ is the electronic mass. The complete absorption coefficient per electron can be found by integrating over the velocity distribution in the stellar atmosphere, commonly approximated by the Maxwell-Boltzmann distribution. Thus, $\alpha_{\mathrm{ff}}$ becomes

$$
\alpha_{\mathrm{ff}}(H)=\frac{2}{3^{\frac{3}{2}}} \frac{h^{2} e^{2} R}{\pi m_{e}^{3}} \frac{1}{\nu^{3}}\left(\frac{2 m_{e}}{\pi k \mathrm{~T}}\right)^{\frac{1}{2}} .
$$

Finally, from Gaunt and Fowler (1930), the corrected absorption coefficient in $\mathrm{cm}^{2}$ per $\mathrm{H}$ atom is given by 


$$
k\left(H_{\mathrm{ff}}\right)=\frac{\alpha_{\mathrm{ff}}(H) g_{\mathrm{ff}} N_{i} N_{e}}{N_{0}}=\alpha_{0} \lambda^{3} g_{\mathrm{ff}} \frac{\log e}{2 \theta I} 10^{-\theta I}
$$

where $N_{i}, N_{e}$ and $N_{0}$ are the number density of protons, electrons, and hydrogen, $I=h c R, R=\frac{2 \pi^{2} m e^{4}}{h^{3} c}$, and $\theta=\frac{5040}{\mathrm{~T}}$.

Equations 1.23 and 1.26 denote the absorption coefficient due to bound-free and freefree interactions with neutral hydrogen atoms. Other effects also contribute to the continuous opacity, like electron scattering, helium absorption, and the integrated effect of the several and diverse atomic and molecular lines. In cooler stars ionized hydrogen is also an important contributor to the continuum opacity. Although all these effects play a role in the continuous opacity, they will not be discussed here, as most of the thesis is based on stars with effective temperatures and metallicities where the continuous absorption is dominated by hydrogen.

\subsubsection{The line absorption coefficient}

The overall shape of stellar spectra was described above, but the most important portion (for this work at least) is missing: the absorption lines. They are in equation 1.17 as part of the source function, in the absorption coefficient. The physical process responsible for creating absorption lines are the atomic and molecular bound-bound transitions.

In a bound-bound transition, an electron bound to the atom in a given energy level can be excited to a different level, if it absorbs enough energy to cover the energy difference between the levels. For example, a bound-bound transition in a potassium atom in its ground state can occur when a photon with energy $\mathrm{E}=h \nu=h c / \lambda=1.61 \mathrm{eV}$ is absorbed by the atom. In this case there is an electronic transition from the ground state to the first excited level of potassium, and the wavelength of this transition is $\lambda=h c / 1.61=7698 \AA$. This would result in a decrease in the continuum spectral flux at the exact wavelength of the transition (monochromatic line), as photons of that energy are being absorbed by the potassium atoms.

Although this effect is, in some sense, simple and very well quantified, the bound-bound transitions, and thus lines, are also affected by other physical processes like atomic natural broadening, pressure broadening, thermal Doppler broadening, and Stark broadening, and those different processes occur at somewhat different wavelengths, so that the absorption 
line is not monochromatic, but broad and with a complex structure, usually well described by a convolution of Gaussian and Lorentzian profiles.

\subsubsection{Atomic natural absorption}

The natural atomic absorption (NAA) is the name given to the interaction between a light wave function with an ensemble of interacting atomic dipole-charge oscillators, that represents the absorbing atoms in the gas. The solution to the NAA parameter arises from calculating the solution to Maxwell's wave equation and depends on the speed of the wave, that depends upon the magnetic permeability and electric permittivity of the medium. The form of the solution will be that of a harmonic oscillator, including a damping constant. The spectral line is located where the frequency of the oscillator is at its maximum. However, the frequency varies within a given $\Delta \omega$, thus the spectral line will never be perfectly monochromatic, but a broad line even if there are no other effects playing a role in line formation. We show the absorption coefficient $\alpha$ per atom for the natural atomic absorption in equation 1.27

$$
\alpha=\frac{e^{2}}{m c} \frac{\lambda^{2}}{c} \frac{\frac{\gamma \lambda^{2}}{4 \pi c}}{\Delta \lambda^{2}+\left(\frac{\gamma \lambda^{2}}{4 \pi c}\right)^{2}}
$$

where $e$ is the electric charge, $m$ is the electron mass, $c$ the speed of light, $\lambda$ and $\Delta \lambda$ are the central wavelength and the wavelength range in which the line forms (comes from the variation of frequency mentioned above) and $\gamma$ is the damping constant. This equation can be interpreted as a flux profile within a certain frequency interval.

Now, if we integrate $\alpha$ over all frequencies, we obtain the energy per second per atom per square radian absorbed by the total line from the unit $I_{\nu}$ beam.

$$
\int_{0}^{\infty} \alpha d \nu=\int_{-\infty}^{\infty} \alpha d \Delta \nu=\frac{\pi e^{2}}{m c}
$$

Equation 1.28 can be transformed into wavelength units:

$$
\int_{0}^{\infty} \alpha d \lambda=\int_{-\infty}^{\infty} \alpha d \Delta \lambda=\frac{\pi e^{2}}{m c} \frac{\lambda^{2}}{c}
$$

Quantum mechanical solutions to these equations introduce another term, called "the oscillator strength", and equation 1.28 becomes: 


$$
\int_{0}^{\infty} \alpha d \nu=\frac{\pi e^{2}}{m c} f
$$

where $f$ is introduced to account for the probabilistic nature of the atomic transitions, and without it all lines of a given species would have the same profile. It accounts for the quantum mechanical transition probability and can be written as

$$
f=\frac{m c}{\pi e^{2}} B_{\mathrm{lu}} h \nu=7.484 \times 10^{-7} \frac{\mathrm{B}_{\mathrm{lu}}}{\lambda}
$$

where $B_{\text {lu }}$ is the quantum mechanical transition probability, and $\mathrm{l}$ and $\mathrm{u}$ refer to the lower and upper levels of the transition. The oscillator strength is an important atomic property and one of the main sources of uncertainties when estimating chemical abundances in stars. It is most often used in a logarithmic scale, and multiplied by the statistical weight of the atomic level $(\log (g f))$. We use this quantity in such a manner because $f_{\text {emission }} *_{\mathrm{g}_{\mathrm{u}}}=$ $f_{\text {absorption }}{ }^{*} \mathrm{~g}_{1}$, which avoids errors when dealing with absorption or emission lines in the spectra. Although the oscillator strength can be measured in the laboratory for many chemical species, in many cases we still rely on approximations and theoretical quantum mechanical calculations, which bear large uncertainties. In cases when the uncertainties are too large and the theoretical value cannot reproduce the line observed in the solar spectrum, researchers still use what is called the astrophysical $\log (g f)$. This is obtained by systematically changing the $\log (g f)$ until the synthetic line matches the observed solar spectrum (e.g., Erdelyi-Mendes and Barbuy, 1989; Meléndez and Barbuy, 1999; Laverick et al., 2019).

Turning back to equation 1.27, there is a term $\gamma$, which is the damping constant introduced when solving the wave equation in the form of an oscillator. This constant can be calculated from the dipole emission theory and has the form:

$$
\frac{d \mathrm{~W}}{d \mathrm{t}}=-\frac{2 e^{2} \omega^{2}}{3 m c^{3}} \mathrm{~W}=-\gamma \mathrm{W}
$$

The solution of the above equation is given by $\mathrm{W}=\mathrm{W}_{0} \mathrm{e}^{\gamma \mathrm{t}}$, where

$$
\gamma=\frac{2 e^{2} \omega^{2}}{3 m c^{3}}=\frac{0.22}{\lambda^{2}}
$$

with $\lambda$ in centimeters. A quantum approach to solving the damping constant yields 


$$
\gamma_{\mathrm{u}}=4 \pi \sum_{\mathrm{l}<\mathrm{u}} A_{\mathrm{lu}}
$$

with $\mathrm{l}$ smaller than $\mathrm{u}$, where $\mathrm{l}$ and $\mathrm{u}$ are, again, the lower and upper atomic levels. Physically $\gamma$ can be interpreted as the life-time in which an electron will stay at a given level if there are no interactions with it. The final damping constant, the component responsible for the natural broadening, is given by the convolution of $\gamma_{\mathrm{u}}$ and $\gamma_{\mathrm{l}}: \gamma=\gamma_{\mathrm{u}}+\gamma_{\mathrm{l}}$

In Figure 1.5 the behavior of the natural atomic absorption is shown, including the natural atomic broadening of the line.

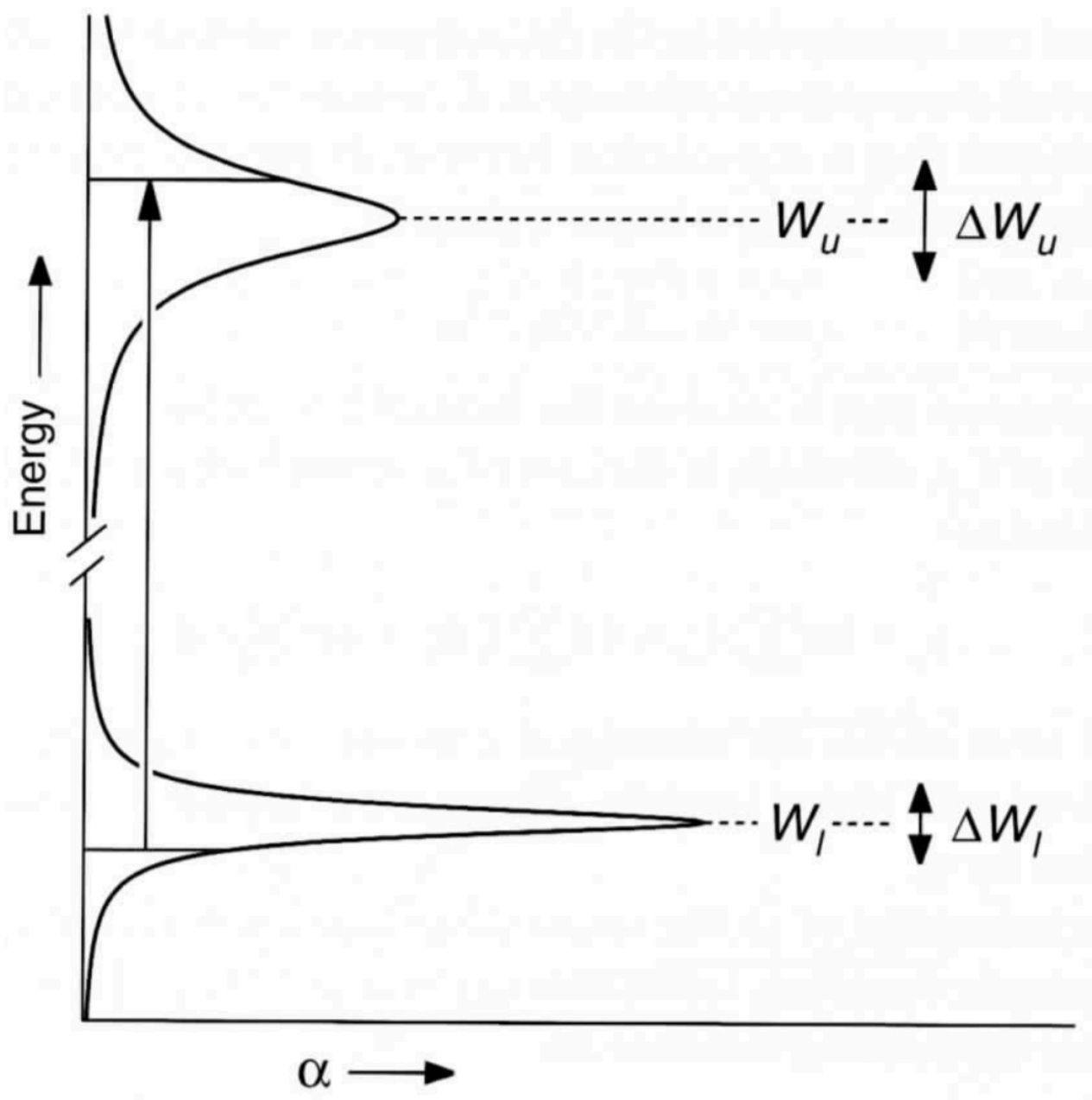

Figure 1.5: The width of the line under natural atomic absorption only. Figure extracted from Gray (2005) 


\subsubsection{Pressure Broadening}

As atoms interact with photons, electrons, other atoms, molecules or ions, the energy of the atomic levels can change for a brief period of time due to the disturbance caused by these encounters. These collisions can be observed in the spectra as line asymmetries, line broadening and shifts in the line, and are widely known as pressure broadening.

The energy change in the atomic levels, introduced by the collisions, depends on the distance between the atom and the colliding particle and can be approximated by

$$
\Delta \mathrm{W}=\frac{\mathrm{a}}{\mathrm{R}^{\mathrm{n}}}
$$

where $\mathrm{a}$ is a constant, $\mathrm{R}$ is the separation between the colliding particles and $\mathrm{n}$ is an integer that depends on the type of the interaction. An example of the energy shift dependence with the distance of the collision is seen in Figure 1.6. The energy change given in equation 1.35 can also be viewed as a change in frequency at the spectrum, and is described by

$$
\Delta \nu=\frac{\mathrm{C}_{\mathrm{n}}}{\mathrm{R}^{\mathrm{n}}}
$$

The constant $\mathrm{C}_{\mathrm{n}}$ must be measured in the laboratory or calculated for each transition, and is also a big source of uncertainty in the line shape. The most important pressure interaction is between a given atom and an atom of a different species, in which case $n=6$ (London, 1930). Hence, the most important pressure broadening constant is known as the $\mathrm{C}_{6}$ constant and is widely used in radiative transfer codes for synthesizing atomic lines. The numerical values for these collisions and the associated broadening are calculated using the classical van der Waals formulation (the approach used in the radiative transfer code MOOG (Sneden, 1973)). The collision associated with charged perturbers is known as the quadratic Stark effect and the associated constant is the $\mathrm{C}_{4}$ constant, also widely used in radiative transfer codes. Examples of numerical values of both $\mathrm{C}_{4}$ and $\mathrm{C}_{6}$ constants, as well as the full mathematical development of the formulas to calculate these constants and broadening, can be seen in chapter 11 of Gray (2005).

The pressure broadening in Gray (2005) is estimated under the impact approximation. This approximation stands in stellar photospheres due to the high temperatures, and under these conditions it is expected that the duration of the collisions is small compared to the 
time between collisions. Under this assumption, the absorption coefficient has the same form as that from the atomic natural absorption (equation 1.27).

$$
\alpha=\operatorname{constant} \frac{\gamma_{\mathrm{n}} / 4 \pi}{\left(\nu-\nu_{0}\right)^{2}+\left(\frac{\gamma_{n}}{4 \pi}\right)^{2}}
$$

In the above equation $\gamma_{n}$ is the damping constant calculated separately for the Stark and van der Waals effects $\left(\gamma_{4}\right.$ and $\left.\gamma_{6}\right)$ :

$$
\gamma_{4}=2 \pi \nu N\left(\frac{\pi^{2} C_{4}}{\nu}\right)^{\frac{2}{3}} \approx 29 \nu^{\frac{1}{3}} C_{4}^{\frac{2}{3}} N
$$

$$
\gamma_{6} \approx 17 \nu^{\frac{3}{5}} C_{6}^{\frac{2}{5}} N
$$

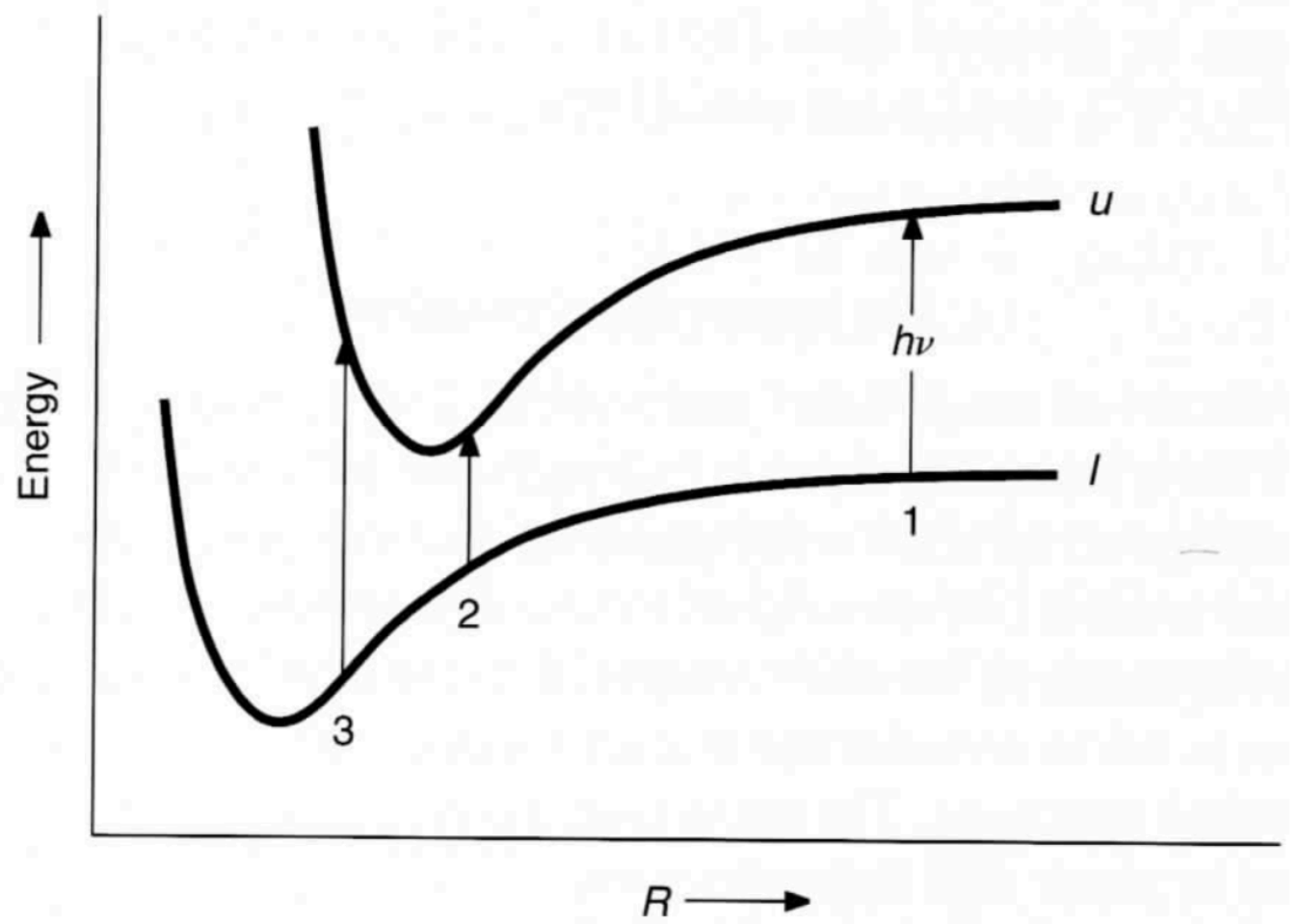

Figure 1.6: The energies of the different atomic levels depends on the distance $\mathrm{R}$ to the perturber. Figure extracted from Gray (2005)

\subsubsection{Doppler Broadening}

All atoms in a star are in motion, mostly due to the their thermal energy. Part of this velocity is on our line of sight, the radial velocity. Every motion in this direction is responsible for a Doppler shift of the line given by 


$$
\frac{\Delta \lambda}{\lambda}=\frac{\Delta \nu}{\nu}=\frac{v_{R}}{c}
$$

where $v_{R}$ is the radial velocity of the atom and $c$ the speed of light. The distribution of $\Delta \lambda$ shapes the absorption coefficient, and depends on the velocity distribution of all the atoms in the stellar atmosphere:

$$
\frac{d \mathrm{~N}}{\mathrm{~N}}=\frac{1}{\pi^{\frac{1}{2}} v_{0}} e^{-\left(\frac{v_{R}}{v_{0}}\right)^{2}} d v_{R}
$$

where $v_{0}$ is given by the temperature variance, $v_{0}^{2}=2 k \mathrm{~T} / \mathrm{m}$, where $k$ is the Boltzmann constant, $\mathrm{T}$ is the temperature of the medium, and $m$ the atomic mass. Using equation 1.40 and 1.41 we estimate the $d \mathrm{~N}$ distribution as

$$
\frac{d \mathrm{~N}}{\mathrm{~N}}=\frac{1}{\pi^{\frac{1}{2}} \Delta \lambda_{D}} e^{-\left(\frac{\Delta \lambda}{\Delta \lambda_{D}}\right)^{2}} d \Delta \lambda
$$

where $\Delta \lambda_{D}$ is the shift in wavelength. The energy from an intensity beam is $\left(\lambda^{2} / c\right) \pi e^{2} f /(m c)$ in wavelength units, times $d \mathrm{~N} / \mathrm{N}$, and gives the absorption coefficient $\alpha$ (below is shown the absorption coefficient both in wavelength and in frequency space):

$$
\begin{aligned}
& \alpha d \lambda=\frac{\pi^{\frac{1}{2}} e^{2}}{m c} f \frac{\lambda_{0}^{2}}{c} \frac{1}{\Delta \lambda_{D}} e^{-\left(\frac{\Delta \lambda}{\Delta \lambda_{D}}\right)^{2}} d \lambda \\
& \alpha d \nu=\frac{\pi^{\frac{1}{2}} e^{2}}{m c} f \frac{1}{\Delta \nu_{D}} e^{-\left(\frac{\Delta \nu}{\Delta \nu_{D}}\right)^{2}} d \nu
\end{aligned}
$$

\subsubsection{Microturbulence Broadening}

Microturbulence is the quantity in the atmospheric models used to account for the nonthermal movements of the gas, in a scale that is small compared to the unit optical depth. The velocity field of these motions is modeled as a Gaussian distribution, similarly to the modeling of the velocity of thermal motions. Thus, the absorption coefficient associated with this effect is the same as in the equations 1.43 , with the velocity substituted by the microturbulence $(\xi)$.

\subsubsection{Mass Absorption Coefficient}

Finally, the form of the absorption coefficient will be a convolution of all coefficients discussed above, which will shape the absorption line: $\alpha=\alpha($ natural $) * \alpha($ Stark $) *$ $\alpha($ vanderWaals $) * \alpha($ Doppler $) * \alpha($ Microturbulence $)$. 
The convolution leads to the Hjerting function $(H(u, a)$, Gray, 2005, page 257). In the Hjerting function the free variable $a$ is the damping parameter, and Figure 1.7 shows its influence on the convoluted equation, which can be directly related to the growth of the wings of a line.

However, the convoluted absorption, in the form of the Hjerting equation, still refers to the damping effects of only one atom. The radiative transfer equation needs the mass absorption coefficient, that is the absorption coefficient due to all the atoms of a given species (and a given ionization) in the stellar atmosphere. Thus, we need to estimate the number of absorbers per unit mass of a given species, which has the form $\mathrm{N} \alpha=l_{\nu} \rho\left(l_{\nu}\right.$ as defined in equation 1.46, where $\mathrm{N} / \rho$ is the number of absorbers per unit mass.

$$
\frac{\mathrm{N}}{\rho}=\frac{\mathrm{N}}{\mathrm{N}_{\mathrm{E}}} \frac{\mathrm{N}_{\mathrm{E}}}{\mathrm{N}_{\mathrm{H}}} \frac{\mathrm{N}_{\mathrm{H}}}{\rho}
$$

where $\mathrm{N} / \mathrm{N}_{E}$ is the fraction of the element $\mathrm{E}$ that can create the absorption line of interest, taking into account the ionization and excitation of the atom $\mathrm{E}, \mathrm{N}_{E} / \mathrm{N}_{H}$ is the number abundance, $A$, of $\mathrm{E}$, and $\mathrm{N}_{H} / \rho$ is the number of hydrogen atoms per unit mass of stellar material, given by

$$
\frac{\rho}{\mathrm{N}_{\mathrm{H}}}=\sum_{j} \frac{\mathrm{N}_{\mathrm{j}}}{\mathrm{N}_{\mathrm{H}}} \mu_{j}=\sum_{j} A_{j} \mu_{j} .
$$

where $A_{j}=\mathrm{N}_{\mathrm{j}} / \mathrm{N}_{\mathrm{H}}$ is the abundance, and $\mu_{j}$ the atomic mass of element $j$. The coefficient can then be written as

$$
l_{\nu}=\frac{\pi^{\frac{1}{2}} e^{2}}{m c} f \frac{H(u, a)}{\Delta \nu_{D}} \frac{\mathrm{N}}{\mathrm{N}_{\mathrm{E}}} \frac{A}{\sum A_{j} \mu_{j}}\left(1-10^{-\chi_{\lambda} \theta}\right)
$$

in units of $\mathrm{cm}^{2} / \mathrm{g}$.

The expression above, added to the continuous absorption coefficient, is how we can calculate theoretical spectral lines when solving the radiative transfer equation.

\subsubsection{The LTE assumption}

In equation 1.46 the term $\mathrm{N} / \mathrm{N}_{\mathrm{E}}$ is the fraction of the element $\mathrm{E}$ capable of absorbing photons at the wavelength of interest, which depends on the electronic population of the upper and lower atomic levels of the transition. To synthesize a line we must estimate 


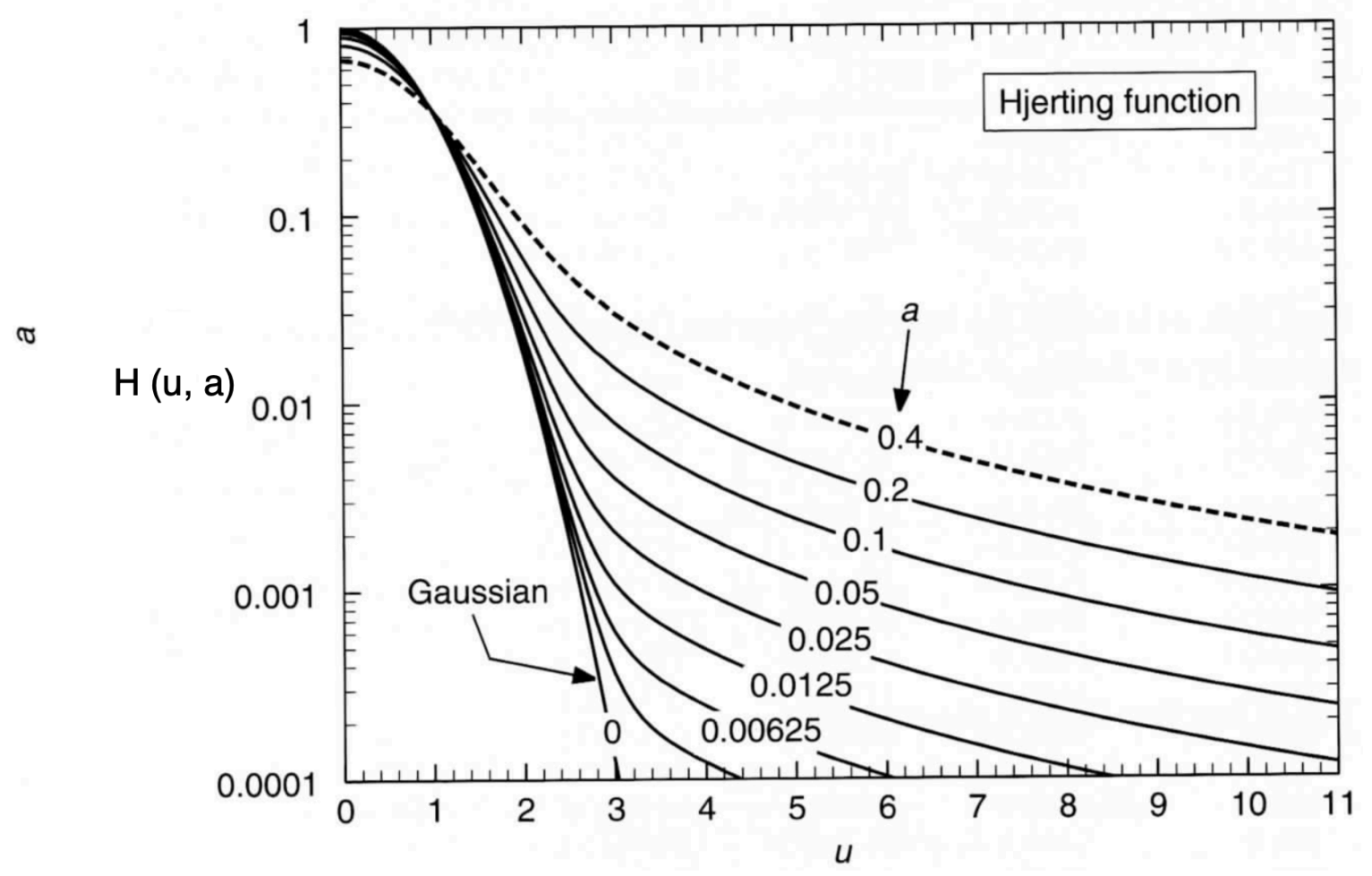

Figure 1.7: The growth of the Hjerting function for different damping parameters. Figure extracted from Gray (2005).

the population on each of the levels, which will be one of the defining elements of the line strength, thus being of crucial interest on abundance determinations.

Most of the work in stellar astrophysics that analyzes absorption and emission lines assume that they are formed under local thermodynamical equilibrium (LTE). Simply put, in LTE we assume that the depth of the stellar atmosphere in which the line of interest forms is under thermodynamical equilibrium (TE). In LTE all material temperatures, i.e., kinetic ion temperature, electron temperature, excitation temperature, and ionization temperature, are equal. This is to say that in thermodynamical equilibrium all atomic, ionic and molecular level populations are given by the Saha-Boltzmann statistics, and defined by the local temperature of the region under equilibrium. In this condition the Boltzmann distribution holds, and the line source function is simplified and given by the Planck function $\left(S_{\nu 0}=B_{\nu 0}\right)$.

Although the assumption might bring great simplifications to the calculation of spectral lines, it is important to portray where, in a stellar atmosphere, does the LTE assumption holds. Extensive tests show that LTE is a good approximation when the ratios between the collisional and radiative transitions are high. 
In LTE, all matter is under equilibrium and when this is true the velocity component in the $\mathrm{x}$ direction is given by the Maxwell distribution:

$$
\left(\frac{n\left(v_{x}\right)}{\mathrm{N}} d v_{x}\right)_{\mathrm{LTE}}=\left(\frac{m}{2 \pi k \mathrm{~T}}\right)^{\frac{1}{2}} e^{-\left(\frac{1}{2}\right) \frac{m v_{x}^{2}}{k \mathrm{~T}}} d v_{x}
$$

where $\mathrm{N}$ is the number of particles with mass $m$ per $\mathrm{cm}^{3}$. And if we ignore the direction component equation 1.47 becomes

$$
\left(\frac{n(v)}{\mathrm{N}} d v\right)_{\mathrm{LTE}}=\left(\frac{m}{2 \pi k \mathrm{~T}}\right)^{\frac{3}{2}} 4 \pi v^{2} e^{-\left(\frac{1}{2}\right) \frac{m v^{2}}{k \mathrm{~T}}} d v .
$$

The main difference between the two equations is that while equation 1.47 is a Gaussian distribution, equation 1.48 has a high-velocity tail due to the $v^{2}$ term. With equation 1.48 we can define the most probable velocity for the particles under temperature $\mathrm{T}$ to be $v_{\mathrm{p}}=\sqrt{\frac{2 k \mathrm{~T}}{m}}$, and the average velocity of the particles is $\langle v\rangle=\sqrt{\frac{3 k \mathrm{~T}}{m}}$.

Now, to calculate the level populations (or rather the ratio between the level populations) we start with the Boltzmann excitation distribution:

$$
\left(\frac{n_{r, s}}{n_{r, t}}\right)_{\mathrm{LTE}}=\frac{g_{r, s}}{g_{r, t}} e^{-\left(\frac{\chi_{r, s}-\chi_{r, t}}{k \mathrm{~T}}\right)}
$$

where $s$ and $t$ subscripts indicate the atomic level and $r$ the ionization stage, $n$ is the number of atoms per $\mathrm{cm}^{3}, \chi$ is the excitation energy measured from the ground level $(r, s=r, 1)$ of stage $r$ and $\chi_{r, s}-\chi_{r, t}=h \nu$ for a radiative transition between levels $(r, s)$ and $(r, t)$, where level $s$ is the higher level.

The Saha distribution for the population ratio between the ground levels of successive ionization stages is

$$
\left(\frac{n_{r+1,1}}{n_{r, 1}}\right)_{\mathrm{LTE}}=\frac{1}{\mathrm{~N}_{\mathrm{E}}} \frac{2 g_{r+1,1}}{g_{r, 1}}\left(\frac{2 \pi m_{e} k \mathrm{~T}}{h^{2}}\right)^{\frac{3}{2}} e^{-\frac{\chi_{r}}{k \mathrm{~T}}},
$$

where the electron density is $\mathrm{N}_{\mathrm{E}}, m_{e}$ is the electron mass, $n_{r+1,1}$ and $n_{r, 1}$ are the population densities of the two ground states of the successive ionization stages $r$ and $r+1, \chi_{r}$ the ionization energy of $r\left(\chi_{r}=h \nu\right.$ for the transition) and $g_{r+1,1}$ and $g_{r, 1}$ the statistical weights of the two ground levels. For the total population of two successive ionization stages the Saha distribution becomes:

$$
\left(\frac{\mathrm{N}_{\mathrm{r}+1}}{\mathrm{~N}_{\mathrm{r}}}\right)_{\mathrm{LTE}}=\frac{1}{\mathrm{~N}_{\mathrm{E}}} \frac{2 \mathrm{U}_{\mathrm{r}+1}}{\mathrm{U}_{\mathrm{r}}}\left(\frac{2 \pi m_{e} k \mathrm{~T}}{h^{2}}\right)^{\frac{3}{2}} e^{-\frac{\chi r}{k \mathrm{~T}}},
$$


where $\mathrm{U}$ is the partition function given by

$$
\mathrm{U}_{\mathrm{r}}=\sum_{\mathrm{s}} \mathrm{g}_{\mathrm{r}, \mathrm{s}} \mathrm{e}^{-\frac{\chi_{\mathrm{r}, \mathrm{s}}}{\mathrm{kT}}}
$$

Combining the Saha and Boltzmann distributions gives the LTE population ratio between a particular level $s$ and the ion state $r$ to which it ionizes by

$$
\left(\frac{n_{r}}{n_{s}}\right)_{\mathrm{LTE}}=\frac{1}{\mathrm{~N}_{\mathrm{E}}} \frac{2 g_{r}}{g_{s}}\left(\frac{2 \pi m_{e} k \mathrm{~T}}{h^{2}}\right)^{\frac{3}{2}} e^{-\frac{\chi_{r s}}{k \mathrm{~T}}},
$$

with $n_{s}$ the total population of level $s, n_{r}$ the number of ions in the ionization level $r$ and $\chi_{r s}=h \nu$ the ionization energy from level $s$ to stage $r$.

As mentioned above, in LTE the interaction with radiation can be simplified, and the line source function can be expressed as the Planck function.

$$
\left[S_{\nu}^{l}\right]_{\mathrm{LTE}}=\frac{2 h \nu^{3}}{c^{2}} \frac{1}{\left(\frac{g_{u} n_{l}}{g_{l} n_{u}}\right)_{\mathrm{LTE}}-1}=\frac{2 h \nu^{3}}{c^{2}} \frac{1}{e^{\frac{h \nu}{k T}}-1}=B_{\nu}(\mathrm{T}) .
$$

This equality is true in every thermal process where the LTE approximation is valid and wherever matter interacts with radiation through the creation and destruction of photons. Now, under LTE, we have a functional form of the line-source function and the methods of estimating the level populations, and we can add them to equation 1.17 and compute a line in LTE.

Details of how the computation of the synthetic line profile proceeds from this point are beyond the scope of this work and can be found in chapter 13 of Gray (2005). However, it was important to show the form of the equations that lead to the calculation of a line profile in LTE and the level populations, because most of the study that will be presented here was based on the analysis of absorption lines under the LTE assumption. However, in Chapter 5 we will discuss line formation where LTE fails.

\subsubsection{Abundance determination}

The chemical abundance of a given element in a star is given by:

$$
A_{x}=\log \left(\frac{N_{x}}{N_{H}}\right)+12
$$

where $X$ is the element of interest, $N_{x}$ and $N_{H}$ are the number of atoms in the stellar atmosphere of the element of interest and of hydrogen. In this scale, the abundance of 
hydrogen is 12 , thus the base of our definition. Now we can subtract the abundance of a given element, iron for example, in the star of interest, from the solar abundance of the same element and retrieve the metallicity definition $[\mathrm{Fe} / \mathrm{H}]$ from Section 1.1.1.

$$
[\mathrm{Fe} / \mathrm{H}]=\log \left(\frac{N_{\mathrm{Fe}}}{N_{H}}\right)-\log \left(\frac{N_{\mathrm{Fe}}}{N_{H}}\right)
$$

As shown in the sections above, the formation of the spectral lines is dependent on the abundance of the elements in the stellar photosphere (equation 1.46). Thus, analyzing spectral lines we can infer the abundance of most elements in stars. The method most commonly used to determine stellar chemical abundances is the curve of growth. This method relies on an existing relation between the increase in the absorption line strength, thus also in the equivalent width (EW or only $\mathrm{W})$, and the increase in chemical abundance in a given stellar atmosphere.

The equivalent width is a measurement of the strength of a given line. It is the width of a box reaching up to the continuum with the same area as the spectral line. The formal definition is in equation 1.57

$$
W=\int \frac{F_{c}-F_{\lambda}}{F_{c}} d \lambda
$$

where the integral is taken throughout the entire line, as exemplified in Figure 1.8.

The most usual way of measuring the EW of a certain line is approximating it by a gaussian, which is a very good approximation for most of the lines and is used throughout our analysis. Returning the discussion on the curve of growth (relation between abundance and equivalent width), it has three phases: the first is where the line is weak and dominated by the Doppler core; in this phase the depth of the line (and EW) grows proportionally to the increase in chemical abundance. The second phase happens as the line depth approaches its maximum value, and the line saturates. Under the assumption of local thermodynamical equilibrium, the minimum flux is fixed by the boundary temperature $\mathrm{T}_{0}$ at $\pi B_{v}\left(\mathrm{~T}_{0}\right)$; in this phase saturation grows asymptotically towards a constant value. In the third, and final phase, the wings of the absorption line become comparable to the continuum opacity $\left(k_{v}\right)$ and the line develops damping wings. An example of the curve of growth and the behavior of a line profile can be seen in Figure 1.9.

From the top panel of Figure 1.9, we can see the relation between EW and the abundance of a given element. The line profile depends on the atmospheric parameters of the 


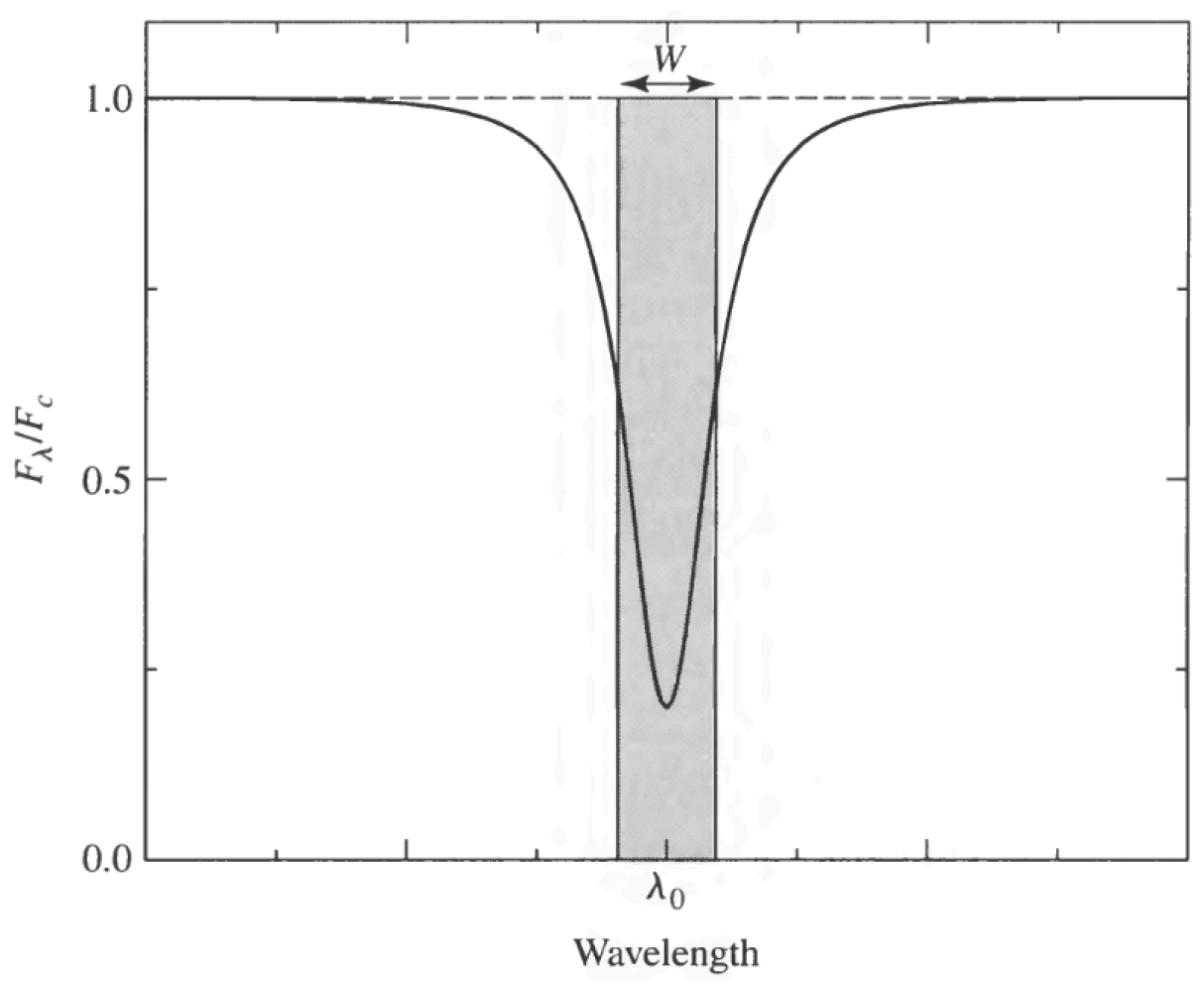

Figure 1.8: The equivalent width is measured as the integral of the relative flux of the spectral line. Figure extracted from Carroll and Ostlie (1996)

star, such as the effective temperature, so that the relation between abundance and EW is complex, and described by equation 1.58 .

$$
\log \left(\frac{W}{\lambda}\right)=B+A_{x}+\log (\mathrm{gf})+\log (\lambda)-\theta_{\mathrm{ex}} \chi-\log \left(\mathrm{k}_{\mathrm{cont}}\right)
$$

where $\mathrm{W}$ is the equivalent width, $\lambda$ is the wavelength of the center of the line, $\mathrm{B}$ is a constant for a given star and a given ion, $A_{x}$ is the abundance (as defined in equation 1.55), $\log (g f)$ is the logarithm of the statistical weight $g$ multiplied by the oscillator strength $f$ of the radiative transition (see section 1.2.3.1), $\chi$ is the excitation potential of that radiative transition, $\theta_{e x}=\frac{5040}{T_{\text {eff }}}$ gives the dependence with the stellar effective temperature, and $k_{\text {cont }}$ is the continuum opacity.

Thus, equation 1.58 shows how the EW of a line changes with chemical abundance, with the properties of the radiative transition and with the temperature of the star. From the behavior of the curve of growth, it is clear that one should try to determine abundances based on EW measured on the first part of the curve, the linear phase, where no saturation problems exist and the abundance can be reliably determined. As a general rule, in the optical we try to measure abundances based on $\mathrm{EW} \leq 120 \mathrm{~m} \AA$ (and if possible, $\mathrm{EW} \leq 100$ 


\section{The abundance dependence}
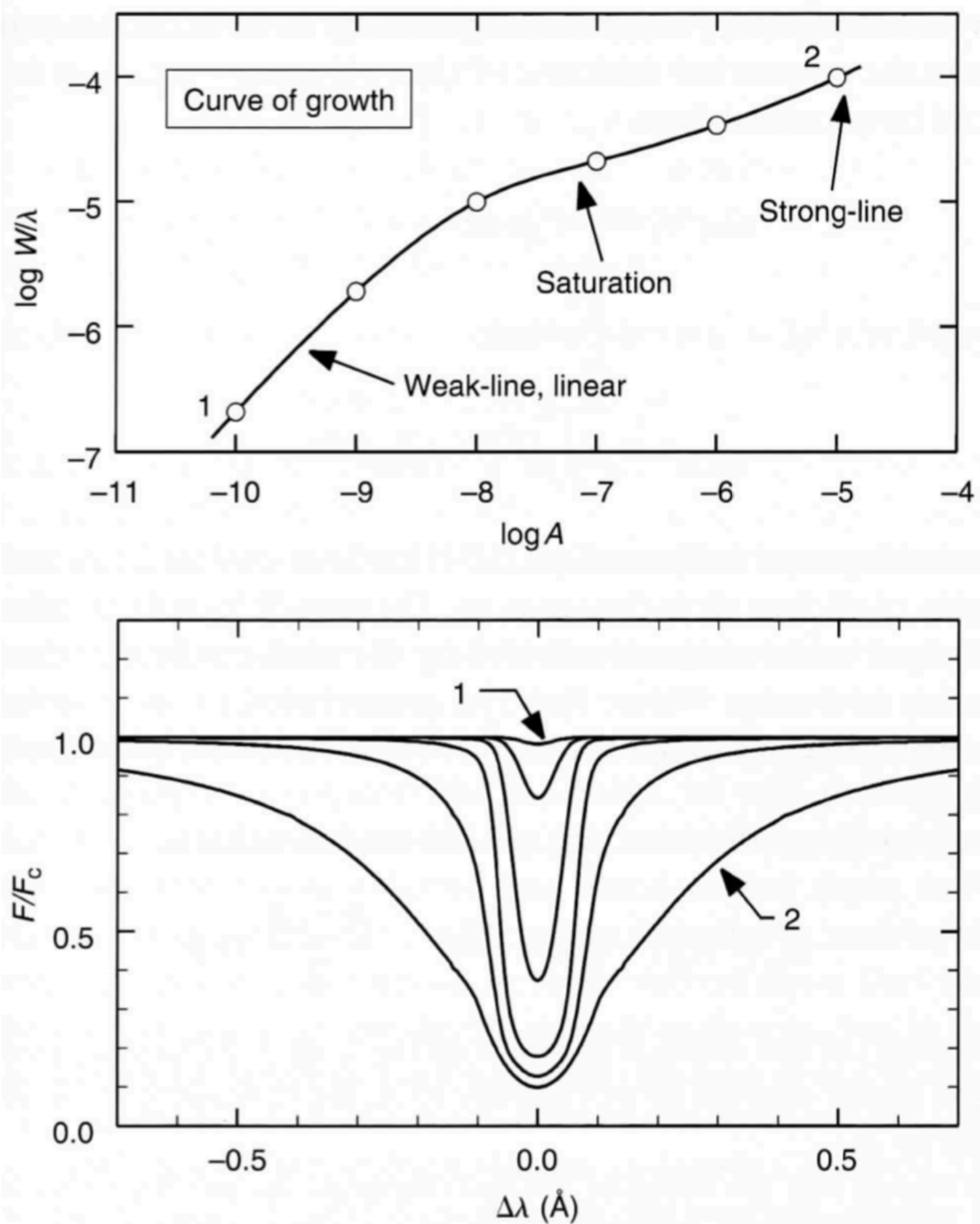

Figure 1.9: Curve of growth (top panel) and the behavior of a line profile (bottom) with the change in chemical abundance. The dots on the curve of growth correspond to the numbers in the line. Figure extracted from Gray (2005) 
$\mathrm{m} \AA$ ) to avoid saturation problems.

Even though we have a good knowledge on how the curve of growth behaves, it is heavily dependent on atomic data $(\log (g f)$ and $\chi)$ and the continuum opacity, which heavily depends on the stellar temperature. So the errors of our abundances also depend on the errors of atomic data (more specifically on the oscillator strength $f$ ), that are often approximate calculations rather than laboratory measurements, and calculations of opacity, which mostly relies on calculations of atomic interactions with hydrogen (section 1.2.2. Because of that, the formal errors of an usual abundance determination are on the order of 0.1 dex, or about $25 \%$ of an abundance determination in a very metal-poor star.

Increasing this precision is the goal of the differential analysis.

\subsubsection{The differential analysis}

The differential analysis, more specifically the line-by-line differential analysis, aims to decrease the errors of the stellar parameters and abundances by analyzing stars that are similar to each other, so that errors from atomic data become less important, or even cancel out.

The first step of a line-by-line differential analysis is to make sure that the spectra of the different stars that are being analyzed are reduced consistently. We must make sure that the wavelength calibration and normalization of all the stars are consistent with each other in all the lines we aim to measure. If the center of each line is consistent, and the normalization is set at the same level in all the stars, we can set the same point of measurement for the EW in all spectra. An example of two spectra consistently reduced around a FeI line is shown in Figure 1.10. As both spectra are consistent, it is possible to set the boundaries to the EW measurement equaly in both stars.

Throughout this work, all the EW measurements (except those from chapter 5) were done manually using the Image Reduction and Analysis Facility (IRAF ${ }^{1}$ ), a software for astronomical data reduction and analysis. Within IRAF we used Gaussian fit in the "splot" task, which is part of the noao.onedspec package. It fits a Gaussian to the line profile and gives us the measured $\mathrm{EW}$ in $\AA$.

With the EW of all stars measured for one given line, instead of estimating the abundance of each star separately, the EWs are compared to the EWs of a standard star. The

\footnotetext{
${ }^{1}$ http://iraf.noao.edu/
} 


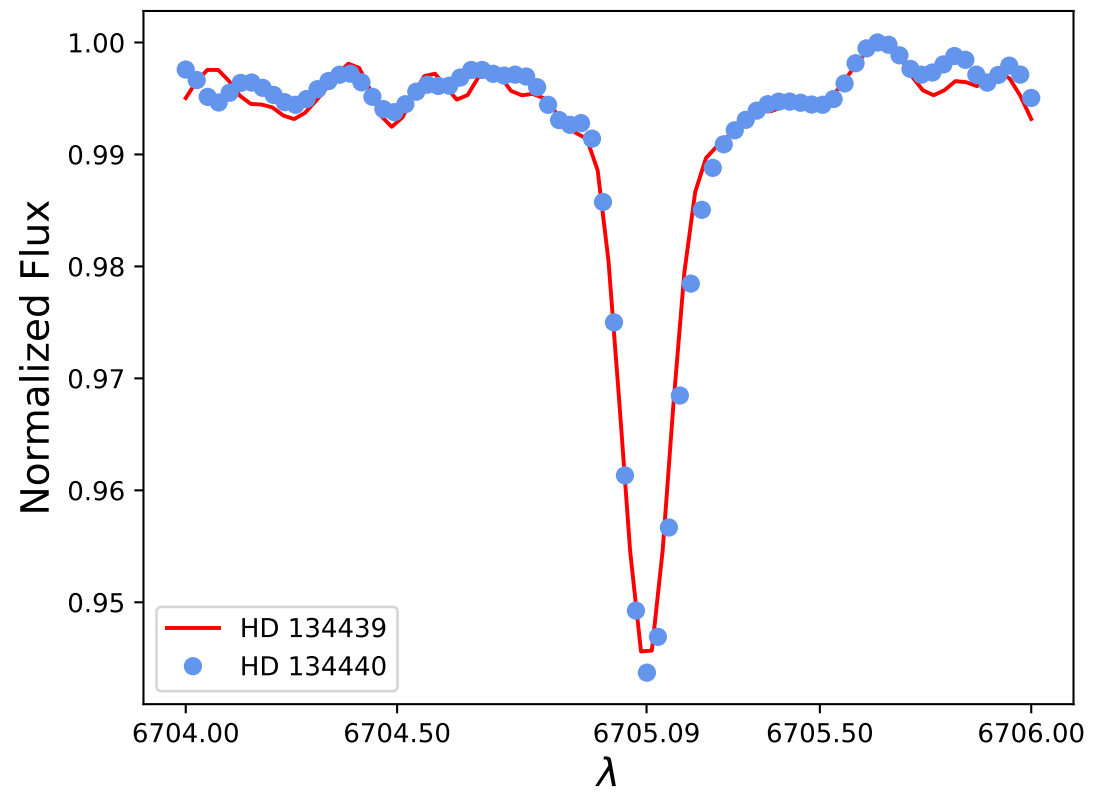

Figure 1.10: The Fe I line at $6705 \AA$ for the twin stars HD 134439 and HD 134440. Two reduced spectra with the line centered at the same wavelength and the continuum normalization set at a same point for a line-by-line differential analysis.

standard star of a differential analysis should be the star that best represents the stars in the sample, with the most reliable stellar parameters (preferably obtained from sources

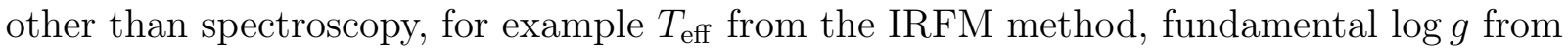
measured parallaxes, and $[\mathrm{Fe} / \mathrm{H}]$ from 3D non-LTE measurements whenever possible) and a good spectrum with high signal to noise ratio $(\mathrm{S} / \mathrm{N})$ and resolution. Thus, the Sun is usually the standard star in most line-by-line differential analysis, as the solar abundances and stellar parameters are the most accurate that astronomers can estimate, and solar spectra are always the best spectra available, with several atlases in the literature with extensive line identifications.

To mathematically demonstrate the advantage of the differential analysis, we will set the Sun as the standard star of comparison and show how equation 1.58 changes when calculating the abundance of an element in one single absorption line. As we are comparing EWs, we subtract the EW of the Sun (our standard star) to the EW of the star we are analyzing (star named AB, for example): 


$$
\begin{array}{r}
\log \left(\frac{\mathrm{W}}{\lambda}\right)_{\mathrm{AB}}-\log \left(\frac{\mathrm{W}}{\lambda}\right)_{\odot}=B+A_{x \mathrm{AB}}+\log (\mathrm{gf})+\log (\lambda)-\theta_{\mathrm{exAB}} \chi \\
-\log \left(\kappa_{\mathrm{cont}}\right)_{\mathrm{AB}}-\mathrm{B}-\mathrm{A}_{\mathrm{x} \odot}-\log (\mathrm{gf})- \\
\log (\lambda)_{\odot}+\theta_{\mathrm{ex} \odot} \chi+\log \left(\kappa_{\mathrm{cont}}\right) \odot \\
\log \left(\frac{\mathrm{W}_{\mathrm{AB}}}{\mathrm{W}_{\odot}}\right)=\mathrm{A}_{\mathrm{xAB}}-\mathrm{A}_{\mathrm{x} \odot}-\chi\left(\theta_{\text {exAB }}-\theta_{\text {ex } \odot}\right)-\log \left(\frac{\kappa_{\text {contAB }}}{\kappa_{\text {cont } \odot}}\right)
\end{array}
$$

From equation 1.59 to equation 1.60, the terms dependent only on atomic data are canceled out, like the $\log (g f)$ of the transition. If the stars being analyzed are twins (we define twin stars as those that have the same effective temperature within $100 \mathrm{~K}$, the same $\log g$ within 0.1 dex, the same metallicity within $0.1 \mathrm{dex}$ ) the terms three and four on the right side of equation 1.60 (namely the two terms that have a stronger dependency on the stellar temperature) will roughly cancel out, and assuming those differences are close to zero, the remaining terms are:

$$
\log \left(\frac{\mathrm{W}_{\mathrm{AB}}}{\mathrm{W}_{\odot}}\right) \approx \mathrm{A}_{\mathrm{xAB}}-\mathrm{A}_{\mathrm{x} \odot}
$$

or:

$$
\delta A_{x} \approx \log \left(\frac{W_{\mathrm{AB}}}{W_{\odot}}\right)
$$

From equations 1.61 or 1.62 we note that the abundance difference between two identical twin stars is only dependent on the difference of the equivalent width of those two stars.

In a line-by-line differential abundance, the $\delta A_{x}$ is done individually for each line and the final differential abundance is taken as the averaged difference of all the lines. If the stars are very similar to each other but not twins, the average opacity around a region will be comparable, but differences in the effective temperature will still exist and the abundance could be given by a less simplified form of equation 1.62 .

$$
\delta A_{x}=\log \left(\frac{W_{\mathrm{AB}}}{W_{\odot}}\right)+\left(\theta_{\mathrm{AB}}-\theta_{\odot}\right)
$$

For stars that are not identical twins, also differences in surface gravity and overall metallicity will have some smaller impact. However, as those differences are small, they can be modelled properly using model atmospheres, so that potential problems in the model atmospheres are roughly canceled out in a differential analysis. 
Thus, in the study of twin stars, it is possible to obtain a much better precision on the differential abundances than for the analysis of absolute abundances (Nissen and Gustafsson, 2018). This technique has been extensively used in solar twins to study chemical imprints of planets in its host stars, Galactic chemical evolution and stellar evolution, and these studies can reach a precision as good as 0.01 dex in some cases (e.g., Meléndez et al., 2009; Ramírez et al., 2009; Tucci Maia et al., 2014, 2016; Biazzo et al., 2015; Spina et al., 2018; Bedell et al., 2018; Botelho et al., 2019).

\subsection{Model atmospheres and radiative transfer code}

This work is almost entirely based on the assumption that the stellar atmosphere can be portrayed by a one dimensional model. As explained in Section 1.2.1, we make the assumption that the stellar photosphere is much smaller than the stellar radius, and we approximate the spectra forming region of the star as being one dimensional and plane parallel. Most of our work is based on MARCS (Gustafsson et al., 2008) model atmospheres, that include one dimensional stratification, hydrostatic equilibrium, mixing-length convection and local thermodynamical equilibrium. MARCS model atmospheres cover the entire range of metallicity, temperature, gravity and microturbulence velocity in which we work, and in their paper they compared their models with those from Castelli and Kurucz (2004), that are also used in this work, and both models agree well.

In practice, the model atmosphere is a table with the temperature, electron and gas pressure as a function of optical depth. We refer the reader to chapter 9 of Gray (2005) and the above papers for details of the assumptions and differences between plane parallel and spherically symmetrical 1D models, and also refer the reader to Magic et al. (2013) and references therein for details on 3D hydrodynamical model atmospheres.

We used the radiative transfer code MOOG (Sneden, 1973) to estimate the chemical abundances. It computes LTE abundances using plane parallel model atmospheres and a linelist with the measured EWs. MOOG was employed via the python wrapper $\mathrm{q}^{2}$ (Ramírez et al., 2014), that creates the entry files for MOOG, including the interpolation of the atmospheric models (both MARCS and Castelli and Kurucz (2004) models are available), and formatting the input linelists. For more information on MOOG, $\mathrm{q}^{2}$, their 
usage, and the entry files each of the programs requires, see their websites ${ }^{2}$.

We also used spectral synthesis in this work, mostly via MOOG. Besides the differences in the parameter file of MOOG, the user must provide a linelist as complete as possible of the region of the line under analysis, so that the program can correctly assess not only the lines of interest but the opacity due to other species (besides hydrogen) and other lines that might be blended or in the wings of the line of interest. More details can be found in the MOOG website.

The above described assumptions, atmospheric models and codes, were used to analyze the chemical evolution of the Milky Way through metal-poor stars, and it is divided as follows: In Chapter 2 we show the impact of using the differential line-by-line analysis when estimating chemical abundances, an extended view of the Reggiani et al. (2016) and Reggiani and Meléndez (2017) publications; in Chapter 3 we analyze a sample of 23 metal-poor stars to study the formation and chemical evolution of the solar neighborhood halo, and is based on Reggiani et al. (2017); Chapter 4 is focused on how chemical tagging can be used to uncover details of Galactic formation and of the nature of stars, and was based on the work published in Reggiani and Meléndez (2018); in Chapter 5 we study the chemical evolution of potassium through non-LTE abundances, based on Reggiani et. al. (2019); and in Chapter 6 we make the concluding remarks. The scientific papers cited above are all attached to the thesis in Apendix A.

${ }^{2}$ https://www.as.utexas.edu/chris/moog.html; https://github.com/astroChasqui/q2_tutorial 
Chapter 2

\section{Differential analysis of metal-poor stars}

\subsection{The first differential analysis in extremely metal-poor stars}

As mentioned in Section 1.1, low metallicity stars hold the key to unlocking many steps of the evolution and formation of the early stages of the Milky Way (Eggen et al., 1962; Searle and Zinn, 1978), or to uncover the rise of neutron-capture elements and their production sites (Audouze and Silk, 1995; Shigeyama and Tsujimoto, 1998; Chieffi and Limongi, 2002; Umeda and Nomoto, 2002; Meynet et al., 2010; Roederer et al., 2016).

Thus, astronomers have been studying metal-poor stars for several decades. Among interesting objects worth noticing, there is the "Keller" star, the most metal-poor star found to date (Keller et al. 2014), a star so poor in any metals that the only element clearly identified in its spectrum was calcium, besides upper limits of a few other chemical species (like Fe I). This star is believed to have been enriched by a single supernova event. Like most stars with metallicities below $[\mathrm{Fe} / \mathrm{H}] \leq-4.0$, the Keller star is also enhanced with carbon (Nordlander et al., 2017).

Stars like that are, however, very difficult to find. Large searches for metal-poor stars have been going on since the late 70's, with a special remark to the prism HK survey of Beers et al. (1985, 1990, 1992) and the Hamburgo/ESO objective prism survey of Christlieb et al. (1999). These surveys have had great success in identifying metal-poor stars based on metallicity markers like the calcium $\mathrm{H}$ and $\mathrm{K}$ lines, but, like most surveys, they provided low-resolution spectra and the metal-poor candidates needed to be confirmed via follow-up observations in larger telescopes with medium and high-resolution spectrographs. Since the advent of these surveys, identification of these stars is improving with higher resolution and higher S/N spectra being available directly from surveys like APOGEE (Majewski et al. 
2007) and SEGUE (Yanny et al., 2009), and photometric surveys allied with machine learning techniques that also improve the detection of such objects (e.g., the PRISTINE survey, Starkenburg et al., 2017). However, high-resolution follow up is still required to measured the detailed chemical patterns of these stars (e.g., Yong et al., 2013a; Siqueira Mello et al., 2014; Li et al., 2015; Marino et al., 2019).

Unfortunately, high-resolution spectra of metal-poor stars are still challenging to obtain, as these stars are mostly located in the Galactic Halo at large distances from us, thus requiring hours of telescope usage, even in 10-meter class telescopes. As telescope hours are expensive, most of the spectra of metal-poor stars are not as high quality as spectra of solar like stars, for example. Lower integration times also lead to a lower S/N ratio, making it difficult to distinguish good unblended and well-defined absorption lines, as the metallicity decreases (Figure 1.1).

Hence, the errors associated with the abundance analysis of metal-poor stars are usually on the order of $25 \%$, as already mentioned in Section 1.1. In order to decrease those errors, we decided to apply, for the first time, the differential technique to the analysis of extremely metal-poor stars, and test if it is able to considerably decrease the final errors even in stars that are not twins. We chose to study two EMP stars for which Keck spectra of highresolution and very high $\mathrm{S} / \mathrm{N}$ ratio was available, taken by J. Melendez for the analysis of isotopic lithium abundances (Asplund and Meléndez, 2008; Lind et al., 2013).

\subsection{Data and stellar parameters}

Two stars were originally analyzed via a line-by-line differential analysis, to assess if the technique can be employed in extremely metal-poor stars (G 64-12 and G 64-37); the results were published in Reggiani et al. (2016). In this thesis, we extended the analysis and included star CD -24 17504 (CD24), for which spectra were taken with the same configuration was also available.

All three stars were observed with the High-Resolution Echelle Spectrometer (HIRES, Vogt et al., 1994), at the Keck 10 meters telescope. Star G 64-12 was observed on June 16, 2005, G 64-37 on January 19, 2006, and CD24 on June 17, 2005. The observations were performed with the same setup using the E4 slit ( $0.4 " \mathrm{x} 7$ "), resulting in a resolving power of $\mathrm{R} \approx 95000$, with a $\mathrm{S} / \mathrm{N}=700$ at $5000 \AA$ and $\mathrm{S} / \mathrm{N}=900$ around the Li $6707 \AA$ 
line in the spectra of stars G 64-12 and G 64-37, but in star CD24 the exposure times were much smaller, resulting in a much reduced (when compared to the other two stars) $\mathrm{S} / \mathrm{N} \approx 150$ at the Li line, leading to more significant errors. The spectra have a wavelength coverage ranging from $3900 \AA$ to $8300 \AA$. The orders were extracted using the Mauna Kea Echelle Extraction (MAKEE ${ }^{1}$ ) package. We performed the Doppler correction and continuum normalization via IRAF.

The linelist was created specifically to analyze these metal-poor stars, taking into consideration the observable lines in the spectra of $\mathrm{G}$ 64-12. We visually inspected the spectra to recognize measurable lines included in the linelist. The atomic data are from the VALD (Ryabchikova et al. 2015) database with updated $\log (g f)$ and hyperfine structure data from Kuruc2 2 , The full linelist is published in Reggiani et al. (2016), see Appendix A. The abundances and stellar parameters were obtained using the curve of growth (Section 1.2.6), via the line-by-line differential technique (Section 1.2.7). The equivalent widths of each line were measured simultaneously for stars G 64-12 and G 64-37, using the splot task in IRAF, fitting Gaussian profiles to the observed lines. When necessary, we employed the "deblend" task for blended lines. In blended lines we also used Gaussian profiles, and the placement of the continuum was given manually. The same linelist and measurement technique was used in CD24, but the EWs were determined later, which might be a source of error due to a possible different placement of the continuum, or points of measurement.

For Reggiani et al. (2016), all the differential stellar parameters and abundances were found "manually" (meaning an analysis that did not rely on the use of any wrapper codes, and directly employed the radiative code and its output), using the Castelli and Kurucz (2004) model atmospheres. We analyzed star CD24 using the $\mathrm{q}^{2}$ code, and for consistency we reanalyzed G 64-12/G 64-37 stars using the same code. For the analysis with $\mathrm{q}^{2}$, we used MARCS atmospheric models which, as mentioned in Section 1.3, are consistent with Castelli and Kurucz (2004) models. Nevertheless, differences in the atmospheric models can lead to differences in the derived stellar parameters and abundances. It is also important to mention that an automated computer program is better on finding the best point of convergence for the stellar parameters than a "manual" analysis could ever be. Thus, this

${ }^{1}$ The package was created by T. A. Barlow and is freely available at
http://www.astro.caltech.edu/ tb/makee/
2 http://kurucz.harvard.edu/linelists.html


could also be a source of uncertainties and differences between the two analysis. However, we will see that the results are indistinguishable within the small errors we obtained.

Star G 64-12 is used as the standard star in the analysis and its stellar parameters are: $T_{\text {eff }}=6463 \mathrm{~K}$ (Infrared flux method, IRFM, Meléndez et al. 2010), $\log (g)=4.26$ (fundamental value, Nissen et al., 2007), and $[\mathrm{Fe} / \mathrm{H}]=-3.20$ and $\xi=1.65 \mathrm{~km} . \mathrm{s}^{-1}$ are from our measurements of the iron lines. Through differential excitation equilibrium we estimated the effective temperature of the two other objects. As can be seen in equations 1.58 and 1.63 , there is a dependence of the line abundance with temperature, and the excitation temperature is found when the abundances of all the lines best agree to each other (when there is no trend between the abundances, differential or not and the excitation potential of the lines). The gravity was determined via differential ionization equilibrium; lines of different ionization stages respond differently to the gravity of the star and the solution is found when the abundances from both ionization stages are the same. The microturbulent velocities were found by allowing no trend of the differential abundances with the reduced equivalent width. The spectroscopic stellar parameters, differential or not, can be determined using any chemical species that have more than one measurable ionization stage lines available, like Fe I and Fe II, or Ti I and Ti II (Meléndez et al., 2012). In our work, we only used iron lines to determine the stellar parameters. In Figure 2.1 we show the determination of the stellar parameters of CD24 given by $\mathrm{q}^{2}$, and Figure 1 from Reggiani et al. (2016) shows the same plots for the "manual" parameters determination of G 64-37.

The stellar parameters of stars G 64-37 and CD24, along with the standard star G 64-12, are given in Table 2.1. Comparing with our "manual" analysis in Reggiani et al. (2016), there is an excellent agreement of the estimated stellar parameters of G 64-37: a negligible difference of $2 \mathrm{~K}$ in $T_{\text {eff }}$, and a 0.02 dex difference in metallicity. Both can be completely disregarded as they are smaller than the estimated errors and our results are, unsurprisingly, the same as our "manual" analysis. Unsurprisingly because $\mathrm{q}^{2}$ is a MOOG wrapper, thus the abundances were found using the same radiative transfer code, and the atmospheric models are comparable. The only difference observed is due to the better capacity of a computer program to simultaneously, and quickly, find all stellar parameters in a more fine-tuned manner. The stellar parameters of G 64-37 derived from the differential analysis are also comparable with the stellar parameters from non-spectroscopic sources, 
cd2417504: 6408, 4.325, -3.18, 1.655 [g64-12]
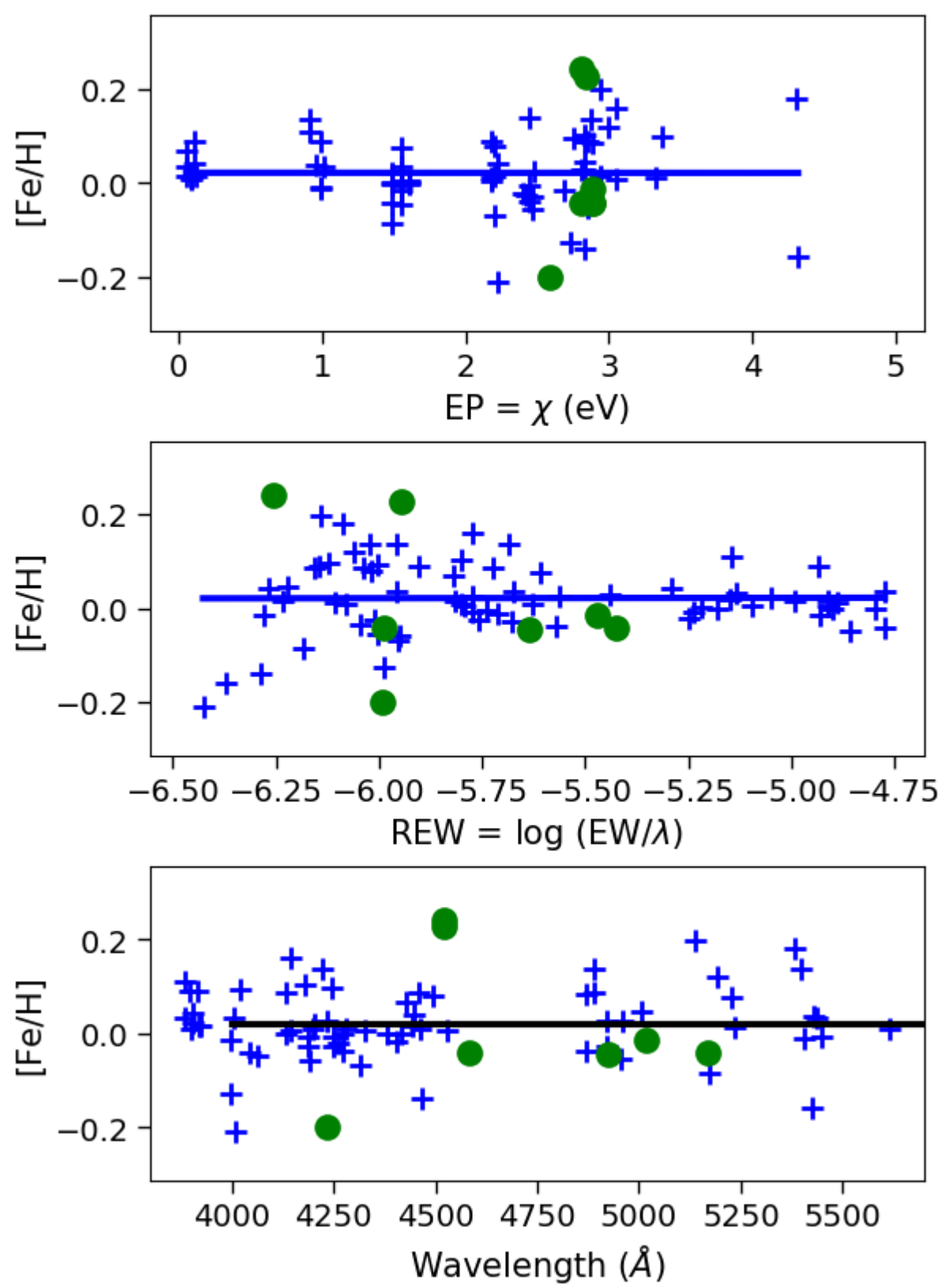

Figure 2.1: Differential excitation equilibrium (top panel), differential abundance as a function of reduced EW (middle panel), and differential abundance as a function of wavelength (lower panel), for the determination of stellar parameters of CD -24 17504 using $q^{2}$. 
Table 2.1 - Stellar parameters and errors of stars G 64-12, G 64-37, and CD -24 17504. The star in boldface was used as the standard star of this analysis.

\begin{tabular}{lccc}
\hline \hline & G 64-12 & G 64-37 & CD24 \\
\hline$T_{\text {eff }}(\mathrm{K})$ & 6463 & 6568 & 6408 \\
$\sigma \mathrm{T}_{\text {eff }}$ & 50 & 45 & 110 \\
$\log (g)\left(\mathrm{g} \mathrm{in} \mathrm{cm} / \mathrm{s}^{2}\right)$ & 4.26 & 4.40 & 4.33 \\
$\sigma \log (g)$ & 0.15 & 0.07 & 0.19 \\
{$[\mathrm{Fe} / \mathrm{H}]$} & -3.20 & -3.02 & -3.18 \\
$\sigma[\mathrm{Fe} / \mathrm{H}]$ & 0.04 & 0.03 & 0.08 \\
$\xi\left(\mathrm{km} . \mathrm{s}^{-1}\right)$ & 1.65 & 1.74 & 1.66 \\
$\sigma \xi$ & 0.06 & 0.08 & 0.19 \\
\hline
\end{tabular}

such as the IRFM temperature of $T_{\text {eff }}=6583 \pm 50 \mathrm{~K}$ from Meléndez et al. (2010), and the $\log g=4.24 \pm 0.15$ from Nissen et al. (2007), which further confirms the reliability of the method.

It is important to stress that we are determining the stellar parameters from Fe lines, and iron is known to suffer from non-LTE on several atomic lines. Iron lines can also be influenced by the surface gravity of the star, which also better accounted in a nonLTE analysis. Fortunately, we are analyzing our stars in a differential analysis and, as demonstrated in equations 1.59 to 1.63 , most atomic and stellar atmospheric data effects are greatly diminished in such an analysis.

\subsection{Errors in a differential analysis}

Before turning ourselves to the abundance results and the conclusions that can be drawn from the stars, we go back to the differential analysis itself and the proposition that it can improve the precision of the abundances. In the last few years different authors have used the differential abundance analysis to evaluate stellar evolution effects (Monroe et al., 2013; Tucci Maia et al., 2015), to recognize planet signatures in chemical abundances of stars (Meléndez et al., 2009; Ramírez et al., 2009; Tucci Maia et al., 2014; Biazzo et al., 2015), to study the chemical evolution of the solar neighborhood (Nissen, 2015; Reggiani et al. 2016), study abundance anomalies in open clusters (Spina et al., 2018) and globular clusters (Yong et al., 2013), and distinct populations in the stellar halo (Nissen and Schuster, 2010; Ramírez et al. 2012; Fishlock et al. 2017), claiming precisions as low as about 0.01 dex 
in solar twins analyses (e.g., Tucci Maia et al., 2014). But, so far, it was unclear if such low errors are achieved because of the technique itself or if the source of precision are the spectra and their high quality. To test the error sources in similar stars, but not twin stars, and using a standard star that does not have as precise parameters and abundances as the Sun, we analyzed only the measurement errors of the pair of stars G 64-12/G 64 -37 observed with HIRES at Keck, and a sample of very metal-poor stars observed with the UVES spectrograph at the VLT telescope.

The UVES sample is composed by 11 stars with $\mathrm{R} \approx 50000$ and $\mathrm{S} / \mathrm{N}=180$ at $500 \mathrm{~nm}$, much lower than the S/N of G 64-12 and G 64-37. The UVES spectra were reduced through the ESO pipeline with Doppler and barycentric velocity corrections done using the IRAF package for python, PyRaf and the spectra were normalized via IRAF. The measurement details and analysis of this sample can be seen in Chapter 3 .

For our precision tests, we calculated the stellar parameters and chemical abundances of both samples in two manners. First, we have performed a classical spectroscopic analysis of the data (excitation equilibrium of the Fe I and Fe II lines to determine $\mathrm{T}_{\text {eff }}, \log g$ from ionization equilibrium, and microturbulent velocity by allowing no trend in line abundances with reduced EW). Abundances were then estimated from the measured EWs, with the "classical" stellar parameters. We also calculated stellar parameters and abundances using the line-by-line differential approach, as described in the section above for stars $\mathrm{G} 64-12 / \mathrm{G}$ 64-37 (see Table 2.1), and we did the same for the UVES sample. The standard star of the UVES sample (HD 338529) was selected due to the availability of stellar parameters from the same non-spectroscopic sources as used in G 64-12, $\mathrm{T}_{\text {eff }}$ from the IRFM given in Meléndez et al. (2010), and $\log g$ from Nissen et al. (2007), with standard errors of $50 \mathrm{~K}$ and 0.15 dex, respectively. For consistency, and a better error assessment, both "classical" and differential analyses were done using the $\mathrm{q}^{2}$ code.

Stellar parameters calculated strictly from spectroscopic methods are known to differ from photometric methods for metal-poor stars, where the spectroscopic temperatures could be up to $300 \mathrm{~K}$ lower than photometric temperatures. The effective temperature of star G 64-12, for example, is $6168 \mathrm{~K}$ when estimated through excitation equilibrium, while the IRFM temperature is $6463 \mathrm{~K}$, and the temperature of star $\mathrm{G} 64-37$ is $6258 \mathrm{~K}$ from classical excitation equilibrium, and $6583 \mathrm{~K}$ from the IRFM (Meléndez et al., 2010). The

\footnotetext{
${ }^{3}$ PyRAF is a product of the Space Telescope Science Institute, which is operated by AURA for NASA
} 
surface gravity experiences more extreme differences, with relative differences up to 1 dex, between the classical ionization equilibrium and the $\log g$ from the absolute magnitude. The use of different sets of stellar parameters (strictly spectroscopic vs differential analysis with a standard star with IRFM temperature and log $g$ from absolute magnitude) also translates into abundance differences of up to 0.15 dex.

In this section, we will not discuss neither the values of the stellar parameters, both classical and differential, nor the abundances themselves. The abundances, and associated analysis, of stars G 64-12 and G 64-37, will be discussed in Section 2.4, and the analysis of the UVES sample will be discussed in Chapter 3. We will only discuss the ratios between the errors of the different analysis methods here, also because of the differences that arise from different analysis techniques, specially those for estimating the stellar parameters.

All the abundance errors here include the observational errors, added in quadrature to the quadrature of the errors due to the stellar parameters uncertainties:

$$
\sigma\left(A_{x}\right)=\sqrt{\left(\frac{\sigma_{o b s}}{\sqrt{N}}\right)^{2}+\sigma_{\mathrm{sp}}^{2}}
$$

where $\sigma\left(A_{x}\right)$ is the error of the abundance $A$ in species $x, \sigma_{o b s}$ is the scatter in the estimated abundances due to the scatter in the measured EWs (either several measurements of a single line, whenever there is only one line available, or a due to different abundances from different lines, whenever 2 or more lines are available for the same species), $N$ is the number of measured lines of the $x$ species, and $\sigma_{\mathrm{sp}}$ is the quadratic error in abundances when changing the stellar parameters of the star by their uncertainties and recalculating the chemical abundances.

We analyze the ratio between the errors of the classical (absolute) and the differential analysis, to estimate the gain in precision by using the differential analysis. This gain is defined as follows:

$$
\sigma_{\mathrm{Z}}=\frac{\sigma_{\mathrm{Z}}^{\text {classical }}}{\sigma_{\mathrm{Z}}^{\text {diff }}},
$$

where $\mathrm{Z}$ is either a stellar parameter $\left(T_{\text {eff }}, \log g,[\mathrm{Fe} / \mathrm{H}]\right.$, or $\left.\xi\right)$ or a chemical abundance $A(\mathrm{X})$. 


\subsubsection{Discussion of the error ratios}

In Figure 2.2 we show the gain in precision of the stellar parameters. From top to bottom we show the gains in $T_{\text {eff }}, \log g,[\mathrm{Fe} / \mathrm{H}]$, and $\xi$. The average gains on $T_{\text {eff }}, \log g$, $[\mathrm{Fe} / \mathrm{H}]$, and microturbulence are $1.43,1.47,1.51$, and 1.49 respectively. We do not compare errors of the standard stars of our samples because they come from different methods $\left(\mathrm{T}_{\text {eff }}\right.$ from the IFRM, and $\log g$ from the absolute magnitude). The overall result clearly indicates that the differential abundance yields more precise stellar parameters, but are dependent on the quality of the spectra, as the better spectra of the G $64-12 / \mathrm{G} 64-37$ sample yielded a precision almost twice as the averaged precision of UVES sample.

In Figure 2.3 we show the gain on precision for the abundances. As can be seen, the average gain for G 64-37 is twice the average gain for the UVES sample. We attribute this difference to the quality of the spectra. The better spectral quality allows us to measure the EWs more consistently between the stars, which, in turn, makes the comparison more precise, thus lowering the error bars. Nevertheless, in both cases, the differential analysis performs much better, to a point where the usual $25 \%$ error on the abundance of a EMP star might be decreased by about a factor of 3. Interestingly, the mean error ratio of $\mathrm{Cr}$ II of the UVES sample is about 1.

We also tested the effects of the differential technique, only applied to the calculation of the abundances, by using the stellar parameters obtained from a classical analysis. In this case there will be no benefit in using the differential analysis. This effect is observed because the estimated stellar parameters of the stars are not as similar as it is necessary for a good application of the differential method, and because the abundance errors become dominated by the much larger errors of the classical stellar parameters. In this analysis, the average gain decreased from 3 to 1.4 in G $64-37$ and from 1.4 to having no gain in the UVES sample.

With this simple, but consistent analysis, we were able to see that a differential analysis can increase the precision of our results by up to three times, depending on the quality of the spectra and measurements. We have also seen that the most influential part of our analysis was the precise determination of the stellar parameters (the better constrained the stellar parameters, the better our final abundance results). 

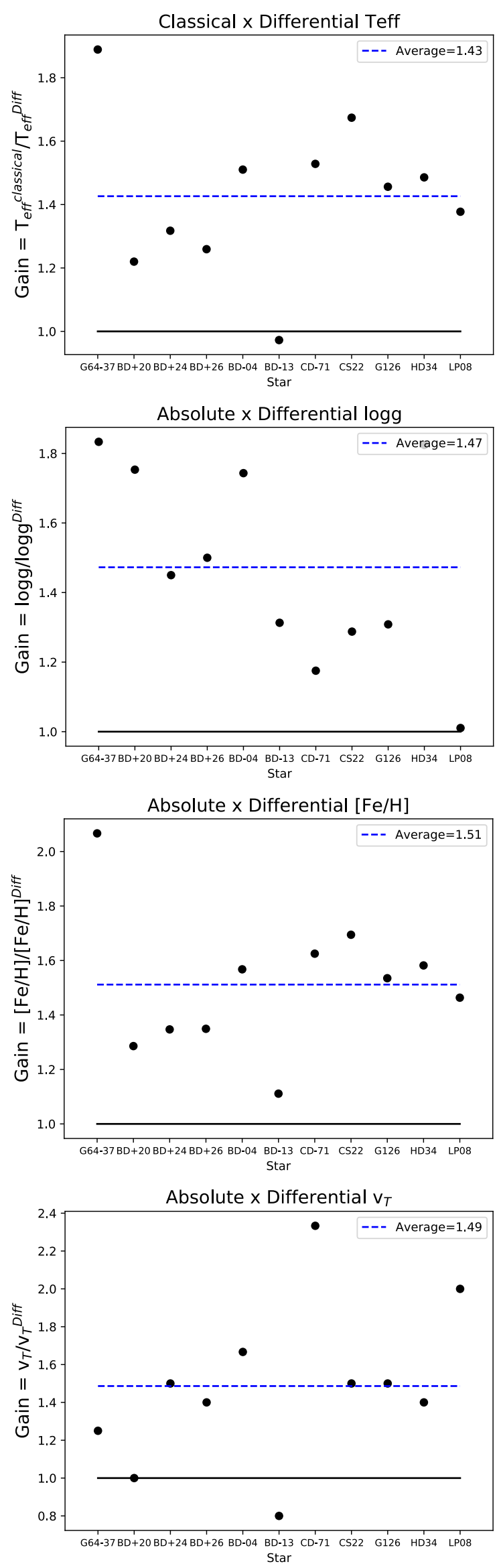

Figure 2.2: Gain in precision in stellar parameters with the differential analysis. The first (from the top), second, third and fourth panels show the precision gain in $T_{\text {eff }}, \log g,[\mathrm{Fe} / \mathrm{H}]$, and $\xi$. 

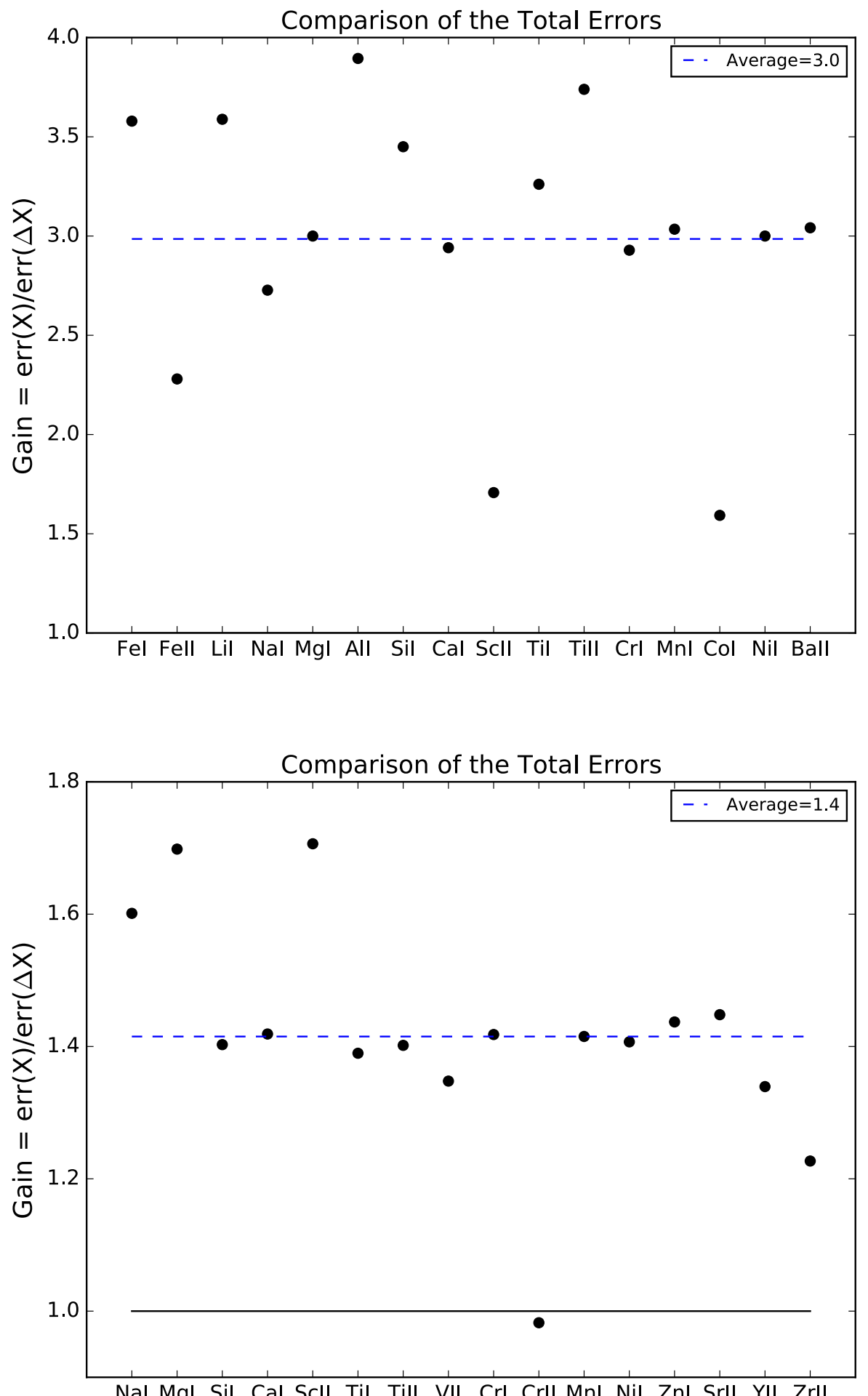

Figure 2.3: Gain in precision in chemical abundances with a differential analysis. Upper panel shows the ratios in star G 64-37, and the lower panel are the gain from the UVES sample. 


\subsection{Abundance analysis}

In the last section, we showed that the small errors in our analysis are directly related both to the differential technique and to the quality of the spectra, and now we return to the abundance analysis of stars G 64-12, G 64-37, and CD24. Our differential $\Delta[\mathrm{X} / \mathrm{H}]$ abundances are in Figure 2.4. We first comment the strikingly difference between the errors of the two stars (G 64-37 and CD24). While G 64-37 has errors smaller than 0.05 dex, the much lower spectral quality along the possible differences in measurements (G 64-12 and CD24 were not measured simultaneously, as explained above), translated in much less precise results of CD24, as can be seen in Table 2.2. Leaving the abundances and errors of CD24 aside for the moment, we turn back to the results drawn from G 64-37.

These differential abundances have errors as small as $\approx 0.01-0.02$ dex, and such big precision can reveal small inhomogeneities that exist between these stars, leading to the identification, for example, of different chemical populations within the very homogeneous halo of our Galaxy, while the errors of a classical technique might make it difficult to say with certainty that these abundance patterns are different. High-quality observations demonstrate that the separation of the halo population via the abundance pattern of $\alpha$ elements, require a precision on the order of 0.05 dex (e.g., Nissen and Schuster, 2010). The differential abundances in the top panel of Figure 2.4 are indicative that G 64-37 belong to a different population than $\mathrm{G} 64-12$, as the confidence $(\Delta[\mathrm{X} / \mathrm{H}] / \sigma)$ of the abundance differences are mostly higher than $2 \sigma$ (see Table 2.2.

From Figure 2.4 and Table 2.3, we see that star CD24 also seems to have a different abundance pattern than G 64-12, but the errors are in general too high to definitely conclude that. The difference in the errors of G 64-37 and CD24, clearly shows the increasing necessity of higher precision studies to understand the formation environments of similar stars. While we can definitely state that G $64-37$ has a different pattern only from the $\Delta[\mathrm{X} / \mathrm{H}]$ values, only the $\Delta[\mathrm{X} / \mathrm{Fe}]$ can differentiate CD24 from $\mathrm{G} 64-12$, as the errors are smaller than the apparent difference between those two stars. However, most of the confidence levels for CD24 are small, with the exception of the strontium abundance, with a confidence level far beyond the $2 \sigma$ threshold.

In Figure 2.5 we show both the differential abundances scaled relative to $\mathrm{Fe}(\Delta[\mathrm{X} / \mathrm{Fe}])$ and the absolute $[\mathrm{X} / \mathrm{Fe}]$. The Fe scaled differential abundances further demonstrate that 

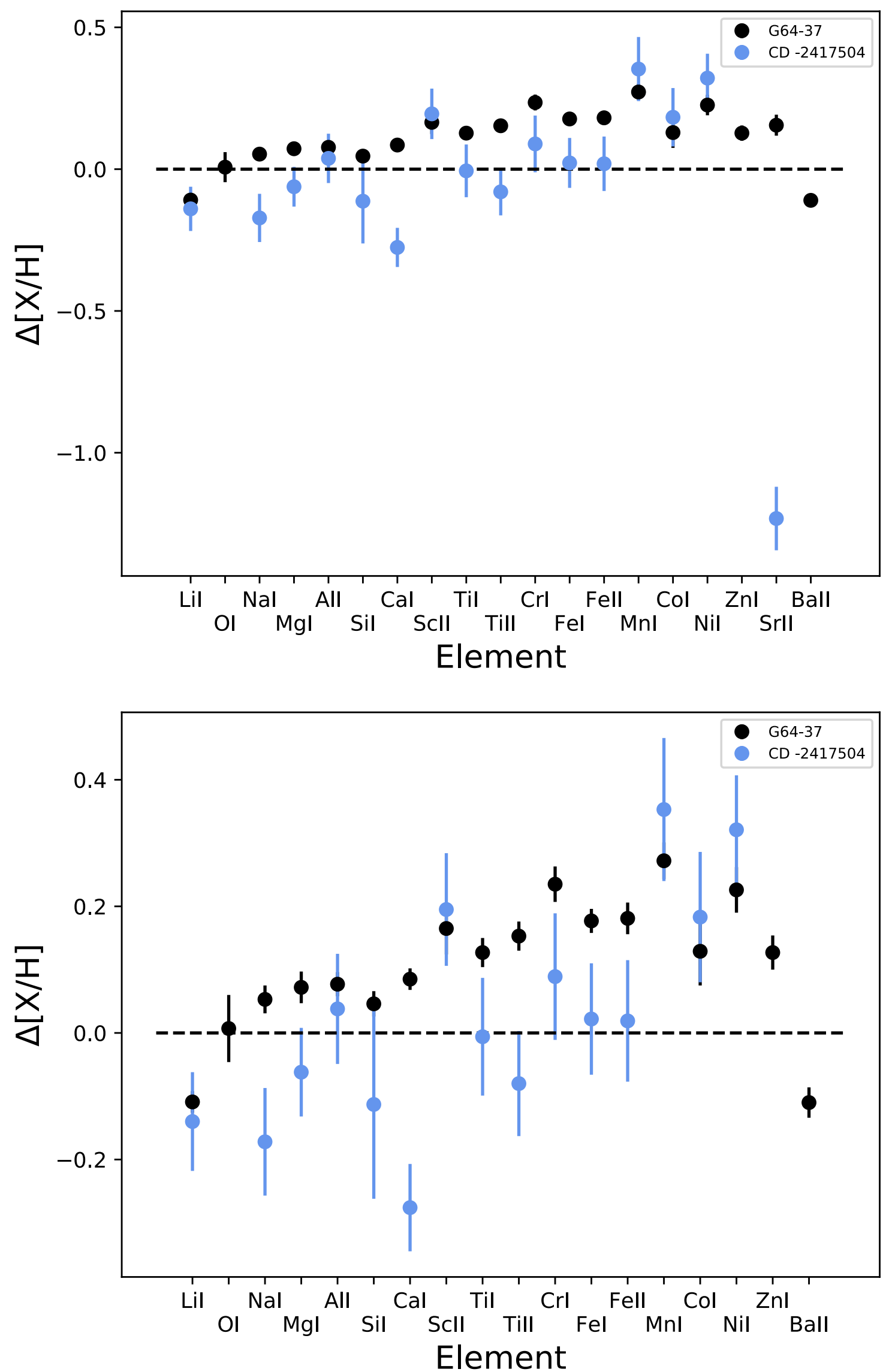

Figure 2.4: Differential abundances of stars G 64-37 and CD -2417504. The top panel shows all differential abundances and in the lower panel $\mathrm{Sr}$ is excluded to better see other elements. 
Table 2.2 - Differential abundances of stars G 64-37 and CD -2417504. The standard star is G 64-12.

\begin{tabular}{lcccc|cccc}
\hline \hline & & \multicolumn{3}{c|}{ G 64-37 } & \multicolumn{3}{c}{ CD -2417504 } \\
Species & $\Delta[\mathrm{X} / \mathrm{H}]$ & $\sigma \Delta[\mathrm{X} / \mathrm{H}]$ & $\Delta[\mathrm{X} / \mathrm{H}] / \sigma$ & {$[\mathrm{X} / \mathrm{Fe}]$} & $\Delta[\mathrm{X} / \mathrm{H}]$ & $\sigma \Delta[\mathrm{X} / \mathrm{H}]$ & $\Delta[\mathrm{X} / \mathrm{H}] / \sigma$ & {$[\mathrm{X} / \mathrm{Fe}]$} \\
\hline LiI & -0.109 & 0.017 & 6.412 & - & -0.140 & 0.078 & 1.795 & - \\
OI & 0.007 & 0.053 & 0.132 & 0.983 & - & - & - & - \\
NaI & 0.053 & 0.022 & 2.409 & -0.296 & -0.172 & 0.085 & 2.024 & -0.366 \\
MgI & 0.072 & 0.025 & 2.880 & 0.290 & -0.062 & 0.070 & 0.886 & 0.311 \\
AlI & 0.077 & 0.019 & 4.053 & -0.795 & 0.038 & 0.087 & 0.437 & -0.679 \\
SiI & 0.046 & 0.020 & 2.300 & -0.378 & -0.113 & 0.149 & 0.758 & -0.382 \\
CaI & 0.085 & 0.017 & 5.000 & 0.324 & -0.276 & 0.069 & 4.000 & 0.118 \\
ScII & 0.165 & 0.041 & 4.024 & 0.069 & 0.195 & 0.089 & 2.191 & 0.254 \\
TiI & 0.127 & 0.023 & 5.522 & 0.511 & -0.006 & 0.093 & 0.065 & 0.533 \\
TiII & 0.153 & 0.023 & 6.652 & 0.415 & -0.080 & 0.083 & 0.964 & 0.337 \\
CrI & 0.235 & 0.028 & 8.393 & -0.114 & 0.089 & 0.100 & 0.890 & -0.105 \\
FeI & 0.177 & 0.019 & 9.316 & -0.085 & 0.022 & 0.088 & 0.250 & -0.085 \\
FeII & 0.181 & 0.025 & 7.240 & -0.031 & 0.019 & 0.096 & 0.198 & -0.038 \\
MnI & 0.272 & 0.029 & 9.379 & -0.638 & 0.353 & 0.113 & 3.124 & -0.402 \\
CoI & 0.129 & 0.054 & 2.389 & 0.266 & 0.183 & 0.103 & 1.777 & 0.475 \\
NiI & 0.226 & 0.036 & 6.278 & -0.022 & 0.321 & 0.086 & 3.733 & 0.228 \\
ZnI & 0.127 & 0.027 & 4.704 & 0.388 & - & - & - & - \\
SrII & 0.155 & 0.037 & 4.189 & -0.034 & -1.232 & 0.112 & 11.000 & -1.266 \\
BaII & -0.110 & 0.024 & -4.583 & -0.439 & - & - & - & - \\
\hline
\end{tabular}


G 64-12 is probably from a different population than the other two objects. Most of the [X/Fe] abundances of G 64-37 and CD24 are quite within the expected abundances for their metallicity range: for example, Reggiani et al. (2017) indicate that the mean abundance ratio for $[\mathrm{Mg} / \mathrm{Fe}]$ is 0.32 dex for metal-poor stars, and from Table $2.3[\mathrm{Mg} / \mathrm{Fe}]_{\mathrm{G} 64-37}=0.296$ and $[\mathrm{Mg} / \mathrm{Fe}]_{\mathrm{CD} 24}=0.311$, quite within the expected value. Other abundance ratios like $[\mathrm{Cr} / \mathrm{Fe}]$ or $[\mathrm{Mn} / \mathrm{Fe}]$ are also very much alike the expected abundances for these metallicity stars. However, we do not discuss this further because the galactic chemical evolution of metal-poor stars will be examined in detail in the next chapter.

It is important to emphasize that for all the $\alpha$-elements the significance of our results for star G 64-37 are above $2 \sigma$, and if the $\alpha$-elements are indeed indicative of different populations (Nissen and Schuster, 2010), G 64-37 and G 64-12 (as similar as they might be) could have originated in differently polluted ISM (different populations). Likewise, $[\mathrm{X} / \mathrm{Fe}]$ abundance ratios of CD24 indicate an abundance pattern different than that of $\mathrm{G}$ 64-12, the standard star, and it is similar to the pattern observed in G 64-37, excluded the higher Z elements (nucleosynthesis of higher Z elements are more dependent in explosion energy of the supernova, which could possibly explain these differences). Thus, G 64-37 and CD24 are likely from the same population, which is the same as saying that they were born in a similarly polluted ISM.

The results presented here illustrate how a differential study can indicate different populations in stars that appear to be similar; the difference in the abundance patterns of these stars can give us important information on the environments where these stars formed and the supernova that enriched them; other possible applications of the differential abundance technique in metal-poor stars include the study of lithium depletium (see, Reggiani et al., 2016), stellar evolution (Reggiani et al., 2017), chemical tagging and the recognition of planetary accretion (Chapter 4, Reggiani and Meléndez, 2018).

\subsection{CEMP-no stars}

The abundance of carbon in stars has been a hot subject in astrophysics for a long time, both for "normal" stars and for stars with spectral signatures of carbon much more prominent (e.g, Bidelman, 1956, Ishida, 1960; Sneden, 1974, Barbuy, 1981; Carbon et al., 1987; Rossi et al., 1999, 2005; Spite et al., 2005, Yong et al., 2013b; Placco et al., 2014; 

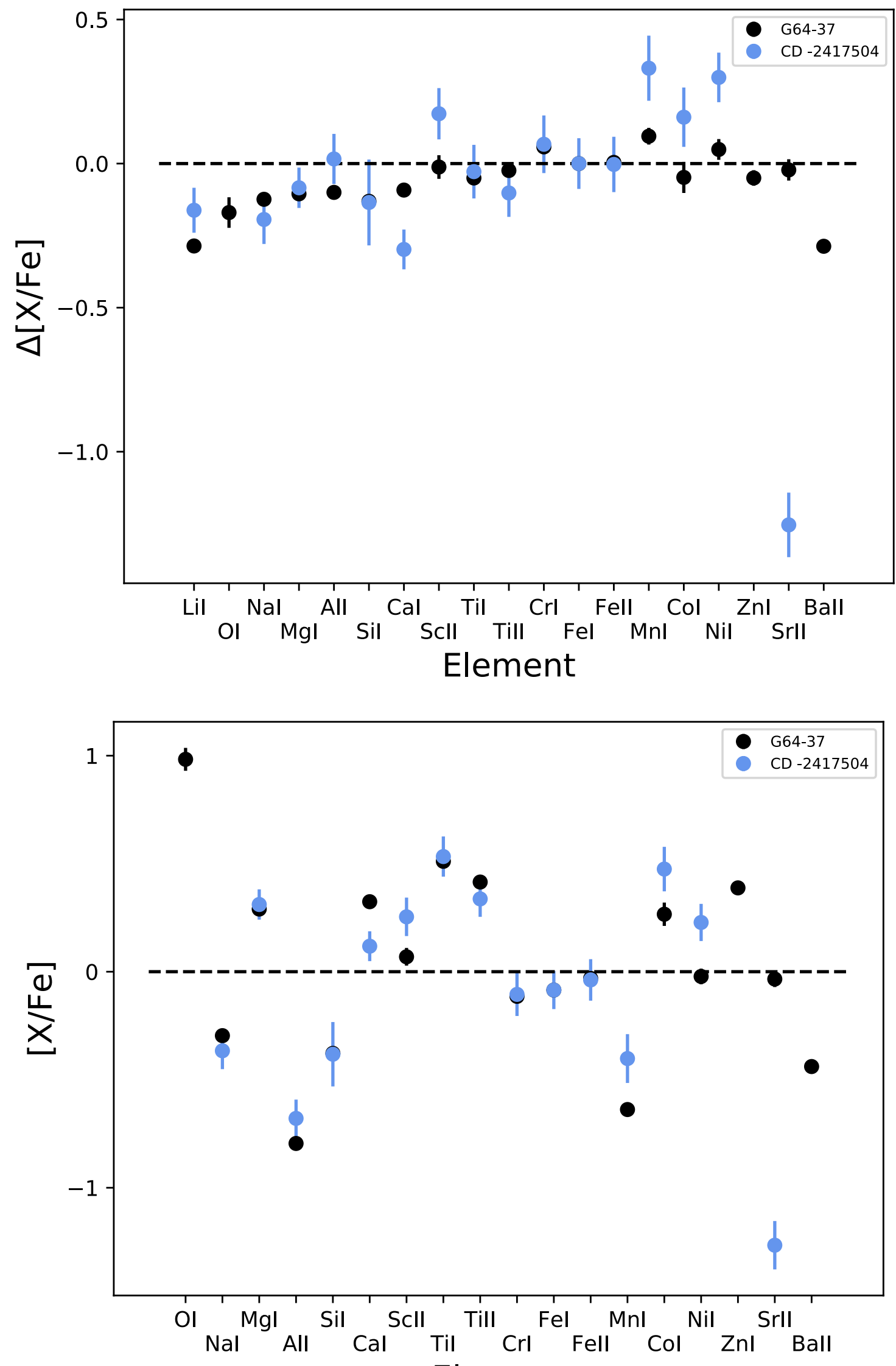

Element

Figure 2.5: Differential [X/Fe] abundances of stars G 64-37 and CD -24 17504 are shown in the upper panel, and absolute $[\mathrm{X} / \mathrm{Fe}]$ abundances are shown in the lower panel. 
Hansen et al. 2016; Norris and Yong, 2019). The stars with unusual high carbon were called "carbon stars". Much more recently, via the large HK survey, Beers et al. (1992) recognized that a big portion of the metal-poor stars had a large ratio of $[\mathrm{C} / \mathrm{Fe}]$. Since this finding there have been several works focused on the carbon abundance in metal-poor stars and their frequency on the galaxy. It was found that the frequency of these carbon rich stars increases with decreasing metallicity (e.g., Rossi et al., 1999; Lee et al., 2013; Placco et al. 2014), reaching as much as $80 \%$ of the metal-poor stars for metallicities below $[\mathrm{Fe} / \mathrm{H}] \leq-4.0$, albeit the exact frequency has been questioned (Cohen et al., 2005). It is thus clear that there is a relation between the nucleosynthesis in the most metal-poor stars, and perhaps even in Pop III stars, and carbon abundances. With the recognition of the importance of the carbon abundance, Beers and Christlieb (2005) proposed a way to classify the carbon enhanced metal-poor stars (CEMP). The classification is shown in Table 2.3. Originally Beers and Christlieb (2005) classified CEMP stars as those with $[\mathrm{C} / \mathrm{Fe}] \geq+1.0$, and this value was later updated to $[\mathrm{C} / \mathrm{Fe}] \geq+0.7$ by Aoki et al. (2007).

Table 2.3 - Nomenclature of stars based on their carbon enhancement (Beers and Christlieb, 2005), with updated [C/Fe] from Aoki et al. (2007).

\begin{tabular}{lrr}
\hline \hline CEMP & {$[\mathrm{C} / \mathrm{Fe}] \geq+0.7$} \\
CEMP-s & {$[\mathrm{C} / \mathrm{Fe}] \geq+0.7$ and $[\mathrm{Ba} / \mathrm{Fe}]>+1.0$ and $[\mathrm{Ba} / \mathrm{Eu}]>+0.5$} \\
CEMP-r & {$[\mathrm{C} / \mathrm{Fe}] \geq+0.7$ and $[\mathrm{Eu} / \mathrm{Fe}]>+1.0$} \\
CEMP-r $/ \mathrm{s}$ & {$[\mathrm{C} / \mathrm{Fe}] \geq+0.7$ and $[\mathrm{Ba} / \mathrm{Eu}]<+0.5$} \\
CEMP-no & {$[\mathrm{C} / \mathrm{Fe}] \geq+0.7$ and $[\mathrm{Ba} / \mathrm{Fe}]<0.0$} \\
\hline
\end{tabular}

The carbon enhancement origin of each type of CEMP stars differ. CEMP-s stars are associated with binary pairs, in which the extra carbon came from accreting material of a companion that has gone through the AGB phase, where it produced both carbon and sprocess (slow neutron-capture process) elements (e.g., Herwig, 2005; Lucatello et al., 2005; Hansen et al., 2015). CEMP-r stars are metal-poor stars enhanced both in carbon and in elements produced in the rapid neutron-capture process (r-process), and both carbon and r-process material are believed to come from their original birth cloud. CEMP-r/s are CEMP stars with enhancement in both s and r-process elements, and their origin is still unclear (e.g., Yoon et al., 2016); the s-process elements might come from a binary companion and the r-process component from the original birth cloud, or both s- and r-process elements might be accounted from their production in the intermediate neutron- 
capture process (i-process, Roederer et al., 2016; Hampel et al., 2016).

CEMP-no stars are enhanced in carbon but not enhanced in any neutron-capture process elements, and their carbon is the original content from their birth cloud. These clouds were enhanced in carbon and, yet, were very metal-poor, and they are believed to be clouds polluted by original massive first-generation stars (Pop III stars, e.g., Yoon et al., 2016). Thus, the identification of these stars, along with a detailed analysis of their abundance patterns, give us the clues that might allow us to constrain the nature of the first generation of stars and their nucleosynthetic processes.

However, it is often very difficult to correctly assess the carbon abundance in metalpoor stars, because at lower metallicities stars the only available feature is the $\mathrm{CH}$ G-band at approximately $4300 \AA$. Peterson and Sneden (1978) showed that this molecular band in warm $\left(T_{\text {eff }}=6500 \mathrm{~K}\right)$ metal-poor stars might be smaller than $2 \%$ of the continuum level, even when the carbon-to-iron ratio is as high as $[\mathrm{C} / \mathrm{Fe}]>+1.0$. Thus, the recognition of EMP stars with a high carbon-to-iron ratio, can become a big challenge for warm dwarfs, as the $\mathrm{S} / \mathrm{N}$ must be extremely high for a definite detection of the carbon band.

Although we did not analyze the absolute [X/Fe] abundances in Reggiani et al. (2016), we show in Table 2.2 the absolute abundance of all the analyzed elements for G 64-37. The low $[\mathrm{Ba} / \mathrm{Fe}]=-0.44$ dex indicates that there is no enhancement of either s- or r-process elements in this star. In Reggiani et al. (2016) we also analyzed the carbon abundances of G 64-12 and G 64-37 via spectral synthesis of the G-band (see Fig. 3 of Reggiani et al. 2016).

A more specific analysis of carbon was performed in Placco et al. (2016), where the carbon abundances were re-estimated via spectral synthesis, along with a spectral synthesis analysis of barium and strontium abundances, elements that indicate the presence of neutron-capture enhancement. They confirmed low abundances of these elements, along with high carbon $([\mathrm{Sr} / \mathrm{Fe}]=+0.05 \mathrm{dex},[\mathrm{Ba} / \mathrm{Fe}]=-0.35 \mathrm{dex}$, and $[\mathrm{C} / \mathrm{Fe}]=+1.12$ dex for $\mathrm{G}$ 64-37), showing that these are CEMP-no stars and with abundances in concordance with ours within the errors. Our analysis of CD24 showed an extremely low strontium abundance of $[\mathrm{Sr} / \mathrm{Fe}]=-1.27$, along with an estimated carbon abundance of $[\mathrm{C} / \mathrm{Fe}]=1.0 \pm 0.2$ $\operatorname{dex}(\mathrm{A}(\mathrm{C})=6.25)$, also from the molecular CH G-band. Thus, this star is also CEMP-no. We did not detect a clear barium line, but our carbon and the CEMP-no classification are in line with the reported by Jacobson and Frebel (2015). 
The analysis of these three stars, with enough confidence to guarantee that the carbon abundances were detections rather than limits, was only possible due to the high quality of our spectra. The availability of such spectra will greatly improve once the next generation of telescopes (ELT, TMT, GMT) and spectrographs become available.

Dwarf stars had not gone through any evolutionary processes that might have created or destroyed any carbon (at least for the mass range in which we are working, $\mathrm{M} \leq 1 \mathrm{M}_{\odot}$ ), or neutron-capture elements, and the chemical composition of these stars still reflects the original composition of their birth. Thus, they make the best type of sites in which to analyze the composition of the early ISM. In this context, in Placco et al. (2016) we showed an analysis of the possible progenitors of these stars (their masses, explosion energy, and chemical yields), supposing that only one supernova event enriched the ISM from which these objects were born. The take away from this exercise is that if these stars ( $\mathrm{G} \mathrm{64-12}$ and G 64-37) came from a birth cloud polluted by a single supernova, they had the same initial mass and explosion energy (within the errors). We did the same with CD24 and the output plot from STARFIT $]^{4}$ is shown in Figure 2.6. We found that the progenitor of this star, assuming it came from an ISM polluted by a single supernova, had an initial mass of $10.5 \mathrm{M}_{\odot}$, and an exploding energy of $0.3 \times 10^{51} \mathrm{ergs}$, very similar to the progenitors of $\mathrm{G} 64-12$ and $\mathrm{G} 64-37\left(10.7-11.9 \mathrm{M}_{\odot}\right)$. Notice, however, that the error bars in these progenitors can explain the differences from G 64-12 and G 64-37, and CD24 (Figures 2.5 and 2.4), that we observe as signals from different populations. It is important to reinforce that we used STARFIT in the usual configuration, in which the upper limit for the fit is the abundance of Zn, thus it is not possible to conjecture upon the differences between the abundances of the neutron-capture elements of CD24, G 64-12, and G 64-37, specially the extremely low abundance of Sr observed in CD24.

Studies such as these, specially when dealing with CEMP-no stars, heavily rely on the carbon abundance, and it is thus extremely important to use the most accurate abundance as possible. The abundances reported above, as most of the carbon abundances in the literature, are based on spectral synthesis of the molecular $\mathrm{CH}$ band at $\approx 430 \mathrm{~nm}$, with 1D atmospheres. Also, all molecular bands are analyzed with the assumption that LTE holds. Thus, ever since CEMP stars were identified, and later classified, the studies are almost entirely based on 1D LTE analysis.

\footnotetext{
${ }^{4}$ http://starfit.org/
} 


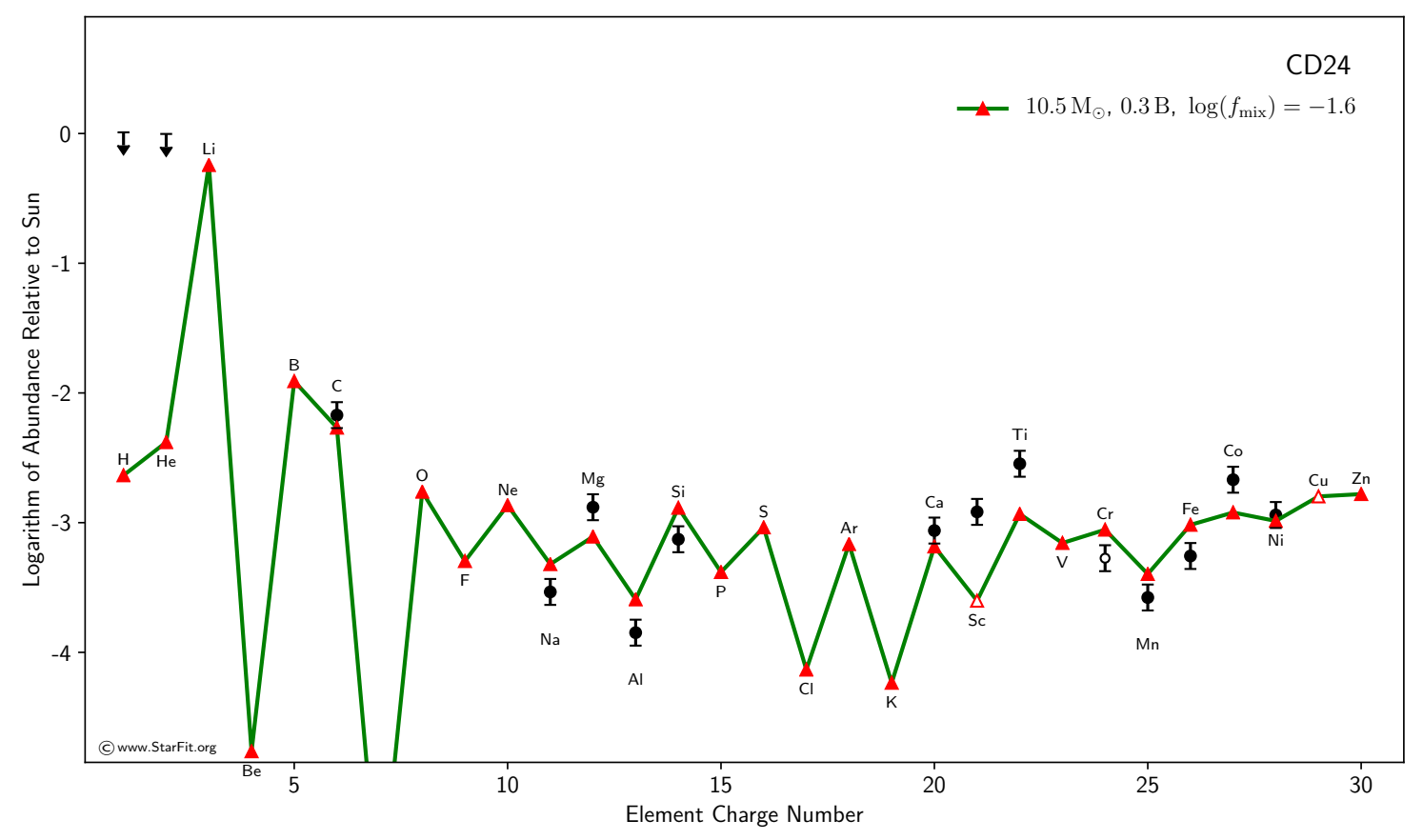

Figure 2.6: STARFIT output showing the progenitor mass and explosion energy of CD24.

There are studies of the 3D effects in the G-band (Gallagher et al., 2016, 2017), but mostly theoretical, and they find that 3D effects might change carbon abundances as much as 1 dex. In a recent work, Amarsi et al. (2019) analyzed up to $7 \mathrm{C}$ I carbon lines in the spectra of 39 stars in 1D LTE, 1D non-LTE , 3D LTE, and 3D non-LTE. For G 64-12, G 6437 , and CD24, their inferred 3D non-LTE carbon abundances were $[\mathrm{C} / \mathrm{Fe}]_{\mathrm{G} 64-12}=0.18$, $[\mathrm{C} / \mathrm{Fe}]_{\mathrm{G} 64-37}=0.10$, and $[\mathrm{C} / \mathrm{Fe}]_{\mathrm{CD} 24}=0.11$, respectively, a considerably lower abundance than the 1D LTE spectral synthesis analysis from the $\mathrm{CH}$ G-band. The first study to systematically apply 3D and non-LTE corrections to the carbon CH G-band is from Norris and Yong (2019). Both these studies show much lower C abundances, and a very high percentage of stars classified as CEMP stars under 1D LTE assumptions, have abundances below the $[\mathrm{C} / \mathrm{Fe}]=+0.7$ threshold, therefore portraying a considerably different picture than previous studies.

The portion of the Miky Way composed by CEMP stars drastically changes when applying the 3D and non-LTE corrections, and conclusions regarding the formation and chemical evolution of our Galaxy can change when using these extremely different methods (1D LTE vs 3D non-LTE ). For example, see the MDF of CEMP stars in Fig. 7 of Norris and Yong (2019). 
In the future, 3D and non-LTE analyses of the $\mathrm{CH}$ band may become available, or at least correction tables for 1D LTE analyses. This will naturally lead to an update in the empirical classification of CEMP stars. Furthermore, the more accurate 3D/non-LTE works will result in better predictions of yields from the first generation stars. If there are drastic changes in the models of Pop III stars, based on revised 3D/non-LTE C abundance, there may be an important revision on the chemical evolution and formation of the Milky Way, the nucleosynthesis of the first elements, and the physical processes that governed the first objects to appear in the Universe. This is something left for future studies on the evolution of carbon in cosmic history.

The discussion in this chapter is mostly based on Reggiani et al. (2016). The paper is attached in Appendix $\mathrm{A}$ and the full discussion, linelists, and abundance tables, can be found there. 
Chapter 3

\section{Chemical evolution of the galaxy}

In Chapter 2, we showed that differential abundances can be used to significantly increase (as much as three times, depending on the quality of the spectra) the precision of the estimated chemical abundances in stars. Here we discuss the implications of applying the technique to a sample of metal-poor halo stars, to study the formation and chemical evolution of the Solar neighborhood halo (inner galactic halo).

\subsection{Formation of the Galactic halo}

Metal-poor stars in the halo of our Galaxy have an excess of $\alpha$-elements in their composition, when compared to the Sun, of $[\alpha / \mathrm{Fe}] \approx+0.4$ dex (e.g., Barbuy, 1988; Gratton et al., 2003; Cayrel et al., 2004; Ryde and Lambert, 2004; Meléndez et al., 2006; Spite et al., 2011; Yong et al., 2013b), that is observed in O, Mg, Si, S, Ca, and Ti. Meanwhile, stars in the thick disk have a lower level of enhancement, and thin disk stars have no sign of $\alpha$-enhancement (Bensby et al., 2014), except for some peculiar stars Adibekyan et al., 2011). Although strictly speaking titanium is not an $\alpha$-element, it is commonly referred to as such due to the similarity in titanium abundance and the abundances of the $\alpha$-elements, but its real nucleosynthetic source is still a matter under debate (e.g., Nomoto et al., 2006; The et al., 2006; Clayton, 2007; Wongwathanarat et al., 2017). As $\alpha$-elements are mainly a nucleosynthetic product of Supernovae type II, when compared to the iron abundance (which is mainly a nucleosynthetic product of type Ia Supernovae), the $[\alpha / \mathrm{Fe}]$ ratio traces the timescales of the chemical evolution and formation of these stars, given that we know how to model the distribution of Supernovae type Ia and type II. Products of more massive stars, and with a shorter lifespan, Supernovae type II started to enrich the ISM earlier than 
Supernovae type Ia.

Thus, if two stars with the same metallicity (iron abundance) have a similar formation history, their level of $\alpha$-enhancement should be similar within measurement errors, while different formation scenarios may be translated into different levels of enhancement. This explains the abundance differences between the halo and thin disk, for example, as the halo is mainly composed by older stars formed when Supernovae type II were more relevant, and thin disk stars belong to a population formed when the ISM already had time to be enriched in material both from Supernovae type Ia and type II.

However, chemical abundances are not the only indicators of different populations and formation scenarios. Carollo et al. (2007) proposed that the Milky Way halo can be divided into two different components: an inner halo with stars that have prograde rotation with velocities up to $50 \mathrm{~km} \cdot \mathrm{s}^{-1}$, closer to the Sun (distances of up to $10-15 \mathrm{kpc}$ ), and a metallicity distribution function $(\mathrm{MDF})$ that peaks at $[\mathrm{Fe} / \mathrm{H}]=-1.6 \mathrm{dex}$; and an outer halo component where stars have a clear retrogade rotation, -40 to $-70 \mathrm{~km} . \mathrm{s}^{-1}$, dominate the region beyond $15-20 \mathrm{kpc}$ and have a MDF that peaks at $[\mathrm{Fe} / \mathrm{H}]=-2.1$ dex. A lot of discussion followed this proposition (e.g., Schönrich et al., 2010; Carollo et al., 2010; Beers et al. 2012), but today it is widely accepted that the Galactic halo can be divided in two components. These two components, an inner and an outer halo are distinguishable in their velocity components, as is seen in Figure 3.1. Such figure shows that there are different components in the histogram of the stellar velocities, where the stars with a more pronounced retrograde rotation are more important at larger distances, associated with the outer halo component, and the stars with prograde velocities dominate for smaller distances, and the different peaks are associated with the inner halo, and with the thick disk. Figure 3.2 shows the same different components using the metallicity distribution function $(\mathrm{MDF})$, where it is clear to see that for larger distances the MDF peaks at $[\mathrm{Fe} / \mathrm{H}]=$ -2.1 , and is associated with the outer halo, and at smaller distances the MDF peaks at $[\mathrm{Fe} / \mathrm{H}]=-1.6$, associated with the inner halo. This indicates that the halo did not form at once and is evidence for the hierarchical formation proposition (e.g., Bullock et al., 2001).

Studying the chemical composition of the inner halo stellar component, Nissen and Schuster (2010) (NS10), and the subsequent studies from Nissen and Schuster (2011); Schuster et al. (2012); Ramírez et al. (2012), and Fishlock et al. (2017), showed that besides the widely accepted components discussed above, there is also a duality of chemical 

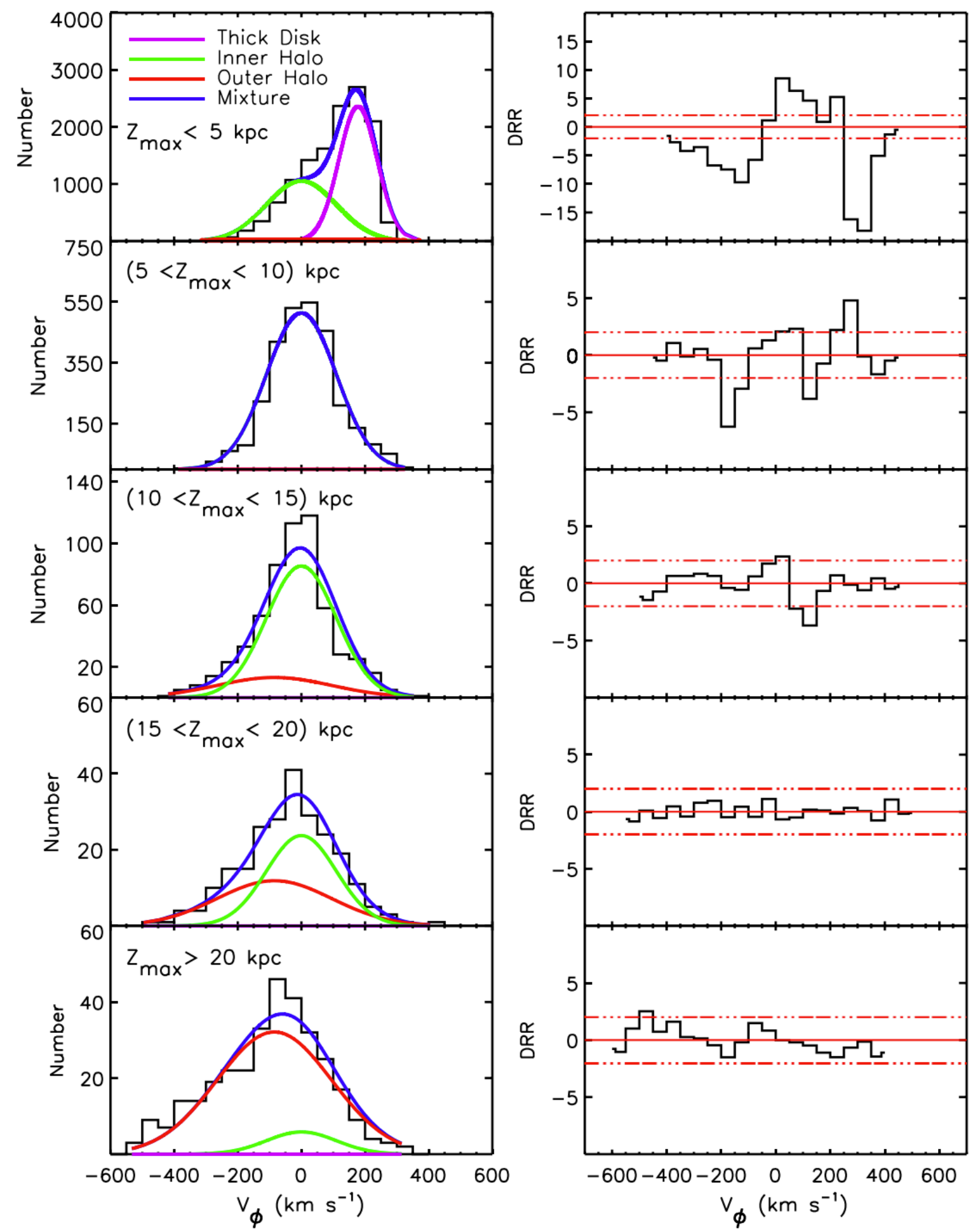

Figure 3.1: Stellar velocity distribution of the inner (green line), outer halo (red line), and thick disk (purple line) for different Galactic heights. Figure extracted from Carollo et al. (2010). 

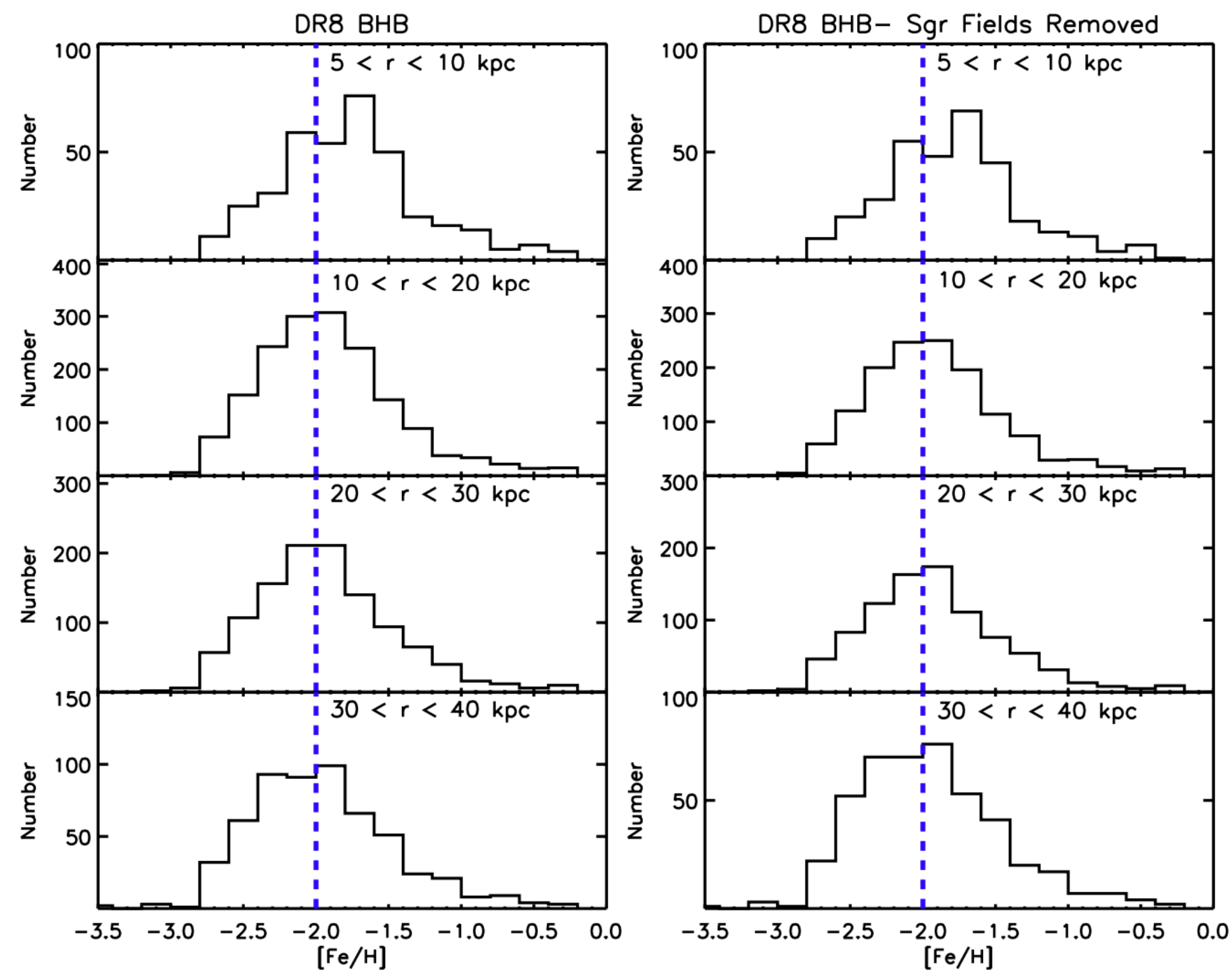

Figure 3.2: Metallicity distribution function of the stellar halo in different Galactic heights from BHB stars with, and without Srg fields removed, at left and right panels, respectively. Figure extracted from Beers et al. (2012). 
abundances within the solar neighborhood halo. In Figure 3.3 we show the main result from Nissen and Schuster $(2010)$ with the distribution of $[\alpha / \mathrm{Fe}]$ as a function of $[\mathrm{Fe} / \mathrm{H}]$ for a sample of stars that reside in close proximity to the Sun.

Note that the metallicity distribution from this sample of stars ends at the mean $[\mathrm{Fe} / \mathrm{H}]$ of the inner halo described above, and has a narrow range in metallicity of $-1.6 \leq[\mathrm{Fe} / \mathrm{H}] \leq$ -0.4 , but there is a clear separation in two well-defined abundance populations. We will refer to these populations from NS10 as the high and low- $\alpha$ populations $([\alpha / \mathrm{Fe}] \approx+0.35$ and $[\alpha / \mathrm{Fe}] \approx+0.20$, respectively). NS10 showed that these two populations are distinguishable in $[\mathrm{Na} / \mathrm{Fe}],[\mathrm{Mg} / \mathrm{Fe}],[\mathrm{Si} / \mathrm{Fe}],[\mathrm{Ca} / \mathrm{Fe}],[\mathrm{Ti} / \mathrm{Fe}]$, and $[\mathrm{Ni} / \mathrm{Fe}]$. It is also possible to distinguish these populations in age space (high- $\alpha$ stars being $2-3$ Gyrs older than the low- $\alpha$ stars) and orbital parameters (Schuster et al. 2012$)$, with high- $\alpha$ stars concentrated in the innermost part of the Galaxy (lower Galactic heights) and less pronounced velocity, and the low- $\alpha$ stars have more pronounced velocity and are located at larger distances (see the velocity components at Figure 3.4). Ramírez et al. (2012) and Fishlock et al. (2017) followed up on the works of NS10 and showed that these populations are also distinguishable via oxygen and scandium chemical abundances.

Before these studies it was widely accepted that stars within the inner (or outer) halo, at a given metallicity, had homogeneous abundance ratios, as the formation scenario expected these stars to be all born from a similarly polluted ISM (either in-situ stars for the inner halo, or acreted stars for the outer halo). This was also accepted because, before Nissen and Schuster (2010), the analysis errors had dominated the spread in abundances; the two populations were only clearly distinguished because the precision in their work reached $\approx$ 0.03 dex, using the differential technique. The conclusion drawn by NS10 and corroborated in later publications is that the low- $\alpha$ stars, concentrated in larger galactic heights, were acreted from dwarf galaxies, while the high- $\alpha$ stars were born in the Milky Way.

This proposition is also supported by the results presented by Suda et al. (2017), that studied the position of the "knee" of the Milky Way and a few neighbor galaxies (Fornax, Sculptor and Draco). The "knee" is a drop in the $\alpha$-element content due to the different timescales between Type Ia and Type II supernovae (e.g., Tinsley, 1979; Matteucci and Brocato, 1990). The timescales of different environments change the position of the knee in the $[\alpha / \mathrm{Fe}]$ vs $[\mathrm{Fe} / \mathrm{H}]$ diagram. Suda et al. (2017) found that the knee of the Milky Way is at $[\mathrm{Fe} / \mathrm{H}]=-1.0$ and that the knee of those smaller galaxies are at $[\mathrm{Fe} / \mathrm{H}] \approx-2.0$. 

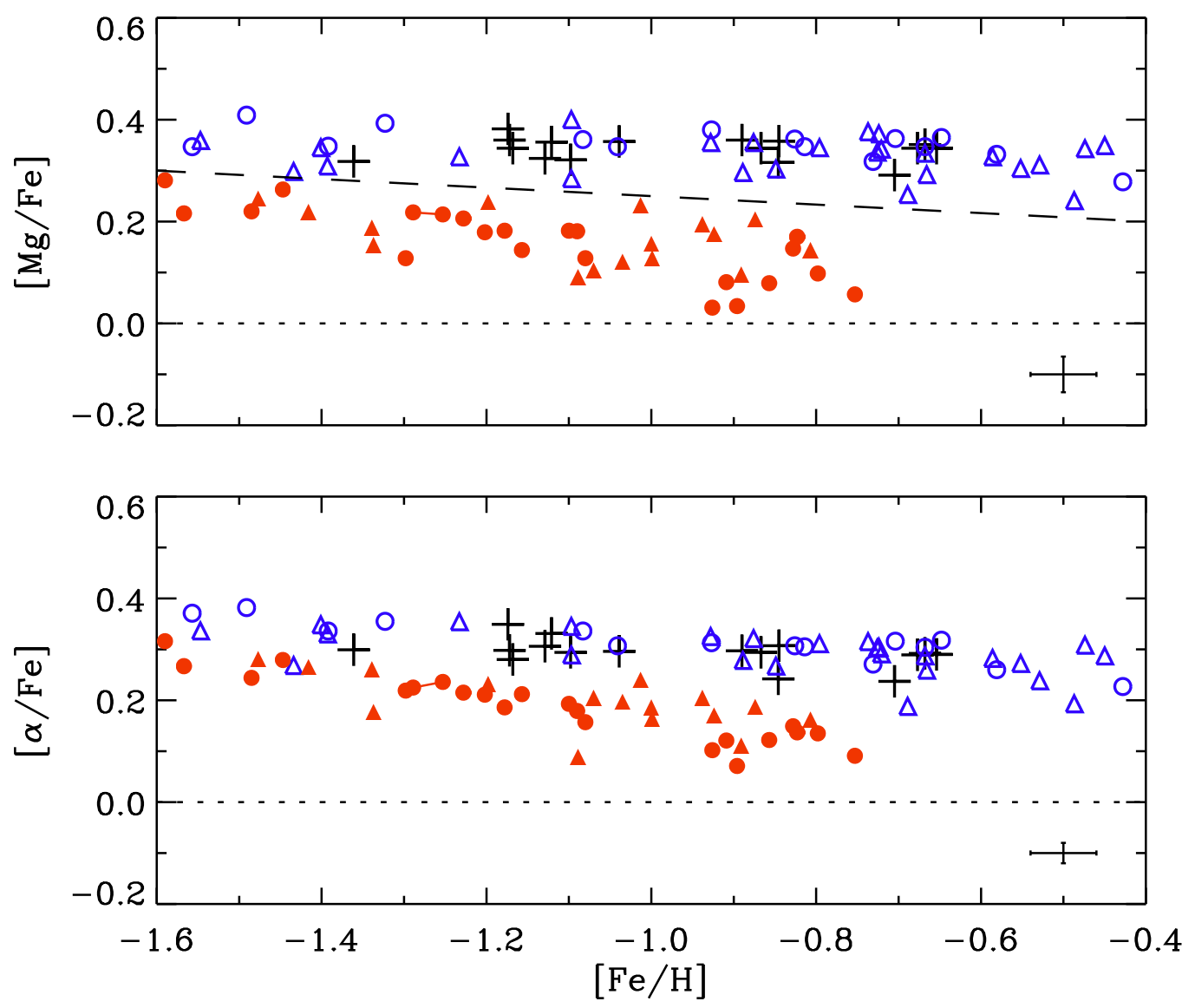

Figure 3.3: Chemical abundances of $\alpha$-elements in the solar neighborhood as a function of metallicity, for thick-disk stars (crosses), high- $\alpha$ halo stars (open blue circles), and low- $\alpha$ halo stars (filled red symbols). Figure extracted from Nissen and Schuster (2010). 


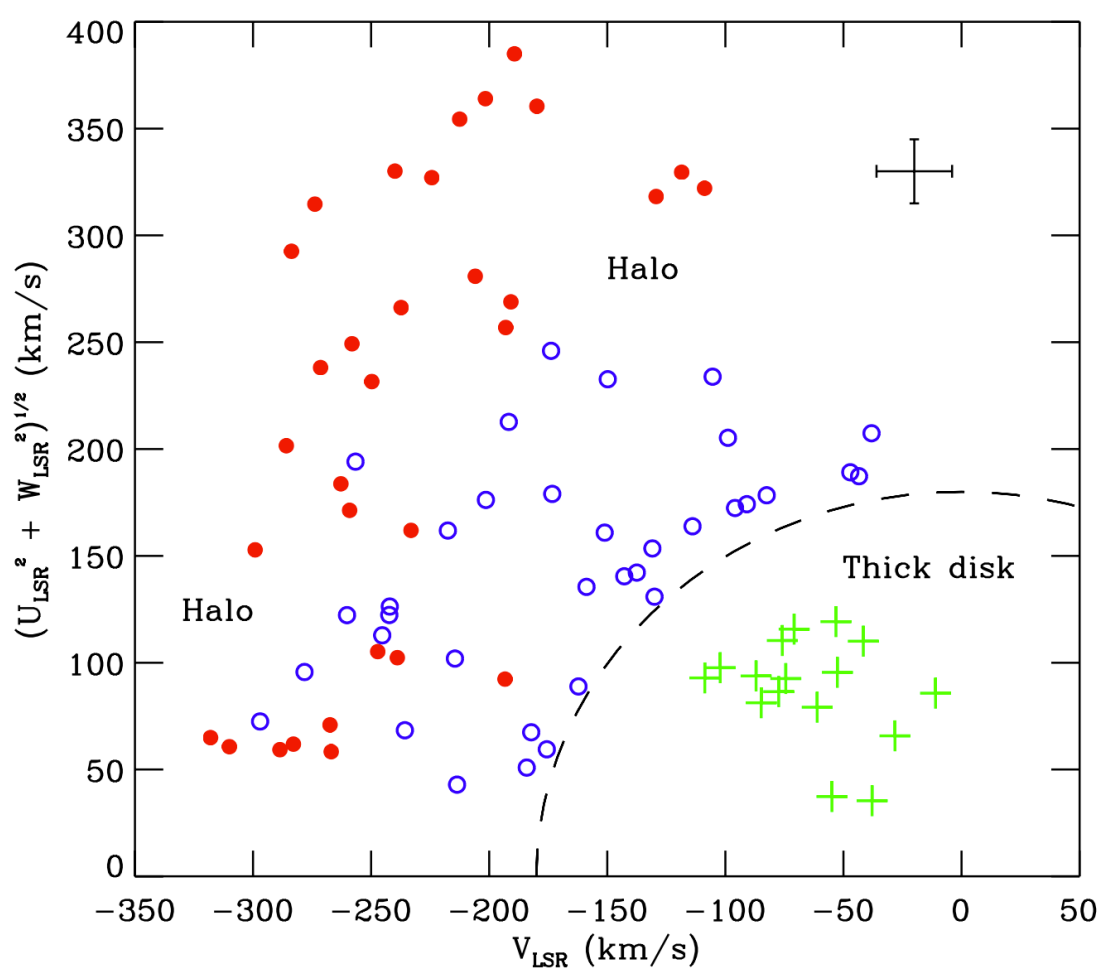

Figure 3.4: Toomre diagram of the high- $\alpha$ (open blue circles) and low- $\alpha$ stars (filled red symbols) populations. Figure extracted from Schuster et al. (2012).

Thus, the low $[\alpha / \mathrm{Fe}]$ stars for $[\mathrm{Fe} / \mathrm{H}]<-1.0$ are unlikely to have formed in the Milky Way.

The chemical studies of NS10 and subsequent works were focused on the same sample with a narrow metallicity range $(-1.6 \leq[\mathrm{Fe} / \mathrm{H}] \leq-0.4)$, at the high metallicity end of the inner halo. As can be seen in Figure 3.3, the focus in these stars make sense, as the separation between the high- and low- $\alpha$ populations get increasingly smaller as the metalicity decreases. Thus, to focus on lower metallicities one must reduce, as much as possible, the measurement errors, and in Section 3.1.2 we show our efforts to extend these previous works to lower metallicity stars.

\subsubsection{Observational data}

Our sample was observed using the UVES spectrograph (Dekker et al., 2000) in the VLT telescope in 2015A (project 095.D-0504(A)). The observed stars were chosen so that we could apply, as best as possible, the differential technique. Thus, we observed stars with previous stellar parameters determinations (although most of the parameters came from lower spectral quality data). Our sample is comprised of stars with $T_{\text {eff }}=6250 \pm 250$ 
$\mathrm{K}, \log g=4.0 \pm 0.5 \mathrm{dex}$, and $-2.8 \leq[\mathrm{Fe} / \mathrm{H}] \leq-1.5$. As we were interested in observing as many stars as possible in the shortest amount of time, still reaching good $\mathrm{S} / \mathrm{N}$ and resolution, all of our stars had magnitudes of $\mathrm{V} \leq 12$, and aiming for a mean $\mathrm{S} / \mathrm{N} \approx 150$, our integration times were up to 2 hours.

The spectra were pre-reduced by the ESO pipeline, and further corrections (barycentric and radial velocity corrections) were done using the IRAF package for python, PyRaf 1 . The normalization of the spectra was done using IRAF. Our final spectra had a resolution of $\mathrm{R} \approx 50000$, with a $\mathrm{S} / \mathrm{N} \approx 130$ at $4000 \AA$ and $\mathrm{S} / \mathrm{N}=250$ at $6000 \AA$, ranging from 3400 to $6800 \AA$.

All the spectra were analyzed via the line-by-line differential technique, both to determine the stellar parameters and to determine the abundances. After an initial analysis and quality assessment of our results, we saw fit to divide the sample in two, to use the differential analysis in narrower ranges of metallicity, and thus chose two standard stars. The standard star for the lower metallicity section in our sample is HD 338529 ( $T_{\text {eff }}=6426 \mathrm{~K}$ from the IRFM, Meléndez et al. (2010), $\log g=4.09$ estimated using GAIA DR1 parallax, and $[\mathrm{Fe} / \mathrm{H}]=-2.29$ and $\xi=1.5 \mathrm{~km} \cdot \mathrm{s}^{-1}$ from our EWs) and CD -4802445 is the standard of the higher metallicity stars in our sample $\left(T_{\text {eff }}=6453 \mathrm{~K}\right.$ from the IRFM, Meléndez et al. (2010), $\log g=4.23$ estimated using GAIA DR1 parallax, and $[\mathrm{Fe} / \mathrm{H}]=-1.96$ and and $\xi=1.5 \mathrm{~km} . \mathrm{s}^{-1}$ from our EWs). The linelist used for this analysis is fully reproduced in Reggiani et al. (2017), which can be seen in Apendix A. The estimated abundances and errors are also given in Reggiani et al. (2017). All the errors of [X/Fe] were estimated as $\sigma=\sqrt{\sigma_{\mathrm{X}}^{2}+\sigma_{\mathrm{Fe}}^{2}}$, with $\sigma_{\mathrm{X}}$ defined as previously shown in equation 2.1 .

We complemented our data with previously measured abundances of dwarf stars with similar stellar parameters from Bonifacio et al. (2009); Andrievsky et al. (2010); Nissen and Schuster (2010, 2011); Schuster et al. (2012); Ramírez et al. (2012), and Fishlock et al. (2017).

\subsubsection{Very metal-poor stars in the solar neighborhood halo}

In Figure 3.5, we show the chemical abundances of our sample of stars for the same elements originally analyzed in NS10. The scatter, mean values, and mean errors, of each of the analyzed elements are shown on top of each panel.

\footnotetext{
${ }^{1}$ PyRAF is a product of the Space Telescope Science Institute, which is operated by AURA for NASA
} 
At the metal-poor end of the NS10 sample we see the abundance differences between the stars of the two populations decreasing, and as the NS10 sample merges with our data there is only a single discernible population at $[\mathrm{Fe} / \mathrm{H}] \lesssim-1.6$. The single population continues into the lower metallicity stars of Bonifacio et al. (2009) and Andrievsky et al. (2010). Within the scatter of our sample, as low as 0.03 dex for $[\mathrm{Ni} / \mathrm{Fe}]$, there is only one recognizable population in the very metal-poor (VMP) regime of the solar neighborhood halo. This indicates that, accepting the proposition from NS10 and the following studies, a merger event responsible for the accretion of stars in the inner halo brought a new stellar population that can only be differentiated, in chemical space, from Milky Way halo stars for metallicities $[\mathrm{Fe} / \mathrm{H}] \gtrsim-1.6$.

Other recent studies on formation of the structures in the Milky Way (e.g., Belokurov et al., 2018; Iorio and Belokurov, 2019) studied the shape of the galactic halo using GAIA DR2 RR Lyrae stars. They showed evidences to support that the inner halo was formed not by several minor accretion events, but by a single massive merger event.

A single massive merger forming the bulk of the inner halo is a better alternative to explain the NS10 populations than multiple smaller merger events, as in the later we should expect multiple stellar populations, with different enrichment histories, being acreted, and we should observe a highly scattered population in $[\alpha / \mathrm{Fe}]$ space instead of two well-defined populations. Thus, going forward with the massive merger alternative, the VMP stars $([\mathrm{Fe} / \mathrm{H}] \leq-2.0)$ of the acreted galaxy must have had a similar enrichment history as those born in-situ (as we can not differentiate them). From the above conclusions, we can argue that the knee of the galaxy that merged with ours was at $[\mathrm{Fe} / \mathrm{H}] \geq-2.0$, as if it was at lower metallicities we would also observe two populations in our VMP stars. There is a higher scatter in the lower metallicity stars, which are from the "First Stars" project (Bonifacio et al., 2009), but, unlike NS10 and our samples, their sample is composed primarily by outer halo stars.

Although there is no clear correlation between the metallicity position of the knee and the total mass of the galaxies in which the knee was measured, Suda et al. (2017) interpret the knee position correlation as either a result of the fact that supernovae type II ejecta not being retained in galaxies with smaller potential wells, or due to a weak star formation rate. Both explanations, however, lead us to assume that a larger galaxy might have a knee at higher metallicities, which is also in line with the major merger scenario from Iorio 
and Belokurov (2019) and with the observed behavior in our data.
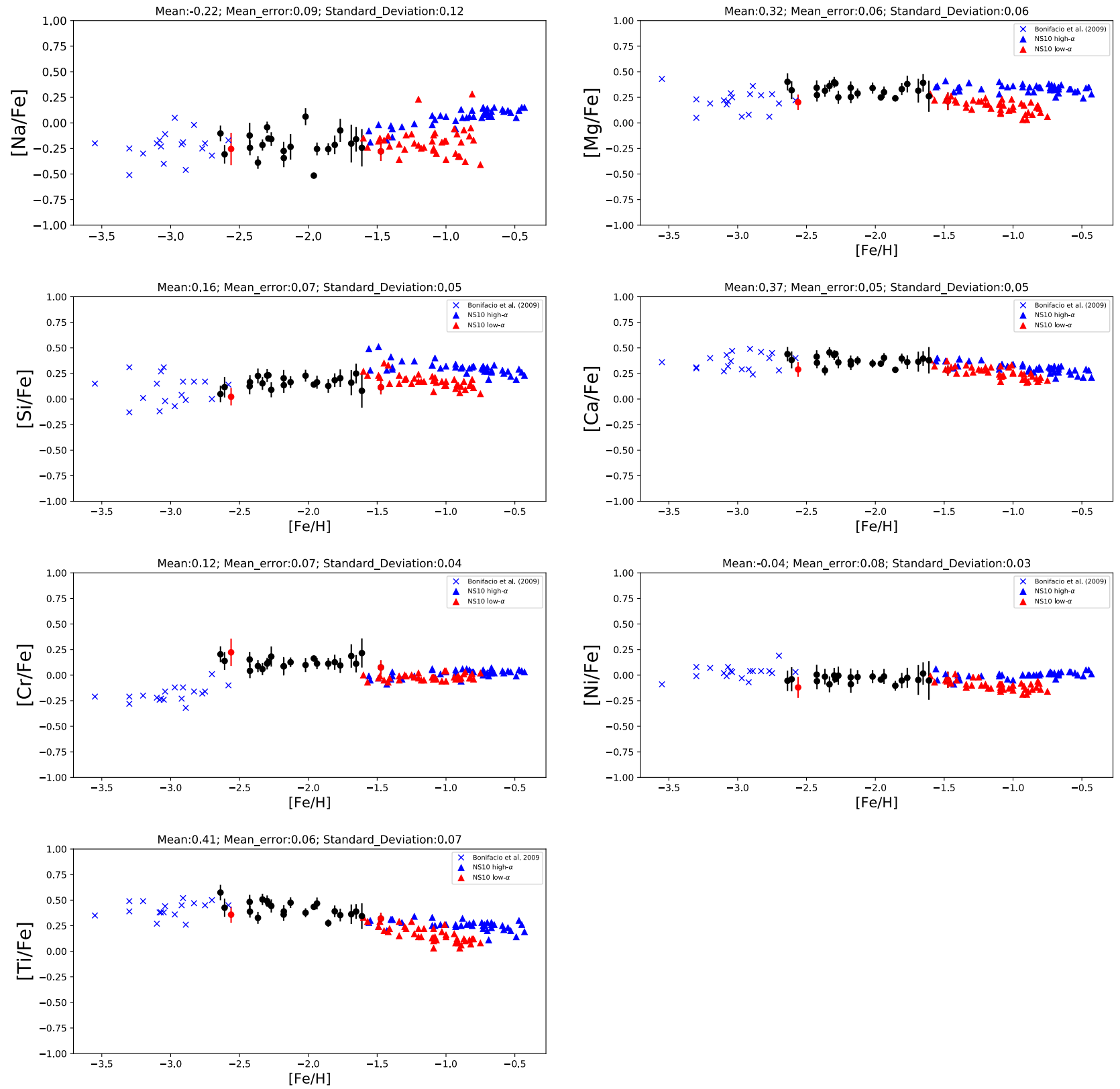

Figure 3.5: Chemical abundances of $\mathrm{Na}, \mathrm{Mg}, \mathrm{Si}, \mathrm{Ca}, \mathrm{Ti}, \mathrm{Cr}$, and $\mathrm{Ni}$ as a function of $[\mathrm{Fe} / \mathrm{H}]$. The black dots are our measured stars, red dots are two Blue Straggler stars we found in our sample, blue and red triangles are the high- $\alpha$ and low- $\alpha$ populations from NS10, and the blue crosses are stars from Bonifacio et al. (2009) and Andrievsky et al. (2010).

The lack of different populations at lower metallicities, along with the two well-defined populations from NS10 for higher metallicities, are evidences of the stellar component that support the single massive merger event proposition (e.g., Iorio and Belokurov, 2019). In this scenario, the stars with $[\mathrm{Fe} / \mathrm{H}]$ lower than the knee of a supposed merging galaxy had 
a very similar, within the observed scatter, chemical evolution as those born in the Milky Way. Thus, assuming that lower $[\mathrm{Fe} / \mathrm{H}]$ stars in the inner halo are a mixture of acreted and born in-situ stars, and as the chemical patterns are very similar, the star formation history (SFH) of both the acreted galaxy and of the galactic halo must had been similar. One might even go further and argue that it is possible that the mass of the accreted galaxy was similar to the mass of the star forming regions of the galactic halo.

Given that this lower $[\mathrm{Fe} / \mathrm{H}]$ stars are very homogenized, our sample can also be used to study Galactic chemical evolution, by comparing our abundances with the outcomes of Galactic evolution models. In Reggiani et al. (2017), we compared a chemical evolution model widely used in the literature with our data.

\subsection{The Galactic Chemical Evolution model}

To study the galactic chemical evolution (GCE) of the solar neighborhood halo, we compared our precise data to the chemical evolution model first presented in Kobayashi et al. (2006), updated in Kobayashi et al. (2011) (K11 model) and finally updated in Zhao et al. (2016) and Sneden et al. (2016), the K15 model. We will briefly go through the main features of the model here, but the main descriptions can be seen in Kobayashi et al. (2011) and Zhao et al. (2016); Sneden et al. (2016). The K11 and K15 models are one-zone models, where instantaneous mixing is assumed. This assumption is likely a good approximation for metallicities $[\mathrm{Fe} / \mathrm{H}] \gtrsim-2.0$, but the inhomogeneity of the ISM when lower metallicity stars formed $([\mathrm{Fe} / \mathrm{H}] \lesssim-2.5$, i.e., the outer halo) would be better described by a chemodynamical model (where the chemical and dynamical evolution are tracked simultaneously). Like in K11, the K15 model used the initial mass function (IMF) description from Kroupa (2001, 2008). It is an IMF that assumes a power-law mass spectrum $\Theta \propto m^{-\alpha}$ (much like the Salpeter (1955) IMF) with $\alpha$ varying for different mass ranges: $\alpha=1.3$ for $0.5 \mathrm{M}_{\odot} \leq \mathrm{M} \leq 50 \mathrm{M}_{\odot}, \alpha=0.3$ for $0.08 \mathrm{M}_{\odot} \leq \mathrm{M} \leq 0.5 \mathrm{M}_{\odot}$, and $\alpha=-0.7$ for $0.01 \mathrm{M}_{\odot} \leq \mathrm{M} \leq 0.08 \mathrm{M}_{\odot}$. In Figure 3.6, we show the MDF of the solar neighborhood and its prediction with different IMFs. We can see that both the Salpeter (1955) and the Kroupa (2008) IMFs are good descriptions for the solar neighborhood stars, with the Kroupa (2008) IMF being slightly better.

In the K11 and K15 models, the chemical enrichment is modelled by adding the contri- 


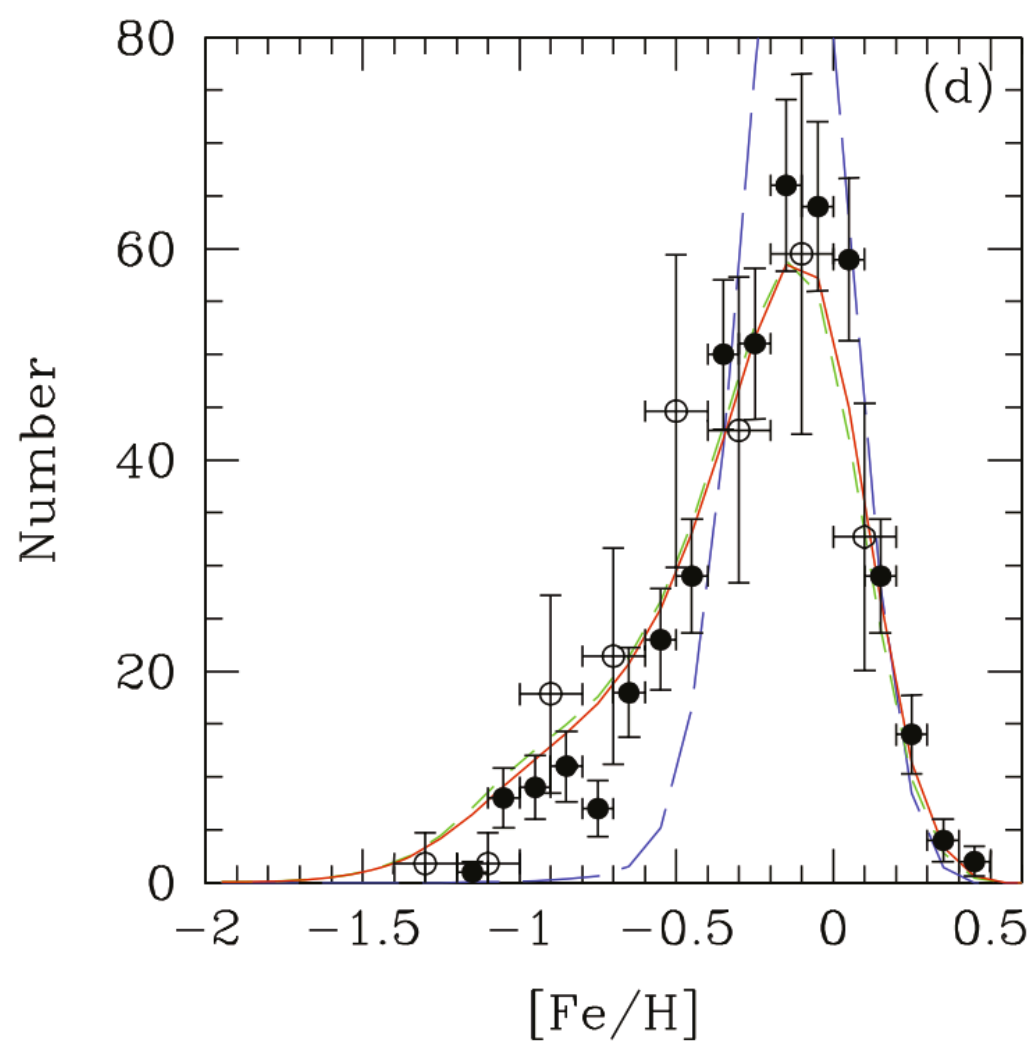

Figure 3.6: Metallicity distribution function of the Solar neighborhood stars and associated predictions with different IMF assumptions. Solid line is the prediction with the Kroupa IMF, the short dashed line with the Salpeter IMF, and long dashed line is the prediction with the Chabrier (2003) IMF. Figure extracted from Kobayashi et al. (2011). 
butions from stars of all mass ranges, as a function of initial masses and metallicities of the stars. The chemical enrichment is given by the yields from multiple sources: Core-collapse supernovae and Hypernovae (core-collapse supernovae with explosion energies of up to 10 times higher than regular supernovae), for initial stellar masses ranging from $M=13 \mathrm{M}_{\odot}$ to $\mathrm{M}=40 \mathrm{M}_{\odot}$. We show an example of their yields in Figure 3.7. Type Ia supernovae ( $S N e I a$ ) is included via the single-degenerate scenario: the white dwarf mass grows via accretion of hydrogen-rich material from a binary companion (a main-sequence star, where the time-scales are $\sim 0.1-1$ Gyrs, or a red giant, lifetimes of $\sim 1-20$ Gyrs). Stellar winds are another source of ISM enrichment, added through the return of the pre-existing heavy elements in the stellar envelope for all stars with masses $\mathrm{M} \gtrsim 1 \mathrm{M}_{\odot}$. Yields of asymptotic giant branch $(\mathrm{AGB})$ stars are added for stars with initial masses of $1 \mathrm{M}_{\odot} \leq \mathrm{M} \leq 6.5 \mathrm{M}_{\odot}$. The yields from super-AGB stars are also added (stars with masses between 8 and 10 $\mathrm{M}_{\odot}$ ). The yields in K11 and K15 also include different isotopic ratios. Although we do not analyze the isotopic ratios in this work, they are a powerful tool to constrain which are the main sources of chemical enrichment in the Galaxy (e.g., Barbuy et al., 1987; Meléndez and Cohen, 2007; Carlos et al., 2018), and also can give light in other problems such as stellar evolution (e.g., Harris and Lambert, 1984; Briley et al., 1997), planet engulfment (Ghezzi et al., 2009) and lithium in metal-poor stars (Smith et al., 1993; Asplund et al., 2006; Lind et al., 2013).

The yields do not include pair-instability supernovae (PISNe, stars with $100 \mathrm{M}_{\odot} \lesssim \mathrm{M} \lesssim$ $300 \mathrm{M}_{\odot}$ that greatly enrich the ISM with heavy metals such as Fe and S), as the expected signature of PISNe had not been observed in metal-poor stars at the time, and also do not include yields from rotating massive stars (although included in one model at Kobayashi et al. (2011), the main model we used in our work did not include these objects). Other enrichment sources not included are magneto-rotational supernovae, neutron star mergers, neutrino driven winds, and electron capture supernovae.

In K11, and K15, the star formation rate (SFR) is proportional to the gas fraction and described by $\frac{1}{\tau_{S}} f_{g}$. Infall of primordial gas is considered, and the ratio differs for the solar neighborhood and for the other components in the Galaxy. Outflow is included for the halo so that it is proportional to the SFR. The SFR in the model is parameterized so that the MDF of the solar neighborhood is reproduced by the model, as it is one of their better constrains. The MDFs of the solar neighborhood, from model and observations, are shown 


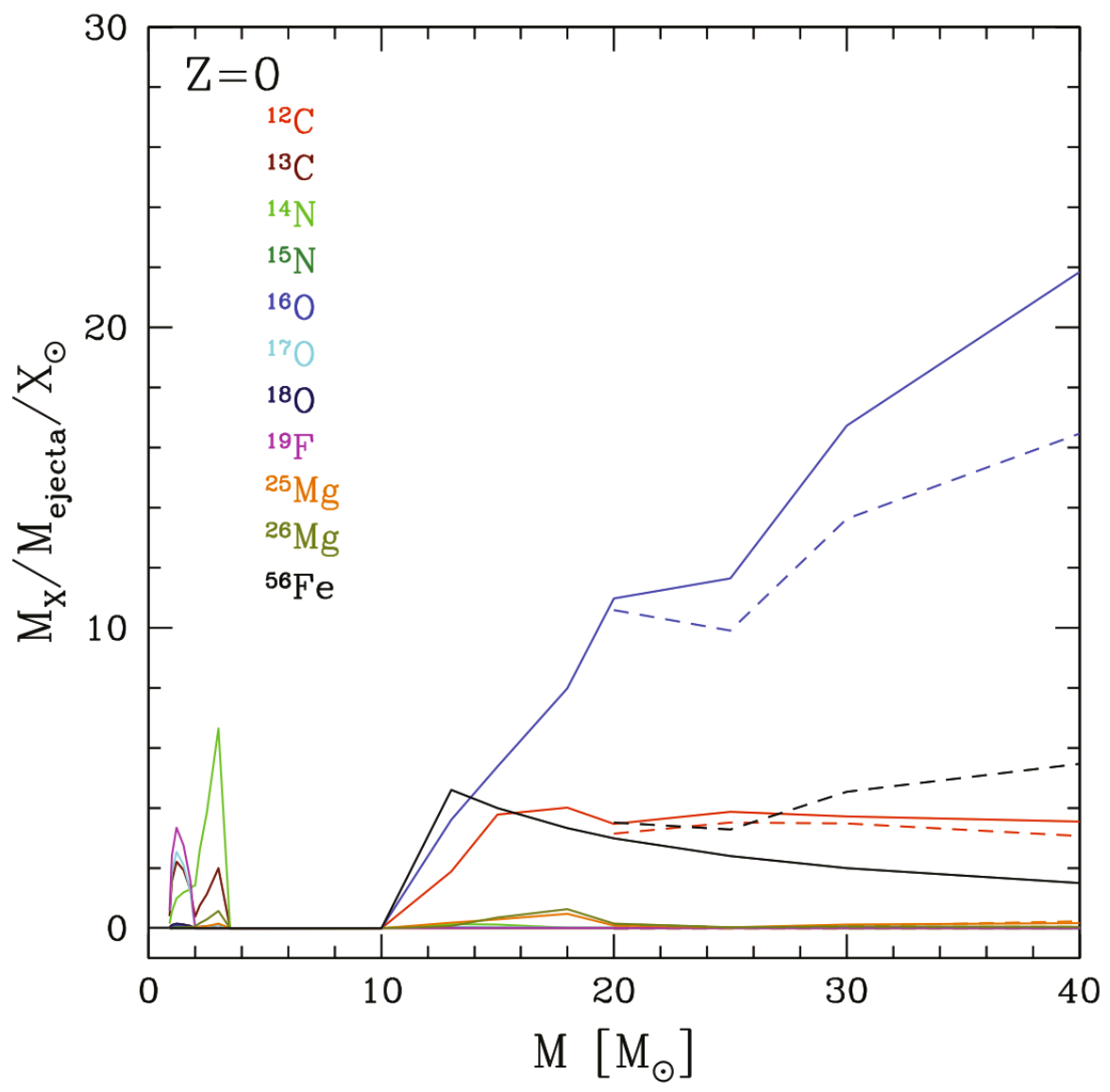

Figure 3.7: Example of supernovae yields as a function of initial stellar masses, for a initial stellar metallicity of $\mathrm{Z}=0$. Figure extracted from Kobayashi et al. (2011). 
in Figure 3.6, and the good fit is indicative of a reliable parametrization.

The output of the models are curves of the Galactic chemical evolution of each element, up to zinc. The K11 and K15 models do not include s-process and r-process enrichment, but an in-depth analysis of the chemical enrichment and enrichment sources of r-process elements can be seen in Haynes and Kobayashi (2019).

\subsubsection{Model vs Observations}

It is difficult to constrain the GCE curves obtained from these models. There are a multitude of different measurements in the literature, with different measurement methods, via stars in different evolutionary stage. In Figure 2 of Reggiani et al. (2017), we showed how different measurements of $[\mathrm{Mg} / \mathrm{Fe}]$, taken from the SAGA ${ }^{2}$ database (Suda et al. 2008, 2011, 2017), are unable to be used as a powerful constraint of the chemical evolution of this element $([\mathrm{Mg} / \mathrm{Fe}])$ because the spread in the data is too large. On that figure we have only chosen data from stars that are similar to those in our work (same range in stellar parameters, see Section 3.1.1), but in order to show that the situation can be even worse we show in Figure 3.8 the spread of $[\mathrm{Ni} / \mathrm{Fe}]$ as a function of metallicity.

In Figure 3.8 we show the entire outcome of the SAGA database, where the different colors represent stars of different evolutionary status, and different classification based on their carbon abundance, and shows over 1615 abundances. The spread we see in the [Ni/Fe] ratio, at Figure 3.8, is as large as about 1 dex. Such largely scattered data can not be used to constrain any model results. We show nickel abundances because it is one of the most well-constrained abundances of all our sample (see Figure 3.5, or Figure 5 of Reggiani et al., 2017), with a standard deviation of only $0.03 \mathrm{dex}$, and our data showed that the chemical enrichment of nickel can be very well explained with the K15 model and the assumptions therein, which we would not be able to do with the data on Figure 3.8. This big spread due to different analysis is observed for any and all elements. In Hinkel et al. (2016), there is an in depth analysis of this fact by analyzing the abundance determinations of a same sample by different groups, employing different analysis techniques.

In this context, we can apply the data described in Section 3.1.1 and the very precise measurements we made, using the line-by-line differential abundance technique, to characterize and constrain the chemical evolution of the solar neighborhood halo by comparing

\footnotetext{
${ }^{2}$ http://sagadatabase.jp/
} 


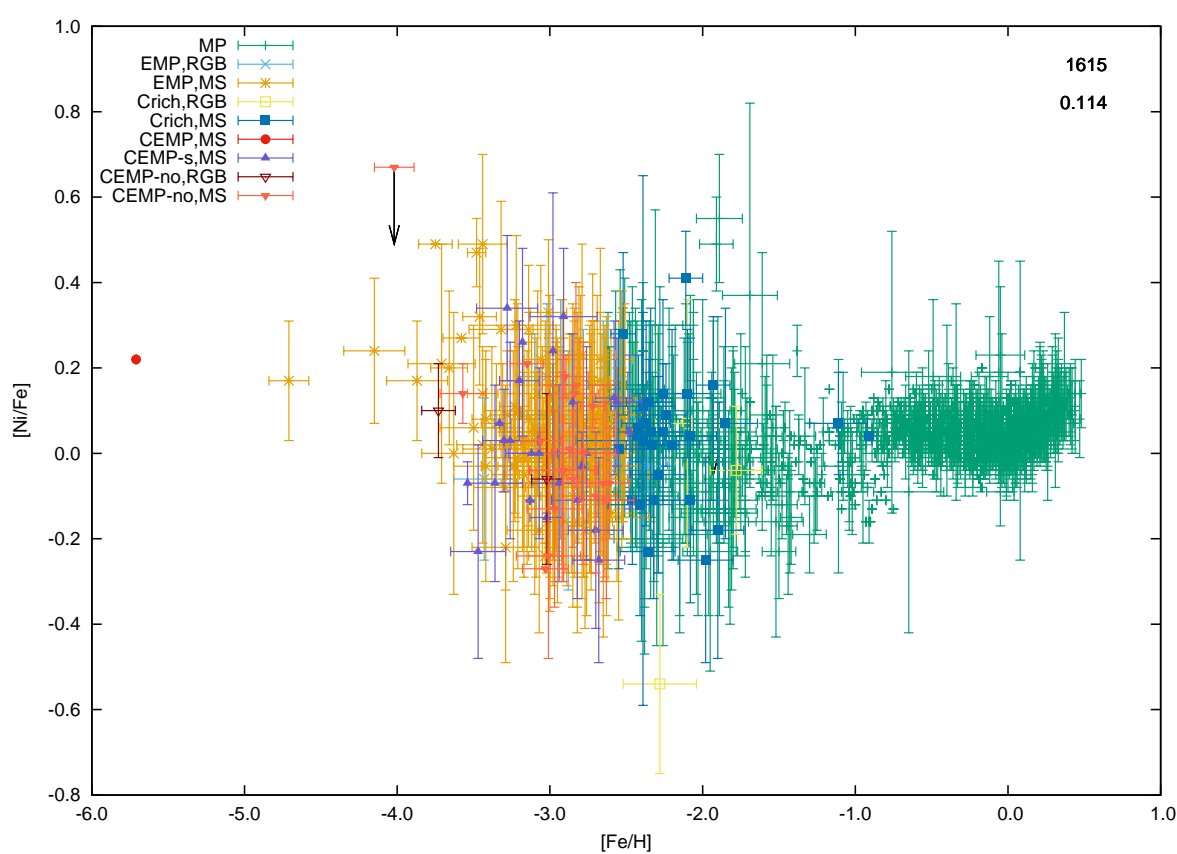

Figure 3.8: Nickel abundances as a function of metallicity. Different colors represent stars in different evolutionary status and different classification based on the carbon abundances. Output plot from the SAGA database.

our abundances with the outcome of the K15 GCE model.

In the context of the halo formation, as there are no evidences of different populations in our sample, we assume that the SFH of the merger event and of the galactic halo are similar, so that the stars observed in this work have all evolved with an ISM similarly enriched. Either that, or all our stars were born in-situ, and the major merger that formed the inner halo is mostly responsible for bringing stars of higher metallicity into the solar neighborhood (the NS10 low- $\alpha$ population). In both cases, the conclusions drawn from the analysis below have the same impact in the history of the chemical evolution of our Galaxy.

\subsection{Constraining GCE with line-by-line differential abundances}

We estimated the chemical abundances of $\mathrm{Na}, \mathrm{Mg}, \mathrm{Al}, \mathrm{Si}, \mathrm{Ca}, \mathrm{Sc}, \mathrm{Ti}, \mathrm{V}, \mathrm{Cr}, \mathrm{Mn}, \mathrm{Fe}$, $\mathrm{Co}, \mathrm{Ni}, \mathrm{Zn}, \mathrm{Sr}, \mathrm{Y}, \mathrm{Zr}$, and Ba. We divided our analysis of all these elements into four: the analysis of the even- $\mathrm{Z}$ light elements, composed by $\mathrm{Mg}, \mathrm{Si}, \mathrm{Ca}$, and $\mathrm{Ti}$, which are the $\alpha$-elements discussed in Section 3.1.2, in the context of the inner-halo formation; the odd-Z 
light elements, composed by $\mathrm{Na}, \mathrm{Al}$, and $\mathrm{Sc}$; the iron-peak elements, V, Cr, Mn, Co, Ni, and Zn (and iron through the metallicity of the stars); and the heavy elements, Sr, Y, $\mathrm{Zr}$, and $\mathrm{Ba}$, all of which mainly trace the evolution of the s-process. Although barium is known to have an r-process component, with $15 \%$ of the solar system Ba coming from the r-process (McWilliam, 1998), and with indications of an r-process component for Sr and $\operatorname{Zr}$ (Battistini and Bensby, 2016), these elements are mainly associated with the s-process.

The full discussion is in Reggiani et al. (2017), but we highlight the main results here. Shown in Figure 3.9 are the abundance ratios $([\mathrm{X} / \mathrm{Fe}])$, as a function of metallicity, of chemical elements that are used to draw some of the most important conclusions, and are explained below. As a general overview of all the chemical elements up to Zn, we found that the abundances are very homogeneous. The high degree of homogeneity in our data suggests that, for the solar neighborhood, the instantaneous mixing assumption is a good approximation. For the lower metallicity stars (Bonifacio et al., 2009; Andrievsky et al. 2010) the scatter is larger but they are not contained within the solar neighborhood.

The model overproduces the $\alpha$-elements $[\mathrm{Mg} / \mathrm{Fe}]$ and $[\mathrm{Si} / \mathrm{Fe}]$. The discrepancy between models and observations for these two elements is also seen in other works (e.g., Zhao et al. 2016), even in a non-LTE analysis. Both our $[\mathrm{Mg} / \mathrm{Fe}]$ and $[\mathrm{Si} / \mathrm{Fe}]$ ratios agree well with other works of halo stars that extend to the metallicity range of our interest, and the reliability of our abundances is further addressed in the paper. On the other hand, we see that the model underproduces titanium, with an abundance difference of $\approx 0.4$ dex, which can not be reconciled by possible non-LTE effects in the abundance analysis. This discrepancy could be partially reduced by further enhancing Ti production in jet-like explosions (Kobayashi et al., 2006). Inducing the enhancement of Ti might, however, further increase the abundance of $\mathrm{Mg}$, Si and Ca. Of all the $\alpha$-elements, the best match between models and observations is for calcium. Calcium production can be very well reproduced by the K15 model, thus the yields from core-collapse supernovae (the main contributor for $\mathrm{Ca}$ ) are well-constrained for this element (to see all the $\alpha$-elements abundance ratios, we refer the reader to Figure 2 of Reggiani et al., 2017).

The odd-Z elements (to see all the odd-Z elements abundance ratios, we refer the reader to Figure 4 of Reggiani et al., 2017) $\mathrm{Na}$ and $\mathrm{Al}$ are highly affected by non-LTE effects, and we tried to correct the abundances as best as possible. Sodium measurements are mainly based on the resonant $5889 \AA$ and $5895 \AA$ lines, and we corrected all abundances in a star- 
by-star basis using the abundance correction grid from (Lind et al., 2011), available at the INSPECT ${ }^{3}$ project. Without the non-LTE corrections our results change drastically, and it is not possible to reconcile neither measurements of the different sources, nor observations and model. The Al I line is also affected by non-LTE effects but, unlike sodium, there was not a source available to individually correct all abundances, and we chose to correct all our stars by +0.65 dex, following the main corrections in Andrievsky et al. (2008) for similar objects. Both $\mathrm{Na}$ and $\mathrm{Al}$ abundances show a small disagreement between model and observations, both higher at the more metal-rich end of the sample. The observed increase in abundance with increasing metallicity is seen in the model but with a smaller slope. For aluminum it would be better if we had access to a full grid of non-LTE corrections, which may be able to decrease the model/observations discrepancy.

The most prominent discrepancy between model and observations for all elements was that found for scandium. It is difficult to assess where the observed discrepancy originates. For all stars with $[\mathrm{Fe} / \mathrm{H}] \geq-1.6$, the $[\mathrm{Sc} / \mathrm{Fe}]$ ratio is in good agreement with the model prediction, but all lower metallicity stars present a much higher Sc abundance. The data from Fishlock et al. (2017), the NS10 sample, were analyzed via spectral synthesis, while our data came from the curve of growth analysis (EW). Although a small difference might come from different measurement methods, this is unable to explain such large discrepancy, specially given that the data from Bonifacio et al. (2009) has as high abundances as ours. We tested for problems in our EW measurement by analyzing the abundance of Sc in stars of higher metallicity (including one that is on the NS10 sample) via EW of the same lines, (see Reggiani and Meléndez, 2018), and the abundances are consistent with the results of Fishlock et al. (2017). Thus, this "jump" in abundances is not caused by our measurement method. Based on the data presented in Zhao et al. (2016), we believe that this is a nonLTE effect, given that their discrepancy between lower and higher metallicity objects is smaller. Non-LTE effects might partially drive the model/observations discrepancy, while $\nu$-induced nucleosynthesis (not included in the K15 model) might be the key to overcome the remaining difference (Kobayashi et al., 2011).

From the iron peak elements (all iron peak elements abundance ratios are shown in Figure 5 of Reggiani et al., 2017) we call the attention to the difference between Cr I and Cr II measurements. The results from Cr I have, much like in Sc, a "jump" in the

\footnotetext{
${ }^{3}$ http://inspect-stars.com/
} 
abundance ratio at $[\mathrm{Fe} / \mathrm{H}] \approx-2.0$, while abundances based on $\mathrm{Cr}$ II are very flat, one of the most homogeneous abundances, with a standard deviation of only 0.04 dex. The additional data does not agree very well with Cr II, and they are more in line with our Cr I estimates (although not entirely), which is expected given that both Bonifacio et al. (2009) and Nissen and Schuster (2010) measured the abundances via Cr I lines. Neutral cromium suffers from strong non-LTE effects due to overionization from low-excitation odd Cr I levels (Bergemann and Cescutti, 2010), and it is thus not reliable to compare LTE estimates of $[\mathrm{CrI} / \mathrm{Fe}]$ with the model results. Thus, we compare the model to the $\mathrm{Cr}$ II estimates, and find that chromium is one of the most well-constrained elements in our analysis.

The downward trend of Co and Zn with metallicity, is also a commonly observed feature (e.g., Cayrel et al., 2004; Kobayashi et al., 2006; Bonifacio et al., 2009; Zhao et al. 2016) that is not reproduced by chemical evolution models. As mentioned before, K15 is a one-zone model and part of the trend observed in Co and Zn (and also the flat correlation of $[\mathrm{Ni} / \mathrm{Fe}])$ could be partially explained by a chemodynamical model, where the inhomogeneties of the ISM can be better traced (Kobayashi and Nakasato, 2011). The $[\mathrm{Co} / \mathrm{Fe}]$ and $[\mathrm{Zn} / \mathrm{Fe}]$ are also indicative of inhomogeneous enrichment from hypernovae (as both Co and Zn nucleosynthesis are very dependent on explosion energy).

The K15 GCE model does not analyze the neutron-capture elements. The heavy elements in our data are mainly produced in the s-process (Busso et al., 1999; Karakas et al., 2014), mostly synthesized in low-mass AGB stars (Busso et al., 2001). At low metallicities there is also an important contribution from fast rotating massive stars (Pignatari et al. 2010; Frischknecht et al., 2016), and their yields are observed as cosmic scatter for more metal-poor stars, as they formed when the ISM was not mixed enough.

We found that the neutron-capture elements are much more scattered than the remaining of the sample, a scatter as high as 0.27 dex for Ba, which might be due to the production of barium both via s- and r-process. In the solar system $85 \%$ of the barium abundance comes from s-process and the remainder is from the r-process (McWilliam, 1998), but more metal-poor stars might have different contributions from each neutroncapture processes and, at least partially, the different contributions might explain the large scatter. The element with the next largest scatter in our sample is strontium, which has been previously shown to have a large spread (McWilliam, 1998; Cayrel et al., 2004; 
François et al., 2007; Bonifacio et al., 2009), and is possibly also produced via r-process (Battistini and Bensby, 2016). That being the case, the spread could be understood in much the same way as the spread in Ba. Yttrium and zirconium are not as scattered as the other heavy elements, but are still much more scattered than the remaining chemical elements, an indicative that s-process elements have important contributions from different enrichment sources (e.g., AGB stars, supernovae type II).

Due to the large dispersion, caused by the diverse nucleosynthetic origins, the linear regression we used to describe the elements up to $\mathrm{Zn}$ is not suitable to understand the cosmic scatter of the neutron-capture elements. Thus, the lines used to describe the dispersion are a non-parametric regression of our data set. One of the most important results is that in the more metal-rich end, where the ISM is better mixed, the non-parametric regressions are closer to the linear regressions. This shows that in earlier cosmic history the sources of enrichment of neuron-capture elements were not as homogeneized as the other elements. From this, we can rule out supernovae type II as the main source of enrichment for s-process elements. If they were the main source, the scatter would have to be much smaller, similarly to the $[\alpha / \mathrm{Fe}]$ abundances.

With our small errors, we were able to study the chemical evolution and formation of the galactic halo by showing that the bulk of the inner halo stellar component, described by our unevolved dwarfs, is composed of stars with a high degree of homogeneity, and we were also able to constrain the chemical evolution by comparing our data to a GCE model. We also drew conclusions about the spectroscopic analysis of different elements (Sc, $\mathrm{Cr}$ ), and about the main enrichment sources of s-process elements.

In the next section we explore the $\mathrm{Li}$ abundance in our sample of stars, adding to our previous measurements the abundances of a larger, complementary, sample of lithium abundances.

\subsection{Lithium and the Spite plateau}

Our lithium abundances have been corrected for non-LTE effects (Lind et al., 2009), via the interface of the INSPECTI project. Our narrow range in metallicity allowed us to measure with precision the position of the Spite plateau (Spite and Spite, 1982) as

\footnotetext{
${ }^{4}$ http://inspect-stars.com/
} 

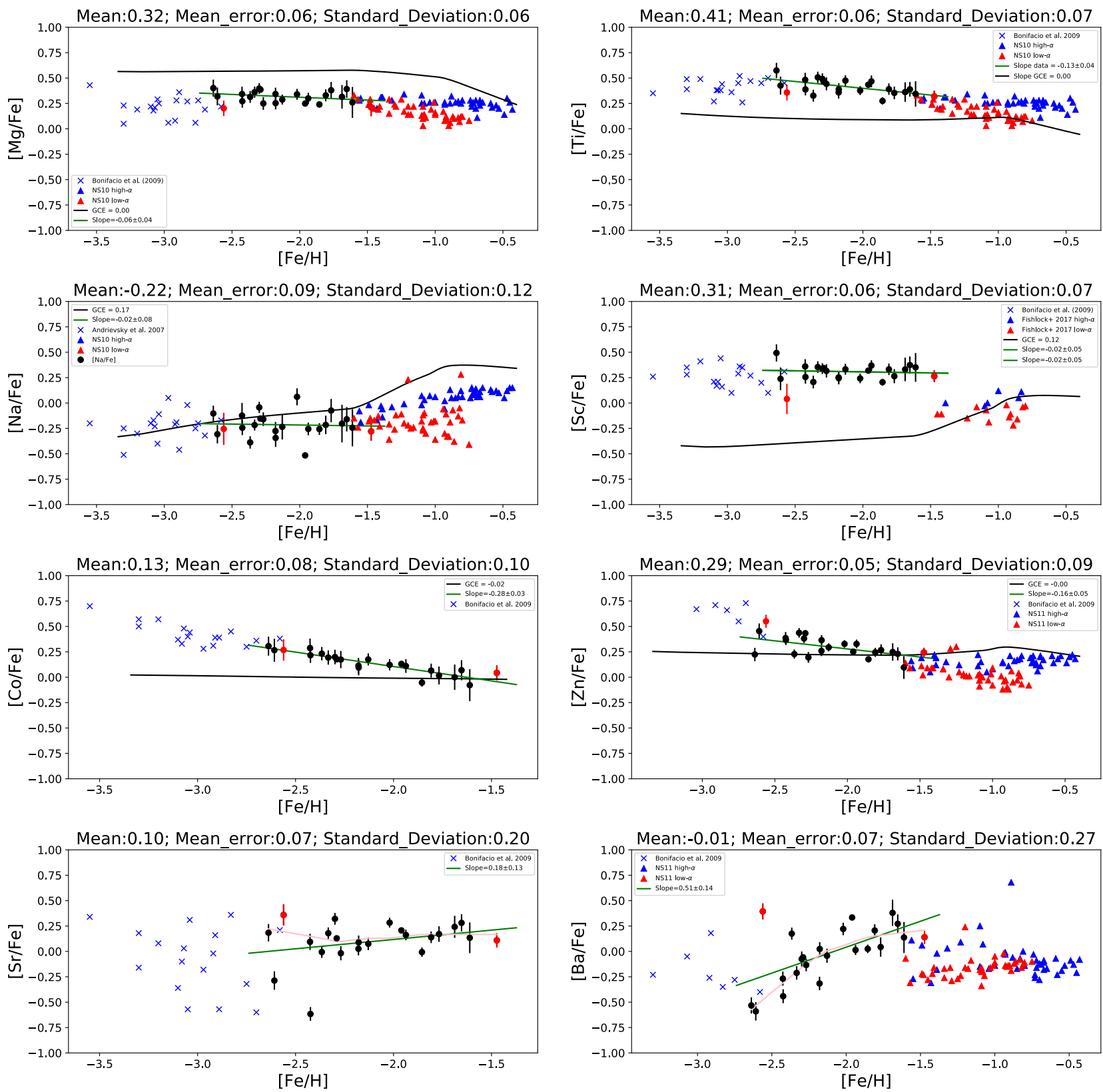

Figure 3.9: Abundances as a function of metallicity. Black and red dots are our measurements, red and blue triangles are the low- and high- $\alpha$ populations of NS10, and blue crosses are the data from the "First Stars" project (Bonifacio et al., 2009; Andrievsky et al., 2010). The black lines are the K15 GCE model prediction, the green lines are the linear regression to our data, and pink lines represent the non-parametric regression. 
$A(\mathrm{Li})=2.27 \pm 0.04$ for stars with $6050 \leq \mathrm{T}_{\text {eff }} / \mathrm{K} \leq 6640$. In Reggiani et al. (2017) we present the lithium abundances as a function of $[\mathrm{Fe} / \mathrm{H}]$ and as a function of stellar mass.

We looked for indications of a broken Li plateau, as reported by Meléndez et al. (2010), but our sample is not statistically large enough to identify differences within a sample that has a relatively small temperature range $(\approx 600 \mathrm{~K})$. We showed the correlation of lithium abundances with mass in our paper, but it is not strong enough to be used as a constraint in models of stellar evolution and lithium depletion, again because our range in stellar mass is very narrow (and the uncertainties relatively large), due to the small range in effective temperature

The Standard Big Bang nucleosyntheis model $(\mathrm{SBBN})$, that predicts $A(\mathrm{Li})=2.64$ (Coc, 2016), is likely not the problem in the disagreement between model predictions and observations (the Spite plateau). Thus, studies of this particular lithium problem are mainly focused on finding solutions that do not involve changes to the SBBN. Among possible explanations we call attention to: a) nuclear physics solutions (Coc et al., 2012); b) massive decaying particles destroying lithium (Olive et al., 2012); c) magnetic effects (Kusakabe and Kawasaki, 2015); d) lithium depletion during the main-sequence evolution, like gravitational settling and rotational induced mixing (e.g., Korn et al., 2006; Charbonnel and Primas, 2005; Richard et al., 2005). None of these models have, so far, been conclusively accepted as the main driver of lithium depletion. Thus, the discussion is still open and new possible solutions being constantly introduced in the literature. In our paper we favored a study from Fu et al. (2015), where the Spite plateau was reproduced with a model that depletes lithium during the pre-main sequence and main-sequence, via microscopic diffusion, overshooting, EUV photoevaporation and late accretion.

Here, we also call the attention to an interesting proposition made in the recent work of Takeda (2019), who introduces a method of analysis that instead of depleting lithium reanalyzes the problem of determining the lithium abundance. Takeda (2019) introduces through a simple toy model, a chromospheric region on top of the stellar photosphere by adding high temperature layers to the stellar atmospheric model. This simple chromospheric model introduces back-radiation to the line forming region at the photosphere, which increases the photoionization, diminishing the available neutral lithium atoms and, thus, the strength of the lithium line. In a regular analysis (even in non-LTE ), which does not include a chromosphere, the line would be considered too small and the abundance inferred 
would be smaller than the real abundance.

His pilot study included only the findings for a fixed $T_{\text {eff }}(6250 \mathrm{~K})$ and $\log g(4.0)$, in a range of metallicities $(-5.0 \leq[\mathrm{Fe} / \mathrm{H}] \leq-1.0)$, well within the atmospheric parameters of our sample, with different temperature structures for the chromosphere. Takeda $(2019)$ showed that this is a promising study, that might be able to reproduce the Spite plateau, thus a more detailed work taking into account variations in stellar parameters, is strongly encouraged.

In Figures 3.10 and 3.11 , we show our Li abundances, along the abundances from Nissen and Schuster (2012). They measured lithium in the dual- $\alpha$ populations and found no apparent difference of lithium between them. The differences in $\mathrm{Li}$ abundance are observable in effective temperature, with an increasingly lower lithium abundance for lower $T_{\text {eff }}$ stars. The correlation of lithium with mass and with total metal content were studied, and indicate, like previously reported in Meléndez et al. (2010), a dependence of depletion with mass.

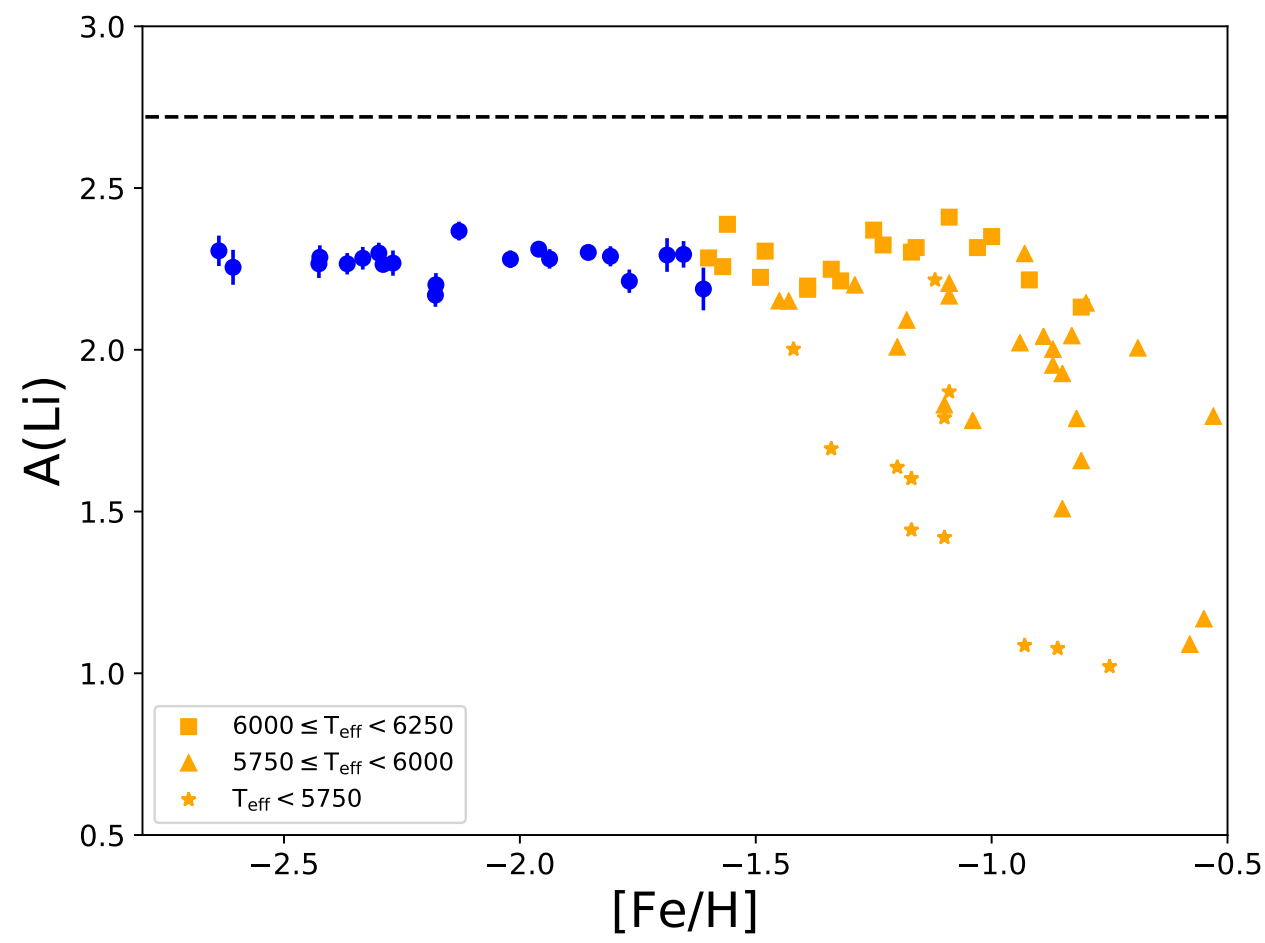

Figure 3.10: Lithium as a function of $[\mathrm{Fe} / \mathrm{H}]$. Blue dots are our data and the orange symbols are from NS12, and the black dashed line is the predicted primordial Li abundance.

We qualitatively analyze the full-set of data in light of the Fu et al. (2015) and the Ta- 


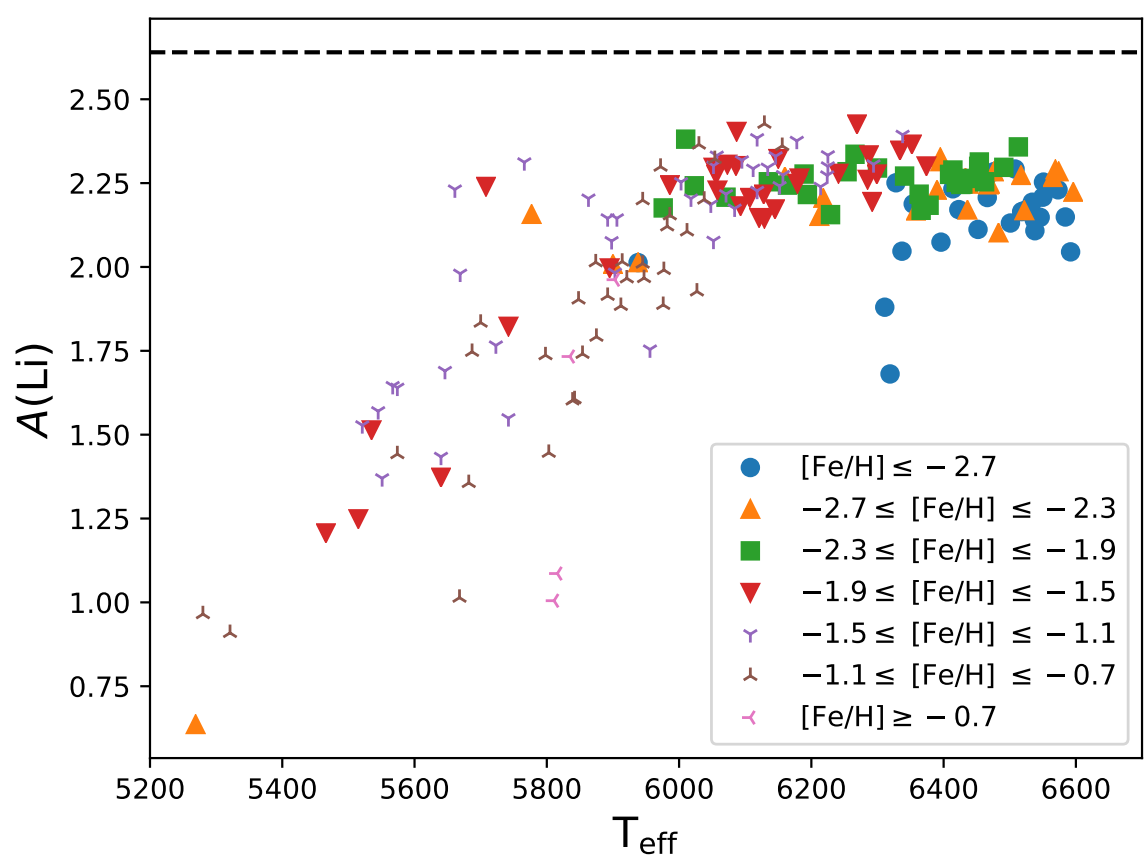

Figure 3.11: Lithium as a function of $T_{\text {eff }}$. Dashed line is predicted primordial Li abundance.

keda (2019) works. Like already pointed out in Reggiani et al. (2017), the model proposed by Fu et al. (2015) to deplete lithium, can reproduce the Spite plateau for stellar masses of our data. In Figure 8 of Fu et al. (2015), they show their lithium abundances as a function of stellar temperature and mass, and the behavior of their model is similar to the full data-set we show in Figure 3.11. The work from Takeda (2019), however, despite of being promising, still lacks further analysis with stellar atmospheres of different parameters. It reproduces the Spite plateau and the correlation of abundance with metallicity, but it is still not possible to argue that it is an explanation for the cosmological lithium problem, as it is a pilot study of only one stellar atmosphere. Takeda $(2019)$ also cautions that this study relies on the assumption that these dwarfs have a chromosphere, based on the detection of a helium line at $10830 \AA$ in Pop II stars (a line associated with the presence of a chromosphere - Takeda and Takada-Hidai (2011)), but there are no other indications of the presence of this structure in such stars and additional data is needed to confirm that this is a plausible explanation for the low Li abundances in Pop II stars.

There is also the problem that either stellar evolution or model atmosphere solutions, need to simultaneously explain the Spite Plateau and the lithium depletion observed over time in solar twins (Ryan et al., 2001, Carlos et al., 2016), which are mainly explained by evolutionary effects, like depletion from rotationally-induced mixing (effects that could 
also explain the lithium depletion in the BSS). Thus, more than explaining only the Spite plateau, a final theory of lithium depletion will also have to shed a light on the lithium in Pop I solar-type stars.

Our data, restricted to a very narrow range of stellar parameters, was able to precisely locate a mean position of the Spite plateau, and with the aid of the data from Nissen and Schuster (2012), we showed that the possible explanations for the lithium problem might have to consider different works, either via stellar evolution or a more complete model atmosphere analysis including the effect of a chromosphere. It is, however, unlikely that new physics or nucleosynthetic theories will play a role in the final solution to the lithium problem, as there is observable depletion not only in Pop II stars, but also in Pop I solar twins of different ages, indicating that the mechanism responsible for our low measurements in theses stars is not restricted to the early cosmic times of our Universe.

During our analysis, more specifically our analysis of lithium, we also found a pair of Blue Straggler stars. Due to their different evolutionary status they are not adequate to be used in the above studies, and they are more thoroughly discussed in the next section. They are represented by the red dots in Figure 3.9 .

\subsection{Blue Straggler Stars}

Blue Straggler stars (BSS) are stars brighter and bluer than the main-sequence turnoff, laying along an extrapolation of the main sequence, mimicking a rejuvenated stellar population (Ferraro et al., 1999; Ryan et al., 2001; Ferraro et al., 2006; Mapelli et al., 2006). They were first identified by Sandage (1953), and they remained a puzzle for many years, but today are known to be a feature of binary evolution. They are commonly identified via photometry in Globular Clusters (GC), standing out as a continuation of the main-sequence, as shown in Figure 3.12 .

There are two mainly accepted scenarios for the formation of BSS: Mass transfer, in a close binary, from an evolved companion (McCrea, 1964); and a collision/merger. Bailyn (1992) claimed that in GC both scenarios take place, but the merger scenario is more favored in denser GCs. From the different forming scenarios, Sarajedini (1993) claims that there are three observable types of BSS: a) binary mass transfer that does not increase the helium abundance, resulting in evolved stars with magnitude typical for its mass; 


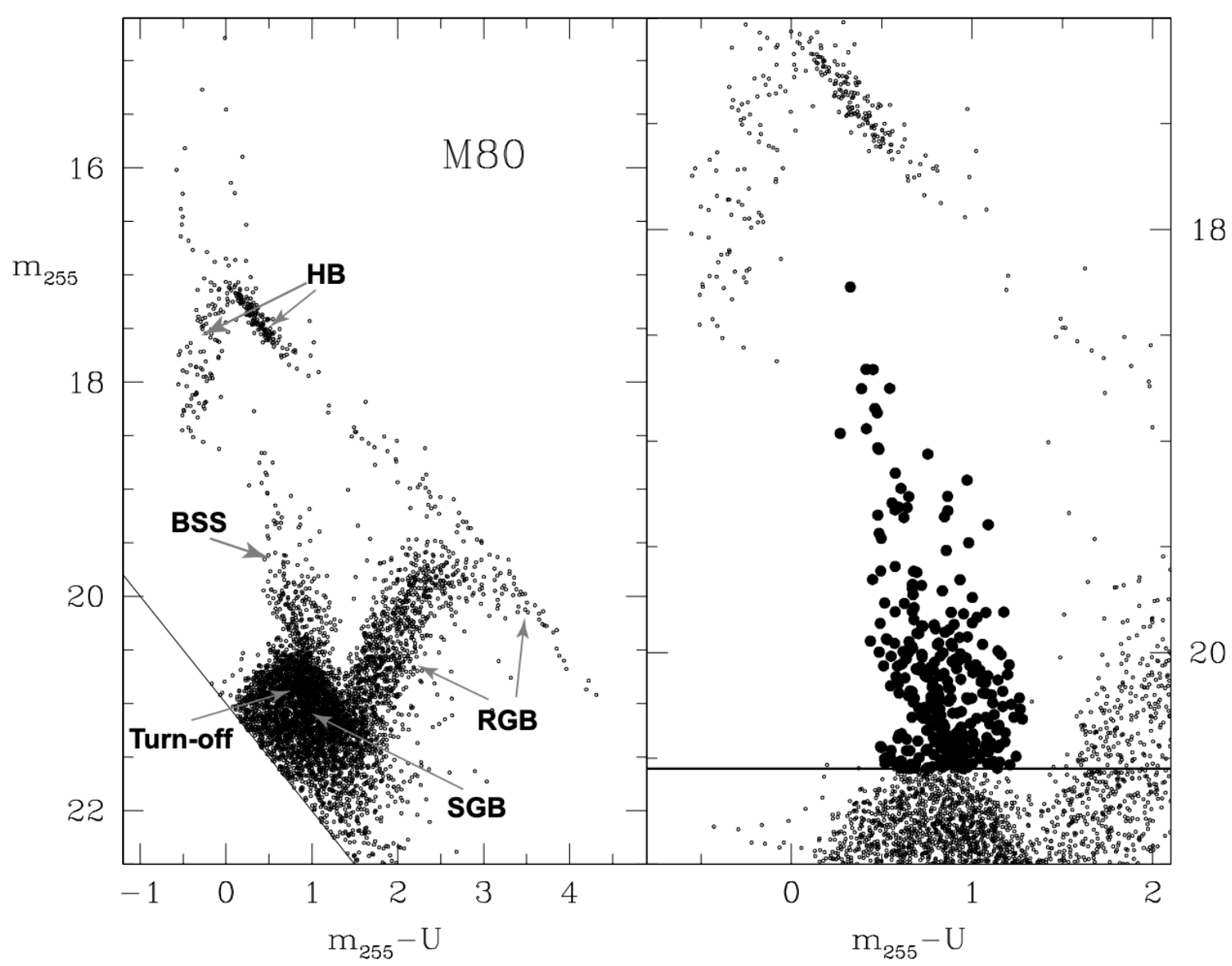

Figure 3.12: HR diagram of the globular cluster M80. Notice the BSS population standing out as a continuation of the main-sequence, shown better in the right panel. Figure extracted from Ferraro et al. (1999). 
b) two stars colliding to form a star with an increased helium abundance in the stellar envelope, resulting in a star with a higher $\mathrm{M}_{\mathrm{V}}$; and c) mass transfer and merging, where the component that overflowed the Roche lobe is completely dispersed into the companion, enriching the envelope of its companion by helium created in its core, resulting in a star with a higher magnitude (although not as high as the magnitude of a BSS created via the collision process only), but being the most unlikely scenario.

Thus, BSS can be identified via their position in the HR diagram. Stellar spectra can be analyzed to find these stars in the field, although it is not a usual technique, as BSS are mostly observed in dense environments (GCs). Ryan et al. (2001) showed that field ultra lithium-poor and beryllium-poor stars are BSS. As lithium and beryllium are fragile, they are easily burned when there is a deepening of the convective zone, which might occur in either scenarios (mass transfer or merger).

From the low lithium abundance (upper limits of $A(\mathrm{Li}) \leq 0.94$ and $A(\mathrm{Li}) \leq 1.36$ for stars HD 340279 and G 66-30, respectively), we identified these stars as BSS. In Figure 3.13 we show the Li line region of the BSS compared to a "normal" turn-off star from our sample ( $\mathrm{G}$ 66-30 was also recently identified as a BSS by Amarsi et al. (2019)).

We analyzed the chemical composition of these stars taking into consideration their evolutionary status, and the most interesting result is the enhanced $\mathrm{Zn}$ abundance in HD 340279, when compared to the other stars in our sample with similar metallicities. If this extra zinc is not from a regular abundance scatter due to an inhomogeneous ISM, which we argue against based on the homogeneity of the remaining stars in our sample, then the enhancement must be directly related to the formation of the BSS. In that case, if the BSS formed under the mass transfer scenario from an AGB companion, then the extra Zn was synthesized in the AGB star. Under that assumption, we can use the zinc enhancement as a secondary tool to determine the initial mass of the AGB companion that formed the BSS. Zinc can be synthesized in AGB stars via the s-process, but not to the level where it become important to the GCE ("Heger and Woosley, 2002; Umeda and Nomoto, 2002; Karakas et al., 2009). The Zn nucleosynthesis becomes important only for AGB stars of initial mass of $\mathrm{M} \geq 3.0 \mathrm{M}_{\odot}$, thus the AGB companion that formed this field BSS must have been massive.

Under that assumption one could also expect to observe an extra enhancement of sprocess material in this star, and indeed the abundances of $[\mathrm{Sr} / \mathrm{Fe}]$ and $[\mathrm{Ba} / \mathrm{Fe}]$, which 

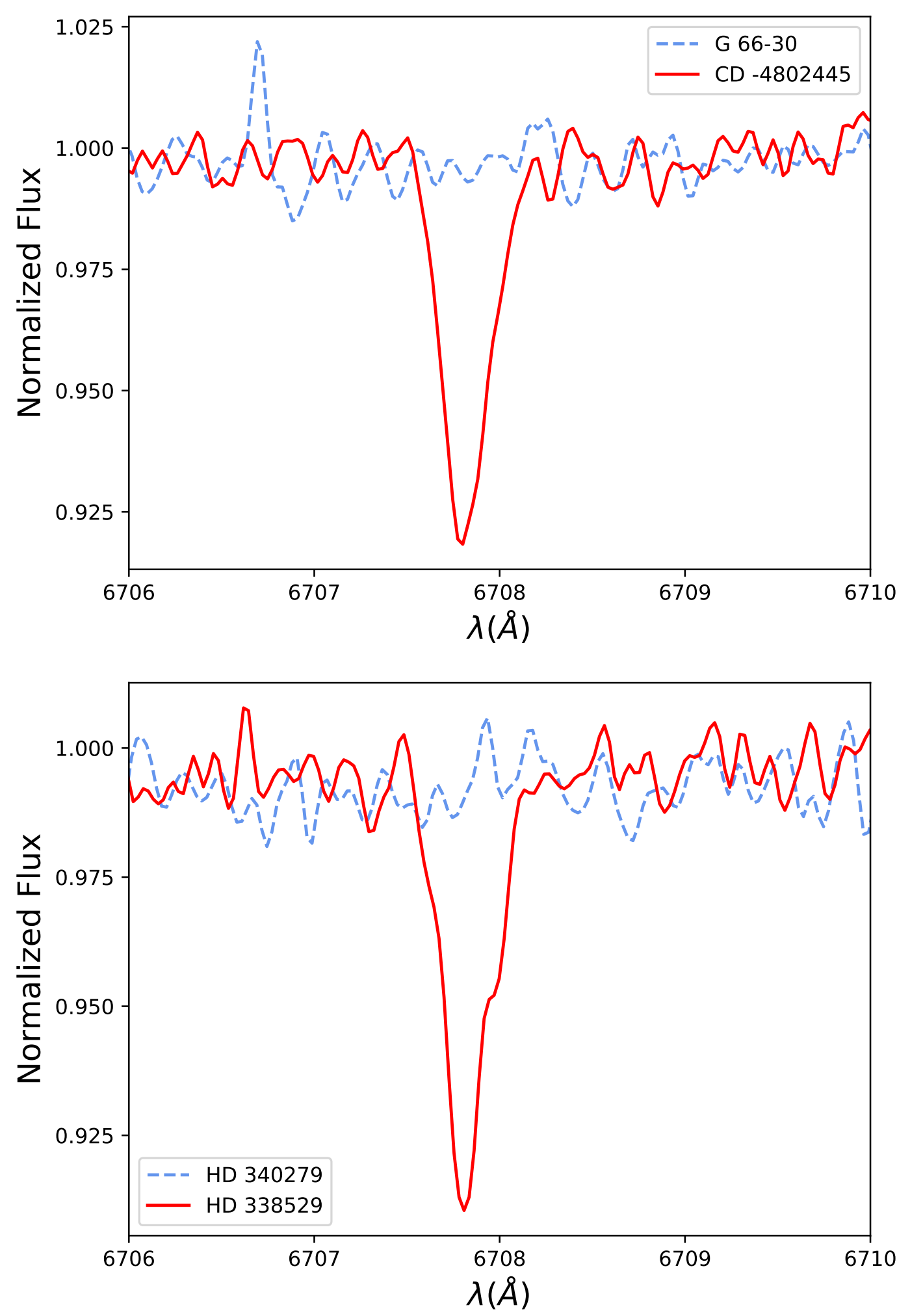

Figure 3.13: Lithium line in BSS (dashed blue lines) and in "normal" turn-off stars (red solid lines). The top panel show stars G 66-30 and CD -48 02445, and the bottom panel stars HD 340279 and HD 338529. 
are shown in the last two panels of Figure 3.9, as well as the [Y/Fe] (Fig. 3.9, Reggiani et al., 2017), are higher than in the other stars of similar metallicity. It is then very likely that HD 340279 became a BSS by accreting mass from an AGB star, massive enough to produce $\mathrm{Zn}$, and the mass transfer from it raised Zn to a point where it stands out from the scatter in our sample. Notice that although AGB stars could produce Li (D'Antona and Ventura, 2010; Maciel and Costa, 2012, 2018), the much higher rotation in BSS due to mass transfer (Ryan et al., 2002), may induce a large Li depletion in the BSS.

As neutron-capture elements have a very big scatter due to the several possible sources of enrichment, and there are large uncertainties involved in estimating the yields from several nucleosynthetic sources for s- and r-process elements, the zinc abundance might be a better, or at least complementary, way of constraining the formation of BSS via chemical abundances, as the much lower scatter in the chemical abundances of stars makes it much easier to recognize when a single star is enhanced. 
Chapter 4

\section{Chemical tagging and Planet signature}

We have discussed how we used the differential abundances to compare stars among each other, showed how the measurement errors can be improved by the differential technique, and applied it to study the formation and chemical evolution of the solar neighborhood halo, with implications for yields of AGB stars, the evolution of lithium through cosmic history and the Spite plateau, and the connection between chemical abundances and the evolution of binary stars (through the analysis of Blue Straggler Stars). As from Section 3.1, the stellar chemical composition can be used to trace the formation of galactic structures, and the better the precision of the data, the most likely it is that we can identify which systems the stellar components resemble. In this Chapter, we continue discussing the halo formation and evolution through the analysis of a binary pair from the galactic halo. We also discuss how precise chemical abundances can be used to find inhomogeneties between pairs of stars, and possible implications to planet formation. This Chapter is based on Reggiani and Meléndez (2018), which can be read in Appendix A

\subsection{Chemical Tagging}

Stars are born in clusters and associations originated in huge molecular clouds (e.g., Fujii and Portegies Zwart, 2016), and presumably these clouds are homogeneous in their chemical composition. The associations where most of the stars are born often disappear after some time due to collisions within the association (that heat and expel stars), collisions with other associations, collisions of dwarf galaxies with our galaxy that disrupt any structure in their path, interactions with other stars and molecular clouds, or even due to shock waves that come from the aftermath of massive stars dying. Regardless of how it 
happens, stars born in a given association are often found in different parts of the Galaxy, having migrated due to different encounters and different gravitational pulls they undergo during their lifetime.

Thus, it is not possible to map out the initial position of the stars in our Galaxy only through their present position, as it will likely have changed considerably throughout their long lifetime. It is even possible that the current kinematics of such stars do not resemble the kinematics of their original association, as stars could be accelerated out of their birth cloud. However, it does not matter where a star is and how long ago it was born when we observe them, if the photospheric chemical abundance still has not changed due to evolutionary processes of the star. When that is the case, there are still observational variables (all or most chemical abundances of the star) that can be traced back to the original birth place of the star. Identifying where a star came from, based on its chemical composition, is what is called chemical tagging, and it has been widely used to identify structures in the Galaxy, to study the chemodynamical evolution of the Galaxy, formation of the Galaxy, and galactic nucleosynthesis (Venn et al., 2004; Carretta et al., 2012; Meléndez et al., 2014; Mitschang et al., 2014; Hogg et al., 2016). However, even with precise abundance measurements, it might be difficult to pinpoint the birthplace of a star, if the star was born at a time where the ISM was very homogenized, because many molecular clouds would have had a similar composition. In those cases, only by using both chemical and kinematic information, we can trace back a star to its original birth location.

It is important for chemical tagging that the stars analyzed are as unevolved as possible, as evolutionary effects, like atomic diffusion, might influence the results (Dotter et al., 2017; Souto et al., 2019; Liu et al., 2019). Another form of avoiding effects of stellar evolution is by analyzing stars in the same evolutionary stages, thus the same effects would have taken place in all analyzed stars (as all analyzed stars have similar masses). Due to these difficulties, and to the extra difficulty of having detailed measurements of a sufficient number of chemical elements, the first study that actually identified a big number of structures within the Galaxy (both previously known and unknown) using only chemical tagging was Hogg et al. (2016).

We analyzed the chemical composition of a pair of binary stars, along with a benchmark star from our galaxy, in order to find where these stars came from. The stars of our interest are the binary pair HD 134439/HD 134440. It had already been suggested that they were 
accreted from a dwarf Galaxy due to their extreme kinematics (Carney et al., 1996). We also highlight the chemical composition study of these stars by Chen and Zhao (2006) and their follow-up study (Chen et al., 2014). They found that these stars have extremely low abundances of the $\alpha$-elements, an indication that they did not originate in the Milky Way. On the other hand, Shigeyama and Tsujimoto (2003) proposed that the extremely low $[\alpha / \mathrm{Fe}]$ in stars is evidence of planet engulfment. Thus, in order to further trace the main reasons of the uncanny abundance of HD 134439/134440, we reanalyzed a full set of its chemical abundances using the line-by-line differential technique.

\subsection{Data, stellar parameters and chemical abundances.}

We observed stars HD 134439/134440 and star HD 103095 (the standard star of our analysis) using HDS, the High-Dispersion Spectrograph (Noguchi et al., 2002), at the 8.2meter Subaru telescope. The observations took place under the Subaru program (9S16ATE005 / o162060), and under the Gemini time exchange program (GU2016A- 005), on May 27 2016, with the standard HDS setups Rb and Yc, with a total wavelength coverage from $4400 \AA$ to $7950 \AA$. The slit width was set to 0.5 ", corresponding to a resolving power of $\mathrm{R}=72000$. The exposure times were 100,1000, and 1500 seconds for HD 103095, HD 134439 and HD 134440, respectively, yielding $\mathrm{S} / \mathrm{N} \approx 250$ at $5000 \AA$ in all stars.

We used the line-by-line differential technique in the analysis of these stars. The standard star (HD 103095) was observed due to its proximity in stellar parameters to the objects of study (from previous analyses), and because it is a benchmark star that represents the chemical abundance of Milky Way halo objects of its metallicity and has been subject of several previous abundance studies (e.g., Smith et al., 1992; Balachandran and Carney, 1996; King, 1997; Meléndez and Cohen, 2007; Jofré et al., 2015). We adopted the stellar parameters from Sitnova et al. (2015) for HD $103095\left(T_{\text {eff }}=5100 \pm 65 \mathrm{~K}\right.$, $\log g=4.65 \pm 0.08 \mathrm{dex}, \xi=0.9 \pm 0.05 \mathrm{~km} / \mathrm{s}$ ), and we found $[\mathrm{Fe} / \mathrm{H}]=-1.35 \pm 0.08 \mathrm{dex}$ from our Fe I and Fe II line measurements - consistent with the adopted metallicity by Sitnova et al. (2015). It is important to mention that the adopted effective temperature of the standard star is in line with the recent interferometric $T_{\text {eff }}=5140 \pm 49 \mathrm{~K}$ from Karovicova et al. (2018).

In the same way as described before, we calculated the stellar parameters of HD 
Table 4.1 - Stellar parameters and errors of stars HD 103095 (reference), HD 134439, HD 134440 and HD 163810 .

\begin{tabular}{lcccc}
\hline \hline & HD 103095 & HD 134439 & HD 134440 & HD 163810 \\
\hline$T_{\text {eff }}(\mathrm{K})$ & $5100 \pm 65$ & $5084 \pm 27$ & $4946 \pm 26$ & $5526 \pm 37$ \\
$\log g$ & $4.65 \pm 0.08$ & $4.66 \pm 0.06$ & $4.68 \pm 0.07$ & $4.56 \pm 0.09$ \\
{$[\mathrm{Fe} / \mathrm{H}]$} & $-1.35 \pm 0.08$ & $-1.43 \pm 0.02$ & $-1.39 \pm 0.02$ & $-1.26 \pm 0.03$ \\
$\xi(\mathrm{km} / \mathrm{s})$ & $0.90 \pm 0.05$ & $1.22 \pm 0.08$ & $1.17 \pm 0.06$ & $0.99 \pm 0.15$ \\
\hline
\end{tabular}

134439/134440 relative to HD 103095 using the differential method, and for consistency check with the large sample of NS10, we also estimated the stellar parameters of star HD 163810, from their sample. For HD 163810, we took spectra from the NS10 original observations, available in the ESO archive, program 071.B-0529. In Figure 4.1, we show the differential excitation equilibrium, and differential abundances as a function of reduced equivalent width, for HD 134440.

In Table 4.1 we show the adopted stellar parameters for the stars of interest and for the consistency check star HD 163810. The stellar parameters for HD 163810 are also in good agreement with the stellar parameters adopted by NS10 and their follow-up studies.

The abundances of C, Na, Mg, Si, Ca, Sc, Ti, V, Cr, Mn, Co, Ni, Cu, Zn, Sr, Y, Ba, along with upper limits of $\mathrm{O}, \mathrm{La}, \mathrm{Ce}, \mathrm{Nd}$, and $\mathrm{Sm}$, were estimated via the line-by-line differential technique. All the abundances are in Table 1 of Reggiani and Meléndez (2018). We did not measure the Eu abundance from our spectra, but we adopted the values from Chen et al. (2014) in our analysis.

\subsubsection{Additional data}

As the abundances of these two stars do not resemble the abundances of the bulk of inner halo stars, in order to find out their true origin we must compare their chemical content to the chemistry of other environments. As we already knew that they have a very low $[\alpha / \mathrm{Fe}]$ ratio, we gathered data from other environments that are also known to have the same feature.

Thus, we compare our abundances with the NS10 (and follow-up studies) results, interested in comparisons with their extra-galactic low- $\alpha$ population (Section 3.1). We also compare our abundance pattern to stars from dwarf spheroidal galaxies (dSph). The 
hd134440: 4946, 4.68, -1.385, 1.17 [hd103095]
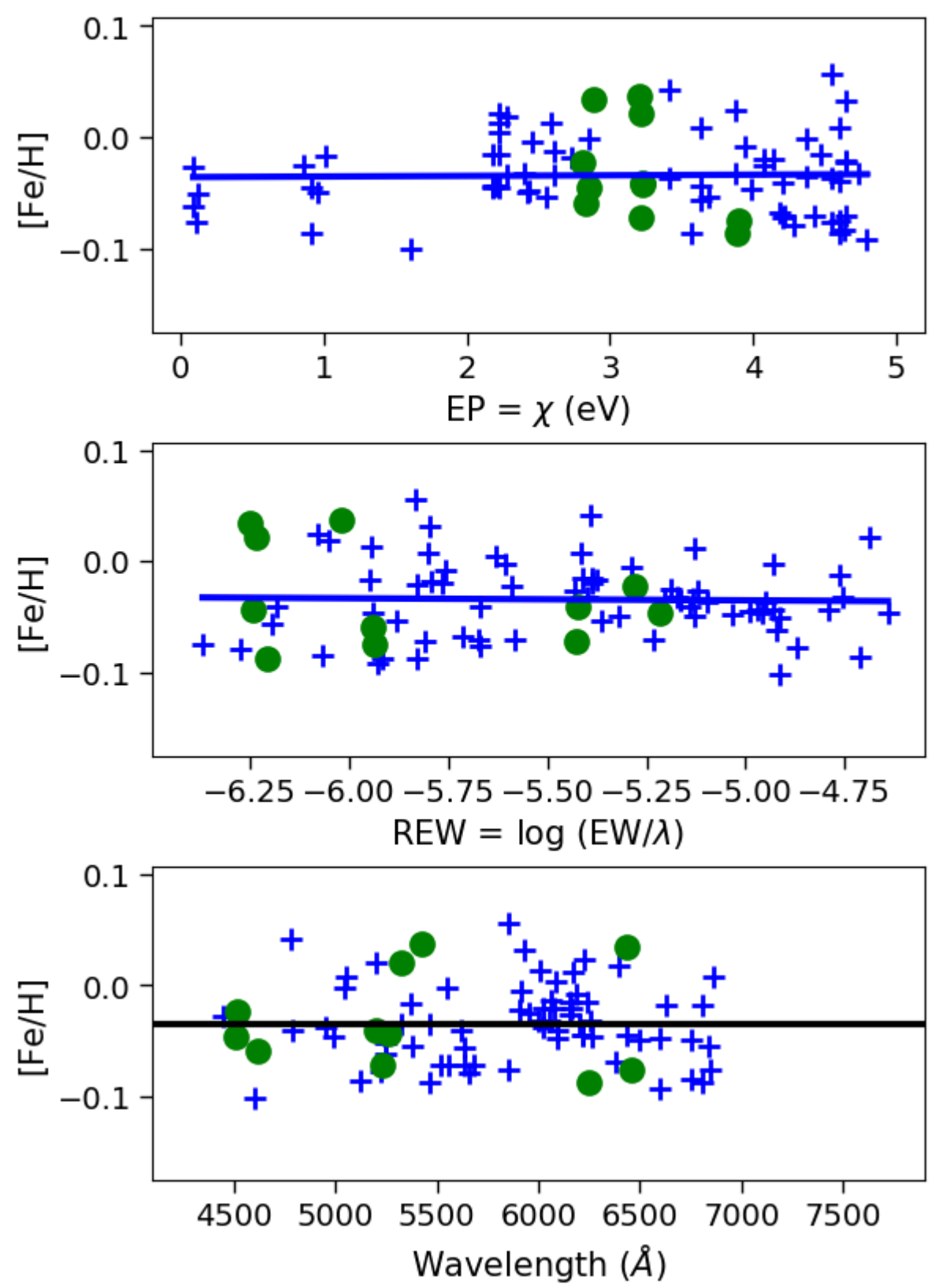

Figure 4.1: Differential equilibrium plots for star HD 134440, relative to the standard star HD 103095. $T_{\text {eff }}=4946 \mathrm{~K}$ obtained via equilibrium of differential abundances with excitation potential in the top panel, $\log g=4.68$ via differential ionization equilibrium, $[\mathrm{Fe} / \mathrm{H}]=-1.39$ via differential Fe abundances, and $\xi=1.17 \mathrm{~km} . \mathrm{s}^{-1}$ via differential abundances vs reduced EW (middle panel). 
abundance pattern of stars from dSph usually have very low $[\alpha / \mathrm{Fe}]$ and also distinctive neutron-capture elemental abundance ratios, from which they can be readily identified (Suda et al., 2017). The data we employed in our work were gathered from Shetrone et al. (2003); Geisler et al. (2005); Monaco et al. (2005); Letarte et al. (2010). It is important to point out that the comparison data are not the ideal stars for a chemical tagging study as, due to technical limits, all the dSph stars are giant stars.

\subsection{The birth environment of HD 134439 and HD 134440}

In Figure 4.2 we show the $[\mathrm{X} / \mathrm{Fe}]$ ratios of $\mathrm{O}, \mathrm{Na}, \mathrm{Mg}, \mathrm{Si}, \mathrm{Ca}, \mathrm{Sc}, \mathrm{Ti}, \mathrm{Cr}, \mathrm{Mn}, \mathrm{Co}, \mathrm{Cu}$, Zn, Y, Ba, La, Ce, Nd, and Eu. Europium abundances are from Chen et al. (2014). Most of these abundances were already shown in Reggiani and Meléndez (2018), but we added data of cobalt and europium, that were not previously shown. Regarding ours and NS (referring to all studies based on the NS10 sample) measurements of abundances in star HD 163810, we found that there is a small offset. The averaged offset of the $\alpha$-elements (to which we included oxygen) is 0.05 dex with a standard deviation of 0.04 dex.

While the abundance ratios for HD 103095 are similar to those of the low- $\alpha$ population being thus representative of the possible accretion event that formed the bulk of the inner halo, the abundance pattern of HD 134439 and HD 134440 have much lower [X/Fe] ratios (mostly observed in $\mathrm{O}, \mathrm{Mg}, \mathrm{Si}$, and $\mathrm{Ca}$ ), and even adding the averaged difference found trough our analysis of HD 163810, the abundances of the binary pair are still lower than the abundance of the low- $\alpha$ population. The staggering low abundance of oxygen was also found by Chen et al. (2014).

The abundance ratios of $\mathrm{Na}, \mathrm{Ti}, \mathrm{Ni}$, and $\mathrm{Zn}$ are slightly below the lower envelope defined by the low- $\alpha$ stars, and both members of the binary system do not show any sizable difference in the abundance pattern of other elements, such as Cr, Mn, and heavy elements Y, Ba, La, Ce, and Eu, when compared to the low and high- $\alpha$ populations, but are slightly lower in Nd (but it is possibly due to measurement errors, as Nd abundances are upper limits and must be viewed with care).

The abundance pattern of dSph are very distinguishable, as they have very low $[\alpha / \mathrm{Fe}]$ in a broad range of metallicities, with knees in different $[\mathrm{Fe} / \mathrm{H}]$ positions, when compared to the knee of the Milky Way (see Section 3.1), and abundances of other elements, such as 
Sc, also lower than those that we see in the stars of our galaxy (e.g., Sbordone et al., 2007; Frebel et al., 2016; Ji et al., 2016; Suda et al., 2017). As already mentioned in Section 3.1, Suda et al. (2017) studied the location of the knee in the Milky Way, Fornax, Sculptor and Draco, showing that the knees for the above dSphs are at lower metallicities than that of the Milky Way. The $\alpha$ patterns of HD 134439 and HD 134440 are very low, and from that we can conclude that these stars must have been born in a galaxy with a knee below $[\mathrm{Fe} / \mathrm{H}] \leq-1.5$, as previously suggested by Chen and Zhao (2006) and Chen et al. (2014). Another chemical element that can be used for chemical tagging is barium (Spitoni et al. 2016), as from their models there is a distinctive difference in barium from dSph's, ultrafaint dwarf galaxies (UfD's) and the Milky Way halo. From our measurements, [Ba/Fe] of the binary pair is consistent with the low- $\alpha$ stars. However, barium is produced both in the s- and r-processes, and the excess, or lack of an excess of barium, might not exclusively indicate the formation environment; it may be more important to distinguish the main sources of nucleosynthesis where the star was born.

$[\mathrm{Ba} / \mathrm{Y}]$ can also be used to find the birth environment of a star, as a high enough value can be linked to a high number of supernovae type II without time for the rise of s-process contributions by AGB stars, like is commonly associated with dSphs (Chen et al., 2014; Tolstoy et al., 2009). The [Ba/Y] ratio of dSphs stars are clearly super-solar, reaching 1 dex for the higher metallicity regime. Although our results are a bit higher than reported by Chen et al. (2014) and Chen and Zhao (2006), our ratios are also sub-solar, but $[\mathrm{Ba} / \mathrm{Y}]$ of HD 134439/134440 are above that of the NS populations. There is a remarkable offset between these stars and the super-solar ratios $([\mathrm{Ba} / \mathrm{Y}] \geq 0.6 \mathrm{dex})$ in $\mathrm{dSph}$ galaxies, pointing to a birth environment that does not favor a high production of heavy relative to light n-capture elements.

We can further constrain the birth of these stars using the diagram on Fig. 14 of Suda et al. (2017). From our $[\mathrm{Ba} / \mathrm{Fe}]$ and the adopted $[\mathrm{Eu} / \mathrm{Fe}]$, we locate both stars on the r-process dominant region, where $[\mathrm{Eu} / \mathrm{Ba}] \geq 0.5$.

It is clear that the abundances of stars HD 134439 and HD 134440 are very different from the abundances of Milky Way stars, specially when looking at the $\alpha$-elements, and thus they must had been born in a different galaxy and then acreted to the Milky Way. They were likely acreted, but not in the same major event that formed the bulk of our inner halo. They come from a different accretion event, an event in which the imprints are not 
commonly observed in our inner halo. It is possible that these stars came to the Milky Way during the accretion of a more massive structure, that might still be unidentified (there are several identified structures within our galaxy that still lack a full chemical description), or it is possible that these stars were heated from their original galaxy and acreted onto the Milky Way, while the bulk of the galaxy where they come from is yet in the process of merging with us. From their abundance pattern, we are searching for a dSph with a knee below $[\mathrm{Fe} / \mathrm{H}] \leq-1.5$, low $[\mathrm{Ba} / \mathrm{Y}]$ ratio (indicating low production of heavy relative to low n-capture elements) and r-process dominated abundance patterns. We proposed that this pair of stars might be from a dSph similar to Fornax, as all the above chemical requirements are met.

\subsection{The Planet/Star connection}

Planets come in various sizes and masses (Earth-like planets, Jupiter-like planets, Neptune-like planets, and several masses and sizes between them) and their formation is still a matter of debate and studies (e.g., Nixon et al., 2018). Regardless of the exact mechanism responsible for planet formation, it is becoming increasingly clear that their formation leave chemical imprints in their host stars (e.g., Meléndez et al., 2009; Ramírez et al., 2011; Tucci Maia et al., 2014; Teske et al., 2015; Liu et al., 2016, 2018; Ghezzi et al., 2010, 2018).

Meléndez et al. (2009) proposed that the trend in chemical abundances with condensation temperature, that they found through a differential analysis of solar twins, was due to the formation of the rocky bodies of our Solar system (Figure 4.3), a claim that is similar to the previous proposition of Shigeyama and Tsujimoto (2003) regarding the explanation of low- $\alpha$ stars. A differential analysis of the binary pair 16 Cyg (in which one of the stars is known to host a giant planet while the other does not) by Ramírez et al. (2011), showed that there is a difference in abundance of $\approx 0.05$ dex between the stars (Figure 4.4), that was attributed to the formation of the giant planet. This system was further studied by Schuler et al. (2011), and Tucci Maia et al. (2014) with better spectroscopic data, both reaching similar conclusions. Nissen et al. (2017) also found clear abundance differences between the components of the 16 Cyg binary pair, which can not be explained by evolutionary models as both stars are in the same evolutionary stage (both have masses of 

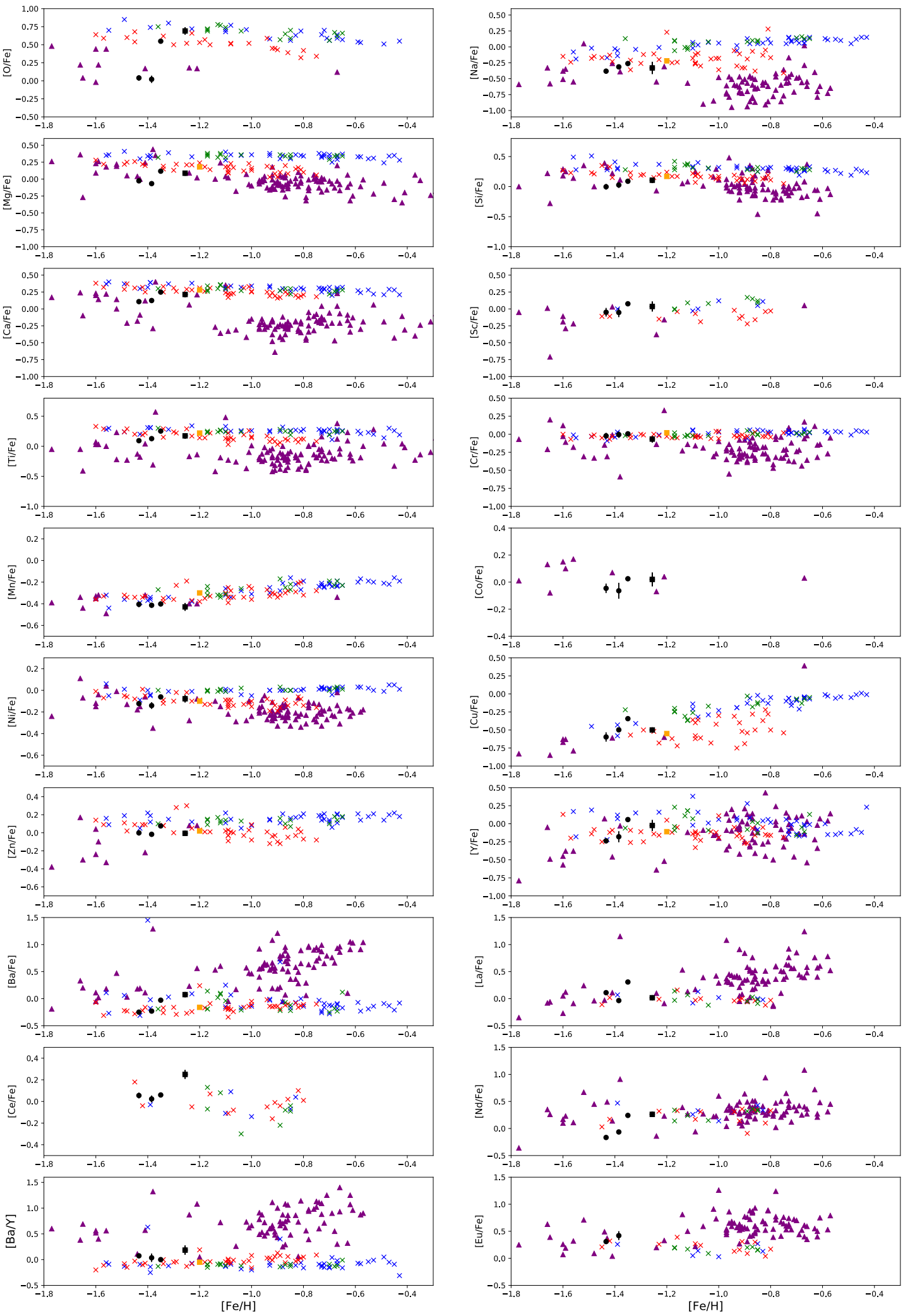

Figure 4.2: The black dots are the abundances of stars HD 103095, HD 134439, and HD 134440. The standard HD 103095 is the most metal-rich of the three stars. The squares are abundances of star HD 163810 (black and orange refer to our measurements and those from the NS10 sample, respectively). The blue, red and green crosses are the high- $\alpha$, low- $\alpha$ and thick-disk populations of NS10, with abundances from Nissen and Schuster (2010, 2011); Ramírez et al. (2012) and Fishlock et al. (2017). The purple triangles are dSphs (Carina, Sculptor, Fornax, and Sagittarius) stars from Shetrone et al. (2003); Geisler et al. (2005); Monaco et al. (2005) and Letarte et al. (2010). 
$\left.\mathrm{M}=1 \mathrm{M}_{\odot}\right)$

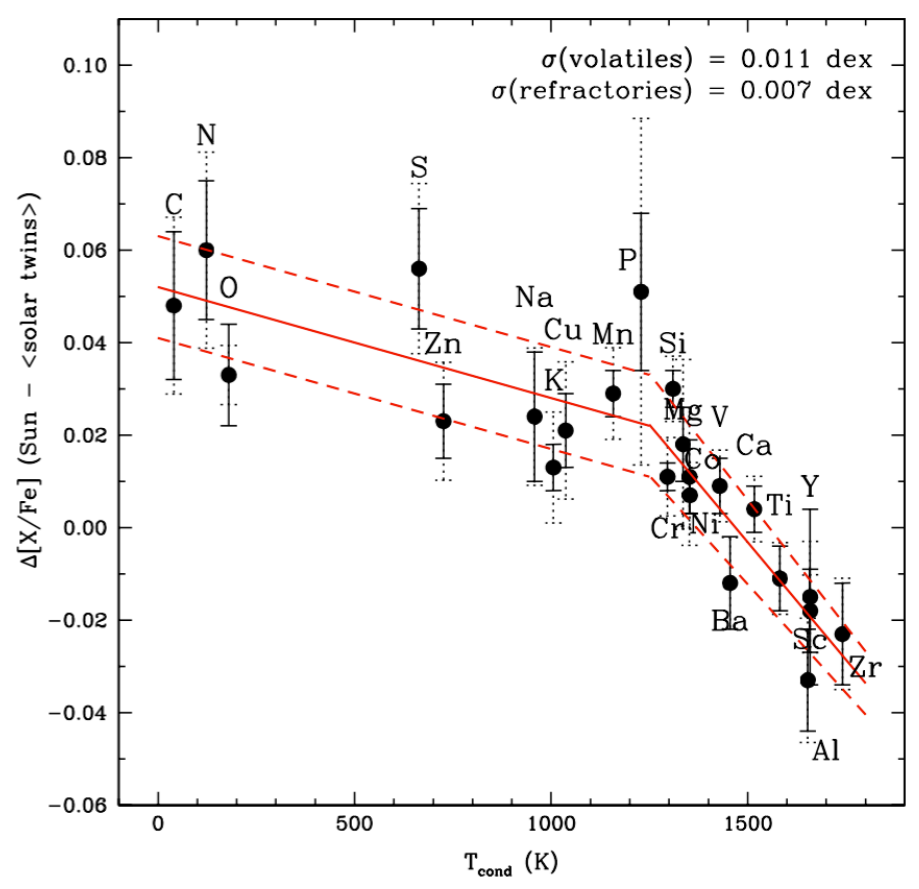

Figure 4.3: Chemical abundances as a function of condensation temperature. The figure shows the differential abundances of the Sun relative to the mean value of solar twins. Figure extracted from Meléndez et al. (2009).

On their study of the abundance anomalies in the binary system of twin stars 16 Cyg, Ramírez et al. (2011) assumed that the chemical differences were due to planet formation, and devised a simple toy model to estimate this effect on the abundance of the host star. They estimated how the metallicity of the star (in $[\mathrm{M} / \mathrm{H}]$ ) would change by the addition of the planet around $16 \mathrm{Cyg} \mathrm{B}$ to the convective zone of its host star (the mass of the planet is known and its composition is assumed to be five times the metallicity of its host star):

$$
\Delta[\mathrm{M} / \mathrm{H}]=\log \left[\frac{(\mathrm{Z} / \mathrm{X})_{\mathrm{cz}} \mathrm{M}_{\mathrm{cz}}+(\mathrm{Z} / \mathrm{X})_{\mathrm{p}} \mathrm{M}_{\mathrm{p}}}{(\mathrm{Z} / \mathrm{X})_{\mathrm{cz}} \mathrm{M}_{\mathrm{cz}}}\right]
$$

where $(\mathrm{Z} / \mathrm{X})_{\mathrm{cz}}$ is the ratio of the fractional abundance of metals $(\mathrm{Z})$ relative to hydrogen $(\mathrm{X})$ in the unperturbed convective zone, $\mathrm{M}_{\mathrm{cz}}$ is the mass of the convective zone, $(\mathrm{Z} / \mathrm{X})_{\mathrm{p}}$ is the metallicity of the planet, and $\mathrm{M}_{\mathrm{p}}$ is the mass of the planet. According to Asplund et al. $(2009),(\mathrm{Z} / \mathrm{X})_{\mathrm{CZ}}^{\odot}=0.018$. Thus, with equation 4.1 it is possible to estimate the mass of a planetary body that can account to the abundance differences between a pair of stars.

Back to our binary pair, Chen and Zhao (2006) found a possible trend in the chemical abundances with condensation temperature for these stars, when separating the abundan- 

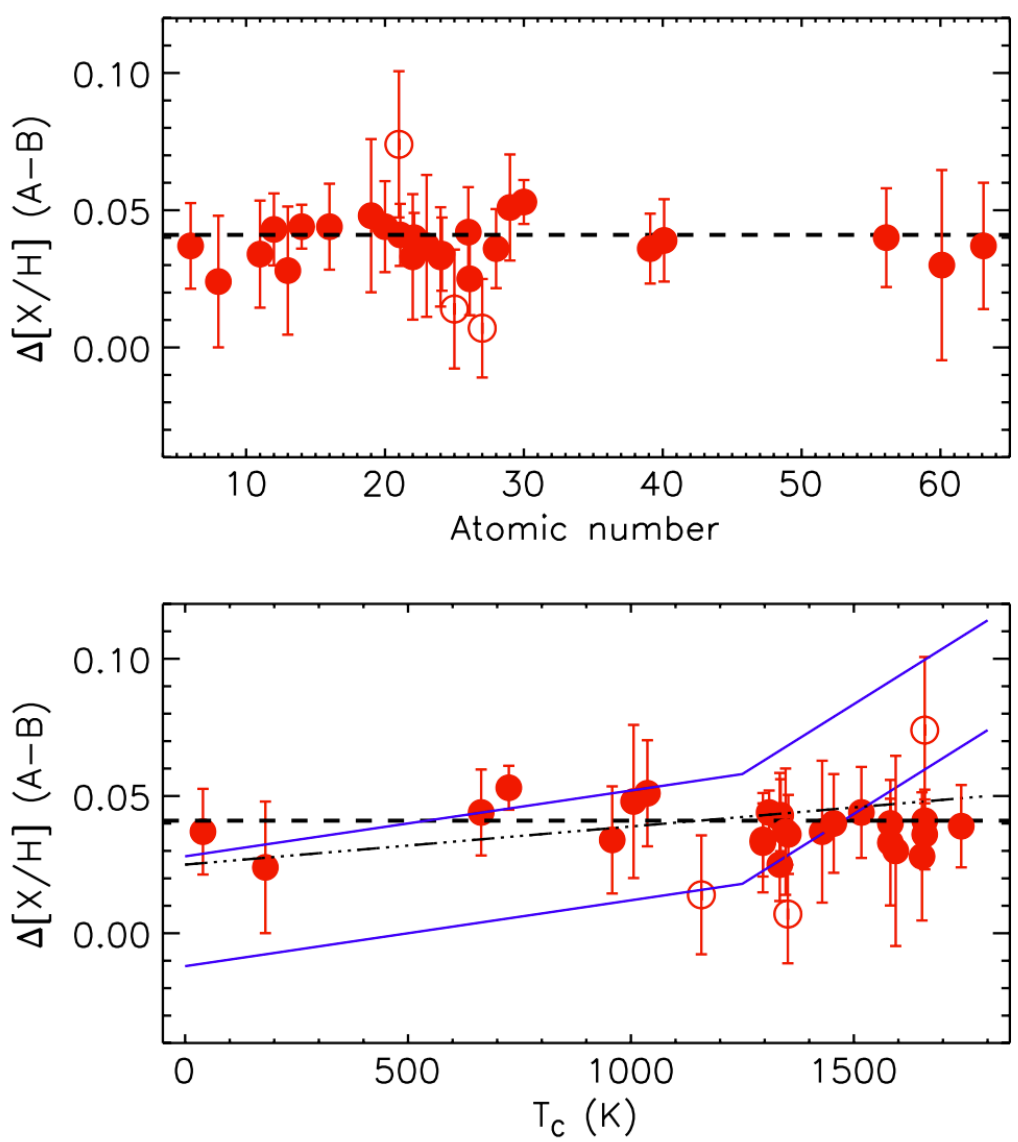

Figure 4.4: Chemical abundances as a function of condensation temperature and atomic number, in a differential analysis of the binary pair 16 Cyg. The blue lines are the trend with condensation temperature found by Meléndez et al. (2009). Figure extracted from Ramírez et al. (2011). 
ces by nucleosynthetic groups, top panel of Figure 4.5 shows the trend with condensation temperature for $\alpha$-elements, and lower panel showing the same trend for the Fe group elements. In this figure there is an increase in abundance with increasing $\mathrm{T}_{\mathrm{C}}$. They attributed this finding as an evidence of formation in an environment of low Type II supernovae with a high dust-to-gas ratio. Chen et al. (2014), however, argued against that possibility, as the beryllium abundances did not support their previous claim.
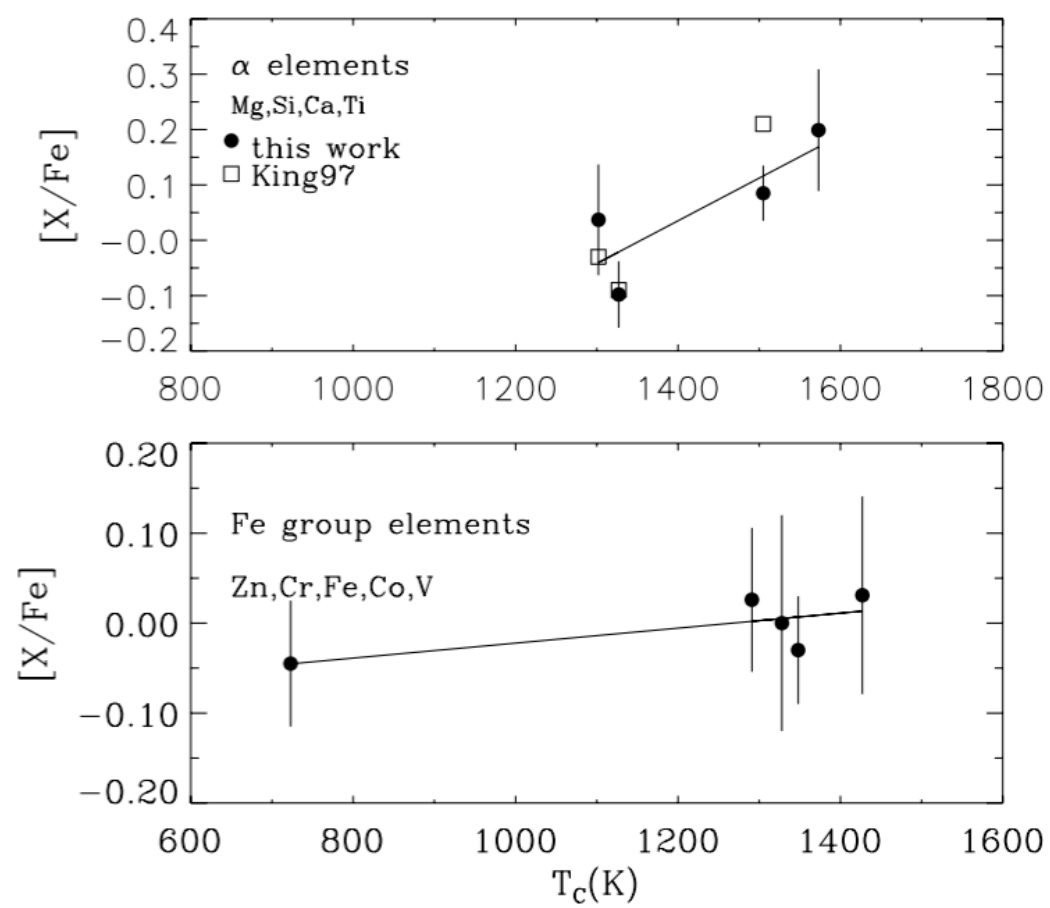

Figure 4.5: Chemical abundances as a function of condensation temperature of stars HD 134439/134440, relative to the solar abundance. The top panel shows the $\alpha$-elements, and the lower panel shows the iron peak elements. Figure extracted from Chen and Zhao (2006).

In light of these conflicting results, we decided to look into possible trends with $\mathrm{T}_{\mathrm{c}}$ and into the relative abundance pattern of these stars. A plot of $[\mathrm{X} / \mathrm{Fe}]$ vs $\mathrm{T}_{\mathrm{c}}$ of our abundances is shown in Figure 3 of Reggiani and Meléndez (2018), and we found that the slopes of the possible trends are unclear and not significant, specially when the standard star used is not the Sun, but HD 103095 (that has a closer abundance pattern, due to its similar metallicity). The significance we found for the slopes are less than $2 \sigma$ for both stars (HD 134439 and HD 134440), which discards the conclusion of Chen and Zhao (2006) that these stars were born in a high dust-to-gas environment, agreeing with the conclusion based on the beryllium abundance by Chen et al. (2014).

As we found no trend with condensation temperature for the differential abundances of 
the binary pair relative to HD 103095, we compared the abundances of these stars against each other, and we show our finding in Figure 4.6. Being binary stars (later confirmed using GAIA DR2 data in El-Badry and Rix, 2018), we expected the abundance difference between these stars to be zero, at least within the errors of our analysis. Instead, we found a mean abundance difference of $\Delta[\mathrm{X} / \mathrm{Fe}]=0.06 \pm 0.01$ (we show the individual abundances differences in Table 2 of Reggiani and Meléndez, 2018). To test the confidence of this result, we performed a t-test to find the likelihood that our result is due to errors in our analysis, finding a low probability $\left(\mathrm{p}=2.05 \times 10^{-6}\right)$ of a zero abundance difference between these stars. The test assumed a mean error of 0.03 dex in each of our abundances, randomly adding (or subtracting) the error $10^{5}$ times, and performed the t-test in each iteration. The final result is the averaged $\mathrm{p}$ value of all iterations. Thus, it is very unlikely that the abundance difference we found is not real. This difference can also be seen from the spectral line we showed in Figure 1.10.

We also found that the lithium abundance of stars HD 134439 and HD 134440 differ by 0.6 dex (the same amount found in a previous study by King (1997)), but unlike the other chemical elements, lithium is more depleted in HD 134440, as is the beryllium abundance reported by Chen et al. (2014) (it was detected in HD 134439 and not in HD 134440). The masses of these stars were estimated to be $0.59 \mathrm{M}_{\odot}$ and $0.58 \mathrm{M}_{\odot}$, and their ages 9.9 and 9.4 Gyrs for HD 134439 and HD 134440, respectively. Thus, it is not possible to attribute these abundance differences to stellar evolution causes.

With all these very interesting and puzzling results, we argued that the abundance differences are due to the engulfment of a planet by HD 134440. This event would increase the photospheric abundance of most elements, and it is also possible that the rotation induced by the accretion could have lead lithium and beryllium deep enough into the star to a temperature layer high enough to burn them. Although we found no indication of extra rotation, it is possible that the star has already reduced its rotation in its long lifetime. Although no planet has been detected in such low metallicity stars so far, Johnson and Li (2012) showed that it is possible to form planets in metal-deficient stars, but the planets would have to be formed in close proximity to its host star, which might increase the probability of an accretion event. Assuming that HD 134440 has indeed engulfed a planet, we used equation 4.1 to estimate the planetary mass that it would have to accrete to create such a difference in abundance, and found that a planet with $\mathrm{M} \approx 0.9 \mathrm{M}_{\mathrm{J}}$ could 
have been engulfed by HD 134440 .

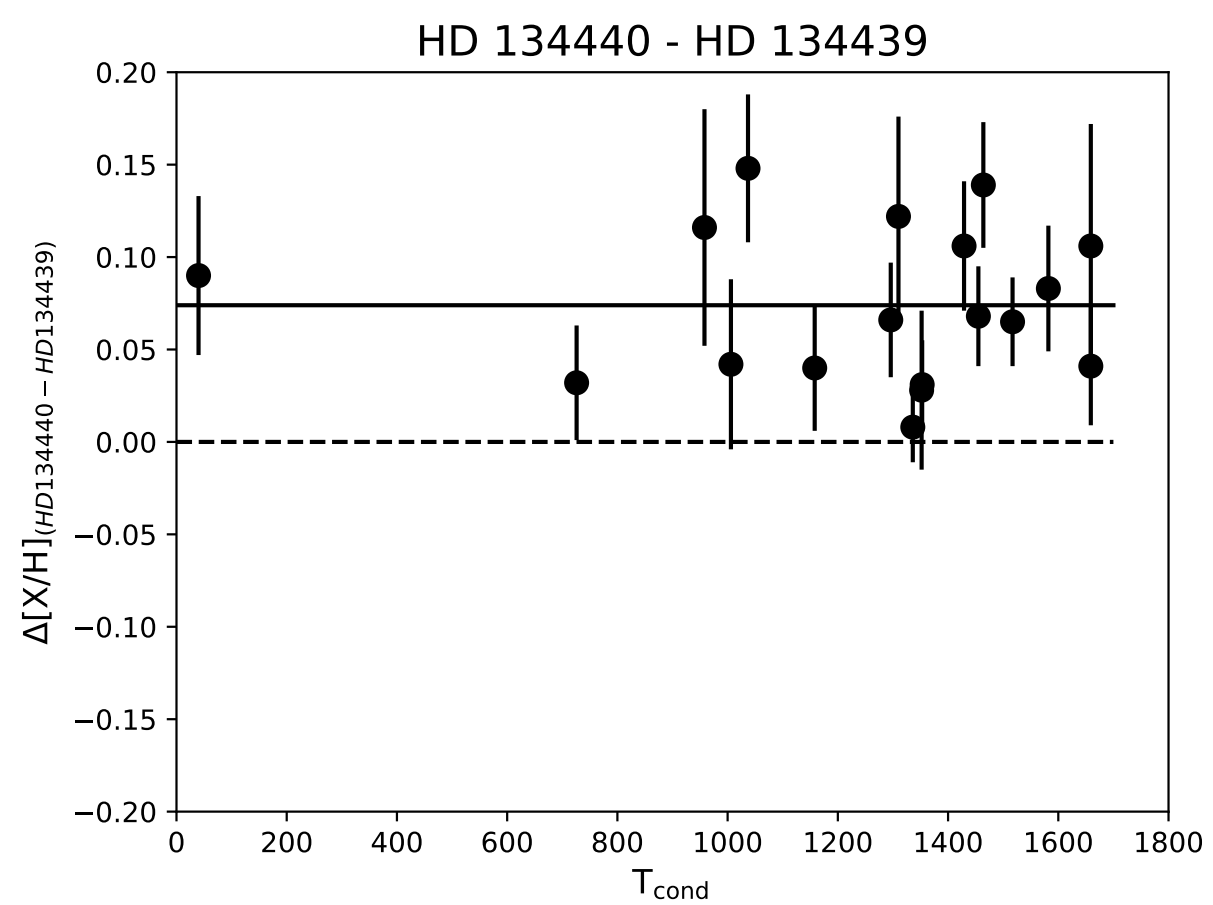

Figure 4.6: Comparison between the chemical abundances of HD 134439 and HD 134440, as a function of condensation temperature. Solid and dashed line are the mean abundance difference found, and the zero abundance difference, respectively. Figure extracted from Reggiani and Meléndez (2018).

More details on the analysis of these stars are in Reggiani and Meléndez (2018), attached in Appendix A of the thesis. 
Chapter 5

\section{Non-LTE analysis of Potassium}

\subsection{Galactic Chemical Evolution of Potassium}

Potassium is a simple alkali metal with $\mathrm{Z}=19$, with an atomic structure that highly resembles that of sodium, and just like sodium, the older GCE models predict abundances much lower than those based on LTE analysis (e.g., Kobayashi et al., 2006, 2011; Zhao et al. 2016, Prantzos et al., 2018). In this Chapter, we address the chemical evolution of potassium. This is based on the paper by Reggiani et al. (2019), attached in Appendix A.

\subsubsection{Theoretical chemical evolution of potassium}

Potassium is one of the elements synthesized in oxygen burning, and its production in supernovae is highly dependent on the production of oxygen through carbon $\left({ }^{12} \mathrm{C}(\alpha, \gamma){ }^{16} \mathrm{O}\right.$ - CNO cycle) and on convective effects during the nucleosynthetic phase. Potassium production is also sensitive to convective overshoot, mixing during the last stages of shell oxygen burning, the stellar density structure near the iron core (stellar mass), initial location of the mass cut, and the fall back mass (Thielemann and Arnett, 1985; Bazan and Arnett, 1994; Arnett, 1994; Woosley and Weaver, 1995).

The main production of $\mathrm{K}$ is either by hydrostatic oxygen shell burning or explosive oxygen burning and the potassium isotopes ${ }^{39} \mathrm{~K}$ and ${ }^{41} \mathrm{~K}$ are produced in these processes only, but the proportion of their production varies with stellar mass. The ${ }^{40} \mathrm{~K}$ isotope can also be produced in the neon and carbon shells, but it is the rarest isotope. The proportion of potassium isotopes in the solar system, as determined from meteorites, are ${ }^{39} \mathrm{~K}=93.132 \%$, ${ }^{40} \mathrm{~K}=0.147 \%$, and ${ }^{39} \mathrm{~K}=6.721 \%$, according to Lodders et al. (2009). Lodders et al. (2009) also found an excellent agreement between the solar photospheric $(\mathrm{A}(\mathrm{K})=5.12$, consistent 
with our solar K abundance) and the meteoritic abundance.

In Figure 5.1 there is an example of potassium production for two different initial stellar masses. It is easy to see that the main isotope $\left({ }^{39} \mathrm{~K}\right)$ is produced in a much larger scale for both models presented.
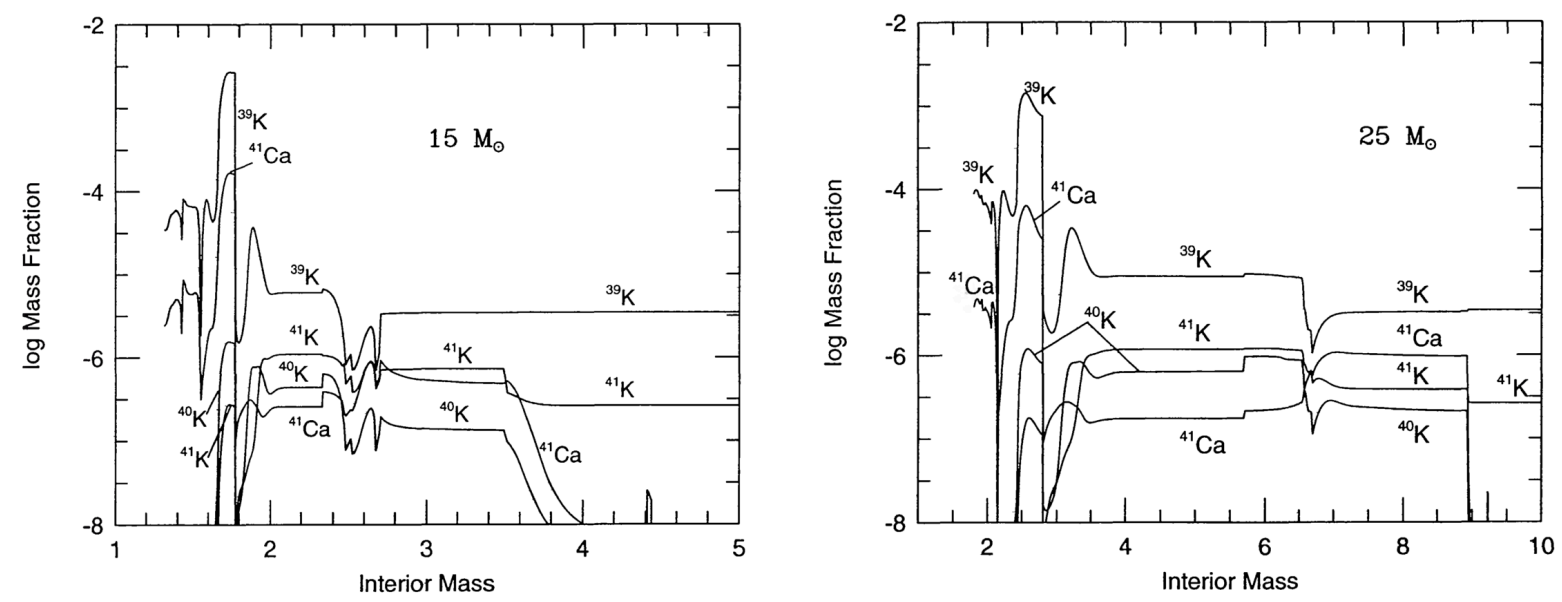

Figure 5.1: Mass fractions in the interiors of a $15 \mathrm{M}_{\odot}$ (left panel) and a $25 \mathrm{M}_{\odot}$ (right panel) star, showing the synthesis of K isotopes. Figure extracted from Woosley and Weaver (1995).

Standard GCE models, which we consider to be older GCE models that do not have the addition of nucleosynthetic events like neutron stars mergers (NSM), rotating stars, or collapsars (i.e., all the nuclesynthetic products in these standard models come primarily from Supernovae types Ia and II, Hypernovae and AGB stars), highly underestimate the production of potassium when compared to observations, as shown in Figure 5.2. We can see that such models can underestimate the abundances by as much as 1 dex, exemplified by the abundances from the K11 and K15 models (Kobayashi et al., 2011; Zhao et al. 2016; Sneden et al., 2016), portrayed with LTE abundances in Figure 14 of Reggiani et al. (2019). Kobayashi et al. (2011) argued that the low abundances in the models are, at least partially, due to the lack of a neutrino process in their modeling.

However, there are models with additional nucleosynthetic processes that are able to considerably raise the output abundance of potassium. That is the case of the recent work from Prantzos et al. (2018) and its GCE model with the addition of yields from rotating massive stars (from now on we will use PT and PTR for the models without and with massive rotating stars). How do these stars produce extra potassium during the oxygen burning at their final stages? Rotating massive stars produce additional seed atoms for the nucleosynthesis of potassium, and other elements like scandium. In rotating massive 


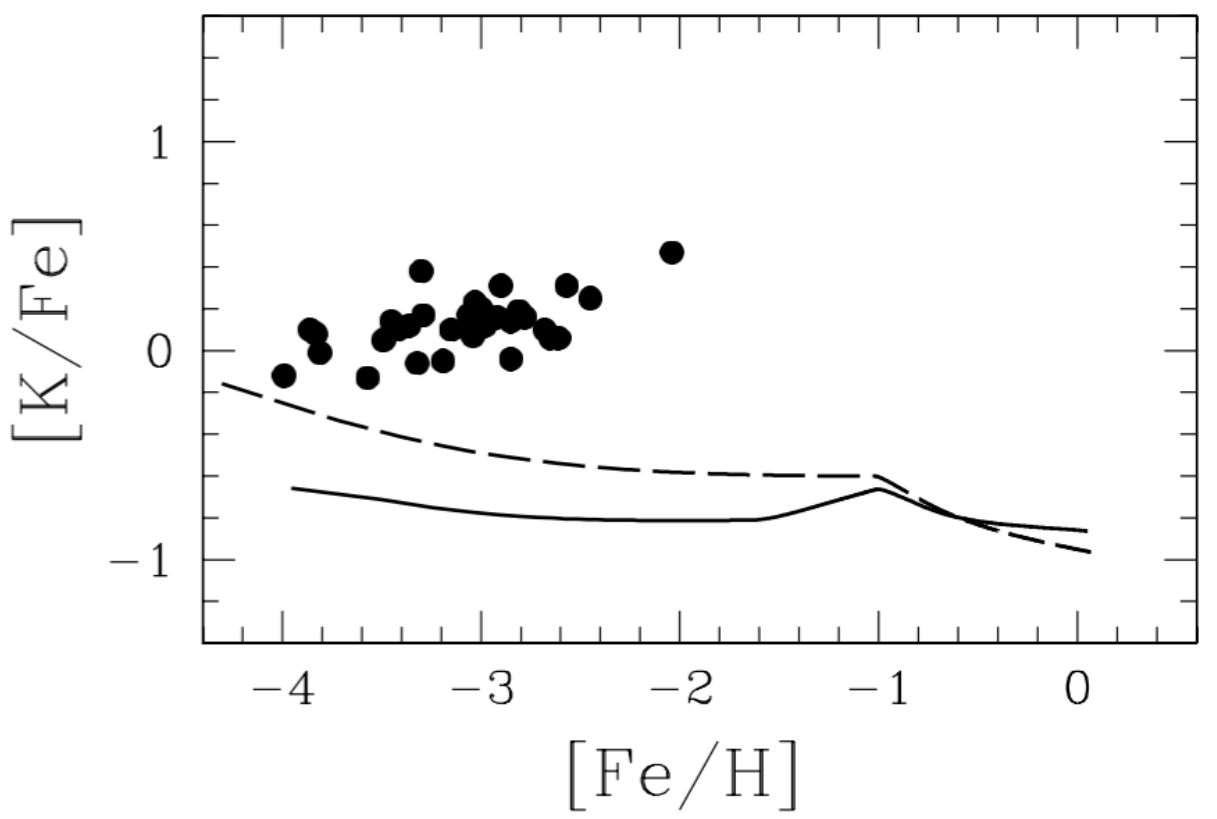

Figure 5.2: Potassium abundance as a function of metallicity. The black dots are observational data and the lines are GCE models. Figure extracted from Kobayashi et al. (2006).

stars, the rotation-induced mixing continuously brings carbon, produced in the helium core via $3 \alpha$ process, to the hydrogen burning shell. This freshly introduced carbon produces oxygen via the CNO cycle, and the freshly produced oxygen becomes extra seed for the production of potassium during oxygen burning.

In Figure 5.3 we show four different GCE models, the K11 and K15 models, and the PT and PTR models. The details of the differences of the K11, K15, and PT(R) models will not be discussed, but it is important to point that the models from Prantzos et al. (2018), like the K11 and K15 models, are one-zone models, with Kroupa IMF and also consider gas infall. Thus, their main assumptions are similar to those discussed for the K11 and K15 models in Section 3.2 .

The mean potassium abundance $([\mathrm{K} / \mathrm{Fe}]$, estimated throughout the entire metallicity regime of the models) vary considerably: -0.73 dex for K11, -0.72 dex in K15, -0.61 dex in PT, and -0.35 dex in PTR. This variation of up to 0.38 dex (between K11 and PTR) already reduced the mean difference from models and observation from $\sim 1$ dex to less than 0.7 dex, a considerably better result. As in this $\mathrm{PhD}$ work the main interest is metal-poor stars, it is also interesting to estimate the mean $\mathrm{K}$ abundances for $[\mathrm{Fe} / \mathrm{H}] \leq-1.0$, which are -0.74 dex, -0.74 dex, -0.73 dex and -0.05 dex, for K11, K15, PT and PTR, respectively. The difference of almost 0.7 dex between the models without and with yields from massive 
rotating stars is impressive. It can diminish the mean difference between models and observations to $\sim 0.3$ dex. There is also a remarkable difference in the behavior of the curves.

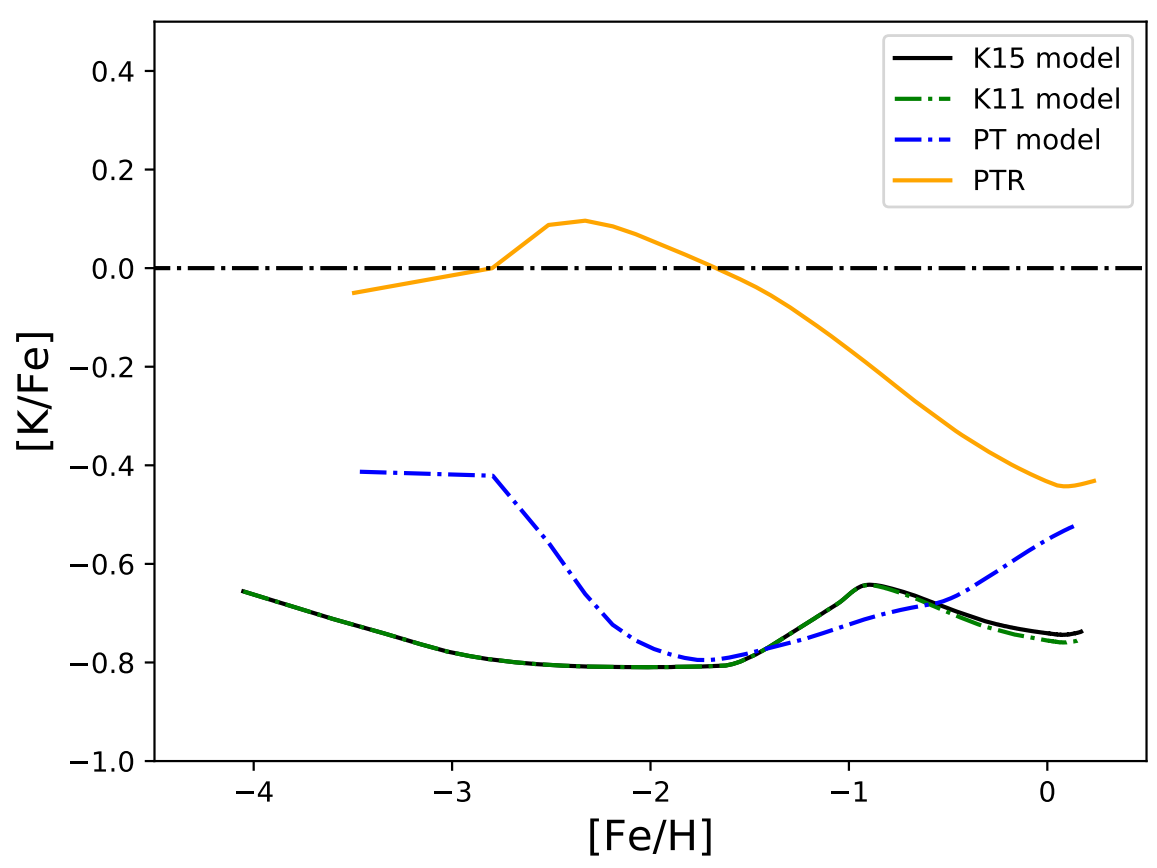

Figure 5.3: Potassium abundance as a function of metallicity. We portray four different models: K11 and K15 (Kobayashi et al., 2011; Zhao et al., 2016; Sneden et al., 2016). PT and PTR are the two one-zone models from (Prantzos et al. 2018) without, and with, the addition of nucleosynthetic yields of rotating massive stars, respectively.

\subsubsection{The observational data}

We discussed, from a simplified modeling perspective, how the differences between models and observations of K could be improved. But as GCE models must be continuously improved, so must our observed abundances.

First, it must be clear that when we refer to "observed abundances", we are actually saying: "abundances estimated through the analysis of stellar spectra", as the determination of chemical abundances involve a series of assumptions on the stellar atmosphere and how the spectral line is formed, as discussed in Chapter 1. Particularly, in Section 1.2.5 we discussed how the radiative transfer codes used throughout this work assume that the spectral lines form under LTE conditions.

Potassium is a difficult element to measure in stellar spectra. Between $3000 \AA$ and 
$10000 \AA$ (UV, visible and near infrared regions accessible through CCD detectors), there are only 4 clearly measurable lines of K: $5801 \AA$, $6939 \AA, 7664 \AA$ and $7698 \AA$. The first two lines are weak and can only be measured in solar-metallicity cool stars, and are in crowded spectral regions, which makes the spectral normalization difficult, and therefore yields a less reliable measurement (either via EW or spectral synthesis). For the other two lines, formation in LTE is not a valid assumption (Bruls et al., 1992; Takeda et al., 2002; Zhang et al., 2006; Andrievsky et al., 2010; Zhao et al., 2016). To make the analysis of potassium harder, the $7664 \AA$ line is heavily blended with telluric $\mathrm{O}_{2}$ and is very difficult to be correctly measured. Thus, stellar potassium data mostly come from the $7698 \AA$ line, which is a strong line, commonly saturated in high metallicity stars. In Figure 5.4 we can see how the line is saturated in the Sun, HD 192263, and Procyon. HD 192263 is a cool $\left(T_{\text {eff }} \sim 5000 \mathrm{~K}\right)$ star with strong damping wings, and Procyon is a hotter $\left(T_{\text {eff }} \sim 6500\right.$ $\mathrm{K}$ ) solar-metallicity star showing damping wings with a strong asymmetry, in which the non-LTE corrections are as large as -0.7 dex. Thus, the LTE assumption is not valid for the analysis of potassium.

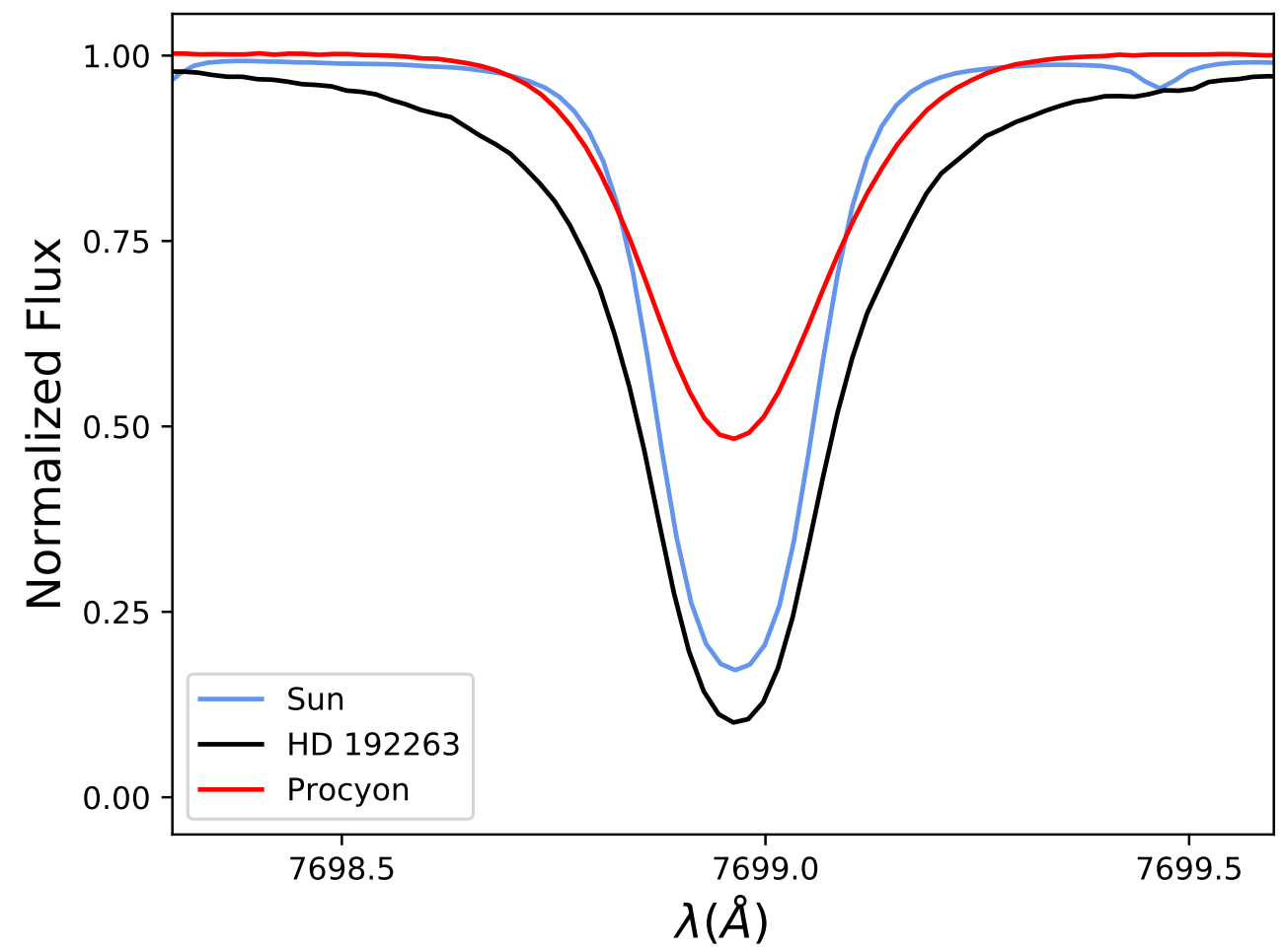

Figure 5.4: The potassium $7698 \AA$ resonance line in the Sun, HD 192263, and Procyon.

Furthermore, observational studies of potassium have shown that it highly differs from 
the other light elements. Spina et al. (2016) studied the nucleosynthetic history of 24 chemical elements in solar twins of the galactic disk and found that all elements, except potassium, have an evolution with age that can be fitted using a hyperbolic, or a 2 piece linear curve, as shown in Figure 5.5. This result might either indicate inhomogeneity in potassium nucleosynthetic evolution, or a problem in the determination of potassium abundances. All K abundances from Spina et al. (2016) were estimated from the strong and saturated $7698 \AA$ line.

Another clear indication of the problem surrounding the potassium analysis, from an observational perspective, is given in Figure 5.6. A differential analysis between the binary stars 16 Cyg A and B is shown, and potassium is indicated by a circle. The top panel shows the LTE differential abundances and in the lower panel the abundances were nonLTE corrected based on the correction grid from Zhang et al. (2006). It clearly shows that the non-LTE corrected abundances are much more in line with what is expected for that condensation temperature (based on the remaining chemical elements). This is yet another example of how non-LTE corrected abundances are more reliable when dealing with the potassium 7698 A line.

The chemical evolution of potassium must be studied further both from the modeling and from the observational perspectives. In Figure 13 of Prantzos et al. (2018), there is an example of the conjunction of these different perspectives. New modeling and yields, along with non-LTE potassium abundances, can reconnect models and observations.

However, the latest published non-LTE abundance correction grid of potassium is from a study made over 10 years ago, Zhang et al. (2006), and their correction grid is based on "only" 100 model atmosphere points. Furthermore, much of the data required for nonLTE modeling has greatly evolved since. Thus, we pursued the creation of a new and improved model atom of potassium, with the most up to date atomic data, in order to create the largest grid of corrections to date. In the next sections we discuss the nonLTE formulation, necessary data to create a model atom, and we present the main results from our work, developed mostly during a 1-year Brazilian CAPES studentship (process number 88881.132145/2016-01) awarded to Henrique Reggiani, as a visitor PhD student at the Max-Planck-Institut für Astronomie (Heidelberg, Germany), under supervision of Dr. Karin Lind. 


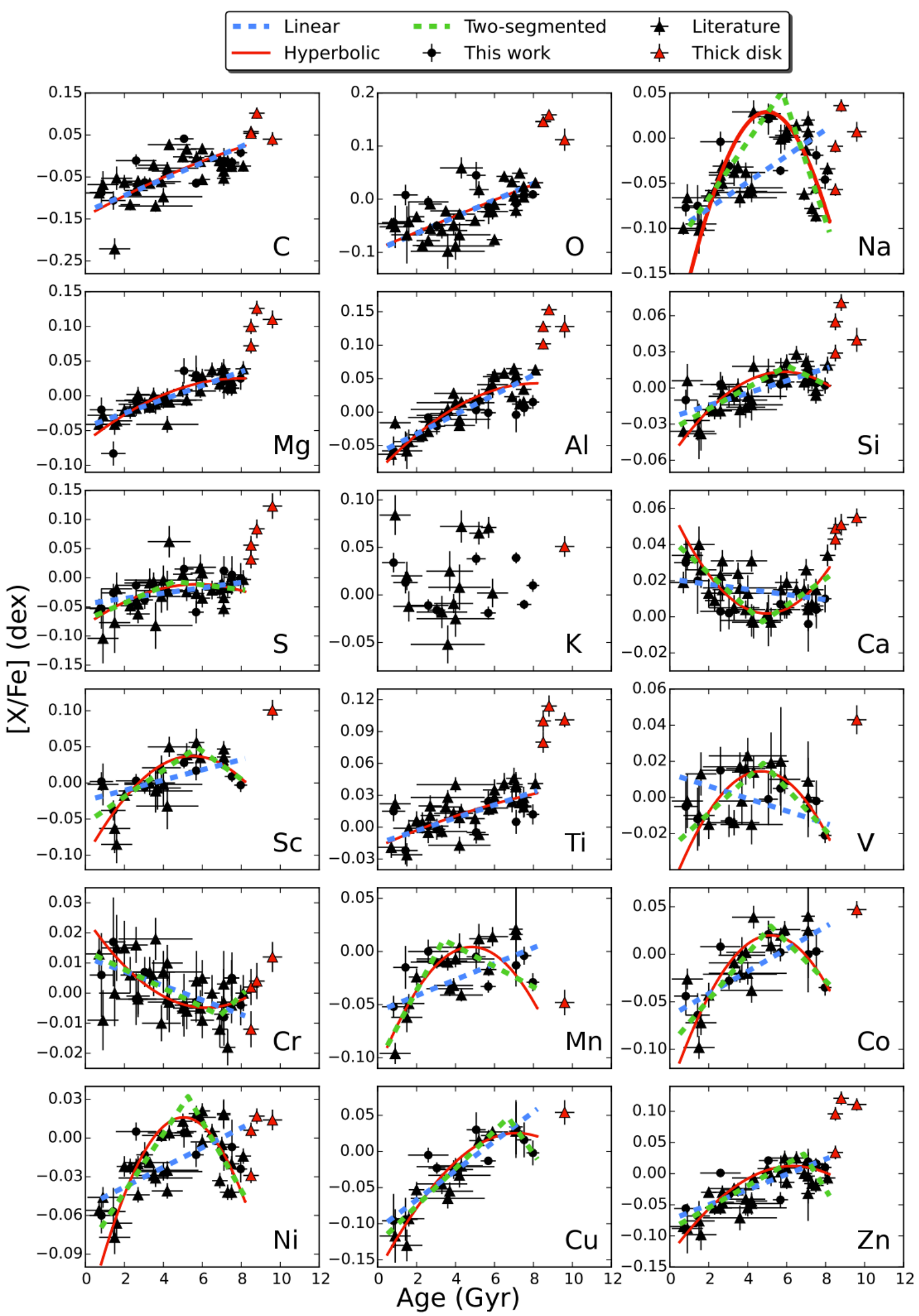

Figure 5.5: Chemical evolution of the elements as a function of stellar age, using solar twins. Figure extracted from Spina et al. (2016). 

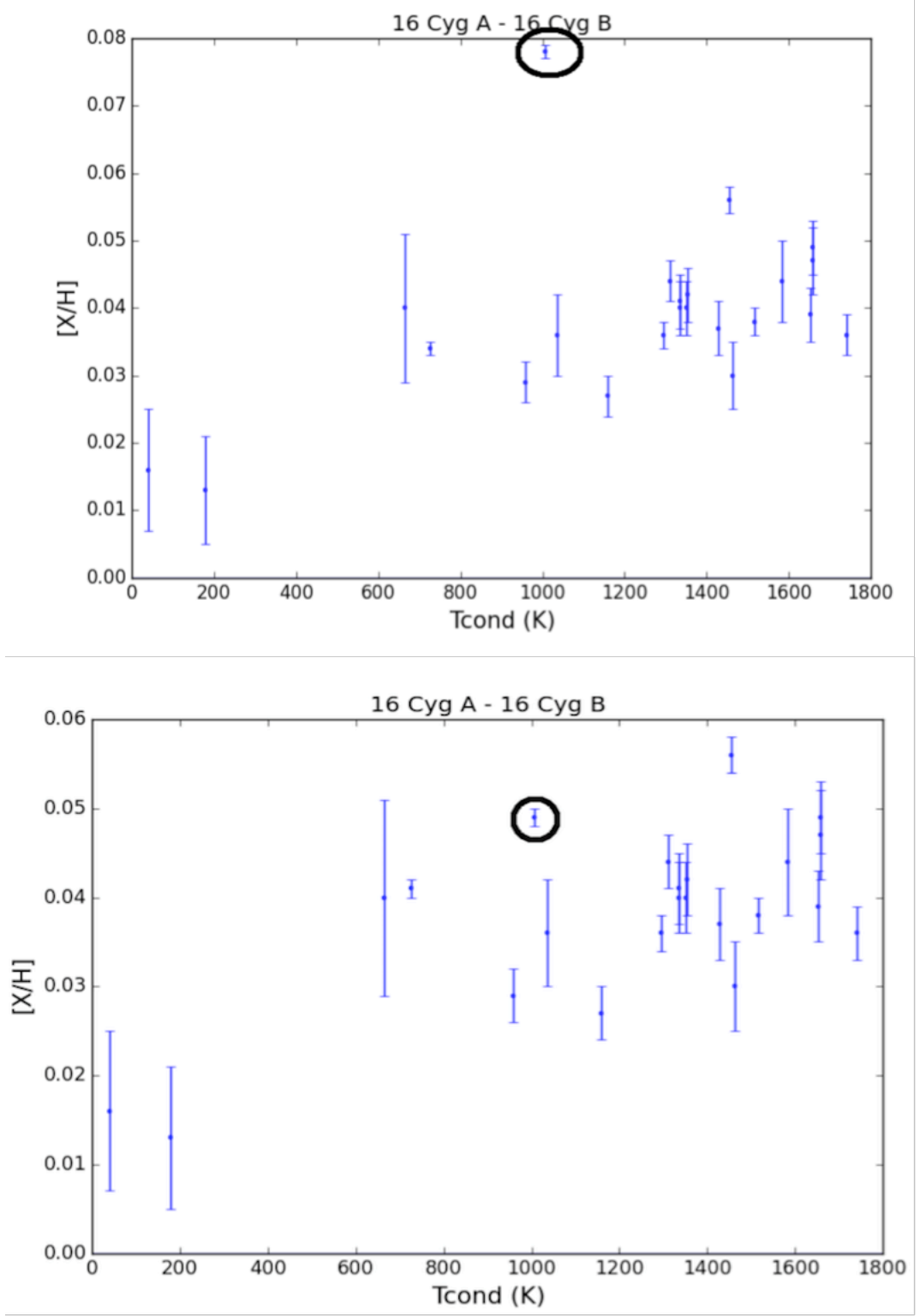

Figure 5.6: Differential abundances between the binary twin stars $16 \mathrm{Cyg}$ A and B, as a function of condensation temperature. M. Tucci-Maia (private communication). 


\subsection{The non-LTE formulation}

In Section 1.2.5 it was shown how the source-function and level populations can be calculated with the assumption that the medium is under local thermodynamical equilibrium; we discussed line source functions and how are used to synthesize a spectral line. However, the LTE assumption is not always valid.

In regions where the mean free path of photons is large, in low-density environments, there are not enough collisions to establish the thermodynamic equilibrium, hence the LTE approximation fails. That is what happens, for example, in the formation of the stronger spectral lines (resonant), formed in the outer layers of the stellar photosphere. In metal-poor stars, where densities are lower due to the limited amount of heavy atoms, the LTE assumption also often fails. Another example is line formation in stars with very high temperatures, like $\mathrm{O}$ type stars, where the radiation field is too strong and more important than collisions (Gray, 2005).

Under these conditions, abundances calculations must be made outside local thermodynamical equilibrium (non-local thermodynamical equilibrium, non-LTE). In non-LTE, the Boltzmann and Saha equations can not be used to estimate the level populations. The interactions between matter and radiation are given by the statistical equilibrium, i.e., atomic level populations do not change over time (Asplund, 2005). The interactions that must be considered in a non-LTE formulation are: the scattering of a photon by an atom, both to atomic excitation or ionization, and to the transfer of the photon energy into kinetic energy; excitation and desexcitation by collisions between atoms and free electrons (emitted photons depend on the kinetic energy of the process); ionization and charge exchange by collision with free electrons; excitation and deexcitation by collision with other atomic species; and ionization and charge transfer due to the collision with other atomic species.

In order to include those processes, it is necessary to build detailed model atoms. The complexity of such modeling depends directly on the number of possible atomic transitions (that depends on the number of electrons in the atom). Due to the increasing complexity towards higher atomic number (Z) atoms, non-LTE studies have, so far, been restricted to few atoms and transitions, with the exceptions of $\mathrm{Fe}$ and $\mathrm{Ti}$, where there are several nonLTE line transitions calculations. Even for Fe and Ti, there are not calculations available 
for all line transitions used in studies of chemical abundances.

When model atoms are ready, the calculations of line transitions in non-LTE involve the simultaneous solution of statistical equilibrium, particle conservation and radiative transfer equations.

\subsubsection{Non-LTE radiative transfer}

Line formation in non-LTE refers to the situation when the radiation fields, in all directions and frequencies, and level populations, do not vary with time. Thus, the statistical equilibrium equation can be written as:

$$
\frac{d n_{i}(\vec{r})}{d t}=\sum_{j \neq i}^{N} n_{j}(\vec{r}) P_{j i}(\vec{r})-n_{i} \sum_{j \neq i}^{N}(\vec{r}) P_{i j}(\vec{r})=0,
$$

where $n_{i}$ is the population of a particular level, $N$ the number of levels important to the population of $n_{i}, j$ connects all the levels and $P_{i j}$ are the transition rates. When in LTE, the equilibrium of the medium states that the transition rates due to collisions with other atoms, ionized or not, with electrons and with radiation, are the same. In the non-LTE case, however, we need to consider these processes separately and the transition rate $P_{i j}$ is the sum of the radiative and collisional transition rates.

$$
P_{i j}=R_{i j}+C_{i j}
$$

where $R_{i j}$ is the contribution from radiative transitions and $C_{i j}$ is the contribution from collisions with other particles (excitation and deexcitation). For bound-bound transitions the radiative rate per particle is split into:

$$
R_{i j}=A_{i j}+B_{i j} \overline{J_{\nu}}
$$

where $A_{i j}$ denotes the radiative emission and $B_{i j}$ denotes the radiative absorption/stimulated emission and $\bar{J}_{\nu_{0}}$ is the mean intensity averaged over the transition profile at any given frequency.

As in LTE, the particles of a given system must be conserved and the second basic equation of the non-LTE formulation is

$$
\sum_{j=1}^{n_{l}} n_{j}=n_{t o t}
$$


And the last equation is the radiative transfer equation:

$$
\begin{aligned}
& \mu \frac{d I_{\nu \mu}}{d z}=-\kappa_{\nu \mu} I_{\nu \mu}+j_{\nu \mu} \\
& \mu \frac{d I_{\nu}(\vec{r}, \mu)}{d \tau_{\nu}(\vec{r})}=-S_{/ n u}(\vec{r})+I_{\nu}(\vec{r}, \mu),
\end{aligned}
$$

where $\mu$ are the directions and $\bar{r}$ the important locations.

The main complication in non-LTE profile calculations is the appearance of the mean intensity $\left(\overline{J_{0}}\right)$ at the statistical equilibrium equation (equation 5.1). Thus, equation 5.1 directly depends on the intensity that is given by equation 5.5 , while this intensity depends on the level populations (through the source function). In LTE the level populations can be calculated separately (equation 1.53), but as it is not possible in non-LTE, the radiative transfer equation must be solved simultaneously with the statistical equilibrium for all relevant frequencies.

We use the non-LTE radiative transfer code MULTI (Carlsson, 1986), version 2.3, to solve the radiative equations. MULTI solves the equations using a multi-dimensional Newton-Raphson iterative scheme. For further details on the calculation method of MULTI, see Carlsson (1986, 1992).

\subsubsection{Atomic levels}

To solve the statistical equilibrium and radiative transfer equations in non-LTE, a wealth of information is necessary, starting with the detailed levels and their respective energies. For our potassium atom, we added all the available information on the NIST ${ }^{1}$ atomic spectra database (Kramida et al. 2019).

The original level information for potassium on NIST comes primarily from Sugar and Corliss (1985) and Sansonetti (2008). The level information necessary to add to the atomic model are the level energy (in $\mathrm{cm}^{-1}$ ), the statistical weight of the level, a label for the level with 20 characters and the ionization stage. All these informations are available on NIST for all levels with principal quantum number up to $n=20$ and orbital quantum number $l=3$. We added 110 fine-structure resolved levels from the NIST database.

Our model atom is complete up to $n=20$ and $l=19$, which is $\approx 0.13 \mathrm{eV}$ below the potassium ionization energy $(4.34 \mathrm{eV})$. The levels not available in NIST are not finestructure resolved and were added for particle number conservation. These levels are not

\footnotetext{
${ }^{1}$ https://www.nist.gov/pml/atomic-spectra-database
} 
connected to each other via bound-bound transitions and, thus, have LTE populations. The energies of these levels were estimated assuming they are Rydberg levels.

A Rydberg atom is an atom excited into a high energy level. It resembles a hydrogen atom and the effect of the core electrons is accounted for as a quantum defect $\delta$. For alkali metals in a Rydberg state, the energy of the level can be described by the principal quantum number and is given by:

$$
E_{b}=-\frac{R_{y}}{n^{2}-\delta}
$$

where $R_{y}=13.6 \mathrm{eV}\left(R_{y}=109736.605 \mathrm{~cm}^{-1}\right), n$ is the principal quantum number and $\delta$ is the quantum defect. Using this formula, most of the higher orbital levels of same principal quantum number have the same energy, and they are added as superlevels, with the same energy and a statistical weight that is the sum of the statistical weight of all the different $l$ levels.

The ground level of K II has also been added to the atomic model. In total, we added 134 levels from the ground level of K I to the ground level of the single ionized potassium.

\subsubsection{Bound-bound transitions}

As mentioned in Section 5.2 , to calculate the non-LTE line formation, we must consider all possible interactions between the atom and radiation, as well as between the $\mathrm{K}$ atom and matter. Radiative bound-bound transitions can be summarized by three main pair of processes: a) photo scattering: excitation immediately followed by an either induced or spontaneous atomic deexcitation, so that the photon created by the deexcitation is often described as being the same photon that initially excited the atom, but redirected into a different direction and, possibly, slightly shifted in frequency; b) photon creation: a collisional excitation followed by spontaneous or induced deexcitation, where the new photon has the energy transferred to it from the initial kinetic energy due to the collision; c) photon destruction: radiative excitation followed by collisional deexcitation, which thermalizes a photon into kinetic energy.

The above processes are relevant, specially if the line forms in a location where scattering is important, because it will suffer from strong non-LTE effects. We limit our discussion of the processes as it is not part of our scope to dive into how these processes are physically treated and how to mathematically describe each of them. It is sufficient to 
explicitly describe how they can be treated (a, b, and c cases above) and to describe how we add these processes to the modeling of our atom.

\subsubsection{Bound-Bound radiative transitions}

To treat the bound-bound radiative transitions, MULTI requires the user to add the upper level and lower levels of the transition, the oscillator strength of the transition $(f)$, the inverse lifetime of the upper level, the van der Waals broadening and the Stark broadening parameters. Besides the atomic information, the user has the freedom to choose how many frequency points will be modelled for each line (this number will control the quality of the synthetic line profile, as well as other aspects like the quality of the estimated EW, but higher number of frequency points take longer to calculate). In Figure 5.7 we show an example of a synthetic potassium line in the IR with sufficient and insufficient frequency points for its modeling.

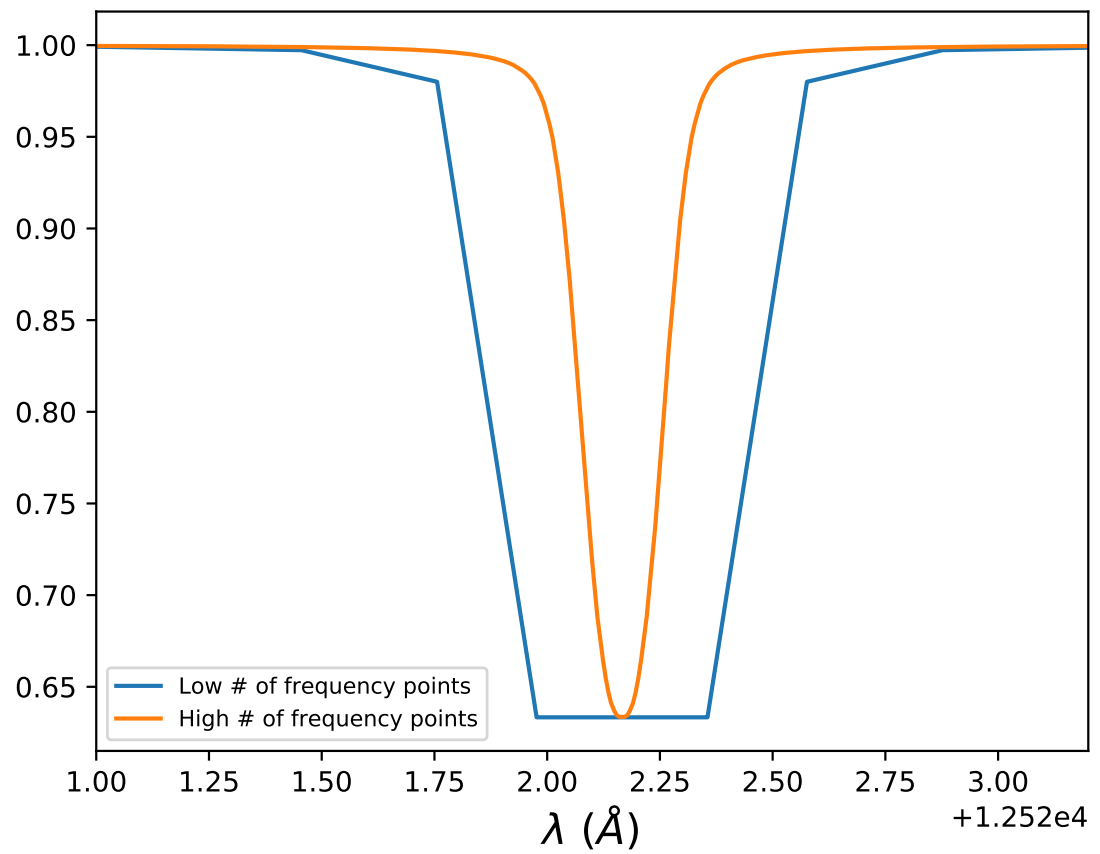

Figure 5.7: Synthetic $12522 \AA$ potassium line modeled with different numbers of frequency points.

Like the atomic levels, most of these transitions are listed in the NIST database, and their original sources are Wiese et al. (1969); Biemont and Grevesse (1973) and Sansonetti (2008). The total number of bound-bound transitions in our model atom is 250 .

With the atomic levels and bound-bound radiative transitions, we can create the Grotian diagram of our atom, a visual representation of the atomic levels and all the radiative 
interactions between them. In Figure 5.8 we show the diagram of the potassium atom, the same diagram presented in Reggiani et al. (2019). We did not add any transition with the Rydberg levels of high quantum numbers. We argue that these transitions are not important to the statistical equilibrium. To come to this conclusion, we removed all available transitions with wavelength longer than $20,000 \AA$ from our model atom and compared the results of an atom without those lines against the atom with all 250 lines, and the results were indistinguishable. Thus, our final model atom is represented in Figure 5.8 .

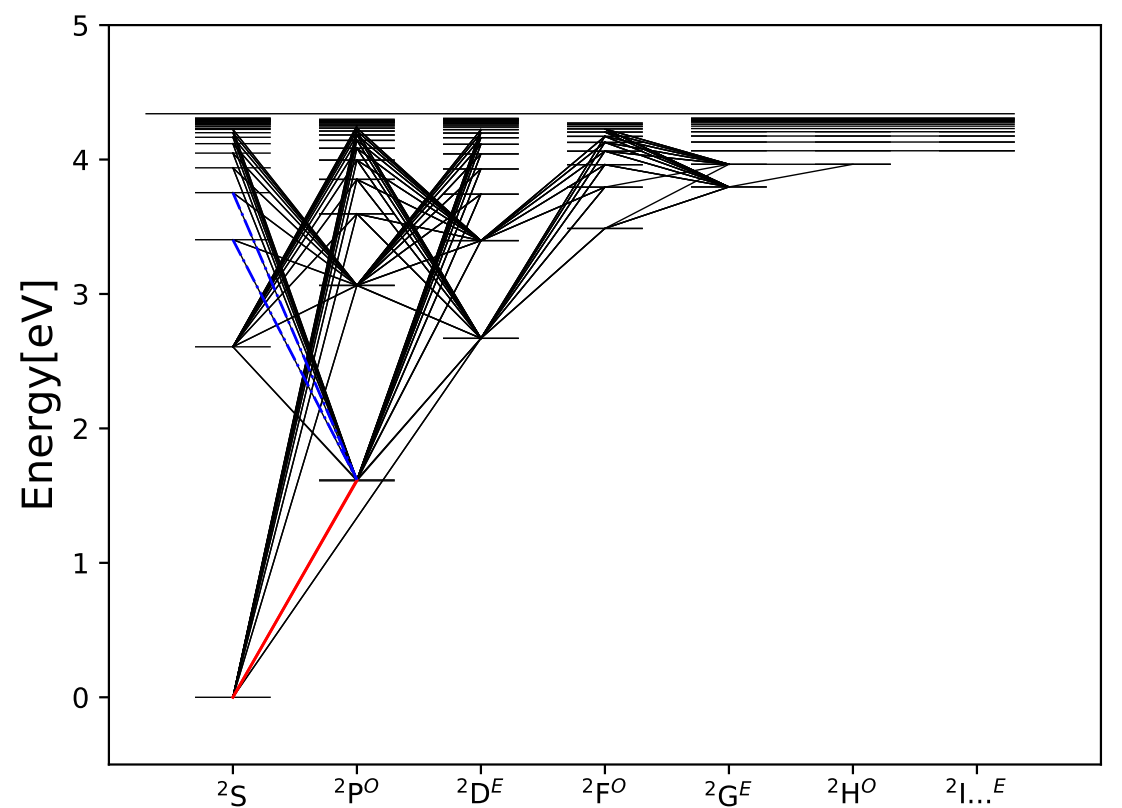

Figure 5.8: Grotian diagram of the potassium atom. The red (7664 and $7698 \AA$ ) ) and blue (5801, and $6939 \stackrel{A}{A})$ lines indicate the transitions that were studied in detail. Figure extracted from Reggiani et al. (2019).

\subsubsection{Collisional excitation}

The bound-bound transitions in the atom are also caused by collisions with matter within the stellar atmosphere. As most of the stellar atmosphere is composed of hydrogen atoms and free electrons, these particles cause the only collisional processes important for the statistical equilibrium.

In MULTI we add the collisional information as the rate coefficient $(q)$, that is the collisional cross section $\sigma$ multiplied by the velocity distribution $v, q=\langle\sigma v>$. As in LTE, the velocity distribution assumed is the Maxwell distribution (equation 1.47). The rate coefficients are then estimated for each transition in a range of different temperatures. 
The temperature intervals assumed for calculating the rate coefficients must be sufficient for MULTI to be able to interpolate the rates in the different layers of the atmospheric model (meaning different temperatures, or atmospheric depths). As we are mainly working with late-type stars and not aiming to produce a non-LTE atom to be used in spectral analysis of extremely hot objects, like $\mathrm{O}$ stars, we added collisional rates for temperatures of $\mathrm{T}=1000,2000,3000,4000,5000,6000,7000,8000,9000,10000,15000$, and 20000 K. Besides the coefficients for each of the selected temperatures, we also need to input the upper and lower levels of the bound-bound transitions.

As the velocity distribution is well known, the remaining problem is to estimate the collisional cross-sections, and that is a very complicated problem. We briefly discuss the data we have employed for the collisional cross-sections of potassium with neutral hydrogen atoms $(\mathrm{H}+\mathrm{K})$ and with free electrons $(\mathrm{e}+\mathrm{K})$.

\subsubsection{3 e+K rates}

Older atomic models for non-LTE modeling of alkali metals have used the e+K collisional rates from the methods by, among others, Park (1971) (hereafter; Park71), and van Regemorter (1962) (hereafter; vanReg62). Park71 described a semi- empirical method based on quantum mechanical calculations and empirical corrections from the, at the time, best available laboratory measurements. Probably one of the most compelling reasons as to why this method is still used today is that it is computationally very cheap. We employed this method in most of the collisional rates and all the calculations can be done in a personal computer, following the recipe in the original paper. Although Park71 is employed in most of our work, the rates estimated via this method are usually not in agreement with most recent methods, specially for the lower atomic levels. The other method usually employed (Zhang et al., 2006; Andrievsky et al., 2010) is given by the formulation of vanReg62, which was designed to estimate the rates of allowed transitions only.

The most recent methods of calculation can yield more reliable results, as the calculations are based on more realistic physical approaches, but they are computationally very expensive. In this work, we employed two recent methods of calculating e+K rates, the convergent close coupling method (CCC, Bray and Stelbovics, 1992) and the B-spline R-matrix method (BSR, Zatsarinny and Bartschat, 2013). Both methods are independent approaches to solve the close coupling equations. Details of the methods can be found 
in the aforementioned papers, and in Reggiani et al. (2019) there is a brief description of both methods and how the final $\mathrm{e}+\mathrm{K}$ coefficients were estimated. These two methods have already been used for modeling non-LTE effects in lithium and magnesium (Osorio et al. 2011; Barklem et al. 2017) and these papers showed that CCC and BSR agree with each other to within a factor of two.

Both methods make more realistic assumptions to the physics involved in the collisional rates, but at the expense of computing time. While we can calculate the Park71 rates for our entire atom, i.e. all bound-bound transitions possible (there are 133 levels before our first ionized level), the e+K rates from the CCC and BSR methods are only available for 15 levels $(4 s-6 d)$. In Figure 2 of Reggiani et al. (2019), we compare the rate coefficients of the CCC, BSR, Park71, and vanReg62 methods and show the excellent agreement between the two close coupling methods, and the differences in the coefficients estimated via the older methods.

Although the interactions estimated with the CCC and BSR methods are only available for a limited number of atomic levels, these levels are the most important for the statistical equilibrium. Besides, the CCC and BSR methods tend to agree more with Park71 and vanReg62 methods for high excitation levels. In our paper, we also demonstrate how these different rate coefficients impacts the line formation, by showing the synthetic line in three different model atmospheres with different atoms, using different e+K rates.

\subsubsection{4 $H+K$ rates}

The situation regarding neutral hydrogen collisions is similar to the electron collision rates. While there are older calculation methods that are reliably used mainly for the interactions with high excitation levels, there are more recent and reliable methods that provide better data at the expense of large computational time. The older method employed in this work, are the rates estimated via the free electron model, in the scattering length approximation, of Kaulakys 1986, 1991). The most up-to-date data, available for levels $4 s-4 f$, were recently published by Yakovleva et al. (2018) and were calculated using the asymptotic two-electron model (LCAO) of Barklem (2016).

For transitions involving low-lying levels (up to level 4f), we adopted the rate coefficients from the LCAO model. Following Amarsi et al. (2018), we added these data to rate coefficients calculated using the free electron model (Eq. 18, Kaulakys, 1991). The rate 
coefficients for interactions between $4 s$ and $4 f$ are dominated by the Kaulakys (1991) rates, as they are higher than the LCAO rates from Yakovleva et al. (2018). The rate coefficients for transitions from or to levels higher than $4 f$ were all calculated using the free electron model alone.

This methodology follows the work by Amarsi et al. (2018), whom analyzed the impact of using different approaches to the inelastic $\mathrm{H}+\mathrm{K}$ collisions. From their study, and other studies that employed both methods separately, the best approach seems to be using both rates $(\mathrm{LCAO}+$ free electron models) together, as in this manner they were better able to reproduce the solar center-to-limb variation of the oxygen triplet (computing the line with a $3 \mathrm{D}$ approach).

\subsubsection{Bound-Free transitions}

The bound-free transitions are the ionizing transitions, when the atom of interest interacts with either photons or matter and sufficient energy is transferred to a given electron so that it can leave the atom. For an electron in the ground level of potassium, for example, it takes $4.34 \mathrm{eV}$ to break the coulomb barrier that keeps it bound to the atom. As before, the important processes for our modeling are the bound-free transitions due to interaction with radiation (photons), called photoionization, and the ionization due to collisions with free electrons and neutral hydrogen.

The bound-free transitions always involve three particles, the two colliding particles (in our case the potassium atom and a photon, free electron or neutral hydrogen) and a electron that breaks free from the atom as the third particle of the interaction. Quoting Rutten (2003): "In this interaction there is partial exchange of kinetic and radiative energy even in radiative transitions, in addition to a possibly non-thermalizing part given by the threshold energy. The latter represents a discrete memory that may be transported non-locally in scattering sequences similarly to the bound-bound excitation energy of a resonance transition. The part above the edge has no memory, sampling the Maxwell distribution anew at each electron capture. This local sampling is analogous to the collisional broadening of bound-bound transitions. Both are described by redistribution over the extinction profile. In the limit of negligible Maxwell contribution (a sharp extinction edge resembling a spectral line in the spectrum) bound-free scattering behaves just as bound-bound scattering. When the Maxwell part dominates over the fixed part (as is the case for $\mathrm{H}$ ionization), 
recombination tends toward the thermal nature of free-free bremsstrahlung."

\subsubsection{Photoionization}

Photoionization is the bound-free process involving the atom of interest, a photon and the electron that is released from the atom. In our modeling, we need to add the lower level of the transition, the collisional cross-section at the photoionization threshold (the threshold is the wavelength in which the photon energy is just enough to ionize the atom but there is no remaining energy transferred as kinetic energy), the wavelength of the photoionization threshold, the minimum wavelength to be considered and a series of collisional cross-sections at different wavelengths. It is possible to add as many wavelength points as one wants, but MULTI will read the cross-sections and wavelengths and will create a function to interpolate this over the entire wavelength until the edge. We added 20 wavelength points for each transition considered (133 transitions).

As for the data, there are two sets included. For the lower levels, $4 s-7 d$, we included the fine-structure resolved calculations from Zatsarinny and Tayal (2010), calculated using the fully relativistic Dirac B-spline R-matrix (DBSR) method. The remaining transitions were estimated using the hydrogenic approximation previously described in equation 1.20 .

In Figure 5.9 we show the difference between the DBSR data and the hydrogenic approximation for the first excited level. The differences from the two approaches are staggering; changing between the two methods has a big effect on the non-LTE synthetic line product, as seen in Section 4.3 of Reggiani et al. (2019).

\subsubsection{Collisional ionization}

The last piece of information we need to add to model our atomic processes are the rate coefficients from collisional induced ionization, namely the ionization of potassium by collision with a neutral hydrogen atom and with free electrons. For these processes, we also have to add the rate coefficients in the same format as the bound-bound transitions induced from collisions, namely the rate coefficients for each temperature $(\mathrm{T}=1000,2000,3000,4000,5000,6000,7000,8000,9000,10000,15000$, and $20000 \mathrm{~K})$.

The ionization induced from collisions with neutral hydrogen atoms are from Yakovleva et al. (2018). The induced ionization rates from collisions with free electrons, were estimated with the neutral atom approximation from Allen (1976): 


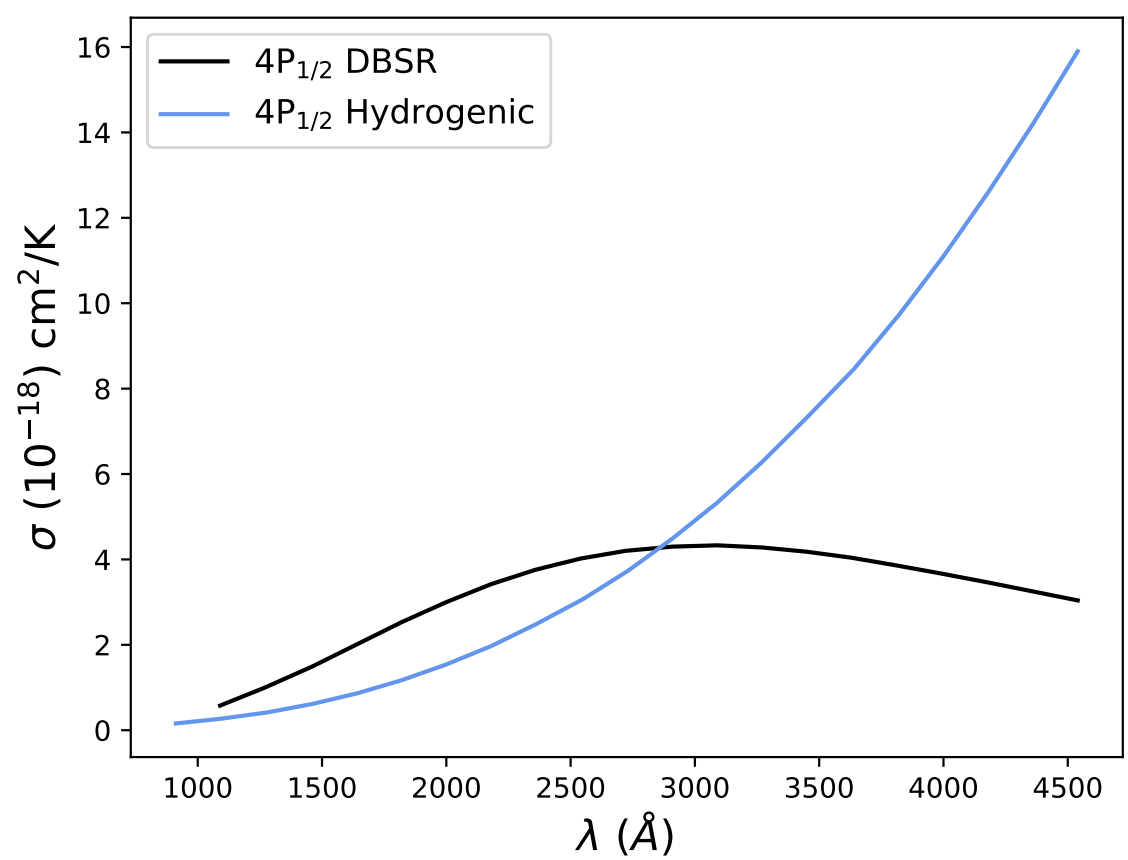

Figure 5.9: Photoionization cross-sections of the first excited level as a function of wavelength. In black the DBSR cross-sections and in blue the hydrogenic values.

$$
\alpha_{\mathrm{bf}}=1.1 \times 10^{-8} \mathrm{nT}^{\frac{1}{2}} \chi_{\mathrm{eV}}^{-2} 10^{\frac{-5040 \chi_{\mathrm{eV}}}{\mathrm{T}}} \text { in } \mathrm{cm}^{3} \mathrm{~s}^{-1}
$$

where $n$ is the principal quantum number of the lower level, $\mathrm{T}$ is the temperature, and $\chi_{\mathrm{eV}}$ is the excitation potential in electron volts.

Our standard model atom, used throughout the study for the main analysis of potassium, included the 250 radiative bound-bound transitions; the CCC rate coefficients of collisions between free electrons and the potassium atoms between levels $4 s$ and $6 d$, with the remaining $\mathrm{e}+\mathrm{K}$ rate coefficients estimated with the method from Park71; the rate coefficients of collisions between potassium and neutral hydrogen between lower levels $(4 \mathrm{~s}$ and $4 f$ ) from Yakovleva et al. (2018) (LCAO model by Barklem (2016)) and from the free electron model of Kaulakys (1991), and the remaining rate coefficients (with levels higher than $4 f$ ) estimated only through the Kaulakys (1991) model; the photoionization cross-sections from $4 s$ to $7 d$ are the fully relativistic DBSR data from Zatsarinny and Tayal (2010), complemented with the hydrogenic approximation given by equation 1.20 , the ionization via collisions with free electrons estimated via equation 5.7, and the ionization via collisions with neutral hydrogen from Yakovleva et al. (2018). 


\subsubsection{Departure Coefficients}

Departure coefficients are defined as the ratio between the level population in non-LTE and in LTE (the Saha-Boltzmann value). They are defined separately for the lower and upper levels of the transitions (Wijbenga and Zwaan, 1972).

$$
b_{l}=\frac{n_{l}}{n_{l}^{\mathrm{LTE}}} \quad b_{u}=\frac{n_{u}}{n_{u}^{\mathrm{LTE}}}
$$

One of the main outputs given in MULTI are the LTE and non-LTE level populations for each level in the model atom. Thus, it is possible to analyze the 134 departure coefficients from our model atom, and qualitatively analyze how large the non-LTE effects will be for a given line. Looking into the departure coefficients of the lower and higher levels of any given transition, it is possible to (qualitatively) see if it is important to use the non-LTE approach to estimate the abundances and line profiles.

Figure 5.10 show examples of departure coefficients of our potassium atom, for three lower levels and one high-excitation level. While it is important to show the lower levels because they are the most affected by non-LTE effects, a higher level is plotted to show that the higher the level the closer to LTE is the population (the higher level was chosen randomly to show this effect). In the top panel, we show departure coefficients calculated in a solar atmosphere, in the middle panel the coefficients calculated in the atmosphere of the metal-poor turn-off star HD 84937, and the last panel shows the departure coefficients of a giant metal-poor star. The departure coefficients are plotted as a function of optical depth. As LTE depends on the density, because regions with more collisions can induce thermodynamical equilibrium, the hypothesis of equilibrium depends on the depth within the stellar atmosphere, and so the non-LTE effects in a given line also depend on the mean formation depth of that line.

The departure coefficients are important not only as a diagnostic tool of the departure from LTE. These coefficients can also be used to actually calculate the non-LTE abundance by correcting the level populations in LTE. This method of calculating non-LTE abundances is used, for example, in the radiative transfer code SME (Piskunov and Valenti, 2017), as the bound-bound line source function can be written as a function of the departure coefficients: 

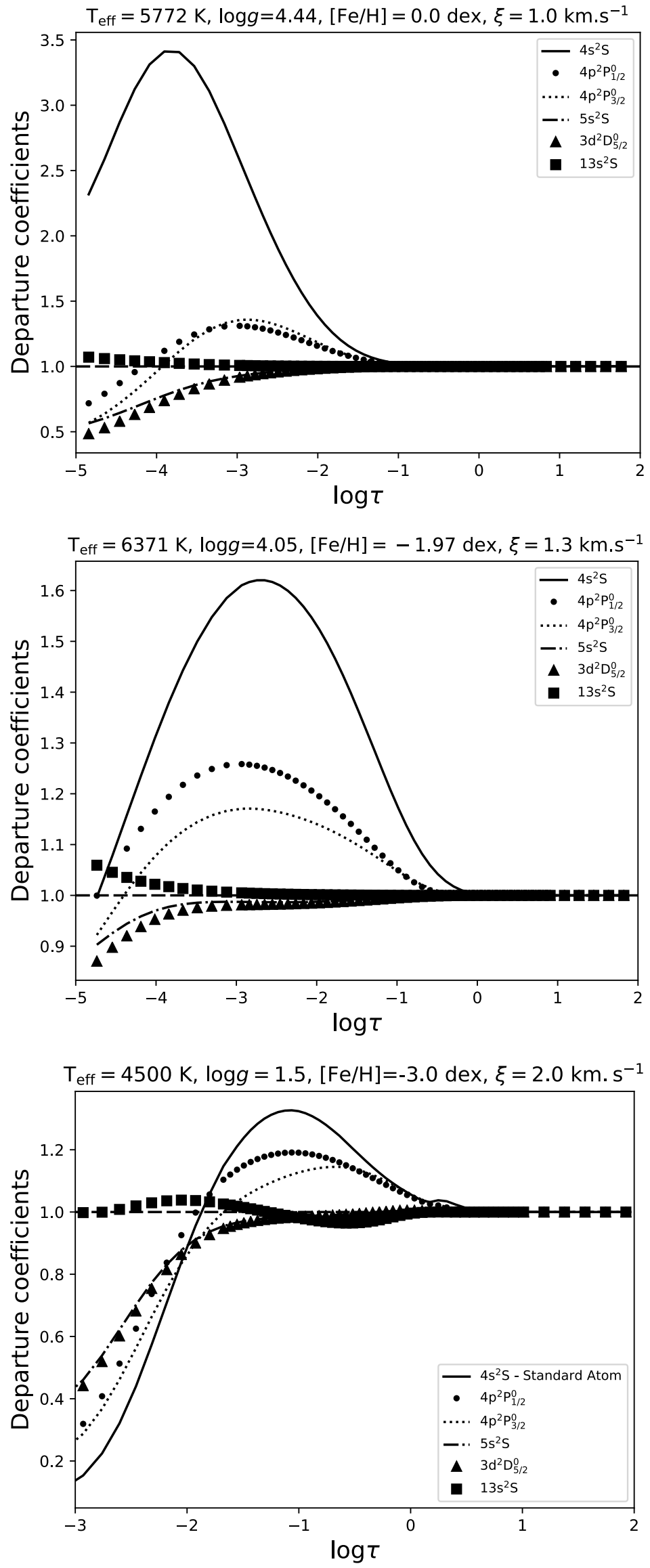

Figure 5.10: Departure coefficients as a function of optical depth. 


$$
S_{\nu_{0}}^{l}=\frac{2 h \nu_{0}^{3}}{c^{2}} \frac{1}{\frac{b_{l}}{b_{u}} e^{\frac{h \nu_{0}}{k T}}-1} .
$$

In the particular case where $\frac{b_{l}}{b_{u}} e^{\frac{h \nu_{0}}{k T}}>>1$, the Wien approximation, the departure from the bound-bound line source function to the Planck function is simply given by the ratio of the departure coefficients.

$$
S_{\nu_{0}}^{l} \approx \frac{b_{u}}{b_{l}} B_{\nu_{0}}
$$

In Figure 5.11 we show the wavelength variation of the non-LTE source function for $\mathrm{T}_{\text {eff }}=10000 \mathrm{~K}$ for different departure coefficients. It is clear to see that the bigger the ratio between non-LTE and LTE level populations, the bigger will be the change in the line source function and the more important will be the non-LTE effects when estimating chemical abundances and stellar parameters (via spectroscopy).

The departure coefficients can also be used to calculate the non-LTE departure of the line opacity in much the same way:

$$
\kappa_{\text {line }}^{\mathrm{NLTE}}=\kappa_{\text {line }}^{\mathrm{LTE}} \frac{b_{l} e^{\frac{h \nu}{k T}}-b_{u}}{e^{\frac{h \nu}{k T}}-1}
$$

In this formulation, the same used in SME, the line source function becomes:

$$
S=\frac{S_{\text {cont }} \kappa_{\text {cont }}+\sum S_{\text {line }} \kappa_{\text {line }}}{\kappa_{\text {cont }}+\sum \kappa_{\text {line }}}
$$

Although we do not employ this method to calculate the non-LTE line profile, it is important to derive the equations to demonstrate that there are different approaches to the problem of estimating non-LTE abundances. It is, however, necessary to create a grid of departure coefficients over different model atmospheres, much like in the development of a grid to directly correct LTE calculated abundances. Although the departure coefficients are a good diagnostic tool to visualize the non-LTE effects, it is easier to see how line formation changes through the analysis of the synthetic lines vs observed lines in wellknown stars. This will be discussed in the next section. 


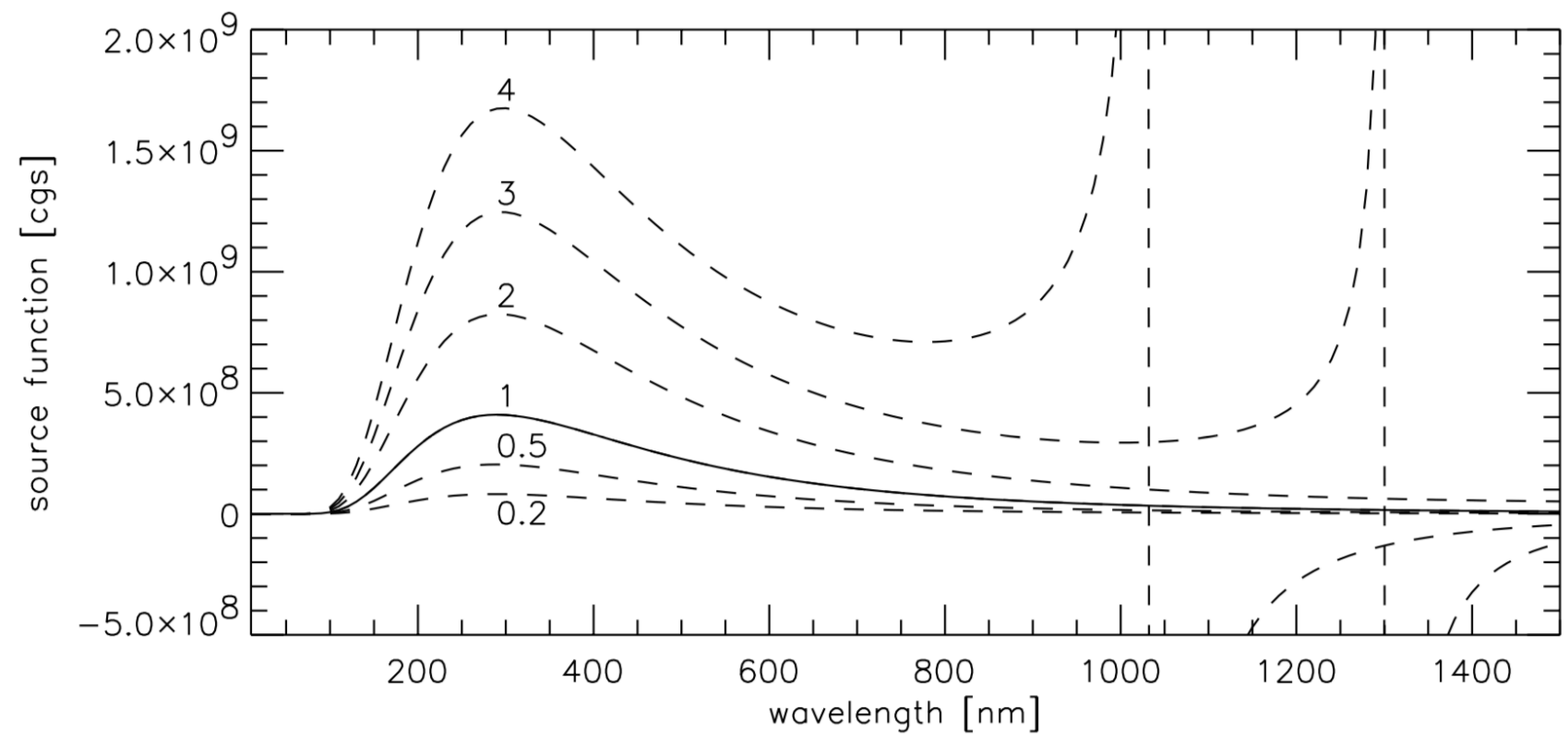

Figure 5.11: Line source functions versus wavelength for different departure coefficients. The solid line is the Planck source function. Figure extracted from Rutten (2003).

\subsection{Discussion}

\subsubsection{Non-LTE effects}

Our results are thoroughly discussed in Reggiani et al. (2019), attached in Appendix A, but we briefly highlight our main results. As mentioned in Sections 5.2.3 through 5.2.4.2, there are different ways to estimate the collisional cross-sections for bound-bound and bound-free transitions. In our work, we created nine different atoms to test the effects of these collisions in the final synthetic line. Our standard atom was described at the end of Section 5.2.3.2, and in the eight other atoms we employed different collisional recipes to the $\mathrm{e}+\mathrm{K}$ rates, the photoionization cross-sections, and removed some of the radiative bound-bound transitions. For details, the differences between the atoms are specified in Table 2 of Reggiani et al (2019).

In Figure 5.12 we show the departure coefficients of the ground state for these different atoms in the solar atmosphere and a giant metal-poor star atmosphere. The most noticeable result in the solar atmosphere is the departure coefficient of the atom with the reduced rate coefficients of collisions between potassium and free electrons. In that case, the non-LTE level populations are smaller than the LTE populations, demonstrating that it might be one of the most important piece of data to the statistical equilibrium. 
Another interesting result that we can see in that figure is that the departure coefficient of the ground state of the atom that only has the resonance bound-bound transitions (transitions from the $4 p^{2} P_{1 / 2}^{0}$ and $4 p^{2} P_{3 / 2}^{0}$ to $4 s^{2} S$ ) has a large departure coefficient of the ground state, and although smaller than that of the standard atom, it is still considerable, translating into a considerable difference in the abundance determination. It demonstrates that the main cause of the non-LTE departures is overpopulation in the ground state due to photon losses in these transitions. This conclusion had already been reached by Bruls et al. (1992) and our results corroborate them. Bruls et al. (1992) also argued that the infrared transitions are important to the statistical equilibrium, but to a lesser degree.

To further demonstrate the importance of both the resonance and infrared transitions (even though the infrared transitions are not as significant), we show the radiative bracket of our bound-bound transitions in Figure 5.13. The radiative brackets are the lower level non-LTE population multiplied by the radiative rate from the lower to the upper level minus the upper level non-LTE population multiplied by the radiative rate from the upper to the lower level. The higher the absolute value, the higher the influence of that particular transition to the equilibrium. As can be seen in Figure 5.13, the most important transitions are the resonance transitions, 7664 and $7698 \AA$ shown in blue, followed by the infrared transitions at $\approx 12,000 \AA$.

Although the behavior of the departure coefficients are the same, it is much less extreme in the giant metal-poor star, and it is also important to point the increased importance of the neutral hydrogen collisions in this atmosphere, to the point where the departure coefficients with the decreased rates are even bigger than in the standard atom, at given atmospheric depths. We aim to demonstrate how the behavior changes when we change the environment in which the non-LTE departures are being estimated.

From the departure coefficients we can see in which transitions the non-LTE effects will be more important, as the bigger the difference in the level populations from LTE to non-LTE, the more (or less if the ratio is below 1) atoms are available to be seeds for a particular transition. But, regardless of how powerful it is as a diagnostic tool, we turn ourselves to the analyzes of the synthetic lines, as they can better demonstrate, visually, the differences between non-LTE and LTE analyses, and the importance of applying the best possible assumptions in an abundance analysis. 

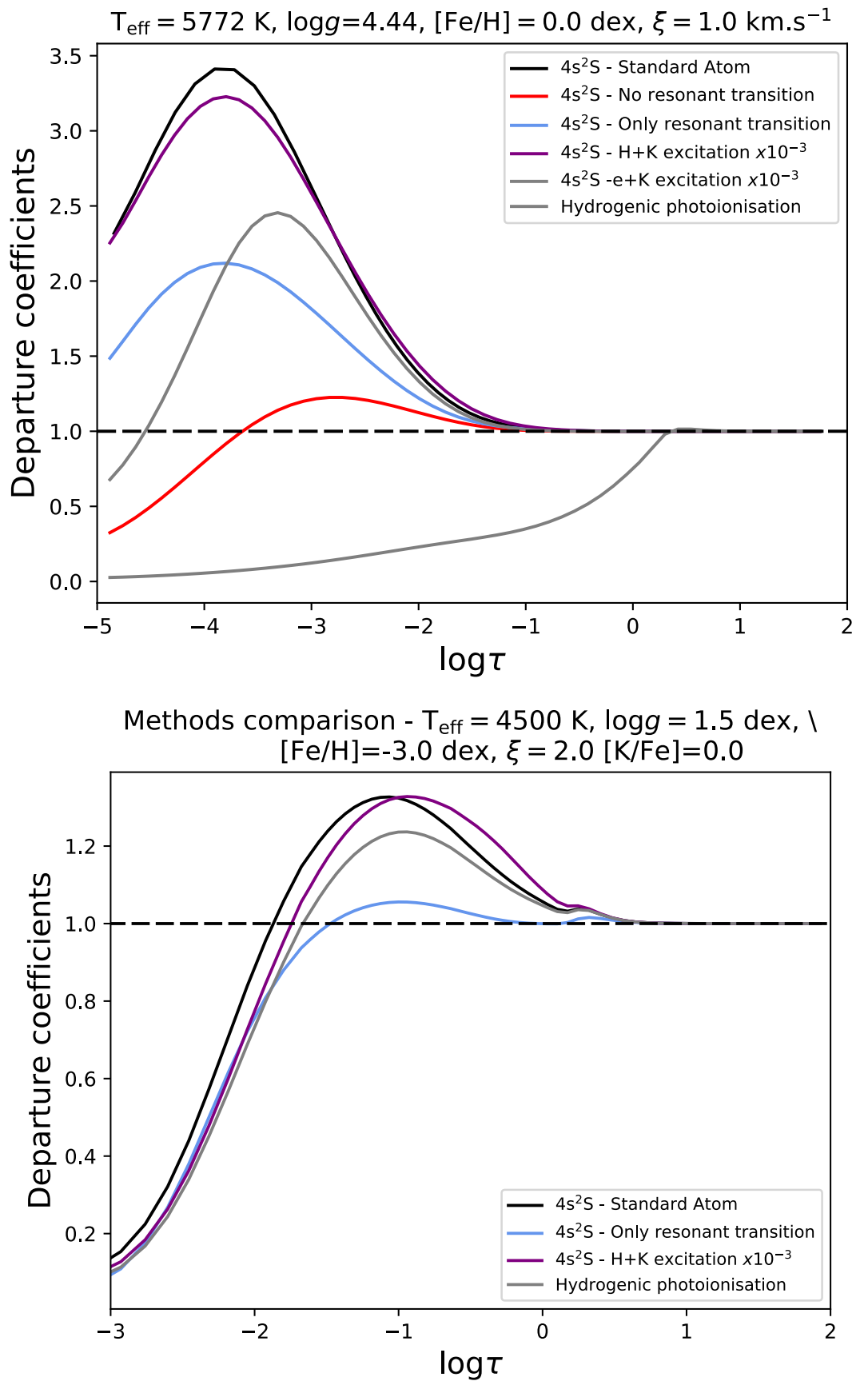

Figure 5.12: Departure coefficients for different configurations of the model atom. The top panel shows the results for the solar atmosphere and the lower panel for a metal-poor giant star atmosphere. 


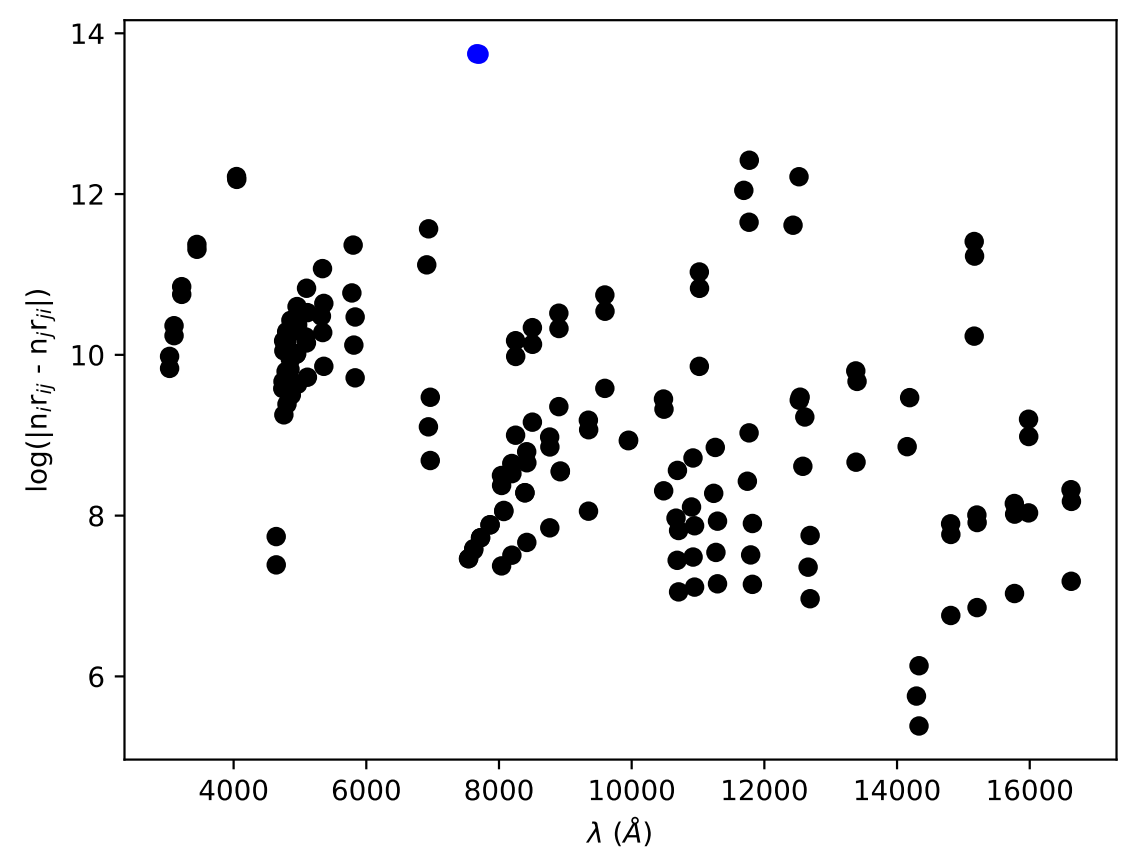

Figure 5.13: The logarithm of the radiative brackets, in a stellar depth of $\log (\tau) \approx-0.6$, as a function of wavelength for our bound-bound transitions. In blue the brackets of the resonance transitions. The radiative brackets were calculated for departures in the solar atmosphere.

\subsubsection{Non-LTE synthetic lines under different cross-sections assumptions}

The MULTI output creates symmetric line profiles, and it provides us with half of the line profile of all bound-bound transitions, and the quality of the profile depends on the number of frequency points chosen for each transition in the model atom (see Figure 5.7). To create the full profile one needs to mirror the "blue" side of the line into the "red" side. The code solves the statistical equilibrium for the transitions in the model atom of interest, and provides the means to understand the differences between LTE and non-LTE modeling of those transitions. As will be further addressed, MULTI does not create full spectral profiles of a region, thus it is not possible to obtain the full description of a line when it is either blended or its wings are not well defined due to the presence of adjacent lines. However, to the interests of this work, the profile from MULTI fits our needs.

In Figure 5.14 (reproduced from our paper), we compare the synthetic lines of three different model atmospheres under LTE, and non-LTE with our different atoms. Our results using the e+K rates from $\mathrm{CCC}$ and BSR methods were indistinguishable, thus only the standard atom $(\mathrm{CCC} \mathrm{e}+\mathrm{K})$ is shown in the figure. We show how a line that is almost 
insensitive to non-LTE (5801 $\AA$ ) behaves similarly under all conditions (except in the case where the non-LTE level populations are smaller than LTE) with variations as small as 0.01 dex.

For the resonance line $(7698 \AA)$ the result is not the same. Changing the recipes for the collisional cross-sections calculations has a non-negligible impact on the synthetic lines, thus on the abundance analysis. Again, the most overwhelming result is the line shalower than the LTE case, when the $\mathrm{e}+\mathrm{K}$ rates are decreased. The importance of the $\mathrm{e}+\mathrm{K}$ rates is also observed when the $\mathrm{CCC}$ or BSR rates are switched to either the Park71 or the vanReg62 method, in which case an abundance analysis yields results as different as 0.11 dex in $[\mathrm{K} / \mathrm{Fe}]$. Hydrogenic photoionization cross-sections also changed the abundance, but not as considerably, and the remaining atoms did not cause drastic changes in the estimated abundance, at least for the solar atmosphere.

Although the abundance differences are smaller in the other two model atmospheres analyzed, the main results are the same, but as the star becomes more metal-poor and the temperature diminishes, the importance of the $\mathrm{e}+\mathrm{K}$ rates diminishes, and the importance of the $\mathrm{H}+\mathrm{K}$ rates increase. This happens because of the availability of free electrons in the atmosphere versus the availability of neutral hydrogen. As the temperature decreases less electrons break from their atoms and, thus, less electrons are available to interact with other atoms in the stellar atmosphere; the same happens as the star gets more and more metal-poor; as less atoms of species other than hydrogen and helium form those stars, there are less electrons to break free from their atoms and interact with other species. As the vanReg62 e+K rates are higher than those of the other methods, at least for the most important transitions (see Fig. 2 of Reggiani et al. 2019), even with a decreased importance of these rates in the giant atmosphere (last panel of Figure 5.14), it is still as important to the equilibrium as the $\mathrm{H}+\mathrm{K}$ rates.

Concerning the source of the non-LTE effects, they are mainly a source-function effect from photon losses. The same effect was observed in the Na D lines (see Lind et al., 2011, Section 3.1), as the overpopulation of the ground state pushes the mean formation depth outward and deepens the lines. The line source function of a line formed by resonance scattering is determined by the radiation field, and thus by the radiative rates in the lines themselves (see Figure 5.13). That also explains why atoms with only the two resonance lines are so similar to the standard atom, i.e., because the source function is the same in 
the two cases. These results are detailed in the Section 4 of our paper.

\subsubsection{Non-LTE synthetic lines in benchmark stars and 3D effects}

We performed an analysis of the performance of our model atom by synthesizing the $7698 \AA$ line and finding the potassium abundances, via EW matching, of the Sun and five other GAIA-ESO benchmark stars. The full analysis and all the fitted lines are shown in our paper, and we reproduce two of our main results here.

First, it is important to show that our model atom is capable to simultaneously find similar abundances for all observable lines of the visible spectrum of a given star. From our EW matching analyzes, we derived a 1D non-LTE solar abundance of $\mathrm{A}(\mathrm{K})=5.11$, from averaging the result of the three optical lines in the solar spectrum. The individual abundances were found to be $A(\mathrm{~K})_{5801 \AA}=5.10, A(\mathrm{~K})_{6939 \AA} \circ=5.10$, and $A(\mathrm{~K})_{7698 \AA}=5.12$. In our paper, we compare these individual abundances with previous works, and here it suffices to say that we have a better agreement between the different lines than previously reported. In Figure 5.15 we show the synthetic lines versus the solar spectrum. Differently than the other benchmark stars, for the Sun we also plot the synthetic line of an infrared (IR) line to demonstrate that our atom can correctly reproduce not only the optical lines, but also transitions in other spectral regions.

As previously mentioned, the MULTI synthetic profile does not include the adjacent regions to the line of interest, and because of that the wings of the optical lines other than the resonant $7698 \AA$ line (which is much stronger than any weak stellar or telluric blend), are not entirely modeled. Nevertheless, the plots only demonstrate that the synthetic lines can correctly reproduce the core of the observed ones, as the wings are mostly composed of blended features and other lines.

We also show the same plot for our analysis of Procyon in Figure 5.16. Although the abundance analysis yielded results that are comparable to former studies $\left(A(K)_{\mathrm{NLTE}}=\right.$ 4.86), there is a major difference from the observed and synthetic spectra at the wings of the line, specially an asymmetry between blue and red wings. This was also observed in the wings of other benchmark stars, such as in the red wing of HD 192263, and we argue that this is a result of convective movements in the star.

This type of convection effect is known and it can be modelled by solving the statistical equilibrium in a radiation-hydrodinamical simulation of a 3D stellar atmosphere Dravins 

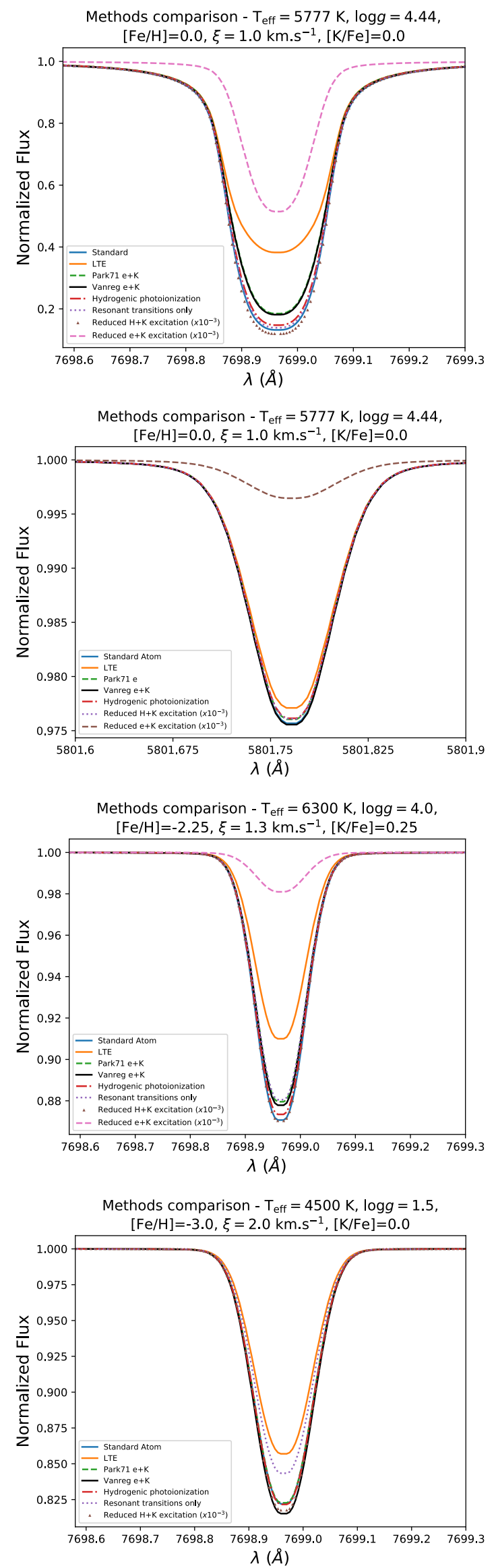

Figure 5.14: First and second panels show the comparison between the synthetic spectral lines using different collisional recipes for the $7698 \AA$ and $5801 \AA$ lines in the 1D solar atmosphere. The third and

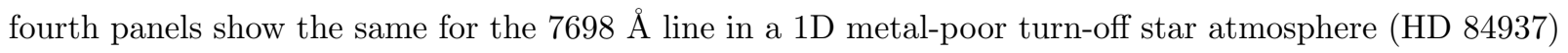
and a 1D metal-poor giant star atmosphere. Figure extracted from Reggiani et al. (2019). 

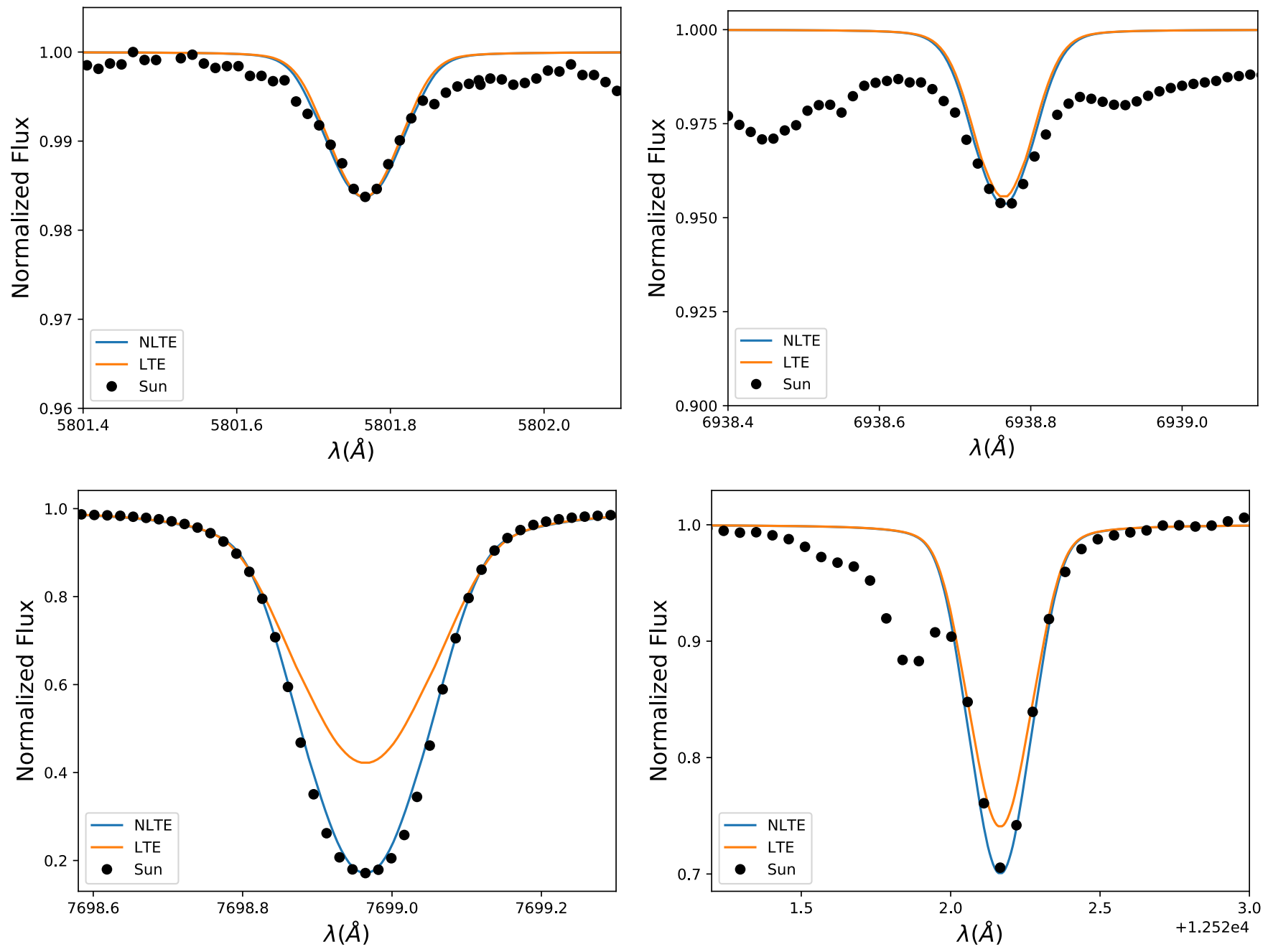

Figure 5.15: Synthetic LTE and non-LTE lines and the observed spectrum of the Sun. Top panels are the 5801 and 6938 optical lines, and the lower panels are the resonant $7698 \AA$ line, and the IR $12522 \AA$ line. Figure extracted from Reggiani et al. (2019). 


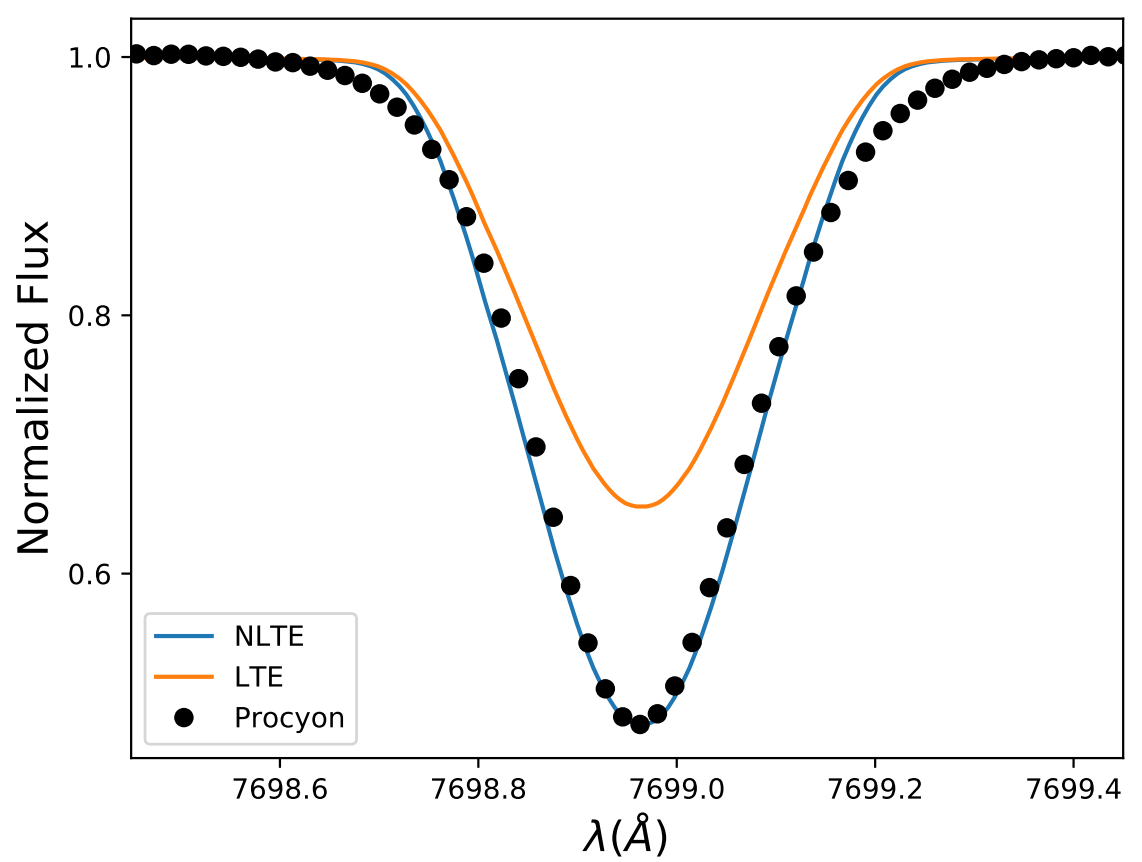

Figure 5.16: Synthetic LTE and non-LTE lines and the observed spectrum of Procyon. Figure extracted from Reggiani et al. (2019).

et al., 1981; Asplund et al., 2000). For the first time, we performed a 3D non-LTE analysis of potassium to test our hypothesis, with one snapshot on a STAGGER (Magic et al. 2013) atmospheric model and using the BALDER (Amarsi et al., 2018) radiative transfer code. The 3D+NLTE profile was computed in collaboration with A. Amarsi, co-autor of our paper, but all other NLTE calculations were entirely performed by H. Reggiani. We show in Figure 5.17 an extended version of the plot presented in our paper. Here it is also shown the 1D LTE and 3D LTE synthetic lines, and we see that the depth of the 1D LTE line is similar to the depth of the 3D non-LTE line, although the wings are completely different, as are the EWs of these different synthetic lines. The 3D LTE line has wings with the asymmetries observed in stellar spectra, such as Procyon, but is too shallow to correctly represent any observed abundance.

Although the 3D non-LTE line is a much better, and physically realistic, representation of the stellar line, the 1D non-LTE line can be used to infer the stellar abundance via EW, as both 3D non-LTE and 1D non-LTE have comparable EWs. 


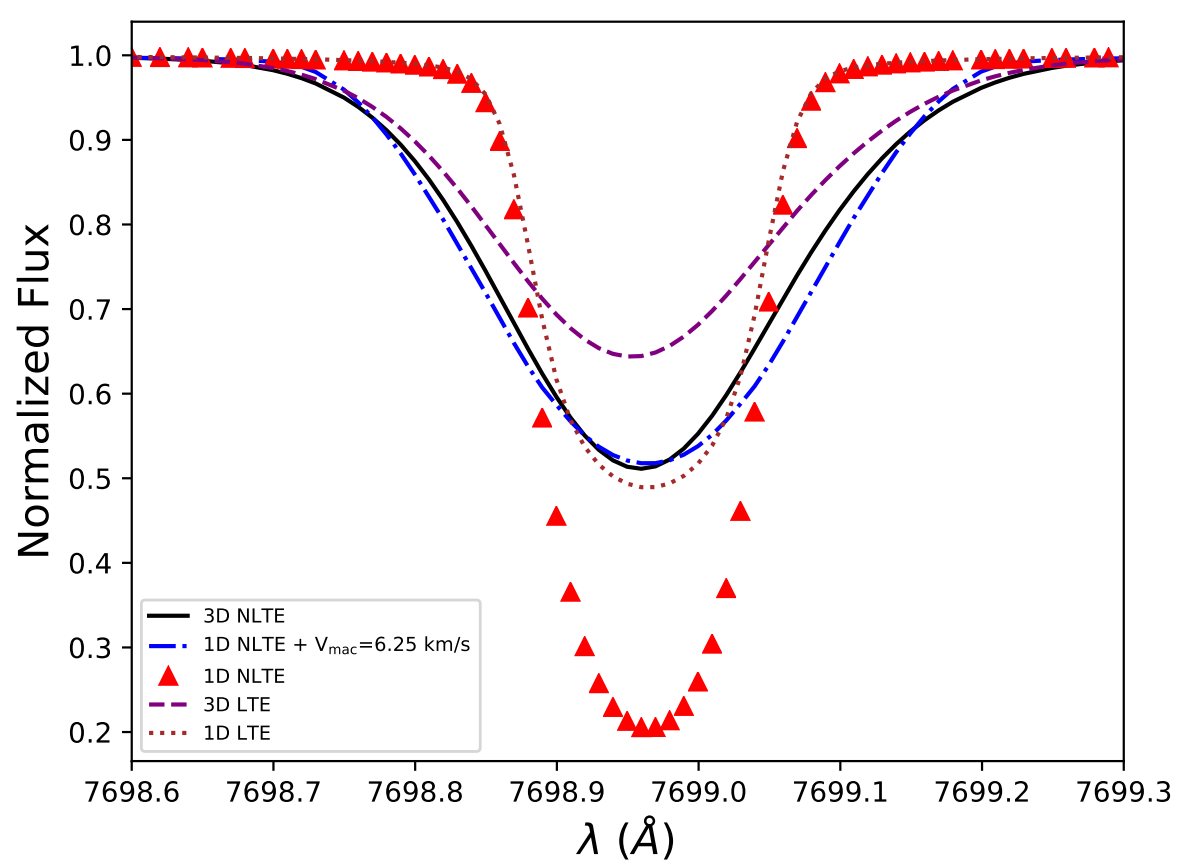

Figure 5.17: The result of our test with a 3D atmospheric model, by synthesizing a star with an atmosphere as close as possible to that of Procyon. We show 3D and 1D synthetic lines.

\subsubsection{Grid of corrections}

After our extensive analysis on the non-LTE effects observed in the potassium visible lines, and the testing of our model atom on several benchmark stars, making sure it can correctly reproduce (besides the 3D effects discussed) the potassium lines in these stars, we computed a table of abundance corrections. One of the possible output options in MULTI is the estimation of both non-LTE and LTE EWs of the synthetic lines. We ran our model atom under 1D MARCS model atmospheres with stellar parameters covering from $4000 \leq \mathrm{T}_{\text {eff }} / \mathrm{K} \leq 8000$ with steps of $500 \mathrm{~K}$. For each $\mathrm{T}_{\text {eff }}$ the surface gravity range is $0.5 \leq \log (\mathrm{g}) \leq 5.0$ dex in steps of 0.5 , varying the metallicities between $-5.00 \leq[\mathrm{Fe} / \mathrm{H}]$ $\leq+0.50$ in steps of $0.25 \mathrm{dex}$, and microturbulence velocities of $\xi_{\mathrm{T}}=1.0,2.0$ and 5.0 $\mathrm{km} \cdot \mathrm{s}^{-1}$. We created a table with each stellar parameter, abundance $(A(\mathrm{~K}))$, non-LTE and LTE EWs. We interpolated the LTE EW and abundances and created a grid of abundance corrections based on the interpolated LTE abundances and the actual non-LTE abundances of each point in our grid.

Our final grid of corrections has the LTE and non-LTE abundances in each model point, the abundance correction $\left(\Delta=A(\mathrm{~K})_{\mathrm{NLTE}}-\mathrm{A}(\mathrm{K})_{\mathrm{LTE}}\right)$, and we also present the non-LTE and LTE EWs in case the user decides to use a different interpolation method and estimate 
his/her own abundance corrections. Although our analysis focused on the $7698 \AA$ line, our grid of corrections also includes other important potassium lines, like the blended $7664 \AA$ line and the infrared line at $15168 \AA$.

We show example results of our grid in Figures 5.18 and 5.19, for the abundance corrections of the $7698 \AA$ and $7664 \AA$ lines, respectively. We can see that the abundance corrections of both lines are equally large (both transitions are from the first excited state to the ground state $-4 p^{2} P_{1 / 2}^{0}$ and $4 p^{2} P_{3 / 2}^{0}$ to $\left.4 p^{2} S\right)$. We show these transitions because they have the strongest non-LTE effects (as seen in the departure coefficients).

Figures 5.18 and 5.19 show that the largest abundance corrections are observed for the largest EWs. The corrections increase as the lines saturate and get smaller as the lines begin to develop damping wings. For giant stars, the process of line saturation happens at lower metallicities, and so do the damping wings, so the corrections are not as large, and decrease at lower metallicities (compared to the dwarfs). As the abundance corrections are largely dependent on the line saturation, there is an indirect dependence with temperature, observed in the abundance corrections of the giant star, where it reaches the largest corrections at lower metallicities, and at higher metallicites the corrections are smaller than for the dwarfs (the cooler is the star, the more neutral atoms will be at the atmosphere). The metallicity trend is also a by-product of the line saturation, as the higher the metallicity, the more potassium will be in the atmosphere, so the line will be deeper and more saturated (which will also depend on the stellar temperature).

\subsubsection{Analysis of GCE models and data under the non-LTE assumption}

In Section 5.1 we saw how the potassium abundance observed is currently very different from the abundances predicted by GCE models. In this section, we will show our results from Reggiani et al. (2019) and how non-LTE corrected observed abundances, along with the new GCE model with yields from rotating massive stars by Prantzos et al. (2018), can partially solve the potassium problem. We analyzed the observed abundances from three different studies: Cayrel et al. (2004), Roederer et al. (2014) and Spina et al. (2016).

In Figure 5.20 the LTE data were estimated using MOOG, from the EWs provided in the aforementioned studies, and the non-LTE data are the LTE abundances corrected using the grid of corrections discussed above. We can clearly see that while the LTE abundances differ in behavior from all the models, with an abundance increase throughout the entire 

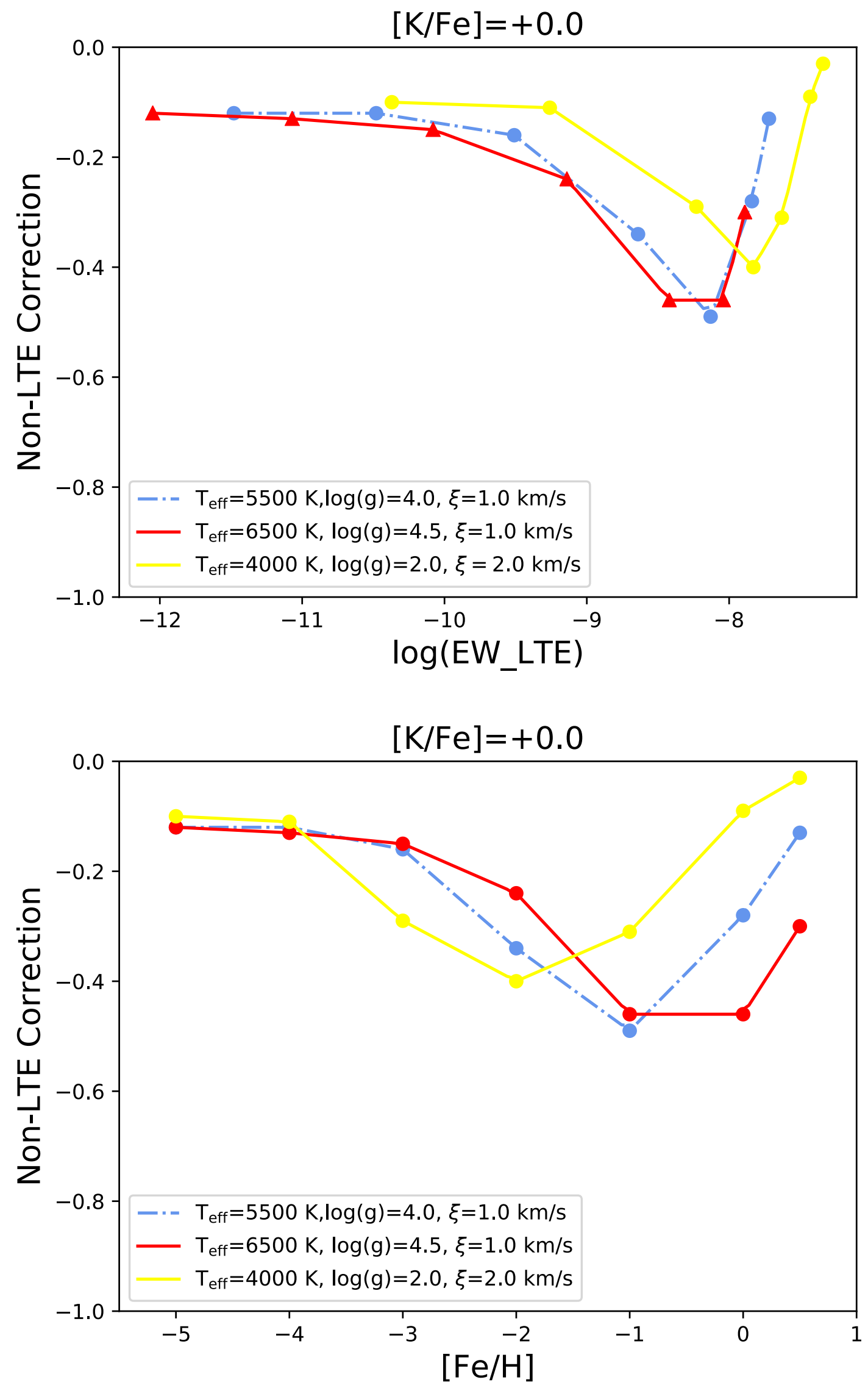

Figure 5.18: Correction grid for the $7698 \AA$ line as a function of EW (top panel) and [Fe/H] (lower panel). 

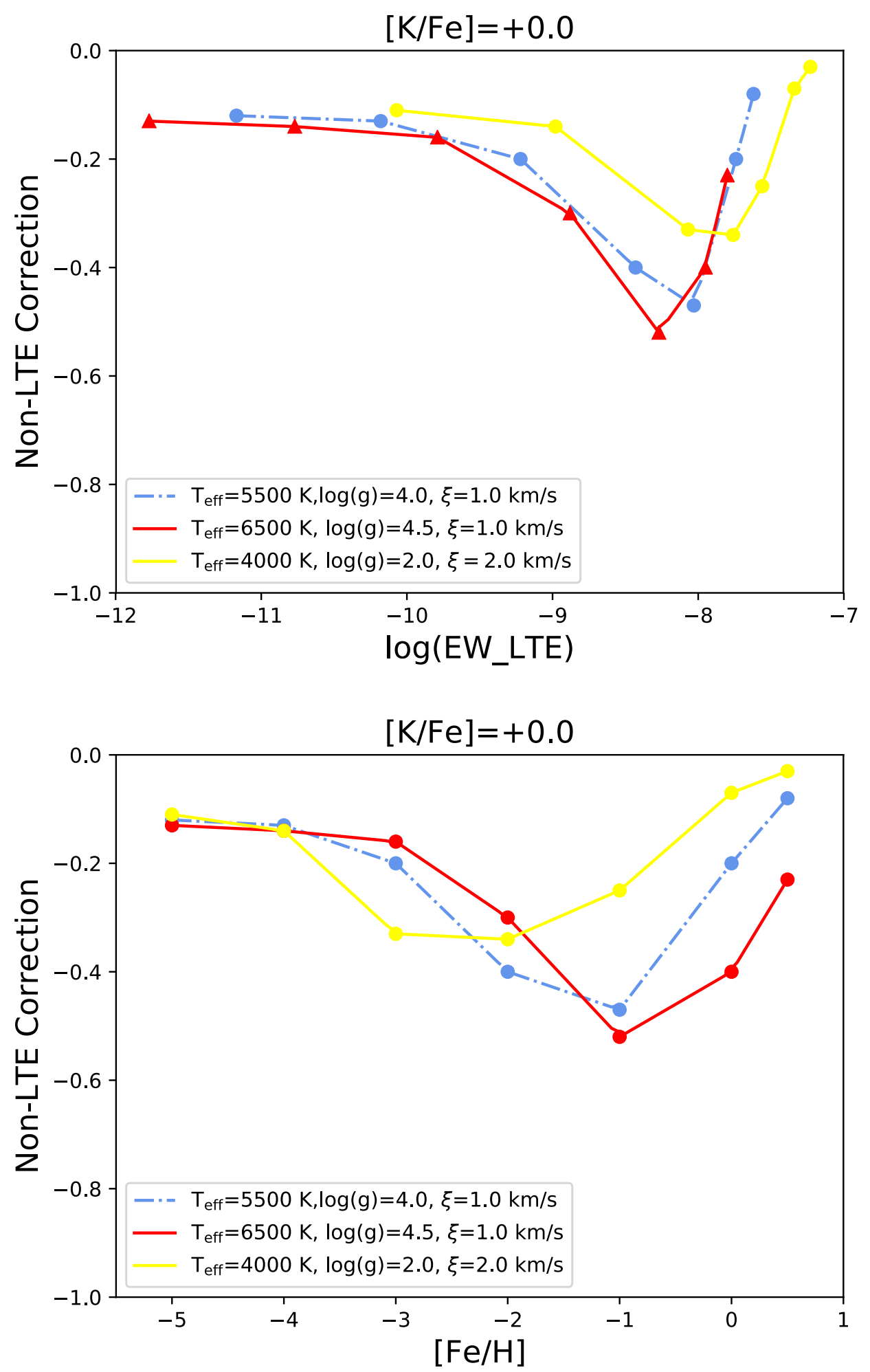

Figure 5.19: Correction grid for the $7664 \AA$ line as a function of EW (top panel) and [Fe/H] (lower panel). 
metallicity range, the behavior of the non-LTE corrected data has a decreasing trend from about $[\mathrm{Fe} / \mathrm{H}]=-2.1$ dex, that clearly fits the behavior of one of the models.

The behavior of our non-LTE corrected data resembles the prediction of the model with rotating massive stars. It is important to say that, although the qualitative behavior of data and model seems to be the same, the abundances from the model are still not as high as the observed abundances, specially the solar twins and the Sun itself. Actually, none of the models can reproduce the $\mathrm{K}$ solar abundance.

These two seemingly conflicting results indicate that yields of potassium, from older models, can be increased almost to the correct amount by including nucleosynthetic sites that are expected to have been more common at the early stages of the Galaxy. Unfortunately, the lack of more direct evidence of highly rotating stars in the early Milky Way, means that the yields do not have a solid ground. Once better constraints on metal-poor rotating massive stars are obtained, we will gather information that might tell if the modeling being introduced can be a real portray of nature; this additional information will allow us to better model these objects, so that we can better understand the observed potassium abundances.

It is hard to correctly model the chemical evolution of the early Galaxy, but we do have another piece of information that might help. The solar twins from Spina et al. (2016) bare an important result: just like Spina et al. (2016) were unable to describe the evolution of $[\mathrm{K} / \mathrm{Fe}]_{\text {LTE }}$ over time, we also could not fit well our $[\mathrm{K} / \mathrm{Fe}]_{\text {NLTE }}$ vs. age (Figure 5.21). In Figure 5.21, we show in blue and red the LTE and non-LTE derived abundances versus stellar age, while the black lines are a 4th degree polynomial that was used to indicate the difference in the scatter of the two methods. However, the non-LTE corrected abundances have a smaller scatter, and this suggests that the NLTE results are more reliable (the closer are the metallicities and ages of the stars, the more similar their chemical abundances should be), so there must be a reason why it is not possible to fit the abundances over time. We argue that this could be due to inhomogeneous potassium enrichment of the medium, or simply because the empirical fits suggested by Spina et al. (2016) are not adequate to reproduce the GCE of potassium.

If yields from massive rotating stars were not fully homogenized in the ISM, the inhomogeneties should be observable as a small abundance scatter in samples of similar stars throughout the Galaxy. However, the scatter in the non-LTE abundances of the solar-twins 

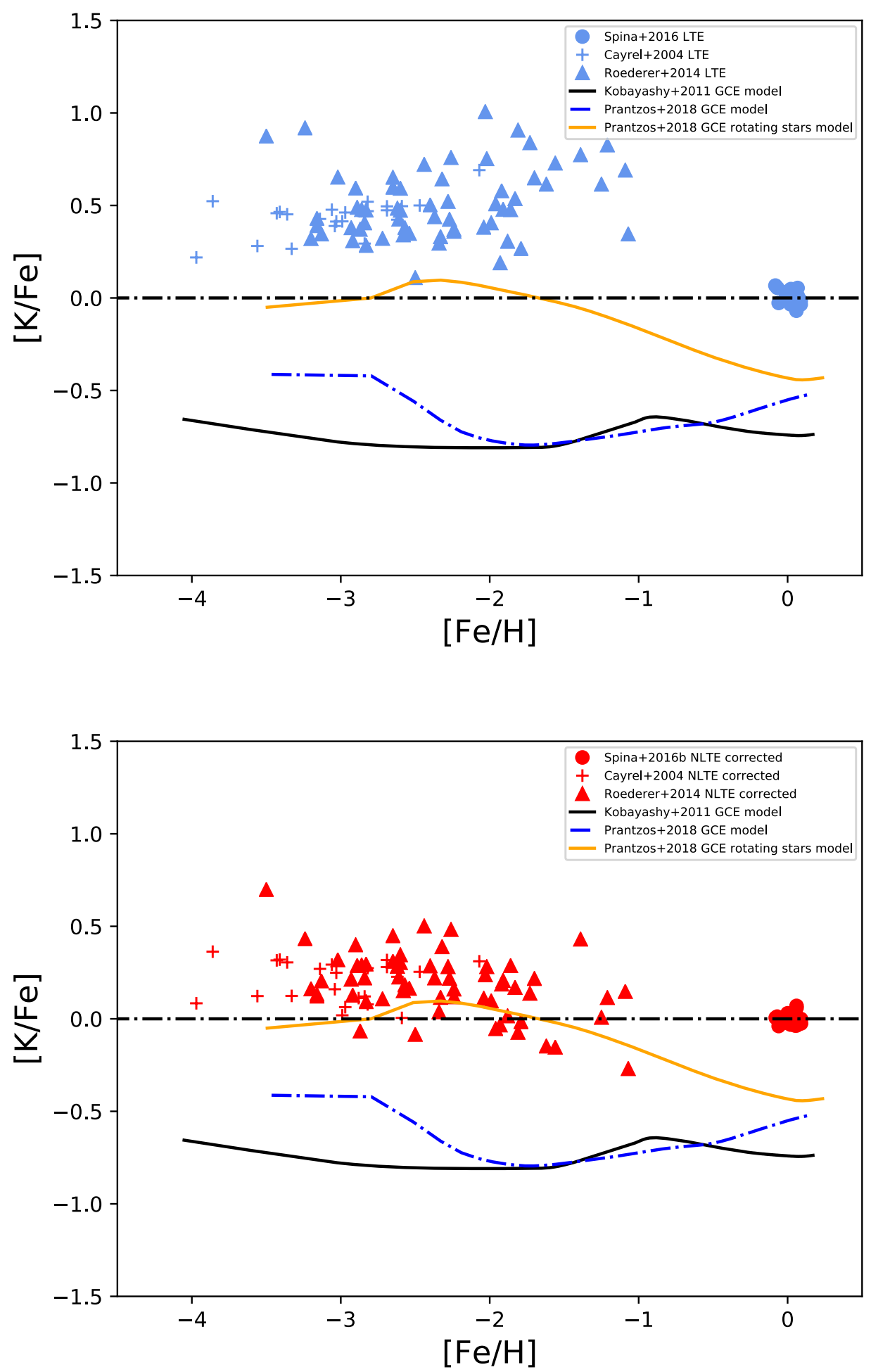

Figure 5.20: LTE abundances are shown in the top panel and non-LTE data in the lower panel. Circles are solar twins from Spina et al. (2016), triangles are from Roederer et al. (2014), and the plus signs are from Cayrel et al. (2004). In black the model from Kobayashi et al. (2011), in blue the base model of Prantzos et al. (2018), and in orange the model with rotating massive stars from Prantzos et al. (2018). Figure extracted from Reggiani et al. (2019). 
is small (0.027 dex), and therefore the observed scatter could just be due to errors in our analysis. A larger sample of twin stars is necessary to conclude whether we are indeed observing cosmic scatter.

It is not yet completely clear that rotating massive stars are the nucleosynthetic source missing from older GCE models, but from our observations and the model from Prantzos et al. (2018), it seems likely that such events, or other similarly rare events with the capability of producing more potassium, might be playing an important role in the chemical evolution of potassium in our galaxy.

The paper describing all the details and results of the analysis can be found in Appendix A, with a full description of the abundances of the benchmark stars and the GCE comparison. Also, in the paper there are more details about the $\mathrm{e}+\mathrm{K}$ rate coefficients calculations and the dependence of the abundance corrections with effective temperature and surface gravity.

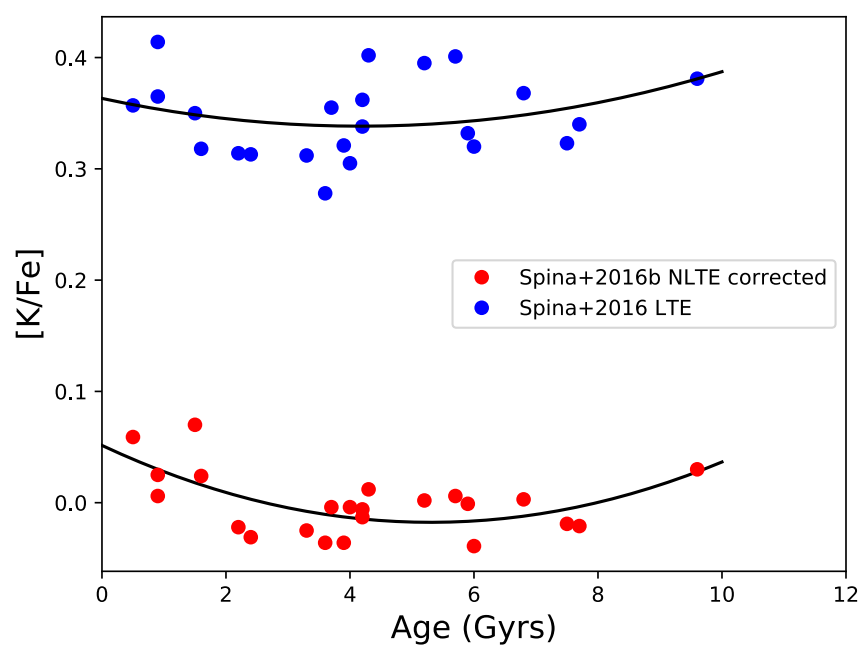

Figure 5.21: LTE and non-LTE abundances of solar twins as a function of age. They were scaled with the absolute LTE $A(\mathrm{~K})_{\mathrm{LTE}}$ and non-LTE $A(\mathrm{~K})_{\mathrm{NLTE}}$, thus their different abundances. Figure extracted from Reggiani et al. (2019). 
Chapter 6

\section{Conclusions}

In this thesis we took steps towards a better understanding of the formation and chemical evolution of the solar neighborhood halo, through a differential analysis. This powerful technique recognized differences in the abundance patterns of halo stars historically known as similar objects, G 64-12, G 64-37, and CD -24 1705. The reduced errors provided both by the quality of our spectra and the analysis technique, proved to be invaluable in recent chemical abundance analyses.

The application of this technique to a large sample of similar stars has been used to show that the bulk of the solar neighborhood halo is composed by a single population in $[\mathrm{Fe} / \mathrm{H}] \leq-1.6$. We argue that if this structure of the Galaxy was formed by one major accretion event (Iorio and Belokurov, 2019), the MDF of the accreted galaxy must have peaked in $[\mathrm{Fe} / \mathrm{H}] \geq-1.6$, and must have had a knee in $[\mathrm{Fe} / \mathrm{H}] \geq-2.0$. The position of this knee is very similar to that of other dSph's (Suda et al., 2017), and any lower metallicity stars that might have been acreted along would have had a nucleosynthetic history similar to the inner halo of the Milky Way, which can be used as a constrain on the mass of that galaxy. It must had been massive enough so that the $[\mathrm{Fe} / \mathrm{H}] \leq-2.0$ stars have a similar abundance pattern to objects born in-situ.

Our VMP sample was also used to study the chemical enrichment of the Galaxy, through comparisons with GCE models. We found evidence of inhomogeneous enrichment by hypernovae, of extra production of $\alpha$-elements in the yields from the GCE model, and of zinc production in AGB stars.

Chemical abundances can also be used to study binary evolution, and the chemical pattern of our two BSS indicate that they were likely created by different mechanisms. While the star enhanced in zinc is likely to have formed via the mass transfer mechanism 
(from an AGB star), the other object is likely to have been formed via collision/merger with a companion that was not yet enriched in elements synthesized in the AGB phase. The former can also be used to constrain the mass of the AGB star, as zinc production becomes important only for AGB stars more massive than $3 \mathrm{M}_{\odot}$.

Regarding the cosmological lithium problem, we argued that the likely cause of the problem is a stellar evolutionary effect, as lithium depletion is observed both in extremely old stars (as the lithium plateau), and in Pop I solar twins. We discussed two recent propositions of lithium depletion, one that argues that lithium is depleted via stellar evolution in the pre-main sequence and main sequence phases, and one that looks at the lithium depletion from a stellar atmosphere point of view. So far, stellar evolution models are favored as they can better describe depletion for stars of different stellar parameters. However, the analysis mechanism proposed by Takeda (2019) is a very interesting new look into an old problem, and, to this author, seems like a very plausible explanation that relies not in new and poorly understood stellar effects, but caused by the lack of chromospheres in model atmospheres.

The inner galactic halo, although mostly composed by stars like those analyzed in Reggiani et al. (2017), also has its uncanny objects, very well represented by HD 134439 and HD 134440. These two stars have an abundance pattern unlike most stars in our galaxy. Their metallicity is representative of the inner halo, but the abundances of $\alpha$-elements, and others, are too low for them to be recognized as a part of the main structure of the inner halo. Their kinematics also drives us to believe that they are not, originally, part of our galaxy. They must have come from a different accretion event than that responsible for the formation of the inner halo, and an event that is not observable as a large structure. This indicate that either they were part of a very small galaxy that left just a few imprints in the Milky Way, or they were heated to the inner halo from a structure that is still in the process of becoming a part of the Milky Way. Their heavy element abundance and $\alpha$-element abundances drives us to conclude that they were likely from a dSph of similar enrichment history to Fornax. A more precise tagging may be possible by adding precise kinematic information (GAIA DR2 and future releases).

Also interesting is the comparison of abundances between the binary pair themselves, that has a big similarity to the planet imprints found in binary twin stars with planets. This lead us to conclude that one of the stars might have accreted a planetary body with 
a mass of $\mathrm{M} \approx 0.9 \mathrm{M}_{\mathrm{J}}$.

Finally, we studied the chemical evolution of potassium from a non-LTE perspective. We created a model atom to study the line formation of this element, as without nonLTE calculations, models and observations are too far to be reconciled. We showed the different influences of atomic data in the non-LTE analysis and how the most up-to-date information is important from the modeling perspective. An analysis of benchmark stars showed us that, at times, a 3D non-LTE treatment of the line formation is necessary to fully account for all the effects in line formation.

From the chemical evolution perspective, we showed that the non-LTE analysis alone is not enough to explain the differences with the GCE models, and it is necessary to add yields from non-standard nucleosynthetic processes to account for the observed abundances (in this case, yields from rotating massive stars). Although the agreement is much better and the non-LTE data and model have a similar behavior throughout the $[\mathrm{Fe} / \mathrm{H}]$ evolution, there still is a systematic difference in abundance that needs to be reconciled. Regarding PopI stars, all models we used in our comparisons are unable to reach the solar observed potassium abundance, indicating that we still lack details to fully comprehend the evolution of potassium. From the observational perspective, non-LTE K abundances have a lower scatter than LTE abundances, showing that non-LTE results are both more accurate and precise.

We finish with two remarks: First, we call the attention to the fact that if the mechanism of adding a chromosphere to model atmospheres proposed in Takeda (2019) proves to be correct, it will have an important effect in all low excitation atoms (including potassium). That could potentially further decrease the observed $\mathrm{K}$ abundance by as much as $0.1-0.2$ dex (somewhat smaller than the observed difference in lithium, because lithium has an excitation potential lower than potassium), and make it closer to the predictions by GCE models; lastly, we call the attention to the importance of the carbon abundances and the question raised at the end of Chapter 2. If our CH G-band molecular abundances are indeed too high (by as much as 1 dex), it will be necessary, as our technical capabilities increase and we start to be able to use 3D and non-LTE abundances in more analyses, to have a second look at our empirical definitions of carbon enhancement. Again, we say that this may not invalidate the current conclusions regarding the fractions of CEMP, as all the measurements made today are done in much the same way, but it would have an 
important impact for modeling the yields from the first stars and their contribution to the early ISM. 


\section{Bibliography}

Abel T., Bryan G. L., Norman M. L., The Formation and Fragmentation of Primordial Molecular Clouds, ApJ, 2000, vol. 540, p. 39

Abel T., Bryan G. L., Norman M. L., The Formation of the First Star in the Universe, Science, 2002, vol. 295, p. 93

Adibekyan V. Z., Santos N. C., Sousa S. G., Israelian G., A new $\alpha$-enhanced super-solar metallicity population, A\&A, 2011, vol. 535, p. L11

Aguado D. S., Allende Prieto C., González Hernández J. I., Rebolo R., J0023+0307: A Mega Metal-poor Dwarf Star from SDSS/BOSS, ApJ, 2018, vol. 854, p. L34

Allen C. W., Astrophysical Quantities, 1976

Amarsi A. M., Barklem P. S., Asplund M., Collet R., Zatsarinny O., Inelastic O+H collisions and the O I $777 \mathrm{~nm}$ solar centre-to-limb variation, A\&A, 2018, vol. 616, p. A89

Amarsi A. M., Nissen P. E., Asplund M., Lind K., Barklem P. S., Carbon and oxygen in metal-poor halo stars, A\&A, 2019, vol. 622, p. L4

Andrievsky S. M., Spite M., Korotin S. A., Spite F., Bonifacio P., Cayrel R., François P., Hill V., Non-LTE abundances of Mg and $\mathrm{K}$ in extremely metal-poor stars and the evolution of $[\mathrm{O} / \mathrm{Mg}],[\mathrm{Na} / \mathrm{Mg}],[\mathrm{Al} / \mathrm{Mg}]$, and $[\mathrm{K} / \mathrm{Mg}]$ in the Milky Way, A\&A, 2010, vol. 509, p. A88

Andrievsky S. M., Spite M., Korotin S. A., Spite F., Bonifacio P., Cayrel R., Hill V., François P., NLTE determination of the aluminium abundance in a homogeneous sample of extremely metal-poor stars, A\&A, 2008, vol. 481, p. 481 
Aoki W., Beers T. C., Christlieb N., Norris J. E., Ryan S. G., Tsangarides S., Carbonenhanced Metal-poor Stars. I. Chemical Compositions of 26 Stars, ApJ, 2007, vol. 655, p. 492

Arnett D., Oxygen-burning hydrodynamics. 1: Steady shell burning, ApJ, 1994, vol. 427, p. 932

Asplund M., New Light on Stellar Abundance Analyses: Departures from LTE and Homogeneity, ARA\&A, 2005, vol. 43, p. 481

Asplund M., Grevesse N., Sauval A. J., Scott P., The Chemical Composition of the Sun, ARA\&A, 2009, vol. 47, p. 481

Asplund M., Lambert D. L., Nissen P. E., Primas F., Smith V. V., Lithium Isotopic Abundances in Metal-poor Halo Stars, ApJ, 2006, vol. 644, p. 229

Asplund M., Ludwig H.-G., Nordlund A., Stein R. F., The effects of numerical resolution on hydrodynamical surface convection simulations and spectral line formation, A\&A, 2000, vol. 359, p. 669

Asplund M., Meléndez J., Primordial and Pre-Galactic Origins of the Lithium Isotopes. In First Stars III , vol. 990 of American Institute of Physics Conference Series, 2008, p. 342

Audouze J., Silk J., The First Generation of Stars: First Steps toward Chemical Evolution of Galaxies, ApJ, 1995, vol. 451, p. L49

Bailyn C. D., Are there two kinds of blue stragglers in globular clusters?, ApJ, 1992, vol. 392 , p. 519

Balachandran S. C., Carney B. W., The Oxygen Abundances in Halo Dwarfs. I. HD 103095, AJ, 1996, vol. 111, p. 946

Barbuy B., Carbon-to-iron ratio in extreme population II stars, A\&A, 1981, vol. 101, p. 365

Barbuy B., Oxygen in 20 halo giants, A\&A, 1988, vol. 191, p. 121

Barbuy B., Spite F., Spite M., Magnesium isotopes in metal-poor and metal-rich stars, A\&A, 1987, vol. 178, p. 199 
Barklem P. S., Excitation and charge transfer in low-energy hydrogen-atom collisions with neutral atoms: Theory, comparisons, and application to Ca, Phys. Rev. A, 2016, vol. 93, p. 042705

Barklem P. S., Osorio Y., Fursa D. V., Bray I., Zatsarinny O., Bartschat K., Jerkstrand A., Inelastic e+Mg collision data and its impact on modelling stellar and supernova spectra, A\&A, 2017, vol. 606, p. A11

Battistini C., Bensby T., The origin and evolution of r- and s-process elements in the Milky Way stellar disk, A\&A, 2016, vol. 586, p. A49

Bazan G., Arnett D., Convection, nucleosynthesis, and core collapse, ApJ, 1994, vol. 433, p. L41

Bedell M., Bean J. L., Meléndez J., Spina L., Ramírez I., Asplund M., Alves-Brito A., dos Santos L., Dreizler S., Yong D., Monroe T., Casagrande L., The Chemical Homogeneity of Sun-like Stars in the Solar Neighborhood, ApJ, 2018, vol. 865, p. 68

Beers T. C., Carollo D., Ivezić Ž., An D., Chiba M., Norris J. E., Freeman K. C., Lee Y. S., Munn J. A., Re Fiorentin P., Sivarani T., Wilhelm R., Yanny B., York D. G., The Case for the Dual Halo of the Milky Way, ApJ, 2012, vol. 746, p. 34

Beers T. C., Christlieb N., The Discovery and Analysis of Very Metal-Poor Stars in the Galaxy, ARA\&A, 2005, vol. 43, p. 531

Beers T. C., Kage J. A., Preston G. W., Shectman S. A., Estimation of stellar metal abundance. I - Calibration of the CA II K index, AJ, 1990, vol. 100, p. 849

Beers T. C., Preston G. W., Shectman S. A., A search for stars of very low metal abundance. I, AJ, 1985, vol. 90, p. 2089

Beers T. C., Preston G. W., Shectman S. A., A search for stars of very low metal abundance. II, AJ, 1992, vol. 103, p. 1987

Belokurov V., Erkal D., Evans N. W., Koposov S. E., Deason A. J., Co-formation of the disc and the stellar halo, MNRAS, 2018, vol. 478, p. 611 
Bensby T., Feltzing S., Oey M. S., Exploring the Milky Way stellar disk. A detailed elemental abundance study of $714 \mathrm{~F}$ and $\mathrm{G}$ dwarf stars in the solar neighbourhood, A\&A, 2014, vol. 562, p. A71

Bergemann M., Cescutti G., Chromium: NLTE abundances in metal-poor stars and nucleosynthesis in the Galaxy, A\&A, 2010, vol. 522, p. A9

Biazzo K., Gratton R., Desidera S., Lucatello S., Sozzetti A., Bonomo A. S., Damasso M., Gandolfi D., Affer L., Boccato C., et. al. The GAPS programme with HARPS-N at TNG. X. Differential abundances in the XO-2 planet-hosting binary, A\&A, 2015, vol. 583, p. A135

Bidelman W. P., The carbon stars. An astrophysical enigma, Vistas in Astronomy, 1956, vol. 2, p. 1428

Biemont E., Grevesse N., Atomic Wavelengths and Transition Probabilities in the Infrared, A\&A, 1973, vol. 27, p. 163

Bond H. E., A Search for Metal-Deficient Stars, ApJS, 1970, vol. 22, p. 117

Bonifacio P., Andersen J., Andrievsky S. M., Barbuy B., Beers T. C., Caffau E., Cayrel R., Depagne E., François P., et al. The ESO Large Programme "First Stars", Astrophysics and Space Science Proceedings, 2009, vol. 9, p. 31

Bonifacio P., Spite M., Cayrel R., Hill V., Spite F., François P., Plez B., Ludwig H.-G., Caffau E., Molaro P., Depagne E., Andersen J., Barbuy B., Beers T. C., Nordström B., Primas F., First stars XII. Abundances in extremely metal-poor turnoff stars, and comparison with the giants, A\&A, 2009, vol. 501, p. 519

Botelho R. B., Milone A. d. C., Meléndez J., Bedell M., Spina L., Asplund M., dos Santos L., Bean J. L., Ramírez I., Yong D., Dreizler S., Alves-Brito A., Yana Galarza J., Thorium in solar twins: implications for habitability in rocky planets, MNRAS, 2019, vol. 482 , p. 1690

Bray I., Stelbovics A. T., Convergent close-coupling calculations of electron-hydrogen scattering, Phys. Rev. A, 1992, vol. 46, p. 6995 
Briley M. M., Smith V. V., King J., Lambert D. L., Isotopic Carbon Abundances in M71, AJ, 1997, vol. 113, p. 306

Bromm V., Coppi P. S., Larson R. B., Forming the First Stars in the Universe: The Fragmentation of Primordial Gas, ApJ, 1999, vol. 527, p. L5

Bromm V., Coppi P. S., Larson R. B., The Formation of the First Stars. I. The Primordial Star-forming Cloud, ApJ, 2002, vol. 564, p. 23

Bromm V., Larson R. B., The First Stars, ARA\&A, 2004, vol. 42, p. 79

Bruls J. H. M. J., Rutten R. J., Shchukina N. G., The formation of helioseismology lines. I - NLTE effects in alkali spectra, A\&A, 1992, vol. 265, p. 237

Bullock J. S., Kravtsov A. V., Weinberg D. H., Hierarchical Galaxy Formation and Substructure in the Galaxy's Stellar Halo, ApJ, 2001, vol. 548, p. 33

Busso M., Gallino R., Lambert D. L., Travaglio C., Smith V. V., Nucleosynthesis and Mixing on the Asymptotic Giant Branch. III. Predicted and Observed s-Process Abundances, ApJ, 2001, vol. 557, p. 802

Busso M., Gallino R., Wasserburg G. J., Nucleosynthesis in Asymptotic Giant Branch Stars: Relevance for Galactic Enrichment and Solar System Formation, ARA\&A, 1999, vol. 37 , p. 239

Carbon D. F., Barbuy B., Kraft R. P., Friel E. D., Suntzeff N. B., Carbon and nitrogen abundances in metal-poor dwarfs of the solar neighborhood, PASP, 1987, vol. 99, p. 335

Carlos M., Karakas A. I., Cohen J. G., Kobayashi C., Meléndez J., A Formation Timescale of the Galactic Halo from Mg Isotopes in Dwarf Stars, ApJ, 2018, vol. 856, p. 161

Carlos M., Nissen P. E., Meléndez J., Correlation between lithium abundances and ages of solar twin stars, A\&A, 2016, vol. 587, p. A100

Carlsson M., A computer program for solving multi-level non-LTE radiative transfer problems in moving or static atmospheres., Uppsala Astronomical Observatory Reports, 1986, vol. 33 
Carlsson M., The MULTI Non-LTE Program (Invited Review). In Cool Stars, Stellar Systems, and the Sun , vol. 26 of Astronomical Society of the Pacific Conference Series, 1992, p. 499

Carney B. W., Laird J. B., Latham D. W., Aguilar L. A., A Survey of Proper Motion Stars. XIII. The Halo Population, AJ, 1996, vol. 112, p. 668

Carollo D., Beers T. C., Chiba M., Norris J. E., Freeman K. C., Lee Y. S., Ivezić Ž., Rockosi C. M., Yanny B., Structure and Kinematics of the Stellar Halos and Thick Disks of the Milky Way Based on Calibration Stars from Sloan Digital Sky Survey DR7, ApJ, 2010, vol. 712 , p. 692

Carollo D., Beers T. C., Lee Y. S., Chiba M., Norris J. E., Wilhelm R., Sivarani T., Marsteller B., Munn J. A., Bailer-Jones C. A. L., Fiorentin P. R., York D. G., Two stellar components in the halo of the Milky Way, Nature, 2007, vol. 450, p. 1020

Carretta E., Bragaglia A., Gratton R. G., Lucatello S., D’Orazi V., Chemical Tagging of Three Distinct Populations of Red Giants in the Globular Cluster NGC 6752, ApJ, 2012, vol. 750 , p. L14

Carroll B. W., Ostlie D. A., An Introduction to Modern Astrophysics, 1996

Castelli F., Kurucz R. L., New Grids of ATLAS9 Model Atmospheres, arXiv Astrophysics e-prints, 2004

Cayrel R., Depagne E., Spite M., Hill V., Spite F., François P., Plez B., Beers T., Primas F., Andersen J., Barbuy B., Bonifacio P., Molaro P., Nordström B., First stars V Abundance patterns from $\mathrm{C}$ to $\mathrm{Zn}$ and supernova yields in the early Galaxy, A\&A, 2004, vol. 416, p. 1117

Chabrier G., Galactic Stellar and Substellar Initial Mass Function, PASP, 2003, vol. 115, p. 763

Charbonnel C., Primas F., The lithium content of the Galactic Halo stars, A\&A, 2005, vol. 442 , p. 961 
Chen Y., King J. R., Boesgaard A. M., The Origin of the Metal-Poor Common Proper Motion Pair HD 134439/134440: Insights from New Elemental Abundances, PASP, 2014, vol. 126, p. 1010

Chen Y. Q., Zhao G., The puzzling abundance pattern of HD 134439 and HD 134440 , MNRAS, 2006, vol. 370, p. 2091

Chieffi A., Limongi M., The Explosive Yields Produced by the First Generation of Core Collapse Supernovae and the Chemical Composition of Extremely Metal Poor Stars, ApJ, 2002, vol. 577, p. 281

Christlieb N., Schörck T., Frebel A., Beers T. C., Wisotzki L., Reimers D., The stellar content of the Hamburg/ESO survey. IV. Selection of candidate metal-poor stars, A\&A, 2008, vol. 484, p. 721

Christlieb N., Wisotzki L., Reimers D., Gehren T., Reetz J., Beers T. C., An Automated Search for Metal-Poor Halo Stars in the Hamburg/ESO Objective-Prism Survey. In The Third Stromlo Symposium: The Galactic Halo , vol. 165 of Astronomical Society of the Pacific Conference Series, 1999, p. 259

Clayton D., Handbook of Isotopes in the Cosmos, 2007

Coc A., Primordial Nucleosynthesis. In Journal of Physics Conference Series , vol. 665 of Journal of Physics Conference Series, 2016, p. 012001

Coc A., Goriely S., Xu Y., Saimpert M., Vangioni E., Standard Big Bang Nucleosynthesis up to CNO with an Improved Extended Nuclear Network, ApJ, 2012, vol. 744, p. 158

Coelho P. R. T., A new library of theoretical stellar spectra with scaled-solar and $\alpha$ enhanced mixtures, MNRAS, 2014, vol. 440, p. 1027

Cohen J. G., Christlieb N., McWilliam A., Shectman S., Thompson I., Melendez J., Wisotzki L., Reimers D., New Extremely Metal-Poor Stars in the Galactic Halo, ApJ, 2008, vol. 672 , p. 320

Cohen J. G., Shectman S., Thompson I., McWilliam A., Christlieb N., Melendez J., Zickgraf F.-J., Ramírez S., Swenson A., The Frequency of Carbon Stars among Extremely Metal-poor Stars, ApJ, 2005, vol. 633, p. L109 
Cyburt R. H., Fields B. D., Olive K. A., Yeh T.-H., Big bang nucleosynthesis: Present status, Reviews of Modern Physics, 2016, vol. 88, p. 015004

D'Antona F., Ventura P., Lithium factories in the Galaxy: novae and AGB stars. In Light Elements in the Universe, vol. 268 of IAU Symposium, 2010, p. 395

de Boer K., Seggewiss W., Stars and Stellar Evolution, 2008

Dekker H., D’Odorico S., Kaufer A., Delabre B., Kotzlowski H., Design, construction, and performance of UVES, the echelle spectrograph for the UT2 Kueyen Telescope at the ESO Paranal Observatory. In Optical and IR Telescope Instrumentation and Detectors , vol. 4008 of Proc. SPIE, 2000, p. 534

Dotter A., Conroy C., Cargile P., Asplund M., The Influence of Atomic Diffusion on Stellar Ages and Chemical Tagging, ApJ, 2017, vol. 840, p. 99

Dravins D., Lindegren L., Nordlund A., Solar granulation - Influence of convection on spectral line asymmetries and wavelength shifts, A\&A, 1981, vol. 96, p. 345

Eggen O. J., Lynden-Bell D., Sandage A. R., Evidence from the motions of old stars that the Galaxy collapsed., ApJ, 1962, vol. 136, p. 748

El-Badry K., Rix H.-W., Imprints of white dwarf recoil in the separation distribution of Gaia wide binaries, MNRAS, 2018, vol. 480, p. 4884

Erdelyi-Mendes M., Barbuy B., Oscillator strengths and damping constants from the solar spectrum at 830-870 NM, A\&AS, 1989, vol. 80, p. 229

Ferraro F. R., Paltrinieri B., Rood R. T., Dorman B., Blue Straggler Stars: The Spectacular Population in M80, ApJ, 1999, vol. 522, p. 983

Ferraro F. R., Sabbi E., Gratton R., Piotto G., Lanzoni B., Carretta E., Rood R. T., Sills A., Fusi Pecci F., Moehler S., Beccari G., Lucatello S., Compagni N., Discovery of Carbon/Oxygen-depleted Blue Straggler Stars in 47 Tucanae: The Chemical Signature of a Mass Transfer Formation Process, ApJ, 2006, vol. 647, p. L53

Fishlock C. K., Yong D., Karakas A. I., Alves-Brito A., Meléndez J., Nissen P. E., Kobayashi C., Casey A. R., Sc and neutron-capture abundances in Galactic low- and high- $\alpha$ field halo stars, MNRAS, 2017, vol. 466, p. 4672 
François P., Depagne E., Hill V., Spite M., Spite F., Plez B., Beers T. C., Andersen J., James G., Barbuy B., Cayrel R., Bonifacio P., Molaro P., Nordström B., Primas F., First stars. VIII. Enrichment of the neutron-capture elements in the early Galaxy, A\&A, 2007, vol. 476 , p. 935

Frebel A., Metal-poor Stars. In New Horizons in Astronomy, vol. 393 of Astronomical Society of the Pacific Conference Series, 2008, p. 63

Frebel A., Stellar Archaeology: New Science with Old Stars, ArXiv e-prints, 2010

Frebel A., Aoki W., Christlieb N., Ando H., Asplund M., Barklem P. S., Beers T. C., Eriksson K., Fechner C., Fujimoto M. Y., Honda S., et. al. Nucleosynthetic signatures of the first stars, Nature, 2005, vol. 434, p. 871

Frebel A., Norris J. E., , 2013 Metal-Poor Stars and the Chemical Enrichment of the Universe. p. 55

Frebel A., Norris J. E., Gilmore G., Wyse R. F. G., The Chemical Evolution of the Bootes I Ultra-faint Dwarf Galaxy, ApJ, 2016, vol. 826, p. 110

Frischknecht U., Hirschi R., Pignatari M., Maeder A., Meynet G., Chiappini C., Thielemann F.-K., Rauscher T., Georgy C., Ekström S., s-process production in rotating massive stars at solar and low metallicities, MNRAS, 2016, vol. 456, p. 1803

Fu X., Bressan A., Molaro P., Marigo P., Lithium evolution in metal-poor stars: from pre-main sequence to the Spite plateau, MNRAS, 2015, vol. 452, p. 3256

Fujii M. S., Portegies Zwart S., The Formation and Dynamical Evolution of Young Star Clusters, ApJ, 2016, vol. 817, p. 4

Gallagher A. J., Caffau E., Bonifacio P., Ludwig H.-G., Steffen M., Homeier D., Plez B., An in-depth spectroscopic examination of molecular bands from 3D hydrodynamical model atmospheres. II. Carbon-enhanced metal-poor 3D model atmospheres, A\&A, 2017, vol. 598, p. L10

Gallagher A. J., Caffau E., Bonifacio P., Ludwig H.-G., Steffen M., Spite M., An indepth spectroscopic examination of molecular bands from 3D hydrodynamical model 
atmospheres. I. Formation of the G-band in metal-poor dwarf stars, A\&A, 2016, vol. 593, p. A48

Gaunt J. A., Fowler R. H., Continuous absorption, Philosophical Transactions of the Royal Society of London. Series A, Containing Papers of a Mathematical or Physical Character, 1930, vol. 229, p. 163

Geisler D., Smith V. V., Wallerstein G., Gonzalez G., Charbonnel C., "Sculptor-ing" the Galaxy? The Chemical Compositions of Red Giants in the Sculptor Dwarf Spheroidal Galaxy, AJ, 2005, vol. 129, p. 1428

Ghezzi L., Cunha K., Smith V. V., de Araújo F. X., Schuler S. C., de la Reza R., Stellar Parameters and Metallicities of Stars Hosting Jovian and Neptunian Mass Planets: A Possible Dependence of Planetary Mass on Metallicity, ApJ, 2010, vol. 720, p. 1290

Ghezzi L., Cunha K., Smith V. V., Margheim S., Schuler S., de Araújo F. X., de la Reza R., Measurements of the Isotopic Ratio ${ }^{6} \mathrm{Li} /{ }^{7} \mathrm{Li}$ in Stars with Planets, ApJ, 2009, vol. 698, p. 451

Ghezzi L., Montet B. T., Johnson J. A., Retired A Stars Revisited: An Updated Giant Planet Occurrence Rate as a Function of Stellar Metallicity and Mass, ApJ, 2018, vol. 860, p. 109

Gratton R. G., Carretta E., Desidera S., Lucatello S., Mazzei P., Barbieri M., Abundances for metal-poor stars with accurate parallaxes. II. alpha -elements in the halo, A\&A, 2003, vol. 406, p. 131

Gray D. F., The Observation and Analysis of Stellar Photospheres, 2005

Gustafsson B., Edvardsson B., Eriksson K., Jørgensen U. G., Nordlund Å., Plez B., A grid of MARCS model atmospheres for late-type stars. I. Methods and general properties, A\&A, 2008, vol. 486, p. 951

Hampel M., Stancliffe R. J., Lugaro M., Meyer B. S., The Intermediate Neutron-capture Process and Carbon-enhanced Metal-poor Stars, ApJ, 2016, vol. 831, p. 171 
Hansen C. J., Nordström B., Hansen T. T., Kennedy C. R., Placco V. M., Beers T. C., Andersen J., Cescutti G., Chiappini C., Abundances of carbon-enhanced metal-poor stars as constraints on their formation, A\&A, 2016, vol. 588, p. A37

Hansen T. T., Andersen J., Nordström B., Beers T. C., Yoon J., Buchhave L. A., The role of binaries in the enrichment of the early Galactic halo. I. r-process-enhanced metal-poor stars, A\&A, 2015, vol. 583, p. A49

Harris M. J., Lambert D. L., Oxygen isotopes in the atmospheres of Betelgeuse and Antares, ApJ, 1984, vol. 281, p. 739

Haynes C. J., Kobayashi C., Galactic simulations of r-process elemental abundances, MNRAS, 2019, vol. 483, p. 5123

Heger A., Woosley S. E., The Nucleosynthetic Signature of Population III, ApJ, 2002, vol. 567 , p. 532

Herwig F., Evolution of Asymptotic Giant Branch Stars, ARA\&A, 2005, vol. 43, p. 435

Hinkel N. R., Young P. A., Pagano M. D., Desch S. J., Anbar A. D., Adibekyan V., BlancoCuaresma S., Carlberg et al. A Comparison of Stellar Elemental Abundance Techniques and Measurements, ApJS, 2016, vol. 226, p. 4

Hogg D. W., Casey A. R., Ness M., Rix H.-W., Foreman-Mackey D., Hasselquist S., Ho A. Y. Q., Holtzman J. A., Majewski S. R., Martell S. L., Mészáros S., Nidever D. L., Shetrone M., Chemical Tagging Can Work: Identification of Stellar Phase-space Structures Purely by Chemical-abundance Similarity, ApJ, 2016, vol. 833, p. 262

Iorio G., Belokurov V., The shape of the Galactic halo with Gaia DR2 RR Lyrae. Anatomy of an ancient major merger, MNRAS, 2019, vol. 482, p. 3868

Ishida K., Statistical Study of Carbon Stars, PASJ, 1960, vol. 12, p. 214

Jacobson H. R., Frebel A., CD -2417504: A New Comprehensive Element Abundance Analysis, ApJ, 2015, vol. 808, p. 53

Ji A. P., Frebel A., Simon J. D., Chiti A., Complete Element Abundances of Nine Stars in the r-process Galaxy Reticulum II, ApJ, 2016, vol. 830, p. 93 
Jofré P., Heiter U., Soubiran C., Blanco-Cuaresma S., Masseron T., Nordlander T., Chemin L., Worley C. C., et. al. Gaia FGK benchmark stars: abundances of $\alpha$ and iron-peak elements, A\&A, 2015, vol. 582, p. A81

Johnson J. L., Li H., The First Planets: The Critical Metallicity for Planet Formation, ApJ, 2012, vol. 751, p. 81

Karakas A. I., Marino A. F., Nataf D. M., Nucleosynthesis in Helium-enriched Asymptotic Giant Branch Models: Implications for Heavy Element Enrichment in $\omega$ Centauri, ApJ, 2014, vol. 784, p. 32

Karakas A. I., van Raai M. A., Lugaro M., Sterling N. C., Dinerstein H. L., Nucleosynthesis Predictions for Intermediate-Mass Asymptotic Giant Branch Stars: Comparison to Observations of Type I Planetary Nebulae, ApJ, 2009, vol. 690, p. 1130

Karovicova I., White T. R., Nordlander T., Lind K., Casagrande L., Ireland M. J., Huber D., Creevey O., Mourard D., et al. Accurate effective temperatures of the metal-poor benchmark stars HD 140283, HD 122563, and HD 103095 from CHARA interferometry, MNRAS, 2018, vol. 475, p. L81

Kaulakys B., Free 'electron model for inelastic collisions between neutral atomic particles and Rydberg atoms, 1986

Kaulakys B., Free electron model for collisional angular momentum mixing of high Rydberg atoms, Journal of Physics B: Atomic, Molecular and Optical Physics, 1991, vol. 24, p. L127

Keller S. C., Bessell M. S., Frebel A., Casey A. R., Asplund M., Jacobson H. R., Lind K., Norris J. E., Yong D., Heger A., Magic Z., da Costa G. S., Schmidt B. P., Tisserand P., A single low-energy, iron-poor supernova as the source of metals in the star SMSS J031300.36-670839.3, Nature, 2014, vol. 506, p. 463

King J. R., Lithium Abundances of the Cool Metal-Poor Dwarfs HD 103095, HD 134439, and HD 134440, PASP, 1997, vol. 109, p. 776

Kobayashi C., Karakas A. I., Umeda H., The evolution of isotope ratios in the Milky Way Galaxy, MNRAS, 2011, vol. 414, p. 3231 
Kobayashi C., Nakasato N., Chemodynamical Simulations of the Milky Way Galaxy, ApJ, 2011, vol. 729 , p. 16

Kobayashi C., Umeda H., Nomoto K., Tominaga N., Ohkubo T., Galactic Chemical Evolution: Carbon through Zinc, ApJ, 2006, vol. 653, p. 1145

Korn A. J., Grundahl F., Richard O., Barklem P. S., Mashonkina L., Collet R., Piskunov N., Gustafsson B., A probable stellar solution to the cosmological lithium discrepancy, Nature, 2006, vol. 442, p. 657

Kramers H. A., XCIII. On the theory of X-ray absorption and of the continuous X-ray spectrum, The London, Edinburgh, and Dublin Philosophical Magazine and Journal of Science, 1923, vol. 46, p. 836

Kramida A., Ralchenko Y., Reader J., NIST ASD Team NIST Atomic Spectra Database (version 5.6.1), https://physics.nist.gov/asd [Tue Jan 29 2019]. National Institute of Standards and Technology, Gaithersburg, MD.), 2019

Kroupa P., On the variation of the initial mass function, MNRAS, 2001, vol. 322, p. 231

Kroupa P., The IMF of Simple and Composite Populations. In Pathways Through an Eclectic Universe, vol. 390 of Astronomical Society of the Pacific Conference Series, 2008, p. 3

Kusakabe M., Kawasaki M., Chemical separation of primordial $\mathrm{Li}^{+}$during structure formation caused by nanogauss magnetic field, MNRAS, 2015, vol. 446, p. 1597

Laverick M., Lobel A., Royer P., Merle T., Martayan C., van Hoof P. A. M., Van der Swaelmen M., David M., Hensberge H., Thienpont E., The Belgian repository of fundamental atomic data and stellar spectra (BRASS). II. Quality assessment of atomic data for unblended lines in FGK stars, A\&A, 2019, vol. 624, p. A60

Lee Y. S., Beers T. C., Masseron T., Plez B., Rockosi C. M., Sobeck J., Yanny B., Lucatello S., Sivarani T., Placco V. M., Carollo D., Carbon-enhanced Metal-poor Stars in SDSS/SEGUE. I. Carbon Abundance Estimation and Frequency of CEMP Stars, AJ, 2013, vol. 146, p. 132 
Letarte B., Hill V., Tolstoy E., Jablonka P., Shetrone M., Venn K. A., Spite M., Irwin M. J., Battaglia G., Helmi A., Primas F., François P., Kaufer A., Szeifert T., Arimoto N., Sadakane K., A high-resolution VLT/FLAMES study of individual stars in the centre of the Fornax dwarf spheroidal galaxy, A\&A, 2010, vol. 523, p. A17

Li H., Aoki W., Zhao G., Honda S., Christlieb N., Suda T., High-resolution spectroscopic studies of ultra metal-poor stars found in the LAMOST survey, PASJ, 2015, vol. 67, p. 84

Lind K., Asplund M., Barklem P. S., Departures from LTE for neutral Li in late-type stars, A\&A, 2009, vol. 503, p. 541

Lind K., Asplund M., Barklem P. S., Belyaev A. K., Non-LTE calculations for neutral Na in late-type stars using improved atomic data, A\&A, 2011, vol. 528, p. A103

Lind K., Melendez J., Asplund M., Collet R., Magic Z., The lithium isotopic ratio in very metal-poor stars, A\&A, 2013, vol. 554, p. A96

Liu F., Asplund M., Yong D., Feltzing S., Dotter A., Meléndez J., Ramírez I., Chemical (in)homogeneity and atomic diffusion in the open cluster M67, arXiv e-prints, 2019

Liu F., Yong D., Asplund M., Feltzing S., Mustill A. J., Meléndez J., Ramírez I., Lin J., Detailed chemical compositions of the wide binary HD 80606/80607: revised stellar properties and constraints on planet formation, A\&A, 2018, vol. 614, p. A138

Liu F., Yong D., Asplund M., Ramírez I., Meléndez J., Gustafsson B., Howes L. M., Roederer I. U., Lambert D. L., Bensby T., The detailed chemical composition of the terrestrial planet host Kepler-10, MNRAS, 2016, vol. 456, p. 2636

Lodders K., Palme H., Gail H.-P., Abundances of the Elements in the Solar System, Landolt Börnstein, 2009, p. 712

London F., Zur Theorie und Systematik der Molekularkräfte, Zeitschrift fur Physik, 1930, vol. 63 , p. 245

Lucatello S., Tsangarides S., Beers T. C., Carretta E., Gratton R. G., Ryan S. G., The Binary Frequency Among Carbon-enhanced, s-Process-rich, Metal-poor Stars, ApJ, 2005, vol. 625 , p. 825 
Maciel W. J., Costa R. D. D., Lithium abundances and metallicities: trends from metalpoor and AGB/RGB stars., Memorie della Societa Astronomica Italiana Supplementi, 2012, vol. 22, p. 103

Maciel W. J., Costa R. D. D., Mass loss rates of Li-rich AGB/RGB stars, Astronomische Nachrichten, 2018, vol. 339, p. 168

Magic Z., Collet R., Hayek W., Asplund M., The Stagger-grid: A grid of 3D stellar atmosphere models. II. Horizontal and temporal averaging and spectral line formation, A\&A, 2013, vol. 560, p. A8

Majewski S. R., Skrutskie M. F., Schiavon R. P., Wilson J. C., O’Connell R. W., Smith V. V., Shetrone M., Cunha K., Frinchaboy P. M., et. al. The Apache Point Observatory Galactic Evolution Experiment (APOGEE). In American Astronomical Society Meeting Abstracts , vol. 39 of Bulletin of the American Astronomical Society, 2007, p. 962

Mapelli M., Sigurdsson S., Ferraro F. R., Colpi M., Possenti A., Lanzoni B., The radial distribution of blue straggler stars and the nature of their progenitors, MNRAS, 2006, vol. 373 , p. 361

Marino A. F., Da Costa G. S., Casey A. R., Asplund M., Bessell M. S., Frebel A., Keller S. C., Lind K., Mackey A. D., et al. Keck HIRES spectroscopy of SkyMapper commissioning survey candidate extremely metal-poor stars, MNRAS, 2019, vol. 485, p. 5153

Matteucci F., Brocato E., Metallicity distribution and abundance ratios in the stars of the Galactic bulge, ApJ, 1990, vol. 365, p. 539

McCrea W. H., Extended main-sequence of some stellar clusters, MNRAS, 1964, vol. 128, p. 147

McWilliam A., Barium Abundances in Extremely Metal-poor Stars, AJ, 1998, vol. 115, p. 1640

Meléndez J., Asplund M., Gustafsson B., Yong D., The Peculiar Solar Composition and Its Possible Relation to Planet Formation, ApJ, 2009, vol. 704, p. L66

Meléndez J., Barbuy B., Oscillator Strengths and Damping Constants for Atomic Lines in the J and H Bands, ApJS, 1999, vol. 124, p. 527 
Meléndez J., Bergemann M., Cohen J. G., Endl M., Karakas A. I., Ramírez I., Cochran W. D., Yong D., MacQueen P. J., Kobayashi C., Asplund M., The remarkable solar twin HIP 56948: a prime target in the quest for other Earths, A\&A, 2012, vol. 543, p. A29

Meléndez J., Casagrande L., Ramírez I., Asplund M., Schuster W. J., Observational evidence for a broken Li Spite plateau and mass-dependent Li depletion, A\&A, 2010, vol. 515, p. L3

Meléndez J., Cohen J. G., Magnesium Isotopes in Metal-poor Dwarfs: The Rise of AGB Stars and the Formation Timescale of the Galactic Halo, ApJ, 2007, vol. 659, p. L25

Meléndez J., Ramírez I., Karakas A. I., Yong D., Monroe T. R., Bedell M., Bergemann M., Asplund M., Tucci Maia M., Bean J., do Nascimento Jr. J.-D., Bazot M., Alves-Brito A., Freitas F. C., Castro M., 18 Sco: A Solar Twin Rich in Refractory and Neutron-capture Elements. Implications for Chemical Tagging, ApJ, 2014, vol. 791, p. 14

Meléndez J., Shchukina N. G., Vasiljeva I. E., Ramírez I., Permitted Oxygen Abundances and the Temperature Scale of Metal-poor Turnoff Stars, ApJ, 2006, vol. 642, p. 1082

Meynet G., Hirschi R., Ekstrom S., Maeder A., Georgy C., Eggenberger P., Chiappini C., Are C-rich ultra iron-poor stars also He-rich?, A\&A, 2010, vol. 521, p. A30

Mitschang A. W., De Silva G., Zucker D. B., Anguiano B., Bensby T., Feltzing S., Quantitative chemical tagging, stellar ages and the chemo-dynamical evolution of the Galactic disc, MNRAS, 2014, vol. 438, p. 2753

Monaco L., Bellazzini M., Bonifacio P., Ferraro F. R., Marconi G., Pancino E., Sbordone L., Zaggia S., The Ital-FLAMES survey of the Sagittarius dwarf spheroidal galaxy. I. Chemical abundances of bright RGB stars, A\&A, 2005, vol. 441, p. 141

Monroe T. R., Meléndez J., Ramírez I., Yong D., Bergemann M., Asplund M., Bedell M., Tucci Maia M., Bean J., Lind K., Alves-Brito A., Casagrande L., Castro M., do Nascimento J.-D., Bazot M., Freitas F. C., High Precision Abundances of the Old Solar Twin HIP 102152: Insights on Li Depletion from the Oldest Sun, ApJ, 2013, vol. 774, p. L32 
Nissen P. E., High-precision abundances of elements in solar twin stars. Trends with stellar age and elemental condensation temperature, A\&A, 2015, vol. 579, p. A52

Nissen P. E., Akerman C., Asplund M., Fabbian D., Kerber F., Kaufl H. U., Pettini M., Sulphur and zinc abundances in Galactic halo stars revisited, A\&A, 2007, vol. 469, p. 319

Nissen P. E., Gustafsson B., High-precision stellar abundances of the elements: methods and applications, A\&A Rev., 2018, vol. 26, p. 6

Nissen P. E., Schuster W. J., Two distinct halo populations in the solar neighborhood. Evidence from stellar abundance ratios and kinematics, A\&A, 2010, vol. 511, p. L10

Nissen P. E., Schuster W. J., Two distinct halo populations in the solar neighborhood. II. Evidence from stellar abundances of $\mathrm{Mn}, \mathrm{Cu}, \mathrm{Zn}, \mathrm{Y}$, and $\mathrm{Ba}, \mathrm{A} \& \mathrm{~A}, 2011$, vol. 530, p. A15

Nissen P. E., Schuster W. J., Two distinct halo populations in the solar neighborhood. IV. Lithium abundances, A\&A, 2012, vol. 543, p. A28

Nissen P. E., Silva Aguirre V., Christensen-Dalsgaard J., Collet R., Grundahl F., Slumstrup D., High-precision abundances of elements in Kepler LEGACY stars. Verification of trends with stellar age, A\&A, 2017, vol. 608, p. A112

Nixon C. J., King A. R., Pringle J. E., The Maximum Mass Solar Nebula and the early formation of planets, MNRAS, 2018, vol. 477, p. 3273

Noguchi K., Aoki W., Kawanomoto S., Ando H., Honda S., Izumiura H., Kambe E., Okita K., Sadakane K., Sato B., Tajitsu A., Takada-Hidai T., Tanaka W., Watanabe E., Yoshida M., High Dispersion Spectrograph (HDS) for the Subaru Telescope, PASJ, 2002, vol. 54, p. 855

Nomoto K., Tominaga N., Umeda H., Kobayashi C., Maeda K., Nucleosynthesis yields of core-collapse supernovae and hypernovae, and galactic chemical evolution, Nuclear Physics A, 2006, vol. 777, p. 424 
Nordlander T., Amarsi A. M., Lind K., Asplund M., Barklem P. S., Casey A. R., Collet R., Leenaarts J., 3D NLTE analysis of the most iron-deficient star, SMSS0313-6708, A\&A, 2017, vol. 597, p. A6

Norris J. E., Bessell M. S., Yong D., Christlieb N., Barklem P. S., Asplund M., Murphy S. J., Beers T. C., Frebel A., Ryan S. G., The Most Metal-poor Stars. I. Discovery, Data, and Atmospheric Parameters, ApJ, 2013, vol. 762, p. 25

Norris J. E., Yong D., The Most Metal-Poor Stars. V. The CEMP-no Stars in 3D and Non-LTE, arXiv e-prints, 2019

Olive K. A., Petitjean P., Vangioni E., Silk J., Higher D or Li: probes of physics beyond the standard model, MNRAS, 2012, vol. 426, p. 1427

O'Shea B. W., Norman M. L., Population III Star Formation in a $\Lambda$ CDM Universe. I. The Effect of Formation Redshift and Environment on Protostellar Accretion Rate, ApJ, 2007, vol. 654 , p. 66

Osorio Y., Barklem P. S., Lind K., Asplund M., The influence of electron collisions on non-LTE Li line formation in stellar atmospheres, A\&A, 2011, vol. 529, p. A31

Park C., Electron impact excitation rate coefficients for hydrogen, helium and alkali atoms., J. Quant. Spec. Radiat. Transf., 1971, vol. 11, p. 7

Peterson R. C., Sneden C., Relative abundances in metal-poor stars. II - The carbon-to-iron ratio, ApJ, 1978, vol. 225, p. 913

Pignatari M., Gallino R., Heil M., Wiescher M., Käppeler F., Herwig F., Bisterzo S., The Weak s-Process in Massive Stars and its Dependence on the Neutron Capture Cross Sections, ApJ, 2010, vol. 710, p. 1557

Piskunov N., Valenti J. A., Spectroscopy Made Easy: Evolution, A\&A, 2017, vol. 597, p. A16

Placco V. M., Beers T. C., Reggiani H., Meléndez J., G64-12 and G64-37 Are Carbonenhanced Metal-poor Stars, ApJ, 2016, vol. 829, p. L24 
Placco V. M., Frebel A., Beers T. C., Stancliffe R. J., Carbon-Enhanced Metal-Poor Star Frequencies in the Galaxy: Corrections for the Effect of Evolutionary Status on Carbon Abundances, ArXiv e-prints, 2014

Placco V. M., Frebel A., Lee Y. S., Jacobson H. R., Beers T. C., Pena J. M., Chan C., Heger A., Metal-poor Stars Observed with the Magellan Telescope. III. New Extremely and Ultra Metal-poor Stars from SDSS/SEGUE and Insights on the Formation of Ultra Metal-poor Stars, ApJ, 2015, vol. 809, p. 136

Prantzos N., Abia C., Limongi M., Chieffi A., Cristallo S., Chemical evolution with rotating massive star yields - I. The solar neighbourhood and the s-process elements, MNRAS, 2018, vol. 476, p. 3432

Ramírez I., Meléndez J., Asplund M., Accurate abundance patterns of solar twins and analogs. Does the anomalous solar chemical composition come from planet formation?, A\&A, 2009, vol. 508, p. L17

Ramírez I., Meléndez J., Bean J., Asplund M., Bedell M., Monroe T., Casagrande L., Schirbel L., Dreizler S., Teske J., Tucci Maia M., Alves-Brito A., Baumann P., The Solar Twin Planet Search. I. Fundamental parameters of the stellar sample, A\&A, 2014, vol. 572, p. A48

Ramírez I., Meléndez J., Chanamé J., Oxygen Abundances in Low- and High- $\alpha$ Field Halo Stars and the Discovery of Two Field Stars Born in Globular Clusters, ApJ, 2012, vol. 757 , p. 164

Ramírez I., Meléndez J., Cornejo D., Roederer I. U., Fish J. R., Elemental Abundance Differences in the 16 Cygni Binary System: A Signature of Gas Giant Planet Formation?, ApJ, 2011, vol. 740, p. 76

Reggiani H., Amarsi A. M., Lind K., Barklem P. S., Zatsarinny O., Bartschat K., Fursa D. V., Bray I., Spina L., Meléndez J., Non-LTE analysis of K I in late-type stars, A\&A, 2019, vol. 627, p. A177

Reggiani H., Meléndez J., Comparison of errors between a differential and a classical abundance analysis, Canadian Journal of Physics, 2017, vol. 95, p. 855 
Reggiani H., Meléndez J., Evidences of extragalactic origin and planet engulfment in the metal-poor twin pair HD 134439/HD 134440, MNRAS, 2018, vol. 475, p. 3502

Reggiani H., Meléndez J., Kobayashi C., Karakas A., Placco V., Constraining cosmic scatter in the Galactic halo through a differential analysis of metal-poor stars, A\&A, 2017, vol. 608, p. A46

Reggiani H., Meléndez J., Yong D., Ramírez I., Asplund M., First high-precision differential abundance analysis of extremely metal-poor stars, A\&A, 2016, vol. 586, p. A67

Richard O., Michaud G., Richer J., Implications of WMAP Observations on Li Abundance and Stellar Evolution Models, ApJ, 2005, vol. 619, p. 538

Roederer I. U., Karakas A. I., Pignatari M., Herwig F., The Diverse Origins of Neutroncapture Elements in the Metal-poor Star HD 94028: Possible Detection of Products of I-Process Nucleosynthesis, ApJ, 2016, vol. 821, p. 37

Roederer I. U., Preston G. W., Thompson I. B., Shectman S. A., Sneden C., Burley G. S., Kelson D. D., A Search for Stars of Very Low Metal Abundance. VI. Detailed Abundances of 313 Metal-poor Stars, AJ, 2014, vol. 147, p. 136

Rossi S., Beers T. C., Sneden C., Carbon Abundances for Metal-Poor Stars Based on Medium-Resolution Spectra. In The Third Stromlo Symposium: The Galactic Halo , vol. 165 of Astronomical Society of the Pacific Conference Series, 1999, p. 264

Rossi S., Beers T. C., Sneden C., Sevastyanenko T., Rhee J., Marsteller B., Estimation of Carbon Abundances in Metal-Poor Stars. I. Application to the Strong G-Band Stars of Beers, Preston, and Shectman, AJ, 2005, vol. 130, p. 2804

Rutten R. J., Radiative Transfer in Stellar Atmospheres, 2003

Ryabchikova T., Piskunov N., Kurucz R. L., Stempels H. C., Heiter U., Pakhomov Y., Barklem P. S., A major upgrade of the VALD database, Phys. Scr, 2015, vol. 90, p. 054005

Ryan S. G., Beers T. C., Kajino T., Rosolankova K., Ultra-Lithium-deficient Halo Stars and Blue Stragglers: A Common Origin?, ApJ, 2001, vol. 547, p. 231 
Ryan S. G., Gregory S. G., Kolb U., Beers T. C., Kajino T., Rapid Rotation of Ultra-Lidepleted Halo Stars and Their Association with Blue Stragglers, ApJ, 2002, vol. 571, p. 501

Ryde N., Lambert D. L., On the Galactic chemical evolution of sulfur, A\&A, 2004, vol. 415, p. 559

Salpeter E. E., The Luminosity Function and Stellar Evolution., ApJ, 1955, vol. 121, p. 161

Sandage A. R., The color-magnitude diagram for the globular cluster M 3., AJ, 1953, vol. 58 , p. 61

Sansonetti J. E., Wavelengths, Transition Probabilities, and Energy Levels for the Spectra of Potassium (KI through KXIX), Journal of Physical and Chemical Reference Data, 2008, vol. 37 , p. 8

Sarajedini A., A Survey of Galactic Globular Clusters for Blue Straggler Stars. In Blue Stragglers, vol. 53 of Astronomical Society of the Pacific Conference Series, 1993, p. 14

Sbordone L., Bonifacio P., Buonanno R., Marconi G., Monaco L., Zaggia S., The exotic chemical composition of the Sagittarius dwarf spheroidal galaxy, A\&A, 2007, vol. 465, p. 815

Schönrich R., Binney J., Dehnen W., Local kinematics and the local standard of rest, MNRAS, 2010, vol. 403, p. 1829

Schuler S. C., Cunha K., Smith V. V., Ghezzi L., King J. R., Deliyannis C. P., Boesgaard A. M., Detailed Abundances of the Solar Twins 16 Cygni A and B: Constraining Planet Formation Models, ApJ, 2011, vol. 737, p. L32

Schuster W. J., Moreno E., Nissen P. E., Pichardo B., Two distinct halo populations in the solar neighborhood. III. Evidence from stellar ages and orbital parameters, A\&A, 2012, vol. 538, p. A21

Searle L., Zinn R., Compositions of halo clusters and the formation of the galactic halo, ApJ, 1978, vol. 225, p. 357 
Shetrone M., Venn K. A., Tolstoy E., Primas F., Hill V., Kaufer A., VLT/UVES Abundances in Four Nearby Dwarf Spheroidal Galaxies. I. Nucleosynthesis and Abundance Ratios, AJ, 2003, vol. 125, p. 684

Shigeyama T., Tsujimoto T., Fossil Imprints of the First-Generation Supernova Ejecta in Extremely Metal-deficient Stars, ApJ, 1998, vol. 507, p. L135

Shigeyama T., Tsujimoto T., Accretion of Dust Grains as a Possible Origin of Metal-poor Stars with Low $\alpha /$ Fe Ratios, ApJ, 2003, vol. 598, p. L47

Silk J., The first stars, MNRAS, 1983, vol. 205, p. 705

Siqueira Mello C., Hill V., Barbuy B., Spite M., Spite F., Beers T. C., Caffau E., Bonifacio P., Cayrel R., François P., Schatz H., Wanajo S., High-resolution abundance analysis of very metal-poor r-I stars, A\&A, 2014, vol. 565, p. A93

Sitnova T., Zhao G., Mashonkina L., Chen Y., Liu F., Pakhomov Y., Tan K., Bolte M., Alexeeva S., Grupp F., Shi J.-R., Zhang H.-W., Systematic Non-LTE Study of the $-2.6 \leq[\mathrm{Fe} / \mathrm{H}] \leq 0.2 \mathrm{~F}$ and $\mathrm{G}$ dwarfs in the Solar Neighborhood. I. Stellar Atmosphere Parameters, ApJ, 2015, vol. 808, p. 148

Smith G., Lambert D. L., Ruck M. J., A fine analysis of calcium and iron lines in the spectrum of the subdwarf Groombridge 1830, A\&A, 1992, vol. 263, p. 249

Smith V. V., Lambert D. L., Nissen P. E., The 6Li/7Li ratio in the metal-poor halo dwarfs HD 19445 and HD 84937, ApJ, 1993, vol. 408, p. 262

Sneden C., Carbon and nitrogen abundances in metal-poor stars., ApJ, 1974, vol. 189, p. 493

Sneden C., Cowan J. J., Kobayashi C., Pignatari M., Lawler J. E., Den Hartog E. A., Wood M. P., Iron-group Abundances in the Metal-poor Main-Sequence Turnoff Star HD 24937, ApJ, 2016, vol. 817, p. 53

Sneden C. A., Carbon and Nitrogen Abundances in Metal-Poor Stars., THE UNIVERSITY OF TEXAS AT AUSTIN., 1973, Ph.D. Thesis 
Souto D., Allende Prieto C., Cunha K., Pinsonneault M., Smith V. V., Garcia-Dias R., Bovy J., García-Hernández D. A., Holtzman J., et. al. Chemical Abundances of Mainsequence, Turnoff, Subgiant, and Red Giant Stars from APOGEE Spectra. II. Atomic Diffusion in M67 Stars, ApJ, 2019, vol. 874, p. 97

Spina L., Meléndez J., Casey A. R., Karakas A. I., Tucci-Maia M., Chemical Inhomogeneities in the Pleiades: Signatures of Rocky-forming Material in Stellar Atmospheres, ApJ, 2018, vol. 863, p. 179

Spina L., Meléndez J., Karakas A. I., Ramírez I., Monroe T. R., Asplund M., Yong D., Nucleosynthetic history of elements in the Galactic disk. [X/Fe]-age relations from highprecision spectroscopy, A\&A, 2016, vol. 593, p. A125

Spite M., Caffau E., Andrievsky S. M., Korotin S. A., Depagne E., Spite F., Bonifacio P., Ludwig H.-G., Cayrel R., François P., Hill V., Plez B., Andersen J., Barbuy B., Beers T. C., Molaro P., Nordström B., Primas F., First stars. XIV. Sulfur abundances in extremely metal-poor stars, A\&A, 2011, vol. 528, p. A9

Spite M., Cayrel R., Plez B., Hill V., Spite F., Depagne E., François P., Bonifacio P., Barbuy B., Beers T., Andersen J., Molaro P., Nordström B., Primas F., First stars $\mathrm{VI}$ - Abundances of $\mathrm{C}, \mathrm{N}, \mathrm{O}, \mathrm{Li}$, and mixing in extremely metal-poor giants. Galactic evolution of the light elements, A\&A, 2005, vol. 430, p. 655

Spite M., Spite F., Lithium abundance at the formation of the Galaxy, Nature, 1982, vol. 297, p. 483

Spitoni E., Vincenzo F., Matteucci F., Romano D., Are ancient dwarf satellites the building blocks of the Galactic halo?, MNRAS, 2016, vol. 458, p. 2541

Starkenburg E., Martin N., Youakim K., Aguado D. S., Allende Prieto C., Arentsen A., Bernard E. J., Bonifacio P., et. al. The Pristine survey - I. Mining the Galaxy for the most metal-poor stars, MNRAS, 2017, vol. 471, p. 2587

Suda T., Hidaka J., Aoki W., Katsuta Y., Yamada S., Fujimoto M. Y., Ohtani Y., Masuyama M., Noda K., Wada K., Stellar Abundances for Galactic Archaeology Database. IV. Compilation of stars in dwarf galaxies, PASJ, 2017, vol. 69, p. 76 
Suda T., Katsuta Y., Yamada S., Suwa T., Ishizuka C., Komiya Y., Sorai K., Aikawa M., Fujimoto M. Y., Stellar Abundances for the Galactic Archeology (SAGA) Database Compilation of the Characteristics of Known Extremely Metal-Poor Stars, PASJ, 2008, vol. 60 , p. 1159

Suda T., Yamada S., Katsuta Y., Komiya Y., Ishizuka C., Aoki W., Fujimoto M. Y., The Stellar Abundances for Galactic Archaeology (SAGA) data base - II. Implications for mixing and nucleosynthesis in extremely metal-poor stars and chemical enrichment of the Galaxy, MNRAS, 2011, vol. 412, p. 843

Sugar J., Corliss C., Atomic energy levels of the iron-period elements: Potassium through Nickel, 1985

Takeda Y., Possibility of chromospheric back-radiation influencing the lithium line formation in Spite plateau stars, A\&A, 2019, vol. 622, p. A107

Takeda Y., Takada-Hidai M., Chromospheres in Metal-Poor Stars Evidenced from the He I $10830 \AA ̊$ Line, PASJ, 2011, vol. 63, p. 547

Takeda Y., Zhao G., Chen Y.-Q., Qiu H.-M., Takada-Hidai M., On the Abundance of Potassium in Metal-Poor Stars, PASJ, 2002, vol. 54, p. 275

Tegmark M., Silk J., Rees M. J., Blanchard A., Abel T., Palla F., How Small Were the First Cosmological Objects?, ApJ, 1997, vol. 474, p. 1

Teske J. K., Ghezzi L., Cunha K., Smith V. V., Schuler S. C., Bergemann M., Abundance Differences between Exoplanet Binary Host Stars XO-2N and XO-2S - Dependence on Stellar Parameters, ApJ, 2015, vol. 801, p. L10

The L.-S., Clayton D. D., Diehl R., Hartmann D. H., Iyudin A. F., Leising M. D., Meyer B. S., Motizuki Y., Schönfelder V., Are ${ }^{44}$ Ti-producing supernovae exceptional?, A\&A, 2006, vol. 450, p. 1037

Thielemann F. K., Arnett W. D., Hydrostatic Nucleosynthesis - Part Two - Core Neon to Silicon Burning and Presupernova Abundance Yields of Massive Stars, ApJ, 1985, vol. 295, p. 604 
Tinsley B. M., Stellar lifetimes and abundance ratios in chemical evolution, ApJ, 1979, vol. 229 , p. 1046

Tolstoy E., Hill V., Tosi M., Star-Formation Histories, Abundances, and Kinematics of Dwarf Galaxies in the Local Group, ARA\&A, 2009, vol. 47, p. 371

Tucci Maia M., Meléndez J., Castro M., Asplund M., Ramírez I., Monroe T. R., do Nascimento Jr. J. D., Yong D., Shallow extra mixing in solar twins inferred from Be abundances, A\&A, 2015, vol. 576, p. L10

Tucci Maia M., Meléndez J., Ramírez I., High Precision Abundances in the 16 Cyg Binary System: A Signature of the Rocky Core in the Giant Planet, ApJ, 2014, vol. 790, p. L25

Tucci Maia M., Ramírez I., Meléndez J., Bedell M., Bean J. L., Asplund M., The Solar Twin Planet Search. III. The $[\mathrm{Y} / \mathrm{Mg}]$ clock: estimating stellar ages of solar-type stars, A\&A, 2016, vol. 590, p. A32

Umeda H., Nomoto K., Nucleosynthesis of Zinc and Iron Peak Elements in Population III Type II Supernovae: Comparison with Abundances of Very Metal Poor Halo Stars, ApJ, 2002, vol. 565 , p. 385

van Regemorter H., Rate of Collisional Excitation in Stellar Atmospheres., ApJ, 1962, vol. 136 , p. 906

Venn K. A., Irwin M., Shetrone M. D., Tout C. A., Hill V., Tolstoy E., Stellar Chemical Signatures and Hierarchical Galaxy Formation, AJ, 2004, vol. 128, p. 1177

Vogt S. S., Allen S. L., Bigelow B. C., Bresee L., Brown B., Cantrall T., Conrad A., Couture M., Delaney C., Epps H. W., et. al. HIRES: the high-resolution echelle spectrometer on the Keck 10-m Telescope. In Instrumentation in Astronomy VIII , vol. 2198 of Proc. SPIE, 1994, p. 362

Wiese W. L., Smith M. W., Miles B. M., Atomic transition probabilities. Vol. 2: Sodium through Calcium. A critical data compilation, 1969

Wijbenga J. W., Zwaan C., Empirical NLTE Analyses of Solar Spectral Lines. I: A Method and Some Applications to Earlier Analyses, Sol. Phys., 1972, vol. 23, p. 265 
Wongwathanarat A., Janka H.-T., Müller E., Pllumbi E., Wanajo S., Production and Distribution of ${ }^{44} \mathrm{Ti}$ and ${ }^{56} \mathrm{Ni}$ in a Three-dimensional Supernova Model Resembling Cassiopeia A, ApJ, 2017, vol. 842, p. 13

Woosley S. E., Weaver T. A., The Evolution and Explosion of Massive Stars. II. Explosive Hydrodynamics and Nucleosynthesis, ApJS, 1995, vol. 101, p. 181

Yakovleva S. A., Barklem P. S., Belyaev A. K., Data on inelastic processes in low-energy potassium-hydrogen and rubidium-hydrogen collisions, MNRAS, 2018, vol. 473, p. 3810

Yanny B., Rockosi C., Newberg H. J., Knapp G. R., Adelman-McCarthy J. K., Alcorn B., Allam S., et al SEGUE: A Spectroscopic Survey of 240,000 Stars with g $=14-20$, AJ, 2009, vol. 137, p. 4377

Yong D., Meléndez J., Grundahl F., Roederer I. U., Norris J. E., Milone A. P., Marino A. F., Coelho P., McArthur B. E., Lind K., Collet R., Asplund M., High precision differential abundance measurements in globular clusters: chemical inhomogeneities in NGC 6752, MNRAS, 2013, vol. 434, p. 3542

Yong D., Norris J. E., Bessell M. S., Christlieb N., Asplund M., Beers T. C., Barklem P. S., Frebel A., Ryan S. G., The Most Metal-poor Stars. II. Chemical Abundances of 190 Metal-poor Stars Including 10 New Stars with $[\mathrm{Fe} / \mathrm{H}] \leq=-3.5$, ApJ, 2013a, vol. 762, p. 26

Yong D., Norris J. E., Bessell M. S., Christlieb N., Asplund M., Beers T. C., Barklem P. S., Frebel A., Ryan S. G., The Most Metal-poor Stars. III. The Metallicity Distribution Function and Carbon-enhanced Metal-poor Fraction, ApJ, 2013b, vol. 762, p. 27

Yoon J., Beers T. C., Placco V. M., Rasmussen K. C., Carollo D., He S., Hansen T. T., Roederer I. U., Zeanah J., Observational Constraints on First-star Nucleosynthesis. I. Evidence for Multiple Progenitors of CEMP-No Stars, ApJ, 2016, vol. 833, p. 20

Yoshida N., Formation of the first generation of stars and blackholes in the Universe, Proceeding of the Japan Academy, Series B, 2019, vol. 95, p. 17

Yoshida N., Omukai K., Hernquist L., Abel T., Formation of Primordial Stars in a $\Lambda$ CDM Universe, ApJ, 2006, vol. 652, p. 6 
Zatsarinny O., Bartschat K., TheB-splineR-matrix method for atomic processes: application to atomic structure, electron collisions and photoionization, Journal of Physics B: Atomic, Molecular and Optical Physics, 2013, vol. 46, p. 112001

Zatsarinny O., Tayal S. S., Photoionization of potassium atoms from the ground and excited states, Phys. Rev. A, 2010, vol. 81, p. 043423

Zhang H. W., Butler K., Gehren T., Shi J. R., Zhao G., NLTE analysis of the solar potassium abundance, A\&A, 2006, vol. 453, p. 723

Zhao G., Mashonkina L., Yan H. L., Alexeeva S., Kobayashi C., Pakhomov Y., Shi J. R., Sitnova T., Tan K. F., Zhang H. W., Zhang J. B., Zhou Z. M., Bolte M., Chen Y. Q., Li X., Liu F., Zhai M., Systematic Non-LTE Study of the $-2.6 \leq[\mathrm{Fe} / \mathrm{H}] \leq 0.2 \mathrm{~F}$ and G Dwarfs in the Solar Neighborhood. II. Abundance Patterns from Li to Eu, ApJ, 2016, vol. 833 , p. 225 
Appendix 

Appendix A

\section{Apendix A}

The scientific papers Reggiani et al. (2016), Reggiani et al. (2017), Reggiani and Meléndez (2018), and Reggiani et al. (2019) from which the thesis was based are attached bellow. 


\title{
First high-precision differential abundance analysis of extremely metal-poor stars ${ }^{\star}$
}

\author{
Henrique Reggiani ${ }^{1}$, Jorge Meléndez ${ }^{1}$, David Yong ${ }^{2}$, Ivan Ramírez ${ }^{3}$, and Martin Asplund ${ }^{2}$ \\ 1 Universidade de São Paulo, Insituto de Astronomia, Geofísica e Ciências Atmosféricas, IAG, Departamento de Astronomia, \\ Rua do Matão 1226, Cidade Universitária, 05508-900 São Paulo, SP, Brazil \\ e-mail: hreggiani@gmail.com \\ 2 The Australian National University, Research School of Astronomy and Astrophysics, Weston, ACT 2611, Australia \\ ${ }^{3}$ University of Texas at Austin, McDonald Observatory and Department of Astronomy, TX, USA
}

Received 23 September 2015 / Accepted 7 December 2015

\section{ABSTRACT}

\begin{abstract}
Context. Studies of extremely metal-poor stars indicate that chemical abundance ratios $[\mathrm{X} / \mathrm{Fe}]$ have a root mean square scatter as low as 0.05 dex (12\%). It remains unclear whether this reflects observational uncertainties or intrinsic astrophysical scatter arising from physical conditions in the interstellar medium at early times.

Aims. We measure differential chemical abundance ratios in extremely metal-poor stars to investigate the limits of precision and to understand whether cosmic scatter or observational errors are dominant.

Methods. We used high-resolution $(R \sim 95000)$ and high signal-to-noise $(S / N=700$ at $5000 \AA)$ HIRES/Keck spectra to determine high-precision differential abundances between two extremely metal-poor stars through a line-by-line differential approach. We determined stellar parameters for the star G64-37 with respect to the standard star G64-12. We performed EW measurements for the two stars for the lines recognized in both stars and performed spectral synthesis to study the carbon abundances.

Results. The differential approach allowed us to obtain errors of $\sigma\left(T_{\text {eff }}\right)=27 \mathrm{~K}, \sigma(\log g)=0.06 \mathrm{dex}, \sigma([\mathrm{Fe} / \mathrm{H}])=0.02 \mathrm{dex}$ and $\sigma\left(v_{t}\right)=0.06 \mathrm{~km} \mathrm{~s}^{-1}$. We estimated relative chemical abundances with a precision as low as $\sigma([\mathrm{X} / \mathrm{Fe}]) \approx 0.01$ dex. The small uncertainties demonstrate that there are genuine abundance differences larger than the measurement errors. The observed Li difference cannot be explained by the difference in mass because the less massive star has more Li.

Conclusions. It is possible to achieve an abundance precision around $\approx 0.01-0.05$ dex for extremely metal-poor stars, which opens new windows on the study of the early chemical evolution of the Galaxy.
\end{abstract}

Key words. stars: abundances - stars: evolution - stars: Population II - Galaxy: abundances - Galaxy: evolution - Galaxy: halo

\section{Introduction}

Extremely metal-poor (EMP) stars (i.e. stars with $[\mathrm{Fe} / \mathrm{H}]<-3$ ) are relics of the early universe and can provide us with precious clues about the chemical evolution and formation of the Galaxy. These objects arguably offer the most powerful insights into the evolution, nucleosynthetic yields, and properties of the first supernovae (Audouze \& Silk 1995; Ryan et al. 1996; Shigeyama \& Tsujimoto 1998; Chieffi \& Limongi 2002; Umeda \& Nomoto 2002).

The most accurate abundance measurements in EMP stars come from Cayrel et al. (2004) and Arnone et al. (2005) with errors for $[\mathrm{X} / \mathrm{Fe}]$ as low as 0.05 dex. A key open question is whether the observed scatter in abundance ratios reflects genuine cosmic scatter or measurement uncertainties. Higher precision abundance studies of EMP stars are needed to clarify this issue, but such measurements are challenging as they require long exposures using $8 \mathrm{~m}$ class telescopes to obtain high-resolution and high signal-to-noise ratio $(\mathrm{S} / \mathrm{N})$ data. To improve our precision we employed the differential technique in our analysis. Recently, the differential technique in twin stars, meaning stars with similar stellar parameters, made it possible to considerably

* Table A.1 is also available at the CDS via anonymous ftp to cdsarc.u-strasbg.fr (130.79.128.5) or via http://cdsarc.u-strasbg.fr/viz-bin/qcat?J/A+A/586/A67 improve the precision achieved in spectroscopic studies because many error sources, such as imprecise $\log (g f)$ values, largely cancel out, allowing a much better precision in the determination of relative stellar parameters and abundances. Studies with this technique have been used to recognize planet signatures on the chemical composition of stars (Meléndez et al. 2009; Ramírez et al. 2009; Tucci Maia et al. 2014; Biazzo et al. 2015), stellar evolution effects (Monroe et al. 2013; Tucci Maia et al. 2015), chemical evolution in the solar neighborhood (Nissen 2015), abundance anomalies in globular clusters (Yong et al. 2013), and distinct populations in the metal-rich halo (Nissen \& Schuster 2010).

Here we explore, for the first time, the chemical composition of two EMP turn-off stars through a strictly differential analysis, achieving an unprecedented precision $(0.01 \mathrm{dex})$ for a few of the analyzed species.

\section{Observations and data reduction}

Spectra of G64-12 and G64-37 were obtained with the High Resolution Echelle Spectrometer (HIRES; Vogt et al. 1994), on the Keck 10 m telescope at Mauna Kea. The star G64-12 was observed on June 16, 2005, and G64-37 on January 19, 2006. The observations were performed with the same setup using the slit E4 $\left(0.4^{\prime \prime} \times 7^{\prime \prime}\right)$, resulting in a resolving power of $R \sim 95000$, 
with a $S / N=700$ at $5000 \AA$ and $S / N=900$ around the Li $6707 \AA$ line. The spectra have a wavelength coverage ranging from $\sim 3900 \AA$ to $8300 \AA$.

The orders were extracted using the Mauna Kea Echelle Extraction $\left(\mathrm{MAKEE}^{1}\right)$ package, especially written to reduce HIRES spectra. We performed the Doppler correction and continuum normalization via IRAF.

\section{Analysis}

We used a line-by-line differential approach to obtain stellar parameters and chemical abundances, as described in our previous works (e.g. Meléndez et al. 2012; Yong et al. 2013; Ramírez et al. 2015). The 2014 version of the local thermodynamic equilibrium (LTE) analysis code MOOG (Sneden 1973) was employed with the Castelli et al. (1997) atmospheric models.

The linelist was created by inspecting each feature to verify that each chosen line could be measured on both spectra. The $\log (g f)$ values and energy levels are from Vienna Atomic Line Database (VALD). The Fe I lines were updated using data from Den Hartog et al. (2014) and transition probabilities for the Fe II lines are from Meléndez \& Barbuy (2009). The Ti II values were updated using Lawler et al. (2013). We note that the choice of $\log (g f)$ values is inconsequential in a differential analysis.

The equivalent widths (EWs) were measured by hand with the splot task in IRAF, using Gaussian profile fits. In order to determine the local continuum we compared each line in the two stars by overplotting the spectra in a $6 \AA$ window.

The complete linelist, including the EWs for both objects, is presented in Table A.1.

G64-12 is used as the standard star for the analysis with the following stellar parameters: $T_{\text {eff }}=6463 \mathrm{~K}$ from the infrared flux method (IRFM; Meléndez et al. 2010), $\log g=$ 4.26 dex from the absolute magnitude (Nissen et al. 2007) ${ }^{2}$ and, using our EWs, we obtained $[\mathrm{Fe} / \mathrm{H}]=-3.20$ dex and $v_{t}=1.65 \mathrm{~km} \mathrm{~s}^{-1}$. We then employed a strictly line-by-line differential approach to obtain the stellar parameters of G64-37. Using the Fe I and Fe II abundances from G64-12 as references we determined $T_{\text {eff }}=6570 \mathrm{~K}$ through differential excitation equilibrium (Fig. 1), consistent with the IRFM value $\left(T_{\text {eff }}=6583 \pm 50 \mathrm{~K}\right.$, Meléndez et al. 2010). We obtained a $\log g=4.40$ dex through differential ionization equilibrium, consistent with Nissen et al. (2007) $(\log g=4.24 \pm 0.15)$. We obtained $v_{t}=1.74 \mathrm{~km} \mathrm{~s}^{-1}$ by allowing no trend in the differential Fe I line abundances with reduced EWs (Fig. 1), and found $[\mathrm{Fe} / \mathrm{H}]=-3.00 \mathrm{dex}$. The errors for the atmospheric parameters are $\sigma\left(T_{\text {eff }}\right)=27 \mathrm{~K}, \sigma(\log g)=0.06 \mathrm{dex}, \sigma([\mathrm{Fe} / \mathrm{H}])=0.02 \mathrm{dex}$, and $\sigma\left(v_{t}=0.06\right) \mathrm{km} \mathrm{s}^{-1}$. They include the degeneracy of stellar parameters and were determined strictly through a differential approach.

Once the stellar parameters of G64-37 were determined through the iron lines, we determined the abundance of the other elements recognized in both spectra: $\mathrm{Li}, \mathrm{O}, \mathrm{Na}, \mathrm{Mg}, \mathrm{Al}, \mathrm{Si}, \mathrm{Ca}$, $\mathrm{Sc}, \mathrm{Ti}, \mathrm{Cr}, \mathrm{Mn}, \mathrm{Fe}, \mathrm{Co} \mathrm{Ni}, \mathrm{Zn}, \mathrm{Sr}$, and Ba. For the elements Li, $\mathrm{Mn}, \mathrm{Co}$, and $\mathrm{Ba}$, hyperfine splitting was accounted for. For $\mathrm{Li}$ we used the linelist described in Meléndez \& Ramírez (2004). For $\mathrm{Mn}$ and Co we employed the linelists from Kurucz ${ }^{3}$ and for Ba we employed the linelist from McWilliam (1998). We present the final differential abundances in Table 1, along with

\footnotetext{
1 The package was created by T. A. Barlow and is freely available at http://www . astro. caltech. edu/ tb/makee/

2 The parallax is too uncertain, and so we adopted the photometric $M_{V}$ from Nissen et al. (2007)

3 http://kurucz.harvard.edu/linelists.html
}
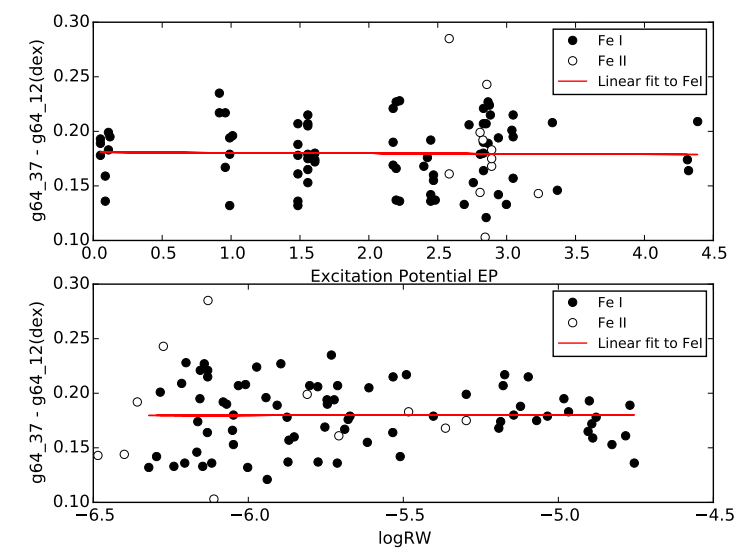

Fig. 1. Differential abundances versus lower excitation potential (top panel) and reduced equivalent widths (lower panel).

the errors from propagating the stellar parameter errors and the observational error. The total errors were calculated by quadratically adding both observational and systematic errors. In the last column of Table 1 we also show the ratio between differential abundances and total errors. This column reveals there are genuine abundance differences, greater than $2 \sigma$ significance for $\Delta[\mathrm{X} / \mathrm{H}]$, for all elements (except oxygen and silicon) between the two stars.

To demonstrate the importance of the differential technique in this work we analyzed the $[\mathrm{Mg} / \mathrm{H}]$ ratio for star $\mathrm{G} 64-12$ in a non-differential way (classic analysis), achieving a much higher total error. The observational error $(\sigma / \sqrt{N})$ alone $(0.059 \mathrm{dex})$ is higher than the total error obtained by using the differential analysis; when added to the parameter uncertainties (0.021 dex) the final error associated with the measurement is $\approx 0.083 \mathrm{dex}$, much higher than the 0.026 dex achieved using the differential technique.

We also present the differential abundance results relative to $\mathrm{Fe}(\Delta[\mathrm{X} / \mathrm{Fe}])$. In this case the errors were derived considering how the error for each stellar parameter behaves in relation to the same error in the iron differential abundance. After this step, we quadratically added the new parameter errors with the observational errors (defined as $\sigma / \sqrt{N}$, where $N$ is the number of measured lines) presented in Table 1 . We can see through the significance of our results (Table 2, Col. 4) that working with $[\mathrm{X} / \mathrm{Fe}]$ has decreased the confidence in the result of some elements when compared to the results of $[\mathrm{X} / \mathrm{H}]$ (Table 1). Eleven out of 17 species exhibit abundance differences (greater than $2 \sigma$ significance) between the two stars for $\Delta[\mathrm{X} / \mathrm{Fe}]$. For the remaining six elements, the majority are heavy elements for which the total error is dominated by observational uncertainties arising from the small numbers of weak spectral lines, as can be seen in Table A.1.

To further show the improvement that the differential technique offers, in Fig. 2 we compare our errors with those obtained by Cayrel et al. (2004, Table 9) using a classical analysis. The dashed line represents the median value of the ratios between the two errors showing that our results are about four times more precise than the aforementioned work.

For carbon it was more appropriate to determine the abundances by spectral synthesis of the $\mathrm{CH}$ band. First, we estimated the macro-turbulent ( $V_{\text {macro }}$ ) velocity of the stars by visually fitting four different iron lines (3920.2 $\AA$, 4005.2 $\AA$, 4045.8 $\AA$, $4063.6 \AA$ ). We determined $V_{\text {macro }}=3.8 \mathrm{~km} \mathrm{~s}^{-1}$ for G64-12 and $V_{\text {macro }}=3.7 \mathrm{~km} \mathrm{~s}^{-1}$ for G64-37. 
H. Reggiani et al.: First high-precision differential abundance analysis of extremely metal-poor stars

Table 1. Relative abundances (G64-37 minus G64-12) and associated uncertainties due to errors in stellar parameters and observations.

\begin{tabular}{lrrrrrrrrr}
\hline \hline Species & $\Delta[\mathrm{X} / \mathrm{H}]$ & $\begin{array}{r}\Delta T_{\text {eff }} \\
+27 \mathrm{~K} \\
(\mathrm{dex})\end{array}$ & $\begin{array}{r}\Delta \log g \\
+0.06 \mathrm{dex} \\
(\mathrm{dex})\end{array}$ & $\begin{array}{r}\Delta v_{t} \\
+0.06 \mathrm{~km} \mathrm{~s}^{-1} \\
(\mathrm{dex})\end{array}$ & $\begin{array}{r}\Delta[\mathrm{Fe} / \mathrm{H}] \\
+0.02 \mathrm{dex} \\
(\mathrm{dex})\end{array}$ & $\begin{array}{c}\text { Param }^{a} \\
(\mathrm{dex})\end{array}$ & Obs $^{b}$ & Total $^{c}$ & $\Delta[\mathrm{X} / \mathrm{H}] / \sigma$ \\
& $(\mathrm{dex})$ & $(\mathrm{dex})$ & \\
\hline Li I & -0.098 & 0.020 & -0.001 & 0.000 & 0.000 & 0.020 & 0.006 & 0.021 & 4.7 \\
C & 0.230 & 0.020 & 0.010 & 0.020 & 0.010 & 0.030 & 0.02 & 0.037 & 6.2 \\
O I & 0.007 & -0.020 & 0.019 & 0.000 & 0.000 & 0.028 & 0.045 & 0.053 & 0.1 \\
Na I & 0.055 & 0.018 & -0.001 & -0.002 & -0.001 & 0.018 & 0.014 & 0.023 & 2.4 \\
Mg I & 0.072 & 0.014 & -0.003 & -0.004 & 0.000 & 0.015 & 0.021 & 0.026 & 2.8 \\
Al I & 0.078 & 0.022 & -0.001 & -0.002 & -0.001 & 0.022 & 0.005 & 0.023 & 3.4 \\
Si I & 0.047 & 0.022 & -0.001 & -0.008 & -0.001 & 0.023 & 0.007 & 0.024 & 1.9 \\
Ca I & 0.086 & 0.016 & -0.001 & -0.002 & 0.000 & 0.016 & 0.010 & 0.019 & 4.5 \\
Sc II & 0.167 & 0.013 & 0.020 & 0.000 & 0.000 & 0.024 & 0.034 & 0.042 & 4.0 \\
Ti I & 0.129 & 0.024 & -0.001 & 0.000 & -0.001 & 0.024 & 0.011 & 0.026 & 5.0 \\
Ti II & 0.155 & 0.010 & 0.019 & -0.001 & 0.000 & 0.021 & 0.007 & 0.023 & 6.7 \\
Cr I & 0.238 & 0.025 & -0.001 & -0.001 & -0.001 & 0.025 & 0.018 & 0.031 & 7.7 \\
Mn I & 0.284 & 0.028 & -0.001 & -0.001 & -0.001 & 0.028 & 0.020 & 0.034 & 8.4 \\
Fe I & 0.180 & 0.022 & -0.001 & -0.003 & 0.000 & 0.022 & 0.003 & 0.022 & 8.3 \\
Fe II & 0.181 & 0.004 & 0.020 & -0.001 & 0.000 & 0.020 & 0.015 & 0.025 & 7.2 \\
Co I & 0.132 & 0.026 & 0.000 & 0.000 & -0.001 & 0.026 & 0.050 & 0.056 & 2.4 \\
Ni I & 0.193 & 0.013 & 0.001 & 0.000 & 0.000 & 0.013 & 0.018 & 0.022 & 8.8 \\
Zn I & 0.127 & 0.014 & 0.004 & -0.001 & -0.001 & 0.015 & 0.039 & 0.042 & 3.0 \\
Sr II & 0.156 & 0.016 & 0.018 & -0.010 & 0.000 & 0.026 & 0.005 & 0.027 & 5.8 \\
Ba II & -0.114 & 0.018 & 0.017 & -0.001 & 0.000 & 0.025 & 0.004 & 0.025 & 4.6 \\
\hline
\end{tabular}

Notes. ${ }^{(a)}$ Errors due to stellar parameters. ${ }^{(b)}$ Observational error, s.e $=\sigma / \sqrt{N}$. ${ }^{(c)}$ Total error, quantified as the quadratic sum of the stellar parameters errors and the observational error.

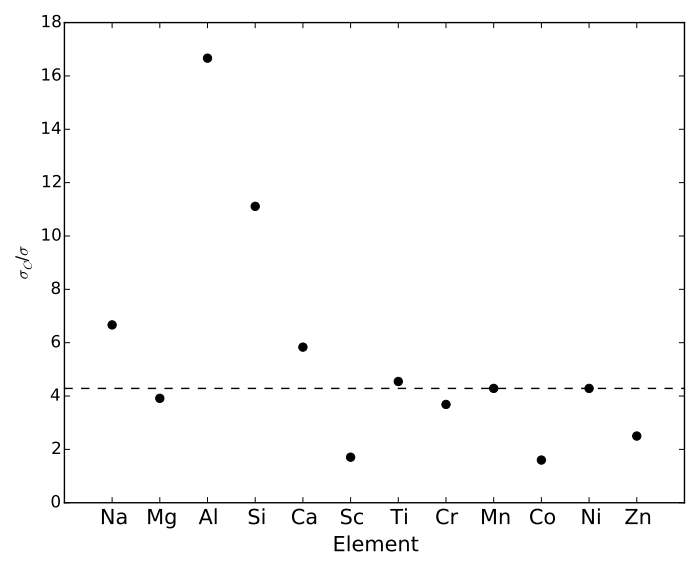

Fig. 2. Ratio between measurement errors from Cayrel et al. (2004) $\left(\sigma_{\mathrm{C}}\right)$ and the errors obtained in this work $(\sigma)$ for a number of elements.

We prepared a linelist, spanning from $4290 \AA$ to $4335 \AA$, specifically for the carbon synthesis using $\mathrm{CH}$ data from Masseron et al. (2014) along with atomic blends for the region from VALD. For each star we synthesized three different regions of the CH band, $4299 \AA$ to $4302 \AA, 4308 \AA$ to $4315 \AA$, and $4322 \AA$ to $4327 \AA$. An example of a best fit for one of the regions, for star G64-12, can be seen in Fig. 3. We averaged the abundance determination for the three regions and determined the abundance difference between the stars. We determined the parameter errors by synthesizing the three regions for each different parameter uncertainty.

We also estimated ages and masses, using the $\mathrm{q} 2$ code (Ramírez et al. 2014). The code fits $\mathrm{Y}^{2}$ isochrones (Yi et al. 2001; Kim et al. 2002) with the adopted stellar parameters. The method estimates the age and mass through a probability distribution approach, as described in Ramírez et al. (2013).
Table 2. $\Delta[\mathrm{X} / \mathrm{Fe}]$ differential abundances (G64-37 - G64-12).

\begin{tabular}{lrrrr}
\hline \hline Species & $\Delta[\mathrm{X} / \mathrm{Fe}]$ & Error & $\Delta[\mathrm{X} / \mathrm{Fe}] / \sigma$ & $\Delta[\mathrm{X} / \mathrm{Fe}]^{C}$ \\
\hline Li I & -0.278 & 0.008 & 34.8 & - \\
C & 0.050 & 0.034 & 1.5 & 0.161 \\
O I & -0.173 & 0.065 & 2.7 & - \\
Na I & -0.125 & 0.015 & 8.3 & - \\
Mg I & -0.108 & 0.023 & 4.7 & -0.112 \\
Al I & -0.102 & 0.006 & 17.0 & - \\
Si I & -0.133 & 0.009 & 14.8 & -0.131 \\
Ca I & -0.094 & 0.012 & 7.8 & -0.106 \\
Sc II & -0.013 & 0.041 & 0.3 & 0.003 \\
Ti & -0.046 & 0.011 & 4.3 & -0.052 \\
Cr I & 0.058 & 0.019 & 3.1 & 0.025 \\
Mn I & 0.104 & 0.021 & 4.9 & 0.057 \\
Co I & -0.048 & 0.050 & 1.0 & 0.021 \\
Ni I & 0.013 & 0.021 & 0.7 & -0.007 \\
Zn I & -0.053 & 0.040 & 1.3 & 0.029 \\
Sr II & -0.024 & 0.022 & 1.1 & 0.048 \\
Ba II & -0.294 & 0.019 & 15.5 & - \\
\hline
\end{tabular}

Notes. ${ }^{(C)}$ Data corrected for Galactic chemical evolution.

For G64-12 we estimated an age of $14.0_{-1.1}^{+0.6} \mathrm{Gyr}$ with a mass $M=0.76_{-0.01}^{+0.01} M_{\odot}$. The best solution for star G64-37 is an age of $10.1_{-2.1}^{+1.2}$ Gyr with a mass $M=0.80_{-0.02}^{+0.02} M_{\odot}$. The error bars represent the $68 \%$ confidence threshold.

It is important to note that we derived the stellar ages through a probability density function (PDF) and obtained that star G6412 is older than 12.9 Gyr with $68 \%$ certainty, and older than about 11.5 Gyr with $92 \%$ certainty. The probability of star G6412 being as young as star G64-37 (10 Gyr) is as low as $0.3 \%$. Star G64-37 is younger than 11.3 Gyr with $68 \%$ certainty, and younger than 12.3 with $92 \%$ certainty. 


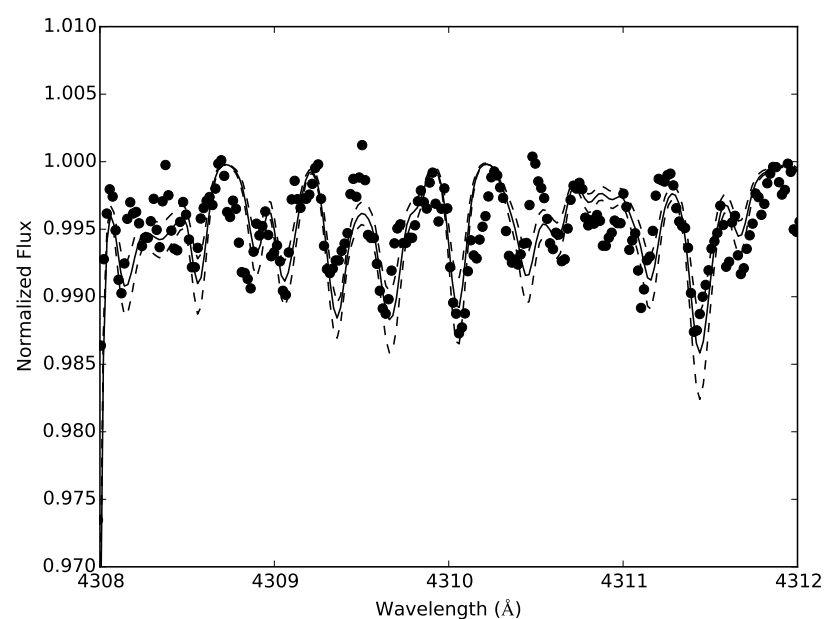

Fig. 3. Best fit of one of the regions synthesized to determine the carbon abundances (star G64-12). The dashed lines are a \pm 0.1 dex difference in $\mathrm{C}$ abundance.

The masses of both stars were also derived trough a PDF and we obtain that star G64-12 is less massive than $0.77 M_{\odot}$ with $68 \%$ certainty and less massive than $0.78 M_{\odot}$ with $92 \%$ certainty. Star G64-37 is more massive than $0.78 M_{\odot}$ with $68 \%$ certainty and more massive than $0.77 M_{\odot}$ with $92 \%$ certainty. The chance of star G64-12 being as massive as G64-37 is only about $4.5 \%$.

Based on our PDF we can say that star G64-12 is older and less massive than star G64-37 with a very high degree of confidence. We note that the difference in age between our pair is similar to the difference in age between "low-alpha" and "highalpha" halo stars at $[\mathrm{Fe} / \mathrm{H}]>-2$ (Schuster et al. 2012).

We checked our stellar parameters and our abundance results using the q2 code, using MARCS model atmospheres (Gustafsson et al. 2008), and the 2014 version of MOOG to compute the curves of growth and obtained consistent results.

\section{Discussion and conclusions}

In Fig. 4 we show our differential abundances. This figure demonstrates that the differential technique is capable of revealing subtle differences in the abundance pattern of metal-poor stars due to the small errors of $\approx 0.01-0.02 \mathrm{dex}$. The precision achieved shows that the pair G64-12/G64-37 have distinct abundance patterns. To compare our results we searched the literature for works that analyzed both stars and have similar S/N and resolution to ours. We found a work from Nissen et al. (2007) and they measured $\Delta[\mathrm{Zn} / \mathrm{H}]=0.19 \pm 0.20$, in good agreement with our results. Fabbian et al. (2009) also found similar stellar parameters, $\Delta[\mathrm{C} / \mathrm{H}]=+0.04 \pm 0.21$ and $\Delta[\mathrm{O} / \mathrm{H}]=-0.03 \pm 0.21$. The difference in carbon abundances might be due to the different techniques used for the determinations; we synthesized CH molecular bands, while Fabbian et al. (2009) measured EWs for CI lines (not available in our spectral coverage), but the values are consistent within the analysis errors. The oxygen abundance agrees with our data, within the errors. Our study, using high-quality observation demonstrates that it is possible to study, for example, the separation of the halo population via the abundance pattern of alpha elements $\mathrm{Mg}, \mathrm{Si}$, and $\mathrm{Ti}$, shown to exist by Nissen $\&$ Schuster (2010) in more metal-rich halo stars.

The differential abundances presented in Table 1 are indicative that these two stars belong to two different populations as there is a significant difference in the abundances of all analyzed elements. In the last column of Table 1 we show the significance
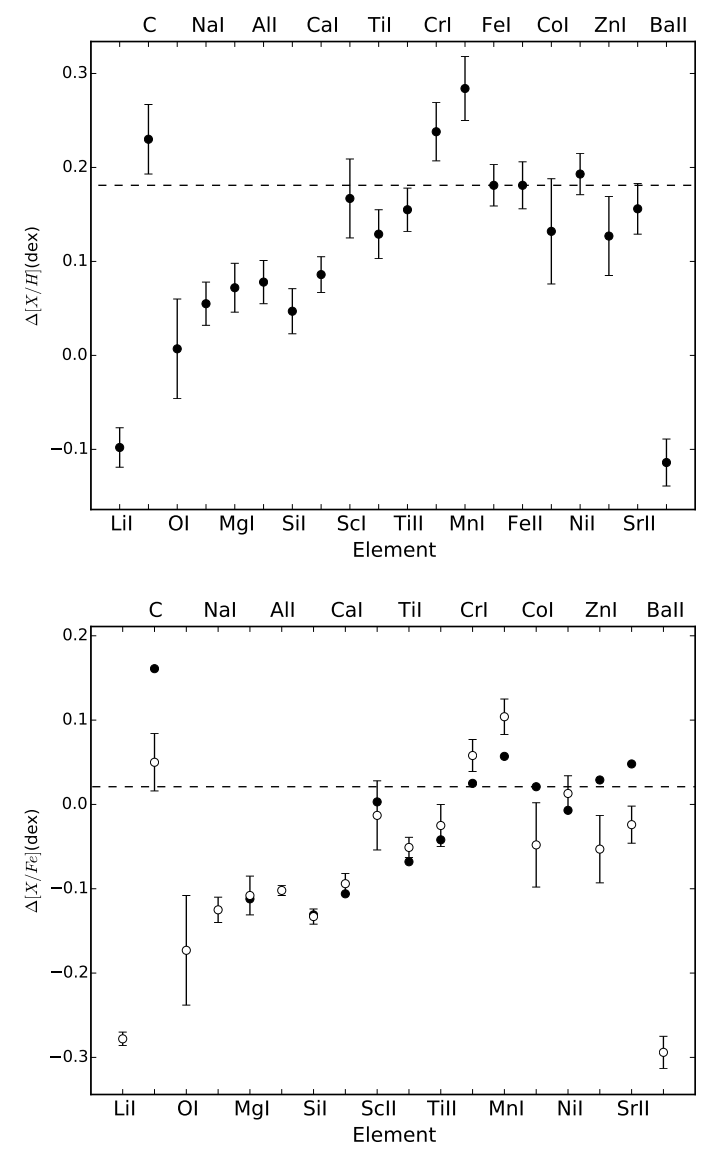

Fig. 4. Top panel: $\Delta[\mathrm{X} / \mathrm{H}]$ abundances. Lower panel: $\Delta[\mathrm{X} / \mathrm{Fe}]$ abundances. Filled circles are the values corrected for Galactic chemical evolution, while the open circles represent the uncorrected abundances (G64-37-G64-12).

of our results and it can be seen that all of our results can be trusted with over $2 \sigma$ confidence, with the exception of oxygen.

By analyzing the $\alpha$-elements, it is possible to see how small the errors must be to separate the stars via differential abundances: $\Delta[\mathrm{Ti} / \mathrm{H}]=0.142 \pm 0.035, \Delta[\mathrm{O} / \mathrm{H}]=0.007 \pm 0.053$, $\Delta[\mathrm{Mg} / \mathrm{H}]=0.072 \pm 0.026$, and $\Delta[\mathrm{Si} / \mathrm{H}]=0.047 \pm 0.024$ are very small. Thus, to distinguish a clear difference we have to achieve errors on the order of (0.01-0.02 dex). There is a small abundance difference between the stars, which indicates that they might belong to distinct halo populations.

As in Nissen \& Schuster (2010) and Ramírez et al. (2012), we can analyze the possibility of distinct halo populations through $[\alpha / \mathrm{Fe}]$ ratios. As can be seen in Table 2, when compared to iron, the differential abundances between these stars are more prominent $(\Delta[\mathrm{O} / \mathrm{Fe}]=-0.173 \pm 0.065, \Delta[\mathrm{Mg} / \mathrm{Fe}]=$ $-0.108 \pm 0.023, \Delta[\mathrm{Si} / \mathrm{Fe}]=-0.133 \pm 0.009$, and $\Delta[\mathrm{Ti} / \mathrm{Fe}]=$ $-0.046 \pm 0.011$ ), which also indicates that they belong to distinct halo populations. It is important to emphasize that for all the $\alpha$-elements the significance of our results are all above $2 \sigma$, including for $[\mathrm{O} / \mathrm{Fe}]$. With this data we find that G64-37, the younger halo star, has lower $[\alpha / \mathrm{Fe}]$, which is in agreement with the results of Schuster et al. (2012).

In order to exclude differences that might arise from Galactic chemical evolution (GCE) we performed linear regressions to the data published in Bonifacio et al. (2009), who performed abundance analyses for stars with similar stellar parameters to the ones used here, but in a wider range of metallicities. Then, we corrected our $[\mathrm{X} / \mathrm{Fe}]$ ratios for the predicted ratio of the linear 
regression. We present the corrected differential abundances, $\Delta[\mathrm{X} / \mathrm{Fe}]^{C}$, for trends in Galactic chemical evolution (GCE) in the last column of Table 2. We note that the GCE corrections are within the error bars of our results and do not change our interpretation, as can also be seen in Fig. 4.

We also estimated the velocity components for the two stars, using an estimated distance from the absolute magnitude by Nissen et al. (2007), proper motion data from van Leeuwen (2007), and radial velocity from Latham et al. (2002). For star G64-12 we obtained $U_{\mathrm{LSR}}=21 \mathrm{~km} \mathrm{~s}^{-1}, V_{\mathrm{LSR}}=$ $-352 \mathrm{~km} \mathrm{~s}^{-1}$, and $W_{\mathrm{LSR}}=-400 \mathrm{~km} \mathrm{~s}^{-1}$ and for star G64$37 U_{\mathrm{LSR}}=231 \mathrm{~km} \mathrm{~s}^{-1}, V_{\mathrm{LSR}}=-369 \mathrm{~km} \mathrm{~s}^{-1}$, and $W_{\mathrm{LSR}}=$ $-77 \mathrm{~km} \mathrm{~s}^{-14}$. We found that both stars have extreme kinematics, falling outside Fig. 3 from Nissen \& Schuster (2010). However, it is important to point out that their study present stars with metallicities $[\mathrm{Fe} / \mathrm{H}]>-1.5$, which are much higher than the stars in this work.

With the small errors achieved, it is also possible to revisit the Li plateau (Spite \& Spite 1982). Meléndez et al. (2010) demonstrated the existence of two plateaus with a break at $[\mathrm{Fe} / \mathrm{H}] \approx-2.5$. With an error of $\sim 0.021$ on our Li differential abundance it will be possible to study a larger sample of stars and determine with higher precision where the break of the $\mathrm{Li}$ plateau is. As the two stars have metallicities that place them on the same plateau, we can compare the absolute differential abundance with the scatter found by Meléndez et al. (2010). The differential $\Delta[\mathrm{Li} / \mathrm{H}]$ abundance found in this study $(0.098 \mathrm{dex})$ is higher than the average scatter $(0.04 \mathrm{dex})$ previously found among stars in that range $([\mathrm{Fe} / \mathrm{H}]<-2.5)$.

In Meléndez et al. (2010) it was argued that the difference in $\mathrm{Li}$ is due to the differences in mass between the stars, as stars with lower masses deplete more lithium (Richard et al. 2005). However, the pair studied here behave unexpectedly: the more metal-poor, older, and less massive star seems to have a higher Li content. To check the result we also performed non-LTE abundance corrections (Lind et al. 2009) and arrived at a differential non-LTE abundance of $\Delta[\mathrm{Li} / \mathrm{H}]=-0.10 \mathrm{dex}$, which shows the consistency of our results. Presently there are only Li diffusion models for $[\mathrm{Fe} / \mathrm{H}] \geq-2$ (Richard et al. 2005). It would be important to extend these models to lower metallicities to test against our high-precision $\mathrm{Li}$ abundances.

The results presented here illustrate how a differential study can help indicate whether lithium is, in fact, being depleted in stars or if physics beyond the primordial nucleosynthesis model is necessary (Fields et al. 2014).

Even after GCE corrections, clear abundance differences remain even among chemical elements produced via similar processes. For example, oxygen is more enhanced than carbon in G64-12, as is also the case of barium and strontium. The difference in the abundance patterns of these stars can give us important information on the environments in which these two stars formed and on the supernovae that enriched them.

We attempted to determine possible supernovae progenitors for our stars. To this end we employed the STARFIT ${ }^{5}$ code (Chan et al., in prep.) with the absolute abundances calculated for our standard star (G64-12) and the absolute values for the standard star plus the differential abundances (Table 1) to study a possible progenitor for star G64-37.
We found no extreme difference between the possible polluting supernovae. The results from STARFIT indicate that the star G64-12 had a progenitor with mas $M=18 M_{\odot}, \log ($ mixing $)=$ -1.0 dex and a remnant of $3.9 M_{\odot}$. The results of G64-37 implied a supernovae with $M=11 M_{\odot}, \log ($ mixing $)=-1.6 \mathrm{dex}$ and a remnant of $1.6 M_{\odot}$.

Our study demonstrates that the advent of precision spectroscopy can open new windows on the study of the early Galaxy, supernovae yields, and the chemical evolution of the Galaxy. With a larger sample of very metal-poor stars we will be able to assess additional questions such as cosmic scatter in the Galactic halo and how the first supernovae enriched our Galaxy.

Acknowledgements. H.R. thanks the CAPES fellowship program. J.M. is grateful for support by FAPESP (2010/50930-6 and 2012/24392-2). D.Y. was supported through an Australian Research Council Future Fellowship (FT140100554). M.A. has been supported by the Australian Research Council grant (FL110100012).

\section{References}

Arnone, E., Ryan, S. G., Argast, D., et al. 2005, A\&A, 430, 507 Audouze, J., \& Silk, J. 1995, AJ, 451, L9

Biazzo, K., Gratton R., Desidera, S., et al. 2015, A\&A, 583, A135

Bonifacio, P., Spite, M., Cayrel, R., et al. 2009, A\&A, 501, 519

Cayrel, R., Depagne, E., Spite, M., et al. 2004, A\&A, 416, 1117

Castelli, F., Gratton, R. G., \& Kurucz, R. L. 1997, A\&A, 318, 841

Chieffi, A., \& Limongi, M. 2002, AJ, 577, 281

Collet, R., Asplund, M., \& Trampedach, R. 2007, A\&A, 469, 687

Den Hartog, E. A., Ruffoni, M. P., et al. 2014, AJ, 215, 23

Fabbian, D., Nissen, P. E., Asplund, M., et al. 2009, A\&A, 500, 1143

Fields, B. D., Molaro, P., \& Sarkar, S. 2014, Chin. Phys., C38, 339

Gustafsson, B., Edvardsson, B., Eriksson, K., et al. 2008, A\&A, 486, 951

Kim, Y-C., Demarque, P., Yi, S., \& Alexander, D. 2002, ApJS, 143, 499

Latham, D. W., Mazeh, T., Carney, B. W., et al. 2002, AJ, 124, 1144

Lawler, J. E., Guzman, A., Wood, M. P., et al. 2013, AJ, 205, 11

van Leeuwen, F. 2007, A\&A, 474, 653

Lind, K., Asplund, M., \& Barklem, P. S. 2009, A\&A, 503, 541

Masseron, T., Plez, B., Van Eck, S., et al. 2014, A\&A, 571, A47

McWilliam, A. 1998, AJ, 115, 1640

Meléndez, J., \& Barbuy, B. 2009, A\&A, 497, 611

Meléndez, J., \& Ramírez, I. 2004, AJ, 615, L33

Meléndez, J., Asplund, M., Gustafsson, B., \& Yong, D. 2009, AJ, 704, L66

Meléndez, J., Casagrande, L., Ramírez, I., et al. 2010, A\&A, 515, L3

Meléndez, J., Bergemann, M., Cohen, J. G., et al. 2012, A\&A, 543, A29

Monroe, T., Meléndez, J., Ramírez, I., et al. 2013, AJ, 774, L32

Nissen, P. E. 2015, A\&A, 579, A52

Nissen, P. E., \& Schuster, W. 2010, A\&A, 511, L10

Nissen, P. E., Akerman, C., Asplund, M., et al. 2007, A\&A, 469, 319

Ramírez, I., Meléndez, J., \& Asplund, M. 2009, A\&A, 508, L17

Ramírez, I., Meléndez, J., \& Chanamé, J. 2012, ApJ, 757, 164

Ramírez, I., Allende Prieto, C., \& Lambert, D. L. 2013, AJ, 764, 78

Ramírez, I., Meléndez, J., Bean, J., et al. 2014, A\&A, 572, A48

Ramírez, I., Khanal, S., \& Aleo, P. 2015, ApJ, 808, 13

Richard, O., Michaud, G., \& Richer, J. 2005, ApJ, 619, 538

Ryan, S. G., Norris, J., \& Beers, T. C. 1996, AJ, 471, 254

Schuster, W. J., Moreno, E., Nissen, P. E., \& Pichardo, B. 2012, A\&A, 538, A21

Shigeyama, T., \& Tsujimoto, T. 1998, AJ, 507, L135

Sneden, C. A. 1973, Ph.D. Thesis, Univ. Texas

Spite, F., \& Spite, M. 1982, A\&A, 115, 357

Tucci Maia, M., Meléndez, J., \& Ramírez, I. 2014, AJ, 790, L25

Tucci Maia, M., Meléndez, J., Castro, M., et al. 2015, A\&A, 576, L10

Umeda, H., \& Nomoto, K. 2002, AJ, 565, 385

Vogt, S. S., Allen, S. L., Bigelow, B. C., et al. 1994, Proc. SPIE, 2198, 362

Yi, S., Demarque, P., Kim, Y.-C., et al. 2001, AJ, 136, 417

Yong, D., Meléndez, J., Grundah, L., et al. 2013, MNRAS, 434, 3542

\footnotetext{
4 The $\mathrm{H}$ parallaxes are too uncertain. Better velocity components will be obtained once Gaia results are released.

5 http://starfit.org/
} 


\section{Appendix A: Additional table}

Table A.1. Linelist used for the abundances determinations.

\begin{tabular}{|c|c|c|c|c|c|}
\hline $\begin{array}{r}\text { Wavelength } \\
(\AA)\end{array}$ & Species & $\begin{array}{r}E P \\
(\mathrm{eV})\end{array}$ & $\begin{array}{r}\log (g f) \\
(\mathrm{dex})\end{array}$ & $\begin{array}{r}\mathrm{G} 64-12(E W) \\
(\mathrm{m} \AA)\end{array}$ & $\begin{array}{r}\mathrm{G} 64-37(E W) \\
(\mathrm{m} \AA)\end{array}$ \\
\hline 3886.282 & 26.0 & 0.052 & -1.076 & 61.40 & 66.20 \\
\hline 3887.048 & 26.0 & 0.915 & -1.144 & 21.20 & 26.10 \\
\hline 3895.656 & 26.0 & 0.110 & -1.670 & 37.70 & 42.00 \\
\hline 3899.707 & 26.0 & 0.087 & -1.531 & 47.10 & 50.40 \\
\hline 3902.946 & 26.0 & 1.557 & -0.466 & 31.30 & 36.00 \\
\hline 3906.480 & 26.0 & 0.110 & -2.243 & 16.60 & 19.70 \\
\hline 3917.181 & 26.0 & 0.990 & -2.155 & 3.60 & 3.90 \\
\hline 3920.258 & 26.0 & 0.121 & -1.746 & 36.10 & 40.90 \\
\hline 3922.912 & 26.0 & 0.052 & -1.651 & 44.50 & 49.40 \\
\hline 3997.392 & 26.0 & 2.727 & -0.479 & 5.00 & 6.70 \\
\hline 3998.053 & 26.0 & 2.692 & -0.910 & 2.00 & 2.30 \\
\hline 4005.242 & 26.0 & 1.557 & -0.610 & 26.00 & 32.00 \\
\hline 4009.713 & 26.0 & 2.223 & -1.252 & 2.20 & 2.50 \\
\hline 4014.531 & 26.0 & 3.047 & -0.587 & 2.10 & 2.80 \\
\hline 4021.867 & 26.0 & 2.758 & -0.729 & 3.00 & 3.60 \\
\hline 4045.812 & 26.0 & 1.485 & 0.280 & 67.50 & 71.20 \\
\hline 4063.594 & 26.0 & 1.557 & 0.062 & 56.20 & 60.50 \\
\hline 4132.058 & 26.0 & 1.608 & -0.675 & 23.10 & 26.90 \\
\hline 4134.678 & 26.0 & 2.831 & -0.649 & 2.90 & 3.70 \\
\hline 4143.415 & 26.0 & 3.047 & -0.204 & 4.60 & 5.60 \\
\hline 4143.868 & 26.0 & 1.557 & -0.511 & 30.80 & 35.30 \\
\hline 4181.755 & 26.0 & 2.831 & -0.371 & 4.90 & 6.60 \\
\hline 4187.039 & 26.0 & 2.449 & -0.548 & 7.10 & 8.10 \\
\hline 4187.795 & 26.0 & 2.425 & -0.554 & 7.10 & 8.80 \\
\hline 4191.431 & 26.0 & 2.469 & -0.666 & 4.90 & 5.90 \\
\hline 4199.095 & 26.0 & 3.047 & 0.155 & 9.10 & 12.30 \\
\hline 4202.029 & 26.0 & 1.485 & -0.708 & 26.90 & 31.70 \\
\hline 4222.213 & 26.0 & 2.449 & -0.967 & 2.70 & 3.50 \\
\hline 4233.603 & 26.0 & 2.482 & -0.604 & 6.20 & 7.10 \\
\hline 4247.426 & 26.0 & 3.368 & -0.239 & 2.40 & 2.90 \\
\hline 4250.119 & 26.0 & 2.469 & -0.405 & 8.70 & 10.30 \\
\hline 4250.787 & 26.0 & 1.557 & -0.714 & 23.00 & 28.20 \\
\hline 4260.474 & 26.0 & 2.399 & 0.109 & 23.30 & 27.40 \\
\hline 4271.154 & 26.0 & 2.449 & -0.349 & 11.50 & 13.20 \\
\hline 4271.761 & 26.0 & 1.485 & -0.164 & 51.20 & 56.60 \\
\hline 4282.403 & 26.0 & 2.176 & -0.779 & 6.10 & 7.70 \\
\hline 4315.085 & 26.0 & 2.198 & -0.965 & 5.10 & 5.80 \\
\hline 4325.762 & 26.0 & 1.608 & 0.006 & 50.30 & 55.50 \\
\hline 4383.545 & 26.0 & 1.485 & 0.200 & 67.40 & 72.20 \\
\hline 4404.750 & 26.0 & 1.557 & -0.142 & 50.10 & 54.90 \\
\hline 4415.123 & 26.0 & 1.608 & -0.615 & 27.20 & 31.70 \\
\hline 4427.310 & 26.0 & 0.052 & -2.924 & 5.10 & 5.90 \\
\hline 4442.339 & 26.0 & 2.198 & -1.255 & 2.30 & 3.20 \\
\hline 4447.717 & 26.0 & 2.223 & -1.342 & 2.00 & 2.80 \\
\hline 4459.118 & 26.0 & 2.176 & -1.279 & 2.40 & 3.30 \\
\hline 4461.653 & 26.0 & 0.087 & -3.210 & 3.20 & 3.40 \\
\hline 4466.552 & 26.0 & 2.831 & -0.600 & 2.90 & 3.80 \\
\hline 4494.563 & 26.0 & 2.198 & -1.136 & 3.30 & 4.00 \\
\hline 4528.614 & 26.0 & 2.176 & -0.822 & 6.60 & 8.00 \\
\hline 4602.941 & 26.0 & 1.485 & -2.209 & 2.00 & 2.20 \\
\hline 4871.318 & 26.0 & 2.865 & -0.363 & 4.40 & 6.20 \\
\hline 4872.138 & 26.0 & 2.882 & -0.567 & 2.60 & 3.60 \\
\hline 4890.755 & 26.0 & 2.875 & -0.394 & 3.70 & 5.20 \\
\hline 4891.492 & 26.0 & 2.851 & -0.112 & 7.10 & 9.50 \\
\hline 4918.994 & 26.0 & 2.865 & -0.342 & 4.70 & 6.10 \\
\hline 4920.503 & 26.0 & 2.832 & 0.068 & 11.90 & 14.40 \\
\hline 4957.299 & 26.0 & 2.851 & -0.408 & 5.10 & 5.70 \\
\hline 4957.597 & 26.0 & 2.808 & 0.233 & 15.90 & 19.60 \\
\hline 5006.119 & 26.0 & 2.832 & -0.638 & 2.50 & 3.50 \\
\hline 5139.463 & 26.0 & 2.940 & -0.509 & 2.20 & 2.60 \\
\hline
\end{tabular}


H. Reggiani et al.: First high-precision differential abundance analysis of extremely metal-poor stars

Table A.1. continued.

\begin{tabular}{|c|c|c|c|c|c|}
\hline $\begin{array}{r}\text { Wavelength } \\
(\AA)\end{array}$ & Species & $\begin{array}{r}E P \\
(\mathrm{eV})\end{array}$ & $\begin{array}{r}\log (g f) \\
(\operatorname{dex})\end{array}$ & $\begin{array}{r}\mathrm{G} 64-12(E W) \\
(\mathrm{mA})\end{array}$ & $\begin{array}{r}\mathrm{G} 64-37(E W) \\
(\mathrm{m} \AA)\end{array}$ \\
\hline 5171.596 & 26.0 & 1.485 & -1.793 & 3.70 & 4.80 \\
\hline 5191.455 & 26.0 & 3.038 & -0.551 & 2.00 & 2.70 \\
\hline 5192.344 & 26.0 & 2.998 & -0.421 & 3.20 & 3.70 \\
\hline 5227.190 & 26.0 & 1.557 & -1.228 & 10.10 & 12.80 \\
\hline 5232.940 & 26.0 & 2.940 & -0.058 & 7.20 & 9.40 \\
\hline 5371.490 & 26.0 & 0.958 & -1.645 & 13.80 & 17.40 \\
\hline 5383.369 & 26.0 & 4.312 & 0.645 & 2.80 & 3.70 \\
\hline 5397.128 & 26.0 & 0.915 & -1.993 & 7.50 & 10.00 \\
\hline 5405.775 & 26.0 & 0.990 & -1.844 & 9.70 & 11.50 \\
\hline 5415.199 & 26.0 & 4.386 & 0.642 & 2.30 & 3.30 \\
\hline 5424.068 & 26.0 & 4.320 & 0.520 & 3.10 & 4.00 \\
\hline 5429.697 & 26.0 & 0.958 & -1.879 & 9.60 & 11.10 \\
\hline 5434.524 & 26.0 & 1.011 & -2.122 & 5.00 & 6.20 \\
\hline 5446.917 & 26.0 & 0.990 & -1.914 & 8.40 & 10.30 \\
\hline 5615.644 & 26.0 & 3.332 & 0.050 & 4.00 & 5.50 \\
\hline 4178.862 & 26.1 & 2.583 & -2.510 & 1.90 & 3.10 \\
\hline 4233.172 & 26.1 & 2.583 & -1.970 & 6.80 & 8.30 \\
\hline 4508.288 & 26.1 & 2.856 & -2.440 & 1.60 & 2.40 \\
\hline 4520.224 & 26.1 & 2.807 & -2.650 & 1.50 & 1.80 \\
\hline 4522.634 & 26.1 & 2.844 & -2.250 & 3.20 & $\begin{array}{l}1.00 \\
3.50\end{array}$ \\
\hline 4555.893 & 26.1 & 2.828 & -2.400 & 1.50 & 2.00 \\
\hline 4583.837 & 26.1 & 2.807 & -1.930 & 5.30 & 7.10 \\
\hline 4923.927 & 26.1 & 2.891 & -1.260 & 12.80 & 16.20 \\
\hline 5018.440 & 26.1 & 2.891 & -1.100 & 17.90 & 21.70 \\
\hline 5169.033 & 26.1 & 2.891 & -1.000 & 21.40 & 26.10 \\
\hline 5197.577 & 26.1 & 3.230 & -2.220 & 1.40 & 1.70 \\
\hline 6707.820 & 3.0 & 0.000 & 0.167 & 24.10 & 16.60 \\
\hline 7771.941 & 8.0 & 9.146 & 0.369 & 4.50 & 5.30 \\
\hline 7774.161 & 8.0 & 9.146 & 0.223 & 3.80 & 3.70 \\
\hline 5889.951 & 11.0 & 0.000 & 0.117 & 31.70 & 29.50 \\
\hline 5895.924 & 11.0 & 0.000 & -0.184 & 19.00 & 19.70 \\
\hline 4057.505 & 12.0 & 4.346 & -1.201 & 2.70 & 3.70 \\
\hline 4167.271 & 12.0 & 4.346 & -1.004 & 4.70 & 4.40 \\
\hline 4351.906 & 12.0 & 4.346 & -0.833 & 5.80 & $\begin{array}{l}5.70 \\
5.70\end{array}$ \\
\hline 5167.321 & 12.0 & 2.709 & -1.030 & 45.40 & 47.50 \\
\hline 5172.684 & 12.0 & 2.712 & -0.402 & 75.70 & 76.10 \\
\hline 5183.604 & 12.0 & 2.717 & -0.180 & 89.30 & 89.80 \\
\hline 5528.405 & 12.0 & 4.346 & -0.620 & 8.10 & 9.10 \\
\hline 3944.006 & 13.0 & 0.000 & -0.623 & 17.80 & 18.00 \\
\hline 3961.520 & 13.0 & 0.014 & -0.323 & 24.00 & 23.30 \\
\hline 3905.523 & 14.0 & 1.909 & -0.743 & 55.80 & 54.70 \\
\hline 4226.728 & 20.0 & 0.000 & 0.244 & 86.50 & 86.60 \\
\hline 4283.011 & 20.0 & 1.886 & -0.292 & 5.80 & 6.00 \\
\hline 4289.367 & 20.0 & 1.879 & -0.388 & 4.10 & 4.80 \\
\hline 4318.652 & 20.0 & 1.899 & -0.295 & 5.10 & 6.00 \\
\hline 4425.437 & 20.0 & 1.879 & -0.358 & 4.30 & 4.70 \\
\hline 4435.679 & 20.0 & 1.886 & -0.517 & 3.30 & 3.30 \\
\hline 4454.779 & 20.0 & 1.899 & 0.258 & 14.20 & 14.50 \\
\hline 4455.887 & 20.0 & 1.899 & -0.414 & 3.00 & 3.60 \\
\hline 5588.749 & 20.0 & 2.526 & 0.358 & 5.60 & 6.50 \\
\hline 5594.462 & 20.0 & 2.523 & 0.097 & 3.70 & 3.70 \\
\hline 5857.451 & 20.0 & 2.933 & 0.240 & 2.30 & 2.30 \\
\hline 6122.217 & 20.0 & 1.886 & -0.386 & 4.60 & 5.40 \\
\hline 6162.173 & 20.0 & 1.899 & -0.167 & 8.00 & 8.10 \\
\hline 6439.075 & 20.0 & 2.526 & 0.394 & 6.90 & 7.10 \\
\hline 4246.822 & 21.1 & 0.315 & 0.242 & 13.30 & 14.80 \\
\hline 4314.083 & 21.1 & 0.618 & -0.096 & 3.30 & 4.50 \\
\hline 4320.732 & 21.1 & 0.605 & -0.252 & 2.40 & 2.20 \\
\hline 4374.457 & 21.1 & 0.618 & -0.418 & 1.70 & 1.90 \\
\hline 4400.389 & 21.1 & 0.605 & -0.536 & 1.30 & 1.80 \\
\hline 3958.206 & 22.0 & 0.048 & -0.177 & 3.50 & 4.10 \\
\hline 3989.759 & 22.0 & 0.021 & -0.198 & 3.40 & 3.80 \\
\hline 3998.636 & 22.0 & 0.048 & -0.056 & 4.40 & 4.20 \\
\hline 4305.908 & 22.0 & 0.848 & 0.510 & 3.30 & 3.60 \\
\hline 4533.241 & 22.0 & 0.848 & 0.476 & 2.70 & 3.30 \\
\hline
\end{tabular}


A\&A 586, A67 (2016)

Table A.1. continued.

\begin{tabular}{|c|c|c|c|c|c|}
\hline $\begin{array}{r}\text { Wavelength } \\
(\AA)\end{array}$ & Species & $\begin{array}{r}E P \\
(\mathrm{eV})\end{array}$ & $\begin{array}{r}\log (g f) \\
(\mathrm{dex})\end{array}$ & $\begin{array}{r}\text { G64-12(EW) } \\
(\mathrm{m} \AA)\end{array}$ & $\begin{array}{r}\mathrm{G} 64-37(E W) \\
(\mathrm{m} \AA)\end{array}$ \\
\hline 4534.776 & 22.0 & 0.836 & 0.280 & 2.00 & 2.20 \\
\hline 4535.568 & 22.0 & 0.826 & 0.162 & 0.90 & 1.10 \\
\hline 4981.731 & 22.0 & 0.848 & 0.504 & 3.30 & 3.70 \\
\hline 4991.065 & 22.0 & 0.836 & 0.380 & 2.80 & 2.90 \\
\hline 4999.503 & 22.0 & 0.826 & 0.250 & 2.10 & 2.10 \\
\hline 3900.539 & 22.1 & 1.131 & -0.290 & 26.70 & 29.10 \\
\hline 3913.461 & 22.1 & 1.116 & -0.360 & 23.70 & 27.00 \\
\hline 4012.383 & 22.1 & 0.574 & -1.840 & 4.80 & 5.70 \\
\hline 4028.338 & 22.1 & 1.892 & -0.920 & 1.80 & 2.30 \\
\hline 4290.215 & 22.1 & 1.165 & -0.870 & 8.00 & 9.80 \\
\hline 4300.042 & 22.1 & 1.180 & -0.460 & 17.70 & 19.20 \\
\hline 4301.922 & 22.1 & 1.161 & -1.210 & 4.80 & 6.10 \\
\hline 4312.860 & 22.1 & 1.180 & -1.120 & 5.70 & 6.40 \\
\hline 4395.031 & 22.1 & 1.084 & -0.540 & 20.80 & 24.20 \\
\hline 4399.765 & 22.1 & 1.237 & -1.190 & 3.80 & 4.10 \\
\hline 4417.714 & 22.1 & 1.165 & -1.190 & 4.60 & 4.90 \\
\hline 4443.801 & 22.1 & 1.080 & -0.710 & 15.70 & 17.30 \\
\hline 4450.482 & 22.1 & 1.084 & -1.520 & 2.60 & 3.40 \\
\hline 4468.507 & 22.1 & 1.131 & -0.600 & 16.80 & 19.00 \\
\hline 4501.270 & 22.1 & 1.116 & -0.770 & 13.00 & 14.90 \\
\hline 4533.960 & 22.1 & 1.237 & -0.530 & 14.60 & 16.60 \\
\hline 4549.622 & 22.1 & 1.584 & -0.110 & 18.50 & 21.50 \\
\hline 4563.757 & 22.1 & 1.221 & -0.690 & 10.30 & 11.80 \\
\hline 4571.971 & 22.1 & 1.572 & -0.320 & 13.10 & 15.70 \\
\hline 4589.947 & 22.1 & 1.237 & -2.940 & 1.80 & 1.80 \\
\hline 4254.336 & 24.0 & 0.000 & -0.114 & 15.40 & 19.70 \\
\hline 4274.797 & 24.0 & 0.000 & -0.231 & 12.60 & 16.90 \\
\hline 4289.717 & 24.0 & 0.000 & -0.361 & 9.20 & 12.30 \\
\hline 5206.037 & 24.0 & 0.941 & 0.019 & 4.30 & 6.80 \\
\hline 5208.425 & 24.0 & 0.941 & 0.158 & 7.00 & 8.60 \\
\hline 4030.730 & 25.0 & 0.000 & -1.037 & 5.00 & 6.80 \\
\hline 4033.044 & 25.0 & 0.000 & -1.200 & 3.50 & 5.40 \\
\hline 4034.469 & 25.0 & 0.000 & -1.326 & 2.40 & 3.80 \\
\hline 3995.269 & 27.0 & 0.923 & -2.026 & 3.40 & 4.10 \\
\hline 4121.294 & 27.0 & 0.923 & -0.993 & 3.00 & 2.90 \\
\hline 4025.101 & 28.0 & 4.088 & -1.343 & 1.50 & 2.20 \\
\hline 4810.528 & 30.0 & 4.078 & -0.137 & 1.10 & 1.20 \\
\hline 4077.709 & 38.1 & 0.000 & 0.167 & 44.40 & 47.50 \\
\hline 4215.519 & 38.1 & 0.000 & -0.145 & 32.20 & 35.30 \\
\hline 4554.000 & 56.1 & 0.000 & -1.447 & 6.60 & 4.10 \\
\hline 4934.100 & 56.1 & 0.000 & -1.767 & 3.90 & 2.35 \\
\hline
\end{tabular}




\title{
Comparison of errors between a differential and a classical abundance analysis
}

\author{
Henrique Reggiani and Jorge Meléndez \\ Universidade de São Paulo - Instituto de Astronomia, Geofísica e Ciências \\ Atmosféricas \\ hreggiani@gmail.com
}

\begin{abstract}
The differential abundance analysis method can improve the precision of stellar chemical abundances. The method compares the equivalent widths of a certain line in a star with the same line in a star considered to be a standard representative of its class, using high resolution and high $\mathrm{S} / \mathrm{N}$ spectra. The method has achieved great results by reducing the measurements errors to unprecedent low levels. However, to date, there has not been a consistent analysis on the actual improvements of this method when compared to a classical analysis in metal-poor stars. Here we present a comparison between the errors of a classical stellar analysis and a differential analysis among low metallicity stars.
\end{abstract}

Keywords: Precision spectroscopy; Differential chemical analysis; Stellar abundances; Stellar parameters; Chemical evolution

\section{Introduction}

In the last few years different authors have used the differential abundance analysis to evaluate stellar evolution effects $[6,18]$, to recognize planet signatures in chemical abundances of stars $[3,10,17,1]$, to study the chemical evolution of the solar neighborhood [9,14], study abundance anomalies in stellar clusters [19] and distinct populations in the stellar halo $[8,11,2]$. Using high resolution and high $\mathrm{S} / \mathrm{N}$ spectra previous works have been able to achieve errors as low as 0.004 dex for solar twins [17] and 0.02 dex for metal-poor stars [14]. But it is, so far, unclear whether these low errors are entirely due to the analysis technique, and how much the spectral quality influences the precision achieved. Here we have consistently analyzed the errors of a classical and a differential analysis in two samples of metal-poor stars with different spectral quality.

\section{Samples and method of analysis}

We have analyzed two separate samples. The first sample is composed by the two stars previously described in [14] (Keck spectra with $\mathrm{R} \approx 95000$ and $\mathrm{S} / \mathrm{N}=400$ at $500 \mathrm{~nm}$ ). The second sample is composed by 11 stars observed with UVES/VLT 
$(\mathrm{R} \approx 50000$ and $\mathrm{S} / \mathrm{N}=180$ at $500 \mathrm{~nm})$. The UVES spectra were reduced through the ESO pipeline and further corrections (such as barycentric and radial velocity correction) were done using the IRAF package for python, pyraf, and the spectra were normalized via IRAF (using the same polynomial and polynomial orders when possible). In both samples we measured equivalent widths using the splot task in IRAF.

The line lists used for the analysis were constructed using the VALD ([15]) database along with new $\log (\mathrm{gf})$ values and hyperfine structure data from $\mathrm{Ku}$ rucz $^{1}$. We calculated the stellar parameters and chemical abundances in two manners. First we have performed a classical spectroscopic analysis of the data. We defined the stellar parameters through excitation equilibrium of the Fe I and Fe II lines to determine $\mathrm{T}_{e f f}$, obtained $\log \mathrm{g}$ through ionization equilibrium, and microturbulent velocity by allowing no trend in line abundances with reduced EW. This was performed using the q2 semi automated program [12], which uses the radiative transfer code MOOG [16] to calculate the best solution for the parameters and the errors for each parameter via statistical analysis. After that we calculate all the chemical abundances using q2 and, once again, the program calculates the errors using observational errors and the stellar parameters errors.

We also calculated these quantities using a line-by-line differential analysis, as described in $[5,19,13]$. The differential method compares the chemical abundance of each line in a given star, with the same line of a standard star. This way, error sources cancel out, in particular the $g f$ values. The absolute abundances can be written as: $\log \frac{W}{\lambda}=B+A_{x}+\log (g f)+\log (\lambda)-\theta_{X e x c}-\log k_{\text {cont }}$, and the differential abundances are: $\log \frac{W_{1, i}}{W_{2, i}}=A_{x, i}^{1}-A_{x, i}^{2}-\left(\theta^{1}-\theta^{2}\right)_{X e x c}-\log \frac{k_{\text {cont }}^{1}}{k_{\text {cont }}^{2}}$. We then compare these differential abundances of Fe I to calculate a differential excitation equilibrium and obtain a differential estimation of the $\mathrm{T}_{\text {eff }}$, a differential ionization equilibrium between Fe I and Fe II lines to obtain $\log g$, and microturbulence by allowing no trend between differential line abundances with reduced EW. Note that in order to calculate the differential parameters of any given star it is necessary to know the stellar parameters of the standard star beforehand with the best possible accuracy and precision.

In our work the standard star was selected due to the availability of stellar parameters from non-spectroscopic sources with low errors, $\mathrm{T}_{\text {eff }}$ from the IRFM [4], and $\log g$ from [7], who estimate the surface gravity using the absolute magnitude of the star from fundamental relation $\log \frac{g}{\log g_{\odot}}=\log \frac{M}{M_{\odot}}+$ $4 \log \frac{\mathrm{T}_{\text {eff }}}{\mathrm{T}_{\text {eff }, \odot}+0.4\left(\mathrm{M}_{\mathrm{bol}}-\mathrm{M}_{\mathrm{bol}, \odot}\right)}$, which have errors of $50 \mathrm{~K}$ and 0.15 dex respectively. Using the Fe I and Fe II lines of these stars as reference we performed a line by line differential analysis on the other stars and estimated their stellar parameters, abundances and their respective errors. The effective temperature, $\log g$, microturbulent velocity and metallicity of the remaining stars were calculated with the differential method, as explained above

Stellar parameters calculated strictly from spectroscopic methods are known to differ from photometric methods in metal-poor stars; usually spectroscopic

\footnotetext{
${ }^{1}$ http://kurucz.harvard.edu/linelists.html
} 
temperatures are $\approx 300 \mathrm{~K}$ lower than photometric ones. The effective temperature of star G64-12, for example, is $6168 \mathrm{~K}$ when estimated through excitation equilibrium and $6463 \mathrm{~K}$ from the IRFM[4], and the temperature of star G64-37 is $6258 \mathrm{~K}$ from classical excitation equilibrium. Interestingly, the temperature of this star is $6568 \mathrm{~K}$ from a differential equilibrium, using star G64-12 with IRFM temperature as the standard point of comparison. The surface gravity experiences more extreme differences, with relative differences up to $1 \mathrm{dex}$, between the classical ionization equilibrium to the $\log g$ from the absolute magnitude, and, thus it shows the same large departure when compared to the differential ionization with a standard star with $\log g$ from the absolute magnitude. The use of these different sets of stellar parameters (strictly spectroscopic vs differential analysis with a standard star with IRFM temperature and $\log g$ from absolute magnitude) will also translate into different abundances results, usually a difference on the order of 0.15 dex. As the stellar parameters and abundances results differ when using a classical and differential analysis, we will not focus on their values. We are only interested in the ratio between the errors of the classical analysis and the differential analysis.

All the abundance errors presented here include the observational errors (defined as $\frac{\sigma}{\sqrt{N}}$ ) added in quadrature to the quadrature of the errors due to the stellar parameters errors.

\section{Results and Discussion}

In Fig. 1a we show the $\mathrm{T}_{\text {eff }}$ error ratios for all the stars. In this figure the first star is from the Keck sample (G64-37). We do not compare errors of the standard stars of our samples because they come from different methods ( $\mathrm{T}_{\text {eff }}$ from the IFRM, and $\log g$ from the absolute magnitude).

There is a clear gain, specially for star G64-37 which is one of the Keck sample stars, which have the highest $\mathrm{S} / \mathrm{N}$ and resolution. The average gain on $\mathrm{T}_{\text {eff }}$ is 1.4 , the average gain for $\log \mathrm{g}$ was 1.3 and for microturbulence it was 1.6. In Figs. $1 \mathrm{~b}$ and $1 \mathrm{c}$ we show the gain on precision for the abundances (Fig $1 \mathrm{~b}$ shows the result for the Keck sample and Fig. 1c shows the average gain for each element throughout the entire UVES sample). As can be seen, the average gain for the Keck sample is twice the average gain for the UVES sample. We attribute this difference to the quality of the spectra. The better spectral quality will allow us to measure the EW more consistently between the two stars, which will make the comparison more precise, thus lowering the error bars. Overall it is clear that the differential analysis provides better errors when compared to a classical analysis.

We also compared the overall effect of the stellar parameters analysis in the final chemical abundance errors and concluded that there is no big benefit from a differential analysis if it is carried out with a classic spectroscopic calculation of the stellar parameters (average gain of 1.4 and 1.1 for the Keck and UVES samples respectively). 


\section{Conclusions}

With this simple, but consistent analysis, we were able to see that a differential analysis can increase the precision of our results by up to three times on average, depending on the quality of the spectra and measurements. We have also seen that the most important part of our analysis was the precise determination of the stellar parameters (the better constrained the stellar parameters the better our final abundance results).

\section{References}

1. K. Biazzo,R. Gratton, S. Desidera, et al., A\&A, A135, 583, 2015

2. C. K. Fishlock, D. Yong, et al., MNRAS, 57F, 2017

3. J. Meléndez, M. Asplund, B. Gustafsson, \& D. Yong, AJ, L66, 704, 2009

4. J. Meléndez, L. Casagrande, I. Ramírez, et al., A\&A, L3, 515, 2010

5. J. Meléndez, M. Bergemann, J.G. Cohen, et al., A\&A, A29, 543, 2012

6. T. Monroe, J. Meléndez, I. Ramírez, et al., AJ, L32, 774, 2013

7. P. E. Nissen, C. Akerman, M. Asplund, et al., A\&A, 319, 469, 2007

8. P.E. Nissen \& W. Schuster, A\&A, L10, 511, 2010

9. P. E. Nissen, A\&A, A52, 579, 2015

10. I. Ramírez, J. Meléndez \& M. Asplund, A\&A, L17, 508, 2009

11. I. Ramírez, J. Meléndez \& J. Chanamé, ApJ, 757, 164, 2012

12. I. Ramírez, J. Meléndez, J. Bean, et al., A\&A, A48, 572, 2014

13. I. Ramírez, S. Khanal \& P. Aleo, ApJ, 808, 13, 2015

14. H. Reggiani, J. Meléndez, et al., A\&A, A67, 586, 2016

15. T, Ryabchikova et al., 2015, PhyS, 90e4005R

16. C. A. Sneden, Ph.D. Thesis, Univ. Texas, 1973

17. M. Tucci Maia, J. Meléndez \& I. Ramírez, AJ, L25, 790, 2014

18. M. Tucci Maia, J. Meléndez, M. Castro, et al., A\&A, L10, 576, 2015

19. D. Yong, J. Meléndez, L. Grundah, et al., MNRAS, 434, 3542, 2013 


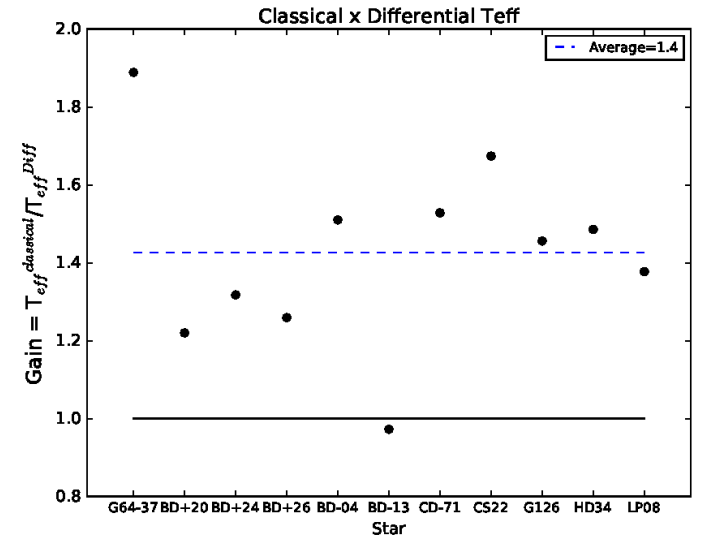

(a) Ratio between $\mathrm{T}_{\text {eff }}$ errors, classical spectrocopic analysis / differential analysis, of all the stars in both samples. G64-37 is the star from the Keck sample.

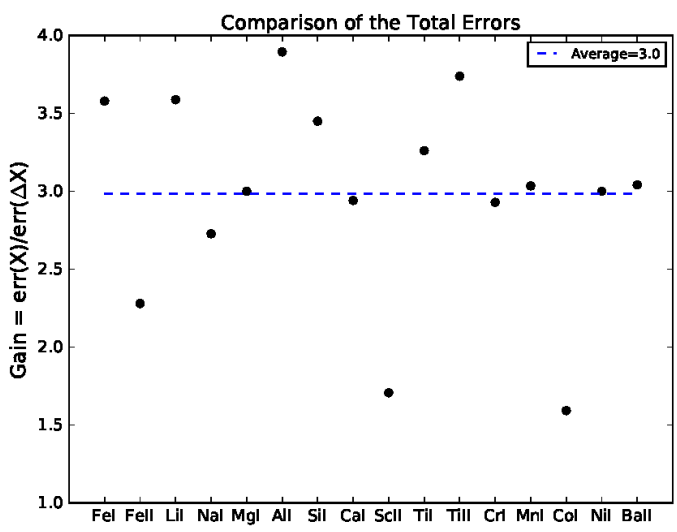

(b) Ratio between errors for chemical abundances (classical analysis/differential analysis) of the Keck sample.

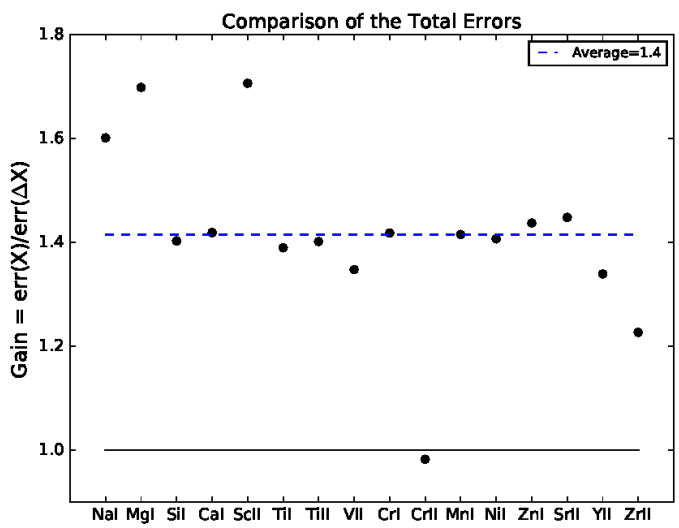

(c) Ratio between errors for chemical abundances (classical analysis/differential analysis) of the UVES sample.

Fig. 1. 


\title{
Constraining cosmic scatter in the Galactic halo through a differential analysis of metal-poor stars ${ }^{\star}$
}

\author{
Henrique Reggiani ${ }^{1,2}$, Jorge Meléndez ${ }^{1}$, Chiaki Kobayashi ${ }^{3}$, Amanda Karakas $^{4}$, and Vinicius Placco ${ }^{5}$ \\ 1 Universidade de São Paulo, Instituto de Astronomia, Geofísica e Ciências Atmosféricas, IAG, Departamento de Astronomia, \\ rua do Matão 1226, Cidade Universitária, 05508-900, SP, Brazil \\ e-mail: hreggiani@gmail.com \\ 2 Max-Planck Institute for Astronomy, Konigstuhl 17, 69117 Heidelberg, Germany \\ 3 Centre for Astrophysics Research, School of Physics, Astronomy and Mathematics, University of Hertfordshire, College Lane, \\ Hatfield AL10 9AB, UK \\ 4 Monash Centre for Astrophysics, School of Physics \& Astronomy, Monash University, Clayton VIC 3800, Australia \\ 5 Department of Physics, JINA Center for the Evolution of the Elements, University of Notre Dame, Notre Dame, IN 46556, USA
}

Received 8 March 2017 / Accepted 1 September 2017

\begin{abstract}
Context. The chemical abundances of metal-poor halo stars are important to understanding key aspects of Galactic formation and evolution.

Aims. We aim to constrain Galactic chemical evolution with precise chemical abundances of metal-poor stars $(-2.8 \leq[\mathrm{Fe} / \mathrm{H}] \leq-1.5)$. Methods. Using high resolution and high S/N UVES spectra of 23 stars and employing the differential analysis technique we estimated stellar parameters and obtained precise LTE chemical abundances.

Results. We present the abundances of $\mathrm{Li}, \mathrm{Na}, \mathrm{Mg}, \mathrm{Al}, \mathrm{Si}, \mathrm{Ca}, \mathrm{Sc}, \mathrm{Ti}, \mathrm{V}, \mathrm{Cr}, \mathrm{Mn}, \mathrm{Co}, \mathrm{Ni}, \mathrm{Zn}, \mathrm{Sr}, \mathrm{Y}, \mathrm{Zr}$, and Ba. The differential technique allowed us to obtain an unprecedented low level of scatter in our analysis, with standard deviations as low as 0.05 dex, and mean errors as low as 0.05 dex for $[\mathrm{X} / \mathrm{Fe}]$.

Conclusions. By expanding our metallicity range with precise abundances from other works, we were able to precisely constrain Galactic chemical evolution models in a wide metallicity range $(-3.6 \leq[\mathrm{Fe} / \mathrm{H}] \leq-0.4)$. The agreements and discrepancies found are key for further improvement of both models and observations. We also show that the LTE analysis of Cr II is a much more reliable source of abundance for chromium, as Cr I has important NLTE effects. These effects can be clearly seen when we compare the observed abundances of Cr I and Cr II with GCE models. While Cr I has a clear disagreement between model and observations, Cr II is very well modeled. We confirm tight increasing trends of Co and $\mathrm{Zn}$ toward lower metallicities, and a tight flat evolution of $\mathrm{Ni}$ relative to Fe. Our results strongly suggest inhomogeneous enrichment from hypernovae. Our precise stellar parameters results in a low star-to-star scatter $(0.04 \mathrm{dex})$ in the $\mathrm{Li}$ abundances of our sample, with a mean value about 0.4 dex lower than the prediction from standard Big Bang nucleosynthesis; we also study the relation between lithium depletion and stellar mass, but it is difficult to assess a correlation due to the limited mass range. We find two blue straggler stars, based on their very depleted $\mathrm{Li}$ abundances. One of them shows intriguing abundance anomalies, including a possible zinc enhancement, suggesting that zinc may have been also produced by a former AGB companion.
\end{abstract}

Key words. stars: abundances - stars: evolution - stars: Population II - Galaxy: abundances - Galaxy: evolution - Galaxy: halo

\section{Introduction}

The information imprinted in the chemical patterns of metalpoor $([\mathrm{Fe} / \mathrm{H}] \leq-1.0)$ stars hold one of the keys to understanding the formation and evolution of the Milky Way in its early stages (Eggen et al. 1962; Searle \& Zinn 1978). These objects arguably offer the most powerful insights into the evolution, nucleosynthetic yields, and properties of the first supernovae, they constrain the shape of the IMF, and provide clues to the rise of the $s$ - and $r$-processes in the Galaxy and the sites that produce them (Audouze \& Silk 1995; Ryan et al. 1996; Shigeyama \& Tsujimoto 1998; Chieffi \& Limongi 2002; Umeda \& Nomoto 2002; Meynet et al. 2010).

\footnotetext{
$\star$ Tables A.1-A.6 are also available at the CDS via anonymous ftp to cdsarc.u-strasbg.fr (130.79.128.5) or via

http://cdsarc.u-strasbg.fr/viz-bin/qcat?]/A+A/608/A46
}

Studies of metal-poor stars are usually focused on extremely metal-poor stars $([\mathrm{Fe} / \mathrm{H}] \leq-3.0-$ EMP) (e.g., Cayrel et al. 2004; Arnone et al. 2005; Cohen et al. 2008; Bonifacio et al. 2009; Hollek et al. 2011; Aoki et al. 2013; Yong et al. 2013; Jacobson et al. 2015), or in CEMP, carbon enhanced metal-poor, stars (e.g., Aoki et al. 2007; Spite et al. 2013; Keller et al. 2014; Placco et al. 2014, 2016a,b), which are the objects most likely to hold the keys to uncover details of the first generation of stars, the Pop III stars. There are also studies of the more metal-rich end of metal-poor stars (Nissen \& Schuster 2010; Schuster et al. 2012; Ramírez et al. 2012; Fishlock et al. 2017), focused on stars of metallicities $[\mathrm{Fe} / \mathrm{H}] \geq-1.5$, which provide evidence of extra-galactic stars in the Milky Way halo.

However, there is a gap in metallicities between $-2.5 \leq$ $[\mathrm{Fe} / \mathrm{H}] \leq-1.5$, where there are few comprehensive studies of accurate chemical abundances and as such there are significant gaps when comparing to models of Galactic chemical 
evolution (e.g., Chiappini et al. 1999, for a comparison using robust statistics; Cescutti 2008, for a stochastic model; Kobayashi \& Nakasato 2011, for a chemodynamical simulation). With this gap in precise abundances, model results are often compared to inhomogeneous works, obtained with different spectral resolutions and analysis methods causing large spreads in the $[\mathrm{X} / \mathrm{Fe}]$ ratios, and making it very difficult for models to be properly constrained.

In this metallicity range mixing in the interstellar medium (ISM) would not have been active long enough to make all observed scatter statistical, as is the case of metal-rich stars. Thus, an extensive spread in the data would indicate the presence of real cosmic scatter and/or inhomogeneous mixing in the ISM, which could be due to the presence of different populations (as found by Nissen \& Schuster 2010). For this reason, studies of these objects can also give us important insights into the accretion of extra-galactic stars by the Milky Way.

However, to uncover such details, we must obtain precisions at the level of 0.05 dex. In order to accomplish that, we make use of the differential technique. Recently, the differential technique in twin stars (meaning stars with similar stellar parameters), made it possible to considerably improve the precision achieved in spectroscopic studies. This was possible because many error sources, such as imprecise $\log (g f)$ values, largely cancel out, allowing a much better precision in the determination of relative stellar parameters and abundances. Studies with this technique have been used to recognize planet signatures on the chemical composition of stars (Meléndez et al. 2009; Ramírez et al. 2009; Tucci Maia et al. 2014; Biazzo et al. 2015), stellar evolution effects (Monroe et al. 2013; Tucci Maia et al. 2015; Carlos et al. 2016), chemical evolution as a function of age in the solar neighborhood (Nissen 2015; Spina et al. 2016), chemical abundance anomalies in globular clusters (Yong et al. 2013) and open clusters (Önehag et al. 2014; Liu et al. 2016b,a), distinct populations in the metal-rich halo (Nissen \& Schuster 2010) and distinct chemical abundances in EMP stars by Reggiani et al. (2016). O'Malley et al. (2017) has also employed a differential analysis for an exploratory work on main sequence $-2.7 \leq[\mathrm{Fe} / \mathrm{H}]-1.4$ stars. The abundance analysis they performed, however, is based on spectra of lower quality than in the present work, acquired using different instrumentation, and only a few elements were explored.

In this context we present a LTE differential study of the chemical abundances of 18 elements ( $\mathrm{Li}, \mathrm{Na}, \mathrm{Mg}, \mathrm{Al}, \mathrm{Si}, \mathrm{Ca}$, Sc, Ti, V, Cr, Mn, Co, Ni, Zn, Sr, Y, Zr and Ba), in 23 metal-poor stars, and compare the results with a chemical evolution model, which we describe in Sect. 4.

The paper is divided as follows: in Sect. 2 we describe observations and data reduction, we detail the stellar parameters in Sect. 3, comparing our parameters to other works in Sect. 4. Chemical abundances and results are shown in Sect. 5, and lithium is studied in Sect. 6. The pair of blue straggler stars are discussed in Sect. 7 and conclusions are presented in Sect. 8.

\section{Observations and data reduction}

\subsection{Sample selection and observations}

All stars observed in this work were selected due to a proximity in their stellar parameters that allowed us to obtain precise abundances through the differential technique. Using the updated catalog of stellar parameters of Ramírez \& Meléndez (2005), we selected 26 stars with previous assessments of stellar parameters within: $T_{\text {eff }}=6250 \pm 250 \mathrm{~K}, \log g=4.0 \pm 0.5$ dex and metallicities $-2.8 \leq[\mathrm{Fe} / \mathrm{H}] \leq-1.5$, and brighter than $V=12$, which assured that we were able to observe all stars in a reasonable time (up to $2 \mathrm{~h}$ of exposure time) and $S / N \sim 150-250$.

The observational data were obtained with the UVES spectrograph (Dekker et al. 2000) at the $8.2 \mathrm{~m}$ VLT telescope, during 2015A (project 095.D-0504(A)). All the spectra were taken with the same instrumental configuration, which guarantees similar spectra quality and improves the precision in a line-by-line differential analysis. The blue side of the spectra has an effective range from $3300 \AA$ to $4500 \AA$, and the red side of the spectra has a range of 4800-6800 $\AA$. We used a $0.8^{\prime \prime}$ slit on both arms of the spectrograph, with a final resolution of $R \approx 50000$ per pixel in both the blue arm and red arms. The average $\mathrm{S} / \mathrm{N}$ of the sample is: $S / N \approx 130$ at $4000 \AA$ and $S / N \approx 250$ at $6000 \AA$.

Of the original 26 observed stars, we removed three from the final analysis. Two of them were too metal-rich, and one star has a very high rotation. All three were excluded from the analysis for not being compatible with the remainder of the sample.

\subsection{Data reduction}

The bias and flat field corrections, order extraction and wavelength calibration, were performed by the UVES-Echelle pipeline. Barycentric and radial velocity corrections were performed automatically via the IRAF package for python (pyraf) and the spectra normalization were performed manually for each spectra via IRAF. After the normalization process the spectra of each star were combined for the abundance analysis.

\section{Stellar parameters}

We have performed manual EW measurements, via the splot task in IRAF using Gaussian profile fitting, for our entire sample, measuring a given line one at a time in all stars, which assures that the continuum placement of a given line is the same for all the stars, reducing the final abundance errors. We employed the semi-automatic q2 ${ }^{1}$ code (Ramírez et al. 2014), with MARCS plane-parallel 1D model atmospheres (Gustafsson et al. 2008) and the 2014 version of the LTE analysis code MOOG (Sneden 1973).

The $\log (g f)$ values and energy levels of our linelist are from the Vienna Atomic Line Database (VALD). The Fe I lines were updated using data from Den Hartog et al. (2014) and transition probabilities for the Fe II lines are from Meléndez \& Barbuy (2009). The Ti II values were updated using Lawler et al. (2013). Nevertheless, we note that the choice of $\log (g f)$ values is inconsequential in a differential analysis.

We started by performing an absolute spectroscopic measurement of the stellar parameters. Using excitation equilibrium for determining $T_{\text {eff }}$, ionization equilibrium for $\log g$, allowing no trend of Fe I line abundances with respect to the reduced EW gave us the microturbulence $\left(v_{\mathrm{T}}\right)$, and using the measured $\mathrm{EW}$, we obtained the initial $[\mathrm{Fe} / \mathrm{H}]$ for all stars.

Analyzing the preliminary spectroscopic results we chose stars HD 338529 and CD-48 2445 as our reference objects because the stellar parameters are in between the initial guess for the parameters of our other targets. We have chosen two different standard stars because the range in metallicity of our complete sample is too large. Thus, we separated the sample into two, with $-2.1 \leq[\mathrm{Fe} / \mathrm{H}] \leq-2.7$ and $-2.1 \leq[\mathrm{Fe} / \mathrm{H}] \leq-1.4$.

https://github.com/astroChasqui/q2 
hd122196 : 6052, 3.66, -1.855, 1.44 [cd-4802445]
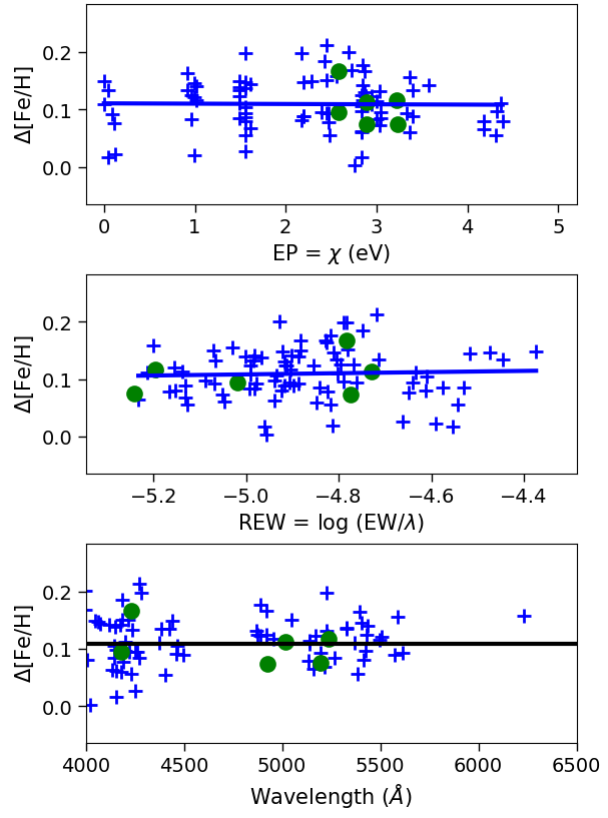

Fig. 1. Differential abundances in HD 122196 versus lower excitation potential (top panel), reduced equivalent widths (middle panel) and wavelength (lower panel). The blue crosses represent the differential Fe I abundances, and the green circles are the differential Fe II abundances.

We opted to use as our initial stellar parameters of HD 338529: $T_{\text {eff }}=6426 \pm 50 \mathrm{~K}$ from the infrared flux method (IRFM, Meléndez et al. 2010), $\log g=4.09 \pm 0.03$ dex from the GAIA parallax and, using our EW, we obtained $[\mathrm{Fe} / \mathrm{H}]=-2.29$ and $v_{\mathrm{T}}=1.5 \mathrm{~km} \mathrm{~s}^{-1}$. The initial stellar parameters of CD-48 2445 are: $T_{\text {eff }}=6453 \pm 50 \mathrm{~K}$ from the IRFM (Meléndez et al. 2010), $\log g=4.23 \pm 0.03$ dex from the GAIA parallax and, using our EW, we obtained $[\mathrm{Fe} / \mathrm{H}]=-1.96$ and $v_{\mathrm{T}}=1.5 \mathrm{~km} \mathrm{~s}^{-1}$.

Then, we employed a strictly line-by-line differential approach (e.g., Reggiani et al. 2016; Meléndez et al. 2012; Yong et al. 2013; Ramírez et al. 2015) to obtain the stellar parameters for the remaining targets. Using as reference the Fe I and Fe II abundances from HD 338529 and CD-48 2445, we determined $T_{\text {eff }}$ through differential excitation equilibrium (e.g., Fig. 1). The $T_{\text {eff }}$ have an overall good agreement with the IRFM values from Meléndez et al. (2010), when available. We obtained the $\log g$ through differential ionization equilibrium, and $v_{t}$ by allowing no trend in the differential Fe I line abundances with reduced EW (e.g., Fig. 1), and found $[\mathrm{Fe} / \mathrm{H}]$ with our line measurements. The errors for the atmospheric parameters include the degeneracy of the parameters and were determined strictly through a differential approach. The adopted stellar parameters, including errors, are provided in Table A.1.

\section{Comparison with other works}

In order to check the consistency of the adopted atmospheric parameters, we have compared them to three different studies. The first is Sitnova et al. (2015) with five stars in common, the second is Bensby et al. (2014) with five stars in common, and Meléndez et al. (2010) with nine stars in common. We have calculated the difference from the atmospheric parameters $\left(T_{\text {eff }}\right.$ and $\log g$ ) of each of the works cited above and our study, and calculated the median (less sensitive to the presence of outliers) of the absolute difference: $\Delta T_{\text {median }}=66 \mathrm{~K}$, and $\Delta \log g_{\text {median }}=0.18$.
For the median discrepancy in temperature between our $T_{\text {eff }}$ and those of the above references we find $\Delta T_{\text {median }}=66 \mathrm{~K}$. This shows that the temperature is within a reasonable agreement between the works cited above, as the median is very similar to our calculated measurement errors and always smaller than the combination between our errors and the errors of the other works.

The median discrepancy in surface gravity is $\Delta \log g_{\text {median }}=$ $0.18 \mathrm{dex}$, which is at the upper limit of our uncertainties, but within the combined error bars of our errors and those from the literature. We note that there are differences both in the methods and data used to estimate this parameter. For example, while we used the more precise GAIA parallaxes for our standard stars, previous works used the more uncertain HIPPARCos parallaxes. For the other stars in our sample we determined $\log g$ with our differential spectroscopic approach, relative to our two standard stars, which are representative of our metal-poor sample. In this regard, we remark that other works use the Sun as a standard star, which might not be the best choice due to the large difference in stellar parameters between the Sun and such metal-deficient stars.

Sitnova et al. (2015) determined $\log g$ by using as a first guess the $\log g$ from HIPPARCos parallaxes and adjusting them to obtain ionization balance, and Bensby et al. (2014) by applying a correction to $\log g$ (from ionization balance) after a comparison between the different methods they used (which included parallaxes measurements from HIPPARCos). Meléndez et al. (2010) determined $T_{\text {eff }}$ using the IRFM, while the surface gravities are from a compilation of literature values.

The largest discrepancies are between our results and the recent work of Sitnova et al. (2015). In their work they have calculated the effective temperatures using different IRFM data sources and performed corrections of up to $50 \mathrm{~K}$ to obtain the ionization balance of $\mathrm{Fe}$ I and Fe II NLTE measurements and remove the slope in $[\mathrm{Fe} / \mathrm{H}]$ vs. $E_{\text {exc }}$ (excitation energies). They also employed a differential approach to calculate the parameters of their non benchmark stars, using the Sun as a standard point of comparison.

\section{Chemical abundances}

All the chemical abundances presented here are the result of a line-by-line differential analysis. The solar abundances used in this work are all from Asplund et al. (2009). We present the abundances in Figs. 2 to 6, and in Tables A.2 to A.5.

In Figs. 2 to 5 we plot the K15 model (Sneden et al. 2016; Zhao et al. 2016), which is the updated Galactic chemical evolution (GCE) of Kobayashi et al. (2011, hereafter K11). We note that Kroupa IMF is applied in K15 and K11 models, while the Salpeter IMF is applied in Kobayashi et al. (2006). These models give almost the same results, except for C, N, Sc, Ti, V, Co, and $\mathrm{Zn}$ (see Sect. 5.1 for more details).

Two stars with extremely low lithium abundances, which could be blue stragglers, are indicated in the figures. Their chemical abundances have not been used to calculate the linear regression slopes of our data, the standard deviations and the mean errors presented in the figures. Although we do not use these values it is important to stress that the errors from their measurement do not increase the mean errors of the remaining sample, as they are similar to the errors of the rest of the sample.

We also added to the figures, when available, data from other precise works on unevolved stars, in order to compare observations and the galactic evolution model in a wider metallicity range $(-3.6 \leq[\mathrm{Fe} / \mathrm{H}] \leq-0.4)$. For EMP stars we add the work from Andrievsky et al. (2007, 2008), Bonifacio et al. (2009), 

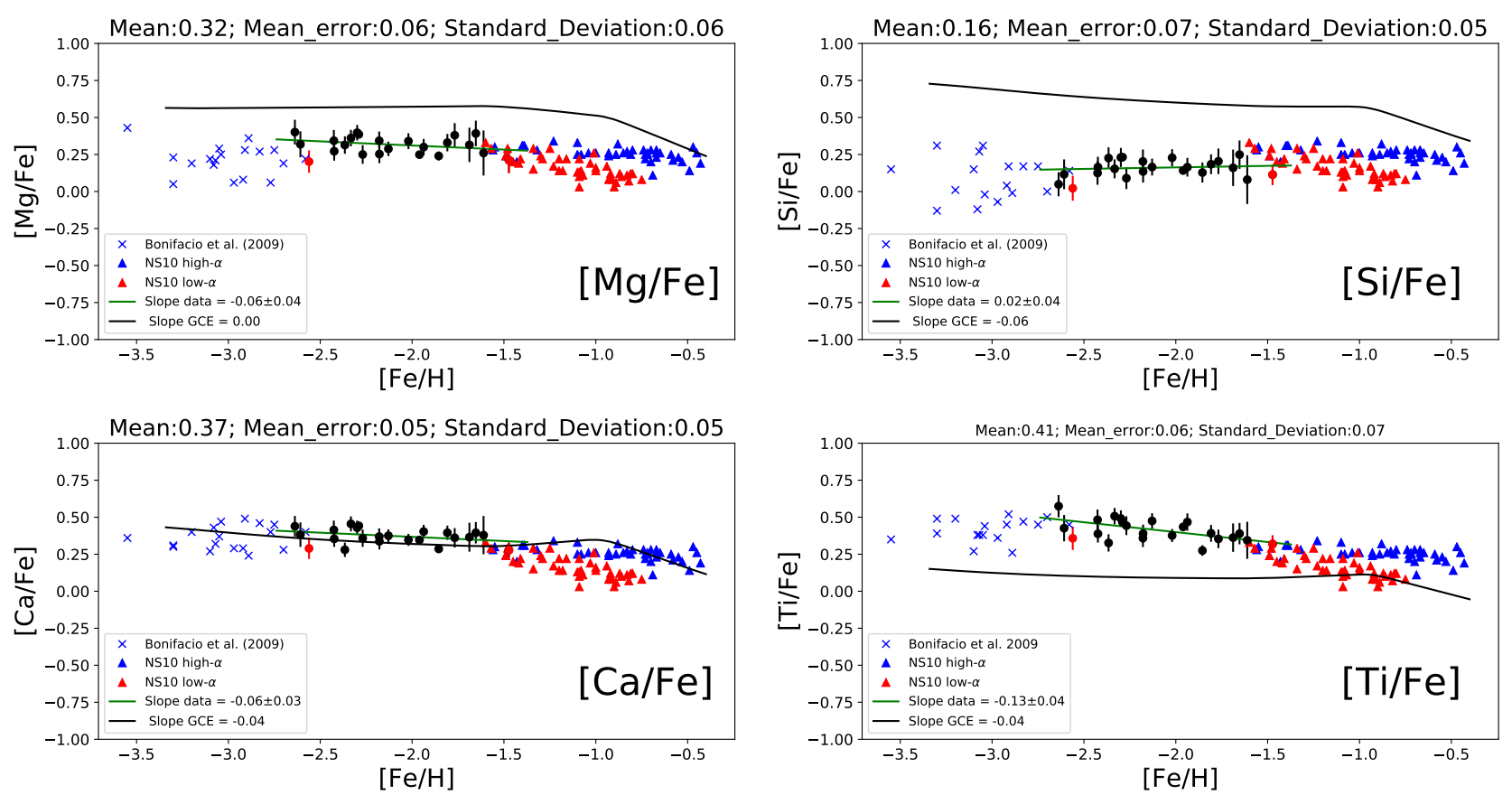

Fig. 2. $[\mathrm{X} / \mathrm{Fe}]$ abundances for $\alpha$-elements $(\mathrm{Mg}, \mathrm{Si}, \mathrm{Ca}$ and $\mathrm{Ti})$. The green line represent the best linear fit to the data and the slope is shown in the plots. The black line is the GCE prediction. The mean differential, our errors and the standard star-to-star scatter (standard deviation) for each element (for our measurements) are shown in top of each panel. The blue crosses are data of EMP stars from Bonifacio et al. (2009), the blue and red triangles are metal-poor stars from Nissen \& Schuster (2010), the black filled circles are the data measured in this work and the red filled circles are the blue straggler stars from this sample.

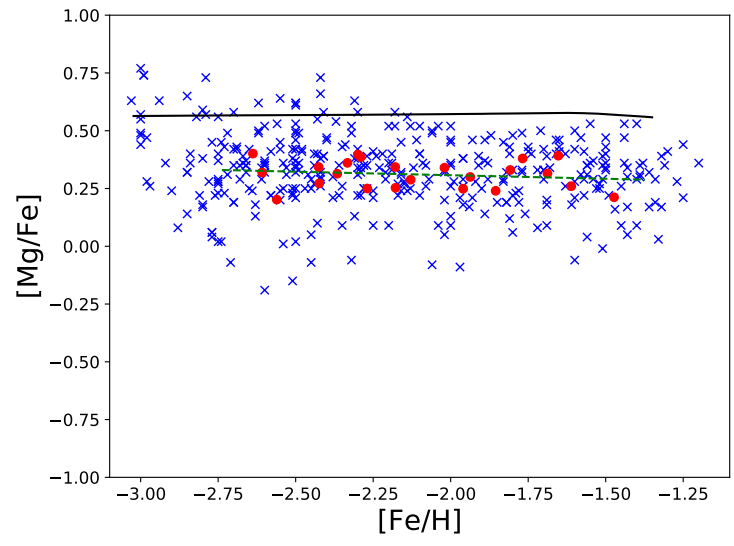

Fig. 3. $[\mathrm{Mg} / \mathrm{Fe}]$ abundances from the SAGA database. Notice the big data dispersion that arises from different analysis methods and samples (blue crosses). The stars selected from the SAGA database have $T_{\text {eff }}=$ $6250 \pm 250$ and $\log g=4.0 \pm 0.5$, as in our sample. For comparison our more precise results are shown by red circles. The black line is the GCE model prediction.

from the First Stars large program turn-off objects, and for the more metal rich end we add data from Nissen \& Schuster (2010, 2011) and Fishlock et al. (2017). We present these latter data in the figures (high- $\alpha$ and low- $\alpha$, as defined in Nissen \& Schuster 2010). We also emphasize that the above studies are based on dwarf stars of comparable atmospheric parameters as our data set.

It is important to stress that the mean values, mean errors, standard deviations, and data slopes are calculated using only our data set. The slopes calculated for the GCE model are the slopes for the $-2.7 \leq[\mathrm{Fe} / \mathrm{H}] \leq-1.5$ region, which is the metallicity covered by our data set.
As mentioned in Sect. 3 we have separated our sample in two. We have scaled the differential abundances of Sample 2, as designated in Table A.1, based on the absolute abundances of the standard star of Sample 2, while the differential abundances of Sample 1 were scaled using the standard star of Sample 1.

\subsection{Light even-Z metals}

The abundances of $\alpha$-elements can be seen in Fig. 2 and Table A.2.

\subsubsection{Magnesium}

Abundances of magnesium were determined based on $8 \mathrm{Mg}$ I lines, and for each star we used only lines with $E W \geq 10 \mathrm{~m} \AA$. As can be seen in Fig. 2 the star-to-star standard deviation of our differential $[\mathrm{Mg} / \mathrm{Fe}]$ measurements is $0.06 \mathrm{dex}$, which is the same as the average error found. The small negative slope of $-0.06 \pm 0.04$ in our results, is in good agreement with the GCE prediction of a flat slope in the observed metallicity region. Previous comparisons with model data, such as the comparison in Kobayashi et al. (2006), are unable to constrain the behavior due to the large scatter in the data. However, the comparison data used in the aforementioned work is based on data from different authors, using different analyses and data sets, resulting in a large dispersion, which means it was not possible to constraint their results based on data with deviations as small as presented in this work.

To exemplify the data dispersion, we have gathered $[\mathrm{Mg} / \mathrm{Fe}]$ data from the SAGA database (Suda et al. 2008, 2011; Yamada et al. 2013) with the following search parameters: $6000 \leq T_{\text {eff }} \leq 6500,3.5 \leq \log g \leq 4.5$ and $-2.8 \leq[\mathrm{Fe} / \mathrm{H}] \leq$ -1.5 , to mimic the coverage in stellar parameters of our sample. We plotted all the data returned, even the same object with 
H. Reggiani et al.: Constraining cosmic scatter in the Galactic halo through a differential analysis of metal-poor stars
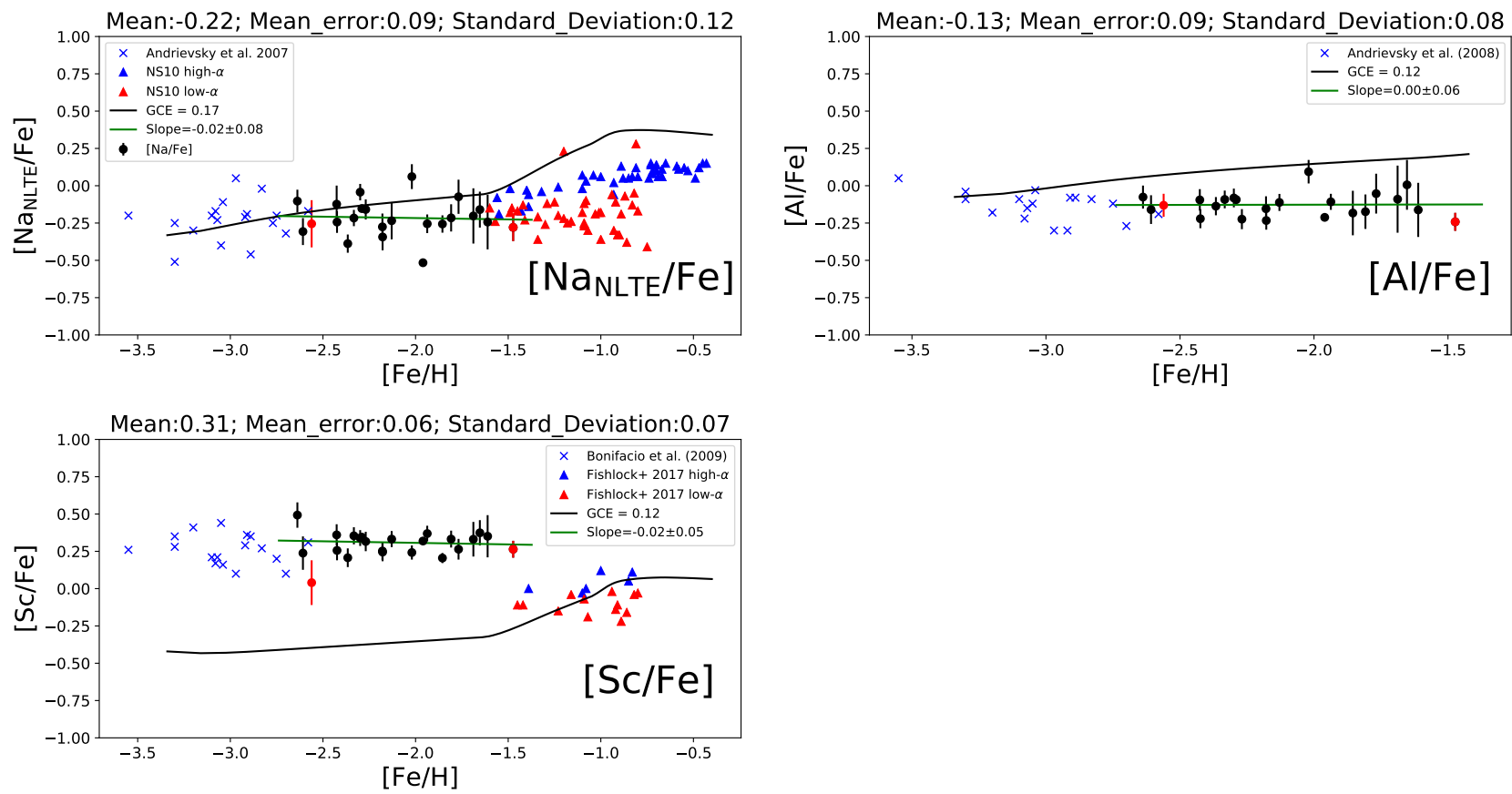

Fig. 4. Same as Fig. 2 for light odd $\mathrm{Z}$ elements (Na, $\mathrm{Al}$ and $\mathrm{Sc}$ ). The data for EMP stars (blue crosses) for $\mathrm{Na}$, $\mathrm{Al}$, and $\mathrm{Sc}$ come from Andrievsky et al. (2007, 2008), Bonifacio et al. (2009) respectively, the data for the metal rich end for Na, and Sc (triangles) are from Nissen \& Schuster (2010), Fishlock et al. (2017) respectively, the black filled circles are the data measured in this work and the red filled circles the two blue straggler stars from this sample.

several measurements, with a total of 364 data points, which can be seen in Fig. 3. Data points from the SAGA database and the stars from our sample are both plotted. We also show the linear fit to our data and the galactic chemical evolution model prediction. As can be seen, the SAGA data agrees with the model predictions within measurement errors, in contrast to our abundance ratios which are not compatible with the GCE model. This happens because the data spread in SAGA is about 1 dex, ranging from $\approx-0.2$ to $\approx+0.8$ dex. With such a high dispersion, it is not possible to precisely constrain model results, as anything within that large range can be fitted.

The deviation between model and observations for $[\mathrm{Mg} / \mathrm{Fe}]$ also extends to the works of Bonifacio et al. (2009) and Nissen \& Schuster (2010), which can be seen in Fig. 2. As in our case, their sample of unevolved stars are very homogeneous. The bigger dispersion in the Bonifacio et al. (2009) results probably arise from the fact that at lower metallicities the ISM may have been more inhomogeneous. It is also interesting to see how the two populations found by Nissen \& Schuster (2010) merge into one at the metal-poor end of their sample and continue without any discernible distinction from our sample toward lower metallicities. These data are important to verify the galactic chemical evolution model in a wider metallicity range. We notice that the GCE model starts to match the observational $[\mathrm{Mg} / \mathrm{Fe}]$ data only at the metal-rich end.

The mean absolute value we found for $\mathrm{Mg}$ is $[\mathrm{Mg} / \mathrm{Fe}]=$ $+0.32 \pm 0.06 \mathrm{dex}$, which is within the expected values for the metallicity range and our low scatter is a very good improvement over what has been previously reported. Bonifacio et al. (2009) found a mean value of $[\mathrm{Mg} / \mathrm{Fe}]=+0.21$ dex with a standard deviations of 0.10 dex. This observed difference is likely due to the different samples, analyses and errors. The difference from our work and the study of giant EMP stars by Cayrel et al. (2004) is smaller. They found a mean $[\mathrm{Mg} / \mathrm{Fe}] \approx+0.27 \pm 0.10$ dex. But, as mentioned by Bonifacio et al. (2009), part of this discrepancy may be due to problems in line measurements of Cayrel et al. (2004), also described in Sect. 3.3 of Andrievsky et al. (2010).

Our mean $[\mathrm{Mg} / \mathrm{Fe}]=0.32$ dex is also in agreement with the mean $[\mathrm{Mg} / \mathrm{Fe}]=0.29 \pm 0.07$ dex found by Zhao et al. (2016) in their NLTE analysis (in the same metallicity region). The departures from NLTE are small for Mg (Zhao et al. 2016), hence the very good agreement with our results. They also found an offset between their NLTE abundances and the K15 GCE model of $\sim 0.25$ dex, which is the same as the mean discrepancy of our data $(0.24$ dex $)$.

We note that Zhao et al. (2016) showed that $[\mathrm{O} / \mathrm{Fe}]$ is consistent with the $\mathrm{K} 15 \mathrm{GCE}$ model at $[\mathrm{Fe} / \mathrm{H}] \sim-1$. This means that the model $[\mathrm{O} / \mathrm{Mg}]$ is inconsistent with these observations, which is not a problem of galaxy evolution but of nucleosynthesis yields. There is an uncertainty in $\mathrm{C}(\alpha, \gamma) \mathrm{O}$ reaction, and the observations of stellar abundances suggest that the rate adopted in Kobayashi et al. (2006) yields (1.3 times the value given in Caughlan \& Fowler 1988) is not correct. To constrain the rate, it is necessary to use 3D and NLTE analysis for both $\mathrm{Mg}$ and $\mathrm{O}$.

\subsubsection{Silicon}

Our silicon abundances are based on only one measured line (3905.523 $\AA$ ), which is blended with $\mathrm{CH}$, but is the only line that can be detected in all of our stars. Silicon has a mean error of 0.07 dex that is higher than the star-to-star standard deviation of 0.05 . The calculated $0.02 \pm 0.04$ slope in our data is flatter when compared to the model prediction of -0.06 slope. The galactic chemical evolution model has a plateau at about $0.6 \mathrm{dex}$ and there is a large offset between the data and the model of $\approx 0.2$ dex. The mean abundance we found, $[\mathrm{Si} / \mathrm{Fe}]=+0.16$, is in agreement with the data from both (Bonifacio et al. 2009, $0.09 \mathrm{dex}$ ) and (Nissen \& Schuster 2010, $0.25 \mathrm{dex}$ ), thus our work connects the low and high-metallicity studies. 

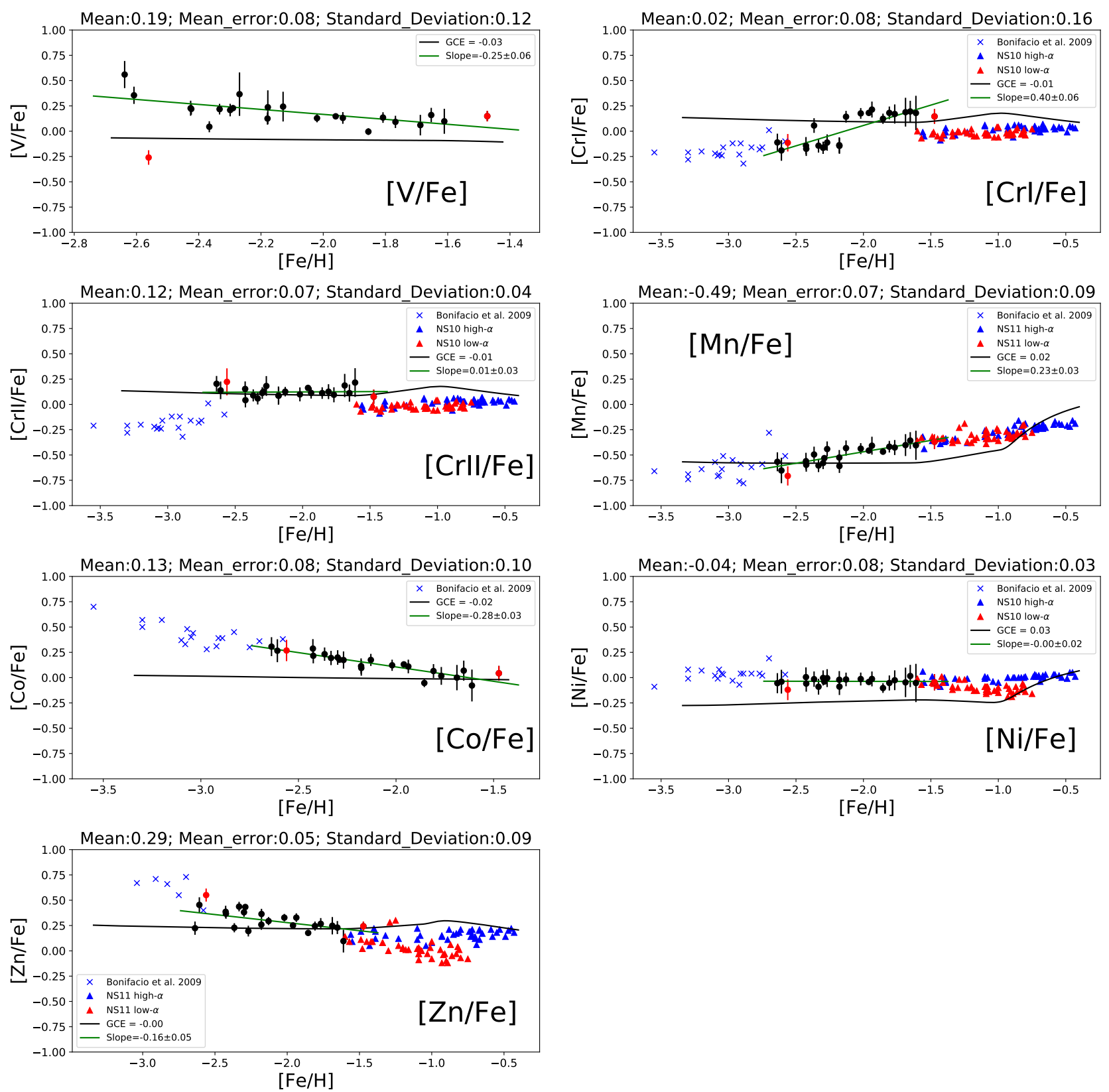

Fig. 5. Same as Fig. 2 for iron peak elements (V, Cr, Mn, Co, Ni and Zn). The blue crosses are data from Bonifacio et al. (2009), the red and blue triangles are the Nissen \& Schuster $(2010,2011)$ measurements, the black filled circles are the data measured in this work and the red filled circles the two blue straggler stars from this sample.

The discrepancy between model and observations seen in this work are also observed in Zhao et al. (2016). Their mean NLTE abundances for the region $-2.6 \leq[\mathrm{Fe} / \mathrm{H}] \leq-1.4$ is +0.32 dex, which still has a considerable difference from the mean $\approx 0.59$ dex given by the model in the same region. Thus, although there are important NLTE corrections to be made in the silicon abundances, the difference between model and observations are not reconciled by the more accurate abundance estimate provided by the NLTE calculations.

\subsubsection{Calcium}

We measured $\mathrm{Ca}$ abundances from $\mathrm{Ca}$ I lines. We only considered lines with $E W \geq 10 \mathrm{m \AA}$. Calcium is one of the best fits between all the data sets and GCE model predictions. As can be seen in Fig. 2 the agreement between data and predictions is impressive. It is also remarkable that the calcium slope is the same as magnesium. The GCE model agrees with almost all of our data within the error bars $(0.05$ dex $)$, which are considerably lower than previous works; notice also that our error is the same as the star-to-star standard deviation of our sample. On the more metal-rich end, we see that the high- $\alpha$ population is in better agreement with the model predictions, which support the conclusion by Nissen \& Schuster (2010) that the low$\alpha$ stars might have originated in a different environment (dwarf spheroidal galaxies). At the metal-poor end we see that the data from Bonifacio et al. (2009) agrees well with the model predictions, but once again has a higher dispersion, which is not uncommon to EMP stars.

The slope we found from our data set is the same as that found for $\mathrm{Mg}$, which is produced via the same mechanism as $\mathrm{Ca}$, but the $\mathrm{Ca}$ slope has a better agreement between data and model 
H. Reggiani et al.: Constraining cosmic scatter in the Galactic halo through a differential analysis of metal-poor stars
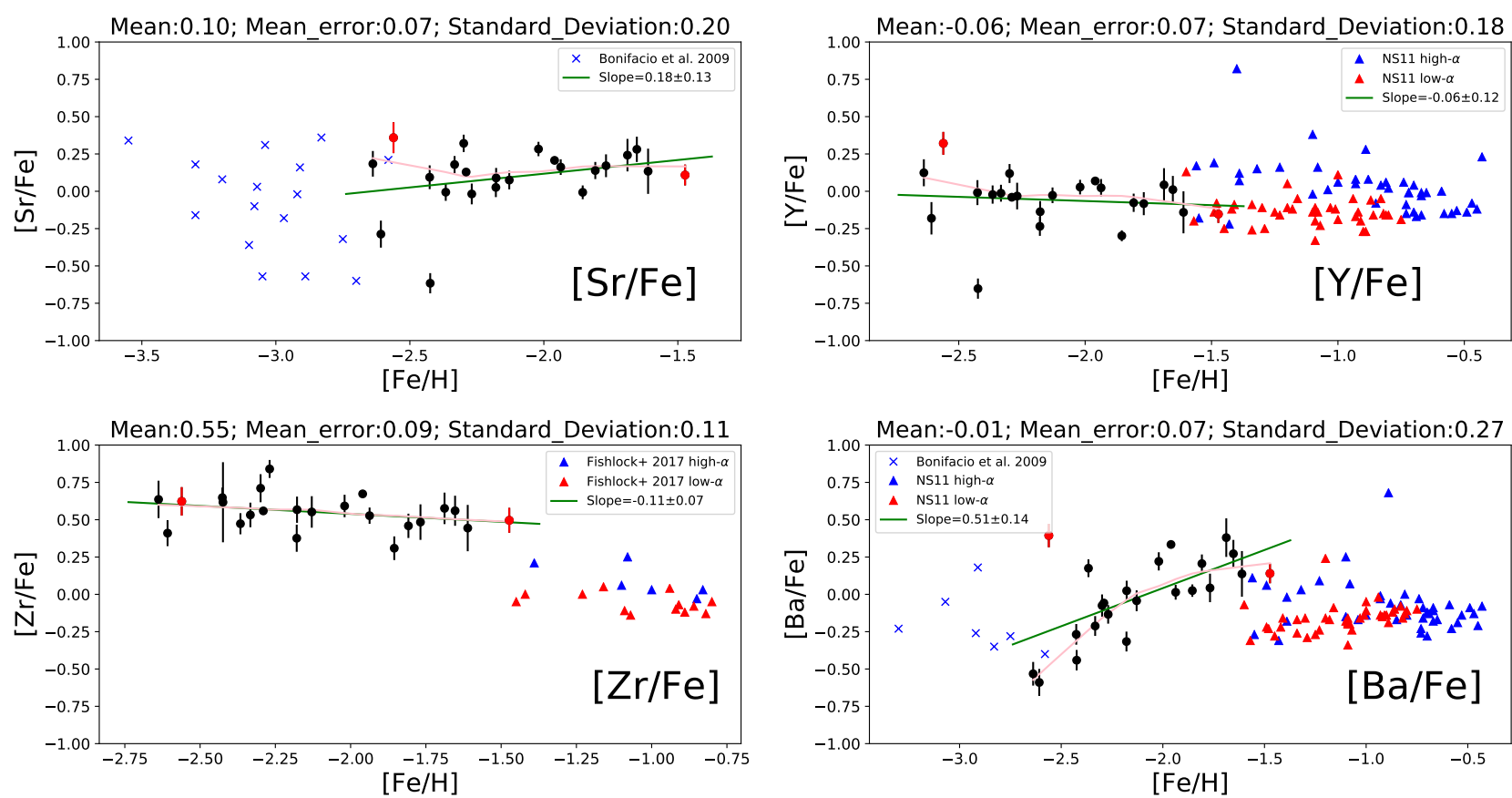

Fig. 6. Same as Fig. 2 for neutron-capture elements (Sr, Y, Zr and Ba). For Sr and Ba we show the Bonifacio et al. (2009) data for EMP stars as blue crosses, the blue and red triangles on Y, Ba and $\mathrm{Zr}$ are from Nissen \& Schuster (2011), Fishlock et al. (2017) respectively, the black filled circles are the data measured in this work and the red filled circles the two blue straggler stars from this sample.

prediction. Our mean $[\mathrm{Ca} / \mathrm{Fe}]=0.37$ dex agrees very well with Kobayashi et al. (2006), which predicts a plateau of $[\mathrm{Ca} / \mathrm{Fe}] \approx$ $0.27-0.39$ for the metallicity range $-3 \leq[\mathrm{Fe} / \mathrm{H}] \leq-1$, which also matches the mean value from Arnone et al. (2005) $([\mathrm{Ca} / \mathrm{Fe}]=+0.37)$. As seen before (Bonifacio et al. 2009), we find a small difference between our work with dwarfs and the study with giants of Cayrel et al. (2004), that found a somewhat lower value in their sample. Zhao et al. (2016) reported a mean $[\mathrm{Ca} / \mathrm{Fe}]=0.32 \pm 0.08$, that also agrees with our own results and the K15 model.

\subsubsection{Titanium}

There were approximately 50 lines of titanium measured, including Ti I and Ti II. The differential results for both species are very homogeneous (the mean values of $\mathrm{Ti}$ I and $\mathrm{Ti}$ II differ in 0.04 dex only). Our Ti data has a slope of $-0.13 \pm 0.04$ and the galactic evolution model predicts a slope of -0.04 . There is also an offset of $\approx 0.4$ dex between model and our data. Even larger discrepancies had already been seen in Kobayashi et al. (2006), and the effects of jet-like explosions to enhance Ti, first proposed by Maeda \& Nomoto (2003), has been applied in the K15 GCE model plotted here. But these effects are not enough to remove the discrepancy with the observed data.

The mean value (averaging $\mathrm{Ti} \mathrm{I}$ and $\mathrm{Ti}$ II) is $[\mathrm{Ti} / \mathrm{Fe}]=$ $0.41 \mathrm{dex}$. The overall behavior of $\mathrm{Ti}$, also considering the data sets from Bonifacio et al. (2009), Nissen \& Schuster (2010), is a decrease in $[\mathrm{Ti} / \mathrm{Fe}]$ with an increase in metallicity. At the more metal-rich end the model seems to agree with the low- $\alpha$ population, which is in contrast with what is seen in $[\mathrm{Ca} / \mathrm{Fe}]$. If we are to interpret the low- $\alpha$ as a population from another environment, we should expect that $[\mathrm{Ti} / \mathrm{Fe}]$ to be in agreement with the high- $\alpha$ population, born in the Milky Way. Thus, the model and observations of Ti also do not match at the more metal-rich end, although the discrepancy is smaller.
The discrepancy between observations and the K15 model also extends to the NLTE analysis of Zhao et al. (2016), although they found a somewhat smaller abundance $[\mathrm{Ti} / \mathrm{Fe}]=0.30 \pm$ 0.05 , decreasing the discrepancy with the model. We emphasize that, within the errors, the results of Zhao et al. (2016) are compatible with ours.

The results presented in Fig. 2 do not indicate the presence of any extra-galactic objects or different populations other than regular Milky Way metal-poor stars in our sample of very metalpoor objects. However, it is important to stress that the star-tostar scatter in our abundances is similar to the abundance errors, thus, to fully discard the presence of separate populations, it is necessary to obtain better data, with higher $\mathrm{S} / \mathrm{N}$ and spectral resolution, which will improve the errors to a level below the current observed star-to-star scatter, hence bringing tighter constraints on the true cosmic scatter in metal-poor stars. The comparison between GCE model and observations show that there still is a discrepancy of $\sim 0.3 \mathrm{dex}$ in $\mathrm{Mg}, \mathrm{Si}$, and $\mathrm{Ti}$ predictions, but $\mathrm{Ca}$ nucleosynthesis seems to be very well defined in the K15 model, matching the observations from EMP to almost solar metallicity stars.

\subsection{Light odd-Z metals}

All the odd-Z light elements results can be seen in Fig. 4 and Table A.3.

\subsubsection{Sodium}

The LTE differential results have the largest star-to-star standard deviation among the light elements (0.17 dex) and a mean error of 0.09 dex with a slope of $+0.35 \pm 0.09$, much higher than the 0.17 predicted by the GCE model. Such high star-to-star standard deviation and steep slope had already been reported by other authors (e.g., Cayrel et al. 2004). The absolute mean 
value we found $[\mathrm{Na} / \mathrm{Fe}]=+0.22 \mathrm{dex}$, is much higher than the -0.07 dex predicted by the GCE model.

It is well known, however, that there are considerable NLTE departures for sodium that can change the estimated abundances up to 0.5 dex (Baumueller et al. 1998), especially on the resonant 588.9 and $589.5 \mathrm{~nm}$ lines that were used to determine the LTE differential abundances. We corrected our abundances using the Lind et al. (2011) data, available trough the Inspect ${ }^{2}$ project. These corrections were done to each of the resonant lines, which suffer large NLTE effects and saturate on the more metal-rich stars. After taking the useful suggestions from the referee we also added measurements of the 568.2 and $568.8 \mathrm{~nm}$ lines (which are less sensitive to NLTE departures) when the lines were clearly measurable - stars with $[\mathrm{Fe} / \mathrm{H}] \geq-2.1(\mathrm{ex}-$ cept for a couple of stars where the lines were not detected due to bad $\mathrm{S} / \mathrm{N}$ in that region of the spectra). The final $[\mathrm{Na} / \mathrm{Fe}]$ abundances are listed in Table A.3 and shown in Fig. 4.

With the NLTE corrections, the behavior of our sample is in good agreement with the data from other works, making a solid "bridge" between the EMP and MP stars, and the slope $(-0.02 \pm 0.08)$ is in better agreement with the model. The starto-star standard deviation of $0.12 \mathrm{dex}$ is also similar to the scatter of 0.13 dex found by Andrievsky et al. (2007). Since Na production highly depends on the initial metallicity of progenitor stars, it is very unlikely to have a negative slope in the $[\mathrm{Na} / \mathrm{Fe}]$ evolution. It is also important to see how the observations begin to deviate from the model for metallicities $[\mathrm{Fe} / \mathrm{H}] \geq-2.2$, probably due to problems in the NLTE treatment of the data. Better measurements of the weaker $568 \mathrm{~nm}$ lines in all the stars could possibly alleviate the issue.

The considerable NLTE corrections had a major effect on the mean $[\mathrm{Na} / \mathrm{Fe}]$. The LTE mean is $[\mathrm{Na} / \mathrm{Fe}]=+0.22 \mathrm{dex}$, while the NLTE result is $\left[\mathrm{Na}_{\mathrm{NLTE}} / \mathrm{Fe}\right]=-0.22 \mathrm{dex}$. In contrast to the halo data shown in Kobayashi et al. (2006), the scatter in our results are small. It is also worth mentioning that with the applied corrections the scatter in our data is smaller, which might validate the adopted NLTE corrections.

Sodium abundances from Zhao et al. (2016) are well reproduced by the K15 model, but their observations have a larger scatter than what we find here. We also see the Na overproduction for higher metallicities in the K15 model, when compared to their measurements, also seen in the $[\mathrm{Na} / \mathrm{Fe}]$ shown here, from Nissen \& Schuster (2010). We note the $\mathrm{Na}$ overproduction of AGB stars has been solved in the Karakas (2010) yields, and this problem is likely to be caused by the metallicity dependence of core-collapse supernovae.

\subsubsection{Aluminum}

As for sodium, NLTE effects play an important role in aluminum abundances in metal-poor stars. It can be seen from the lower panel of Fig. 2 of Andrievsky et al. (2008) that in the temperature, surface gravity, and metallicity range of our stars, the NLTE corrections are almost constant (they range from 0.6 to $0.7 \mathrm{dex}$ ). This is a similar result to what was seen in the correction grid provided by Baumueller \& Gehren (1997). Thus, we applied the same correction of +0.65 dex to all our stars, which left the differential abundances, and errors, unaltered. However, it is important to use these corrections with caution, because as pointed by Andrievsky et al. (2008), the shapes of the LTE and NLTE profiles are different, therefore spectral synthesis is more appropriate than NLTE corrections. As we did not have

\footnotetext{
2 http://inspect-stars.com/
}

access to the NLTE spectral synthesis of $\mathrm{Al}$, we chose to use NLTE corrections to assess their effect on the GCE.

The Al data scatter and the mean error are on the same level (0.09 and 0.08 dex), which indicates a very small, if any, astrophysical scatter for $\mathrm{Al}$ in our sample. The scatter we found is similar to the low standard deviation of the turnoff objects published in Andrievsky et al. (2008; 0.09 dex), both our work and that of Andrievsky et al. (2008) have a lower scatter than the LTE work by Cayrel et al. $\left(2004 ; \sigma_{\mathrm{Al}}=0.21\right)$.

When compared to the GCE model, there is a small disagreement. In Fig. 4 we see that the data from Andrievsky et al. (2008) agrees with the model prediction until $[\mathrm{Fe} / \mathrm{H}] \sim-3$. For higher metallicities there is a disagreement of up to $0.25 \mathrm{dex}$ and the overall behavior is a flat slope. Notice that the more metal-rich stars from Andrievsky et al. (2008) are in good agreement with our data. Albeit there is discrepancy between the model predictions and our data, which fall lower than predicted, a more proper NLTE approach should be followed to confirm this behavior. Although there is a discrepancy, our results have a much smaller scatter than previous works and, along with quality data on different metallicities ranges, will be important to improve GCE models.

Zhao et al. (2016) found a similar result in his NLTE analysis. The Al trend in the K15 model is similar to the Na trend, and there is an offset of $\sim 0.25$ dex between data and model. Our offset is slightly higher ( $0.29 \mathrm{dex})$, but the overall behavior of the data is similar and supports the previous work. The mismatch of [Na/Al] at low metallicity might also be related to the mismatch of $[\mathrm{O} / \mathrm{Mg}]$ ratios.

\subsubsection{Scandium}

Our Sc abundances were measured using Sc II lines. We corrected the lines for hyperfine structure using HFS data from Kurucz ${ }^{3}$ linelists. The scatter in our sample is 0.07 dex, which is the same as reported by Cayrel et al. (2004) and also very similar to the $\sim 0.1$ dex standard deviation on Bonifacio et al. (2009). One could expect a higher scatter for Sc abundance, compared to other elements, due to the fact that the nucleosynthesis of this element is heavily dependent on the mass of the progenitor (Chieffi \& Limongi 2002). We, however, find the scatter of Sc to be at the same level as in the other light elements. The overall behavior of our data points is consistent with previous results from Cayrel et al. (2004) and follow closely what was found by Bonifacio et al. (2009) and seems to be quite in good agreement with the trend they found.

It is intriguing to observe that both Bonifacio et al. (2009) and our work have $[\mathrm{Sc} / \mathrm{Fe}]$ ratios significantly higher than the metal-rich halo stars studied by Fishlock et al. (2017), which are from the high and low- $\alpha$ populations from Nissen \& Schuster (2010). Their analysis was based on spectral synthesis and $\chi^{2}$ minimization of one Sc II line, while ours was performed via analysis of the curve of growth, both using MOOG (Sneden 1973). Although at first sight we could think of systematic errors, our curve of growth LTE analysis of Sc in a star from the Nissen \& Schuster (2010), shows that indeed the metal-rich halo stars have $[\mathrm{Sc} / \mathrm{Fe}]$ ratios slightly lower than solar (Reggiani \& Melendez, in prep.).

There is a disagreement between scandium measurements (ours and Bonifacio et al. 2009) and the galactic chemical evolution model, but such a difference was already reported in Kobayashi et al. (2006). In that work there is a difference of

\footnotetext{
3 http://kurucz.harvard.edu/linelists.html
} 
almost 1 dex, while the difference with our data is about 0.7 dex This is likely due to the effect of jet-like explosions applied to the K15 model (Sneden et al. 2016). Sc yields could be more enhanced by the $v$ process (Kobayashi et al. 2011), which is not included in the K15 model. Interestingly the GCE model agrees better with the data from Fishlock et al. (2017) for the more metal-rich stars.

The $[\mathrm{Sc} / \mathrm{Fe}]$ data by Zhao et al. (2016) follows a similar behavior to our data, Bonifacio et al. (2009) and Fishlock et al. (2017), showing a disagreement with the K15 model at low metallicities and an agreement for the metal-rich objects. However, the Zhao et al. (2016) scandium abundances are lower ( $[\mathrm{Sc} / \mathrm{Fe}] \sim 0.2$ dex versus our $[\mathrm{Sc} / \mathrm{Fe}] \sim 0.3 \mathrm{dex})$, and do not have a big offset between the more metal-poor and more metalrich stars, thus having a somewhat smaller difference when compared to the K15 model. That indicates that a NLTE treatment is more accurate for Sc measurements for more metal-poor stars.

\subsection{Iron-peak}

The results for the iron-peak elements are presented in Fig. 5 , and in Table A.4.

\subsubsection{Vanadium}

Vanadium abundances were calculated from V II lines with hyperfine structure data from Wood et al. (2014). There are only a few abundances of V for halo stars in Kobayashi et al. (2006) and the data points are scattered, which shows the difficulty in comparing model results with actual data. Our results indicate a slope of $-0.25 \pm 0.06$, that is steeper than the GCE predictions. The data results are also somewhat higher than the GCE model, as previously seen in Kobayashi et al. (2006) and in the K15 model with the effects of jet-like explosions. As for Sc, $\mathrm{V}$ yields could be enhanced by the $v$ process (Kobayashi et al. 2011). The mean error and scatter are on the same level. It is puzzling the extremely lower $\mathrm{V}$ abundance of one of the blue straggler stars; this will be discussed further in Sect. 7.

\subsubsection{Chromium}

In previous works, such as Cayrel et al. (2004), Cr is found to have a positive slope, meaning a decreasing abundance with decreasing metallicity. Bonifacio et al. (2009) also found a similar behavior for their turnoff stars (see their Fig. 8). Our Cr I data is consistent with that behavior but presents a steeper slope $(0.40 \pm 0.06$ against a 0.12 reported by Cayrel et al. 2004). All the chromium results from Cr I (Bonifacio et al. 2009; this work; and Nissen \& Schuster 2010) are inconsistent with GCE model predictions and our $\mathrm{Cr}$ I abundances vary from lower to higher than the model, for the more metal-poor and more metal-rich end of our sample, respectively.

The behavior of $\mathrm{Cr}$ II is, however, very different. In our sample the slope of $\mathrm{Cr}$ II is $0.01 \pm 0.03$, which is consistent with the -0.01 GCE model results, as can be seen in Fig. 5 . This difference between $\mathrm{CrI}$ and $\mathrm{Cr}$ II measurements had already been reported in Kobayashi et al. (2006), Lai et al. (2008), Bonifacio et al. (2009). As in Kobayashi et al. (2006), we consider the LTE analysis of Cr II to be better than Cr I in LTE, to trace the chemical evolution of this element. The star-to-star standard deviation of $\mathrm{Cr}$ II is smaller than the errors $(0.04 \mathrm{dex}$ and 0.07 dex respectively) and this scatter is among the lowest of our sample. In Fig. 5 we show $\mathrm{Cr}$ I and Cr II abundances in different panels, for our data and those of Bonifacio et al. (2009) and Nissen \& Schuster (2010), together with the GCE model, which predicts roughly a flat plateau in $[\mathrm{Cr} / \mathrm{Fe}]$. It is clear than the agreement is better for $\mathrm{Cr}$ II. The lesser agreement for $\mathrm{Cr}$ I is probably due to NLTE effects.

\subsubsection{Manganese}

Manganese abundances were calculated using hyperfine components from Kurucz ${ }^{3}$. In Fig. 5 we can see that the mean error and the scatter for $\mathrm{Mn}$ are 0.07 and 0.09 dex, suggesting the scatter might have an astrophysical origin. Our results are in agreement with the star-to-star scatter in the metal-poor giants of Cayrel et al. (2004). The steep slope we found for Mn $(0.23 \pm 0.03)$ is much steeper than GCE model prediction, but it seems to be in good agreement with data from Bonifacio et al. (2009), Nissen \& Schuster (2011). Thus, our [Mn/Fe] measurements connect well with lower and higher metallicity data.

Manganese is an odd- $Z$ element and Mn yields depends on the progenitor metallicity, but such a steep increase in abundance with increasing metallicity was not seen in the GCE model. When comparing our data with the scattered data plot seen in (Kobayashi et al. 2006, see their Fig. 22) one can notice that their data also has higher values of Mn when compared to the model, but the scatter is big and makes it difficult to assess whether the model agrees or not with those earlier literature values.

\subsubsection{Cobalt}

We have also used Kurucz's HFS data for Co abundances. We see in Fig. 5 that the cobalt abundances decrease with increasing metallicity throughout the entire metallicity range being analyzed. Our data have a steep slope of $-0.28 \pm 0.03$, which is steeper than the -0.02 value predicted by GCE model. The disagreement between data and GCE model is also seen in Kobayashi et al. (2006). Our errors are at the same level as the scatter, but our data is more precise than previous works.

\subsubsection{Nickel}

Although nickel is synthesized in the same process as Co, in the complete Si burning region, Cayrel et al. (2004), Lai et al. (2008), Bonifacio et al. (2009) had already reported that while $[\mathrm{Co} / \mathrm{Fe}]$ decreases with increasing $[\mathrm{Fe} / \mathrm{H}],[\mathrm{Ni} / \mathrm{Fe}]$ remains flat. We also found a flat slope for nickel $(0.00 \pm 0.02)$, which is consistent with the 0.02 predicted by the GCE model, and data from different metallicity ranges. There is an impressive flat plateau for the $[\mathrm{Ni} / \mathrm{Fe}]$ ratios between $-3.6 \leq[\mathrm{Fe} / \mathrm{H}] \leq-0.4$, indicating a very homogeneous nickel production throughout cosmic history. It is also important to stress that the $[\mathrm{Ni} / \mathrm{Fe}]$ star-to-star scatter $(0.04 \mathrm{dex})$ is significantly smaller than the error $(0.08 \mathrm{dex})$.

\subsubsection{Zinc}

Zinc is also mainly produced in the complete Si burning region, but can also be produced in neutron capture processes in more metal-rich stars (Kobayashi et al. 2006), and there is a negligible portion of $\mathrm{Zn}$ being produced in electron-capture supernovae (Kobayashi et al., in prep.). Depending on the neutrino physics, Co can be enhanced instead of $\mathrm{Zn}$ by electron-capture supernovae (Pllumbi et al. 2015). Both Cayrel et al. (2004) and Bonifacio et al. (2009) found a slope similar to previous results, indicating a formation processes consistent with complete silicon burning. We see the same behavior as in Co (complete Si burning), with a steep slope $(-0.16 \pm 0.05)$ against a flat model 
prediction, going from the Bonifacio et al. (2009) data, all the way to a metallicity of $[\mathrm{Fe} / \mathrm{H}]=-0.4$. When we consider our data set alone there is one data point (the more metal-poor blue straggler) that has a higher abundance, and being a blue straggler this effect could be interpreted as the result of a possible different nucleosynthetic origin. However, when considering also the data from Bonifacio et al. (2009), it seems that the higher zinc abundance of this object is just an effect of cosmic scatter. More data on blue stragglers (BSS) zinc abundances are necessary in order to say if the higher abundance of this star has anything to do with the BSS phenomena.

It is important to note that this GCE model is a so-called one-zone model where instantaneous mixing is assumed. This assumption is valid probably for $[\mathrm{Fe} / \mathrm{H}]>-2$, but not for $[\mathrm{Fe} / \mathrm{H}]<-2.5$ where chemical enrichment should take place inhomogeneously and EMP stars are enriched only by one or two supernovae (Audouze \& Silk 1995). The increasing trends of $\mathrm{Co}$ and $\mathrm{Zn}$ (and the flat trend of $\mathrm{Ni}$ relative to $\mathrm{Fe}$ ) may be explained more realistically, via chemodynamical simulations (e.g., Kobayashi \& Nakasato 2011). From a nucleosynthetic point of view, both $[\mathrm{Co} / \mathrm{Fe}]$ and $[\mathrm{Zn} / \mathrm{Fe}]$ increase with higher explosion energy (i.e., hypernovae, Kobayashi et al. 2006) and it is possible to predict some variation in $\mathrm{Co} / \mathrm{Zn}$. With higher energy, the Fe production mass is larger, but because of the larger amount of $\mathrm{H}$ mixed into the ejecta, the $[\mathrm{Fe} / \mathrm{H}]$ of the EMP stars can be smaller (Nomoto et al. 2013). Our tight trend of Co and (less tight) trend of $\mathrm{Zn}$ is suggestive of inhomogeneous enrichment from hypernovae. $\mathrm{Ni} / \mathrm{Fe}$ does not depend on the explosion energy nor on mass very much, and the flat trend with the small scatter gives strong constraints on the mixing-fallback mechanism of core-collapse supernovae (both for supernovae and hypernovae).

\subsection{Neutron-capture elements}

The abundances of the heavy elements $\mathrm{Sr}, \mathrm{Y}, \mathrm{Zr}$ and $\mathrm{Ba}$ can be seen in Fig. 6, and are shown in Table A.5. The GCE model we have been using to compare our data does not go further than $\mathrm{Zn}$. Heavier elements are predominantly produced by neutron capture events (Meyer 1994). The two main neutron capture processes are the rapid neutron capture process ( $r$-process) and the slow neutron capture process ( $s$-process; Busso et al. 1999; Karakas \& Lattanzio 2014), where most of the $s$-process production occurs in low-mass AGB stars (Busso et al. 2001). The $s$-process can also occur in fast rotating massive stars (Pignatari et al. 2010; Frischknecht et al. 2016), which may have an important contribution at low metallicity. The yields of $s$-process elements depend on masses and initial compositions of these stars, and the result of these processes can be observed as cosmic scatter for more metal-poor stars, formed before the interstellar medium properly mixed the material, and a more statistical scatter for the more metal-rich stars where mixing in the ISM had more time to work. While there are uncertainties surrounding the details of the $s$-process, the site is reasonably well understood, in contrast to the $r$-process. The origin of the $r$-process is unknown and it could occur in different sites, such as SNe II or neutron stars mergers (Cowan \& Sneden 2004; Thielemann et al. 2011; Ji et al. 2016). There is also a third possible mechanism to produce heavy elements, an intermediate neutron capture process, which takes places in neutron flux densities between the $\mathrm{s}$ and $\mathrm{r}$ processes, called the $i$-process (Cowan \& Rose 1977; Hampel et al. 2016). There are evidences of $i$-process in the metal-poor stars nucleosynthetic history (Herwig et al. 2014; Roederer et al. 2016).
These uncertainties in the production sites of $i$-process and $r$-process elements, along with a limited number of published yields of $s$-process from metal-poor stellar models limit the capability of modeling such elements. All these difficulties increase the importance of precise chemical abundances of as many stars as possible with broad wavelength coverage.

\subsubsection{Strontium}

Strontium abundances were calculated from two Sr II lines, which are not significantly affected by NLTE effects according to Hansen et al. (2013). The authors show that accurate Sr II abundances can be obtained if reliable effective temperatures and surface gravities are available, such as in our case. The steep positive slope is mainly due to two more metal-poor stars that appear to have an extremely lower $\mathrm{Sr}$ abundance. This lower abundance, almost 1 dex for the most $\mathrm{Sr}$ deficient star, could suggest that this star was formed in an environment where AGB stars had not been activated yet, which would greatly decrease the $s$-process element production and become apparent in its abundance pattern. However, it is important to stress that the scatter in $\mathrm{Sr}$ is the second biggest in our measurements, lower only to the scatter in barium. The scatter becomes more clear when considering also the data from Bonifacio et al. (2009), which allows us to see that the two low Sr stars in our sample are probably just other examples of the very big large spread in [ $\mathrm{Sr} / \mathrm{Fe}]$. This scatter has been previously reported (McWilliam 1998; Cayrel et al. 2004; François et al. 2007; Lai et al. 2008; Bonifacio et al. 2009) and was confirmed in the NLTE analysis of Andrievsky et al. (2011). As pointed out by Andrievsky et al. (2011), the scatter of strontium decreases at higher metallicities, which agrees with our observations. Overall, the data suggests that the scatter in $[\mathrm{Sr} / \mathrm{Fe}]$ decrease for $[\mathrm{Fe} / \mathrm{H}]>-2.4$. Unfortunately, even with high precision data available, the current errors on stellar yields do not allow us to draw conclusions about the chemical evolution of strontium in the early Galaxy (Hansen et al. 2013), and the nucleosynthetic sites in which it might be produced.

\subsubsection{Yttrium}

According to Hannaford et al. (1982): "the effects due to isotopic splitting and hyperfine structure in yttrium are insignificant, because there is only one stable isotope, and the hyperfine splitting is very small, typically less than $1 \mathrm{~mA}$ ". Thus, yttrium abundances were calculated from five Y II lines, without hyperfine or isotopic corrections. As with $\mathrm{Sr}$, there is significant scatter. The slope is almost flat, but with a big uncertainty, and we see one star with much lower [Y/Fe], which is the same object that deviates almost 1 dex in $\mathrm{Sr}$, showing that this star indeed does have lower $s$-process abundances.

The scatter in Y abundances also extends to the higher metallicity sample of Nissen \& Schuster (2011), but there is a very well defined separation between their low and high- $\alpha$ populations. It is difficult to assess if our data follow a similar behavior because the high scatter we observe is only present in the neutron-capture elements, not the $\alpha$-elements, as seen in Nissen \& Schuster $(2010,2011)$. Also, the scatter seems largest for $[\mathrm{Fe} / \mathrm{H}]<-2.4$.

\subsubsection{Zirconium}

Zirconium abundances were obtained from three $\mathrm{Zr}$ II lines and it has the smallest deviation among the heavy elements in this 
work. The calculated slope is negative, but the mean error is closest to the star-to-star standard deviation than any other heavy element.

Among the neutron-capture elements, $\mathrm{Zr}$ is the element that deviates the most from the more metal-rich sample of Fishlock et al. (2017). Their data show a much lower mean abundance of this element, and differently from the other neutroncapture elements, it does not seem to be a connection between their higher metallicity sample and our lower metallicity range. It is unclear if this is a result of the nucleosynthetic history of the element or due systematic differences in the analyses.

\subsubsection{Barium}

The last heavy element analyzed in our sample is barium. We have applied isotopic splitting corrections from McWilliam (1998). In the solar system $\mathrm{Ba}$ is mainly produced via the $s$-process (85\%, McWilliam 1998), while the remainder is produced via the $r$-process. However, this production scenario can be different for metal-poor stars, where the $r$-process might have more significant contribution. Our results indicate a very steep slope for $\mathrm{Ba}$, not consistent with the other $s$-process elements. This is mainly due to differences in the most metalpoor end of our sample, which have consistently lower abundances, perhaps bringing insights on $s$-process nucleosynthesis. Considering the large scatter observed, our $[\mathrm{Ba} / \mathrm{Fe}]$ ratios are consistent with the metal-poor sample from Bonifacio et al. (2009) and partly consistent with the metal-rich sample by Nissen \& Schuster (2011), albeit most of their sample seem to group around $[\mathrm{Ba} / \mathrm{Fe}] \sim-0.2$.

This rather large scatter in $[\mathrm{Ba} / \mathrm{Fe}]$ could be partly due to NLTE effects. As pointed out in Andrievsky et al. (2009), the NLTE corrections in this metallicity regime rapidly increase with increasing temperature. Thus, even in a homogeneous sample like ours there might be important NLTE corrections in barium abundances. Andrievsky et al. (2009) showed that, even with the NLTE calculations, there is considerable scatter in barium abundances, which support a complex evolution throughout cosmic time, with the possible additional contribution of the $r$-process (e.g., François et al. 2007). It is also important to stress that the low $\mathrm{Sr}$ and low Y star, also has a lower Ba abundance, compared to the other object with the same $[\mathrm{Fe} / \mathrm{H}]$, although the difference is not as considerable.

The $[\mathrm{X} / \mathrm{Fe}]$ abundances of heavy elements in our sample show a very big dispersion. The star-to-star scatter are greater than all the mean errors and also much higher than in the other elements studied. This higher scatter, and diverse nucleosynthesis origins, indicate that the results point to cosmic scatter. This suggests that a linear regression might not be the best function to describe the chemical evolution of these elements. Thus, we added a non-parametric regression to our data set, which can be seen as the pink lines in all panels of Fig. 6. We used a LOWESS function, which fits simple models to localized subsets of data, models that are used to build the function that best describes the variation in the data, point by point. The LOWESS regression works better with large data sets, but we have applied it to our sample of heavy element abundances in order to see the difference between a local regression and a linear regression.

The LOWESS function, Fig. 6, indicates that where the interstellar medium had more time to mix the materials (the more metal-rich end) the linear regression and the non-parametric regressions are close to each other and, as the metallicity decreases the two regressions deviate. Our smaller error bars and precise abundances will be important to constrain the nucleosynthesis of

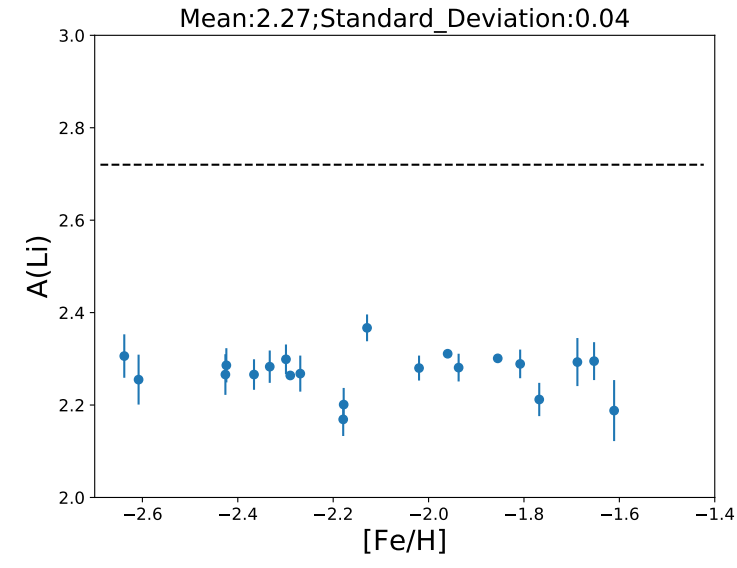

Fig. 7. $A(\mathrm{Li})$ abundances of our sample. The dotted black line represents the Planck+BBN prediction (Coc et al. 2014b; Cyburt et al. 2016).

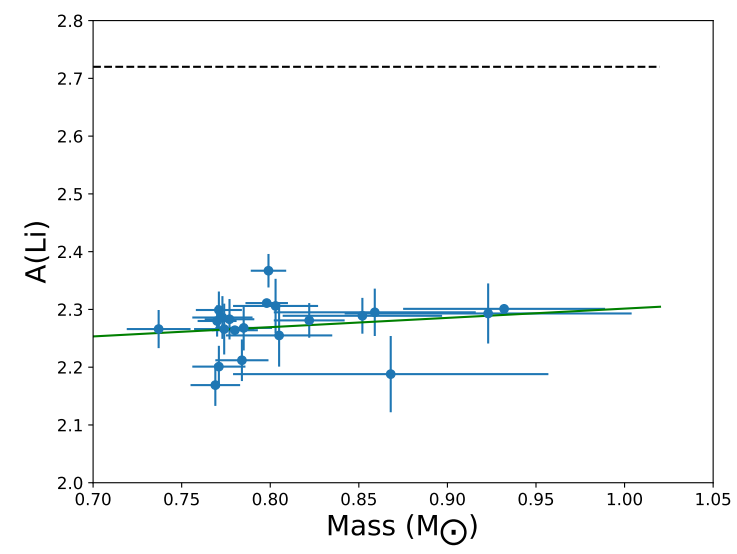

Fig. 8. Lithium vs. stellar mass. The green line represents a linear fit to the data. The black dotted line is the Planck and BBN prediction (Coc et al. 2014b; Cyburt et al. 2016).

these elements and will help to constrain the rise of the $s$-process in the Galaxy.

\section{Lithium}

Spite \& Spite (1982) found that warm metal-poor stars have a constant lithium abundance, and interpreted their finding as relic lithium from primordial nucleosynthesis. However, Big Bang nucleosynthesis (BBN) predictions, along with baryon density observations from Planck, predicts $A(\mathrm{Li})=2.67$ (Coc et al. 2014b; Cyburt et al. 2016), which is $\approx 0.4$ dex higher than what is observed in metal-poor stars (e.g., Spite \& Spite 1982; Asplund et al. 2006; Bonifacio et al. 2007; Meléndez et al. 2010; Spite et al. 2015). This discrepancy has been the focus of many different studies over the years. Possible explanations include new physics (e.g., Coc et al. 2009, 2014a; Iocco et al. 2009; Kohri \& Santoso 2009; Civitarese \& Mosquera 2013; Salvati et al. 2016; Hou et al. 2017) and stellar evolution effects (Richard et al. 2005; Fu et al. 2015).

Our lithium abundances are computed in NLTE, following Lind et al. (2009), and are presented in Fig. 7. The stellar masses from Fig. 8 were estimated using the q2 code, which uses Y2 isochrones (Yi et al. 2001; Kim et al. 2002) to the adopted stellar parameters and their errors. The code estimates the masses using probability distribution functions (see Ramírez et al. 2013, for more details). The lithium abundances and estimated masses can be seen in Table A.6. 
Our results have a very low scatter $(0.04 \mathrm{dex})$ and the mean value $A(\mathrm{Li})=+2.27$ is compatible with measurements of similar metal-poor stars, such as those studied in Meléndez et al. (2010). As can be seen, the plateau is very well defined and the deviations are within our measurement errors.

Models of lithium depletion based on stellar evolution, such as Richard et al. (2005), predict that the least massive stars will be more depleted in Li. Meléndez et al. (2010) shows the existence of a correlation with the initial stellar mass using Richard et al. (2005) model predictions. The correlation found is especially good for the stars in the same metallicity range as this work. In Fig. 8 we show lithium abundances against the mass of the stars, based on Y2 isochrones. However, most of our targets have very similar masses which makes it very difficult to assess if there is any trend with mass.

For stars in the mass range we are working on $\left(0.7-0.8 M_{\odot}\right)$, Fu et al. (2015) were able to reproduce the Spite plateau by invoking pre-stellar lithium depletion. In their model they take into consideration microscopic diffusion, overshooting, UV radiation photoevaporation and late accretion during the pre main sequence and main sequence phases. These effects are responsible for the lithium depletion in their model, which happens mainly in the pre main sequence phase and, to a lower extent, at the main sequence phase. Fu et al. (2015) calculated a $A(\mathrm{Li}) \approx 2.26$, for stars with ages ranging from 10 to $12 \mathrm{Gyr}$ (see Fig. 8 of Fu et al.), and reproduced the spite plateau over metallicities ranging from $-3.5 \leq[\mathrm{M} / \mathrm{H}] \leq-1.5$.

We also point out that stars with an even lower lithium abundance might have suffered effects from rotationally-induced mixing. Such effects have already been shown to deplete lithium in solar like stars (Carlos et al. 2016; Ryan et al. 2002) and might also be important to explain the lower lithium abundances found in some metal-poor stars, which might be the case of the two blue straggler stars found in our sample (see Sect. 7).

\section{The blue straggler stars}

Blue straggler stars (BSS) are main-sequence stars significantly bluer than the main-sequence turnoff population they belong to (Ryan et al. 2001). Due to the color difference from the regular main sequence stars, they are usually identified in globular clusters. Field BSS however are harder to identify because it is difficult to establish other main sequence stars with a common origin, to be used as standards in a color comparison. However, this identification is possible through other means, as employed by Santucci et al. (2015), who identified approximately 8000 BSS stars using color cuts, FWHM of the hydrogen spectral lines and stellar parameters.

Blue stragglers can also be identified via spectroscopy by using their Li or Be abundances. As showed by Ryan et al. (2001), halo ultra lithium-deficient stars can be BSS. We identified stars HD 340279 and G66-30 as blue stragglers based on their Li abundances, as was firstly done by Ryan et al. (2001). BS stars show much lower Li content when compared to stars of similar metallicity.

There are two possible scenarios for the formation of BS stars. In one of them the star is recipient of mass transfer from a more evolved AGB companion (the McCrea 1964, scenario), and in the second there was a collision with a companion. In both scenarios angular momentum is transferred to the BS star. This additional momentum can extend the convection zone, which is a possible explanation for part of the Li depletion. Blue straggler stars may also have enhanced $s$-process material, if it underwent mass transfer from an AGB companion. As stellar collisions will

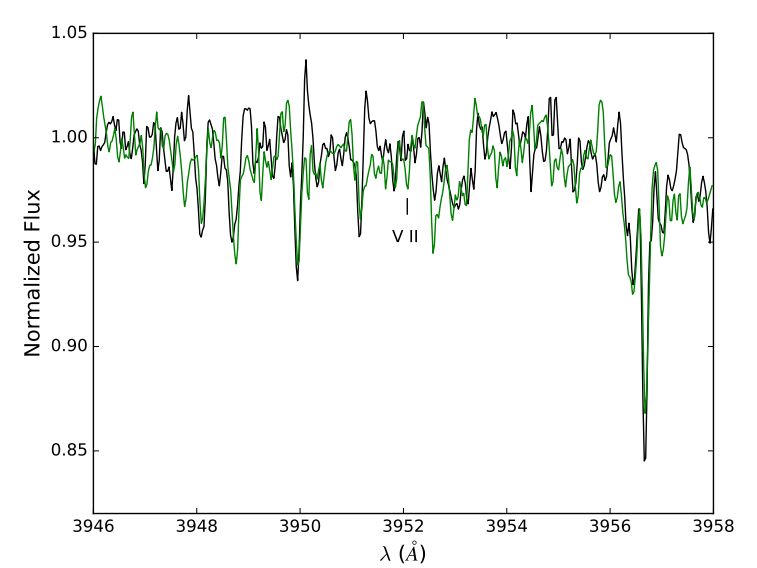

Fig. 9. Spectra around the V II line in 3952.02 for stars HD 340279 and $\mathrm{BD}+262621$.

only occur in very dense environments, such as the core of globular clusters (Sills et al. 2009), it is more likely that the blue straggler stars found in the field have suffered mass transfer rather than collided.

We measured an upper limit to the Li abundance of star HD 340279 of $A(\mathrm{Li}) \leq 0.94$ and $A(\mathrm{Li}) \leq 1.3$ for $\mathrm{G} 66-30$. Ryan et al. (2001) determined an upper limit of $A(\mathrm{Li}) \leq 1.39$ for star HD 340279 and Boesgaard (2007) determined a conservative upper limit of $A(\mathrm{Li}) \leq 1.5$ to $\mathrm{G} 66-30$. In both cases the stars are identified as BSS trough their ultra-deficient Li abundances compared to stars of similar effective temperature.

Boesgaard (2007) has also showed that $\mathrm{G}$ 66-30 is beryllium poor. They determined an upper limit of $A(\mathrm{Be})<-1.0$, which is below the expected value for Li normal stars, which also led to the conclusion that additional momentum has extended the convection zone and further depleted both elements.

Although there is a clear difference in $\mathrm{Li}$ abundance, not all other elements show such a clear difference, as can be seen in Figs. 2 to 6 (BS stars are the red objects). In Fig. 2 we see that the more metal-poor BSS may have a slight underabundance of $\alpha$-elements, while star G 66-30 is within the overall trends when the errors are taken into consideration.

The same effect happens when we look at the odd-Z light element Sc (Fig. 4), which is lower in HD 340279 when compared to stars of similar metallicity.

Star HD 340279 has another very puzzling peculiarity. Its vanadium abundance is lower than the abundances of all other stars. The calculated abundance is 0.6 dex lower when compared to the linear regression. It is not clear why there is such an underabundance, as the other BSS has a normal vanadium abundance and HD 340279 has normal abundances of the other iron peak elements, except for zinc. In Fig. 9 we show the spectra of stars HD 340279 and BD+26 2621, which have similar metallicities, around the $3952 \mathrm{~V}$ II line. We can see that the vanadium line of star BD+26 2621 is identified but in HD 340279 the line is barely visible. However, we caution the reader that the noise in our spectra is on the same level as the vanadium lines. Improved spectra are necessary to confirm this peculiarity.

The BSS star HD 340279 might also be enhanced in zinc if compared exclusively with the rest of the stars of very similar metallicity, including the $[\mathrm{Fe} / \mathrm{H}]=-2.58$ star from Bonifacio et al. (2009). The zinc abundance of this star is also considerably higher than the linear regression predicts. However, if compared to the abundances of the more metal-poor stars of 
Bonifacio et al. (2009), one could attribute the enhancement to cosmic scatter.

Considering the possibility of $\mathrm{Zn}$ enhancement in HD 340279, we can discuss its cause in light of the nucleosynthetic processes that a BSS star undergoes. While studying nucleosynthesis in Pop III stars, Heger \& Woosley (2002) and Umeda \& Nomoto (2002), first proposed the possibility of Zn being produced by the $s$-process. Although zinc production in AGB stars is not large enough to cause comprehensive changes to the GCE (e.g., Karakas et al. 2009), it is possible that a star that underwent mass transfer from an AGB can show enhanced $\mathrm{Zn}$. Under that assumption the excess of zinc in BSS could be another tool to estimate the mass of the AGB progenitor, as production of $\mathrm{Zn}$ in the AGB phase is more important in intermediate $M \geq 3 M_{\odot}$ AGB stars. Zinc is at the beginning of the $s$-process chain but overall production is low, on the order of $[\mathrm{Zn} / \mathrm{Fe}]<0.3$. The exception is in intermediate mass AGB stars where $[\mathrm{Zn} / \mathrm{Fe}] \sim 0.5$.

In order to confirm if there is an excess of $\mathrm{Zn}$ in this star or if that is just an effect of cosmic scatter, it is of extreme importance to obtain more $\mathrm{Zn}$ abundances of BSS stars, providing tools to constrain a possible enhancement in the BSS process, or to exclude the possibility. Strontium, yttrium and barium are very enhanced in HD 340279. It indicates a very big influence of $s$-process nucleosynthesis in this star. G 66-30 on the other hand, does not seem to have an enhanced $s$-process and the abundance is low when compared to the other objects.

Unfortunately the spectroscopic works on BSS that we found are usually focused on one or just a few elements, as for example in Ferraro et al. (2006, 2016). We emphasize the importance of more comprehensive studies on the abundance patterns of BSS.

\section{Conclusions}

Previous studies of metal-poor stars in the halo are mainly focused on the most metal-poor end, $[\mathrm{Fe} / \mathrm{H}] \leq-2.5$ (e.g., Ryan et al. 1996; Norris et al. 2001; Cayrel et al. 2004; Yong et al. 2013; Norris et al. 2013). Our study, on the other hand, has metallicities $[\mathrm{Fe} / \mathrm{H}] \geq-2.7$. Our sample was chosen to obtain good spectra of similar stars, allowing us to seek precise abundances.

The differential analysis technique allow us to greatly decrease the data scatter, and also the errors of the differential chemical abundances. The well-defined trends we observe in our results are, for most elements, compatible with what has been previously found at lower precision.

The small scatter in our data set shows that the chemical evolution was, overall, very homogeneous. Among $\alpha$-elements, our data for $\mathrm{Ca}$ is in excellent agreement with the GCE model while we found $\sim 0.2$ dex offset for $\mathrm{Mg}$, in concordance with the NLTE study of Zhao et al. (2016). We do not see any indication of different populations in $\alpha$-elements, as found by Nissen \& Schuster (2010), below their metallicity threshold. If the stars in their sample were acreted from satellite galaxies, the fact that we do not see these populations in our sample can indicate that the main accretion events started to take place only after SNe Ia already had time to increase the overall metallicity to $[\mathrm{Fe} / \mathrm{H}] \approx-1.5$. This is supported by the fact that the separation between the two populations is mostly seen in the more metal-rich end, and seems to become homogeneous at the metal-poor end of their sample.

The very good homogeneity we observe in the $\alpha$-elements is also seen in odd-light elements and iron peak elements. The $\mathrm{Co}$ and $\mathrm{Zn}$ trends at very low metallicities also suggests an inhomogeneous enrichment with hypernovae.
Our differential LTE analysis shows a very good reliability even when compared to a full NLTE analysis. As shown, the results we obtain are very similar to the NLTE analysis of Zhao et al. (2016), and the comparison with the K15 GCE model they performed are very similar to those presented in this work. Although there is a very good agreement, in some cases the NLTE approach decreases the discrepancy between model and observations, as is the case of scandium, where there is a mean difference of $\approx 0.1$ dex between ours and their abundances. In the case of calcium, however, the results are very much alike and both NLTE and LTE have an impressive agreement with the K15 model. It is also important to stress that the values we compared to the K15 model are our mean values, and also the difference between data and model is based on our own measurements. Zhao et al. (2016) studied a broader metallicity range and thus is internally consistent, unlike our comparisons that are less homogeneous as employed data from other works, however overall there seems to be a good connection between our work and lower and higher metallicities.

LTE calculations of $\mathrm{Cr}$ II are much more reliable than $\mathrm{Cr}$ I. As shown by Bergemann \& Cescutti (2010), Cr I suffers from strong NLTE effects due to the over-ionization from the lowexcitation odd Cr I levels, which is more severe in more metalpoor stars, explaining the positive slope seen in the $\mathrm{Cr}$ I data, while Cr II NLTE effects on abundances are negligible for dwarfs. Taking $\mathrm{Cr}$ II as the indicative of $[\mathrm{Cr} / \mathrm{Fe}]$, we see a good agreement between GCE predictions and observed abundances for the entire metallicity range $(-3.6 \leq[\mathrm{Fe} / \mathrm{H}] \leq-0.4)$, indicative of the good understanding of $\mathrm{Cr}$ nucleosynthesis.

The bigger offsets between observational data and GCE predictions are seen for elements with an already known deviation, such as $\mathrm{Co}$ or $\mathrm{Mn}$, which are in agreement with other observational works (e.g, Cayrel et al. 2004; Bonifacio et al. 2009) but somehow still far from GCE models. For these elements there might be important NLTE effects that are not being taken into consideration in this work, such as for $\mathrm{Mn}$ (Bergemann \& Gehren 2008). This disagreement between observation and GCE predictions is not seen in Kobayashi et al. (2006), for example, as the absolute [X/Fe] values for most elements agree with observations, because the observations are spread due to results from different authors, making the comparison samples inhomogeneous. This is also shown in Fig. 3, by the big dispersion we found using data from the SAGA database. The Co and $\mathrm{Zn}$ trends also suggests an inhomogeneous enrichment with hypernovae.

Although AGB stars do not produce enough zinc to influence Galactic chemical evolution, the fact that the BS star HD 340279 may be enhanced in zinc, suggests that this could be an important tracer of the AGB progenitor masses of BS stars. Also, further observations of neutron-capture elements for this star might be beneficial to constrain the origin of $\mathrm{Zn}$ in this object.

It is also worth commenting on the analysis of star WISE J072543.88-235119.7, a high proper motion star crossing the Galactic plane with a bound retrograde orbit. Scholz et al. (2015) used a spectrum of lower resolution and S/N than ours, finding stellar parameters that are roughly consistent with the parameters found in our work. They suggested that this star might be a good target for follow-up high-resolution spectroscopy. In our results we did not find any distinctive chemical peculiarity in this star. The abundance pattern seems to be in good agreement with the remaining of our sample. Spite et al. (2015) performed a spectroscopic analysis of this star, calculating the effective temperature using a different method (H $\alpha$ fitting), resulting in a different set of stellar parameters. The differences in the stellar 
parameters translated into the abundance differences between our work and theirs. We verified this by recalculating the abundances using our equivalent widths and their stellar parameters, showing a mean difference of $0.08 \mathrm{dex}$ in $A(\mathrm{X})$, which can be easily explained by the measurement errors of both works. Thus, the differences between our measurements arise from the difference in the stellar parameters. They conclude through the $\mathrm{Li}$ abundance that despite the extreme kinematics the star might have formed in situ, which is in agreement with our findings, as it has an abundance pattern resembling that of our own Galaxy.

Here we used a line-by-line differential work to better constrain the chemical evolution of the Galaxy in a metallicity range that does not have many high precision works. Our abundances, along with the data from works in other metallicity ranges, allow us to do a comprehensive comparison of observational data of stars with similar atmospheric parameters, to the K15 Galactic evolution model. Our careful analysis yields precise and accurate data, which have small errors and low scatter, being thus important to better constrain future developments of GCE models. Finally, we encourage NLTE calculations in further works, in particular for the elements $\mathrm{Na}, \mathrm{Al}, \mathrm{Si}, \mathrm{Sc}$, and $\mathrm{Ba}$.

Acknowledgements. This work was based on observations collected at the European Organisation for Astronomical Research in the Southern Hemisphere under ESO program 095.D-0504(A). We would like to thank the referee for his/her valuable inputs. H.R. thanks a CAPES fellowship and CAPES PDSE program (88881.132145/2016-01). J.M. thanks support by FAPESP (2012/24392-2 and 2014/18100-4) and CNPq (Productivity Fellowship). V.M.P. acknowledges partial support for this work from the National Science Foundation under Grant No. PHY-1430152 (JINA Center for the Evolution of the Elements).

\section{References}

Andrievsky, S. M., Spite, M., Korotin, S. A., et al. 2007, A\&A, 464, 1081 Andrievsky, S. M., Spite, M., Korotin, S. A., et al. 2008, A\&A, 481, 481 Andrievsky, S. M., Spite, M., Korotin, S. A., et al. 2009, A\&A, 494, 1083 Andrievsky, S. M., Spite, M., Korotin, S. A., et al. 2010, A\&A, 509, A88 Andrievsky, S. M., Spite, F., Korotin, S. A., et al. 2011, A\&A, 530, A105 Aoki, W., Beers, T. C., Christlieb, N., et al. 2007, ApJ, 655, 492

Aoki, W., Beers, T. C., Lee, Y. S., et al. 2013, AJ, 145, 13

Arnone, E., Ryan, S. G., Argast, D., Norris, J. E., \& Beers, T. C. 2005, A\&A, 430, 507

Asplund, M., Lambert, D. L., Nissen, P. E., Primas, F., \& Smith, V. V. 2006, ApJ, 644, 229

Asplund, M., Grevesse, N., Sauval, A. J., \& Scott, P. 2009, ARA\&A, 47, 481

Audouze, J., \& Silk, J. 1995, ApJ, 451, L49

Baumueller, D., \& Gehren, T. 1997, A\&A, 325, 1088

Baumueller, D., Butler, K., \& Gehren, T. 1998, A\&A, 338, 637

Bensby, T., Feltzing, S., \& Oey, M. S. 2014, A\&A, 562, A71

Bergemann, M., \& Cescutti, G. 2010, A\&A, 522, A9

Bergemann, M., \& Gehren, T. 2008, A\&A, 492, 823

Biazzo, K., Gratton, R., Desidera, S., et al. 2015, A\&A, 583, A135

Boesgaard, A. M. 2007, ApJ, 667, 1196

Bonifacio, P., Molaro, P., Sivarani, T., et al. 2007, A\&A, 462, 851

Bonifacio, P., Spite, M., Cayrel, R., et al. 2009, A\&A, 501, 519

Busso, M., Gallino, R., \& Wasserburg, G. J. 1999, ARA\&A, 37, 239

Busso, M., Gallino, R., Lambert, D. L., Travaglio, C., \& Smith, V. V. 2001, ApJ, 557,802

Carlos, M., Nissen, P. E., \& Meléndez, J. 2016, A\&A, 587, A100

Caughlan, G. R., \& Fowler, W. A. 1988, Atomic Data Nucl. Data Tables, 40, 283

Cayrel, R., Depagne, E., Spite, M., et al. 2004, A\&A, 416, 1117

Cescutti, G. 2008, A\&A, 481, 691

Chiappini, C., Matteucci, F., Beers, T. C., \& Nomoto, K. 1999, ApJ, 515, 226

Chieffi, A., \& Limongi, M. 2002, ApJ, 577, 281

Civitarese, O., \& Mosquera, M. E. 2013, Nucl. Phys. A, 898, 1

Coc, A., Olive, K. A., Uzan, J.-P., \& Vangioni, E. 2009, Phys. Rev. D, 79, 103512

Coc, A., Pospelov, M., Uzan, J.-P., \& Vangioni, E. 2014a, Phys. Rev. D, 90, 085018

Coc, A., Uzan, J.-P., \& Vangioni, E. 2014b, J. Cosmol. Astropart. Phys., 10, 050 Cohen, J. G., Christlieb, N., McWilliam, A., et al. 2008, ApJ, 672, 320

Cowan, J. J., \& Rose, W. K. 1977, ApJ, 212, 149

Cowan, J. J., \& Sneden, C. 2004, in Origin and Evolution of the Elements, Carnegie Observatories Centennial Symp., 27
Cyburt, R. H., Fields, B. D., Olive, K. A., \& Yeh, T.-H. 2016, Rev. Mod. Phys., 88,015004

Dekker, H., D’Odorico, S., Kaufer, A., Delabre, B., \& Kotzlowski, H. 2000, in Optical and IR Telescope Instrumentation and Detectors, eds. M. Iye, \& A. F. Moorwood, Proc. SPIE, 4008, 534

Den Hartog, E. A., Ruffoni, M. P., Lawler, J. E., et al. 2014, ApJS, 215, 23

Eggen, O. J., Lynden-Bell, D., \& Sandage, A. R. 1962, ApJ, 136, 748

Ferraro, F. R., Sabbi, E., Gratton, R., et al. 2006, ApJ, 647, L53

Ferraro, F. R., Lapenna, E., Mucciarelli, A., et al. 2016, ApJ, 816, 70

Fishlock, C. K., Yong, D., Karakas, A. I., et al. 2017, MNRAS, 466, 4672

François, P., Depagne, E., Hill, V., et al. 2007, A\&A, 476, 935

Frischknecht, U., Hirschi, R., Pignatari, M., et al. 2016, MNRAS, 456, 1803

Fu, X., Bressan, A., Molaro, P., \& Marigo, P. 2015, MNRAS, 452, 3256

Gustafsson, B., Edvardsson, B., Eriksson, K., et al. 2008, A\&A, 486, 951

Hampel, M., Stancliffe, R. J., Lugaro, M., \& Meyer, B. S. 2016, ApJ, 831, 171

Hannaford, P., Lowe, R. M., Grevesse, N., Biemont, E., \& Whaling, W. 1982, ApJ, 261, 736

Hansen, C. J., Bergemann, M., Cescutti, G., et al. 2013, A\&A, 551, A57

Heger, A., \& Woosley, S. E. 2002, ApJ, 567, 532

Herwig, F., Woodward, P. R., Lin, P.-H., Knox, M., \& Fryer, C. 2014, ApJ, 792, L3

Hollek, J. K., Frebel, A., Roederer, I. U., et al. 2011, ApJ, 742, 54

Hou, S. Q., He, J. J., Parikh, A., et al. 2017, ApJ, 834, 165

Iocco, F., Mangano, G., Miele, G., Pisanti, O., \& Serpico, P. D. 2009, Phys. Rep., 472, 1

Jacobson, H. R., Keller, S., Frebel, A., et al. 2015, ApJ, 807, 171

Ji, A. P., Frebel, A., Simon, J. D., \& Chiti, A. 2016, ApJ, 830, 93

Karakas, A. I., \& Lattanzio, J. C. 2014, PASA, 31, e030

Karakas, A. I., van Raai, M. A., Lugaro, M., Sterling, N. C., \& Dinerstein, H. L. 2009, ApJ, 690, 1130

Keller, S. C., Bessell, M. S., Frebel, A., et al. 2014, Nature, 506, 463

Kim, Y.-C., Demarque, P., Yi, S. K., \& Alexander, D. R. 2002, ApJS, 143, 499

Kobayashi, C., \& Nakasato, N. 2011, ApJ, 729, 16

Kobayashi, C., Umeda, H., Nomoto, K., Tominaga, N., \& Ohkubo, T. 2006, ApJ, 653, 1145

Kobayashi, C., Izutani, N., Karakas, A. I., et al. 2011, ApJ, 739, L57

Kohri, K., \& Santoso, Y. 2009, Phys. Rev. D, 79, 043514

Lai, D. K., Bolte, M., Johnson, J. A., et al. 2008, ApJ, 681, 1524

Lawler, J. E., Guzman, A., Wood, M. P., Sneden, C., \& Cowan, J. J. 2013, ApJS, 205, 11

Lind, K., Asplund, M., \& Barklem, P. S. 2009, A\&A, 503, 541

Lind, K., Asplund, M., Barklem, P. S., \& Belyaev, A. K. 2011, A\&A, 528, A103

Liu, F., Asplund, M., Yong, D., et al. 2016a, MNRAS, 463, 696

Liu, F., Yong, D., Asplund, M., Ramírez, I., \& Meléndez, J. 2016b, MNRAS, 457, 3934

Maeda, K., \& Nomoto, K. 2003, ApJ, 598, 1163

McCrea, W. H. 1964, MNRAS, 128, 147

McWilliam, A. 1998, AJ, 115, 1640

Meléndez, J., \& Barbuy, B. 2009, A\&A, 497, 611

Meléndez, J., Asplund, M., Gustafsson, B., \& Yong, D. 2009, ApJ, 704, L66

Meléndez, J., Casagrande, L., Ramírez, I., Asplund, M., \& Schuster, W. J. 2010, A\&A, 515, L3

Meléndez, J., Bergemann, M., Cohen, J. G., et al. 2012, A\&A, 543, A29

Meyer, B. S. 1994, ARA\&A, 32, 153

Meynet, G., Hirschi, R., Ekstrom, S., et al. 2010, A\&A, 521, A30

Monroe, T. R., Meléndez, J., Ramírez, I., et al. 2013, ApJ, 774, L32

Nissen, P. E. 2015, A\&A, 579, A52

Nissen, P. E., \& Schuster, W. J. 2010, A\&A, 511, L10

Nissen, P. E., \& Schuster, W. J. 2011, A\&A, 530, A15

Nomoto, K., Kobayashi, C., \& Tominaga, N. 2013, ARA\&A, 51, 457

Norris, J. E., Ryan, S. G., \& Beers, T. C. 2001, ApJ, 561, 1034

Norris, J. E., Yong, D., Bessell, M. S., et al. 2013, ApJ, 762, 28

O'Malley, E., McWilliam, A., Chaboyer, B., \& Thompson, I. 2017, ApJ, 838, 90

Önehag, A., Gustafsson, B., \& Korn, A. 2014, A\&A, 562, A102

Pignatari, M., Gallino, R., Heil, M., et al. 2010, ApJ, 710, 1557

Placco, V. M., Beers, T. C., Roederer, I. U., et al. 2014, ApJ, 790, 34

Placco, V. M., Beers, T. C., Reggiani, H., \& Meléndez, J. 2016a, ApJ, 829, L24

Placco, V. M., Frebel, A., Beers, T. C., et al. 2016b, ApJ, 833, 21

Pllumbi, E., Tamborra, I., Wanajo, S., Janka, H.-T., \& Hüdepohl, L. 2015, ApJ, 808,188

Ramírez, I., \& Meléndez, J. 2005, ApJ, 626, 446

Ramírez, I., Meléndez, J., \& Asplund, M. 2009, A\&A, 508, L17

Ramírez, I., Meléndez, J., \& Chanamé, J. 2012, ApJ, 757, 164

Ramírez, I., Allende Prieto, C., \& Lambert, D. L. 2013, ApJ, 764, 78

Ramírez, I., Meléndez, J., Bean, J., et al. 2014, A\&A, 572, A48

Ramírez, I., Khanal, S., Aleo, P., et al. 2015, ApJ, 808, 13

Reggiani, H., Meléndez, J., Yong, D., Ramírez, I., \& Asplund, M. 2016, A\&A, 586, A67 
H. Reggiani et al.: Constraining cosmic scatter in the Galactic halo through a differential analysis of metal-poor stars

Richard, O., Michaud, G., \& Richer, J. 2005, ApJ, 619, 538

Roederer, I. U., Karakas, A. I., Pignatari, M., \& Herwig, F. 2016, ApJ, 821, 37

Ryan, S. G., Norris, J. E., \& Beers, T. C. 1996, ApJ, 471, 254

Ryan, S. G., Beers, T. C., Kajino, T., \& Rosolankova, K. 2001, ApJ, 547, 231

Ryan, S. G., Gregory, S. G., Kolb, U., Beers, T. C., \& Kajino, T. 2002, ApJ, 571, 501

Salvati, L., Pagano, L., Lattanzi, M., Gerbino, M., \& Melchiorri, A. 2016, J. Cosmol. Astropart. Phys., 8, 022

Santucci, R. M., Placco, V. M., Rossi, S., et al. 2015, ApJ, 801, 116

Scholz, R.-D., Heber, U., Heuser, C., et al. 2015, A\&A, 574, A96

Schuster, W. J., Moreno, E., Nissen, P. E., \& Pichardo, B. 2012, A\&A, 538, A21

Searle, L., \& Zinn, R. 1978, ApJ, 225, 357

Shigeyama, T., \& Tsujimoto, T. 1998, ApJ, 507, L135

Sills, A., Karakas, A., \& Lattanzio, J. 2009, ApJ, 692, 1411

Sitnova, T., Zhao, G., Mashonkina, L., et al. 2015, ApJ, 808, 148

Sneden, C. A. 1973, Ph.D. Thesis, University of Texas, Austin

Sneden, C., Cowan, J. J., Kobayashi, C., et al. 2016, ApJ, 817, 53
Spina, L., Meléndez, J., Karakas, A. I., et al. 2016, A\&A, 593, A125 Spite, F., \& Spite, M. 1982, A\&A, 115, 357

Spite, M., Caffau, E., Bonifacio, P., et al. 2013, A\&A, 552, A107

Spite, M., Spite, F., Caffau, E., \& Bonifacio, P. 2015, A\&A, 582, A74

Suda, T., Katsuta, Y., Yamada, S., et al. 2008, PASJ, 60, 1159

Suda, T., Yamada, S., Katsuta, Y., et al. 2011, MNRAS, 412, 843

Thielemann, F.-K., Arcones, A., Käppeli, R., et al. 2011, Prog. Part. Nucl. Phys., 66, 346

Tucci Maia, M., Meléndez, J., \& Ramírez, I. 2014, ApJ, 790, L25

Tucci Maia, M., Meléndez, J., Castro, M., et al. 2015, A\&A, 576, L10

Umeda, H., \& Nomoto, K. 2002, ApJ, 565, 385

Wood, M. P., Lawler, J. E., Den Hartog, E. A., Sneden, C., \& Cowan, J. J. 2014, ApJS, 214, 18

Yamada, S., Suda, T., Komiya, Y., Aoki, W., \& Fujimoto, M. Y. 2013, MNRAS, 436, 1362

Yi, S., Demarque, P., Kim, Y.-C., et al. 2001, ApJS, 136, 417

Yong, D., Meléndez, J., Grundahl, F., et al. 2013, MNRAS, 434, 3542

Zhao, G., Mashonkina, L., Yan, H. L., et al. 2016, ApJ, 833, 225 


\section{Appendix A: Stellar parameters and chemical abundances}

Table A.1. Stellar parameters for each star.

\begin{tabular}{lrrrrrrrr}
\hline \hline Star & $\begin{array}{r}T_{\text {eff }} \\
(\mathrm{K})\end{array}$ & $\begin{array}{r}\sigma T_{\text {eff }} \\
(\mathrm{K})\end{array}$ & $\begin{array}{r}\log g \\
(\mathrm{dex})\end{array}$ & $\begin{array}{r}\sigma \log g \\
(\mathrm{dex})\end{array}$ & $\begin{array}{r}v_{\mathrm{T}} \\
\left(\mathrm{km} \mathrm{s}^{-1}\right)\end{array}$ & $\begin{array}{r}\sigma v_{\mathrm{T}} \\
\left(\mathrm{km} \mathrm{s}^{-1}\right)\end{array}$ & $\begin{array}{r}{[\mathrm{Fe} / \mathrm{H}]} \\
(\mathrm{dex})\end{array}$ & $\begin{array}{r}\sigma[\mathrm{Fe} / \mathrm{H}] \\
(\mathrm{dex})\end{array}$ \\
\hline BD+20 3603 & 6229 & 50 & 4.09 & 0.08 & 1.29 & 0.04 & -2.179 & 0.042 \\
BD+24 1676 $^{1}$ & 6438 & 63 & 4.13 & 0.10 & 1.54 & 0.04 & -2.426 & 0.049 \\
BD+26 2621 & 6470 & 81 & 4.51 & 0.12 & 1.34 & 0.05 & -2.608 & 0.063 \\
BD-04 3208 & 6433 & 49 & 4.11 & 0.07 & 1.55 & 0.03 & -2.333 & 0.037 \\
BD-13 3442 & 6569 & 73 & 4.36 & 0.12 & 1.67 & 0.05 & -2.638 & 0.054 \\
CD-71 1234 & 6421 & 53 & 4.31 & 0.09 & 1.46 & 0.03 & -2.424 & 0.040 \\
BPS CS 22943-0095 & 6414 & 46 & 4.27 & 0.07 & 1.42 & 0.04 & -2.299 & 0.036 \\
G 126-521 & 6462 & 57 & 4.28 & 0.09 & 1.47 & 0.04 & -2.269 & 0.043 \\
HD 338529* & 6426 & 50 & 4.09 & 0.03 & 1.50 & 0.05 & -2.290 & 0.050 \\
HD 340279 & 6493 & 70 & 4.52 & 0.09 & 1.29 & 0.05 & -2.561 & 0.055 \\
LP 894-1 & 6378 & 53 & 4.26 & 0.09 & 1.37 & 0.03 & -2.178 & 0.041 \\
WISE J072543.88-235119.7 & 6160 & 45 & 4.42 & 0.09 & 1.30 & 0.04 & -2.366 & 0.038 \\
BD+01 3597 & 6435 & 44 & 4.04 & 0.07 & 1.57 & 0.03 & -1.937 & 0.035 \\
BD+02 4651 & 6241 & 43 & 3.89 & 0.09 & 1.49 & 0.03 & -1.808 & 0.036 \\
CD-48 2445 & 6453 & 50 & 4.23 & 0.03 & 1.50 & 0.05 & -1.960 & 0.050 \\
G 66-30 & 6638 & 47 & 4.36 & 0.09 & 1.52 & 0.05 & -1.473 & 0.038 \\
G 126-62 & 6145 & 90 & 3.91 & 0.18 & 1.13 & 0.15 & -1.611 & 0.097 \\
HD 59392 & 6056 & 72 & 3.72 & 0.11 & 1.28 & 0.10 & -1.688 & 0.075 \\
HD 74000 & 6341 & 39 & 4.19 & 0.06 & 1.46 & 0.03 & -2.020 & 0.031 \\
HD 84937 & 6513 & 44 & 4.17 & 0.06 & 1.61 & 0.04 & -2.129 & 0.032 \\
HD 108177 & 6107 & 50 & 4.04 & 0.06 & 1.17 & 0.07 & -1.768 & 0.050 \\
HD 110621 & 6182 & 56 & 3.9 & 0.11 & 1.34 & 0.07 & -1.653 & 0.054 \\
HD 122196 & 6052 & 52 & 3.66 & 0.07 & 1.44 & 0.05 & -1.855 & 0.048 \\
\hline
\end{tabular}

Notes. The standard stars are in bold and with *. Superscript numbers 1 and 2 represent the samples compared to the standard stars HD 338529 and CD-48 2445, respectively.

Table A.2. Abundances of the $\alpha$-elements.

\begin{tabular}{|c|c|c|c|c|c|c|c|c|}
\hline Star & {$[\mathrm{Mg} / \mathrm{Fe}]$} & $\sigma_{-}[\mathrm{Mg} / \mathrm{Fe}]$ & {$[\mathrm{Si} / \mathrm{Fe}]$} & $\sigma_{-}[\mathrm{Si} / \mathrm{Fe}]$ & {$[\mathrm{Ca} / \mathrm{Fe}]$} & $\sigma_{-}[\mathrm{Ca} / \mathrm{Fe}]$ & {$[\mathrm{Ti} / \mathrm{Fe}]$} & $\sigma_{-}[\mathrm{Ti} / \mathrm{Fe}]$ \\
\hline $\mathrm{BD}+203603$ & 0.343 & 0.062 & 0.203 & 0.081 & 0.369 & 0.056 & 0.358 & 0.06 \\
\hline $\mathrm{BD}+241676$ & 0.343 & 0.072 & 0.125 & 0.08 & 0.414 & 0.063 & 0.482 & 0.069 \\
\hline $\mathrm{BD}+262621$ & 0.319 & 0.088 & 0.116 & 0.101 & 0.382 & 0.083 & 0.426 & 0.089 \\
\hline BD-04 3208 & 0.361 & 0.055 & 0.153 & 0.064 & 0.455 & 0.051 & 0.507 & 0.056 \\
\hline BD-13 3442 & 0.401 & 0.084 & 0.049 & 0.081 & 0.439 & 0.07 & 0.574 & 0.075 \\
\hline CD-71 1234 & 0.272 & 0.064 & 0.165 & 0.069 & 0.354 & 0.054 & 0.388 & 0.059 \\
\hline BPS CS 22943-0095 & 0.397 & 0.052 & 0.23 & 0.064 & 0.429 & 0.048 & 0.491 & 0.055 \\
\hline G $126-52$ & 0.25 & 0.063 & 0.09 & 0.073 & 0.358 & 0.058 & 0.443 & 0.064 \\
\hline HD 338529 & 0.386 & 0 & 0.232 & 0 & 0.442 & 0 & 0.465 & 0 \\
\hline HD 340279 & 0.202 & 0.076 & 0.022 & 0.084 & 0.289 & 0.071 & 0.358 & 0.079 \\
\hline LP 894-1 & 0.253 & 0.062 & 0.135 & 0.075 & 0.337 & 0.055 & 0.389 & 0.061 \\
\hline WISE J072543.88-235119.7 & 0.314 & 0.059 & 0.226 & 0.072 & 0.28 & 0.049 & 0.326 & 0.059 \\
\hline $\mathrm{BD}+013597$ & 0.3 & 0.055 & 0.164 & 0.063 & 0.403 & 0.046 & 0.467 & 0.058 \\
\hline $\mathrm{BD}+024651$ & 0.33 & 0.059 & 0.184 & 0.067 & 0.395 & 0.048 & 0.391 & 0.057 \\
\hline CD-48 2445 & 0.249 & 0 & 0.142 & 0 & 0.346 & 0 & 0.434 & 0 \\
\hline G 66-30 & 0.212 & 0.087 & 0.114 & 0.069 & 0.278 & 0.051 & 0.319 & 0.058 \\
\hline G 126-62 & 0.26 & 0.152 & 0.08 & 0.164 & 0.379 & 0.129 & 0.344 & 0.125 \\
\hline HD 59392 & 0.315 & 0.116 & 0.16 & 0.123 & 0.365 & 0.098 & 0.362 & 0.096 \\
\hline HD 74000 & 0.34 & 0.054 & 0.228 & 0.058 & 0.347 & 0.041 & 0.376 & 0.044 \\
\hline HD 84937 & 0.288 & 0.049 & 0.165 & 0.057 & 0.375 & 0.043 & 0.475 & 0.052 \\
\hline HD 108177 & 0.38 & 0.082 & 0.204 & 0.086 & 0.361 & 0.066 & 0.353 & 0.062 \\
\hline HD 110621 & 0.392 & 0.086 & 0.249 & 0.096 & 0.395 & 0.072 & 0.389 & 0.072 \\
\hline HD 122196 & 0.24 & 0.03 & 0.128 & 0.068 & 0.286 & 0.024 & 0.275 & 0.036 \\
\hline
\end{tabular}


H. Reggiani et al.: Constraining cosmic scatter in the Galactic halo through a differential analysis of metal-poor stars

Table A.3. Abundances of the light odd $\mathrm{Z}$ elements.

\begin{tabular}{lrrrrrr}
\hline \hline Star & {$[\mathrm{Na} / \mathrm{Fe}]_{\text {NLTE }}$} & $\sigma_{-}[\mathrm{Na} / \mathrm{Fe}]$ & {$\left[\mathrm{Al}_{\mathrm{NLTE}} / \mathrm{Fe}\right]$} & $\sigma_{-}\left[\mathrm{Al}_{\mathrm{NLTE}} / \mathrm{Fe}\right]$ & {$[\mathrm{Sc} / \mathrm{Fe}]$} & $\sigma_{-}[\mathrm{Sc} / \mathrm{Fe}]$ \\
\hline BD+203603 & -0.276 & 0.089 & -0.154 & 0.083 & 0.252 & 0.060 \\
BD+24 1676 & -0.123 & 0.124 & -0.095 & 0.072 & 0.360 & 0.071 \\
BD+26 2621 & -0.307 & 0.091 & -0.160 & 0.096 & 0.238 & 0.112 \\
BD-04 3208 & -0.216 & 0.054 & -0.092 & 0.059 & 0.354 & 0.057 \\
BD-13 3442 & -0.103 & 0.076 & -0.076 & 0.077 & 0.493 & 0.085 \\
CD-71 1234 & -0.243 & 0.072 & -0.221 & 0.064 & 0.256 & 0.066 \\
BPS CS 22943-0095 & -0.043 & 0.055 & -0.082 & 0.063 & 0.339 & 0.053 \\
G 126-52 & -0.159 & 0.067 & -0.224 & 0.067 & 0.316 & 0.065 \\
HD 338529 & -0.153 & 0 & -0.093 & 0 & 0.345 & 0 \\
HD 340279 & -0.255 & 0.158 & -0.131 & 0.077 & 0.040 & 0.150 \\
LP 894-1 & -0.344 & 0.090 & -0.233 & 0.062 & 0.245 & 0.062 \\
WISE J072543.88-235119.7 & -0.388 & 0.061 & -0.137 & 0.061 & 0.207 & 0.063 \\
BD+01 3597 & -0.255 & 0.062 & -0.108 & 0.054 & 0.369 & 0.052 \\
BD+02 4651 & -0.215 & 0.091 & -0.174 & 0.115 & 0.332 & 0.057 \\
CD-48 2445 & -0.516 & 0 & -0.212 & 0 & 0.319 & 0 \\
G 66-30 & -0.279 & 0.092 & -0.242 & 0.060 & 0.264 & 0.057 \\
G 126-62 & -0.243 & 0.183 & -0.162 & 0.181 & 0.351 & 0.141 \\
HD 59392 & -0.203 & 0.185 & -0.090 & 0.225 & 0.331 & 0.116 \\
HD 74000 & 0.061 & 0.083 & 0.094 & 0.079 & 0.242 & 0.047 \\
HD 84937 & -0.234 & 0.125 & -0.112 & 0.057 & 0.332 & 0.054 \\
HD 108177 & -0.074 & 0.114 & -0.053 & 0.134 & 0.264 & 0.069 \\
HD 110621 & -0.160 & 0.120 & 0.006 & 0.167 & 0.374 & 0.085 \\
HD 122196 & -0.257 & 0.058 & -0.183 & 0.149 & 0.205 & 0.034 \\
\hline
\end{tabular}




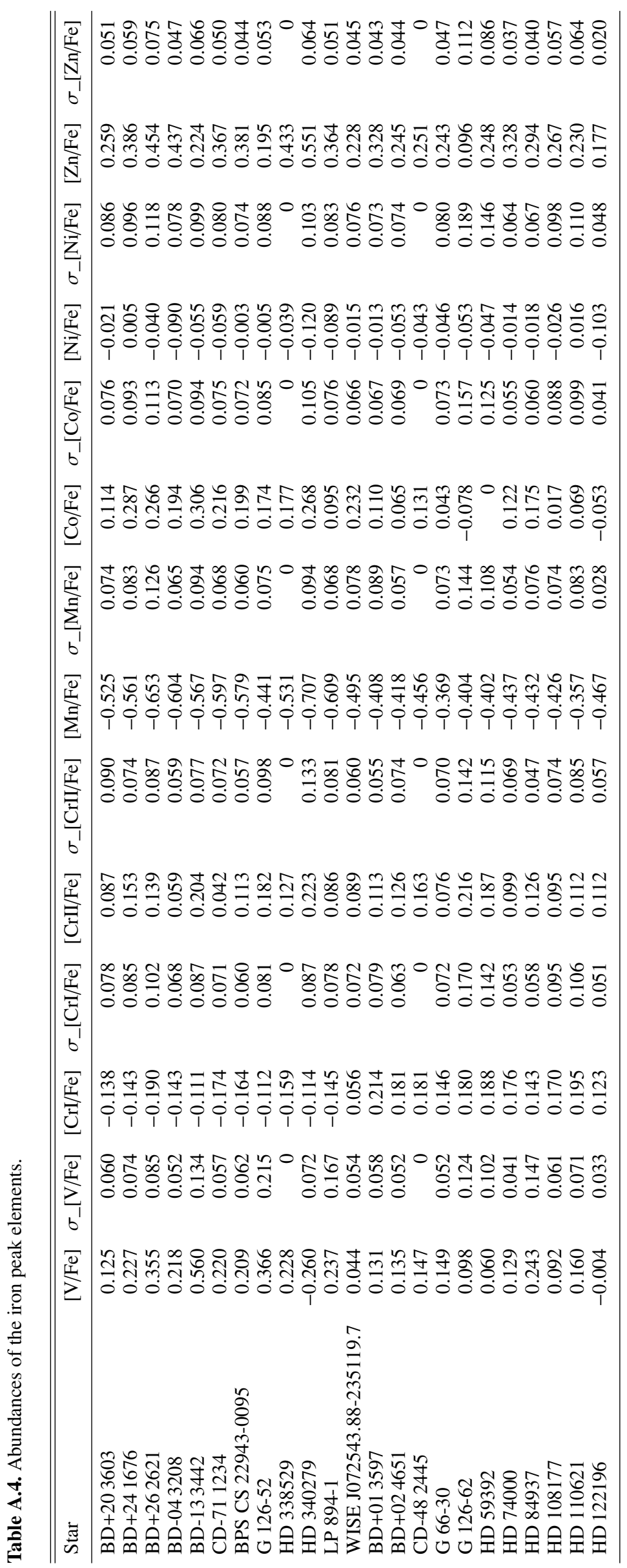


H. Reggiani et al.: Constraining cosmic scatter in the Galactic halo through a differential analysis of metal-poor stars

Table A.5. Abundances of the neutron-capture elements.

\begin{tabular}{lrrrrrrrr}
\hline \hline Star & {$[\mathrm{Sr} / \mathrm{Fe}]$} & $\sigma_{-}[\mathrm{Sr} / \mathrm{Fe}]$ & {$[\mathrm{Y} / \mathrm{Fe}]$} & $\sigma_{-}[\mathrm{Y} / \mathrm{Fe}]$ & {$[\mathrm{Zr} / \mathrm{Fe}]$} & $\sigma_{-}[\mathrm{Zr} / \mathrm{Fe}]$ & {$[\mathrm{Ba} / \mathrm{Fe}]$} & $\sigma_{-}[\mathrm{Ba} / \mathrm{Fe}]$ \\
\hline BD+20 3603 & 0.026 & 0.065 & -0.235 & 0.064 & 0.376 & 0.091 & -0.316 & 0.067 \\
BD+24 1676 & 0.094 & 0.080 & -0.009 & 0.084 & 0.648 & 0.067 & -0.268 & 0.069 \\
BD+26 2621 & -0.287 & 0.091 & -0.181 & 0.109 & 0.410 & 0.087 & -0.590 & 0.091 \\
BD-04 3208 & 0.179 & 0.057 & -0.013 & 0.057 & 0.532 & 0.082 & -0.212 & 0.066 \\
BD-13 3442 & 0.184 & 0.086 & 0.124 & 0.090 & 0.636 & 0.125 & -0.532 & 0.079 \\
CD-71 1234 & -0.616 & 0.068 & -0.652 & 0.068 & 0.617 & 0.268 & -0.441 & 0.068 \\
BPS CS 22943-0095 & 0.321 & 0.058 & 0.119 & 0.064 & 0.711 & 0.094 & -0.075 & 0.074 \\
G 126-52 & -0.018 & 0.07 & -0.032 & 0.089 & 0.840 & 0.060 & -0.134 & 0.062 \\
HD 338529 & 0.129 & 0 & -0.040 & 0 & 0.559 & 0 & -0.058 & 0 \\
HD 340279 & 0.359 & 0.103 & 0.321 & 0.076 & 0.623 & 0.095 & 0.394 & 0.078 \\
LP 894-1 & 0.089 & 0.067 & -0.137 & 0.071 & 0.567 & 0.088 & 0.024 & 0.067 \\
WISE J072543.88-235119.7 & -0.006 & 0.058 & -0.022 & 0.061 & 0.473 & 0.071 & 0.175 & 0.060 \\
BD +013597 & 0.162 & 0.052 & 0.023 & 0.059 & 0.527 & 0.055 & 0.014 & 0.005 \\
BD +024651 & 0.139 & 0.057 & -0.077 & 0.061 & 0.459 & 0.081 & 0.206 & 0.061 \\
CD-48 2445 & 0.207 & 0 & 0.069 & 0 & 0.673 & 0 & 0.334 & 0 \\
G 66-30 & 0.109 & 0.070 & -0.153 & 0.059 & 0.496 & 0.083 & 0.140 & 0.066 \\
G 126-62 & 0.134 & 0.151 & -0.141 & 0.141 & 0.444 & 0.155 & 0.137 & 0.153 \\
HD 59392 & 0.242 & 0.109 & 0.043 & 0.112 & 0.576 & 0.105 & 0.380 & 0.129 \\
HD 74000 & 0.283 & 0.048 & 0.028 & 0.049 & 0.592 & 0.075 & 0.221 & 0.061 \\
HD 84937 & 0.075 & 0.062 & -0.027 & 0.052 & 0.552 & 0.106 & -0.043 & 0.070 \\
HD 108177 & 0.171 & 0.076 & -0.083 & 0.077 & 0.484 & 0.119 & 0.043 & 0.095 \\
HD 110621 & 0.281 & 0.085 & 0.011 & 0.092 & 0.560 & 0.101 & 0.272 & 0.093 \\
HD 122196 & -0.007 & 0.046 & -0.298 & 0.036 & 0.309 & 0.079 & 0.025 & 0.042 \\
\hline
\end{tabular}

Table A.6. Lithium abundances and mass estimates for our stars.

\begin{tabular}{lrrr}
\hline \hline Star & $\begin{array}{r}A(\mathrm{Li}) \\
(\mathrm{dex})\end{array}$ & $\begin{array}{r}\sigma A(\mathrm{Li}) \\
(\mathrm{dex})\end{array}$ & $\begin{array}{r}\text { Mass } \\
\left(M_{\odot}\right)\end{array}$ \\
\hline BD+20 3603 & 2.169 & 0.036 & 0.769 \\
BD+24 1676 & 2.266 & 0.044 & 0.774 \\
BD+26 2621 & 2.255 & 0.054 & 0.805 \\
BD-04 3208 & 2.283 & 0.035 & 0.777 \\
BD-13 3442 & 2.306 & 0.047 & 0.803 \\
CD-71 1234 & 2.286 & 0.037 & 0.773 \\
BPS CS 22943-0095 & 2.299 & 0.032 & 0.771 \\
G 126-52 & 2.268 & 0.039 & 0.785 \\
HD 338529 & 2.264 & 0.035 & 0.780 \\
LP 894-1 & 2.201 & 0.036 & 0.771 \\
WISE J072543.88-235119.7 & 2.266 & 0.033 & 0.737 \\
BD+01 3597 & 2.281 & 0.030 & 0.822 \\
BD+02 4651 & 2.289 & 0.031 & 0.852 \\
CD-48 2445 & 2.311 & 0.032 & 0.798 \\
G 126-62 & 2.188 & 0.066 & 0.868 \\
HD 59392 & 2.293 & 0.052 & 0.923 \\
HD 74000 & 2.280 & 0.027 & 0.770 \\
HD 84937 & 2.367 & 0.029 & 0.799 \\
HD 108177 & 2.212 & 0.036 & 0.784 \\
HD 110621 & 2.295 & 0.041 & 0.859 \\
HD 122196 & 2.301 & 0.020 & 0.932 \\
\hline
\end{tabular}

Notes. We note that the two blue straggler stars are not included in this table. 


\section{Appendix B: Linelist}

Table B.1. Linelist used for the abundances determinations.

\begin{tabular}{|c|c|c|c|}
\hline $\begin{array}{r}\text { Wavelength } \\
(\AA)\end{array}$ & Species & $\begin{array}{r}\mathrm{EP} \\
(\mathrm{eV})\end{array}$ & $\begin{array}{r}\log (g f) \\
(\operatorname{dex})\end{array}$ \\
\hline 3902.95 & 26.0 & 1.56 & -0.47 \\
\hline 3906.48 & 26.0 & 0.11 & -2.24 \\
\hline 3917.18 & 26.0 & 0.99 & -2.16 \\
\hline 3920.26 & 26.0 & 0.12 & -1.75 \\
\hline 3922.91 & 26.0 & 0.05 & -1.65 \\
\hline 3927.92 & 26.0 & 0.11 & -1.52 \\
\hline 3930.30 & 26.0 & 0.09 & -1.49 \\
\hline 3940.88 & 26.0 & 0.96 & -2.60 \\
\hline 3949.95 & 26.0 & 2.18 & -1.25 \\
\hline 3977.74 & 26.0 & 2.20 & -1.12 \\
\hline 3997.39 & 26.0 & 2.73 & -0.48 \\
\hline 3998.05 & 26.0 & 2.69 & -0.91 \\
\hline 4005.24 & 26.0 & 1.56 & -0.61 \\
\hline 4021.87 & 26.0 & 2.76 & -0.73 \\
\hline 4045.81 & 26.0 & 1.49 & 0.28 \\
\hline 4063.59 & 26.0 & 1.56 & 0.06 \\
\hline 4071.74 & 26.0 & 1.61 & -0.02 \\
\hline 4118.55 & 26.0 & 3.57 & 0.22 \\
\hline 4134.68 & 26.0 & 2.83 & -0.65 \\
\hline 4143.42 & 26.0 & 3.05 & -0.20 \\
\hline 4143.87 & 26.0 & 1.56 & -0.51 \\
\hline 4147.67 & 26.0 & 1.49 & -2.10 \\
\hline 4154.50 & 26.0 & 2.83 & -0.69 \\
\hline 4154.81 & 26.0 & 3.37 & -0.40 \\
\hline 4156.80 & 26.0 & 2.83 & -0.81 \\
\hline 4175.64 & 26.0 & 2.85 & -0.83 \\
\hline 4181.76 & 26.0 & 2.83 & -0.37 \\
\hline 4187.04 & 26.0 & 2.45 & -0.55 \\
\hline 4187.80 & 26.0 & 2.43 & -0.55 \\
\hline 4191.43 & 26.0 & 2.47 & -0.67 \\
\hline 4199.10 & 26.0 & 3.05 & 0.16 \\
\hline 4202.03 & 26.0 & 1.49 & -0.71 \\
\hline 4216.18 & 26.0 & 0.00 & -3.36 \\
\hline 4222.21 & 26.0 & 2.45 & -0.97 \\
\hline 4227.43 & 26.0 & 3.33 & 0.27 \\
\hline 4233.60 & 26.0 & 2.48 & -0.60 \\
\hline 4238.81 & 26.0 & 3.40 & -0.23 \\
\hline 4250.12 & 26.0 & 2.47 & -0.41 \\
\hline 4250.79 & 26.0 & 1.56 & -0.71 \\
\hline 4260.47 & 26.0 & 2.40 & 0.11 \\
\hline 4271.15 & 26.0 & 2.45 & -0.35 \\
\hline 4271.76 & 26.0 & 1.49 & -0.16 \\
\hline 4282.40 & 26.0 & 2.18 & -0.78 \\
\hline 4375.93 & 26.0 & 0.00 & -3.03 \\
\hline 4383.55 & 26.0 & 1.49 & 0.20 \\
\hline 4404.75 & 26.0 & 1.56 & -0.14 \\
\hline 4427.31 & 26.0 & 0.05 & -2.92 \\
\hline 4442.34 & 26.0 & 2.20 & -1.26 \\
\hline 4459.12 & 26.0 & 2.18 & -1.28 \\
\hline 4461.65 & 26.0 & 0.09 & -3.21 \\
\hline 4466.55 & 26.0 & 2.83 & -0.60 \\
\hline 4494.56 & 26.0 & 2.20 & -1.14 \\
\hline 4871.32 & 26.0 & 2.87 & -0.36 \\
\hline
\end{tabular}

Notes. The linelist is formatted to be used with the radiative transfer code MOOG (Sneden 1973), and also include the hyperfine splitting, indicated by the negative wavelengths.
Table B.1. continued.

\begin{tabular}{|c|c|c|c|}
\hline $\begin{array}{r}\text { Wavelength } \\
(\AA)\end{array}$ & Species & $\begin{array}{r}\mathrm{EP} \\
(\mathrm{eV})\end{array}$ & $\begin{array}{r}\log (g f) \\
(\mathrm{dex})\end{array}$ \\
\hline 4872.14 & 26.0 & 2.88 & -0.57 \\
\hline 4890.76 & 26.0 & 2.88 & -0.39 \\
\hline 4891.49 & 26.0 & 2.85 & -0.11 \\
\hline 4918.99 & 26.0 & 2.87 & -0.34 \\
\hline 4920.50 & 26.0 & 2.83 & 0.07 \\
\hline 4957.30 & 26.0 & 2.85 & -0.41 \\
\hline 5049.82 & 26.0 & 2.28 & -1.36 \\
\hline 5133.69 & 26.0 & 4.18 & 0.14 \\
\hline 5139.25 & 26.0 & 3.00 & -0.74 \\
\hline 5139.46 & 26.0 & 2.94 & -0.51 \\
\hline 5162.27 & 26.0 & 4.18 & 0.02 \\
\hline 5171.60 & 26.0 & 1.49 & -1.79 \\
\hline 5191.46 & 26.0 & 3.04 & -0.55 \\
\hline 5194.94 & 26.0 & 1.56 & -2.09 \\
\hline 5216.27 & 26.0 & 1.61 & -2.15 \\
\hline 5226.86 & 26.0 & 3.04 & -0.56 \\
\hline 5227.19 & 26.0 & 1.56 & -1.23 \\
\hline 5232.94 & 26.0 & 2.94 & -0.06 \\
\hline 5266.56 & 26.0 & 3.00 & -0.39 \\
\hline 5328.04 & 26.0 & 0.92 & -1.47 \\
\hline 5328.53 & 26.0 & 1.56 & -1.85 \\
\hline 5369.96 & 26.0 & 4.37 & 0.54 \\
\hline 5383.37 & 26.0 & 4.31 & 0.65 \\
\hline 5397.13 & 26.0 & 0.92 & -1.99 \\
\hline 5405.78 & 26.0 & 0.99 & -1.84 \\
\hline 5415.20 & 26.0 & 4.39 & 0.64 \\
\hline 5424.07 & 26.0 & 4.32 & 0.52 \\
\hline 5429.70 & 26.0 & 0.96 & -1.88 \\
\hline 5434.52 & 26.0 & 1.01 & -2.12 \\
\hline 5455.61 & 26.0 & 1.01 & -2.09 \\
\hline 5497.52 & 26.0 & 1.01 & -2.85 \\
\hline 5506.78 & 26.0 & 0.99 & -2.80 \\
\hline 5572.84 & 26.0 & 3.40 & -0.28 \\
\hline 5586.76 & 26.0 & 3.37 & -0.12 \\
\hline 5615.64 & 26.0 & 3.33 & 0.05 \\
\hline 6230.72 & 26.0 & 2.56 & -1.28 \\
\hline 4178.86 & 26.1 & 2.58 & -2.51 \\
\hline 4233.17 & 26.1 & 2.58 & -1.97 \\
\hline 4508.29 & 26.1 & 2.86 & -2.44 \\
\hline 4923.93 & 26.1 & 2.89 & -1.26 \\
\hline 5018.44 & 26.1 & 2.89 & -1.10 \\
\hline 5197.58 & 26.1 & 3.23 & -2.22 \\
\hline 5234.63 & 26.1 & 3.22 & -2.28 \\
\hline 6707.82 & 3.0 & 0.00 & 0.17 \\
\hline 5889.95 & 11.0 & 0.00 & 0.12 \\
\hline 5895.92 & 11.0 & 0.00 & -0.18 \\
\hline 3329.92 & 12.0 & 2.71 & -1.93 \\
\hline 3336.67 & 12.0 & 2.72 & -1.23 \\
\hline 3986.75 & 12.0 & 4.35 & -1.44 \\
\hline 4167.27 & 12.0 & 4.35 & -1.00 \\
\hline 4351.91 & 12.0 & 4.35 & -0.83 \\
\hline 5167.32 & 12.0 & 2.71 & -1.03 \\
\hline 5172.68 & 12.0 & 2.71 & -0.40 \\
\hline 5183.60 & 12.0 & 2.72 & -0.18 \\
\hline 5528.41 & 12.0 & 4.35 & -0.62 \\
\hline 3944.01 & 13.0 & 0.00 & -0.62 \\
\hline 3961.52 & 13.0 & 0.01 & -0.32 \\
\hline 3905.52 & 14.0 & 1.91 & -0.74 \\
\hline
\end{tabular}


H. Reggiani et al.: Constraining cosmic scatter in the Galactic halo through a differential analysis of metal-poor stars

Table B.1. continued.

\begin{tabular}{|c|c|c|c|}
\hline $\begin{array}{r}\text { Wavelength } \\
(\AA)\end{array}$ & Species & $\begin{array}{r}\mathrm{EP} \\
(\mathrm{eV})\end{array}$ & $\begin{array}{r}\log (g f) \\
(\text { dex })\end{array}$ \\
\hline 4226.73 & 20.0 & 0.00 & 0.24 \\
\hline 4283.01 & 20.0 & 1.89 & -0.29 \\
\hline 4289.37 & 20.0 & 1.88 & -0.39 \\
\hline 4298.99 & 20.0 & 1.89 & -0.51 \\
\hline 4302.53 & 20.0 & 1.90 & 0.29 \\
\hline 4318.65 & 20.0 & 1.90 & -0.30 \\
\hline 4425.44 & 20.0 & 1.88 & -0.36 \\
\hline 4435.68 & 20.0 & 1.89 & -0.52 \\
\hline 4454.78 & 20.0 & 1.90 & 0.26 \\
\hline 4455.89 & 20.0 & 1.90 & -0.41 \\
\hline 5265.56 & 20.0 & 2.52 & -0.15 \\
\hline 5588.75 & 20.0 & 2.53 & 0.36 \\
\hline 5594.46 & 20.0 & 2.52 & 0.10 \\
\hline 5857.45 & 20.0 & 2.93 & 0.24 \\
\hline 6102.72 & 20.0 & 1.88 & -0.79 \\
\hline 6122.22 & 20.0 & 1.89 & -0.39 \\
\hline 6162.17 & 20.0 & 1.90 & -0.17 \\
\hline 6439.08 & 20.0 & 2.53 & 0.39 \\
\hline 6493.78 & 20.0 & 2.52 & -0.11 \\
\hline 3736.90 & 20.1 & 3.15 & -0.17 \\
\hline 3736.90 & 20.1 & 3.15 & -0.17 \\
\hline 3353.72 & 21.1 & 0.37 & -0.35 \\
\hline-3353.72 & 21.1 & 0.37 & -0.52 \\
\hline-3353.72 & 21.1 & 0.37 & -1.09 \\
\hline-3353.73 & 21.1 & 0.37 & -0.73 \\
\hline-3353.73 & 21.1 & 0.37 & -0.91 \\
\hline-3353.73 & 21.1 & 0.37 & -2.09 \\
\hline-3353.73 & 21.1 & 0.37 & -1.00 \\
\hline-3353.73 & 21.1 & 0.37 & -0.88 \\
\hline-3353.73 & 21.1 & 0.37 & -1.67 \\
\hline-3353.73 & 21.1 & 0.37 & -1.42 \\
\hline-3353.73 & 21.1 & 0.37 & -0.93 \\
\hline-3353.73 & 21.1 & 0.37 & -1.44 \\
\hline-3353.73 & 21.1 & 0.37 & -1.12 \\
\hline-3353.73 & 21.1 & 0.37 & -1.29 \\
\hline-3353.73 & 21.1 & 0.37 & -1.20 \\
\hline 3368.93 & 21.1 & 0.01 & -2.00 \\
\hline-3368.93 & 21.1 & 0.01 & -1.30 \\
\hline-3368.93 & 21.1 & 0.01 & -0.81 \\
\hline-3368.94 & 21.1 & 0.01 & -1.56 \\
\hline-3368.94 & 21.1 & 0.01 & -1.19 \\
\hline-3368.94 & 21.1 & 0.01 & -1.11 \\
\hline-3368.94 & 21.1 & 0.01 & -1.29 \\
\hline-3368.94 & 21.1 & 0.01 & -1.31 \\
\hline-3368.94 & 21.1 & 0.01 & -1.56 \\
\hline 3572.52 & 21.1 & 0.02 & -1.08 \\
\hline-3572.52 & 21.1 & 0.02 & -0.28 \\
\hline-3572.52 & 21.1 & 0.02 & -0.89 \\
\hline-3572.52 & 21.1 & 0.02 & -0.50 \\
\hline-3572.52 & 21.1 & 0.02 & -1.08 \\
\hline-3572.53 & 21.1 & 0.02 & -0.84 \\
\hline-3572.53 & 21.1 & 0.02 & -0.79 \\
\hline-3572.53 & 21.1 & 0.02 & -0.89 \\
\hline-3572.53 & 21.1 & 0.02 & -0.86 \\
\hline-3572.53 & 21.1 & 0.02 & -1.18 \\
\hline-3572.53 & 21.1 & 0.02 & -0.84 \\
\hline-3572.53 & 21.1 & 0.02 & -0.96 \\
\hline-3572.53 & 21.1 & 0.02 & -1.81 \\
\hline-3572.53 & 21.1 & 0.02 & -0.86 \\
\hline
\end{tabular}

Table B.1. continued.

\begin{tabular}{|c|c|c|c|}
\hline $\begin{array}{r}\text { Wavelength } \\
(\AA)\end{array}$ & Species & $\begin{array}{r}\mathrm{EP} \\
(\mathrm{eV})\end{array}$ & $\begin{array}{r}\log (g f) \\
(\text { dex })\end{array}$ \\
\hline-3572.53 & 21.1 & 0.02 & -1.18 \\
\hline-3572.53 & 21.1 & 0.02 & -0.96 \\
\hline-3572.53 & 21.1 & 0.02 & -1.66 \\
\hline-3572.53 & 21.1 & 0.02 & -1.18 \\
\hline 3576.34 & 21.1 & 0.01 & -1.07 \\
\hline-3576.34 & 21.1 & 0.01 & -0.50 \\
\hline-3576.34 & 21.1 & 0.01 & -0.91 \\
\hline-3576.34 & 21.1 & 0.01 & -0.89 \\
\hline-3576.34 & 21.1 & 0.01 & -1.07 \\
\hline-3576.34 & 21.1 & 0.01 & -0.92 \\
\hline-3576.34 & 21.1 & 0.01 & -1.59 \\
\hline-3576.34 & 21.1 & 0.01 & -0.91 \\
\hline-3576.34 & 21.1 & 0.01 & -1.09 \\
\hline-3576.34 & 21.1 & 0.01 & -3.02 \\
\hline-3576.34 & 21.1 & 0.01 & -0.92 \\
\hline-3576.34 & 21.1 & 0.01 & -1.27 \\
\hline-3576.34 & 21.1 & 0.01 & -1.09 \\
\hline 3590.47 & 21.1 & 0.02 & -2.89 \\
\hline-3590.47 & 21.1 & 0.02 & -1.89 \\
\hline-3590.47 & 21.1 & 0.02 & -1.15 \\
\hline-3590.47 & 21.1 & 0.02 & -2.48 \\
\hline-3590.47 & 21.1 & 0.02 & -1.71 \\
\hline-3590.47 & 21.1 & 0.02 & -1.33 \\
\hline-3590.48 & 21.1 & 0.02 & -2.24 \\
\hline-3590.48 & 21.1 & 0.02 & -1.68 \\
\hline-3590.48 & 21.1 & 0.02 & -1.53 \\
\hline-3590.48 & 21.1 & 0.02 & -2.10 \\
\hline-3590.48 & 21.1 & 0.02 & -1.74 \\
\hline-3590.48 & 21.1 & 0.02 & -1.80 \\
\hline-3590.48 & 21.1 & 0.02 & -2.00 \\
\hline-3590.48 & 21.1 & 0.02 & -1.92 \\
\hline-3590.48 & 21.1 & 0.02 & -2.22 \\
\hline 3613.82 & 21.1 & 0.02 & -0.13 \\
\hline-3613.83 & 21.1 & 0.02 & -0.25 \\
\hline-3613.83 & 21.1 & 0.02 & -1.06 \\
\hline-3613.83 & 21.1 & 0.02 & -0.39 \\
\hline-3613.83 & 21.1 & 0.02 & -0.86 \\
\hline-3613.83 & 21.1 & 0.02 & -2.28 \\
\hline-3613.83 & 21.1 & 0.02 & -0.55 \\
\hline-3613.83 & 21.1 & 0.02 & -0.79 \\
\hline-3613.83 & 21.1 & 0.02 & -1.87 \\
\hline-3613.83 & 21.1 & 0.02 & -0.73 \\
\hline-3613.83 & 21.1 & 0.02 & -0.80 \\
\hline-3613.83 & 21.1 & 0.02 & -1.66 \\
\hline-3613.84 & 21.1 & 0.02 & -0.97 \\
\hline-3613.84 & 21.1 & 0.02 & -0.86 \\
\hline-3613.84 & 21.1 & 0.02 & -1.55 \\
\hline-3613.84 & 21.1 & 0.02 & -1.31 \\
\hline-3613.84 & 21.1 & 0.02 & -0.98 \\
\hline-3613.84 & 21.1 & 0.02 & -1.53 \\
\hline-3613.84 & 21.1 & 0.02 & -1.16 \\
\hline-3613.84 & 21.1 & 0.02 & -1.64 \\
\hline 3645.30 & 21.1 & 0.02 & -2.17 \\
\hline-3645.30 & 21.1 & 0.02 & -1.37 \\
\hline-3645.31 & 21.1 & 0.02 & -1.98 \\
\hline-3645.31 & 21.1 & 0.02 & -1.59 \\
\hline-3645.31 & 21.1 & 0.02 & -2.17 \\
\hline-3645.31 & 21.1 & 0.02 & -1.93 \\
\hline-3645.31 & 21.1 & 0.02 & -1.88 \\
\hline
\end{tabular}


Table B.1. continued.

\begin{tabular}{|c|c|c|c|}
\hline $\begin{array}{r}\text { Wavelength } \\
(\AA)\end{array}$ & Species & $\begin{array}{r}\mathrm{EP} \\
(\mathrm{eV})\end{array}$ & $\begin{array}{r}\log (g f) \\
(\text { dex })\end{array}$ \\
\hline-3645.31 & 21.1 & 0.02 & -1.98 \\
\hline-3645.32 & 21.1 & 0.02 & -1.95 \\
\hline-3645.32 & 21.1 & 0.02 & -2.27 \\
\hline-3645.32 & 21.1 & 0.02 & -1.93 \\
\hline-3645.32 & 21.1 & 0.02 & -2.05 \\
\hline-3645.32 & 21.1 & 0.02 & -2.90 \\
\hline-3645.32 & 21.1 & 0.02 & -1.95 \\
\hline-3645.32 & 21.1 & 0.02 & -2.27 \\
\hline-3645.32 & 21.1 & 0.02 & -2.05 \\
\hline-3645.32 & 21.1 & 0.02 & -2.75 \\
\hline-3645.32 & 21.1 & 0.02 & -2.27 \\
\hline 3651.78 & 21.1 & 0.08 & -1.80 \\
\hline-3651.78 & 21.1 & 0.08 & -1.23 \\
\hline-3651.79 & 21.1 & 0.08 & -1.64 \\
\hline-3651.79 & 21.1 & 0.08 & -1.62 \\
\hline-3651.79 & 21.1 & 0.08 & -1.80 \\
\hline-3651.80 & 21.1 & 0.08 & -1.65 \\
\hline-3651.80 & 21.1 & 0.08 & -2.32 \\
\hline-3651.80 & 21.1 & 0.08 & -1.64 \\
\hline-3651.81 & 21.1 & 0.08 & -1.82 \\
\hline-3651.81 & 21.1 & 0.08 & -3.75 \\
\hline-3651.81 & 21.1 & 0.08 & -1.65 \\
\hline-3651.81 & 21.1 & 0.08 & -2.00 \\
\hline-3651.81 & 21.1 & 0.08 & -1.82 \\
\hline 4246.81 & 21.1 & 0.32 & -0.88 \\
\hline-4246.81 & 21.1 & 0.32 & -0.31 \\
\hline-4246.82 & 21.1 & 0.32 & -0.72 \\
\hline-4246.82 & 21.1 & 0.32 & -0.70 \\
\hline-4246.82 & 21.1 & 0.32 & -0.88 \\
\hline-4246.83 & 21.1 & 0.32 & -0.73 \\
\hline-4246.83 & 21.1 & 0.32 & -1.40 \\
\hline-4246.83 & 21.1 & 0.32 & -0.72 \\
\hline-4246.83 & 21.1 & 0.32 & -0.90 \\
\hline-4246.83 & 21.1 & 0.32 & -2.83 \\
\hline-4246.83 & 21.1 & 0.32 & -0.73 \\
\hline-4246.83 & 21.1 & 0.32 & -1.08 \\
\hline-4246.83 & 21.1 & 0.32 & -0.90 \\
\hline 4314.08 & 21.1 & 0.62 & -2.89 \\
\hline-4314.08 & 21.1 & 0.62 & -1.68 \\
\hline-4314.08 & 21.1 & 0.62 & -2.49 \\
\hline-4314.08 & 21.1 & 0.62 & -0.75 \\
\hline-4314.08 & 21.1 & 0.62 & -1.47 \\
\hline-4314.08 & 21.1 & 0.62 & -2.28 \\
\hline-4314.08 & 21.1 & 0.62 & -0.87 \\
\hline-4314.08 & 21.1 & 0.62 & -2.17 \\
\hline-4314.08 & 21.1 & 0.62 & -1.41 \\
\hline-4314.08 & 21.1 & 0.62 & -1.41 \\
\hline-4314.08 & 21.1 & 0.62 & -1.01 \\
\hline-4314.09 & 21.1 & 0.62 & -2.15 \\
\hline-4314.09 & 21.1 & 0.62 & -1.16 \\
\hline-4314.09 & 21.1 & 0.62 & -2.25 \\
\hline-4314.09 & 21.1 & 0.62 & -1.59 \\
\hline-4314.09 & 21.1 & 0.62 & -1.47 \\
\hline-4314.09 & 21.1 & 0.62 & -1.35 \\
\hline-4314.09 & 21.1 & 0.62 & -1.78 \\
\hline-4314.09 & 21.1 & 0.62 & -1.92 \\
\hline-4314.09 & 21.1 & 0.62 & -1.58 \\
\hline 4320.73 & 21.1 & 0.61 & -2.59 \\
\hline-4320.73 & 21.1 & 0.61 & -2.18 \\
\hline-4320.73 & 21.1 & 0.61 & -1.80 \\
\hline
\end{tabular}

Table B.1. continued.

\begin{tabular}{|c|c|c|c|}
\hline $\begin{array}{r}\text { Wavelength } \\
(\AA)\end{array}$ & Species & $\begin{array}{r}\mathrm{EP} \\
(\mathrm{eV})\end{array}$ & $\begin{array}{r}\log (g f) \\
(\operatorname{dex})\end{array}$ \\
\hline-4320.73 & 21.1 & 0.61 & -1.94 \\
\hline-4320.73 & 21.1 & 0.61 & -1.70 \\
\hline-4320.73 & 21.1 & 0.61 & -1.59 \\
\hline-4320.73 & 21.1 & 0.61 & -1.62 \\
\hline-4320.73 & 21.1 & 0.61 & -1.44 \\
\hline-4320.73 & 21.1 & 0.61 & -1.38 \\
\hline-4320.73 & 21.1 & 0.61 & -1.41 \\
\hline-4320.73 & 21.1 & 0.61 & -1.92 \\
\hline-4320.73 & 21.1 & 0.61 & -1.50 \\
\hline-4320.73 & 21.1 & 0.61 & -1.23 \\
\hline-4320.73 & 21.1 & 0.61 & -1.03 \\
\hline-4320.73 & 21.1 & 0.61 & -0.85 \\
\hline 4324.98 & 21.1 & 0.60 & -2.15 \\
\hline-4324.99 & 21.1 & 0.60 & -1.71 \\
\hline-4324.99 & 21.1 & 0.60 & -1.44 \\
\hline-4324.99 & 21.1 & 0.60 & -1.34 \\
\hline-4324.99 & 21.1 & 0.60 & -1.45 \\
\hline-4324.99 & 21.1 & 0.60 & -1.46 \\
\hline-4325.00 & 21.1 & 0.60 & -1.71 \\
\hline-4325.00 & 21.1 & 0.60 & -1.26 \\
\hline-4325.00 & 21.1 & 0.60 & -0.97 \\
\hline 4374.45 & 21.1 & 0.62 & -2.10 \\
\hline-4374.45 & 21.1 & 0.62 & -1.11 \\
\hline-4374.45 & 21.1 & 0.62 & -1.89 \\
\hline-4374.45 & 21.1 & 0.62 & -1.27 \\
\hline-4374.46 & 21.1 & 0.62 & -1.82 \\
\hline-4374.46 & 21.1 & 0.62 & -2.10 \\
\hline-4374.46 & 21.1 & 0.62 & -1.45 \\
\hline-4374.46 & 21.1 & 0.62 & -1.82 \\
\hline-4374.46 & 21.1 & 0.62 & -1.89 \\
\hline-4374.46 & 21.1 & 0.62 & -1.65 \\
\hline-4374.46 & 21.1 & 0.62 & -1.87 \\
\hline-4374.46 & 21.1 & 0.62 & -1.82 \\
\hline-4374.46 & 21.1 & 0.62 & -1.87 \\
\hline-4374.46 & 21.1 & 0.62 & -1.98 \\
\hline-4374.46 & 21.1 & 0.62 & -2.11 \\
\hline-4374.46 & 21.1 & 0.62 & -1.82 \\
\hline-4374.46 & 21.1 & 0.62 & -2.21 \\
\hline-4374.46 & 21.1 & 0.62 & -2.34 \\
\hline-4374.46 & 21.1 & 0.62 & -1.87 \\
\hline-4374.46 & 21.1 & 0.62 & -2.36 \\
\hline-4374.46 & 21.1 & 0.62 & -2.21 \\
\hline-4374.46 & 21.1 & 0.62 & -1.98 \\
\hline 4400.38 & 21.1 & 0.61 & -2.01 \\
\hline-4400.38 & 21.1 & 0.61 & -1.81 \\
\hline-4400.38 & 21.1 & 0.61 & -1.20 \\
\hline-4400.39 & 21.1 & 0.61 & -1.76 \\
\hline-4400.39 & 21.1 & 0.61 & -1.43 \\
\hline-4400.39 & 21.1 & 0.61 & -1.79 \\
\hline-4400.39 & 21.1 & 0.61 & -1.72 \\
\hline-4400.39 & 21.1 & 0.61 & -2.01 \\
\hline-4400.39 & 21.1 & 0.61 & -1.89 \\
\hline-4400.39 & 21.1 & 0.61 & -2.10 \\
\hline-4400.39 & 21.1 & 0.61 & -1.81 \\
\hline-4400.40 & 21.1 & 0.61 & -2.73 \\
\hline-4400.40 & 21.1 & 0.61 & -2.11 \\
\hline-4400.40 & 21.1 & 0.61 & -1.76 \\
\hline-4400.40 & 21.1 & 0.61 & -2.59 \\
\hline-4400.40 & 21.1 & 0.61 & -1.89 \\
\hline-4400.40 & 21.1 & 0.61 & -1.79 \\
\hline
\end{tabular}


H. Reggiani et al.: Constraining cosmic scatter in the Galactic halo through a differential analysis of metal-poor stars

Table B.1. continued.

\begin{tabular}{|c|c|c|c|}
\hline $\begin{array}{r}\text { Wavelength } \\
(\AA)\end{array}$ & Species & $\begin{array}{r}\mathrm{EP} \\
(\mathrm{eV})\end{array}$ & $\begin{array}{r}\log (g f) \\
(\text { dex })\end{array}$ \\
\hline-4400.40 & 21.1 & 0.61 & -2.11 \\
\hline 4415.54 & 21.1 & 0.60 & -1.86 \\
\hline-4415.55 & 21.1 & 0.60 & -1.71 \\
\hline-4415.55 & 21.1 & 0.60 & -1.72 \\
\hline-4415.55 & 21.1 & 0.60 & -1.29 \\
\hline-4415.56 & 21.1 & 0.60 & -1.89 \\
\hline-4415.56 & 21.1 & 0.60 & -1.69 \\
\hline-4415.56 & 21.1 & 0.60 & -2.39 \\
\hline-4415.56 & 21.1 & 0.60 & -3.81 \\
\hline-4415.56 & 21.1 & 0.60 & -2.07 \\
\hline-4415.57 & 21.1 & 0.60 & -1.89 \\
\hline-4415.57 & 21.1 & 0.60 & -1.72 \\
\hline-4415.57 & 21.1 & 0.60 & -1.71 \\
\hline-4415.57 & 21.1 & 0.60 & -1.86 \\
\hline 3635.46 & 22.0 & 0.00 & 0.05 \\
\hline 3653.49 & 22.0 & 0.05 & 0.22 \\
\hline 3729.81 & 22.0 & 0.00 & -0.35 \\
\hline 3741.06 & 22.0 & 0.02 & -0.21 \\
\hline 3904.78 & 22.0 & 0.90 & 0.28 \\
\hline 3958.21 & 22.0 & 0.05 & -0.18 \\
\hline 3989.76 & 22.0 & 0.02 & -0.20 \\
\hline 3998.64 & 22.0 & 0.05 & -0.06 \\
\hline 4305.91 & 22.0 & 0.85 & 0.51 \\
\hline 4981.73 & 22.0 & 0.85 & 0.50 \\
\hline 4991.07 & 22.0 & 0.84 & 0.38 \\
\hline 4999.50 & 22.0 & 0.83 & 0.25 \\
\hline 3302.10 & 22.1 & 0.15 & -2.36 \\
\hline 3321.70 & 22.1 & 1.23 & -0.31 \\
\hline 3335.19 & 22.1 & 0.12 & -0.42 \\
\hline 3340.34 & 22.1 & 0.11 & -0.54 \\
\hline 3348.84 & 22.1 & 0.12 & -1.15 \\
\hline 3349.40 & 22.1 & 0.05 & 0.53 \\
\hline 3372.79 & 22.1 & 0.01 & 0.28 \\
\hline 3388.75 & 22.1 & 1.24 & -1.10 \\
\hline 3409.81 & 22.1 & 0.03 & -1.98 \\
\hline 3456.38 & 22.1 & 2.06 & -0.10 \\
\hline 3491.05 & 22.1 & 0.11 & -1.15 \\
\hline 3573.73 & 22.1 & 0.57 & -1.49 \\
\hline 3596.05 & 22.1 & 0.61 & -1.03 \\
\hline 3641.33 & 22.1 & 1.24 & -0.71 \\
\hline 3659.76 & 22.1 & 1.58 & -0.53 \\
\hline 3685.20 & 22.1 & 0.61 & 0.13 \\
\hline 3759.29 & 22.1 & 0.61 & 0.28 \\
\hline 3776.05 & 22.1 & 1.58 & -1.25 \\
\hline 3813.39 & 22.1 & 0.61 & -1.83 \\
\hline 3900.54 & 22.1 & 1.13 & -0.29 \\
\hline 3981.99 & 22.1 & 0.57 & -2.91 \\
\hline 4025.13 & 22.1 & 0.61 & -2.14 \\
\hline 4028.34 & 22.1 & 1.89 & -0.92 \\
\hline 4053.82 & 22.1 & 1.89 & -1.13 \\
\hline 4300.04 & 22.1 & 1.18 & -0.46 \\
\hline 4301.92 & 22.1 & 1.16 & -1.21 \\
\hline 4312.86 & 22.1 & 1.18 & -1.12 \\
\hline 4320.95 & 22.1 & 1.17 & -1.80 \\
\hline 4394.06 & 22.1 & 1.22 & -1.78 \\
\hline 4395.03 & 22.1 & 1.08 & -0.54 \\
\hline 4399.77 & 22.1 & 1.24 & -1.19 \\
\hline 4443.80 & 22.1 & 1.08 & -0.71 \\
\hline 4450.48 & 22.1 & 1.08 & -1.52 \\
\hline
\end{tabular}

Table B.1. continued.

\begin{tabular}{|c|c|c|c|}
\hline $\begin{array}{r}\text { Wavelength } \\
(\AA)\end{array}$ & Species & $\begin{array}{r}\mathrm{EP} \\
(\mathrm{eV})\end{array}$ & $\begin{array}{r}\log (g f) \\
(\text { dex })\end{array}$ \\
\hline 4468.51 & 22.1 & 1.13 & -0.60 \\
\hline 4501.27 & 22.1 & 1.12 & -0.77 \\
\hline 4805.09 & 22.1 & 2.06 & -0.96 \\
\hline 5129.16 & 22.1 & 1.89 & -1.24 \\
\hline 5226.54 & 22.1 & 1.57 & -1.26 \\
\hline 5336.79 & 22.1 & 1.58 & -1.59 \\
\hline 3592.01 & 23.1 & 1.10 & -2.60 \\
\hline-3592.02 & 23.1 & 1.10 & -2.19 \\
\hline-3592.02 & 23.1 & 1.10 & -1.95 \\
\hline-3592.02 & 23.1 & 1.10 & -1.61 \\
\hline-3592.02 & 23.1 & 1.10 & -1.42 \\
\hline-3592.02 & 23.1 & 1.10 & -1.81 \\
\hline-3592.02 & 23.1 & 1.10 & -1.39 \\
\hline-3592.02 & 23.1 & 1.10 & -1.71 \\
\hline-3592.02 & 23.1 & 1.10 & -1.45 \\
\hline-3592.02 & 23.1 & 1.10 & -1.63 \\
\hline-3592.02 & 23.1 & 1.10 & -0.87 \\
\hline-3592.02 & 23.1 & 1.10 & -1.04 \\
\hline-3592.02 & 23.1 & 1.10 & -1.93 \\
\hline-3592.02 & 23.1 & 1.10 & -1.24 \\
\hline-3592.02 & 23.1 & 1.10 & -1.51 \\
\hline 3951.95 & 23.1 & 1.48 & -1.39 \\
\hline-3951.95 & 23.1 & 1.48 & -2.13 \\
\hline-3951.95 & 23.1 & 1.48 & -1.56 \\
\hline-3951.96 & 23.1 & 1.48 & -3.13 \\
\hline-3951.96 & 23.1 & 1.48 & -1.94 \\
\hline-3951.96 & 23.1 & 1.48 & -1.76 \\
\hline-3951.96 & 23.1 & 1.48 & -2.71 \\
\hline-3951.96 & 23.1 & 1.48 & -1.91 \\
\hline-3951.96 & 23.1 & 1.48 & -2.03 \\
\hline-3951.97 & 23.1 & 1.48 & -2.47 \\
\hline-3951.97 & 23.1 & 1.48 & -1.97 \\
\hline-3951.97 & 23.1 & 1.48 & -2.45 \\
\hline-3951.97 & 23.1 & 1.48 & -2.33 \\
\hline-3951.97 & 23.1 & 1.48 & -2.15 \\
\hline-3951.97 & 23.1 & 1.48 & -2.23 \\
\hline 3578.69 & 24.0 & 0.00 & 0.41 \\
\hline 4254.34 & 24.0 & 0.00 & -0.11 \\
\hline 4274.80 & 24.0 & 0.00 & -0.23 \\
\hline 4289.72 & 24.0 & 0.00 & -0.36 \\
\hline 5206.04 & 24.0 & 0.94 & 0.02 \\
\hline 3342.58 & 24.1 & 2.46 & -0.74 \\
\hline 3358.49 & 24.1 & 2.46 & -0.59 \\
\hline 3382.68 & 24.1 & 2.46 & -0.95 \\
\hline 3408.76 & 24.1 & 2.48 & -0.39 \\
\hline 3315.66 & 28.0 & 0.11 & -1.23 \\
\hline 4030.73 & 25.0 & 0.00 & -1.04 \\
\hline-4030.75 & 25.0 & 0.00 & -1.96 \\
\hline-4030.75 & 25.0 & 0.00 & -1.18 \\
\hline-4030.76 & 25.0 & 0.00 & -3.17 \\
\hline-4030.76 & 25.0 & 0.00 & -1.78 \\
\hline-4030.76 & 25.0 & 0.00 & -1.34 \\
\hline-4030.77 & 25.0 & 0.00 & -2.82 \\
\hline-4030.77 & 25.0 & 0.00 & -1.75 \\
\hline-4030.77 & 25.0 & 0.00 & -1.52 \\
\hline-4030.78 & 25.0 & 0.00 & -2.70 \\
\hline-4030.78 & 25.0 & 0.00 & -1.82 \\
\hline-4030.78 & 25.0 & 0.00 & -1.74 \\
\hline-4030.78 & 25.0 & 0.00 & -2.00 \\
\hline
\end{tabular}


Table B.1. continued.

\begin{tabular}{|c|c|c|c|}
\hline $\begin{array}{r}\text { Wavelength } \\
(\AA)\end{array}$ & Species & $\begin{array}{r}\mathrm{EP} \\
(\mathrm{eV})\end{array}$ & $\begin{array}{r}\log (g f) \\
(\text { dex })\end{array}$ \\
\hline-4030.78 & 25.0 & 0.00 & -2.77 \\
\hline-4030.78 & 25.0 & 0.00 & -2.03 \\
\hline 4033.04 & 25.0 & 0.00 & -1.20 \\
\hline-4033.05 & 25.0 & 0.00 & -1.98 \\
\hline-4033.06 & 25.0 & 0.00 & -1.98 \\
\hline-4033.06 & 25.0 & 0.00 & -1.46 \\
\hline-4033.06 & 25.0 & 0.00 & -1.82 \\
\hline-4033.07 & 25.0 & 0.00 & -1.82 \\
\hline-4033.07 & 25.0 & 0.00 & -1.79 \\
\hline-4033.07 & 25.0 & 0.00 & -1.81 \\
\hline-4033.08 & 25.0 & 0.00 & -1.81 \\
\hline-4033.08 & 25.0 & 0.00 & -2.24 \\
\hline-4033.08 & 25.0 & 0.00 & -1.91 \\
\hline-4033.08 & 25.0 & 0.00 & -1.91 \\
\hline-4033.09 & 25.0 & 0.00 & -2.94 \\
\hline-4033.09 & 25.0 & 0.00 & -2.17 \\
\hline-4033.09 & 25.0 & 0.00 & -2.17 \\
\hline 4034.47 & 25.0 & 0.00 & -1.33 \\
\hline-4034.47 & 25.0 & 0.00 & -2.02 \\
\hline-4034.47 & 25.0 & 0.00 & -2.97 \\
\hline-4034.48 & 25.0 & 0.00 & -1.54 \\
\hline-4034.49 & 25.0 & 0.00 & -1.87 \\
\hline-4034.49 & 25.0 & 0.00 & -2.59 \\
\hline-4034.49 & 25.0 & 0.00 & -1.81 \\
\hline-4034.50 & 25.0 & 0.00 & -2.41 \\
\hline-4034.50 & 25.0 & 0.00 & -1.89 \\
\hline-4034.50 & 25.0 & 0.00 & -2.22 \\
\hline-4034.50 & 25.0 & 0.00 & -2.37 \\
\hline-4034.50 & 25.0 & 0.00 & -2.05 \\
\hline 3405.07 & 27.0 & 0.43 & -1.59 \\
\hline-3405.08 & 27.0 & 0.43 & -1.39 \\
\hline-3405.08 & 27.0 & 0.43 & -1.37 \\
\hline-3405.08 & 27.0 & 0.43 & -1.38 \\
\hline-3405.08 & 27.0 & 0.43 & -1.26 \\
\hline-3405.08 & 27.0 & 0.43 & -1.59 \\
\hline-3405.09 & 27.0 & 0.43 & -1.24 \\
\hline-3405.09 & 27.0 & 0.43 & -1.22 \\
\hline-3405.09 & 27.0 & 0.43 & -1.37 \\
\hline-3405.09 & 27.0 & 0.43 & -1.07 \\
\hline-3405.10 & 27.0 & 0.43 & -1.23 \\
\hline-3405.10 & 27.0 & 0.43 & -1.26 \\
\hline-3405.10 & 27.0 & 0.43 & -0.90 \\
\hline-3405.11 & 27.0 & 0.43 & -1.31 \\
\hline-3405.11 & 27.0 & 0.43 & -1.22 \\
\hline-3405.12 & 27.0 & 0.43 & -0.74 \\
\hline-3405.12 & 27.0 & 0.43 & -1.52 \\
\hline-3405.12 & 27.0 & 0.43 & -1.23 \\
\hline-3405.13 & 27.0 & 0.43 & -0.60 \\
\hline-3405.14 & 27.0 & 0.43 & -1.31 \\
\hline-3405.15 & 27.0 & 0.43 & -0.46 \\
\hline-3405.16 & 27.0 & 0.43 & -1.52 \\
\hline 3412.32 & 27.0 & 0.51 & -1.51 \\
\hline-3412.32 & 27.0 & 0.51 & -1.31 \\
\hline-3412.33 & 27.0 & 0.51 & -1.78 \\
\hline-3412.33 & 27.0 & 0.51 & -1.14 \\
\hline-3412.33 & 27.0 & 0.51 & -1.71 \\
\hline-3412.33 & 27.0 & 0.51 & -0.99 \\
\hline-3412.33 & 27.0 & 0.51 & -1.51 \\
\hline-3412.33 & 27.0 & 0.51 & -0.86 \\
\hline
\end{tabular}

Table B.1. continued.

\begin{tabular}{|c|c|c|c|}
\hline $\begin{array}{r}\text { Wavelength } \\
(\AA)\end{array}$ & Species & $\begin{array}{r}\mathrm{EP} \\
(\mathrm{eV})\end{array}$ & $\begin{array}{r}\log (g f) \\
(\text { dex })\end{array}$ \\
\hline-3412.33 & 27.0 & 0.51 & -2.41 \\
\hline-3412.33 & 27.0 & 0.51 & -1.41 \\
\hline-3412.33 & 27.0 & 0.51 & -0.75 \\
\hline-3412.34 & 27.0 & 0.51 & -1.36 \\
\hline-3412.34 & 27.0 & 0.51 & -2.25 \\
\hline-3412.34 & 27.0 & 0.51 & -0.64 \\
\hline-3412.34 & 27.0 & 0.51 & -1.37 \\
\hline-3412.34 & 27.0 & 0.51 & -2.25 \\
\hline-3412.35 & 27.0 & 0.51 & -1.44 \\
\hline-3412.35 & 27.0 & 0.51 & -2.35 \\
\hline-3412.35 & 27.0 & 0.51 & -1.65 \\
\hline-3412.36 & 27.0 & 0.51 & -2.55 \\
\hline-3412.36 & 27.0 & 0.51 & -2.95 \\
\hline 3412.59 & 27.0 & 0.00 & -3.76 \\
\hline-3412.60 & 27.0 & 0.00 & -3.36 \\
\hline-3412.61 & 27.0 & 0.00 & -2.46 \\
\hline-3412.61 & 27.0 & 0.00 & -3.16 \\
\hline-3412.62 & 27.0 & 0.00 & -2.25 \\
\hline-3412.62 & 27.0 & 0.00 & -3.06 \\
\hline-3412.62 & 27.0 & 0.00 & -1.45 \\
\hline-3412.62 & 27.0 & 0.00 & -2.18 \\
\hline-3412.63 & 27.0 & 0.00 & -3.06 \\
\hline-3412.63 & 27.0 & 0.00 & -1.56 \\
\hline-3412.63 & 27.0 & 0.00 & -2.17 \\
\hline-3412.63 & 27.0 & 0.00 & -2.22 \\
\hline-3412.63 & 27.0 & 0.00 & -1.67 \\
\hline-3412.63 & 27.0 & 0.00 & -3.22 \\
\hline-3412.64 & 27.0 & 0.00 & -2.32 \\
\hline-3412.64 & 27.0 & 0.00 & -1.80 \\
\hline-3412.64 & 27.0 & 0.00 & -2.52 \\
\hline-3412.64 & 27.0 & 0.00 & -1.95 \\
\hline-3412.64 & 27.0 & 0.00 & -2.59 \\
\hline-3412.64 & 27.0 & 0.00 & -2.12 \\
\hline-3412.64 & 27.0 & 0.00 & -2.32 \\
\hline 3431.55 & 27.0 & 0.10 & -3.52 \\
\hline-3431.56 & 27.0 & 0.10 & -3.11 \\
\hline-3431.56 & 27.0 & 0.10 & -2.41 \\
\hline-3431.57 & 27.0 & 0.10 & -2.89 \\
\hline-3431.57 & 27.0 & 0.10 & -2.21 \\
\hline-3431.57 & 27.0 & 0.10 & -2.77 \\
\hline-3431.57 & 27.0 & 0.10 & -2.16 \\
\hline-3431.58 & 27.0 & 0.10 & -1.57 \\
\hline-3431.58 & 27.0 & 0.10 & -2.72 \\
\hline-3431.58 & 27.0 & 0.10 & -2.18 \\
\hline-3431.58 & 27.0 & 0.10 & -1.71 \\
\hline-3431.58 & 27.0 & 0.10 & -2.75 \\
\hline-3431.58 & 27.0 & 0.10 & -2.27 \\
\hline-3431.58 & 27.0 & 0.10 & -2.46 \\
\hline-3431.58 & 27.0 & 0.10 & -1.88 \\
\hline-3431.59 & 27.0 & 0.10 & -2.07 \\
\hline-3431.59 & 27.0 & 0.10 & -2.72 \\
\hline-3431.59 & 27.0 & 0.10 & -2.33 \\
\hline 3433.04 & 27.0 & 0.63 & -1.33 \\
\hline-3433.04 & 27.0 & 0.63 & -1.16 \\
\hline-3433.04 & 27.0 & 0.63 & -1.16 \\
\hline-3433.04 & 27.0 & 0.63 & -1.03 \\
\hline-3433.04 & 27.0 & 0.63 & -1.03 \\
\hline-3433.04 & 27.0 & 0.63 & -1.35 \\
\hline-3433.04 & 27.0 & 0.63 & -1.15 \\
\hline
\end{tabular}


H. Reggiani et al.: Constraining cosmic scatter in the Galactic halo through a differential analysis of metal-poor stars

Table B.1. continued.

\begin{tabular}{|c|c|c|c|}
\hline $\begin{array}{r}\text { Wavelength } \\
(\AA)\end{array}$ & Species & $\begin{array}{r}\mathrm{EP} \\
(\mathrm{eV})\end{array}$ & $\begin{array}{r}\log (g f) \\
(\text { dex })\end{array}$ \\
\hline-3433.04 & 27.0 & 0.63 & -1.15 \\
\hline-3433.04 & 27.0 & 0.63 & -0.74 \\
\hline 3449.14 & 27.0 & 0.58 & -1.74 \\
\hline-3449.14 & 27.0 & 0.58 & -1.49 \\
\hline-3449.15 & 27.0 & 0.58 & -1.49 \\
\hline-3449.15 & 27.0 & 0.58 & -3.39 \\
\hline-3449.15 & 27.0 & 0.58 & -1.29 \\
\hline-3449.15 & 27.0 & 0.58 & -1.29 \\
\hline-3449.15 & 27.0 & 0.58 & -2.15 \\
\hline-3449.15 & 27.0 & 0.58 & -1.22 \\
\hline-3449.16 & 27.0 & 0.58 & -1.22 \\
\hline-3449.16 & 27.0 & 0.58 & -1.43 \\
\hline-3449.16 & 27.0 & 0.58 & -1.25 \\
\hline-3449.17 & 27.0 & 0.58 & -1.25 \\
\hline-3449.17 & 27.0 & 0.58 & -1.03 \\
\hline-3449.17 & 27.0 & 0.58 & -1.44 \\
\hline-3449.19 & 27.0 & 0.58 & -1.44 \\
\hline-3449.19 & 27.0 & 0.58 & -0.74 \\
\hline 3449.38 & 27.0 & 0.43 & -2.14 \\
\hline-3449.38 & 27.0 & 0.43 & -2.34 \\
\hline-3449.38 & 27.0 & 0.43 & -2.34 \\
\hline-3449.38 & 27.0 & 0.43 & -2.13 \\
\hline-3449.38 & 27.0 & 0.43 & -2.12 \\
\hline-3449.39 & 27.0 & 0.43 & -2.12 \\
\hline-3449.39 & 27.0 & 0.43 & -1.99 \\
\hline-3449.39 & 27.0 & 0.43 & -2.01 \\
\hline-3449.41 & 27.0 & 0.43 & -2.01 \\
\hline-3449.41 & 27.0 & 0.43 & -1.82 \\
\hline-3449.41 & 27.0 & 0.43 & -1.97 \\
\hline-3449.42 & 27.0 & 0.43 & -1.97 \\
\hline-3449.42 & 27.0 & 0.43 & -1.65 \\
\hline-3449.42 & 27.0 & 0.43 & -1.98 \\
\hline-3449.44 & 27.0 & 0.43 & -1.98 \\
\hline-3449.44 & 27.0 & 0.43 & -1.49 \\
\hline-3449.44 & 27.0 & 0.43 & -2.06 \\
\hline-3449.47 & 27.0 & 0.43 & -2.06 \\
\hline-3449.47 & 27.0 & 0.43 & -1.35 \\
\hline-3449.47 & 27.0 & 0.43 & -2.27 \\
\hline-3449.49 & 27.0 & 0.43 & -2.27 \\
\hline-3449.49 & 27.0 & 0.43 & -1.21 \\
\hline 3453.47 & 27.0 & 0.43 & -1.05 \\
\hline-3453.48 & 27.0 & 0.43 & -0.93 \\
\hline-3453.48 & 27.0 & 0.43 & -1.50 \\
\hline-3453.48 & 27.0 & 0.43 & -0.81 \\
\hline-3453.49 & 27.0 & 0.43 & -1.29 \\
\hline-3453.49 & 27.0 & 0.43 & -0.70 \\
\hline-3453.49 & 27.0 & 0.43 & -2.50 \\
\hline-3453.49 & 27.0 & 0.43 & -1.19 \\
\hline-3453.50 & 27.0 & 0.43 & -0.59 \\
\hline-3453.50 & 27.0 & 0.43 & -2.29 \\
\hline-3453.51 & 27.0 & 0.43 & -1.16 \\
\hline-3453.51 & 27.0 & 0.43 & -0.50 \\
\hline-3453.51 & 27.0 & 0.43 & -2.26 \\
\hline-3453.52 & 27.0 & 0.43 & -1.17 \\
\hline-3453.52 & 27.0 & 0.43 & -0.41 \\
\hline-3453.52 & 27.0 & 0.43 & -2.34 \\
\hline-3453.53 & 27.0 & 0.43 & -1.25 \\
\hline-3453.54 & 27.0 & 0.43 & -0.32 \\
\hline-3453.54 & 27.0 & 0.43 & -2.54 \\
\hline
\end{tabular}

Table B.1. continued.

\begin{tabular}{|c|c|c|c|}
\hline $\begin{array}{r}\text { Wavelength } \\
(\AA)\end{array}$ & Species & $\begin{array}{r}\mathrm{EP} \\
(\mathrm{eV})\end{array}$ & $\begin{array}{r}\log (g f) \\
(\text { dex })\end{array}$ \\
\hline-3453.55 & 27.0 & 0.43 & -1.47 \\
\hline-3453.56 & 27.0 & 0.43 & -2.92 \\
\hline 3594.83 & 27.0 & 0.17 & -2.62 \\
\hline-3594.83 & 27.0 & 0.17 & -2.37 \\
\hline-3594.84 & 27.0 & 0.17 & -2.37 \\
\hline-3594.84 & 27.0 & 0.17 & -4.27 \\
\hline-3594.84 & 27.0 & 0.17 & -2.17 \\
\hline-3594.85 & 27.0 & 0.17 & -2.17 \\
\hline-3594.85 & 27.0 & 0.17 & -3.03 \\
\hline-3594.85 & 27.0 & 0.17 & -2.10 \\
\hline-3594.86 & 27.0 & 0.17 & -2.10 \\
\hline-3594.86 & 27.0 & 0.17 & -2.31 \\
\hline-3594.86 & 27.0 & 0.17 & -2.13 \\
\hline-3594.87 & 27.0 & 0.17 & -2.13 \\
\hline-3594.87 & 27.0 & 0.17 & -1.91 \\
\hline-3594.87 & 27.0 & 0.17 & -2.32 \\
\hline-3594.89 & 27.0 & 0.17 & -2.32 \\
\hline-3594.89 & 27.0 & 0.17 & -1.62 \\
\hline 3845.45 & 27.0 & 0.92 & -0.66 \\
\hline-3845.46 & 27.0 & 0.92 & -0.77 \\
\hline-3845.46 & 27.0 & 0.92 & -0.88 \\
\hline-3845.46 & 27.0 & 0.92 & -1.01 \\
\hline-3845.47 & 27.0 & 0.92 & -1.16 \\
\hline-3845.47 & 27.0 & 0.92 & -1.33 \\
\hline-3845.47 & 27.0 & 0.92 & -1.67 \\
\hline-3845.47 & 27.0 & 0.92 & -1.53 \\
\hline-3845.47 & 27.0 & 0.92 & -1.46 \\
\hline-3845.47 & 27.0 & 0.92 & -1.39 \\
\hline-3845.47 & 27.0 & 0.92 & -1.80 \\
\hline-3845.47 & 27.0 & 0.92 & -1.38 \\
\hline-3845.47 & 27.0 & 0.92 & -1.43 \\
\hline-3845.47 & 27.0 & 0.92 & -1.73 \\
\hline-3845.47 & 27.0 & 0.92 & -1.53 \\
\hline-3845.48 & 27.0 & 0.92 & -2.43 \\
\hline-3845.48 & 27.0 & 0.92 & -2.27 \\
\hline-3845.48 & 27.0 & 0.92 & -2.27 \\
\hline-3845.48 & 27.0 & 0.92 & -2.37 \\
\hline-3845.48 & 27.0 & 0.92 & -2.57 \\
\hline-3845.48 & 27.0 & 0.92 & -2.97 \\
\hline 3873.07 & 27.0 & 0.43 & -3.64 \\
\hline-3873.07 & 27.0 & 0.43 & -3.24 \\
\hline-3873.08 & 27.0 & 0.43 & -3.04 \\
\hline-3873.08 & 27.0 & 0.43 & -2.94 \\
\hline-3873.08 & 27.0 & 0.43 & -2.94 \\
\hline-3873.08 & 27.0 & 0.43 & -3.10 \\
\hline-3873.09 & 27.0 & 0.43 & -2.40 \\
\hline-3873.09 & 27.0 & 0.43 & -2.20 \\
\hline-3873.09 & 27.0 & 0.43 & -2.10 \\
\hline-3873.09 & 27.0 & 0.43 & -2.47 \\
\hline-3873.09 & 27.0 & 0.43 & -2.05 \\
\hline-3873.10 & 27.0 & 0.43 & -2.06 \\
\hline-3873.10 & 27.0 & 0.43 & -2.20 \\
\hline-3873.10 & 27.0 & 0.43 & -2.13 \\
\hline-3873.10 & 27.0 & 0.43 & -2.34 \\
\hline-3873.10 & 27.0 & 0.43 & -2.00 \\
\hline-3873.11 & 27.0 & 0.43 & -1.83 \\
\hline-3873.11 & 27.0 & 0.43 & -1.68 \\
\hline-3873.12 & 27.0 & 0.43 & -1.55 \\
\hline-3873.13 & 27.0 & 0.43 & -1.44 \\
\hline
\end{tabular}


Table B.1. continued.

\begin{tabular}{|c|c|c|c|}
\hline $\begin{array}{r}\text { Wavelength } \\
(\AA)\end{array}$ & Species & $\begin{array}{r}\mathrm{EP} \\
(\mathrm{eV})\end{array}$ & $\begin{array}{r}\log (g f) \\
(\text { dex })\end{array}$ \\
\hline-3873.13 & 27.0 & 0.43 & -1.33 \\
\hline 3995.27 & 27.0 & 0.92 & -2.03 \\
\hline-3995.27 & 27.0 & 0.92 & -1.96 \\
\hline-3995.27 & 27.0 & 0.92 & -1.76 \\
\hline-3995.28 & 27.0 & 0.92 & -2.66 \\
\hline-3995.28 & 27.0 & 0.92 & -1.76 \\
\hline-3995.28 & 27.0 & 0.92 & -1.56 \\
\hline-3995.28 & 27.0 & 0.92 & -2.50 \\
\hline-3995.28 & 27.0 & 0.92 & -1.66 \\
\hline-3995.28 & 27.0 & 0.92 & -1.39 \\
\hline-3995.29 & 27.0 & 0.92 & -2.50 \\
\hline-3995.29 & 27.0 & 0.92 & -1.61 \\
\hline-3995.29 & 27.0 & 0.92 & -1.24 \\
\hline-3995.30 & 27.0 & 0.92 & -2.60 \\
\hline-3995.30 & 27.0 & 0.92 & -1.62 \\
\hline-3995.30 & 27.0 & 0.92 & -1.11 \\
\hline-3995.31 & 27.0 & 0.92 & -2.80 \\
\hline-3995.31 & 27.0 & 0.92 & -1.69 \\
\hline-3995.31 & 27.0 & 0.92 & -1.00 \\
\hline-3995.33 & 27.0 & 0.92 & -3.20 \\
\hline-3995.33 & 27.0 & 0.92 & -1.90 \\
\hline-3995.33 & 27.0 & 0.92 & -0.89 \\
\hline 4121.29 & 27.0 & 0.92 & -0.99 \\
\hline-4121.30 & 27.0 & 0.92 & -1.10 \\
\hline-4121.31 & 27.0 & 0.92 & -1.21 \\
\hline-4121.31 & 27.0 & 0.92 & -1.34 \\
\hline-4121.32 & 27.0 & 0.92 & -2.00 \\
\hline-4121.32 & 27.0 & 0.92 & -1.49 \\
\hline-4121.32 & 27.0 & 0.92 & -1.79 \\
\hline-4121.32 & 27.0 & 0.92 & -1.66 \\
\hline-4121.32 & 27.0 & 0.92 & -1.72 \\
\hline-4121.33 & 27.0 & 0.92 & -1.86 \\
\hline-4121.33 & 27.0 & 0.92 & -1.71 \\
\hline-4121.33 & 27.0 & 0.92 & -2.13 \\
\hline-4121.33 & 27.0 & 0.92 & -1.76 \\
\hline-4121.33 & 27.0 & 0.92 & -1.86 \\
\hline-4121.33 & 27.0 & 0.92 & -2.06 \\
\hline-4121.34 & 27.0 & 0.92 & -2.76 \\
\hline-4121.34 & 27.0 & 0.92 & -3.30 \\
\hline-4121.34 & 27.0 & 0.92 & -2.60 \\
\hline-4121.34 & 27.0 & 0.92 & -2.60 \\
\hline-4121.34 & 27.0 & 0.92 & -2.70 \\
\hline-4121.34 & 27.0 & 0.92 & -2.90 \\
\hline 3365.76 & 28.0 & 0.42 & -1.19 \\
\hline 3380.57 & 28.0 & 0.42 & -0.17 \\
\hline 3380.87 & 28.0 & 0.28 & -1.34 \\
\hline 3391.04 & 28.0 & 0.00 & -1.05 \\
\hline 3452.89 & 28.0 & 0.11 & -0.91 \\
\hline 3458.46 & 28.0 & 0.21 & -0.22 \\
\hline 3461.65 & 28.0 & 0.03 & -0.35 \\
\hline 3472.54 & 28.0 & 0.11 & -0.81 \\
\hline 3492.95 & 28.0 & 0.11 & -0.25 \\
\hline 3587.93 & 28.0 & 0.03 & -2.34 \\
\hline 3597.70 & 28.0 & 0.21 & -1.10 \\
\hline 3610.46 & 28.0 & 0.11 & -1.15 \\
\hline 3612.73 & 28.0 & 0.28 & -1.41 \\
\hline 3619.39 & 28.0 & 0.42 & 0.04 \\
\hline 3775.57 & 28.0 & 0.42 & -1.39 \\
\hline 3783.52 & 28.0 & 0.42 & -1.31 \\
\hline 3807.14 & 28.0 & 0.42 & -1.21 \\
\hline
\end{tabular}

Table B.1. continued.

\begin{tabular}{|c|c|c|c|}
\hline $\begin{array}{r}\text { Wavelength } \\
(\AA)\end{array}$ & Species & $\begin{array}{r}\mathrm{EP} \\
(\mathrm{eV})\end{array}$ & $\begin{array}{r}\log (g f) \\
(\text { dex })\end{array}$ \\
\hline 3858.29 & 28.0 & 0.42 & -0.94 \\
\hline 5476.90 & 28.0 & 1.83 & -0.89 \\
\hline 4810.53 & 30.0 & 4.08 & -0.31 \\
\hline 4077.71 & 38.1 & 0.00 & 0.17 \\
\hline 4215.52 & 38.1 & 0.00 & -0.15 \\
\hline 3600.74 & 39.1 & 0.18 & 0.28 \\
\hline 3611.04 & 39.1 & 0.13 & 0.11 \\
\hline 3710.29 & 39.1 & 0.18 & 0.46 \\
\hline 3774.33 & 39.1 & 0.13 & 0.21 \\
\hline 3788.69 & 39.1 & 0.10 & -0.07 \\
\hline 3991.13 & 40.1 & 0.76 & -0.31 \\
\hline 3998.97 & 40.1 & 0.56 & -0.52 \\
\hline 4149.20 & 40.1 & 0.80 & -0.04 \\
\hline 4934.10 & 56.1 & 0.00 & -1.77 \\
\hline-4934.06 & 56.1 & 0.00 & -1.84 \\
\hline-4934.07 & 56.1 & 0.00 & -2.54 \\
\hline-4934.12 & 56.1 & 0.00 & -1.84 \\
\hline-4934.13 & 56.1 & 0.00 & -1.84 \\
\hline-4934.10 & 56.1 & 0.00 & -1.26 \\
\hline-4934.05 & 56.1 & 0.00 & -1.61 \\
\hline-4934.07 & 56.1 & 0.00 & -2.30 \\
\hline-4934.12 & 56.1 & 0.00 & -1.61 \\
\hline-4934.13 & 56.1 & 0.00 & -1.61 \\
\hline-4934.10 & 56.1 & 0.00 & -0.29 \\
\hline 6141.70 & 56.1 & 0.70 & -3.63 \\
\hline-6141.70 & 56.1 & 0.70 & -3.40 \\
\hline-6141.70 & 56.1 & 0.70 & -2.49 \\
\hline-6141.70 & 56.1 & 0.70 & -2.26 \\
\hline-6141.70 & 56.1 & 0.70 & -3.46 \\
\hline-6141.70 & 56.1 & 0.70 & -3.22 \\
\hline-6141.70 & 56.1 & 0.70 & -1.68 \\
\hline-6141.70 & 56.1 & 0.70 & -2.39 \\
\hline-6141.70 & 56.1 & 0.70 & -2.16 \\
\hline-6141.70 & 56.1 & 0.70 & -1.45 \\
\hline-6141.70 & 56.1 & 0.70 & -1.70 \\
\hline-6141.70 & 56.1 & 0.70 & -1.18 \\
\hline-6141.70 & 56.1 & 0.70 & -0.22 \\
\hline-6141.70 & 56.1 & 0.70 & -2.51 \\
\hline-6141.70 & 56.1 & 0.70 & -1.89 \\
\hline-6141.70 & 56.1 & 0.70 & -2.27 \\
\hline-6141.70 & 56.1 & 0.70 & -1.66 \\
\hline-6141.70 & 56.1 & 0.70 & -2.46 \\
\hline-6141.70 & 56.1 & 0.70 & -2.14 \\
\hline-6141.70 & 56.1 & 0.70 & -1.90 \\
\hline-6141.70 & 56.1 & 0.70 & -2.23 \\
\hline 6496.90 & 56.1 & 0.60 & -2.00 \\
\hline-6496.91 & 56.1 & 0.60 & -2.76 \\
\hline-6496.91 & 56.1 & 0.60 & -2.37 \\
\hline-6496.89 & 56.1 & 0.60 & -3.07 \\
\hline-6496.91 & 56.1 & 0.60 & -2.37 \\
\hline-6496.89 & 56.1 & 0.60 & -2.37 \\
\hline-6496.90 & 56.1 & 0.60 & -1.92 \\
\hline-6496.90 & 56.1 & 0.60 & -1.48 \\
\hline-6496.91 & 56.1 & 0.60 & -2.53 \\
\hline-6496.91 & 56.1 & 0.60 & -2.13 \\
\hline-6496.89 & 56.1 & 0.60 & -2.83 \\
\hline-6496.91 & 56.1 & 0.60 & -2.13 \\
\hline-6496.89 & 56.1 & 0.60 & -2.13 \\
\hline-6496.90 & 56.1 & 0.60 & -1.69 \\
\hline-6496.90 & 56.1 & 0.60 & -0.52 \\
\hline
\end{tabular}




\title{
Evidences of extragalactic origin and planet engulfment in the metal-poor twin pair HD 134439/HD 134440
}

\author{
Henrique Reggiani ${ }^{1,2 \star}$ and Jorge Meléndez ${ }^{1 \star}$ \\ ${ }^{1}$ Universidade de São Paulo, Instituto de Astronomia, Geofísica e Ciências Atmosféricas, IAG, Departamento de Astronomia, Rua do Matão 1226, Cidade \\ Universitária, 05508-900, SP, Brazil \\ ${ }^{2}$ Max-Planck Institute for Astronomy, Konigstuhl 17, D-69117 Heidelberg, Germany
}

Accepted 2018 January 8. Received 2018 January 5; in original form 2017 October 25

\begin{abstract}
Recent studies of chemical abundances in metal-poor halo stars show the existence of different populations, which is important for studies of Galaxy formation and evolution. Here, we revisit the twin pair of chemically anomalous stars HD 134439 and HD 134440, using high resolution $(R \sim 72000)$ and high $\mathrm{S} / \mathrm{N}$ ratio $(\mathrm{S} / \mathrm{N} \sim 250) \mathrm{HDS} /$ Subaru spectra. We compare them to the well-studied halo star HD 103095, using the line-by-line differential technique to estimate precise stellar parameters and LTE chemical abundances. We present the abundances of C, $\mathrm{O}, \mathrm{Na}, \mathrm{Mg}, \mathrm{Si}, \mathrm{Ca}, \mathrm{Sc}, \mathrm{Ti}, \mathrm{V}, \mathrm{Cr}, \mathrm{Mn}, \mathrm{Co}, \mathrm{Ni}, \mathrm{Cu}, \mathrm{Zn}, \mathrm{Sr}, \mathrm{Y}, \mathrm{Ba}, \mathrm{La}, \mathrm{Ce}, \mathrm{Nd}$, and Sm. We compare our results to the precise abundance patterns of Nissen \& Schuster (2010) and data from dwarf Spheroidal galaxies (dSphs). We show that the abundance pattern of these stars appears to be closely linked to that of $\mathrm{dSphs}$ with $[\alpha / \mathrm{Fe}]$ knee below $[\mathrm{Fe} / \mathrm{H}]<-1.5$. We also find a systematic difference of $0.06 \pm 0.01$ dex between the abundances of these twin binary stars, which could be explained by the engulfment of a planet, thus suggesting that planet formation is possible at low metallicities $([\mathrm{Fe} / \mathrm{H}]=-1.4)$.
\end{abstract}

Key words: stars: abundances - stars: chemically peculiar-planetary systems - Galaxy: evolution-Galaxy: formation.

\section{INTRODUCTION}

Uncovering the details of our Galaxy's formation is one of the key studies in modern astronomy. Nissen \& Schuster (2010, hereafter NS10) identified the existence of two populations in $[\alpha / \mathrm{Fe}]$, $[\mathrm{Na} / \mathrm{Fe}],[\mathrm{Ni} / \mathrm{Fe}]$, and in stellar kinematics, around the solar neighbourhood for metallicities in the range of $-1.6 \leq[\mathrm{Fe} / \mathrm{H}] \leq-0.8$, and attributed the differences between the populations to the environments in which these stars formed. While one usually attributes the high- $\alpha$ population as stars formed locally in the Milky Way, the low- $\alpha$ stars could have been formed in dwarf galaxies and latter accreted by our larger Galaxy.

In subsequent studies, Nissen \& Schuster (2011, hereafter NS11) showed that the distinction between the two populations also extends to other chemical elements $([\mathrm{Cu} / \mathrm{Fe}],[\mathrm{Zn} / \mathrm{Fe}]$, and $[\mathrm{Ba} / \mathrm{Y}])$, and Ramírez, Meléndez \& ChanameÌ (2012) and Fishlock et al. (2017) extended the previous studies to O, Sc, and neutron-capture elements. NS11 attributed this separation to the different star formation rates (SFRs) of the two environments. In a high SFR environment, mostly massive stars and type II supernovae would contribute to the chemical enrichment and the ratio $[\alpha / \mathrm{Fe}]$ increases to form

\footnotetext{
^E-mail: hreggiani@gmail.com/hreggiani@usp.br (HR); jorge.melendez@ iag.usp.br (JM)
}

the high- $\alpha$ population in situ, and in a low-SFR environment, such as that of dwarf galaxies, a slower chemical enrichment with type Ia supernovae and low-mass AGB stars results in lower $[\alpha / \mathrm{Fe}]$ ratios and other distinct abundance ratios.

The pair of high proper motion stars HD 134439 and HD 134440 were suggested to be accreted from another galaxy by Carney et al. (1996), based on an analysis of their kinematics. An abundance study by King (1997) showed low $[\alpha / \mathrm{Fe}]$ ratios, reinforcing the idea that the stars were not formed in the Milky Way, but rather in an environment like a dSph. Shigeyama \& Tsujimoto (2003) proposed that stars with low $[\alpha / \mathrm{Fe}]$ ratios may have engulfed planets or planetesimals. Analysis of more elements by Chen \& Zhao (2006) confirmed a distinctive low abundance of $\alpha$-elements, concluding that the environment in which these stars were formed had few type II SNe and a high dust-to-gas ratio, meaning that the pair may have been formed in a dusty $\mathrm{dSph}$ medium. Based on the analysis of $\mathrm{Be}, \mathrm{C}, \mathrm{N}, \mathrm{O}, \mathrm{Ag}$, and $\mathrm{Eu}$, complemented with previous abundances of other elements, Chen, King \& Boesgaard (2014) discussed the above scenarios (dSph and planet accretion), and proposed that although an environment such as a dSph could be responsible for some of the abundance ratios, some undetermined nucleosynthetic processes may be responsible for some apparent anomalous abundance ratios in the neutron-capture elements.

The puzzling abundance ratios that Chen \& Zhao (2006) found for these stars could be connected to the recent findings of different halo 
populations (NS10) or the different patterns seen in stars of dSphs (e.g. Tolstoy, Hill \& Toii 2009; Suda et al. 2017). Indeed, as pointed out by Suda et al. (2017), the distinctive $\alpha$-elements abundance pattern $([\alpha / \mathrm{Fe}]<0)$ is also observed in stars of dSphs such as Fornax (Letarte et al. 2010; Lemasle et al. 2014), Carina (Lemasle et al. 2012; Venn et al. 2012; Fabrizio et al. 2015), Sculptor (Geisler et al. 2005), Draco (Shetrone, Bolte \& Stetson 1998; Shetrone, Côté \& Sargent 2001; Cohen \& Huang 2009), Sextans (Shetrone et al. 2001; Aoki et al. 2009), and Sagittarius (Bonifacio et al. 2000, 2004; Sbordone et al. 2007) (see fig. 10 of Suda et al. 2017).

In light of recent measurements of chemical abundances in the halo and dSphs, we revisit the high proper-motion pair HD 134439 and HD 134440, using new data and precise abundances obtained through the differential technique. In Section 2, we detail the data used in this work and the spectra reduction processes. In Section 3, we describe the determination of stellar parameters. In Section 4.1, we compare the stellar chemical composition to the results of the low- and high- $\alpha$ halo stars of NS10, NS11, Ramírez et al. (2012), and Fishlock et al. (2017). In Section 4.2, we discuss the connection with dSphs using abundances from Shetrone et al. (2003), Geisler et al. (2005), Monaco et al. (2005), and Letarte et al. (2010). In Section 4.3, we assess possible trends with condensation temperature. In Section 4.4, we discuss a possible planet signature and our conclusions are presented in Section 5.

\section{OBSERVATIONS AND DATA REDUCTION}

We observed three stars (HD 103095, HD 134439, and HD 134439) at the $8.2 \mathrm{~m}$ SUBARU telescope, with the high dispersion spectrograph HDS (Noguchi et al. 2002), Subaru programme (9S16ATE005/o162060), under the Gemini time exchange programme (GU2016A-005). We observed the targets on 05/27/2016, with the standard HDS set-ups $\mathrm{Rb}$ and $\mathrm{Yc}$, with a total wavelength coverage of $4400 \AA-7950 \AA$. The slit width was set to 0.5 arcsec, corresponding to a resolving power of $R=72000$. The exposure times were 100, 1000, and $1500 \mathrm{~s}$ for HD 103095, HD 134439, and HD 134440, respectively, yielding an $\mathrm{S} / \mathrm{N} \sim 250$ at $5000 \AA$.

In order to make a good differential analysis, it is necessary to use a very well-known star as a standard point of comparison for the measurements. With that in mind, we observed the star HD 103095 as our standard for the objects of our study (HD 134439/134440 pair). This star has been measured several times and there are detailed spectroscopic analysis that can be traced back to as far as Tomkin (1972, and references therein). The stellar parameters of the chosen star must be as similar as possible to the other objects of study, thus rendering HD 103095 a very good choice for this study (as will be further shown later in the paper).

We also obtained a spectrum from the ESO archive for the star HD 163810 (ESO programme 071.B-0529). This UVES/VLT data have a wavelength coverage of 4000-7800 $\AA$, with a spectral resolution of $R=47000$ and $\mathrm{S} / \mathrm{N} \sim 250$ at $5000 \AA$.

HD 163810 was analysed in NS10, NS11, and Ramírez et al. (2012). Their measurements place the pattern of this star in the low- $\alpha$ population. Their adopted stellar parameters $\left(T_{\text {eff }}=5501 \mathrm{~K}\right.$, $\log g=4.56 \mathrm{dex},[\mathrm{Fe} / \mathrm{H}]=-1.20 \mathrm{dex}$, and $v_{T}=1.30 \mathrm{~km} \mathrm{~s}^{-1}$ ) are relatively close to the stellar parameters of the binary pair we are studying, so we also analyse this star to use its abundances as a consistency check of our results.

The carbon measurements of HD 103095, HD 134439, and HD 134440 were done via spectral synthesis of the $4300 \AA$ carbon
$G$-band feature, using HIRES/Keck data from the KOA archive, ${ }^{1}$ project $\mathrm{N} 31 \mathrm{H}$. To synthesize the $\mathrm{CH}$ lines, we used the spectral region 4295-4350 $\AA$, which has an $\mathrm{S} / \mathrm{N} \sim 100$.

The HDS/Subaru data were reduced in real-time by the Subaru staff using IRAF scripts, performing flat, bias corrections, spectral order extractions, and wavelength calibrations. Later, we also corrected the spectra for barycentric and radial velocities and normalized the spectra. The UVES/VLT data obtained had been reduced by the ESO pipeline, which performed order extraction, flat-field and bias corrections, and wavelength calibration. The remaining processing (barycentric and radial velocity corrections) were done using the IRAF package for PYTHON, PYRAF, ${ }^{2}$ and the normalization of the spectrum was done with IRAF. The HIRES/Keck data are provided in reduced format already, processed through the $\mathrm{MAKEE}^{3}$ package. Further processing (Doppler correction and normalization) was done with IRAF.

\section{STELLAR PARAMETERS AND CHEMICAL ABUNDANCES}

We have performed manual EW measurements via the splot task in IRAF, using Gaussian profile fitting. It is also important to stress that a given line was measured consecutively in all three stars, setting a consistent continuum, resulting thus in reliable precise measurements. An example of the linelist with the measured EW can be seen in Table A1, and the full linelist can be found online. The EWs were analysed employing the semi-automatic code q2 (Ramírez et al. 2014), which uses EW to fit abundances through curves-ofgrowth employing the ABFIND function of MOOG (Sneden 1973), with MARCS plane-parallel 1D model atmospheres (Gustafsson et al. 2008) to estimate stellar parameters and abundances.

The determination of stellar parameters and chemical abundances was done via a line-by-line differential analysis (Meléndez et al. 2012; Yong et al. 2013; Ramírez et al. 2015; Reggiani et al. 2016). As mentioned, we chose to observe HD 103095 as the standard star of the differential analysis. We adopted the stellar parameters of HD 103095 based on the recent detailed work by Sitnova et al. (2015): $T_{\text {eff }}=5100 \pm 65 \mathrm{~K}, \log g=4.65 \pm 0.08 \mathrm{dex}, v_{T}=$ $0.90 \pm 0.05 \mathrm{~km} \mathrm{~s}^{-1}$, and we adopted $[\mathrm{Fe} / \mathrm{H}]=-1.35 \pm 0.08 \mathrm{dex}$ from our $\mathrm{Fe}$ I and $\mathrm{Fe}$ II line measurements, which agrees within the errors with the $[\mathrm{Fe} / \mathrm{H}]=-1.26 \pm 0.08$ iron abundance determined by Sitnova et al. (2015).

Using the $\mathrm{Fe}$ I and $\mathrm{Fe}$ II abundances as reference, we employed the differential technique and obtained the model atmospheric parameters of the other three stars; these are summarized in Table 1. The stellar parameters we determined for star HD $163810\left(T_{\text {eff }}=5526 \mathrm{~K}\right.$, $\log g=4.56 \mathrm{dex},[\mathrm{Fe} / \mathrm{H}]=-1.26 \mathrm{dex}$, and $v_{T}=0.99 \mathrm{~km} \mathrm{~s}^{-1}$ ) are in good agreement with the atmospheric parameters derived by NS10 (shown in Section 2). This excellent agreement between the stellar parameters of HD 163810 from NS10 and ours provides reliability to the comparison of our abundance pattern to theirs.

The differential chemical abundances of 24 elements, including some neutron capture elements (C, O, Na, Mg, Si, K, Ca, Sc, Ti, V,

\footnotetext{
${ }^{1}$ The Keck Observatory archive (KOA) is a joint development between the W. M. Keck Observatory (WMKO) located in Waimea, Hawaii and the NASA Exoplanet Science Institute (NExScl) located in Pasadena, California. https://www2.keck.hawaii.edu/koa/public/koa.php

${ }^{2}$ PYRAF is a product of the Space Telescope Science Institute, which is operated by AURA for NASA.

${ }^{3}$ http://www.astro.caltech.edu/ tb/makee/
} 
Table 1. Stellar parameters and chemical abundances of the sample. The standard star is highlighted. $T_{\text {eff }}$ is in $\mathrm{K}, \log g$ in dex, $[\mathrm{Fe} / \mathrm{H}]$ in dex, $v_{T}$ in $\mathrm{km} \mathrm{s}^{-1}$, and the $[\mathrm{X} / \mathrm{Fe}]$ in $\operatorname{dex}$.

\begin{tabular}{|c|c|c|c|c|}
\hline$[\mathrm{X} / \mathrm{Fe}]$ & HD 103095 & HD 163810 & HD 134439 & HD 134440 \\
\hline$T_{\text {eff }}$ & 5100 & 5526 & 5084 & 4946 \\
\hline$\sigma T_{\text {eff }}$ & 65 & 47 & 27 & 26 \\
\hline $\log g$ & 4.65 & 4.56 & 4.66 & 4.68 \\
\hline$\sigma \log g$ & 0.08 & 0.09 & 0.06 & 0.07 \\
\hline$[\mathrm{Fe} / \mathrm{H}]$ & -1.35 & -1.26 & -1.43 & -1.39 \\
\hline$\sigma[\mathrm{Fe} / \mathrm{H}]$ & 0.08 & 0.03 & 0.02 & 0.02 \\
\hline $\mathrm{v}_{T}$ & 0.90 & 0.99 & 1.22 & 1.17 \\
\hline$\sigma v_{\mathrm{T}}$ & 0.05 & 0.15 & 0.08 & 0.06 \\
\hline $\mathrm{C}$ & -0.35 & - & -0.15 & -0.13 \\
\hline$\sigma \mathrm{C}$ & 0.100 & - & 0.150 & 0.130 \\
\hline $\mathrm{O}$ & 0.549 & 0.691 & $\leq 0.040$ & $\leq-0.020$ \\
\hline$\sigma \mathrm{O}$ & 0.077 & 0.051 & 0.039 & 0.051 \\
\hline $\mathrm{Na}$ & -0.262 & -0.331 & -0.382 & -0.314 \\
\hline$\sigma \mathrm{Na}$ & 0.050 & 0.096 & 0.039 & 0.031 \\
\hline $\mathrm{Mg}$ & 0.117 & 0.087 & -0.026 & -0.067 \\
\hline$\sigma \mathrm{Mg}$ & 0.044 & 0.032 & 0.022 & 0.023 \\
\hline $\mathrm{Si}$ & 0.089 & 0.106 & -0.003 & 0.027 \\
\hline$\sigma \mathrm{Si}$ & 0.071 & 0.045 & 0.048 & 0.049 \\
\hline K & 0.339 & 0.454 & 0.143 & 0.136 \\
\hline$\sigma \mathrm{K}$ & 0.094 & 0.129 & 0.059 & 0.051 \\
\hline $\mathrm{Ca}$ & 0.249 & 0.214 & 0.108 & 0.124 \\
\hline$\sigma \mathrm{Ca}$ & 0.055 & 0.039 & 0.024 & 0.024 \\
\hline $\mathrm{Sc}$ & 0.076 & 0.036 & -0.049 & -0.053 \\
\hline$\sigma \mathrm{Sc}$ & 0.043 & 0.074 & 0.064 & 0.069 \\
\hline $\mathrm{Ti}$ & 0.291 & 0.261 & 0.093 & 0.127 \\
\hline$\sigma \mathrm{Ti}$ & 0.042 & 0.049 & 0.037 & 0.039 \\
\hline $\mathrm{V}$ & 0.138 & 0.153 & 0.049 & 0.106 \\
\hline$\sigma \mathrm{V}$ & 0.086 & 0.063 & 0.036 & 0.035 \\
\hline $\mathrm{Cr}$ & 0.006 & -0.067 & -0.022 & -0.005 \\
\hline$\sigma \mathrm{Cr}$ & 0.098 & 0.053 & 0.092 & 0.139 \\
\hline $\mathrm{Mn}$ & -0.402 & -0.429 & -0.405 & -0.414 \\
\hline$\sigma \mathrm{Mn}$ & 0.052 & 0.036 & 0.027 & 0.021 \\
\hline $\mathrm{Co}$ & 0.025 & 0.020 & -0.046 & -0.064 \\
\hline$\sigma \mathrm{Co}$ & 0.052 & 0.051 & 0.035 & 0.059 \\
\hline $\mathrm{Ni}$ & -0.061 & -0.078 & -0.123 & -0.141 \\
\hline$\sigma \mathrm{Ni}$ & 0.028 & 0.034 & 0.018 & 0.031 \\
\hline $\mathrm{Cu}$ & -0.343 & -0.500 & -0.597 & -0.498 \\
\hline$\sigma \mathrm{Cu}$ & 0.071 & 0.033 & 0.065 & 0.033 \\
\hline $\mathrm{Zn}$ & 0.076 & -0.006 & -0.001 & -0.017 \\
\hline$\sigma \mathrm{Zn}$ & 0.038 & 0.024 & 0.030 & 0.027 \\
\hline $\mathrm{Sr}$ & -0.377 & -0.238 & -0.620 & -0.530 \\
\hline$\sigma \mathrm{Sr}$ & 0.081 & 0.048 & 0.034 & 0.034 \\
\hline $\mathrm{Y}$ & 0.058 & -0.024 & -0.237 & -0.180 \\
\hline$\sigma \mathrm{Y}$ & 0.046 & 0.076 & 0.033 & 0.077 \\
\hline $\mathrm{Ba}$ & -0.028 & 0.075 & -0.249 & -0.230 \\
\hline$\sigma \mathrm{Ba}$ & 0.048 & 0.048 & 0.028 & 0.030 \\
\hline $\mathrm{La}$ & $\leq 0.308$ & $\leq 0.017$ & $\leq 0.111$ & $\leq-0.035$ \\
\hline $\mathrm{Ce}$ & $\leq 0.060$ & $\leq 0.250$ & $\leq 0.055$ & $\leq 0.023$ \\
\hline $\mathrm{Nd}$ & $\leq 0.242$ & $\leq 0.262$ & $\leq-0.166$ & $\leq-0.064$ \\
\hline $\mathrm{Sm}$ & $\leq 0.741$ & $\leq 0.587$ & $\leq 0.364$ & $\leq 0.459$ \\
\hline
\end{tabular}

$\mathrm{Cr}, \mathrm{Mn}, \mathrm{Fe}, \mathrm{Co}, \mathrm{Ni}, \mathrm{Cu}, \mathrm{Zn}, \mathrm{Sr}, \mathrm{Y}, \mathrm{Ba}, \mathrm{La}, \mathrm{Ce}, \mathrm{Nd}$, and $\mathrm{Sm}$ ), were estimated. We have used the differential results, along with the absolute measurements of the standard star, and estimated $[\mathrm{X} / \mathrm{Fe}]$ ratios. The $[\mathrm{X} / \mathrm{Fe}]$ ratios and their errors are displayed in Table 1 ; it is important to stress that the abundances of $\mathrm{O}, \mathrm{La}, \mathrm{Ce}, \mathrm{Nd}$, and $\mathrm{Sm}$ are upper limits, as they were derived from very weak lines.

We tested the possibility of enhanced rotation in one of the stars by estimating the vsini of stars HD 103095, HD 134439, and HD 134440. We chose four very well-defined Fe I lines (4602, 5250, 4944, and $5247 \AA$ ) and performed a spectral synthesis to
Table 2. Differential abundances $[\mathrm{X} / \mathrm{H}]$ for $\mathrm{HD}$ 134440 minus HD 134439.

\begin{tabular}{lll}
\hline Element & {$[\mathrm{X} / \mathrm{H}]$} & $\sigma[\mathrm{X} / \mathrm{H}]$ \\
\hline $\mathrm{C}$ & 0.090 & 0.043 \\
$\mathrm{Na}$ & 0.116 & 0.064 \\
$\mathrm{Mg}$ & 0.008 & 0.019 \\
$\mathrm{Si}$ & 0.122 & 0.054 \\
$\mathrm{~K}$ & 0.042 & 0.046 \\
$\mathrm{Ca}$ & 0.065 & 0.024 \\
$\mathrm{Sc}$ & 0.041 & 0.032 \\
$\mathrm{Ti}$ & 0.083 & 0.034 \\
$\mathrm{~V}$ & 0.106 & 0.035 \\
$\mathrm{Cr}$ & 0.066 & 0.031 \\
$\mathrm{Mn}$ & 0.040 & 0.034 \\
$\mathrm{Co}$ & 0.028 & 0.043 \\
$\mathrm{Ni}$ & 0.031 & 0.024 \\
$\mathrm{Cu}$ & 0.148 & 0.040 \\
$\mathrm{Zn}$ & 0.032 & 0.031 \\
$\mathrm{Sr}$ & 0.139 & 0.034 \\
$\mathrm{Y}$ & 0.106 & 0.066 \\
$\mathrm{Ba}$ & 0.068 & 0.027 \\
\hline
\end{tabular}

the lines (with the stellar parameters and $\mathrm{Fe}$ abundance previously estimated via equivalent widths), varying the vsini until achieving the best fit. The synthesis was done with the radiative transfer code MOOG (Sneden 1973), using the 'r' option for the line smoothing, which includes the limb darkening (which we adopted to be $0.6)$, the FWHM of the Gaussian ( $=0.07 \AA$, based on the resolution of our spectra), the macroturbulent velocity (the average result from the following equations: $\mathrm{V}_{\text {macro }}=13.499-0.00707 \times \mathrm{T}_{\text {eff }}+$ $9.2422 \times 10^{-7} \times \mathrm{T}_{\text {eff }}^{2}, V_{\text {macro }}=3.5+\left(T_{\text {eff }}-5777\right) / 650$ and $V_{\text {macro }}$ $=3.5+\left(T_{\text {eff }}-5777\right) / 388$; Meléndez et al. 2012), and the vsini.

Subsequently, we performed a new differential analysis on star HD 134440, with star HD 134439 as the standard star. In this analysis, we used the model stellar parameters previously determined (Table 1). So the abundance differences were estimated through the comparison of each measured line of HD 134440 against the same line on HD 134439. The abundance differences we estimated in this new comparison can be seen in Table 2. This new analysis is used only to directly compare the abundances of the binary pair and our interpretation on planet signatures (Section 4.4).

\section{DISCUSSION}

\subsection{Comparison with the low- and high- $\alpha$ NS10/11 populations.}

In Figs 1 and 2, we show the [X/Fe] ratios of $\mathrm{O}, \mathrm{Na}, \mathrm{Mg}, \mathrm{Si}, \mathrm{Ca}$, $\mathrm{Ti}, \mathrm{Cr}, \mathrm{Ni}, \mathrm{Mn} \mathrm{Cu}, \mathrm{Zn}, \mathrm{Y}, \mathrm{Ba}, \mathrm{Sc}, \mathrm{La}, \mathrm{Ce}$, and $\mathrm{Nd}$, along with abundances of the low- and high- $\alpha$ populations from NS10 and NS11, complemented with abundances from Fishlock et al. (2017) and Ramírez et al. (2012) (we refer to the above four references as 'NS'), and a sample of abundances from stars of dSphs, from the works of Shetrone et al. (2003), Geisler et al. (2005), Monaco et al. (2005), and Letarte et al. (2010).

We also show both ours and NS measurements of the star HD 163810. Although the measurement from the different studies are similar, there is a small offset between the NS measurements and ours. We averaged this difference for the $\alpha$-elements [including oxygen, which uses the same NLTE corrections as the work of Ramírez et al. (2012)] and found a mean offset of 0.05 dex with a standard deviation of 0.04 dex. 

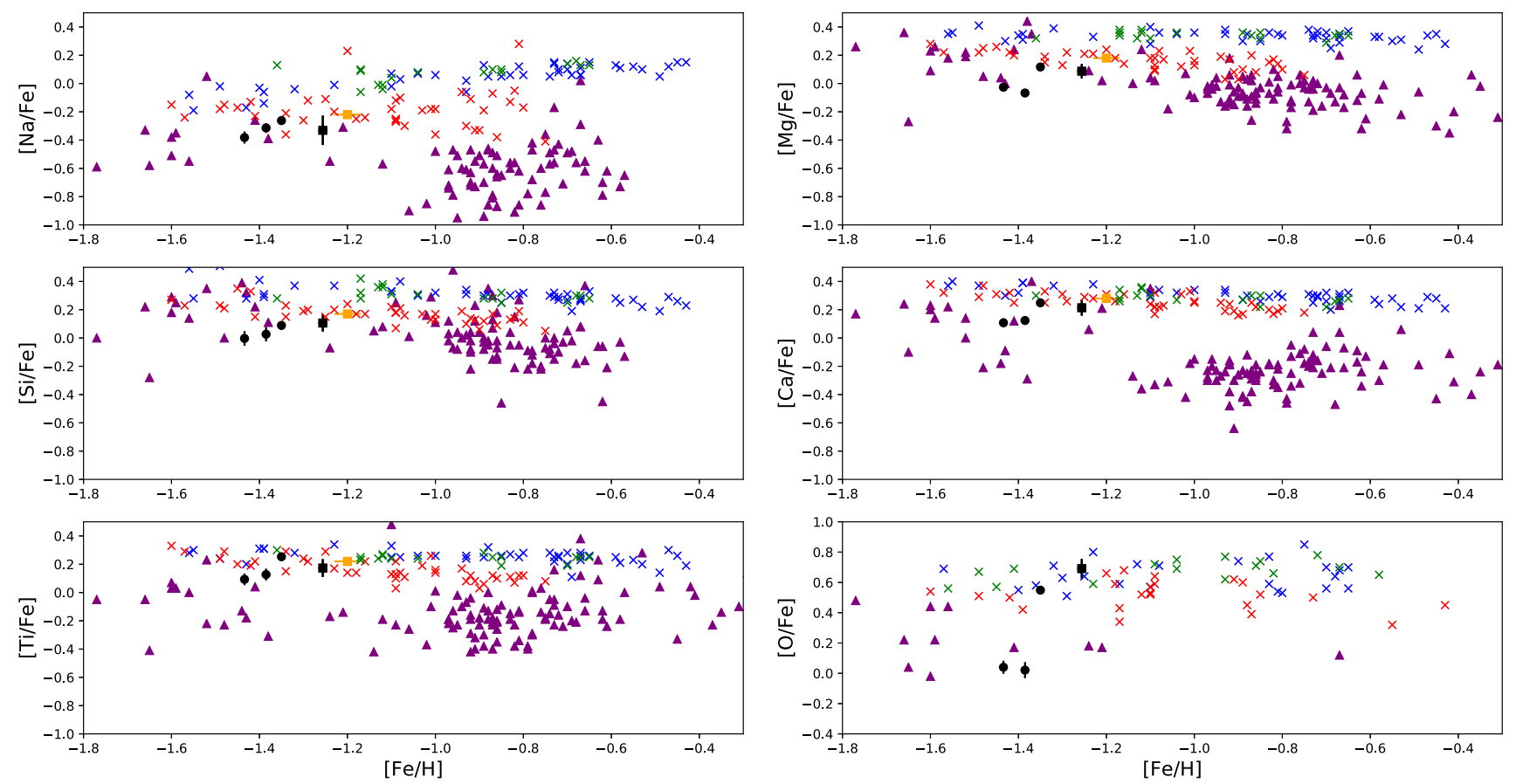

Figure 1. $[\mathrm{X} / \mathrm{Fe}]$ ratios for $\alpha$-elements, Ti and Na. Stars HD 103095, HD 134439, and HD 134440 are plotted as black circles. The standard HD 103095 is the most metal rich of the three stars. The squares represent the star HD 163810 (black and orange colours refer to our measurements and those from NS, respectively). The blue, red, and green crosses are the high- $\alpha$, low- $\alpha$, and thick disc populations of NS. The purple triangles are dSphs (Carina, Sculptor, Fornax, and Sagittarius) stars from Shetrone et al. (2003), Geisler et al. (2005), Monaco et al. (2005), and Letarte et al. (2010).

The black circles are the abundance pattern of stars HD 103095, HD 134439, and HD 134440. The abundance pattern of star HD 103095 closely follows that of the low- $\alpha$ population from NS10. There is a remarkable difference between this star and the pair HD 134439 and HD 134440, specially the [O/Fe] ratio. Our very low oxygen abundance for this pair also agrees with the previous analysis by Chen et al. (2014), who used OH lines in the ultraviolet, in which the HD 134439/HD 134440 binary have [O/Fe] = $-0.26 \pm 0.13$, which is below the most deficient $\alpha$-element in their analysis.

The abundance patterns of HD 134439 and HD 134440 are not compatible with the low- $\alpha$ population of NS for $\mathrm{O}, \mathrm{Mg}, \mathrm{Si}, \mathrm{Ca}$, as can be seen in Fig. 1. Even after adding the systematic difference of 0.05 dex between our measurements and the values from NS, this metal-poor pair still shows $[\mathrm{X} / \mathrm{Fe}]$ ratios below those of the $\alpha$-poor population of NS10. The striking very low- $\alpha$ abundance pattern that we have determined has been previously reported by Chen et al. (2014, table 12) for $\mathrm{O}, \mathrm{Mg}, \mathrm{Si}$ and $\mathrm{Ca}$, but while they compared the abundance pattern of the pair HD 134439/HD 134440 to the overall abundance pattern of halo stars, we have made a more precise comparison, using a line-by-line analysis relative to the star HD 103095, which has similar stellar parameters, and with observations obtained with the same observational set-up. Also, our comparison of the $\alpha$-poor star HD 163810 from NS indicates that HD 103095 is part of the low- $\alpha$ population. Thus, our work reinforces the previous results by Chen et al. (2014) and demonstrates that the binary HD $134439 /$ HD 134440 is definitely below the low- $\alpha$ population from NS10.

It is important to stress that, although the behaviour of $[\mathrm{Ti} / \mathrm{Fe}]$ can be similar to the $[\alpha / \mathrm{Fe}]$ ratios, the nucleosynthetic origin of $\mathrm{Ti}$ is still a matter of debate (e.g. Nomoto et al. 2006; The et al. 2006; Clayton 2007; Wongwathanarat et al. 2017). For Na, Ti, Ni, and Zn, the abundances are consistent with the low- $\alpha$ population, with their ratios being slightly below the lower envelope defined by the low- $\alpha$ stars. Both members of the binary system do not show any sizable difference in the abundance pattern of other elements, such as $\mathrm{Cr}$, $\mathrm{Mn}$, and heavy elements $\mathrm{Y}, \mathrm{Ba}, \mathrm{La}$, and $\mathrm{Ce}$, when compared to the low- and high- $\alpha$ populations.

Albeit the low $\mathrm{O}$ and $\mathrm{Mg}$ may be suggestive of an origin in a halo globular cluster, the low $\mathrm{Na}$ in the pair discards such an hypothesis, as discussed by Chen et al. (2014).

\subsection{Comparison with dwarf spheroidal galaxies}

Studies of chemical abundances in dSph (e.g. Sbordone et al. 2007; Frebel et al. 2016; Ji et al. 2016b; Suda et al. 2017) show that the stars born in these environments have a distinctive abundance pattern of low $[\alpha / \mathrm{Fe}]$ in a broad range of metallicities, from almost solar to the ultra metal-poor region. Other elements such as Sc also show low abundance ratios.

The abundance pattern of different dSph can be used to better constrain the birth place of the HD 134439/HD 134440 pair. A first approach is to study the position of the knee in different extragalactic systems. The 'knee' is a drop in the $\alpha$-element content due to the different time-scales between type Ia and type II supernovae (Tinsley 1979; Mateucci \& Brocato 1990). The particular time-scales of different environments influences the position of the knee in the $[\alpha / \mathrm{Fe}]$ versus $[\mathrm{Fe} / \mathrm{H}]$ diagram, which can be used as a chemical tag for the formation environment of the stars. Suda et al. (2017) studied the location of the knee in the Milky Way, Fornax, Sculptor, and Draco, showing that the knees for the above dSphs are at lower metallicities than that of the Milky Way (see table 2 of Suda et al. 2017). In this context, the distinctive $\alpha$-element pattern of HD 134439/HD 134440 resembles the pattern of a dSph with a $[\alpha / \mathrm{Fe}]$ knee below $[\mathrm{Fe} / \mathrm{H}] \leq-1.5$, as previously suggested by Chen \& Zhao (2006) and Chen et al. (2014). 

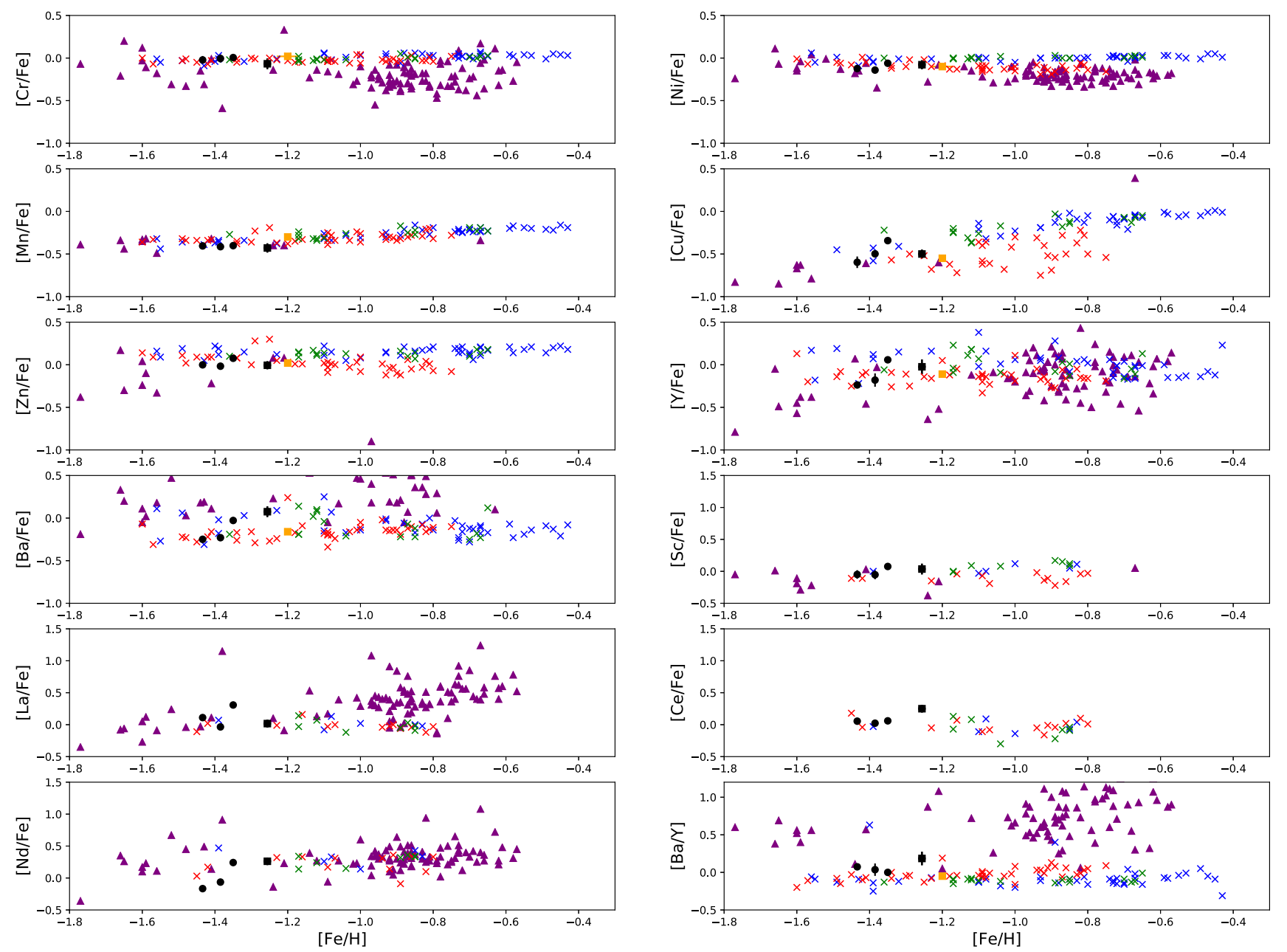

Figure 2. [X/Fe] ratios as in Fig. 1 for $\mathrm{Cr}, \mathrm{Mn}, \mathrm{Ni}, \mathrm{Cu}, \mathrm{Zn}, \mathrm{Y}, \mathrm{Ba}, \mathrm{Sc}, \mathrm{La}, \mathrm{Ce}, \mathrm{Nd}$, and [Ba/Y].

Spitoni et al. (2016) argues that the best chemical element to tag the birth environment of a star might be barium. From their models, there is a distinctive difference in barium from dSphs, ultra-faint dwarf galaxies (UfDs), and the Milky Way halo. From our measurements, however, the $[\mathrm{Ba} / \mathrm{Fe}]$ ratio for the binary pair is consistent with the low- $\alpha$ stars (which are believed to be accreted stars) but not consistent with the several measurements of dSphs. Barium has more than one nucleosynthetic origin (it can be produced both in s and r-processes) and the barium excess or lack of excess might not exclusively indicate the formation environment; it may be more important to distinguish the main sources of nucleosynthesis in a given environment.

The $[\mathrm{Ba} / \mathrm{Y}]$ ratio is also a good indicator of the origin of a given star. A high enough value can be linked to a high SN II environment, with not enough time for the rise of s-process based on AGB stars, like is commonly associated with dSphs (Tolstoy et al. 2009; Chen et al. 2014). This can be clearly seen in our Fig. 2, in which the $[\mathrm{Ba} / \mathrm{Y}]$ ratio of dSphs stars are clearly super-solar, reaching $1 \mathrm{dex}$ for the higher metallicity regime. Although our results are a bit higher than reported by Chen et al. (2014) and Chen \& Zhao (2006) $([\mathrm{Ba} / \mathrm{Y}]=-0.01,-0.05$ for HD 134439 and HD 134440, respectively, against $[\mathrm{Ba} / \mathrm{Y}]=-0.13,-0.08$ found by Chen et al. (2014), for HD 134439 and HD 134440, respectively), our result is consistent with theirs as the ratios we report are sub-solar.
As can be seen in the last panel of Fig. 2, the ratios we found are somewhat above the NS11 measurements (although within the errors), as is also the case of our measurements of the comparison star HD 163810. If we subtract from our other objects the offset between our measurements and that by NS11 for HD 163810, the $[\mathrm{Ba} / \mathrm{Y}]$ ratios for HD 103095, HD 134439, and HD 134440 agree perfectly with the NS11 ratios, thus being in agreement with the low- $\alpha$ and high- $\alpha$ Galactic halo. However, as already pointed by Chen et al. (2014), there is a remarkable offset between these stars and the super-solar $([\mathrm{Ba} / \mathrm{Y}] \sim 0.6 \mathrm{dex})$ ratios observed in $\mathrm{dSph}$ galaxies, pointing to a birth environment that does not favour a high production of heavy relative to light $n$-capture elements.

We can further constrain the birth environment of these stars using the diagram on fig. 14 of Suda et al. (2017). We adopt the $[\mathrm{Eu} / \mathrm{Fe}] \leq 0.31$ for HD 134439 and $[\mathrm{Eu} / \mathrm{Fe}] \leq 0.42$ for HD 134440 from Chen et al. (2014) and our measurements for [Ba/Fe], which result in both stars being located on the r-process dominant region, where $[\mathrm{Eu} / \mathrm{Ba}] \geq 0.5$.

We used chemical tagging to constrain the birth environment of these stars. We searched for a dSph galaxy with a knee below $[\mathrm{Fe} / \mathrm{H}] \leq-1.5$, low $[\mathrm{Ba} / \mathrm{Y}]$ ratio (indicating low production of heavy relative to low $n$-capture elements) and with stars presenting r-process dominated patterns. We propose that this pair of stars might be from an environment similar to Fornax, as all the above chemical requirements, as well as kinematic constraints, are met. 


\subsection{Trend with condensation temperature}

Chen \& Zhao (2006) analysed the elemental abundances versus condensation temperature $\left(T_{\text {cond }}\right)$ of these stars and attributed their abundance pattern to a formation in an environment of low type II supernovae with a high dust-to-gas ratio. On their subsequent study (Chen et al. 2014), they argue against that possibility as the beryllium abundances do not support their previous claim. To verify these results, we show in the upper panel of Fig. 3 a plot of the $[\mathrm{X} / \mathrm{Fe}]$ abundances versus condensation temperature for both stars. As the slopes are not significant $\left(3.55 \times 10^{-4} \pm 2.42 \times 10^{-4}\right.$ and $-5.51 \times 10^{-6} \pm 2.49 \times 10^{-4}$, for the blue and black fits in the upper panel), no clear trend with condensation temperature can be seen from this data. The large scatter is likely caused by the comparison of halo stars (with a distinct abundance pattern nonetheless) to the Sun.

Instead of using the Sun as the standard, a more appropriate comparison can be done using a star with a closer nucleosynthetic history, as is the case of HD 103095. Thus, we present the differential abundances HD 134439/HD 134440 - HD 103095. In that case the scatter is considerably reduced and no correlation with condensation temperature (middle and lower panels of Fig. 3 ) is observed (slopes of $-1.71 \times 10^{-4} \pm 1.00 \times 10^{-4}$ and $-4.54 \times 10^{-5} \pm 8.87 \times 10^{-5}$, significant only at the $\sim 1.7 \sigma$ and $\sim 0.5 \sigma$ levels for HD 134439 and HD 134440, respectively). Thus, the birth environment of the pair do not have a significant dust effect, as also concluded by Chen et al. (2014) through the analysis of the Be abundance, unlike previous suggestions by Chen $\&$ Zhao (2006), who compared the binary pair to the Sun. Our comparison to a standard metal-poor halo star of similar metallicity is more appropriate than the comparison to the Sun $([\mathrm{X} / \mathrm{Fe}])$, which is a thin disc star with quite a different nucleosynthetic history.

\subsection{Planet signature}

It has been claimed that the low- $\alpha$ abundance along with a trend in condensation temperature can be indicative of the engulfment of planetesimals. Chen et al. (2014) argued against this possibility as it is very unlikely that both stars of the pair engulfed the same mass of planetesimals as they have very similar abundance patterns. However, as can be seen in Fig. 4, there is a mean difference of $0.06 \pm 0.01 \mathrm{dex}$ (weighted abundance and weighted error, from $\mathrm{C}$ to $\mathrm{Ba}$, and excluding the upper limits) between the abundances of the pair. As these stars were likely born from the same cloud, as also indicated by their kinematics, the abundance difference between these stars should be zero rather than 0.06 dex. Notice that the differences plotted in Fig. 2 were calculated through a new differential analysis of these stars. In order to better constrain the differences between the stars, we used the stellar parameters previously obtained and recalculated the differential abundances between them, using HD 134439 as the standard star; the differential abundances are shown in Table 2.

To test the probability of finding such abundance differences from zero, we performed a $t$-test, to estimate the probability that two different samples have the same averaged value. The control sample for our test is a sample of zeros (assuming that the binary components have the same abundances). We obtained a $T=7.51$, which is the calculated difference represented in units of standard error. The higher $T$ represents a higher probability of a real difference between the samples. From the test, we obtained a probability $p=1.03 \times 10^{-8}$ of obtaining such a high $T$ value. Thus, it is extremely unlikely that the abundance difference of these stars is 0 .
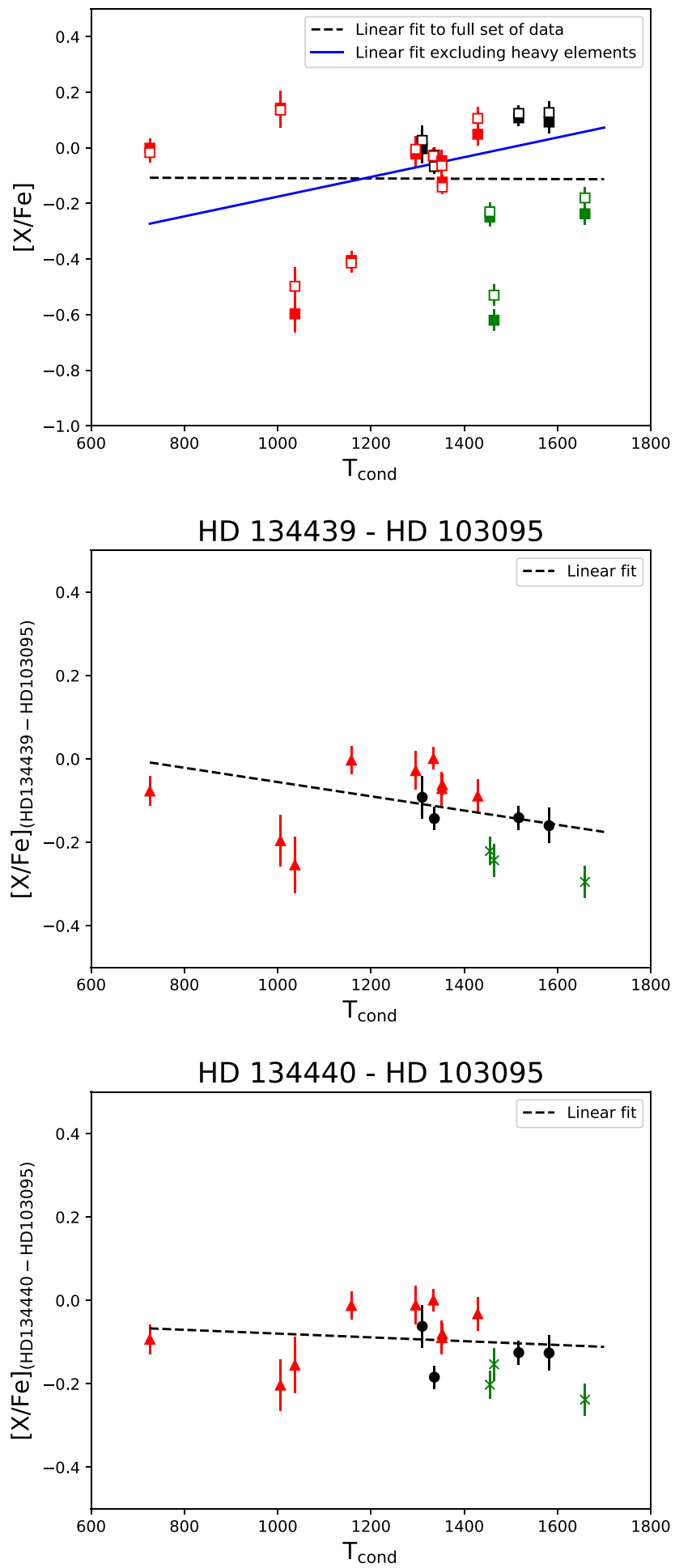

Figure 3. Chemical abundances versus $T_{\text {cond }}$. In the top panel, the $[\mathrm{X} / \mathrm{Fe}]$ for HD 134439 and HD 134440 are shown with filled and open squares, respectively. The middle and lower panels are the differential $[\mathrm{X} / \mathrm{Fe}](\mathrm{HD}$ 134439/HD $134440-$ HD 103095) ratios versus $T_{\text {cond }}$. On three panels, the black colours represent the $\alpha$-elements, the red symbols are the ironpeak elements, and the green points represent the $n$-capture elements $(\mathrm{Sr}$, $\mathrm{Y}$, and $\mathrm{Ba}$ ). The dashed black lines on all panels are the fits to the entire set of abundances and the blue dashed line on the top panel is the fit to the abundances excluding the neutron capture elements. 


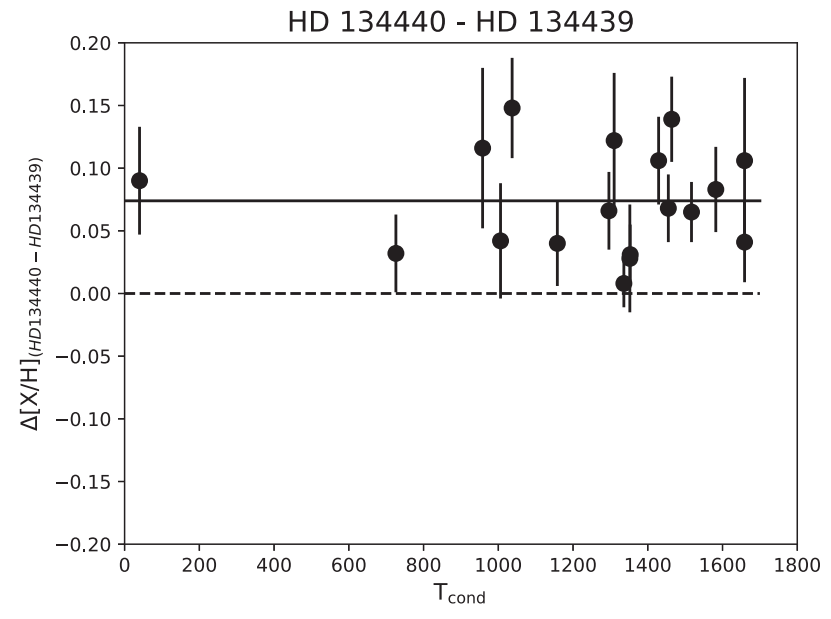

Figure 4. Differential $\Delta[\mathrm{X} / \mathrm{H}]$ abundances between the binaries (HD 134440 - HD 134439) from carbon to barium. The solid line is the fit to the entire set of abundances. The dashed line represents the mean difference in chemical composition that the stars would have if they were chemically identical.

We also performed the $t$-test varying 100000 times the abundances with randomly generated errors from 0 to $0.03 \mathrm{dex}$, and obtained the average $T=5.88$ and $p=2.05 \times 10^{-6}$. Thus, even considering the errors, it is very unlikely that the difference we found could arise just by chance.

Abundance differences have also been found by Chen et al. (2014; table 12), but they found some chemical elements to be less abundant in HD 134440, while others are less abundant in HD 134439; this could be due to differences in the temperature scales between the two works. Chen et al. (2014) adopted the stellar parameters from King (1997), which have a temperature contrast of $215 \mathrm{~K}$ between the components, while we found a difference of $138 \mathrm{~K}$. As mentioned by Chen et al. (2014), the newer IFRM temperatures of Casagrande et al. (2010) have a smaller temperature difference between the components, of only $127 \mathrm{~K}$, or about $90 \mathrm{~K}$ cooler than the adopted by Chen \& Zhao (2006). Notice that our spectroscopic temperature difference $(138 \mathrm{~K})$ is only $11 \mathrm{~K}$ away from the photometric temperature difference obtained from the IRFM by Casagrande et al. (2010), reinforcing thus the reliability of our temperature scale and our spectroscopic measurements. Also, the fact that all our abundance differences (HD 134440 - HD 134439) are consistently of the same sign, reinforces the reliability of the offset we found between the binaries.

In their study of the binary pair of solar twins $16 \mathrm{Cyg} \mathrm{A}$ and B (planet host), Ramírez et al. (2011) also found a similar abundance difference between the components of 0.04 dex. Schuler et al. (2011) did not find abundance differences in $16 \mathrm{Cyg}$, but further work with higher resolving power corroborated a systematic abundance difference between 16 Cyg A and B (Tucci Maia, Meléndez \& Ramírez 2014), a finding also confirmed at lower precision by Mishenina et al. (2016) and at extremely high precision by two recent works using spectra with $R=115000$ (Nissen et al. 2017) and $R=160000$ (Tucci Maia et al. 2018). As both components were formed from the same natal cloud, and both are twins, the abundance differences are likely related to planets. Other precise works on twin binaries with planets show abundance differences between the components of the binary pair, which are likely related to planets (Biazzo et al. 2015; Ramírez et al. 2015; Teske et al. 2016a; Teske, Khanal \& Ramírez 2016b; Saffe et al. 2017).
Sun-like stars deplete lithium as they age (e.g. Do Nascimento et al. 2009; Denissenkov 2010; Carlos, Nissen \& Meléndez 2016; Reddy \& Lambert 2017), but planets could also have an effect on the stellar Li abundances, causing either an enhancement (e.g. Sandquist et al. 2002; Aguilera-Gómez et al. 2016; Carlos et al. 2016; Meléndez et al. 2017; Saffe et al. 2017) or a depletion (e.g. Théado \& Vauclair 2012; Deal, Richard \& Vauclair 2015; Gonzalez 2015). Although planet accretion should increase the lithium content of the star, depending on the conditions of the accretion it could actually deplete Li due to thermohaline mixing (Théado \& Vauclair 2012) or to increased rotation due to transfer of angular momentum.

The HD 134439/HD 134440 lithium abundances were studied by King (1997), who found a lithium difference of 0.6 dex between them (HD 134440 being Li depleted), and claimed that the observed Li difference could be due to large differences in temperature (implying a significant mass difference) between the two components. We have also synthesized the lithium $6707.7 \AA$ line and found similar results; for HD $103095 \epsilon(\mathrm{Li})=+0.37 \pm 0.15$, on HD 1334439, $\epsilon(\mathrm{Li})=+0.55 \pm 0.15$, and $\epsilon(\mathrm{Li}) \leq-0.05$ in HD 134440. However, we find no significant difference in the stellar parameters to account for the lithium differences. Using the q 2 code (Ramírez et al. 2014), we estimated the masses and ages of these stars and both are very similar: 0.59 and $0.58 \mathrm{M}_{\odot}$ and 9.9 and $9.4 \mathrm{Gyr}$ for HD 134439 and HD 134440, respectively.

Thus, we see no evidence that would corroborate a stellar evolution explanation for the low lithium content on HD 134440. Instead, the low Li in HD 134440 could be due to a planet engulfment event. As mentioned above, under some conditions a planet engulfment event could trigger thermohaline convection and cause Li destruction (Deal et al. 2015). Interestingly, Chen et al. (2014) barely detected Be in HD 134439, while it was not present at all in HD 134440. This means that the induced extra-mixing due to the possible planet engulfment event was high enough that not only Li but also Be was depleted in HD 134440. The planet engulfment would also lead to an increase in the abundance of all metals, as we seem to detect in HD 134440, which seem enhanced in all chemical elements, in comparison to its companion HD 134439 .

It is unclear whether the rotation rate would be largely enhanced or not due to a planet engulfment event. In order to verify a possible increased rotation rate in HD 134440, we estimated vsini for HD 103095, HD 134439, and HD 134440, as previously mentioned in Section 3. We found vsini $=1.2 \pm 0.5,1.9 \pm 0.5$, and $2.0 \pm 0.5 \mathrm{~km} \mathrm{~s}^{-1}$ for HD 103095, HD 134439, and HD 134440, respectively. Thus, we see no significant difference in the rotation velocities of the binary pair, which means that the planet engulfment did not affect much the rotation rate of HD 134440. However, we cannot fully discard an increase in the rotation rate due to the unknown inclination angles.

Johnson \& $\mathrm{Li}$ (2012) estimated the critical metallicity for planet formation as $[\mathrm{Fe} / \mathrm{H}]_{\text {crit }}=-1.5+\log (r / 1 \mathrm{au})$, where $r$ is the distance of the planet to its host star in astronomical units (au). As the metallicity of the binary pair is $[\mathrm{Fe} / \mathrm{H}]=-1.4$, it means that it could form planets inside $\sim 1.3$ au, albeit this distance depends on the conditions for planet formation at low metallicities, which are still uncertain.

In light of the aforementioned discussion, we seem to detect, for the first time, the effect of planet engulfment in a metal-poor star. The star HD 134440 seems richer in the measured chemical elements, as the result of the planet engulfment, which may also explain the $\mathrm{Li}$ and Be difference amongst the two components of this binary pair. 


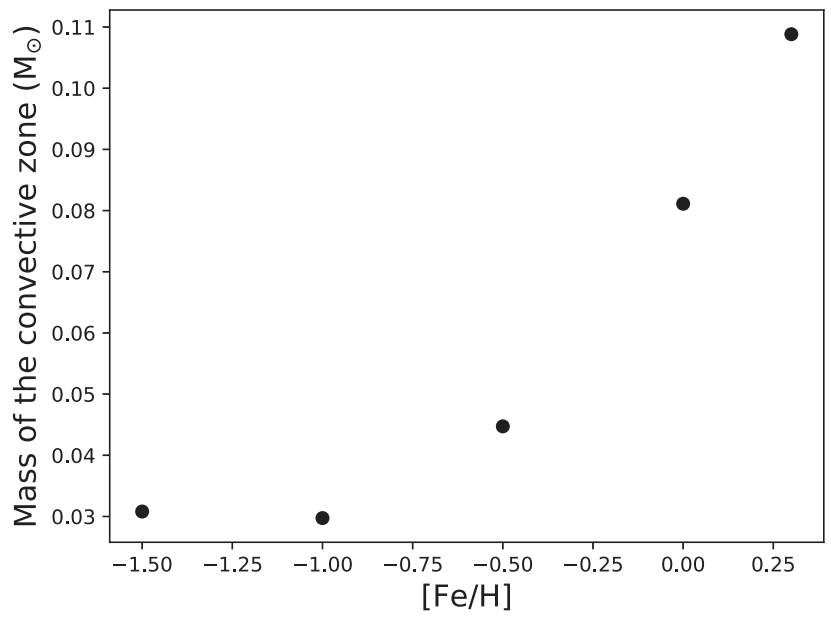

Figure 5. Mass of the convective zone for stars of $0.6 \mathrm{M}_{\odot}$ and $9.5 \mathrm{Gyr}$, at different metallicities, from the YAPSI tracks with a mixing length of 1.92 .

As the two stars (HD 134439 and HD 134440) are most likely from the same birth cloud, we assume that the difference in chemical abundance is due to a planet that might have formed and later engulfed. We estimate a possible mass of the engulfed planet using the same toy model proposed by Ramírez et al. (2011, equation 1). We assume the metal composition of the planet $(\mathrm{Z} / \mathrm{X})$ to be five times the initial composition of the star, which we adopt to be the same metallicity we observe today in HD 134439. With those assumptions and using a mass of $0.03 \mathrm{M}_{\odot}$ for the convective zone of HD 134440 (from the YAPSI $1^{4}$ evolutionary tracks, see Fig. 5), we estimate a planetary mass of $M \sim 0.9 M_{\mathrm{J}}$ that could have been engulfed by HD 134440 . Notice that as the convection zone is small at low metallicities (Fig. 5), planets can have an important effect on the chemical composition of metal-poor stars.

\section{CONCLUSIONS}

We conclude that the binary pair HD 134439/HD 134440 do not belong to either the low- $\alpha$ nor the high- $\alpha$ halo populations proposed by NS10, but follow more closely the $\alpha$ and the $r$-process dominant pattern of stars seen in dwarf galaxies such as Fornax. We also see evidence for the possible accretion of a $0.9 M_{\mathrm{J}}$ planet by $\mathrm{HD}$ 134440. If confirmed, it could corroborate that planetary formation can occur down to metallicities of $[\mathrm{Fe} / \mathrm{H}] \sim-1.5$.

\section{ACKNOWLEDGEMENTS}

H.R. thanks a CAPES fellowship and the CAPES PDSE programme (88881.132145/2016-01). J.M. thanks support by FAPESP (2012/24392-2 and 2014/18100-4) and CNPq (Productivity fellowship). We also thank Dr. Akito Tajitsu for his important support and initial data reduction during the observations with the Subaru Telescope. This study is based (in part) on data collected at Subaru Telescope, which is operated by the National Astronomical Observatory of Japan.

\footnotetext{
${ }^{4}$ http://www.astro.yale.edu/yapsi/download_grids.html
}

\section{REFERENCES}

Aguilera-Gómez C., Chanamé J., Pinsonneault M. H., Carlberg J. K., 2016, ApJ, 829, 127

Aoki W. et al., 2009, A\&A, 502, 569

Biazzo K. et al., 2015, A\&A, 583, A135

Bonifacio P., Hill V., Molaro P., Pasquini L., DiMarcantonio P., Santin P., 2000, A\&A, 359, 663

Bonifacio P., Sbordone L., Marconi G., Pasquini L., Hill V., 2004, A\&A, 414,503

Carlos M., Nissen P. E., Meléndez J., 2016, A\&A, 587, A100

Carney B. W., Laird J. B., Latham D. W., Aguilar L. A., 1996, AJ, 112, $668 \mathrm{C}$

Casagrande L., Ramírez I., Meléndez J., Bessell M., Asplund M., 2010, A\&A, 512, A54

Chen Y. Q., Zhao G., 2006, MNRAS, 370, 2091

Chen Y., King J. R., Boesgaard A. M., 2014, PASP, 126, 1010C

Clayton D., 2007, Handbook of Isotopes in the Cosmos. Cambridge Univ. Press, Cambridge

Cohen J. G., Huang W., 2009, ApJ, 701, 1053

Deal M., Richard O., Vauclair S., 2015, A\&A, 584, A105

Denissenkov P. A., 2010, ApJ, 719, 28

Do Nascimento J. D., Jr, Castro M., Meléndez J., Bazot M., Théado S., Porto de Mello G. F., de Medeiros J. R., 2009, A\&A, 501, 687

Fabrizio M. et al., 2015, A\&A, 580, A18

Fishlock C. K., Yong D., Karakas A. I., Alves-Brito A., Meléndez J., Nissen P. E., Kobayashi C., Casey A. R., 2017, MNRAS, 466, 4672

Frebel A., Norris J. E., Gilmore G., Wyse R. F. G., 2016, ApJ, 826, 110

Geisler D., Smith V. V., Wallerstein G., Gonzalez G., Charbonnel C., 2005, AJ, 129, 1428

Gonzalez G., 2015, MNRAS, 446, 1020

Gustafsson B., Edvardsson B., Eriksson K., Jørgensen U. G., Nordlund Å., Plez B., 2008, A\&A, 486, 951

Ji A. P., Frebel A., Simon J. D., Chiti A., 2016b, ApJ, 830, 93

Johnson J. L., Li H., 2012, ApJ, 751, 81

King J. R., 1997, AJ, 113, 2302

Lemasle B. et al., 2012, A\&A, 538, A100

Lemasle B. et al., 2014, A\&A, 572, A88

Letarte B. et al., 2010, A\&A, 523, A17

Matteucci F., Brocato E., 1990, ApJ, 365, 539

Meléndez J. et al., 2012, A\&A, 543, A29

Meléndez J. et al., 2017, A\&A, 597, A34

Mishenina T., Kovtyukh V., Soubiran C., Adibekyan V. Z., 2016, MNRAS, 462,1563

Monaco L., Bellazzini M., Bonifacio P., Ferraro F. R., Marconi G., Pancino E., Sbordone L., Zaggia S., 2005, A\&A, 441, 141

Nissen P. E., Schuster W. J., 2010, A\&A, 511, L10 (NS10)

Nissen P. E., Schuster W. J., 2011, A\&A, 530, A15 (NS11)

Nissen P. E., Silva Aguirre V., Christensen-Dalsgaard J., Collet R., Grundahl F., Slumstrup D., 2017, A\&A, 608, A112

Noguchi K. et al., 2002, PASJ, 54, 855

Nomoto K., Tominaga N., Umeda H., Kobayashi C., Maeda K., 2006, Nucl. Phys. A, 777, 424

Ramírez I., Meléndez J., Cornejo D., Roederer I. U., Fish J. R., 2011, AJ, 740,76

Ramírez I., Meléndez J., Chanamé J., 2012, ApJ, 757, 164

Ramírez I. et al., 2014, A\&A, 572, A48

Ramírez I. et al., 2015, ApJ, 808, 13

Reddy A. B. S., Lambert D. L., 2017, ApJ, 845, 151

Reggiani H., Meléndez J., Yong D., Ramírez I., Asplund M., 2016, A\&A, 586, A67

Saffe C., Jofré E., Martioli E., Flores M., Petrucci R., Jaque Arancibia M., 2017, A\&A, 604, L4

Sandquist E. L., Dokter J. J., Lin D. N. C., Mardling R. A., 2002, ApJ, 572, 1012

Sbordone L., Bonifacio P., Buonanno R., Marconi G., Monaco L., Zaggia S., 2007, A\&A, 465, 815 
Schuler S. C., Cunha K., Smith V. V., Ghezzi L., King J. R., Deliyannis C. P., Boesgaard A. M., 2011, ApJ, 737, L32

Shetrone M. D., Bolte M., Stetson P. B., 1998, AJ, 115, 1888

Shetrone M. D., Côté P., Sargent W. L. W., 2001, ApJ, 548, 592

Shetrone M. D., Venn K., Tolstoy A., Primas F., Hill V., Kaufer A., 2003, AJ, 125, 684

Shigeyama T., Tsujimoto T., 2003, ApJ, 598, L47

Sitnova T. et al., 2015, ApJ, 808, 148

Sneden C. A., 1973, PhD thesis, Univ. Texas

Spitoni E., Vincenzo F., Matteucci F., Romano D., 2016, MNRAS, 458, 2541

Suda T. et al., 2017, PASJ, 69, 76

Teske J. K., Shectman S. A., Vogt S. S., Díaz M., Butler R. P., Crane J. D., Thompson I. B., Arriagada P., 2016a, AJ, 152, 167

Teske J. K., Khanal S., Ramírez I., 2016b, ApJ, 819, 19

Théado S., Vauclair S., 2012, ApJ, 744, 123

The L.-S. et al., 2006, A\&A, 450, 1037

Tinsley B. M., 1979, ApJ, 229, 1046

Tolstoy E., Hill V., Toii M., 2009, ARA\&A, 47, 371

Tomkin J., 1972, MNRAS, 156, 349

Tucci Maia M., Meléndez J., Ramírez I., 2014, AJ, 790, L25

Table A1. Example of the linelist used for the abundances determinations, formatted to be used with the radiative transfer code Moog (Sneden 1973), and also include the hyperfine splitting, indicated by the negative wavelengths. The full linelist can be found online.

\begin{tabular}{|c|c|c|c|c|c|c|c|}
\hline $\begin{array}{l}\text { Wavelength } \\
(\AA)\end{array}$ & Species & $\begin{array}{c}\mathrm{EP} \\
(\mathrm{eV})\end{array}$ & $\begin{array}{c}\log (g f) \\
(\operatorname{dex})\end{array}$ & $\begin{array}{c}\text { EW_HD } 103095 \\
(\mathrm{m \AA})\end{array}$ & $\begin{array}{c}\text { EW_HD } 134439 \\
(\mathrm{m \AA})\end{array}$ & $\begin{array}{c}\text { EW_HD } 134440 \\
(\mathrm{m \AA})\end{array}$ & $\begin{array}{c}\text { EW_HD } 163810 \\
(\mathrm{m \AA})\end{array}$ \\
\hline 4445.471 & 26 & 0.087 & -5.441 & 25.6 & 24.5 & 33.7 & 11.7 \\
\hline 4779.439 & 26 & 3.415 & -2.020 & 15.3 & 12.3 & 19.5 & 7.7 \\
\hline 4788.757 & 26 & 3.237 & -1.763 & 31.1 & 29.4 & 34.5 & 22.4 \\
\hline 4950.106 & 26 & 3.417 & -1.560 & 35.7 & 33.1 & 39.6 & 25.6 \\
\hline 5044.211 & 26 & 2.851 & -2.058 & 49.5 & 43.3 & 59.3 & 27.7 \\
\hline 5054.642 & 26 & 3.640 & -1.921 & 16.5 & 13.1 & 19.5 & 9.8 \\
\hline 5127.359 & 26 & 0.915 & -3.307 & 86.4 & 86.1 & 99.6 & 63.9 \\
\hline 5198.711 & 26 & 2.223 & -2.135 & 87.0 & 84.2 & 106.5 & 57.0 \\
\hline
\end{tabular}

This paper has been typeset from a $\mathrm{T}_{\mathrm{E}} \mathrm{X} / \mathrm{L}_{\mathrm{A}} \mathrm{E} \mathrm{X}$ file prepared by the author.
Tucci Maia M., Meléndez J., Spina L., Lorenzo-Oliveira D., 2018, MNRAS, submitted

Venn K. A. et al., 2012, ApJ, 751, 102

Wongwathanarat A., Janka H.-T., Müller E., Pllumbi E., Wanajo S., 2017, ApJ, 842, 13

Yong D. et al., 2013, MNRAS, 434, 3542

\section{SUPPORTING INFORMATION}

Supplementary data are available at MNRAS online.

Table A1. Example of the linelist used for the abundances determinations.

Please note: Oxford University Press is not responsible for the content or functionality of any supporting materials supplied by the authors. Any queries (other than missing material) should be directed to the corresponding author for the article.

\section{APPENDIX：LINELIST}




\title{
Non-LTE analysis of $\mathrm{K} \mathrm{I}$ in late-type stars ${ }^{\star}$
}

\author{
Henrique Reggiani ${ }^{1,2}$, Anish M. Amarsi ${ }^{2}$, Karin Lind ${ }^{2,3}$, Paul S. Barklem ${ }^{4}$, Oleg Zatsarinny ${ }^{5}$, Klaus Bartschat $^{5}$, \\ Dmitry V. Fursa ${ }^{6}$, Igor Bray ${ }^{6}$, Lorenzo Spina ${ }^{7}$, and Jorge Meléndez ${ }^{1}$

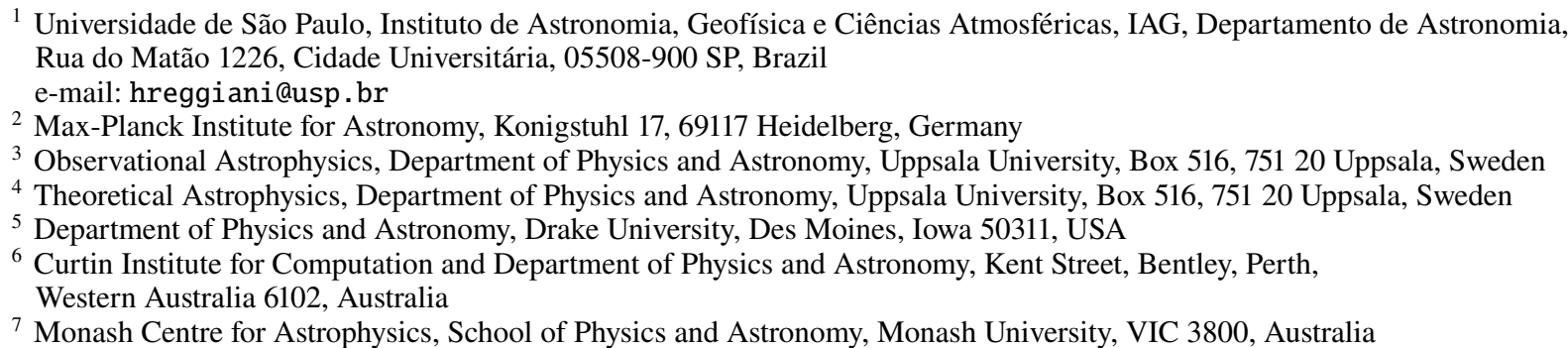

Received 29 January 2019 / Accepted 7 June 2019

\begin{abstract}
Context. Older models of Galactic chemical evolution (GCE) predict $[\mathrm{K} / \mathrm{Fe}]$ ratios as much as 1 dex lower than those inferred from stellar observations. Abundances of potassium are mainly based on analyses of the $7698 \AA$ resonance line, and the discrepancy between GCE models and observations is in part caused by the assumption of local thermodynamic equilibrium (LTE) in spectroscopic analyses.

Aims. We study the statistical equilibrium of K I, focusing on the non-LTE effects on the $7698 \AA$ line. We aim to determine how non-LTE abundances of potassium can improve the analysis of its chemical evolution, and help to constrain the yields of GCE models. Methods. We construct a new model $\mathrm{K}$ I atom that employs the most up-to-date atomic data. In particular, we calculate and present inelastic e+K collisional excitation cross-sections from the convergent close-coupling (CCC) and the $B$-Spline $R$-matrix (BSR) methods, and $\mathrm{H}+\mathrm{K}$ collisions from the two-electron model (LCAO). We constructed a fine, extended grid of non-LTE abundance corrections based on 1D MARCS models that span $4000<T_{\text {eff }} / \mathrm{K}<8000,0.50<\log g<5.00,-5.00<[\mathrm{Fe} / \mathrm{H}]<+0.50$, and applied the corrections to potassium abundances extracted from the literature.

Results. In concordance with previous studies, we find severe non-LTE effects in the $7698 \AA$ line. The line is stronger in non-LTE and the abundance corrections can reach approximately $-0.7 \mathrm{dex}$ for solar-metallicity stars such as Procyon. We determine potassium abundances in six benchmark stars, and obtain consistent results from different optical lines. We explore the effects of atmospheric inhomogeneity by computing for the first time a full 3D non-LTE stellar spectrum of K I lines for a test star. We find that 3D modeling is necessary to predict a correct shape of the resonance $7698 \AA$ line, but the line strength is similar to that found in 1D non-LTE.

Conclusions. Our non-LTE abundance corrections reduce the scatter and change the cosmic trends of literature potassium abundances. In the regime $[\mathrm{Fe} / \mathrm{H}] \lesssim-1.0$ the non-LTE abundances show a good agreement with the GCE model with yields from rotating massive stars. The reduced scatter of the non-LTE corrected abundances of a sample of solar twins shows that line-by-line differential analysis techniques cannot fully compensate for systematic LTE modelling errors; the scatter introduced by such errors introduces a spurious dispersion to K evolution.
\end{abstract}

Key words. stars: abundances - stars: late-type - line: formation - Galaxy: evolution - Galaxy: abundances

\section{Introduction}

Potassium is an alkali metal with an atomic structure very similar to that of sodium (so similar that they were mistakenly believed to be the same element until after the eighteenth century). However, $\mathrm{K}$ is typically an order-of-magnitude less abundant than $\mathrm{Na}$ and its spectral fingerprint in late-type stars is accordingly weaker and much less studied. Potassium has three stable isotopes $\left({ }^{39} \mathrm{~K},{ }^{40} \mathrm{~K}\right.$ and $\left.{ }^{41} \mathrm{~K}\right)$; all produced via hydrostatic oxygen shell burning and explosive oxygen burning in massive stars, with a relative proportion that depends on the stellar mass (Woosley \& Weaver 1995). The lightest isotope is dominant

* Data are only available at the CDS via anonymous ftp to cdsarc.u-strasbg.fr $(130.79 .128 .5)$ or via http://cdsarc. u-strasbg.fr/viz-bin/qcat?J/A+A/627/A177 with $93 \%$ occurrence in solar-system meteorites (Lodders \& Palme 2009). Because of this, and the negligible isotopic shifts of atomic K lines (Clayton 2007), to our knowledge potassium isotopic ratios have not yet been measured in stars.

Assuming that there are no additional nucleosynthetic production sites of $\mathrm{K}$, there is a clear shortage in the supernova yields, as evidenced by the existence of a large discrepancy between models of chemical evolution and observed $\mathrm{K}$ abundances obtained via stellar spectroscopy (Zhao et al. 2016; Sneden et al. 2016). To resolve this discrepancy, the supernova yields for $\mathrm{K}$ would need to be empirically increased by as much as twice what current theory would suggest (e.g., Takeda et al. 2002; Romano et al. 2010). Kobayashi et al. (2011) speculate that the underproduction of $\mathrm{K}$ in the models is at least partially due to the lack of a neutrino process. On the other hand, yields of rotating massive stars improve the agreement between models 
and observations, especially in the metal-poor $([\mathrm{Fe} / \mathrm{H}] \leq-2.0)$ regime, where the scatter of the observed abundances starts to increase and the model predictions match at least the lower envelope (Prantzos et al. 2018).

The discrepancy between models of chemical evolution and observed potassium abundances may also in part be caused by systematic errors in modeling the main potassium abundance diagnostic, the resonance K I 7664 and $7698 \AA$ doublet. In practice, heavy blends with telluric $\mathrm{O}_{2}$ make it difficult to correctly assess the potassium abundances using the $7664 \AA$ line, meaning that most of the measurements of potassium come from the $7698 \AA$ line. Although there are two other observable K I lines in the optical spectra (5801 and $6939 \AA$ ), these are usually weak and can only be measured in cool $\left(T_{\text {eff }} \lesssim 6000 \mathrm{~K}\right)$ highmetallicity $([\mathrm{Fe} / \mathrm{H}] \sim+0.0)$ stars, and are therefore not used as a diagnostic of the potassium abundance in most studies.

In 1975 astronomers already knew that the $7698 \AA$ line was sensitive to departures from local thermodynamic equilibrium (LTE; de La Reza \& Mueller 1975; Bruls et al. 1992; Takeda et al. 2002; Zhang et al. 2006; Andrievsky et al. 2010; Zhao et al. 2016). Previous Galactic chemical evolution (GCE) studies have demonstrated that LTE potassium abundances can be more than 1 dex higher than those predicted by existing models (Kobayashi et al. 2006; Prantzos et al. 2018). Takeda et al. (2002) studied the departures from LTE in the K I $7698.9 \AA$ line across a grid of 100 atmospheric models. They found non-LTE corrections spanning from -0.2 to -0.7 dex, with a strong sensitivity to effective temperature, which was also confirmed in later works (Takeda et al. 2009; Andrievsky et al. 2010). Thus, non-LTE modeling can significantly decrease the discrepancy between models and observations (e.g., Kobayashi et al. 2006, 2011; Romano et al. 2010; Prantzos et al. 2018).

Non-LTE abundances for $\mathrm{K}$ are computed, as is common for late-type stars, under the trace element assumption that neglects feedback on the atmospheric structure. Solving the statistical equilibrium equations requires a wealth of atomic data; in particular radiative and collisional transition probabilities. The main uncertainties in non-LTE analyses of potassium have hitherto originated from the photoionization cross-sections, the inelastic $\mathrm{H}+\mathrm{K}$ collisions, and the inelastic e+K collisions; however, the situation has recently improved. Potassium, atomic number 19, falls just outside of the scope of the Opacity Project (Seaton 1996; Badnell et al. 2005), and previous studies have employed hydrogenic approximations. However, Zatsarinny \& Tayal (2010) calculated photoionization cross-sections with the fully relativistic Dirac B-spline R-matrix (DBSR) method. Furthermore, Yakovleva et al. (2018) recently presented new inelastic $\mathrm{H}+\mathrm{K}$ collisions using the new asymptotic two-electron model of Barklem (2016); this recipe predicts rates that are in reasonable agreement with fully ab-initio quantum mechanical calculations for low-excitation transitions of lithium, sodium, and magnesium, in particular for the processes with the largest rates.

Rates for inelastic e+K collisional excitation have, in the past, typically been estimated using the semi-empirical recipe of Park (1971), hereafter Park71, or the semi-empirical formula from van Regemorter (1962), hereafter vanReg62. Several more accurate methods now exist. In this study, we present new data based on two modern close-coupling methods, namely the convergent close coupling method (CCC) and the B-spline R-matrix method (BSR). Osorio et al. (2011) and Barklem et al. (2017) demonstrated that the rate coefficients calculated via these two methods tend to agree better than a factor of two for lithium and magnesium.
With the goal of improving potassium abundance determinations, here we study the non-LTE effects in potassium. To that end, we construct a new model $\mathrm{K}$ I atom that employs more accurate atomic data than before. In particular, we calculate inelastic $\mathrm{e}+\mathrm{K}$ collisional excitation cross-sections from the $\mathrm{CCC}$ and BSR methods and we also employ improved photoionization cross-sections and inelastic $\mathrm{H}+\mathrm{K}$ collisions from the literature. In Sect. 2 we present and discuss the calculations of the inelastic $\mathrm{e}+\mathrm{K}$ collisional excitation cross-sections from the $\mathrm{CCC}$ and BSR methods, and in Sect. 3 we present the atomic model. In Sect. 4 we discuss the departures from LTE, and compare results among different collisional and photoionization recipes. In Sect. 5 we show the line fits of our non-LTE model against the solar spectrum and the abundance analysis of benchmark stars (HD 103095, HD 84937, HD 140283, HD 192263 and Procyon). In Sect. 7 we describe our non-LTE grid of corrections. In Sect. 8 we discuss the implications for Galactic chemical evolution and we conclude in Sect. 9.

\section{Inelastic e+K collisional excitation}

Calculations of collisional excitation of $\mathrm{K}$ by electron impacts were performed with two state-of-the-art close-coupling methods, the CCC and the BSR. These methods and calculations are described below, in Sects. 2.1 and 2.2. In Sect. 2.3 the resulting data are described, and are compared in Sect. 3.3, along with older calculations.

\subsection{Convergent close coupling method}

The $\mathrm{e}+\mathrm{K} \mathrm{CCC}$ calculations are based on the generalization of the e-H formalism (Bray \& Stelbovics 1992) to quasi oneelectron targets such as atomic $\mathrm{Li}, \mathrm{Na}$, and K (Bray 1994). The valence electron is treated as the active electron on top of a frozen Hartree-Fock core. Additionally, virtual excitation of the core electrons is treated via phenomenological local polarization potentials. Their parameters are adjusted to yield the optimally accurate one-electron excitation energies of the valence electron for each target orbital angular momentum $l$.

The $\mathrm{e}+\mathrm{K}$ system has been considered previously (Stockman et al. 1998, 1999, 2001), which demonstrated the applicability of the CCC approach to the collision system at all energies. The key issue with the CCC method is to choose a sufficiently large number of Laguerre-based states for convergence in the required physical quantities of interest to the desired level of precision. Convergence considerations are energy and transition dependent. To make the presentation simpler we take a single large Laguerre basis $N_{l}$ chosen to generate sufficiently accurate states for the transitions and energies of interest. Specifically, we take the maximum orbital angular momentum $l_{\max }=6$, and take $N_{l}=40-l$. Such a choice leads to $n \leq 9$ physical eigenstates with the remainder being negative- and positive-energy pseudostates. Some of the states generated have very high energies and may be excluded from the calculations, depending on the incident electron energy. Having chosen the Laguerre basis, the calculations proceed as described in Bray (1994).

\subsection{B-spline R-matrix method}

An overview of the $B$-spline $R$-matrix (BSR), which is a different and entirely independent implementation for solving the closecoupling equations, can be found in Zatsarinny \& Bartschat (2013). The calculations were performed with an extended version of the computer code (Zatsarinny 2006) that allows for 
the inclusion of a sufficient number of physical target states as well as continuum pseudostates in the intermediate-energy regime. Like CCC, this $R$-matrix with pseudo-states implementation is expected to provide a converged (with the number of states included) solution of the close-coupling equations, with the remaining differences between the CCC and BSR results most likely being related to a slightly different target description.

All target states considered in the present calculations have the quasi-one-electron structure (core)nl, with the core configuration $\mathrm{K}^{+}\left(1 s^{2} 2 s^{2} 2 p^{6} 3 s^{2} 3 p^{6}\right)$. We started the structure-part of the problem by generating the core orbitals from a Hartree-Fock (HF) calculation for $\mathrm{K}^{+}$. The principal correlation effects in the atomic states are related to the core-valence interaction. In many calculations for alkali-metal atoms (see also the CCC description above), a phenomenological one-electron core polarization potential is typically added to account for this effect. Although such a potential simplifies the calculations significantly and can provide accurate excitation energies and oscillator strengths, the question still remains as to how well the model potential can simulate the entire core-valence correlation, including nondipole contributions. In the present BSR approach, we therefore chose to include the core-valence correlation ab initio through the polarized-pseudostate approach. This method is described in detail in our previous calculation for photoionization of potassium (Zatsarinny \& Tayal 2010).

Specifically, the target states were expanded as

$\Psi\left(3 p^{6} n l, L S\right)=\mathcal{A}\left[\Phi\left(3 p^{6}\right) P(n l)\right]^{L S}+\mathcal{A} \sum_{k=1}^{3}\left[\phi_{p}^{k} P\left(n^{\prime} l^{\prime}\right)\right]^{L S}$,

where $\mathcal{A}$ is the antisymmetrization operator while the $\phi_{p}^{k}$ are the polarized pseudostates that describe the dipole, quadrupole, and octupole polarization of the $3 p^{6}$ core, respectively. Their structure and the corresponding polarizabilities are discussed in Zatsarinny \& Tayal (2010). The unknown functions $P(n l)$ for the outer valence electron were expanded in a $B$-spline basis, and the corresponding equations were solved subject to the condition that the wave function vanishes at the $R$-matrix boundary, which is chosen such that exchange effects between the projectile and the target electrons outside the box are negligible. The $B$-spline coefficients for the valence orbitals $P(n l)$ were obtained by diagonalizing the $N$-electron atomic Hamiltonian. We included $165 B$-splines of order 8 in the present calculations. Choosing $a=80 a_{0}$ (with $a_{0}=0.529 \times 10^{-10} \mathrm{~m}$ denoting the Bohr radius), we obtained a good description for all low-lying states of $\mathrm{K}$ up to $8 \mathrm{~s}$ (Zatsarinny \& Tayal 2010) regarding both level energies and oscillator strengths. The deviations in the recommended excitation energies (NIST 2018) were less than approximately $0.01 \mathrm{eV}$ for all levels. Nevertheless, in the subsequent scattering calculations the calculated excitation energies were adjusted to the experimental ones to remove any uncertainties related to the slightly different excitation thresholds.

The above scheme is also able to generate continuum pseudostates that lie above the ionization threshold. The scattering calculations were carried out by using a fully parallelized version of the BSR complex (Zatsarinny 2006). These $R$-matrices with pseudostate calculations are similar to the recent BSR calculations for e-Be (Zatsarinny et al. 2016) and e-Mg (Barklem et al. 2017) collisions. More computational details are given in those papers. The final close-coupling expansions contained 284 target states, including 30 bound states plus 254 continuum pseudostates with orbital angular momenta up to $L=4$, which covered the target continuum up to $50 \mathrm{eV}$ above the first ionization limit. We calculated partial waves for total orbital angular momenta up to $L_{\max }=50$ numerically. Overall, with the
Table 1. Physical states for which data are provided from the CCC and BSR calculations, along with experimental excitation energies $\left(E_{\text {expt }}\right)$ from NIST (2018).

\begin{tabular}{rrrr}
\hline \hline Index & State & $g$ & $\begin{array}{r}E_{\text {expt }} \\
(\mathrm{eV})\end{array}$ \\
\hline 1 & $4 s$ & 2 & 0.000 \\
2 & $4 p$ & 6 & 1.615 \\
3 & $5 s$ & 2 & 2.607 \\
4 & $3 d$ & 10 & 2.670 \\
5 & $5 p$ & 6 & 3.064 \\
6 & $4 d$ & 10 & 3.397 \\
7 & $6 s$ & 2 & 3.403 \\
8 & $4 f$ & 14 & 3.487 \\
9 & $6 p$ & 6 & 3.596 \\
10 & $5 d$ & 10 & 3.743 \\
11 & $7 s$ & 2 & 3.754 \\
12 & $5 f$ & 14 & 3.795 \\
13 & $5 g$ & 18 & 3.796 \\
14 & $7 p$ & 6 & 3.853 \\
15 & $6 d$ & 10 & 3.930 \\
\hline
\end{tabular}

various total spins and parities, this involved 204 partial waves. We considered all transitions between the lowest 17 physical states. The principal difficulty for initially excited states was the slow convergence of the partial wave expansions for transitions between close-lying levels. When needed, we employed a top-up procedure based on the Coulomb-Bethe approximation.

\section{3. $e+K$ rate coefficients}

In non-LTE applications, the rate coefficient is required, which is calculated by folding the cross sections $\sigma$ produced in the CCC and BSR calculations with the velocity distribution, assumed here to be the Maxwell distribution. The relevant equations are given in Barklem et al. (2017), and the data presented here are similar in form. The effective collision strengths $\Upsilon_{i j}$ from the $\mathrm{CCC}$ and BSR methods are calculated for transitions between the 15 lowest-lying states of $\mathrm{K}$, which includes all states up to $6 \mathrm{~d}$ at $3.93 \mathrm{eV}$; this is the complete set of low-lying states that are included in both calculations. The states and their experimental energies are listed in Table 1. The effective collision strength calculations are done for temperatures $T$ ranging from 1000 to $10000 \mathrm{~K}$ in steps of $1000 \mathrm{~K}$, with additional results for $500 \mathrm{~K}$ at the cool end, and 15000 and $20000 \mathrm{~K}$ at the hot end. The data are only provided electronically at CDS. The data presented there are given as matrices, following the ordering of indexes in Table 1 (i.e., the transition 1-2 corresponds to element $(1,2)$ ), one matrix for each temperature.

The data from the CCC and BSR methods are generally in very good agreement, with the location (offset) and scale (scatter) of the ratio $\Upsilon_{i j}(\mathrm{CCC}) / \Upsilon_{i j}(\mathrm{BSR})$, assuming a log-normal distribution, 1.03 and 0.17, respectively (see Barklem et al. 2017). This indicates a mean offset of only $3 \%$ and scatter of $17 \%$. In Fig. 2, we compare the CCC and BSR data to each other and to older calculations.

\section{Non-LTE model}

We performed non-LTE modeling in the trace-element approximation of the optical spectral lines of KI using 1D planeparallel MARCS (Gustafsson et al. 2008) model atmospheres. 


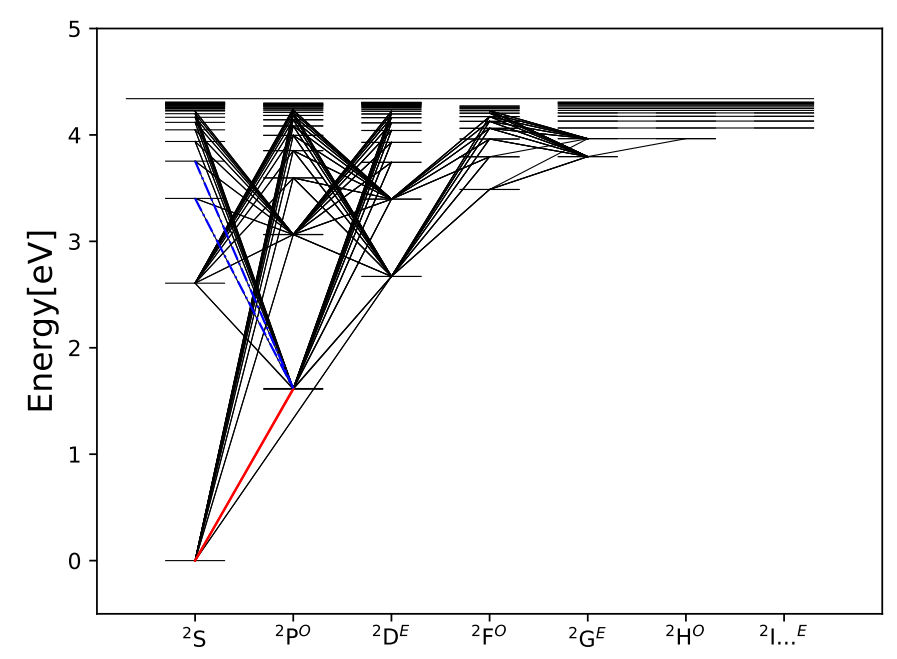

Fig. 1. Grotrian diagram of $\mathrm{K}$ I. In solid red we indicate resonance transitions (both 7664 and $7698 \AA$ ) and the blue dashed lines the 5801 and $6939 \AA$ A transitions.

The statistical equilibrium and spectra were calculated using version 2.3 of the radiative transfer code MULTI (Carlsson 1986, 1992).

\subsection{Energy levels and radiative transitions}

Our potassium model is complete up to $0.13 \mathrm{eV}$ below the first ionization energy $(4.34 \mathrm{eV})$, with all available levels with configurations up to principal quantum number $n=20$ and the K II ground level. We have a total of 134 levels in our atom, out of which 110 are fine-structure-resolved energy levels from the NIST (2018) database, which comes primarily from Sugar \& Corliss (1985) and Sansonetti (2008). The NIST (2018) database is complete up to orbital quantum number $l=3$, and the missing high- $l$ data were computed under the assumption that they are Rydberg levels (24 nonfine-structure-resolved levels).

The oscillator strengths of the allowed bound-bound radiative transitions were taken from Wiese et. al. (1969), Biemont \& Grevesse (1973), and Sansonetti (2008). The total number of bound-bound transitions considered is 250 . The Grotrian diagram of our atomic model can be seen in Fig. 1. All boundbound transitions involving the Rydberg levels are disregarded as it was seen that transitions with wavelengths larger than around $20000 \AA$ had negligible impact on the statistical equilibrium. Therefore, these levels were added so that the code computes the partition function as accurately as possible. Whenever available, broadening data were added from the VALD database (Ryabchikova et al. 2015) or from Barklem et al. (1998).

\subsection{Photoionization cross sections}

The photoionization cross-sections of all levels between 4 and 7s are fine-structure resolved and were taken from Zatsarinny \& Bartschat (2008) and Zatsarinny \& Tayal (2010), calculated using the fully relativistic DBSR method. The photoionization cross-sections of the remaining levels were calculated using the hydrogenic approximation for bound-free transitions (Gray 2005, Eq. (8.4)). For higher levels, the cross sections of the two methods are more compatible with each other compared to the lower levels, and they start to diverge for increasingly higher wavelengths. Details and examples of the cross-sections can be seen in Figs. 1-6 of Zatsarinny \& Tayal (2010).

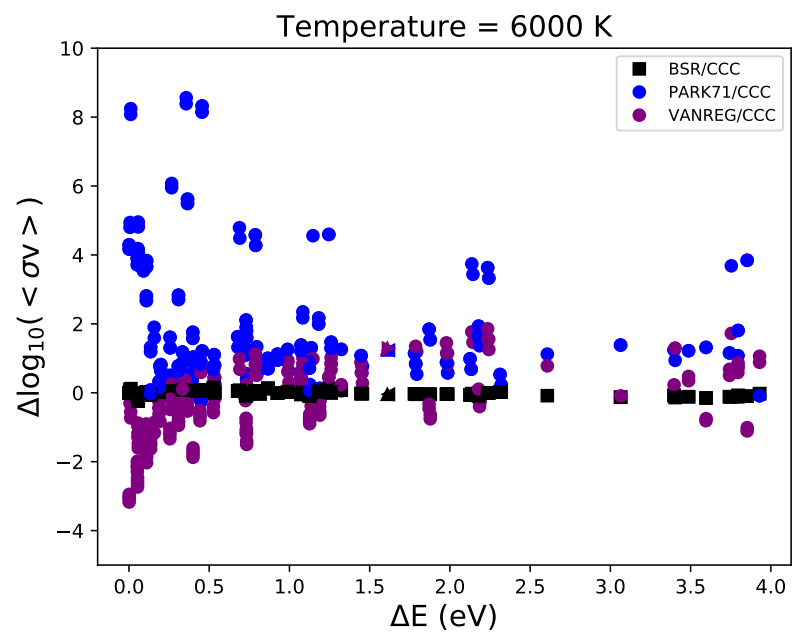

Fig. 2. Ratio between e+K rate coefficients calculated through different methods. The triangles are the rates of the resonance transitions.

\subsection{Collisional data}

The inelastic e+K collisions have a large impact on the statistical equilibrium of potassium. As discussed in Sect. 2, the inelastic $\mathrm{e}+\mathrm{K}$ collision rates for transitions between low-lying levels $4 \mathrm{~s}$ and $6 \mathrm{~d}$ were calculated using the CCC and BSR methods. Although CCC was employed in our standard atom, the e+K rates calculated via BSR were also tested, and the results with either method are indistinguishable; see Sect. 4.1. The rate coefficients for transitions from or to levels higher than $6 \mathrm{~d}$ were all calculated using the Park71 method.

As mentioned in Sect. 2.3, in Fig. 2 we compare the e+K rate coefficients calculated with the CCC, BSR, Park71, and vanReg62 methods at $6000 \mathrm{~K}$. We can see that CCC and BSR are in very good agreement, while the Park71 and vanReg62 methods differ from the two more recent and accurate methods. These disagreements lead to differences in the statistical equilibrium, and hence the synthetic lines, which are further explored in Sect. 4.1. We note that the rate coefficients of the resonance transitions $4 \mathrm{p}^{3 / 2}-4 \mathrm{~s}^{1 / 2}$ and $4 \mathrm{p}^{1 / 2}-4 \mathrm{~s}^{1 / 2}$ calculated via both the Park 71 and the vanReg62 methods are very similar to each other (blue and purple triangles in Fig. 2).

The inelastic $\mathrm{H}+\mathrm{K}$ collisions also influence the statistical equilibrium of potassium. For transitions involving low-lying levels (up to level 4f), we adopted the rate coefficients from Yakovleva et al. (2018), which are based on the LCAO model of Barklem (2016). Following Amarsi et al. (2018), we added these data to rate coefficients calculated using the free electron model in the scattering length approximation (Eq. (18), Kaulakys 1991). The rate coefficients for transitions from or to levels higher than $6 \mathrm{~d}$ were all calculated using the free electron model alone.

Finally, inelastic $\mathrm{e}+\mathrm{K}$ collisional ionization was calculated using the empirical formula in Chap. 3 of Allen (1976). These rates are important for guaranteeing LTE populations deep in the stellar atmosphere.

\section{Non-LTE effects on Potassium}

\subsection{Test models}

Below we present the results of test calculations for the cases of different inelastic e+K collisional rates (CCC, BSR, Park71, and the vanReg62 calculations), and different photoionization calculations (the DBSR method by Zatsarinny \& Tayal (2010) and the 
Table 2. Different atoms created to compare the collisions and photoionization recipes.

\begin{tabular}{|c|c|c|c|c|c|c|c|c|}
\hline \multirow[t]{2}{*}{ Atom designation } & \multicolumn{4}{|c|}{ Electron excitation } & \multicolumn{2}{|c|}{ Neutral hydrogen collisions } & \multicolumn{2}{|l|}{ Photoionization } \\
\hline & $\mathrm{CCC}$ & BSR & Park71 & vanReg62 & Barklem (2016) & Kaulakys (1991) & Zatsarinny \& Tayal (2010) & Hydrogenic \\
\hline (a) Standard atom & $\mathrm{X}$ & & $X$ & & $X$ & $X$ & $\mathrm{X}$ & $\mathrm{X}$ \\
\hline (b) $\mathrm{BSR} \mathrm{e}+\mathrm{K}$ & & $X$ & $X$ & & $X$ & $\mathrm{X}$ & $X$ & $\mathrm{X}$ \\
\hline (c) Park71 e+K & & & $\mathrm{X}$ & & $\mathrm{X}$ & $\mathrm{X}$ & $\mathrm{X}$ & $\mathrm{X}$ \\
\hline (d) Hydrogenic photoionization & $\mathrm{X}$ & & $\mathrm{X}$ & & $\mathrm{X}$ & $X$ & & $\mathrm{X}$ \\
\hline (e) Resonance transitions only & $\mathrm{X}$ & & $X$ & & $\mathrm{X}$ & $X$ & $\mathrm{X}$ & $\mathrm{X}$ \\
\hline ( $f$ ) No resonance transitions & $X$ & & $X$ & & $\mathrm{X}$ & $X$ & $\mathrm{X}$ & $X$ \\
\hline (g) Reduced $\mathrm{H}+\mathrm{K}$ excitation & $\mathrm{X}$ & & $\mathrm{X}$ & & $\mathrm{x} 10^{-3}$ & $\mathrm{x} 10^{-3}$ & $\mathrm{X}$ & $\mathrm{X}$ \\
\hline (h) Reduced e+K excitation & $\mathrm{x} 10^{-3}$ & & $\mathrm{x} 10^{-3}$ & & $\mathrm{X}$ & $\mathrm{X}$ & $\mathrm{X}$ & $\mathrm{X}$ \\
\hline (i) vanReg62 $\mathrm{e}+\mathrm{K}$ & & & & $X$ & $X$ & $X$ & $X$ & $\mathrm{X}$ \\
\hline
\end{tabular}

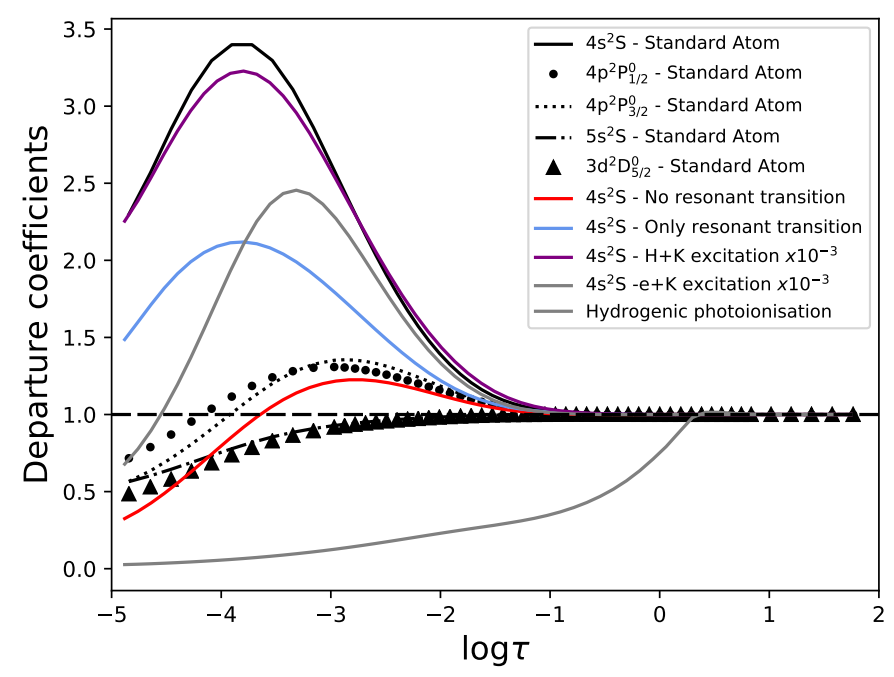

Fig. 3. Departure coefficients for the low-excited K I levels in the solar atmosphere.

hydrogenic approximation). Our standard atom is atom $a$, discussed in Sect. 3. The collisions employed in each atom can be seen in Table 2. Atoms $e$ and $f$ have the same collisions as the standard atom but the first only has the resonance $4 p^{3 / 2}-4 s^{1 / 2}$ and $4 \mathrm{p}^{1 / 2}-4 \mathrm{~s}^{1 / 2}$ radiative transitions and the second has all other transitions except those. Atom $g$ has the $\mathrm{H}+\mathrm{K}$ excitation rates decreased by a factor of $10^{-3}$; and atom $h$ has the e+K excitation rates decreased by a factor of $10^{-3}$. The last atom (atom $i$ ) uses the vanReg62 e+K collisions.

\subsection{Departure coefficients}

In Fig. 3 we show a number of departure coefficients, $b_{\mathrm{k}}=n_{\mathrm{NLTE}} /$ $n_{\text {LTE}}$, in the solar atmosphere, for our standard atom and for a number of our test atoms (Sect. 4.1), designed to test the main contributors to the non-LTE effects in potassium.

From Fig. 3 and from previous works (e.g., Zhang et al. 2006), we see there is a strong overpopulation of the $\mathrm{K}_{\mathrm{I}}$ ground state and the two first excited states $\left(4 \mathrm{~s}, 4 \mathrm{p}^{1 / 2}\right.$ and $\left.4 \mathrm{p}^{3 / 2}\right)$. The major drivers of this overpopulation are the resonance transitions due to photon losses. This was previously demonstrated in Sect. 4.3 of Bruls et al. (1992). According to their study, the infrared lines are also important in the statistical equilibrium (but to a much lower degree) and as such, Fig. 3 illustrates that there are departures from LTE even when the resonance transitions are switched off (test atom $f$ ).
The departure coefficients also illustrate that the inelastic $\mathrm{H}+\mathrm{K}$ excitation is of lesser importance on solar metallicity than the inelastic $\mathrm{e}+\mathrm{K}$ excitation, in line with previous studies for alkali metals (Lind et al. 2009, 2011). This is further discussed in the following section through the analysis of synthetic lines.

\subsection{Effects on spectral lines}

To generate the synthetic spectral lines we employed MARCS (Gustafsson et al. 2008) 1D model atmospheres of the Sun with a potassium abundance of $A(\mathrm{~K})=5.11$, a metal-poor star with the same stellar parameters determined for HD 84937 in Peterson et al. (2017), and adopted by Spite et al. (2017), with a potassium abundance of $A(\mathrm{~K})=3.08\left(T_{\text {eff }}=6300 \mathrm{~K}, \log g=\right.$ $4.00,[\mathrm{Fe} / \mathrm{H}]=-2.25$ and $\xi=1.3 \mathrm{~km} \mathrm{~s}^{-1}$ ), and a giant star with $T_{\text {eff }}=4500 \mathrm{~K}, \log g=1.5,[\mathrm{Fe} / \mathrm{H}]=-3.00, \xi=2.0 \mathrm{~km} \mathrm{~s}^{-1}$, and $[\mathrm{K} / \mathrm{Fe}]=0.0$.

We first discuss how the different collisional and radiative data affect line formation in the solar atmosphere. The results for the 5801 and $7968 \AA$ lines are shown in the first and second panels of Fig. 4. We note that for all tests described in this section, the standard atom $(a)$ and the atom replacing CCC collisions with BSR collisions $(b)$ produce indistinguishable line profiles; as such we do not plot the results of the latter atom.

In our analysis, the high-excitation $5801 \AA$ line is almost insensitive to departures from LTE. Consequently it is insensitive to the details of the non-LTE modeling, with variations of only around 0.01 dex. Unfortunately, the line is very weak and can only be detected in very high-resolution and high-signal-to-noise ratio $(\mathrm{S} / \mathrm{N})$ spectra of stars with solar metallicity or higher, and solar effective temperature or cooler. Therefore, it is unsuitable for abundance analysis in representative samples. This $5801 \AA$ line is however very sensitive to the large decrease in $\mathrm{e}+\mathrm{K}$ excitation of atom $(h)$, a case in which the line is almost not formed and is much weaker than in LTE.

The resonance line has a different behavior. While the wings are insensitive to departures from LTE, the core is significantly deeper in non-LTE, as a result of the overpopulation of the ground state as we discussed in Sect. 4.2. There is a nonnegligible difference between the line cores when using different collisional recipes.

Concerning the sensitivity of the non-LTE effects to the atomic data, the most prominent difference to the resonance line is observed when we decrease the e+K excitation rates by $10^{-3}$ (atom $(h)$ ). In this case the statistical equilibrium changes to the point where the line is not as deep as the LTE case, due to an increased importance of the photoionization - an effect also observed in the departure coefficient. The second largest 

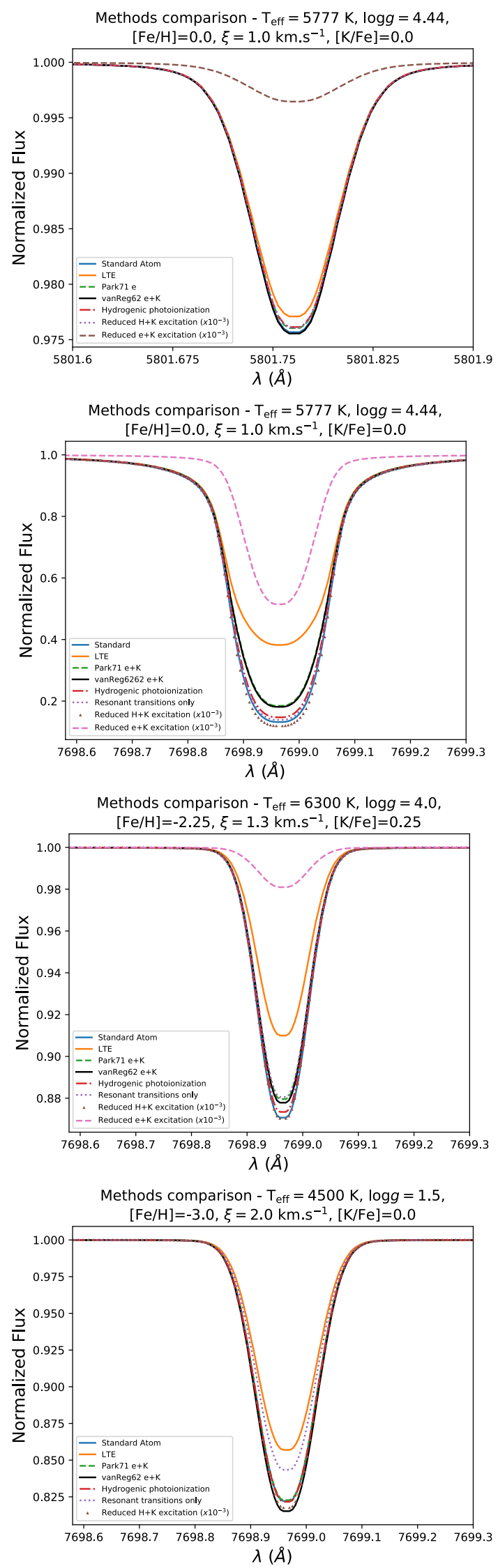

Fig. 4. First and second panels: comparison between the synthetic spectral lines using different collisional recipes for the 5801 and $7698 \AA$ lines in the 1D solar atmosphere. Third and fourth panels: the same but for a 1D metal-poor atmosphere (HD 84937) and a giant star 1D atmosphere, respectively. difference is seen when the CCC collisions are replaced with the Park71 or vanReg62 collisions (atoms $(c)$ and $(i)$ ): an abundance difference of $\Delta=+0.11$ dex with respect to the standard atom $(a)$. The third largest difference is seen when adopting hydrogenic photoionization cross-sections $(d)$ : an abundance difference of $\Delta=+0.03$ dex with respect to the standard atom $(a)$. When synthesizing the line with inefficient $\mathrm{H}+\mathrm{K}$ collisions $(g)$ the line core is deeper than the line from the standard atom $(a)$, but the absolute abundance difference is the same as that produced by the atom with hydrogenic photoionization (atom $(d)$ ). The atom with only two radiative transitions, namely the resonance lines $(e)$, has virtually the same abundance as our standard atom.

The non-LTE effects are mainly a source-function effect caused by photon losses (resonance scattering). Like in the $\mathrm{Na} \mathrm{D}$ lines (see Sect 3.1 of Lind et al. 2011), the overpopulation of the ground state pushes the mean formation depth outward, deepening the lines - but only slightly. The main effect that deepens the spectral line is the sub-thermal line source function. The line source function of a line formed by pure resonance scattering is determined by the radiation field, and therefore by the radiative rates in the lines themselves. This is why the atom with only the resonance lines $(e)$ performs so similarly to the standard atom (a), that is, because the source function is the same in the two cases.

In the third panel of Fig. 4 we show the synthetic spectral lines in a metal-poor turn-off star. Similarly to the solar atmosphere case, for HD 84937, compared to the standard atom (a), the non-LTE effects change the most when the e+K collisions are reduced $(h)$, whereas reducing the $\mathrm{H}+\mathrm{K}$ collisions $(g)$ has only a small impact. The largest abundance differences $(\Delta=+0.04 \mathrm{dex})$ are for atoms $(c),(e)$, and $(i)$. The third largest is for atom $(d)(\Delta=+0.01 \mathrm{dex})$. The general difference between the synthetic lines in HD 84937 is smaller than in the solar atmosphere, because in a metal-poor atmosphere the hydrogen collisions are more relevant to the statistical equilibrium, owing to the reduced number of free electrons.

The stars analyzed in this work are late-type stars, in which the $\mathrm{H}+\mathrm{K}$ excitation is expected to be less important than in giant stars. In the last panel of Fig. 4 we tested the differences in a giant star. In this case the lines using the standard and the (c) atoms are indistinguishable. This happens because in this metal-poor giant star there are not as many free electrons and the contribution of this process becomes less important. Although less important, the effect of the vanReg62 e+K collision rates, which are mostly higher than the other methods employed, can be observed as it increases the depth of the line core. Nevertheless, as the importance of the $\mathrm{e}+\mathrm{K}$ collisions decreases, we observe that the importance of the $\mathrm{H}+\mathrm{K}$ excitation increases: compared to the standard atom $(a)$, there is an abundance difference of $\delta \approx 0.03$ dex after reducing the efficiency of the $\mathrm{H}+\mathrm{K}$ collisions $(g)$. This can be contrasted with the late-type metalpoor star HD 84937 (third panel of Fig. 4), where no appreciable difference is observed.

In this section, we showed that the use of improved calculations of electron collisions is the most influential factor in the non-LTE line profile of dwarfs. The CCC and BSR calculations give comparable results, but substituting these calculations with the older Park71 and vanReg62 methods considerably changes the strength of the synthetic lines. Improved photoionization cross-section calculations with the DBSR method also had nonnegligible differences in the final line depth of the synthetic lines, and are important for accurate abundance determinations through the $7698 \AA$ resonance line. 
Table 3. Stellar parameters of the sample.

\begin{tabular}{lrrrrr}
\hline \hline Star & $T_{\text {eff }}(\mathrm{K})$ & $\log g\left(g\right.$ in $\left.\mathrm{cm} \mathrm{s}^{-2}\right)$ & $\xi\left(\mathrm{km} \mathrm{s}^{-1}\right)$ & {$[\mathrm{Fe} / \mathrm{H}]$} & Broadening $^{(*)}\left(\mathrm{km} \mathrm{s}^{-1}\right)$ \\
\hline Sun & $5772^{(a)}$ & $4.44^{(a)}$ & $1.0^{(b)}$ & $0.00^{(c)}$ & 3.5 \\
HD 192263 & $4998^{(d)}$ & $4.61^{(d)}$ & $0.66^{(b)}$ & $-0.05^{(e)}$ & 3.0 \\
Procyon & $6556^{(f)}$ & $4.01^{(f)}$ & $1.85^{(g)}$ & $-0.02^{(h)}$ & 6.0 \\
HD 103095 & $5140^{(i)}$ & $4.69^{(j)}$ & $0.9^{(k)}$ & $-1.13^{(l)}$ & 1.0 \\
HD 140283 & $5787^{(i)}$ & $3.66^{(m)}$ & $1.6^{(g)}$ & $-2.28^{(n)}$ & 3.8 \\
HD 84937 & $6371^{(d)}$ & $4.05^{(o)}$ & $1.3^{(p)}$ & $-1.97^{(n)}$ & 5.0 \\
\hline
\end{tabular}

Notes. ${ }^{(*)}$ Includes both macroturbulence velocity and rotation in one Gaussian broadening kernel.

References. ${ }^{(a)}$ Reference value from Prša et al. (2016); ${ }^{(b)}$ Spina et al. (2016); ${ }^{(c)}$ Asplund et al. (2009); ${ }^{(d)}$ IRFM value from Casagrande et al. (2011); ${ }^{(e)}$ Andreasen et al. (2017); ${ }^{(f)}$ fundamental value from Chiavassa et al. (2012); ${ }^{(g)}$ Pancino et al. (2017); ${ }^{(h)}\langle 3 \mathrm{D}\rangle$ non-LTE Fe II from Bergemann et al. (2012); ${ }^{\left({ }^{i}\right)}$ fundamental value from Karovicova et al. (2018); ${ }^{(j)}$ fundamental value from Bergemann \& Gehren $(2008) ;{ }^{(k)}$ Reggiani \& Meléndez (2018); ${ }^{(l)}$ 1D LTE Fe II from Ramírez et al. (2013) with $\langle 3 D\rangle$ non-LTE corrections from Amarsi et al. (2016); ${ }^{(m)}$ Gaia Collaboration $(2018) ;{ }^{(n)} 3 \mathrm{D}$ non-LTE value from Amarsi et al. (2016); ${ }^{(o)}$ fundamental value from VandenBerg et al. (2014); ${ }^{(p)}$ Spite et al. (2017).

\section{Abundance analysis of $\mathrm{K}$ in benchmark stars}

We further tested our standard atom by modeling the K I optical lines in different stellar atmospheres. We analyzed the Sun and the following benchmark stars of the GAIA-ESO spectroscopic survey: HD 84937, HD 103095, HD 192263, HD 140283 and Procyon. For the Sun we use the Stenflo (2015) flux solar atlas, and the spectra of the remaining objects are high-resolution $(\mathrm{R} \approx 220000)$ PEPSI spectra (Strassmeier, Ilyin \& Weber 2018); these are available fully reduced and continuum normalized ${ }^{1}$. The stellar parameters of the stellar model atmospheres can be seen in Table 3.

To determine the potassium abundances of the stars we match synthetic equivalent widths (EWs) to observed ones. We tested both Gaussian fitting and full line integration for measuring the EWs in the observed spectra and the methods have similar outcomes. We convolved (Gaussian Kernel) the synthetic spectra of the best abundance to account for rotational and macroturbulence velocities using the PyAstronomy ${ }^{2}$ python package. Both are treated as a free parameter but for a first guess we calculate the macoturbulent velocity using the trend with $T_{\text {eff }}$ described in Gray (2005).

We analyzed three observable lines in the optical spectral region: the K I 5801.7, 6938.7, and 7698.9 $\AA$ lines, and for completeness added the $12522 \AA$ infrared line in the Sun. The atomic data of the transitions are in Table 4. We used standard MARCS (Gustafsson et al. 2008) model atmospheres. In contrast with Zhang et al. (2006) and Scott et al. (2015) we did not analyze the K I 4044.1 and $7664 \AA$ lines because they are heavily blended. We show the fitted lines in Figs. 5-9.

We estimate a lower bound on the modeling errors through the line-to-line scatter of the three observable lines in the Sun and HD 192263. In the Sun, the scatter is only 0.02 dex, but it is up to 0.05 dex in HD 192263, possibly due to the strong damping wings and difficulties in determining the abundance through the resonance line in this star (both are further discussed below). Most of our analyzed stars do not show such strong damping wings and asymmetries observed in HD 192263, and therefore the uncertainties are not as high. Thus, we estimate a lower error of 0.03 dex for our measurements.

\footnotetext{
1 https://pepsi.aip.de/?page_id=552

2 www.hs.uni-hamburg.de/DE/Ins/Per/Czesla/PyA/PyA/ index.html
}

Table 4. Atomic data of the transitions.

\begin{tabular}{lrrrrr}
\hline \hline$\lambda(\AA)$ & Transition & $\mathrm{E}(\mathrm{eV})$ & $\log (g f)$ & $\sigma / a_{0}^{2}$ & $\alpha$ \\
\hline 5801.75 & $4 \mathrm{p}^{2} \mathrm{P}_{3 / 2}^{0}-7 \mathrm{~s}^{2} \mathrm{~S}_{1 / 2}$ & $1.617-3.753$ & -1.605 & - & - \\
6938.77 & $4 \mathrm{p}^{2} \mathrm{P}_{3 / 2}^{0}-6 \mathrm{~s}^{2} \mathrm{~S}_{1 / 2}$ & $1.617-3.403$ & -1.252 & 1721 & 0.294 \\
7698.96 & $4 \mathrm{~s}^{2} \mathrm{~S}_{1 / 2}-4 \mathrm{p}^{2} \mathrm{P}_{1 / 2}^{0}$ & $0.000-1.610$ & -0.176 & 485 & 0.232 \\
12522.16 & $4 \mathrm{p}^{2} \mathrm{P}_{3 / 2}^{0}-5 \mathrm{~s}^{2} \mathrm{~S}_{1 / 2}$ & $1.617-2.607$ & -0.134 & 1260 & 0.183 \\
\hline
\end{tabular}

Notes. The broadening of the lines via elastic collisions with hydrogen are represented via $\sigma$, the cross-section at the velocity of $10^{4} \mathrm{~m} \mathrm{~s}^{-1}$, and $\alpha$, the exponent with which the cross-section varies with velocity $\left(v^{-\alpha}\right.$, Anstee \& O'Mara 1995), and both $\sigma$ and $\alpha$ are from Barklem et al. (1998). For the $5801 \AA$ we use the Unsold's method (Unsold 1955), scaled to a factor of 1.5 .

\subsection{Sun}

For the Sun we derived a 1D non-LTE abundance of $A(\mathrm{~K})=$ 5.11 from averaging the result of the best EW match of the three optical lines in the solar spectrum. In our analysis the individual abundances of each line found were $A(\mathrm{~K})_{5801} \AA=$ $5.10 \mathrm{dex}, A(\mathrm{~K})_{6939 \AA}=5.10 \mathrm{dex}$, and $A(\mathrm{~K})_{7698 \AA}=5.12$. Zhang et al. (2006) did not find, for these same lines, abundances in such good agreement. Their abundances vary up to 0.09 dex between the optical $\left(A(\mathrm{~K})_{5801 \AA}=5.15, A(\mathrm{~K})_{6939 \AA}=5.06\right.$, and $\left.A(\mathrm{~K})_{7698 \AA}=5.14\right)$. With 1D MARCS model atmospheres (such as those used here) Scott et al. (2015) analyzed the lines $5801 \AA$ and $6939 \AA$, finding $A(\mathrm{~K})_{5801} \AA=5.15$ with a non-LTE correction of $-0.03 \mathrm{dex}$, in good agreement with our abundance. For the other line in common they determined $A(\mathrm{~K})_{6939} \AA=5.09$, and applied a correction of -0.03 dex, a somewhat smaller abundance than what we found (0.04 dex) but still within their expected error of 0.05 dex for potassium.

For our plot we needed to apply a change in the original normalization of the 5801 and $6939 \AA$ lines. As already pointed out by Zhang et al. (2006) there are terrestrial blends around the $5801 \AA$ line and a very uncertain continuum at the $6939 \AA$ region due to a number of contributions from different lines. We optimized the continuum of those lines based on adjacent regions (5811 and $6935 \AA$ ).

It must be noted that adjacent features were not synthesized, and the potassium lines at 5801, 6939, and $12522 \AA$ were synthesized with the best abundance and a broadening parameter 

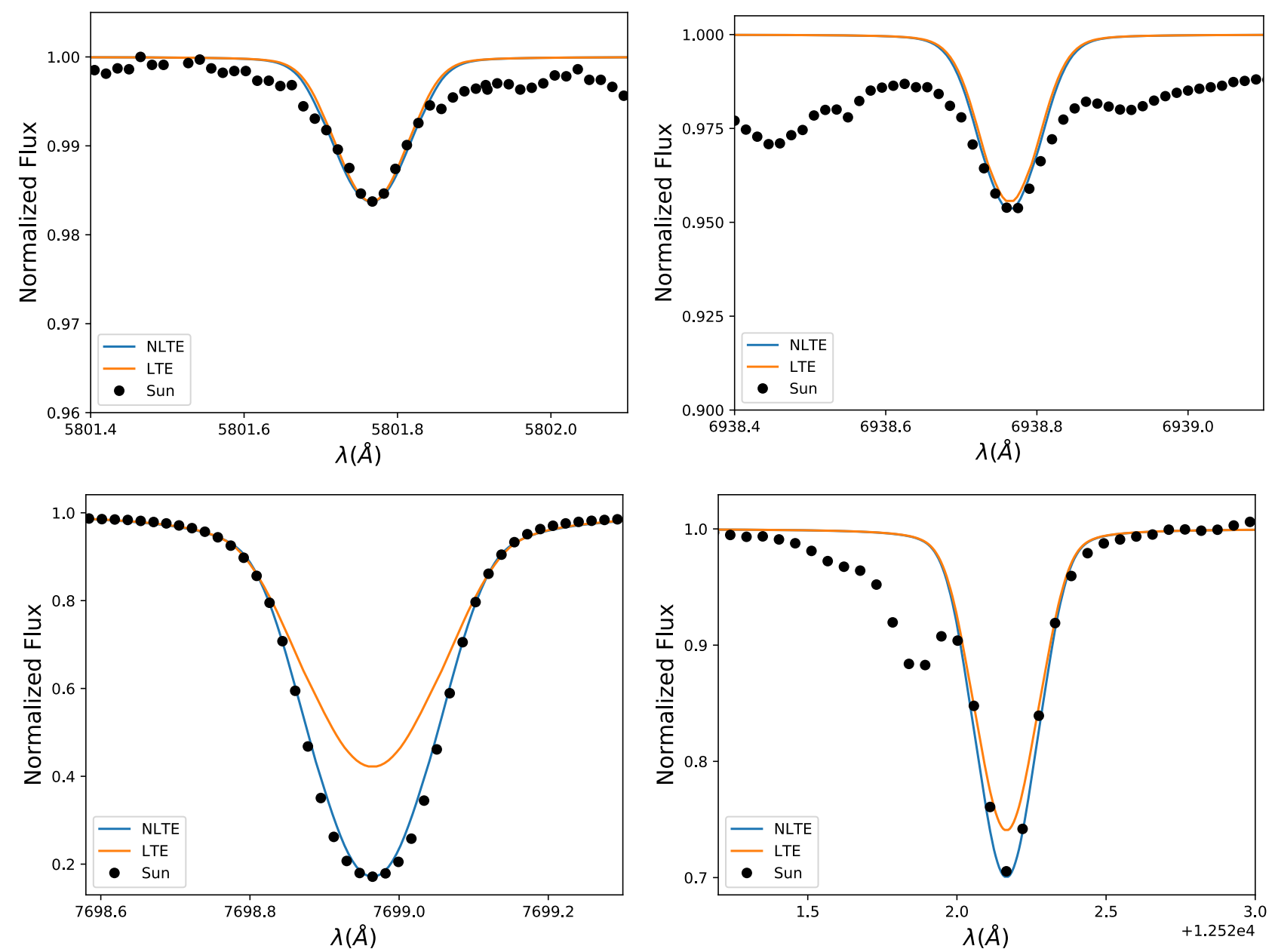

Fig. 5. Non-LTE abundance fit of the 5801, 6939, 7698, and $12522 \AA$ lines (top left, top right, lower left, and lower right, respectively) at the Sun with an abundance of $A(\mathrm{~K})=5.11$. The LTE lines of the same abundance are also shown in the plots.

determined via the fitting of the $7698 \AA$ line. As mentioned above, the abundances were not calculated via synthetic spectra. We separately measured the EW of each observed line and matched them to the EWs of the synthetic lines to find the abundances. Thus, the fits shown in Fig. 5 were not used to estimate the abundances, and the line profiles are shown only to demonstrate that our EW abundance can reproduce the lines. As can be seen, the synthetic line fits to the solar spectra are very good. We also show the LTE line of the same abundance as a comparison and one can see that although the differences between the LTE and non-LTE methods for the 5801 and $6939 \AA$ lines are very small (to fit the lines with LTE one needs an abundance $\approx 0.01$ dex higher than the non-LTE abundance), the difference observed for the resonance $7698 \AA$ line is very large. In the case of the resonance line, the LTE assumption fails completely and it is not possible to correctly reproduce the spectral line, even when considerably increasing the abundance of $\mathrm{K} \mathrm{I}$ to $A(\mathrm{~K}) \approx 5.44$ (our best LTE abundance via EW). We emphasize the importance of taking non-LTE effects into account when studying the GCE of potassium using the resonance line (e.g., Takeda et al. 2002, 2009; Zhang et al. 2006; Andrievsky et al. 2010; Scott et al. 2015).

\section{2. $H D 84937$}

HD 84937 is a low-metallicity $([\mathrm{Fe} / \mathrm{H}]=-1.97)$, bright, main sequence turn-off star that is commonly used as a standard representative of the abundance pattern of similar-metallicity field stars.

Spite et al. (2017) analyzed the abundance pattern of this star and measured a LTE potassium abundance of $A(\mathrm{~K})=3.40$ from the resonance $7698 \AA$ line. These latter authors applied a non-LTE abundance correction of -0.2 dex from Zhang et al. (2006), finally advocating $A(\mathrm{~K})=3.20$ for this line in HD 84937 .

In non-LTE, we measured $A(\mathrm{~K})=3.15$, while our best LTE abundance is $A(\mathrm{~K})=3.33$, a difference of $\Delta=-0.18 \mathrm{dex}$, in excellent agreement with the correction from the model atom described in Zhang et al. (2006) and used in Spite et al. (2017). In Fig. 6 we show the non-LTE abundance along with the LTE synthetic line of the same abundance for comparison. As can be seen, the non-LTE abundance that was found fits the stellar spectrum very well. The small asymmetry in the red wing of the observed spectral line is due to convective motions in the stellar atmosphere, the same conclusion as drawn from the analysis of this line by Smith et al. (2001). The best LTE line was also able to reproduce the observed line and the best non-LTE is indistinguishable from the best LTE line when plotted together.

\section{3. $H D 103095$}

HD 103095 is a metal-poor K-type dwarf, commonly used as a standard star of the inner Halo. This star is an $\alpha$-poor star with $[\mathrm{Mg} / \mathrm{Fe}]=0.12$ dex (Reggiani \& Meléndez 2018), which is lower than usually found for stars of such metallicity. 


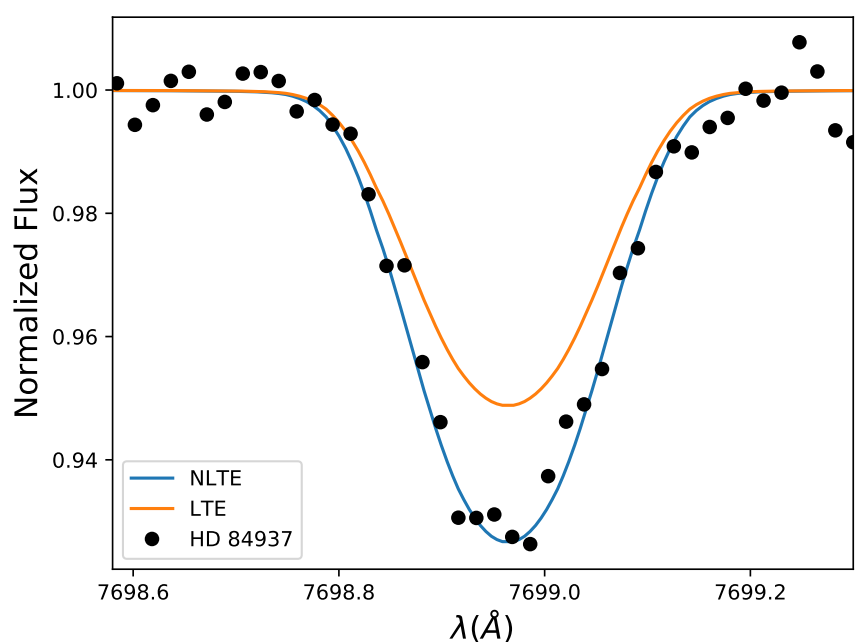

Fig. 6. Non-LTE abundance fit of the $7698 \AA$ A line for HD 84937 with an abundance of $A(\mathrm{~K})=3.15$. The LTE line of the same abundance is also shown in the plot.

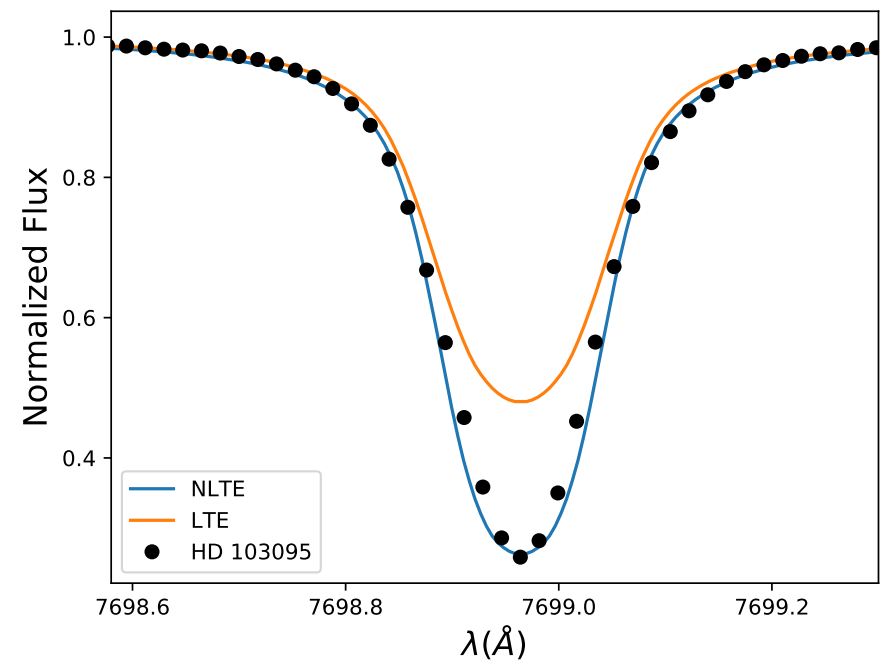

Fig. 7. Non-LTE abundance fit of the $7698 \AA$ line for HD 103095 with an abundance of $A(\mathrm{~K})=4.00$. The LTE line of the same abundance is also shown in the plot.

When assuming LTE, we determined an abundance $A(\mathrm{~K})=$ 4.26 for this star. When the non-LTE modeling was used instead, the best match of EW was found for an abundance of $A(\mathrm{~K})=$ 4.00 , a difference of -0.26 dex. The non-LTE synthetic line, the observed spectra, and the comparison LTE synthetic line can be seen in Fig. 7. The spectral line in HD 103095 is very well reproduced under non-LTE, except for a small discrepancy in the near-to-core region, which might be caused by 3D effects (further discussed in Sect. 6). As for LTE, our best abundance estimate was not able to correctly reproduce the line.

\section{4. $H D 140283$}

HD 140283 is a bright very metal-poor, high-velocity subgiant in the solar neighborhood. Its proximity to the Sun made it the first star with spectroscopic confirmation of chemical abundances lower than what is found in the Sun (Chamberlain \& Aller 1951; Sandage 2000). Its brightness and proximity to the Sun means it has a well-determined parallax and also a well-determined age. Furthermore, it is one of the oldest stars with very reliable age estimation (Bond et al. 2013).

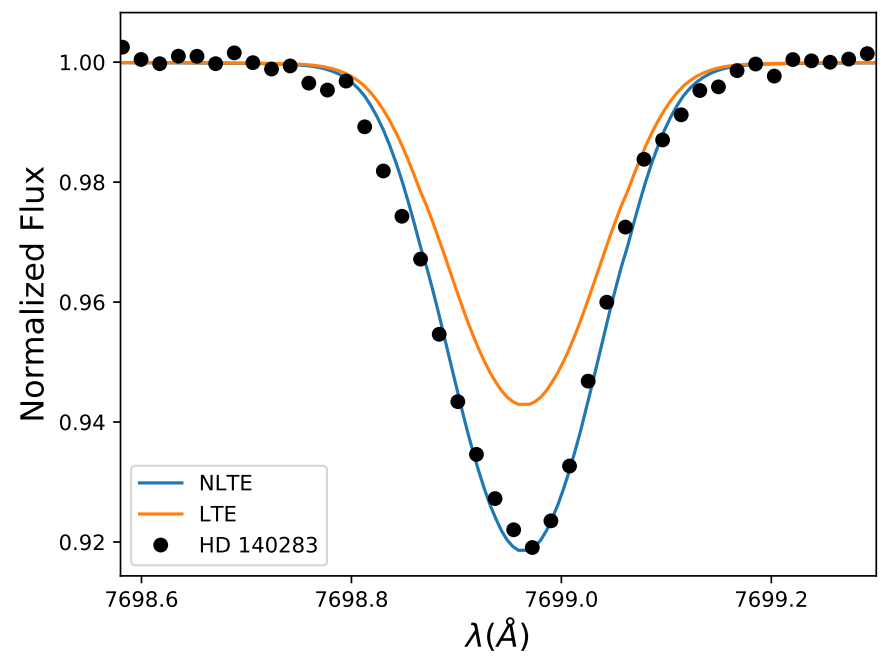

Fig. 8. Non-LTE abundance fit of the $7698 \AA$ line for HD 140283 with an abundance of $A(\mathrm{~K})=2.77$. The LTE line of the same abundance is also shown in the plot.

The best LTE abundance we found for HD 140283 was $A(\mathrm{~K})=2.95$ and the final non-LTE abundance is a very low abundance of $A(\mathrm{~K})=2.77$ (or $[\mathrm{K} / \mathrm{Fe}]=-0.06 \mathrm{dex}$ ), which is lower than the mean non-LTE abundance we found for stars in the metallicity regime $-2.2 \leq[\mathrm{Fe} / \mathrm{H}] \leq-2.5$ of $[\mathrm{K} / \mathrm{Fe}]=$ +0.21 dex; see Fig. 14. This lower-than-usual abundance was not expected, but the LTE abundance we found matches the LTE abundance given by the radiative-transfer code MOOG (Sneden 1973) using the same stellar information.

Although the best abundance is not as high as we expected for the metallicity of the star, the abundance we found fits very well to the observed spectra and the resulting profile appears to be very reliable. Like in HD 84937, if the best LTE abundance is plotted on top of the best non-LTE abundance the two lines are almost indistinguishable, with the non-LTE line being very slightly deeper. In Fig. 8 we show the non-LTE and LTE line of $\mathrm{K}$ with an abundance of $A(\mathrm{~K})=2.77$.

\section{5. $H D 192263$}

HD 192263 is a cool dwarf star of nearly solar metallicity, and is also a Gaia-ESO benchmark star. Under its atmospheric conditions, the $7698 \AA$ line develops strong damping wings and it is possible to visually perceive the asymmetries at the wings of the line, as shown in Fig. 9. It is difficult to reproduce the potassium line even under non-LTE.

In this star, it is possible to detect not only the resonance line, but also the other two clean potassium spectral lines. We therefore determined the abundance of this star by measuring the EW of the three lines $(5801,6939$, and $7698 \AA$ ) and found the nonLTE abundances of $A(\mathrm{~K})=5.07,5.02$, and 5.01, respectively. The adopted value is the averaged value of $A(\mathrm{~K})=5.03$.

The LTE abundances of the 5801 and $6939 \AA$ lines were found to be $A(\mathrm{~K})=5.04$, which is consistent with the non-LTE abundances. The $7698 \AA$ LTE abundance is 5.26, a non-LTE correction of -0.23 dex.

We show the spectra and the synthetic lines of the 5801, 6939, and $7698 \AA$ lines in Fig. 9. The observed spectra can be reproduced by the non-LTE synthetic spectra but in HD 192263 the synthetic line of the best LTE abundance found could not reproduce either the core or the wings of the observed line. Both in LTE and non-LTE there is an asymmetry at the red wing 

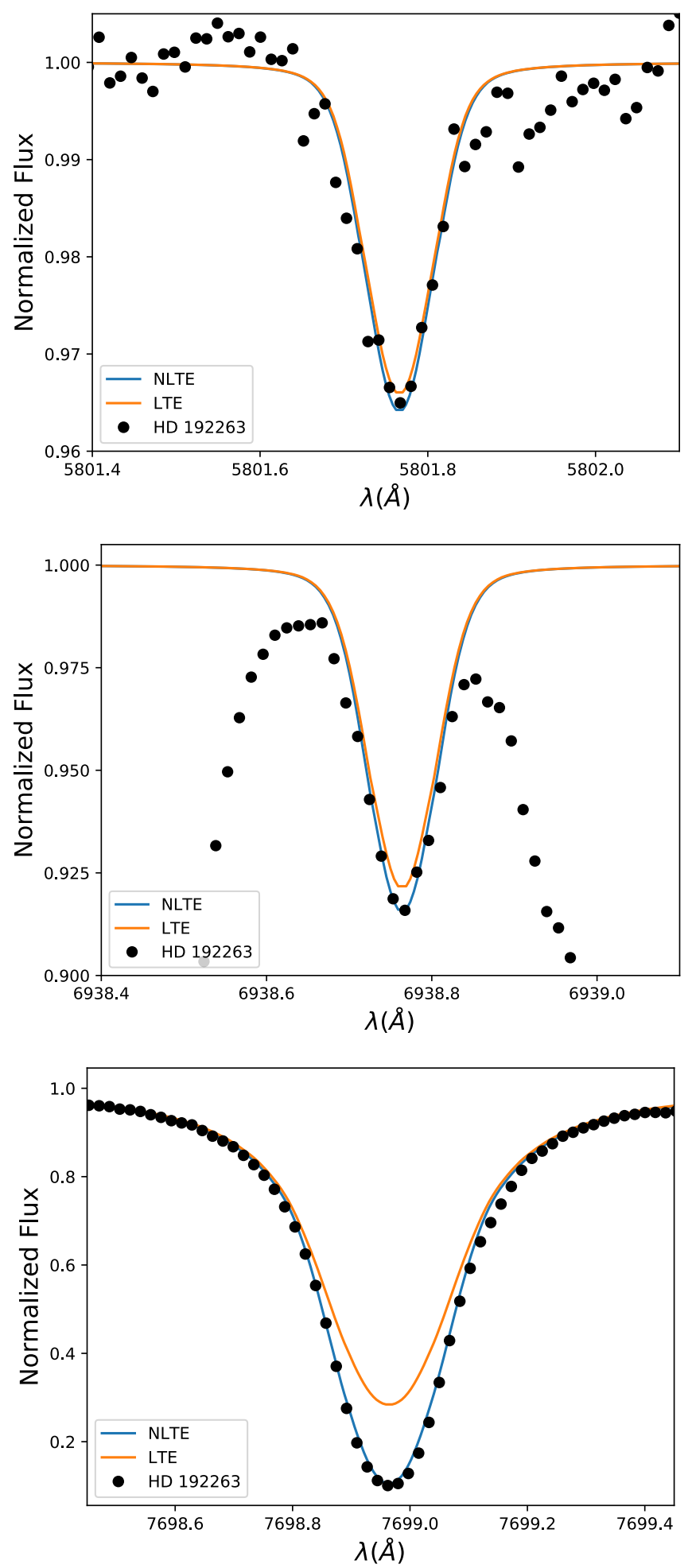

Fig. 9. Non-LTE abundance fit of the 5801, 6939, and $7698 \AA$ lines (top, middle, and lower panels, respectively) for HD 192263 with an abundance of $A(\mathrm{~K})=5.03$. The LTE lines of the same abundance are also shown in the plots.

that has the same form as the asymmetry observed in Procyon (Sect. 5.6 and further discussed in Sect. 6).

\subsection{Procyon}

Procyon is a solar metallicity F-type star very close to the Sun, in which the only observable $\mathrm{K}$ line is the resonance line $7698 \AA$.

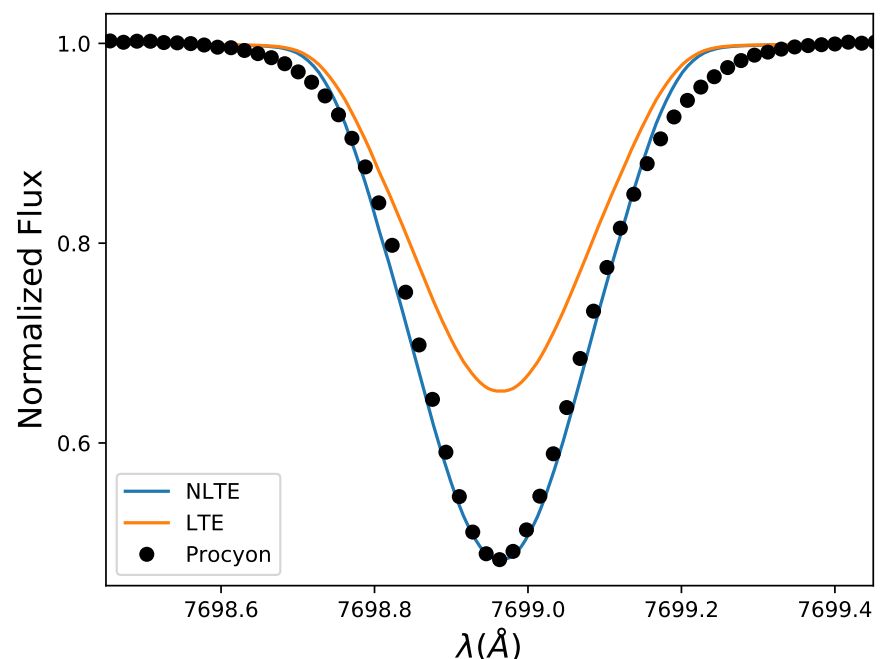

Fig. 10. Non-LTE abundance fit of the $7698 \AA$ line for Procyon with an abundance of $A(\mathrm{~K})=4.86$. The LTE line of the same abundance is also shown in the plot.

The wings of the line are very broad and difficult to correctly model. The non-LTE abundance, based on the EW of the line, fails to reproduce the wings; the red wing is more broadened than the blue wing, as also observed in HD 192263, an effect that is known to be a result of granulation in the stellar atmosphere (e.g., Dravins et al. 1981). Takeda et al. (1996) were able to model the wings of Procyon, but they applied corrections by hand to the red wing of the line and to the line core in order to correct what they believed to be unfavorable instrumental effects in their measurements.

As can be seen in Fig. 10 the line core is well represented by the non-LTE synthetic line, while the broadened wings of the observational spectra diverge near the continuum level. We found a non-LTE abundance of $A(\mathrm{~K})=4.86$ and a LTE abundance of $A(\mathrm{~K})=5.54$, a correction of -0.68 dex. Takeda et al. (1996) analyzed the potassium abundance in Procyon and also found a non-LTE correction of approximately similar to $-0.7 \mathrm{dex}$, although their LTE and non-LTE abundances are higher than what we found. We note again that even in non-LTE a 1D model could not simultaneously reproduce the core and the wings of the potassium $7698 \AA$ line in Procyon. As before, we also show the LTE line of the same abundance $(A(\mathrm{~K})=4.86)$ in Fig. 10.

\section{Three-dimensional non-LTE}

In Sect. 5 we demonstrated that there are severe (1D) non-LTE effects on the potassium resonance line, and that non-LTE methods are therefore needed to obtain reliable estimates of potassium abundances. This was particularly observed through the abundances of the Sun and HD 192263, in which one can measure all three optical lines, including those not heavily affected by non-LTE effects. In those cases only a non-LTE analysis can simultaneously give us consistent abundances in all observable lines.

However, although we can correctly reproduce the core of the $7698 \AA$ line, the wings are not as well reproduced, an effect that is observable in the spectra of HD 192263 (Sect. 5.3) and Procyon (Sect. 5.6). In Procyon one can clearly see the existence of an asymmetry between the blue and red wings of the line.

These types of asymmetries are associated with convection effects and can only be correctly modeled by using a 3D 


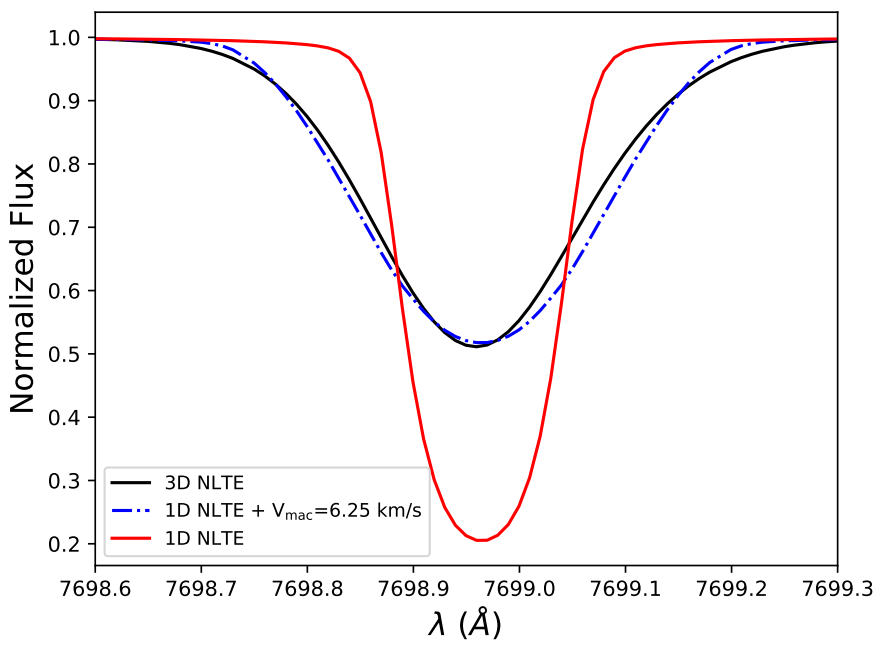

Fig. 11. Synthetic non-LTE $7698 \AA$ potassium line under different atmospheric model assumptions (1D and 3D).

radiation-hydrodynamical simulation of the stellar atmosphere (Dravins et al. 1981; Asplund et al. 2000, 2004). A great amount of effort has been invested in the modeling of the convectioninduced asymmetry of the lithium resonance line, which is similar to potassium in many respects. In particular, many have attempted to disentangle the imprint of convection with possible absorption in the red wing due to ${ }^{6} \mathrm{Li}$ (e.g., Smith et al. 2001; Asplund et al. 2006). The importance of accounting for non-LTE effects combined with the 3D line formation of lithium has also been emphasized (Cayrel et al. 2004; Lind et al. 2013).

The asymmetric shape of the potassium resonance line has been studied in the context of solar granulation for decades (e.g., Marmolino et al. 1987). However, although there have been studies modeling the resonance line formation using 3D LTE models (e.g., Nissen et al. 2000; Scott et al. 2015) there is, to the best of our knowledge, no published 3D non-LTE study of potassium. Although a full quantitative analysis is outside the scope of this project, we explored the effects with the radiative-transfer code BALDER (Amarsi et al. 2018). We ran one snapshot of a full 3D non-LTE calculation based on a STAGGER (Magic et al. 2013) model atmosphere with $T_{\text {eff }}=6437 \mathrm{~K}, \log g=4.0,[\mathrm{Fe} / \mathrm{H}]=0.0$, and $[\mathrm{K} / \mathrm{Fe}]=0.0$, which correspond to similar parameters to those of Procyon.

Our results showed that $1 \mathrm{D}$ non-LTE can only partially reproduce the wings seen in 3D after we added strong macroturbulent broadening effect of $V_{\mathrm{MAC}} \approx 6.25 \mathrm{~km} \mathrm{~s}^{-1}$ (dashed line), and even so the 1D non-LTE does not fully reproduce the wings seen in $3 \mathrm{D}$, particularly the asymmetry of the line.

The 3D non-LTE feature that we synthesized indicates that the misrepresentation of the wings in our 1D non-LTE analysis is due to unaccounted-for $3 \mathrm{D}$ effects. Although the asymmetries of the potassium resonance wings are much better represented in full 3D non-LTE we argue that the EW analysis of the 1D non-LTE yields comparable results.

\section{Non-LTE corrections grid}

Using our standard atom we produced a grid of non-LTE corrections, for model atmospheres of different stellar parameters. Our grid was computed for models with effective temperatures in the range $4000 \leq T_{\text {eff }} / \mathrm{K} \leq 8000$ with steps of $500 \mathrm{~K}$; for each $T_{\text {eff }}$ the surface gravity range is $0.5 \leq \log (\mathrm{g}) \leq 5.0 \mathrm{dex}$ in steps of 0.5 , and we also vary the metallicities in the range
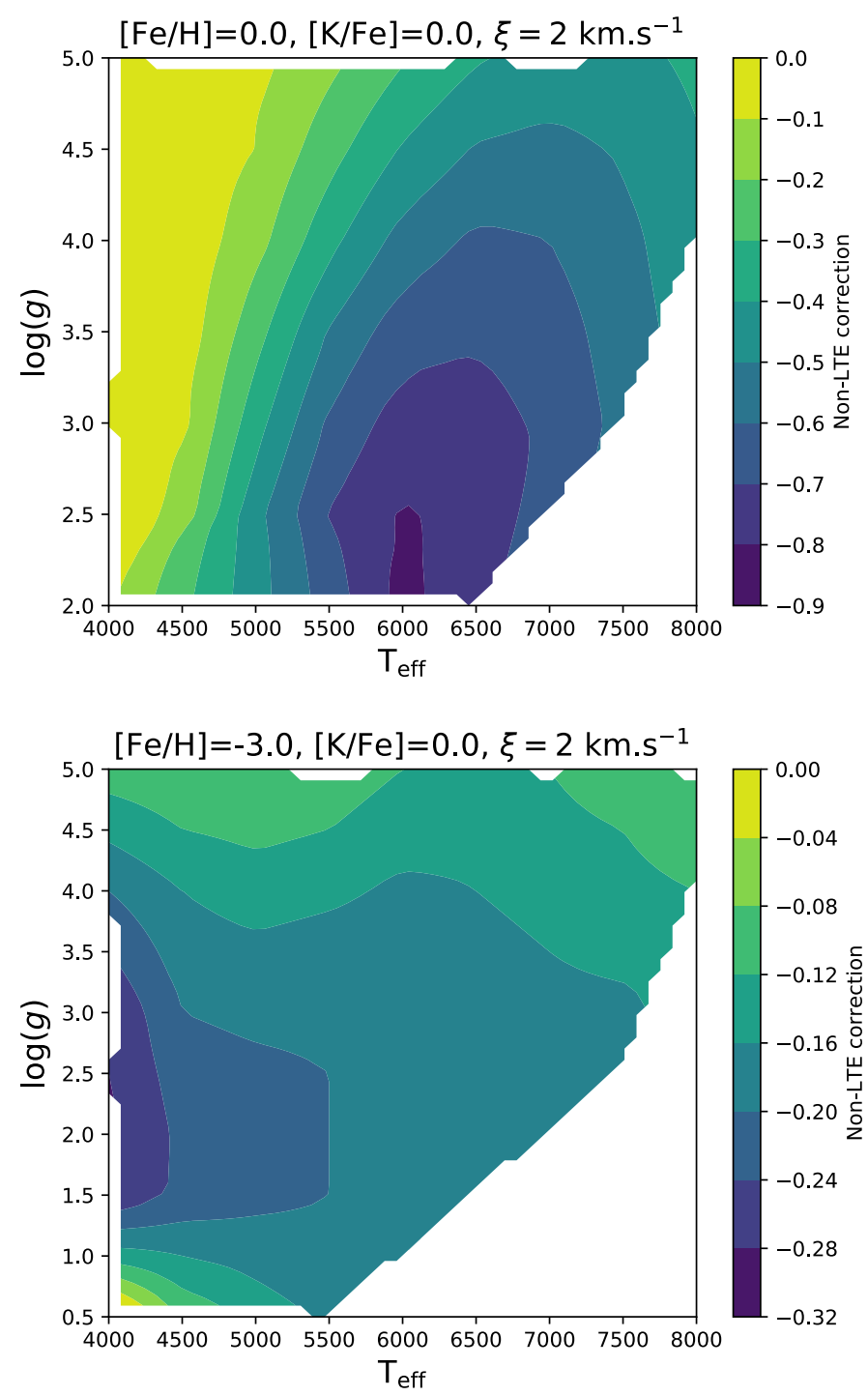

Fig. 12. Contour diagram illustrating the abundance corrections in $[\mathrm{Fe} / \mathrm{H}]=0.0$ (upper panel) and $[\mathrm{Fe} / \mathrm{H}]=-3.0$ (lower panel) for the $7698 \AA$ A line.

$-5.00 \leq[\mathrm{Fe} / \mathrm{H}]+0.50$ in steps of 0.25 and use microturbulence velocities of $\xi=1.0,2.0$, and $5.0 \mathrm{~km} \mathrm{~s}^{-1}$. We determined synthetic spectral lines of potassium abundances varying in the range $-1.25 \leq[\mathrm{K} / \mathrm{Fe}] \leq+1.25$, and estimated the LTE and nonLTE EWs in each case. Thus, we obtained a final grid with synthetic LTE and non-LTE EWs for each calculated abundance in each model atmosphere.

In Fig. 12 we show an example of the non-LTE corrections for solar metallicity varying the stellar parameters. The abundance correction for a Sun-like star is approximately -0.3 dex and for stars like Procyon the abundance correction is as high as -0.7 dex.

From the top panel of Fig. 13 we can see that the correction is very dependent on line strength, and the apparent dependence with temperature seen in Fig. 12 is an indirect effect. The extremely high non-LTE corrections for certain stellar atmospheres shows us that it is imperative to apply non-LTE corrections when analyzing the abundances of potassium from the resonance lines.

In the lower panel of Fig. 13 we show the dependence with metallicity of two model atmospheres for a $\mathrm{K}$ abundance of 

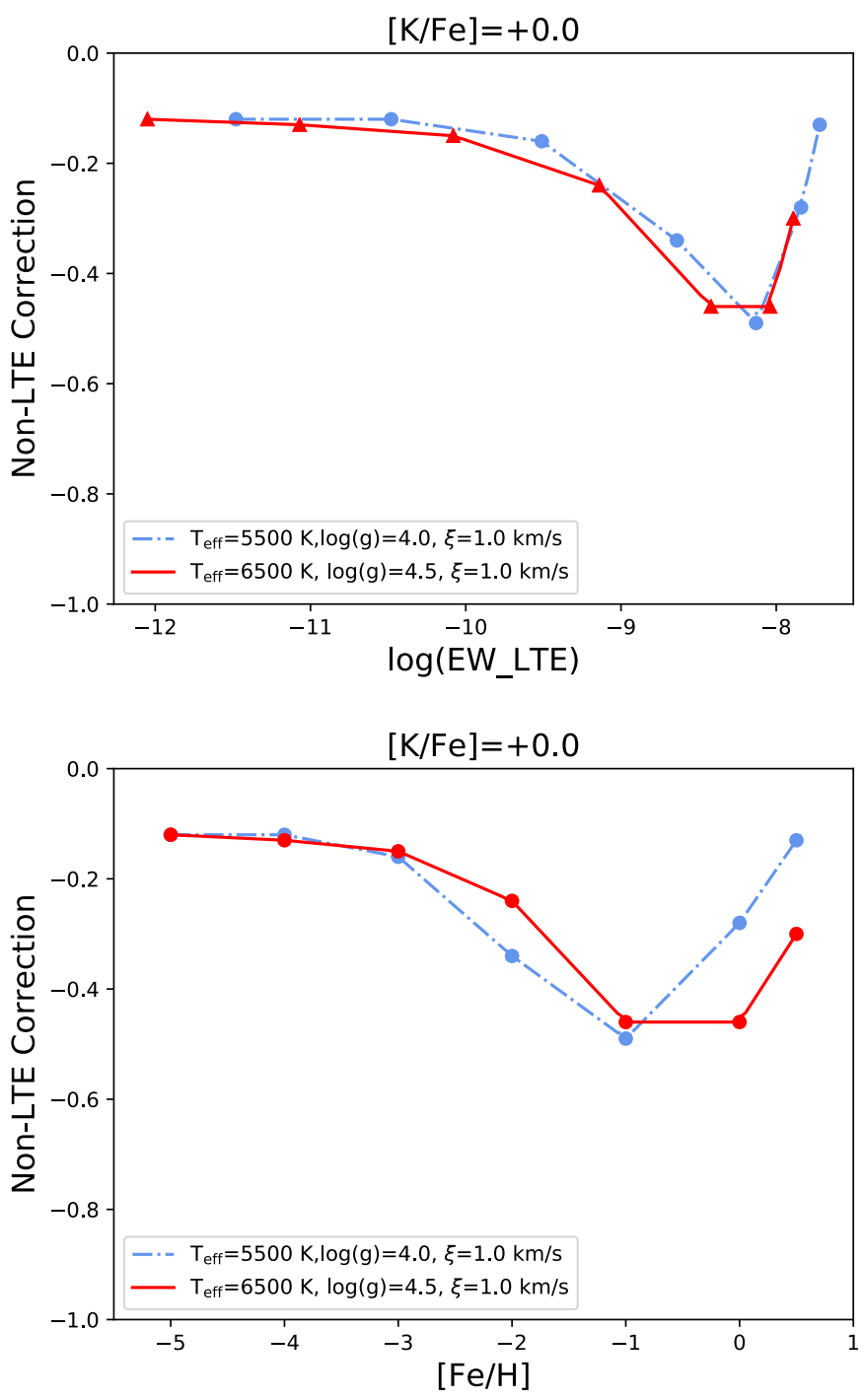

Fig. 13. Top panel: non-LTE corrections variation with EW. Lower panel: non-LTE correction variation with $[\mathrm{Fe} / \mathrm{H}]$. In both panels we have two different model atmospheres and both show the corrections for the $7698 \AA$ A line.

$[\mathrm{K} / \mathrm{Fe}]=0.0$. An important dependence with metallicity starts to appear at $[\mathrm{Fe} / \mathrm{H}]$ greater than -2.0 ; this can be directly related to the line becoming stronger for higher metallicities and saturating. As the metallicity increases and the line gets stronger, the corrections are larger and peak at approximately solar metallicity before decreasing again. However, we have not found any measurements of potassium in stars with metallicities higher than solar, and therefore this decrease in the corrections after $[\mathrm{Fe} / \mathrm{H}]=0.0$ has not been applied in any observational data to test its compatibility with the GCE models.

We also computed the corrections for the resonance $7664 \AA$ line and the infrared lines 15163 and $15168 \AA$ (which are in the APOGEE range). Abundances from the resonance $7664 \AA$ line must also be non-LTE corrected as the corrections are on the same order as the corrections computed for the $7698 \AA$ line, but the infrared lines do not have such strong non-LTE dependence (the mean correction of the $15168 \AA$ for $[\mathrm{K} / \mathrm{Fe}]=0.0$ at the same models presented in Fig. 13 are -0.03 and -0.06 dex).

Our final grid is publicly available at the CDS as an electronic table.

\section{Chemical evolution of Potassium}

\subsection{Metal-poor stars}

We applied our grid of corrections to a series of abundances from several publications in order to study the evolution of potassium in our galaxy. We show these results and compare them to the K11 (Kobayashi et al. 2011), K15 (Zhao et al. 2016; Sneden et al. 2016) GCE models, and the models from Prantzos et al. (2018) with and without yields from massive rotating stars.

We determined abundances under LTE using the 1D LTE code MOOG (Sneden 1973) based on three sets of published EWs: Cayrel et al. (2004), Roederer et al. (2014), and Spina et al. (2016). We then determined the non-LTE abundances by applying non-LTE abundance corrections from our grid. Andrievsky et al. (2010) recently reanalyzed the Cayrel et al. (2004) sample with their non-LTE corrections and we compared our results to theirs, finding a mean difference of approximately $-0.1 \mathrm{dex}$ in the final non-LTE abundances (with their abundances being higher).

Figure 14 and Table A.1 show both the $[\mathrm{K} / \mathrm{Fe}]_{\mathrm{LTE}}$ and the $[\mathrm{K} / \mathrm{Fe}]_{\text {NLTE }}$ results for the above-mentioned samples. In the upper panel of Fig. 14 we see the behavior of the LTE abundances. For all data there is a large discrepancy between the abundances of models and observations, reaching up to 1 dex. For $[\mathrm{Fe} / \mathrm{H}] \lesssim-1.0$, the LTE potassium abundances show an increasing trend with $[\mathrm{Fe} / \mathrm{H}]$, which is similar to what is predicted by the Kobayashi et al. (2011) model, but differs from the behavior of the Prantzos et al. (2018) models.

The non-LTE abundances however show a different result. In the region $[\mathrm{Fe} / \mathrm{H}] \lesssim-1.0$, there is no longer a trend of increasing potassium abundances with increasing $[\mathrm{Fe} / \mathrm{H}]$; rather, the potassium abundances gradually decrease. The model of rotating massive stars by Prantzos et al. (2018) appears to correctly reproduce the observations, although the mean observed abundance is slightly higher than the model. Non-LTE corrected abundances and the model of massive rotating stars show the same behavior: a small increase in abundances between $-3 \leq[\mathrm{Fe} / \mathrm{H}] \leq-2$ followed by a decrease in abundances with increasing metallicity. On the other hand, the models from Kobayashi et al. (2011) and the Prantzos et al. (2018) model without rotation underestimate the production of potassium and their yields clearly fail to reproduce the observations.

\subsection{Solar twins}

The solar twin sample of Spina et al. (2016) sits at $[\mathrm{Fe} / \mathrm{H}] \approx 0.0$ in Fig. 14. Correcting the abundances for non-LTE effects does not have a significant effect here. This is because these abundances were measured in a line-by-line differential analysis with respect to the Sun. To first order, the non-LTE errors in the analysis of the solar twin spectra cancel with the non-LTE errors in the analysis of the solar spectrum. However, the non-LTE corrections lead to a reduction in the scatter of the potassium abundances, as we discuss below.

In their high-precision differential analysis of solar twins (stars with atmospheric parameters similar to the solar parameters $-\Delta \mathrm{T}_{\text {eff }} \pm 100 \mathrm{~K}, \Delta \log (\mathrm{g}) \pm 0.1 \mathrm{dex}, \Delta[\mathrm{Fe} / \mathrm{H}] \pm 0.1 \mathrm{dex}$ and mass within $\approx 5 \%$ of the solar mass) Spina et al. (2016) analyzed 20 elements and for all of them, except potassium, they found a tight relationship between stellar age and $[\mathrm{X} / \mathrm{Fe}]$, with strongly varying slope depending on the element. With this in mind we reanalyzed their sample of solar twins and the same plot of $[\mathrm{K} / \mathrm{Fe}]$ versus age can be seen in Fig. 15. The trends with stellar age of the LTE and the non-LTE abundances are the same. Being 

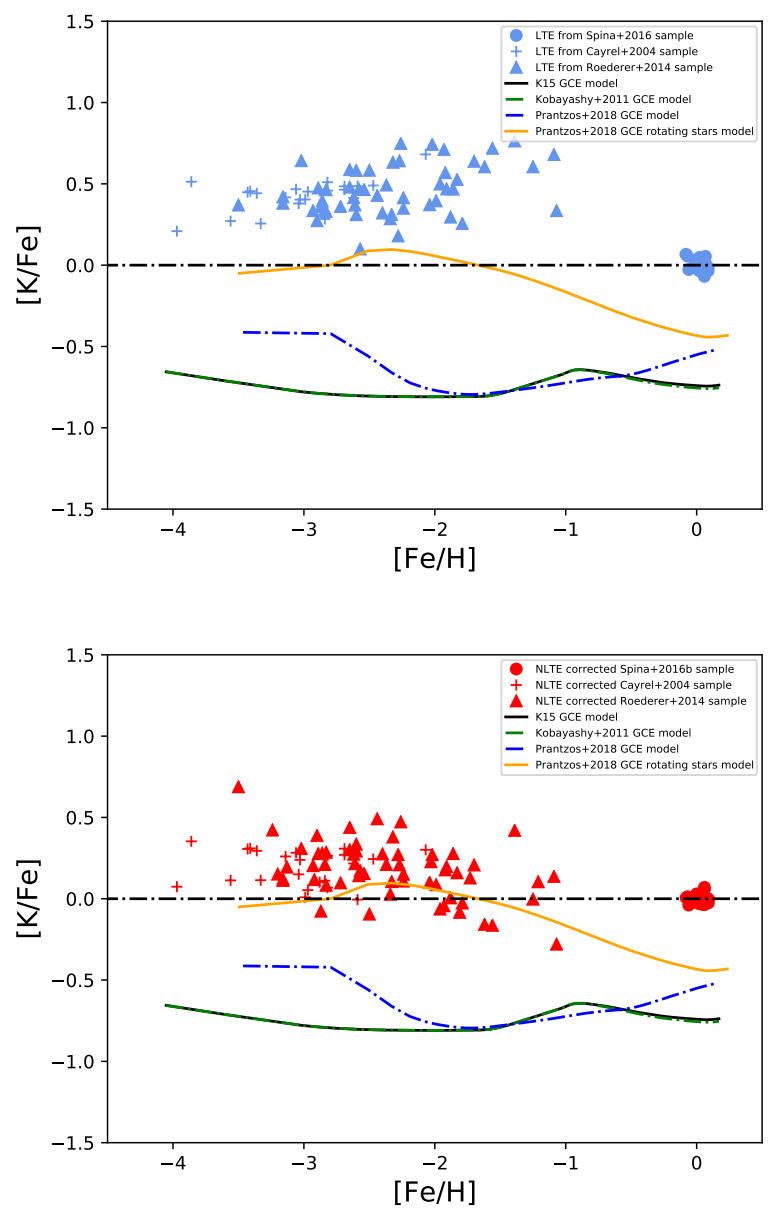

Fig. 14. Top panel: LTE abundances calculated using MOOG and the EWs from Cayrel et al. (2004), Roederer et al. (2014), and Spina et al. (2016). Lower panel: non-LTE corrected abundances (from the LTE abundances in the upper panel). The LTE and non-LTE corrected abundances (in $\mathrm{A}(\mathrm{K})$ ), are shown in Table A.1. In both panels we also show three different GCE models: in black the model from Kobayashi et al (2011); in dashed blue and orange the models from Prantzos et al. (2018) without and with yields from massive rotating stars, respectively.

solar twins, not only are the abundances very similar to one another, but also the non-LTE corrections. Although this result was expected, we call attention to the fact that the non-LTE abundances have a smaller scatter than the LTE abundances. This means that the non-LTE abundances might be more reliable, as the abundances of solar twins are very homogeneous and similar to the solar abundance (Bedell et al. 2018). This is also important because it shows that even in a differential abundance analysis of solar twins, non-LTE corrections play an important role in explaining the evolution of the elements by decreasing the scatter of abundance measurements, which might further improve fits of their behavior. The reduced scatter is important to correctly assess the homogeneity of chemical evolution and therefore to draw more precise conclusions on the nucleosynthetic mechanisms that drive potassium evolution throughout cosmic history. The reduced scatter is also important to assess the homogeneity of different stellar populations (e.g., thin/thick disk) and stellar associations (clusters or binaries), which is also relevant for chemical tagging, a popular application of stellar spectroscopy.

As already mentioned, Spina et al. (2016) found a function able to describe $[\mathrm{X} / \mathrm{Fe}]$ abundances over cosmic time for all elements analyzed, apart from potassium. We also tried to find a function to describe $[\mathrm{K} / \mathrm{Fe}]$ over time with our lower scatter

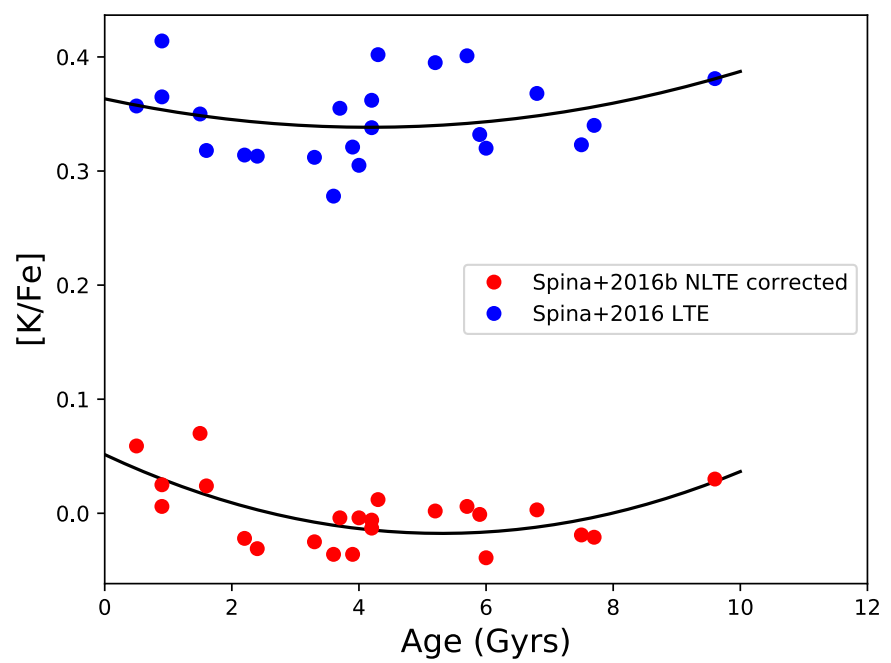

Fig. 15. In red: non-LTE abundances of the Spina et al. (2016) sample. In light blue: scaled LTE abundances. The offset is due to the use of the same solar potassium abundance LTE and non-LTE.

non-LTE abundances, but as in Spina et al. (2016) we did not find a function that can describe its behavior. Figure 15 shows a fourth-degree polynomial function that was used only to demonstrate the smaller scatter of the non-LTE data. The standard deviations of the non-LTE-corrected and the LTE abundances are $0.027 \mathrm{dex}$ and $0.035 \mathrm{dex}$, respectively, an improvement of $\sim 23 \%$. Within $1 \sigma$ the LTE and non-LTE fits are the same.

It is interesting how there is no possible fit to the potassium abundances over time, as found for the other elements. This might indicate an inhomogeneous enrichment of potassium, although the small scatter we observe might be caused by errors in the analysis. If indeed the ISM was not fully homogenized with $\mathrm{K}$, the small abundance differences among stars born in different sites in the Galaxy could explain this uncanny inability to fit the abundances. This behavior could be explained by a GCE where rotating massive stars play a role. As the occurrence of such objects is not as common as, for example, type II supernovae, rotating massive stars might be able to produce these localized small inhomogeneities, which would depend on a localized mass function and the production of the rotating massive stars. This localized effect would not be seen in most of the other commonly measured elements (such as $\mathrm{C}, \mathrm{O}, \mathrm{Na}$, $\mathrm{Mg}, \mathrm{Al}, \mathrm{Si}$, etc) because, as one can see in Fig. 13 of Prantzos et al. (2018), most of them have an evolution that is not significantly influenced by massive rotating stars, as both models (with and without the massive rotating stars) have virtually the same results. However, we caution that such small scatter might also be caused by errors in our analysis, and that the inability to fit the solar-twin data might simply be because the empirical fits suggested are not adequate.

\section{Conclusions}

We built a new model atom of potassium in order to test the non-LTE effects in stellar abundances estimations. We used state-of-the-art calculations of neutral hydrogen and electronic collisional cross-sections and photoionization cross-sections.

The use of improved calculations of electron collisions were the most influential factor in the non-LTE line profile of the latetype stars and both CCC and BSR calculations give comparable results. The choice between the different $\mathrm{H}+\mathrm{K}$ collisions and the DBSR photoionization cross-sections also had non-negligible 
and similar effects on the final line depth of the synthetic lines, and their contribution is more relevant to the analysis of giant stars. The use of different collisional recipes can lead to differences of up to $0.1 \mathrm{dex}$ in the abundance analysis. With the aid of an atom with only the resonance transitions we concluded that the major driver of the non-LTE effects is the photon losses in the 7664 and $7698 \AA$ lines.

As test cases for the quality of our synthetic spectra, we derived the potassium abundance of the Sun and other benchmark stars. We found that our non-LTE synthetic spectra are a good fit to the stellar spectra, as opposed to what is seen in LTE. When available the abundances were derived from the three optical spectral lines and the results are very homogeneous with the non-LTE approach, and vary considerably if every line is analyzed in LTE. Through this analysis we also showed that there are important 3D effects that can be observed via the asymmetries in the wings of the potassium line. The differences of the 1D and 3D line profiles were shown for a test case and we see that EW analyses of 1D non-LTE and 3D non-LTE lines give similar results, which is valuable to validate the grid of corrections that was developed for this work.

Finally, we used our grid of corrections on three sets of potassium data to analyze the chemical evolution of the Galaxy. We see that the yields of the common models (Fig. 14) are not enough to explain the observational data. However, the model with yields from rotating massive stars by Prantzos et al. (2018) is able to partially reproduce the core of the observed abundances, especially the decrease in abundance for metallicities higher than $[\mathrm{Fe} / \mathrm{H}]$ of around $-2.5 \mathrm{dex}$, which we also observe in our non-LTE results from the Roederer et al. (2014) sample. All the GCE models fail to reproduce the observed abundances at solar metallicity. However, the decreased scatter in the solar twins is an important result as it shows that the LTE differential abundance is not fully free of systematic uncertainties due to line formation responsible for part of the observed inhomogeneities, and will help constrain the nucleosynthetic mechanism mainly responsible for potassium production in this narrow metallicity range.

Acknowledgements. We thank the referee for their careful reading of the manuscript, which helped us to improve its quality. H.R. thanks his PDSE program (88881.132145/2016-01). This study was financed in part by the Coordenação de Aperfeiçoamento de Pessoal de Nível Superior - Brasil (CAPES) Finance Code 001. A.M.A. and K.L. acknowledge funds from the Alexander von Humboldt Foundation in the framework of the Sofja Kovalevskaja Award endowed by the Federal Ministry of Education and Research, and K.L. also acknowledges funds from the Swedish Research Council (grant 2015-004153) and Marie Skłodowska Curie Actions (cofund project INCA 600398). P.S.B acknowledges financial support from the Swedish Research Council and the project grant "The New Milky Way" from the Knut and Alice Wallenberg Foundation. The work of O.Z. and K.B. was supported by the United States National Science Foundation under grant Nos. PHY-1403245, PHY-1520970, and PHY-1803844. The numerical BSR calculations were performed on STAMPEDE at the Texas Advanced Computing Center. They were made possible by the XSEDE allocation No. PHY-090031. L.S. is supported from the Australian Research Council (Discovery Project 170100521). J.M. thanks support of FAPESP (2014/18100-4, 2018/04055-8) and CNPq (Bolsa de Produtividade) This work has made use of the VALD database, operated at Uppsala University, the Institute of Astronomy RAS in Moscow, and the University of Vienna.

\section{References}

Allen, C. W. 1976, Astrophysical Quantities, 3rd edn. (London: AthlonePress), Amarsi, A. M., Lind, K., Asplund, M., Barklem, P. S., \& Collet, R. 2016, MNRAS, 463, 1518

Amarsi, A. M., Barklem, P. S., Asplund, M., Collet, R., \& Zatsarinny, O. 2018, A\&A, 616, A89

Andreasen, D. T., Sousa, S. G., Tsantaki, M., et al. 2017, A\&A, 600, A69
Andrievsky, S. M., Spite, M., Korotin, S. A., et al. 2010, A\&A, 509, A88 Anstee, S. D., \& O'Mara, B. J. 1995, MNRAS, 276, 859

Asplund, M., Nordlund, A., Trampedach, R., Allende Prieto, C., \& Stein, R. F. 2000, A\&A, 359, 729

Asplund, M., Grevesse, N., Sauval, A. J., Allende Prieto, C., \& Kiselman, D. 2004, A\&A, 417, 751

Asplund, M., Lambert, D. L., Nissen, P. E., Primas, F., \& Smith, V. V. 2006, ApJ, 644, 229

Asplund, M., Grevesse, N., Sauval, A. J., \& Scott, P. 2009, ARA\&A, 47, 481

Badnell, N. R., Bautista, M. A., Butler, K., et al. 2005, MNRAS, 360, 458

Barklem, P. S. 2016, Phys. Rev. A, 93, 042705

Barklem, P. S., Anstee, S. D., \& O’Mara, B. J. 1998, PASA, 15, 336

Barklem, P. S., Belyaev, A. K., Guitou, M., et al. 2011, A\&A, 530, A94

Barklem, P. S., Osorio, Y., Fursa, D. V., et al. 2017, A\&A, 606, A11

Bedell, M., Bean, J. L., Meléndez, J., et al. 2018, ApJ, 865, 68

Bergemann, M., \& Gehren, T. 2008, A\&A, 492, 823

Bergemann, M., Lind, K., Collet, R., Magic, Z., \& Asplund, M. 2012, MNRAS, 427, 27

Biemont, E., \& Grevesse, N. 1973, A\&A, 27, 163

Bond, H. E., Nelan, E. P., VandenBerg, D. A., Schaefer, G. H., \& Harmer, D. 2013, ApJ, 765, L12

Bray, I, 1994, Phys. Rev. A, 49, 1066

Bray, I., \& Stelbovics, A. T. 1992, Phys. Rev. A, 46, 6995

Bray, I., \& Stelbovics, A. T. 1995, Comput. Phys. Commun., 85, 1

Bruls, J. H. M. J., Rutten, R. J., \& Shchukina, N. G. 1992, A\&A, 265, 237

Casagrande, L., Schönrich, R., Asplund, M., et al. 2011, A\&A, 530, A138

Carlsson, M. 1986, Upps. Astron. Obs. Rep., 33

Carlsson, M. 1992, Cool Stars, Stellar Systems, and the Sun, ASP Conf. Ser. 26 499

Cayrel, R., Depagne, E., Spite, M., et al. 2004, A\&A, 416, 1117

Chamberlain, J. W., \& Aller, L. H. 1951, ApJ, 114, 52

Chiavassa, A., Bigot, L., Kervella, P., et al. 2012, A\&A, 540, A5

Clayton, D. 2007, Handbook of Isotopes in the Cosmos (Cambridge, UK: Cambridge University Press)

de La Reza, R., \& Mueller, E. A. 1975, Sol. Phys., 43, 15

Dravins, D., Lindegren, L., \& Nordlund, A. 1981, A\&A, 96, 345

Drawin, H. W. 1968, Z. Phys., 211, 404

Drawin, H. W. 1969, Z. Phys., 225, 483

Gaia Collaboration 2018, VizieR Online Data Catalog: I/345

Gray, D. F. 2005, The Observation and Analysis of Stellar Photospheres, 3rd Edn. (Cambridge: Cambridge University Press)

Gustafsson, B., Edvardsson, B., Eriksson, K., et al. 2008, A\&A, 486, 951

Lind, K., Asplund, M., \& Barklem, P. S. 2009, A\&A, 503, 541

Lind, K., Asplund, M., Barklem, P. S., \& Belyaev, A. K. 2011, A\&A, 528, A103

Lind, K., Melendez, J., Asplund, M., Collet, R., \& Magic, Z. 2013, A\&A, 554, A96

Lodders, K., \& Palme, H. 2009, Meteorit. Planet. Sci. Suppl., 72, 5154

Karovicova, I., White, T. R., Nordlander, T., et al. 2018, MNRAS, 475, L81

Kaulakys, B. 1986, Sov. Phys. JETP, 64, 229

Kaulakys, B. 1991, J. Phys. B At. Mol. Phys., 24, L127

Kobayashi, C., Umeda, H., Nomoto, K., Tominaga, N., \& Ohkubo, T. 2006, ApJ, 653, 1145

Kobayashi, C., Izutani, N., Karakas, A. I., et al. 2011, ApJ, 739, L57

Kramida, A., Ralchenko, Yu., Reader, J., \& NIST ASD Team 2018, NIST Atomic Spectra Database (ver. 5.6.1), Available at: https://physics.nist.gov/ asd (2018, December 10). National Institute of Standards and Technology, Gaithersburg, MD. https://doi .org/10.18434/T4W30F

Magic, Z., Collet, R., \& Asplund, M. 2013, EAS Pub. Ser., 63, 367

Marmolino, C., Roberti, G., \& Severino, G. 1987, Sol. Phys., 108, 21

Moutou, C., Vigan, A., Mesa, D., et al. 2017, A\&A, 602, A87

Nissen, P. E., Asplund, M., Hill, V., \& D'Odorico, S. 2000, A\&A, 357, L49

Osorio, Y., Barklem, P. S., Lind, K., \& Asplund, M. 2011, A\&A, 529, A31

Pancino, E., Lardo, C., Altavilla, G., et al. 2017, A\&A, 598, A5

Park, C. 1971, J. Quant. Spectr. Rad. Transf., 11, 7

Peterson, R. C., Kurucz, R. L., \& Ayres, T. R. 2017, ApJS, 229, 23

Prantzos, N., Abia, C., Limongi, M., et al. 2018, MNRAS, 476, 3432

Prša, A., Harmanec, P., Torres, G., et al. 2016, AJ, 152, 41

Ramírez, I., Allende Prieto, C., \& Lambert, D. L. 2013, ApJ, 764, 78

Reggiani, H., \& Meléndez, J. 2018, MNRAS, 475, 3502

Reggiani, H., Meléndez, J., Kobayashi, C., Karakas, A., \& Placco, V. 2017, A\&A, 608, A46

Roederer, I. U., Preston, G. W., Thompson, I. B., et al. 2014, AJ, 147, 136

Romano, D., Karakas, A. I., Tosi, M., \& Matteucci, F. 2010, A\&A, 522, A32

Ryabchikova, T., Piskunov, N., Kurucz, R. L., et al. 2015, Phys. Scr, 90, 054005

Sandage, A. 2000, PASP, 112, 293

Sansonetti, J. E. 2008, J. Phys. Chem. Ref. Data, 37, 8 
Schweinzer, J., Brandenburg, R., Bray, I., et al. 1999, At. Data Nucl. Data Tables, 72,239

Scott, P., Grevesse, N., Asplund, M., et al. 2015, A\&A, 573, A25

Seaton, M. 1996, The Observatory, 116, 177

Sitnova, T., Zhao, G., Mashonkina, L., et al. 2015, ApJ, 808, 148

Smith, V. V., Vargas-Ferro, O., Lambert, D. L., \& Olgin, J. G. 2001, AJ, 121, 453

Sneden, C. A. 1973, PhD Thesis, University of Texas, Austin, USA

Sneden, C., Cowan, J. J., Kobayashi, C., et al. 2016, ApJ, 817, 53

Spina, L., Meléndez, J., Karakas, A. I., et al. 2016, A\&A, 593, A125

Spite, M., Peterson, R. C., Gallagher, A. J., Barbuy, B., \& Spite, F. 2017, A\&A 600, A 26

Stenflo, J. O. 2015, A\&A, 573, A74

Stockman, K. A., Karaganov, V., Bray, I., \& Teubner, P. J. O. 1998, J. Phys. B At. Mol. Opt. Phys., 31, L867

Stockman, K. A., Karaganov, V., Bray, I., \& Teubner, P. J. O. 1999, J. Phys. B At. Mol. Opt. Phys., 2, 3003

Stockman, K. A., Karaganov, V., Bray, I., \& Teubner, P. J. O. 2001, J. Phys. B At. Mol. Opt. Phys., 34, 1105

Strassmeier, K. G., Ilyin, I., \& Weber, M. 2018, A\&A, 612, A45

Sugar, J., \& Corliss, C. 1985, Atomic energy levels of the iron-period elements: Potassium through Nickel (Washington: American Chemical Society)
Takeda, Y., Kato, K.-I., Watanabe, Y., \& Sadakane, K. 1996, PASJ, 48, 511

Takeda, Y., Zhao, G., Chen, Y.-Q., Qiu, H.-M., \& Takada-Hidai, M. 2002, PASJ, 54,275

Takeda, Y., Kaneko, H., Matsumoto, N., et al. 2009, PASJ, 61, 563

Unsold, A. 1955, Physik der Sternatmospharen, MIT besonderer Berucksichtigung der Sonne (Berlin: Springer)

van Regemorter, H. 1962, ApJ, 136, 906

VandenBerg, D. A., Bond, H. E., Nelan, E. P., et al. 2014, ApJ, 792, 110

Wiese, W. L., Smith, M.W., \& Miles, B. M. 1969, NSRDS-NBS (Washington, D.C.: US Department of Commerce), 22

Woosley, S. E., \& Weaver, T. A. 1995, ApJS, 101, 181

Yakovleva, S. A., Barklem, P. S., \& Belyaev, A. K. 2018, MNRAS, 473, 3810

Zatsarinny, O. 2006, Comput. Phys. Commun., 174, 273

Zatsarinny, O., \& Bartschat, K. 2008, Phys. Rev. A, 77, 062701

Zatsarinny, O., \& Tayal, S. S. 2010 Phys. Rev. A, 81, 043423

Zatsarinny, O., \& Bartschat, K. 2013, J. Phys. B, 46, 112001

Zatsarinny, O., Bartschat, K., Fursa, D. V., \& Bray, I. 2016 J. Phys. B, 49, 235701

Zhang, H. W., Butler, K., Gehren, T., Shi, J. R., \& Zhao, G. 2006, A\&A, 453, 723

Zhao, G., Mashonkina, L., Yan, H. L., et al. 2016, ApJ, 833, 225 


\section{Appendix A: Non-LTE corrected abundances}

Table A.1. Non-LTE corrected abundances.

\begin{tabular}{|c|c|c|c|c|c|c|}
\hline Star & $T_{\text {eff }}(\mathrm{K})$ & $\log g\left(g\right.$ in $\left.\mathrm{cm} \mathrm{s}^{-2}\right)$ & {$[\mathrm{Fe} / \mathrm{H}]$} & $\xi\left(\mathrm{km} \mathrm{s}^{-1}\right)$ & $K_{\mathrm{NLTE}}$ & $K_{\mathrm{LTE}}$ \\
\hline $18 \mathrm{Sco}$ & 5818 & 4.46 & 0.05 & 1.00 & 5.14 & 5.48 \\
\hline HIP 56948 & 5793 & 4.47 & 0.02 & 1.00 & 5.10 & 5.44 \\
\hline HIP 102152 & 5724 & 4.36 & -0.01 & 1.00 & 5.08 & 5.44 \\
\hline HD 20630 & 5744 & 4.49 & 0.06 & 1.10 & 5.23 & 5.53 \\
\hline HD 202628 & 5831 & 4.51 & 0.00 & 1.00 & 5.13 & 5.47 \\
\hline HIP 30502 & 5736 & 4.42 & -0.06 & 1.00 & 5.01 & 5.37 \\
\hline HIP 73815 & 5800 & 4.33 & 0.03 & 1.10 & 5.14 & 5.50 \\
\hline HIP 77883 & 5701 & 4.37 & 0.02 & 1.00 & 5.11 & 5.45 \\
\hline HIP 89650 & 5853 & 4.42 & -0.01 & 1.10 & 5.09 & 5.46 \\
\hline HD 9986 & 5827 & 4.44 & 0.09 & 1.00 & 5.17 & 5.51 \\
\hline HD 13531 & 5653 & 4.53 & 0.02 & 1.20 & 5.15 & 5.45 \\
\hline HD 13931 & 5895 & 4.29 & 0.07 & 1.10 & 5.19 & 5.58 \\
\hline HD 32963 & 5768 & 4.37 & 0.09 & 1.00 & 5.20 & 5.53 \\
\hline HD 33636 & 5963 & 4.47 & -0.08 & 1.10 & 5.04 & 5.44 \\
\hline HD 43162 & 5661 & 4.53 & 0.06 & 1.20 & 5.24 & 5.52 \\
\hline HD 45184 & 5873 & 4.41 & 0.07 & 1.00 & 5.18 & 5.54 \\
\hline HD 87359 & 5700 & 4.47 & 0.07 & 0.90 & 5.18 & 5.48 \\
\hline HD 95128 & 5904 & 4.35 & 0.02 & 1.10 & 5.13 & 5.53 \\
\hline HD 98618 & 5845 & 4.42 & 0.05 & 1.10 & 5.12 & 5.48 \\
\hline HD 106252 & 5885 & 4.42 & -0.07 & 1.10 & 5.05 & 5.44 \\
\hline HD 140538 & 5704 & 4.48 & 0.06 & 0.90 & 5.13 & 5.45 \\
\hline HD 143436 & 5825 & 4.43 & 0.04 & 1.00 & 5.14 & 5.49 \\
\hline HD 112257 & 5686 & 4.30 & -0.00 & 0.90 & 5.14 & 5.49 \\
\hline HD 2796 & 4950 & 1.50 & -2.47 & 2.10 & 2.88 & 3.13 \\
\hline HD 122563 & 4600 & 1.10 & -2.82 & 2.00 & 2.36 & 2.75 \\
\hline HD 186478 & 4700 & 1.30 & -2.59 & 2.00 & 2.52 & 3.01 \\
\hline $\mathrm{BD}+173248$ & 5250 & 1.40 & -2.07 & 1.50 & 3.34 & 3.72 \\
\hline BD -18 5550 & 4750 & 1.40 & -3.06 & 1.80 & 2.33 & 2.52 \\
\hline BS 16477-003 & 4900 & 1.70 & -3.36 & 1.80 & 2.04 & 2.19 \\
\hline BS 17569-049 & 4700 & 1.20 & -2.88 & 1.90 & 2.33 & 2.69 \\
\hline CS 22169-035 & 4700 & 1.20 & -3.04 & 2.20 & 2.22 & 2.45 \\
\hline CS 22172-002 & 4800 & 1.30 & -3.86 & 2.20 & 1.60 & 1.76 \\
\hline CS 22873-055 & 4550 & 0.70 & -2.99 & 2.20 & 2.13 & 2.52 \\
\hline CS 22873-166 & 4550 & 0.90 & -2.97 & 2.10 & 2.19 & 2.59 \\
\hline CS 22892-052 & 4850 & 1.60 & -3.03 & 1.90 & 2.32 & 2.48 \\
\hline CS 22896-154 & 5250 & 2.70 & -2.69 & 1.20 & 2.69 & 2.88 \\
\hline CS 22897-008 & 4900 & 1.70 & -3.41 & 2.00 & 2.01 & 2.15 \\
\hline CS 22948-066 & 5100 & 1.80 & -3.14 & 2.00 & 2.23 & 2.39 \\
\hline CS 22949-037 & 4900 & 1.50 & -3.97 & 1.80 & 1.21 & 1.35 \\
\hline CS 22952-015 & 4800 & 1.30 & -3.43 & 2.10 & 1.99 & 2.13 \\
\hline CS 22953-003 & 5100 & 2.30 & -2.84 & 1.70 & 2.38 & 2.55 \\
\hline CS 22956-050 & 4900 & 1.70 & -3.33 & 1.80 & 1.89 & 2.04 \\
\hline CS 22966-057 & 5300 & 2.20 & -2.62 & 1.40 & 2.71 & 2.90 \\
\hline CS 22968-014 & 4850 & 1.70 & -3.56 & 1.90 & 1.66 & 1.82 \\
\hline CS 29495-041 & 4800 & 1.50 & -2.82 & 1.80 & 2.55 & 2.80 \\
\hline CS 29518-051 & 5200 & 2.60 & -2.69 & 1.40 & 2.73 & 2.91 \\
\hline HD 94028 & 5730 & 3.70 & -1.81 & 1.00 & 3.22 & 4.20 \\
\hline HD 175305 & 4920 & 2.30 & -1.56 & 1.40 & 3.39 & 4.27 \\
\hline HD 11582 & 5020 & 2.20 & -2.03 & 1.40 & 3.31 & 4.08 \\
\hline $\mathrm{BD}+292356$ & 4710 & 1.75 & -1.62 & 1.50 & 3.33 & 4.09 \\
\hline HD 201891 & 5840 & 4.10 & -1.21 & 1.10 & 4.00 & 4.72 \\
\hline HD 45282 & 5230 & 2.90 & -1.73 & 1.40 & 3.51 & 4.21 \\
\hline G 161-073 & 5680 & 3.90 & -1.07 & 1.10 & 3.76 & 4.38 \\
\hline $\mathrm{BD}+191185 \mathrm{~A}$ & 5440 & 4.30 & -1.25 & 1.10 & 3.86 & 4.46 \\
\hline HD 126238 & 4750 & 1.65 & -1.96 & 1.60 & 3.09 & 3.65 \\
\hline
\end{tabular}

Notes. Stellar parameters and LTE abundances are from Spina et al. (2016); Cayrel et al. (2004) and Roederer et al. (2014). 
H. Reggiani et al.: Non-LTE analysis of K I in late-type stars

Table A.1. continued.

\begin{tabular}{|c|c|c|c|c|c|c|}
\hline Star & $T_{\text {eff }}(\mathrm{K})$ & $\log g\left(g\right.$ in $\left.\mathrm{cm} \mathrm{s}^{-2}\right)$ & {$[\mathrm{Fe} / \mathrm{H}]$} & $\xi\left(\mathrm{km} \mathrm{s}^{-1}\right)$ & $K_{\mathrm{NLTE}}$ & $\overline{K_{\mathrm{LTE}}}$ \\
\hline G 153-064 & 5630 & 4.15 & -1.09 & 0.90 & 4.16 & 4.70 \\
\hline CS $22873-128$ & 4710 & 1.20 & -3.24 & 1.60 & 2.29 & 2.78 \\
\hline HD 178443 & 5170 & 1.45 & -2.02 & 1.90 & 3.36 & 3.83 \\
\hline BD -15 5781 & 4550 & 0.70 & -2.87 & 1.70 & 2.16 & 2.60 \\
\hline G 126-062 & 5970 & 3.85 & -1.70 & 1.10 & 3.62 & 4.05 \\
\hline HD 31128 & 5630 & 3.60 & -1.92 & 1.10 & 3.37 & 3.76 \\
\hline HD 219617 & 5730 & 3.70 & -1.83 & 1.30 & 3.44 & 3.81 \\
\hline CS 22955-110 & 6710 & 3.45 & -1.39 & 1.90 & 4.14 & 4.48 \\
\hline CS 22185-007 & 4730 & 1.30 & -3.02 & 1.60 & 2.40 & 2.73 \\
\hline CS 22182-047 & 5640 & 3.60 & -1.99 & 1.10 & 3.21 & 3.52 \\
\hline CS 22892-052 & 4690 & 1.15 & -3.16 & 1.50 & 2.07 & 2.37 \\
\hline HD 188510 & 5210 & 4.10 & -1.88 & 0.80 & 3.23 & 3.52 \\
\hline BD -01 2582 & 4920 & 1.80 & -2.65 & 1.50 & 2.76 & 3.05 \\
\hline HD 122196 & 5880 & 3.80 & -1.79 & 1.20 & 3.29 & 3.58 \\
\hline CS 29495-005 & 5990 & 3.75 & -2.26 & 1.10 & 3.32 & 3.60 \\
\hline CS 29513-032 & 6080 & 3.85 & -1.91 & 1.30 & 3.40 & 3.67 \\
\hline G 090-025 & 5150 & 4.05 & -2.04 & 0.90 & 3.17 & 3.44 \\
\hline CS 30492-110 & 4660 & 1.05 & -3.16 & 1.80 & 2.06 & 2.33 \\
\hline CS 29514-017 & 5270 & 2.80 & -2.34 & 1.20 & 2.80 & 3.06 \\
\hline CS 29513-014 & 5440 & 1.55 & -2.32 & 2.00 & 3.17 & 3.42 \\
\hline HD 108317 & 5030 & 2.10 & -2.60 & 1.40 & 2.85 & 3.09 \\
\hline G 090-003 & 5680 & 3.60 & -2.24 & 1.20 & 2.98 & 3.22 \\
\hline G 025-024 & 5670 & 3.55 & -2.28 & 1.70 & 3.10 & 3.34 \\
\hline HD 119516 & 5660 & 1.90 & -1.93 & 1.90 & 3.14 & 3.36 \\
\hline CS 22943-095 & 6140 & 3.80 & -2.44 & 1.40 & 3.16 & 3.38 \\
\hline CS 22881-036 & 5940 & 3.70 & -2.37 & 1.10 & 2.95 & 3.17 \\
\hline HD 19445 & 5820 & 3.65 & -2.40 & 1.20 & 2.98 & 3.20 \\
\hline CS 22968-026 & 5850 & 3.65 & -2.33 & 1.20 & 2.88 & 3.10 \\
\hline HD 13979 & 4830 & 1.60 & -2.72 & 1.60 & 2.49 & 2.70 \\
\hline CS 22958-065 & 6020 & 3.75 & -2.24 & 1.30 & 3.02 & 3.23 \\
\hline CS 22884-020 & 6040 & 3.75 & -2.27 & 1.40 & 3.05 & 3.25 \\
\hline CS 22894-004 & 5920 & 3.65 & -2.65 & 1.50 & 2.90 & 3.10 \\
\hline CS 22945-028 & 4900 & 1.75 & -2.89 & 1.50 & 2.50 & 2.70 \\
\hline CS 22958-074 & 5800 & 3.60 & -2.62 & 1.40 & 2.76 & 2.96 \\
\hline CS 22886-012 & 5650 & 3.50 & -2.61 & 1.40 & 2.71 & 2.92 \\
\hline CS 22885-203 & 5820 & 3.60 & -2.57 & 1.30 & 2.71 & 2.91 \\
\hline CS 22186-002 & 5500 & 3.35 & -2.50 & 1.10 & 2.52 & 2.71 \\
\hline CS 22186-017 & 5770 & 3.55 & -2.90 & 1.30 & 2.60 & 2.79 \\
\hline CS 22942-011 & 4930 & 1.85 & -2.83 & 1.20 & 2.36 & 2.55 \\
\hline CS 29514-018 & 5990 & 3.70 & -2.58 & 1.20 & 2.67 & 2.86 \\
\hline CD -36 1052 & 6030 & 2.05 & -1.86 & 3.30 & 3.53 & 3.72 \\
\hline CS 30339-015 & 5840 & 3.60 & -2.86 & 1.40 & 2.53 & 2.72 \\
\hline CS 22896-015 & 5080 & 2.25 & -2.84 & 1.20 & 2.48 & 2.67 \\
\hline $\mathrm{BD}+241676$ & 6140 & 3.75 & -2.54 & 1.40 & 2.72 & 2.91 \\
\hline HE $0938+0114$ & 6030 & 3.65 & -2.92 & 1.20 & 2.31 & 2.49 \\
\hline CS 22951-059 & 5120 & 2.35 & -2.83 & 1.50 & 2.56 & 2.74 \\
\hline CS 22183-031 & 4850 & 1.60 & -3.50 & 1.60 & 2.30 & 2.47 \\
\hline CS 22956-106 & 6410 & 3.90 & -2.60 & 1.80 & 2.80 & 2.97 \\
\hline CS 22893-010 & 5150 & 2.45 & -2.93 & 1.40 & 2.38 & 2.55 \\
\hline CS 29502-092 & 4820 & 1.50 & -3.20 & 1.50 & 2.06 & 2.22 \\
\hline G $190-015$ & 4950 & 3.85 & -3.13 & 1.50 & 2.17 & 2.31 \\
\hline
\end{tabular}

